$37 \begin{aligned} & \text { Schriften aus der Fakultät Geistes- und Kulturwissenschaften } \\ & \text { der Otto-Friedrich-Universität Bamberg }\end{aligned}$

\title{
Zwischen den Zeilen
}

Eigentumslose Haushalte in fränkischen Verzeichnissen aus vor- und frühstatistischer Zeit

Thomas Wenderoth

72.

conthro Pature?

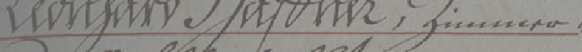

6. Hitrints Dinft, Coglofium,

fit torttr, 32 . Pofor alt,

72. Heter Pitfler, Qailuw jaget.

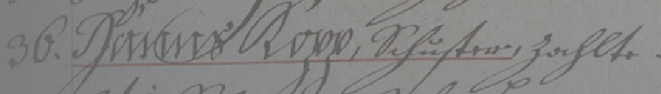

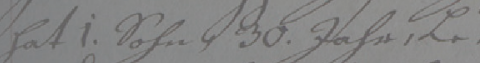

Intwarof fite, binj if

i. Pofu, 20. Tor alst,
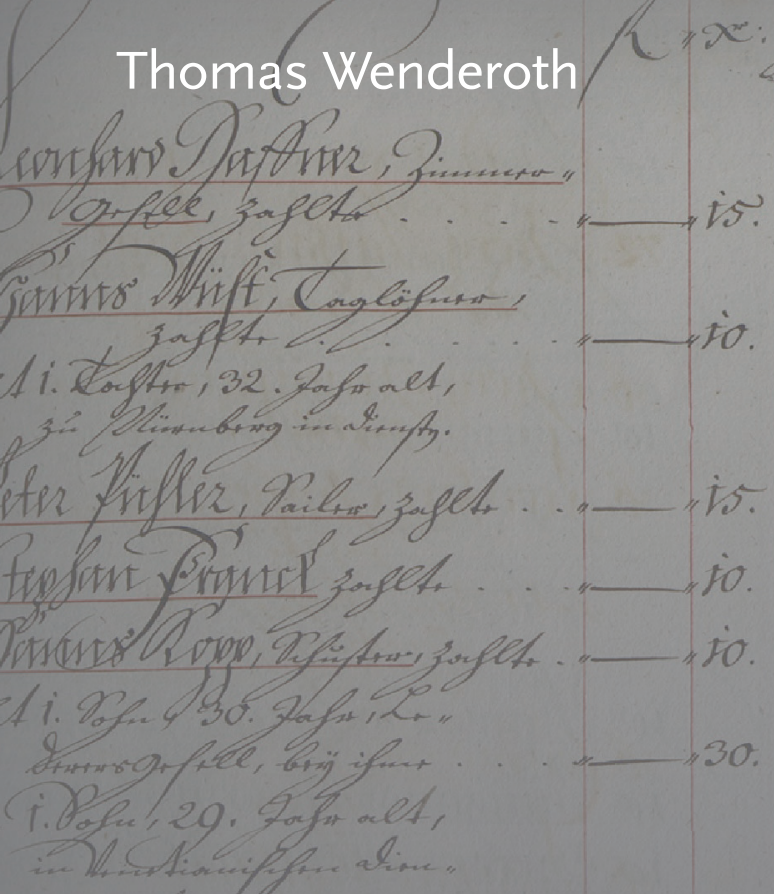

juitugtint

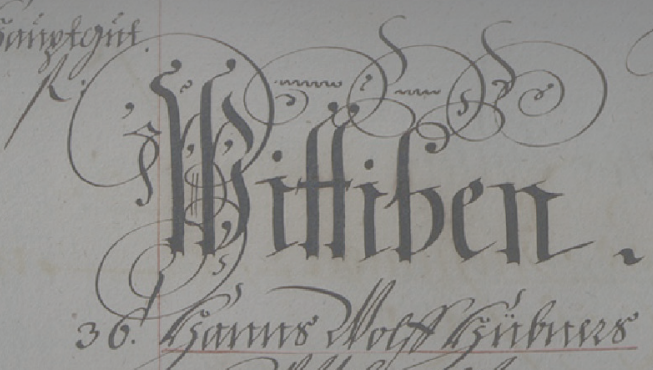

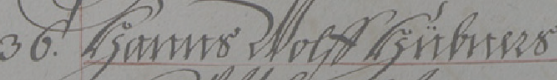

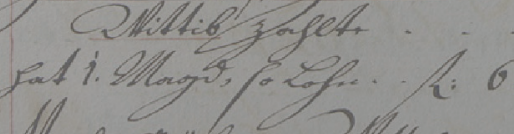

Hol 330 
37 Schriften aus der Fakultät Geistes- und Kulturwissenschaften der Otto-Friedrich-Universität Bamberg 
Schriften aus der Fakultät Geistes- und Kulturwissenschaften der Otto-Friedrich-Universität Bamberg

Band 37 


\section{Zwischen den Zeilen}

Eigentumslose Haushalte in fränkischen Verzeichnissen aus vor- und frühstatistischer Zeit

Thomas Wenderoth

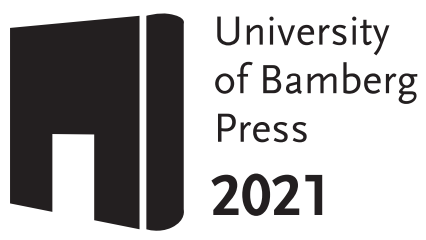


Bibliographische Information der Deutschen Nationalbibliothek

Die Deutsche Nationalbibliothek verzeichnet diese Publikation in der Deutschen Nationalbibliographie; detaillierte bibliographische Informationen sind im Internet über http://dnb.d-nb.de abrufbar.

Dieses Werk ist als freie Onlineversion über das Forschungsinformationssystem (FIS; https://fis.uni-bamberg.de) der Universität Bamberg erreichbar. Das Werk ausgenommen Cover, Zitate und Abbildungen - steht unter der CC-Lizenz CC-BY.

$$
\text { (c) (1) }
$$

Lizenzvertrag: Creative Commons Namensnennung 4.0

https://creativecommons.org/licenses/by/4.0/deed.de

Herstellung und Druck: docupoint Magdeburg Umschlaggestaltung: University of Bamberg Press

Titelbild: Erfassung der „Türkensteuer“ 1716/17 in Altdorf bei Nürnberg, Seite mit Einträgen zu Beständnern (männlichen Mietern) und Wittiben (weiblichen Mietern); StAN, Rst. N, Bauernverzeichnisse 7, fol. 330

University of Bamberg Press, Bamberg 2021

http://www.uni-bamberg.de/ubp

ISSN: $1866-7627$

ISBN: 978-3-86309-843-8 (Druckausgabe)

eISBN: 978-3-86309-844-5 (Online-Ausgabe)

URN: urn:nbn:de:bvb:473-irb-524398

DOI: https://doi.org/10.20378/irb-52439 


\section{Inhaltsverzeichnis}

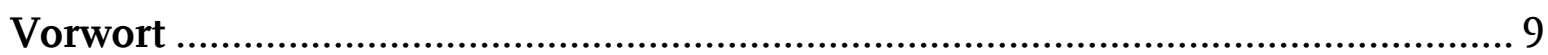

$1 \quad$ Eingrenzung des Themas, Forschungsstand ….......................................... 11

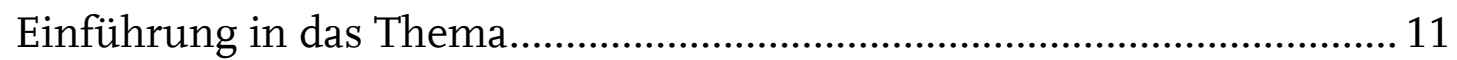

Leben in Gemeinschaft - die räumliche Hierarchie des Wohnens............. 15

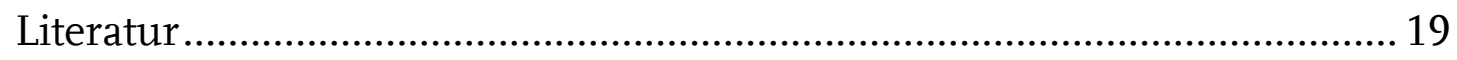

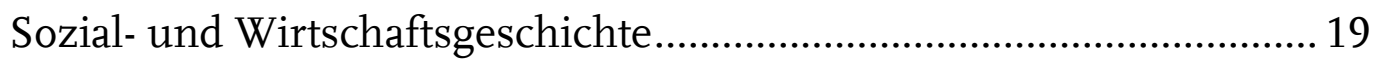

Stadtgeschichte mit einem sozialtopografischen Ansatz ....................... 22

Bevölkerungsgeschichte .......................................................................... 23

Edierte Quellen, Häuserbücher ............................................................. 25

Literatur zu eigentumslosen Einwohnern in fränkischen Städten ....... 27

2 Bezeichnungen der Eigentumslosen in den Quellen ................................. 29

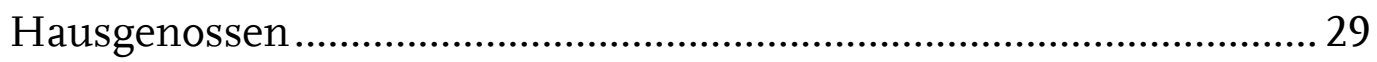

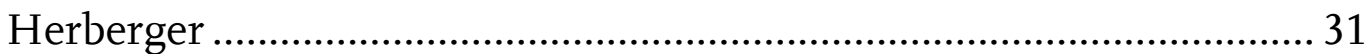

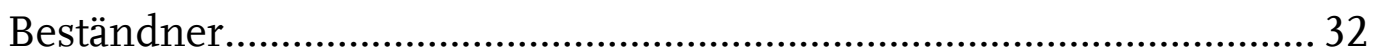

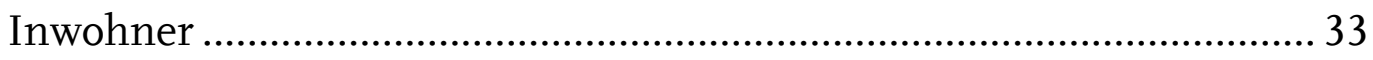

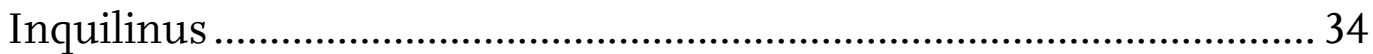

Schutzverwandte, Verspruchleute, Mundleute ……………………....... 34

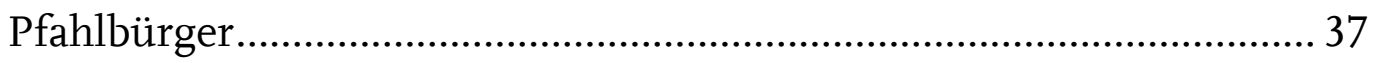

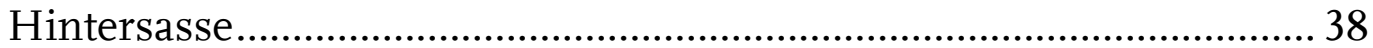

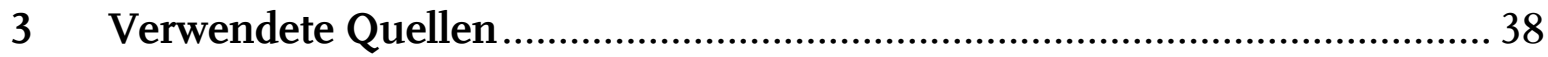

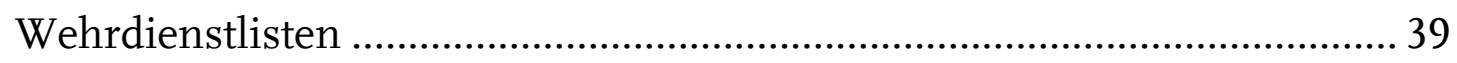

Nürnberger Grabenbuch......................................................................... 39

Verzeichnis der wehrfähigen Bevölkerung Nürnberg 1431 ................... 40

Kopfsteuerlisten........................................................................................ 41

Reichssteuerregister 1497 - Der Gemeine Pfenning.............................. 41

Türkensteuerregister 1716/17............................................................... 45

Quartiergeldbeiträge Nürnberg 1798 .................................................... 47

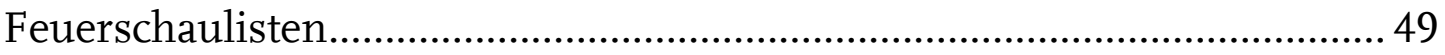

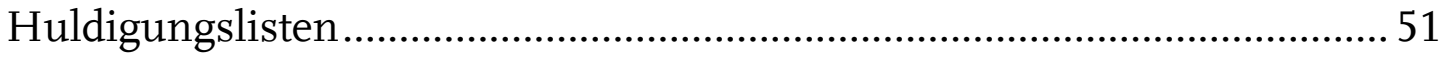

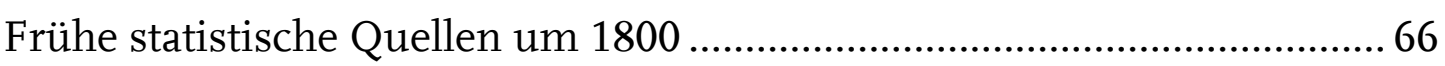




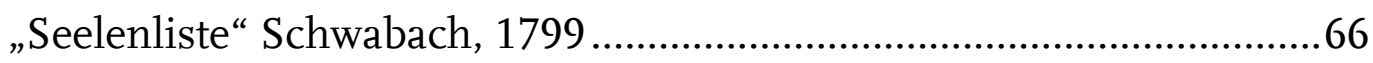

„Verzeichniß aller im Rezatkreise enthaltenen Ortschaften“.................67

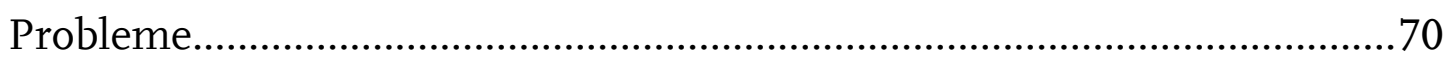

$4 \quad$ Rechtliche Stellung der eigentumslosen Bevölkerung ................................77

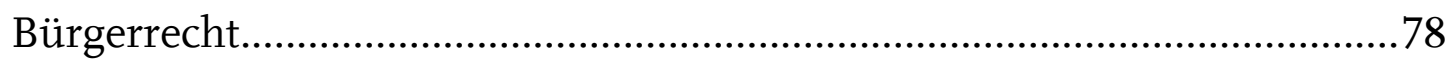

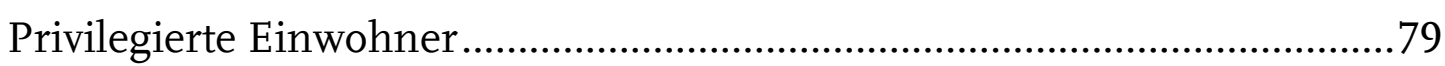

Eingeschränkte bürgerliche Rechte ................................................................... 80

Ohne bürgerliche Rechte: Gäste und Illegalität .............................................85

5 Ökonomische und soziale Stellung eigentumsloser Haushalte ..................89

Unterschicht - Handwerker und Taglöhner ................................................89

Staats-, Kommunal- und Hofbedienstete..........................................................95

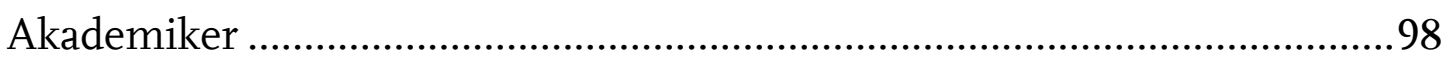

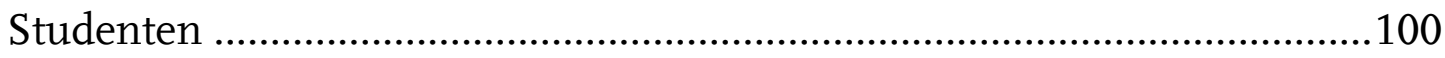

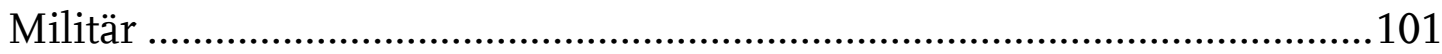

Untermieter, Kostgänger, Bettgeher und Schlafbursche..............................103

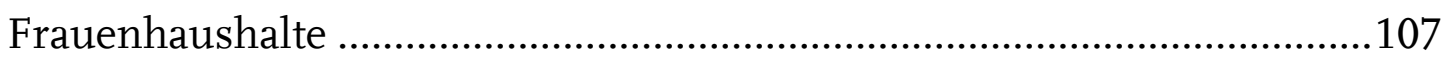

Strukturen eigentumsloser Haushalte am Beispiel Altdorf 1717 ...............115

6 Einfluss der Stadtgröße auf den Anteil eigentumsloser Haushalte...........119

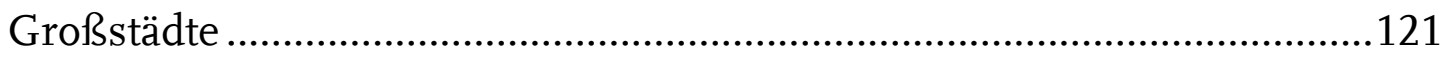

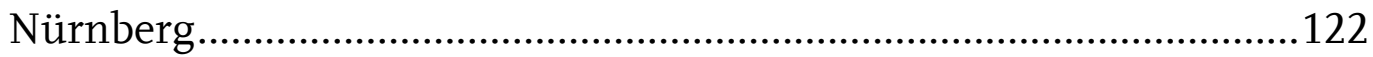

Eigentumslose Haushalte in anderen Großstädten .............................136

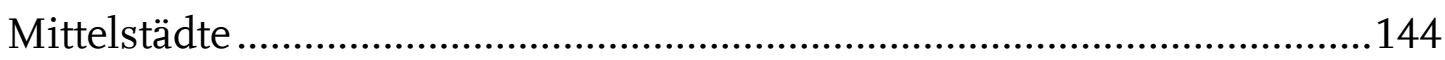

Ehemalige fränkische Reichsstädte ......................................................144

Hohenzollerische Städte in Mittelfranken ...........................................146

Mittelstädte außerhalb Frankens .....................................................149

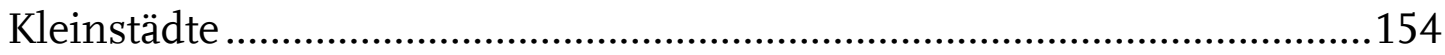

Mieteranteil in fränkischen Kleinstädten 1497 ......................................155

Mieteranteil in fränkischen Kleinstädten der Barockzeit........................162

Mieteranteil in fränkischen Landstädten um 1800...............................175

Entwicklung zwischen 1497 und 1700 am Beispiel Erlangens............182

Zusammenfassung: Bevölkerungsstruktur fränkischer Kleinstädte...185

Kleinstädte außerhalb Frankens .......................................................187 
7 Einfluss der Stadtfunktion auf den Anteil eigentumsloser Haushalte ..... 191

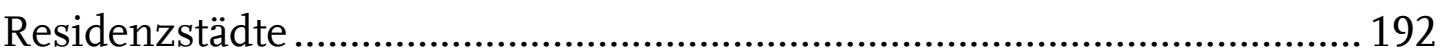

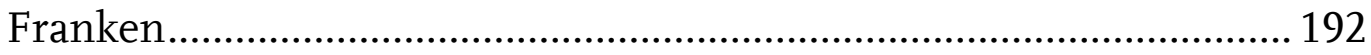

Residenzstädte außerhalb Frankens .................................................. 198

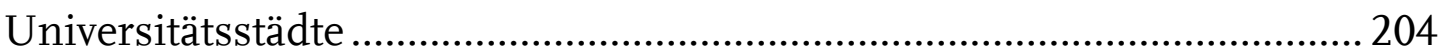

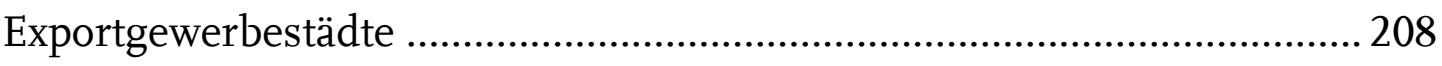

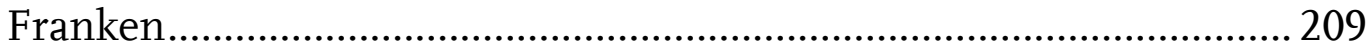

Vergleich zwischen Exportgewerbe- und Ackerbürgerstädten............. 225

Exportgewerbestädte in Kursachsen und Westfalen ............................. 226

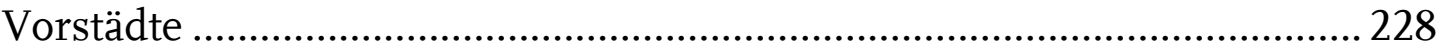

8 Resümee

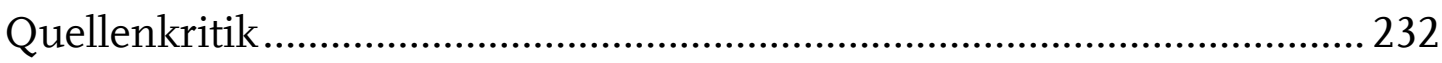

Bevölkerungsgeschichtliche Ergebnisse ..................................................... 232

Differenzierung der Städte .......................................................................... 234

Sozialgeschichtliche Ergebnisse............................................................... 237

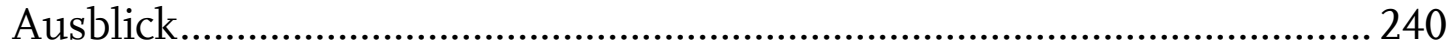

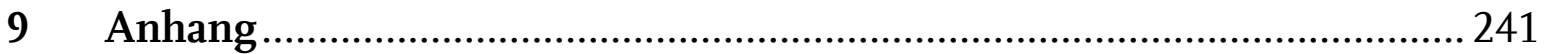

Unveröffentlichte Quellen ....................................................................... 241

Gedruckte Quellen ........................................................................................ 243

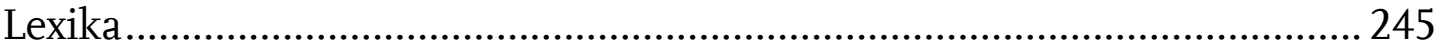

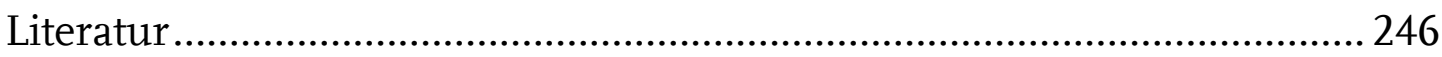

Abkürzungen .............................................................................................. 264

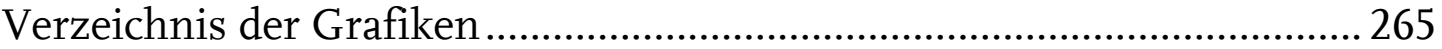

Verzeichnis der Tabellen und Tabellenanhang …………............................ 265

Karte zu den fränkischen Städten ................................................................. 275 



\section{Vorwort}

In dieser Publikation geht es um die Sichtbarmachung der „Unsichtbaren“. Da die eigentumslose Bevölkerung aus verschiedenen Gründen, die weiter unten dargelegt werden, in vielen historischen Bevölkerungsregistern nicht verzeichnet ist, bleiben diese Menschen zumeist auch in der Forschung und in unserem Bild von den Lebensumständen früherer Generationen unsichtbar.

Im Rahmen meines Habilitationsvorhabens an der Bauhaus-Universität Weimar zum Mietwohnen in der Stadt vor 1800 habe ich mich umfassend mit dem quantitativen Anteil eigentumsloser Menschen auseinandergesetzt. ${ }^{1}$ Ich musste feststellen, dass zu dieser Fragestellung bisher keine ausreichenden Untersuchungen vorliegen, auf die ich meine bau- und architekturgeschichtlichen Forschungen hätte stützen können. Ohne das Vorhandensein von eigentumslosen Haushalten lässt sich die Entstehung und Entwicklung des Mietwohnungsbaus nicht erklären. Dieses Desiderat hat mich motiviert, die eigentumslose Bevölkerung früherer Jahrhunderte sichtbar zu machen. Dabei musste ich feststellen, dass sich eigentumslose Menschen auch in den historischen Listen häufig nur beim Lesen zwischen den Zeilen finden lassen, sie tatsächlich auch beim ersten Blick in die Archivalien unsichtbar bleiben.

Da es sich bei meinen prosopographischen Forschungen zur eigentumslosen Bevölkerung um ein in sich abgeschlossenes Thema handelt, habe ich mich entschlossen diesen Teilbereich meiner Habilitationsschrift separat zu veröffentlichen. Eine Publikation der architekturbezogenen Forschungsergebnisse ist ebenfalls in Vorbereitung. Dort werden auch weitere sozial- und wirtschaftsgeschichtliche Forschungsergebnisse zu den Mietern einfließen. Die beiden Publikationen ergänzen sich inhaltlich. Für einen umfassenden Einblick in das Thema des Mietwohnens möchte ich zudem auf meine Dissertationsschrift „Mietshaus und Mietwohnung auf dem Land. Verbreitung, Entwicklung und Typologie am Beispiel des Nürnberger Umlandes 1500-1800“, erschienen in der Schriftenreihe des Bayerischen Landesamtes für Denkmalpflege, hinweisen. ${ }^{2}$ In der nun vorliegenden Abhandlung habe ich mich im Wesentlichen auf die statistischen Aspekte konzentriert. Das ist ein zwar eher mühsamer Stoff für den Leser, gleichwohl denke ich, dass sich beim Lesen der ein oder andere Aha-Moment einstellt und für die Mitverfolgung umfangreicher Zahlendiskussionen entschädigt.

\footnotetext{
${ }^{1}$ WENDEROTH 2021.

${ }^{2}$ WENDEROTH 2019a.
} 
Da diese Pubilaktion auch online verfügbar ist, habe ich im Hinblick auf die erweiterten Suchfunktionen auf die Erstellung eines Orts- und Sachregisters verzichtet.

Dankbar bin ich Prof. Dr. Hans-Rudolph Meier, dass er das Habilitationsvorhaben unterstützt hat, sowie meinen Dienstvorgesetzten Prof. Dr. Rainer Drewello und Prof. Dr. Paul Bellendorf, die mir den nötigen wissenschaftlichen und zeitlichen Freiraum gewährten, dieses Thema umfassend zu bearbeiten.

Für Hinweise sowie die unermüdliche Bereitstellung von Archivalien gilt mein besonderer Dank den Mitarbeitern sowohl des Staatsarchivs Nürnberg als auch des Stadtarchivs Nürnberg. Für die Mühe des Korrekturlesens möchte ich mich ganz herzlich bei Hanne Opoku-Pare, Robert Zöllner und Dr. Christine van Eickels bedanken. An erster Stelle muss hier unbedingt meine liebe Ehefrau, Dipl.Ing. Anna Wenderoth, genannt werden, die immer ein offenes Ohr hatte und mich einmal mehr bei der Erstellung des Manuskripts unterstützt hat.

Schließen möchte ich mit einem Liedvers von Martin Schalling aus dem Jahr 1571: „Es ist ja, Herr, dein G'schenk und Gab / mein Leib und Seel und was ich hab / in diesem armen Leben. / Damit ich's brauch zum Lobe dein, zu Nutz und Dienst des Nächsten mein, / wollst mir dein Gnade geben. “"

\footnotetext{
${ }^{3}$ Evangelisches Gesangbuch, Ausgabe für die Evangelisch-Lutherischen Kirchen in Bayern und Thüringen, 1994, Nr. 397, Vers 2.
} 


\section{Eingrenzung des Themas, Forschungsstand}

\section{Einführung in das Thema}

In der historischen Forschung ist die eigentumslose Bevölkerung bisher häufig unberücksichtigt geblieben. Dies gilt im besonderen Maß für die Zeit der Vormoderne. Um hier Licht ins Dunkel zu bringen, bin ich der Frage nachgegangen, inwiefern konkrete Aussagen zum Anteil der eigentumslosen Haushalte vor 1800 möglich sind und welche historischen Quellen zu Hilfe genommen werden können. Im Grunde handelt es sich um eine statistische Fragestellung zu einer Epoche, die keine präzisen statistischen Erhebungen in unserem heutigen Sinn durchgeführt hat. Zur Annäherung an diese Fragestellung bediene ich mich daher verschiedener Personenverzeichnisse aus vor- und frühstatistischer Zeit. Mit der Aufklärung im 18. Jahrhundert ist eine zunehmend präzisere Bevölkerungserfassung zu beobachten, das späte 18. Jahrhundert kann daher schon als ein frühstatistischer Zeitabschnitt aufgefasst werden. Im Fokus meiner Betrachtungen stehen serielle Quellen, darunter verstehe ich Personenverzeichnisse, die im Minimum einen definierten Bevölkerungsteil an einem Ort zu einem festen Zeitpunkt erfassen. Deutlich aussagekräftiger im Hinblick auf eine angestrebte Verallgemeinerung der Ergebnisse sind jedoch serielle Quellen, die möglichst viele Bevölkerungsgruppen an möglichst vielen Orten zu verschiedenen Zeitpunkten erfassen. Letztere bilden das Rückgrat meiner Untersuchung, sie werden gelegentlich durch singuläre Verzeichnisse, die nur die von mir definierte Minimalanforderung an eine serielle Quelle erfüllen, ergänzt.

Die Vernachlässigung der eigentumslosen Bevölkerung in der historischen Forschung hat nicht nur den Blick auf das Phänomen des frühen Mietwohnens verstellt, sondern führt generell zu einer unvollständigen und zuweilen auch verklärenden Sicht auf die Lebenswirklichkeit in vorausgehenden Jahrhunderten. Insbesondere erscheint mir eine prosopographische Forschung nur möglich, wenn neben den Eigentümern auch die eigentumslosen Einwohner Berücksichtigung finden. Mit ursächlich für die rudimentäre Forschung ist die eingeschränkte Quellenlage, denn viele prosopographische Quellen nennen nur die Eigentümer. In anderen Quellen muss man zwischen den Zeilen lesen lernen, um die eigentumslosen Einwohner zu fassen. Denn bei einem genaueren Blick in die Archivalien finden sich doch immer wieder Hinweise und serielle Quellen, die bei entsprechender Interpretation wertvolle Erkenntnisse zum Anteil der eigentumslosen Bevölkerung ermöglichen. Das Wissen um diese Bevölkerungsgruppe hilft auch dort, wo entsprechend aussagefähige Quellen bisher nicht erschlossen worden sind oder tatsächlich fehlen: Zum einen wird damit die Begrenztheit von 
überlieferten Einwohnerzahlen, die in der Regel lediglich auf Eigentümerverzeichnissen basieren, deutlich, zum anderen kann dies Ansporn sein, nach weiteren, bisher nicht gehobenen Quellen zu forschen. Diese Arbeit möchte ich als Anregung an den Leser verstanden wissen, sich weiter mit dem Thema zu beschäftigen. Die Rekonstruktion historischer Einwohnerzahlen kann von einer differenzierteren Betrachtungsweise nur profitieren.

Im Zentrum dieser Arbeit steht die eigentumslose Bevölkerung in fränkischen Städten, soweit ich sie anhand von überlieferten Verzeichnissen ermitteln konnte. Der Begriff der Eigentumslosigkeit bezieht sich im Folgenden konkret auf Wohneigentum. Das Leben im fremden Eigentum bedeutet nicht automatisch absolute Besitzlosigkeit, in Einzelfällen konnte es sogar vorkommen, dass ein Einwohner, der selbst in fremdem Eigentum zur Miete lebte, an anderer Stelle Wohneigentum besaß. Nachdem dies aber eine absolute Ausnahme darstellt, werden auch solche wohlhabenden Einwohner im Folgenden mit unter dem Begriff der Eigentumslosigkeit behandelt.

Grundsätzlich kann die eigentumslose Bevölkerung in sechs Gruppen untergliedert werden: Die erste und zahlenmäßig größte Gruppe bilden die Mieterhaushalte samt den Untermietern. Die zweite Gruppe besteht aus den Bewohnern von Dienstwohnungen, also den Beamten und öffentlich Bediensteten. Eine weitere, dritte Gruppe setzt sich aus dem geistlichen Stand zusammen, wobei Gemeindepfarrer in den historischen Listen häufig mit in der Gruppe der Beamten genannt sind. In diese Gruppe gehören auch die klösterlichen Gemeinschaften. Da Ordensgemeinschaften zum einen in den von mir ausgewerteten Verzeichnissen nicht erfasst sind und zum anderen die untersuchten Städte weit überwiegend protestantisch waren, werde ich auf diese Personengruppe nicht weiter eingehen. Eine vierte Gruppe bilden die kasernierten Soldaten, diese sind in den regulären Verzeichnissen zumeist ebenfalls nicht erfasst. In einer fünften Gruppe finden sich die Dienstboten, Lehrjungen und Gesellen, die mit im Haushalt ihres Arbeitgebers leben und, wenn überhaupt, dort miterfasst sind. In vielen Fällen handelt es sich um eine zeitlich befristete Lebensform im Anschluss an die Kindheit, die mit der Heirat und Gründung eines eigenen Hausstandes endet. Die sechste Gruppe ist in den historischen Quellen rein quantitativ kaum fassbar: Es handelt sich um nichtsesshafte Menschen, die entweder obdachlos waren oder zum fahrenden Volk gehörten. Die letzte Gruppe ist in den von mir ausgewerteten Listen nicht notiert; diese Menschen bleiben ebenfalls unberücksichtigt.

Anhand dieser Auflistung wird deutlich, welche Vielfalt an Lebensformen sich im Zusammenhang mit Eigentumslosigkeit gebildet hat. Für Menschen mit Wohneigentum stellte dagegen in der Regel die Familie die übliche Lebensform dar, der Verheiratung konnte der Witwenstand folgen. Im fortgeschrittenen Alter resultiert aus dem Witwenstand jedoch häufig die Übertragung des Wohneigentums 
an die jüngere Generation und damit der (Wieder-)Eintritt in die Gruppe der eigentumslosen Bevölkerung.

Da in den historischen Verzeichnissen in aller Regel nur klassische Haushalte erfasst sind, finden sich nur ganz selten Hinweise zu den Menschen aus den letzten vier Gruppen. Aber auch die den beiden erstgenannten Gruppen angehörenden Mieterhaushalte, Untermieterhaushalte und Beamtenhaushalte sind nicht immer vollständig erfasst worden. Daraus folgt, dass die von mir ermittelten Zahlen zwangsläufig unvollständig sind. In der überwiegenden Zahl der Städte ohne Klöster und Kasernen werden die Ergebnisse dennoch ein recht genaues Bild vom Anteil der eigentumslosen Haushalte an der Gesamtbevölkerung zeichnen. Um präzisere Ergebnisse zu erzielen, muss die individuelle Überlieferung der jeweiligen Stadt umfassend berücksichtigt werden, was wiederum eine flächenhafte Untersuchung und Vergleichbarkeit der Städte untereinander erschwert, wenn nicht gar vereitelt.

Eben aus diesem Grund habe ich mich bei meiner Forschung auf die genannten seriellen Quellen konzentriert und beziehe nur gelegentlich individuelle Archivalien mit in die Analyse ein.

Der Untersuchungszeitraum erstreckt sich vom 15. Jahrhundert bis ins frühe 19. Jahrhundert. Der Schwerpunkt liegt auf der Zeit nach dem Dreißigjährigen Krieg, da mit den Huldigungslisten nunmehr flächenhafte und serielle Quellen überliefert sind. Die Datenbasis zu flächenhaften Bevölkerungszahlen für das ausgehenden Mittelalter muss generell als gering gelten. Einen Glücksfall stellen für die Forschung daher die Kopfsteuerlisten zur Erhebung des gemeinen Pfennings von 1497 dar, die sich zumindest für die beiden hohenzollerischen Territorien oberhalb und unterhalb Gebürgs weitgehend erhalten haben. Auch in diesen Listen ist jedoch nur eine Teilgruppe der eigentumslosen Bevölkerung erfasst. Für das Gebiet unterhalb Gebürgs, dem späteren Markgraftum BrandenburgAnsbach, wurden als nachfolgende Quelle die barockzeitlichen Huldigungslisten von mir bearbeitet. Der Untersuchungszeitraum schließt ab mit der Auswertung des Alphabetischen Verzeichnisses für den Rezatkreis von 1818, das ebenfalls flächenhaft Daten überliefert.

Darüber hinaus werden, um das Bild abzurunden, für einige Städte weitere Quellen zu Rate gezogen. Hier ist zuallererst Nürnberg als die fränkische Metropole zu nennen. Für Nürnberg hat sich das Reichssteuerregister von 1497 nur unvollständig erhalten. In der Barockzeit erfolgte keine Huldigung der reichsstädtischen Bevölkerung, damit fehlen auch die entsprechenden Listen. Das Gleiche gilt für die nürnbergischen Landstädte. Hier kann als Ersatz für die Huldigungslisten immerhin ein Kopfsteuerverzeichnis aus den Jahren 1717/18 zu Hilfe genommen werden und damit ein Vergleich zwischen den hohenzollerisch-ansbachischen und den nürnbergischen Städten erfolgen. 
Der geographische Schwerpunkt der Untersuchung liegt im heutigen Regierungsbezirk Mittelfranken. Die Kerngruppe der untersuchten Orte bilden die Städte des ehemaligen Fürstentums Brandenburg-Ansbach, wie sie in den Huldigungslisten überliefert sind. Da die herangezogenen Verzeichnisse die sich im Lauf der Jahrhunderte verändernde territoriale Ordnung widerspiegeln, werden phasenweise auch weitere fränkische Städte außerhalb dieser Kerngruppe mit betrachtet. Die Überlieferung des Reichssteuerregisters enthält zum Beispiel neben den Städten des späteren Fürstentums Brandenburg-Ansbach (unterhalb Gebürgs) auch die Städte des Fürstentums Brandenburg-Bayreuth (oberhalb Gebürgs). Um die Aussagefähigkeit der Quelle nicht zu schmälern, habe ich beschlossen sämtliche Städte auszuwerten. Das Alphabetische Verzeichnis des Rezatkreises deckt weitestgehend das Gebiet des heutigen Regierungsbezirks Mittelfranken ab. Nach der Einverleibung Frankens durch Bayern und der damit verbundenen territorialen Neugliederung wurden alle kleineren Herrschaften mediatisiert und aus den diversen, zumeist nicht in sich abgeschlossenen, Territorien 1806 ein zusammenhängender Bezirk gebildet. Dieser umfasst als größte ehemalige Herrschaftsgebiete das Fürstentum Ansbach, das Territorium der Reichsstadt Nürnberg und das Hochstift Eichstätt sowie weitere kleine Reichsstädte, Grafschaften, Reichsritterschaften etc. Die vergrößerte Städtezahl bietet damit automatisch auch Vergleichsmöglichkeiten zwischen den Städten des Fürstentums Ansbach und den Städten in den ehemaligen Nachbarterritorien.

Zum allergrößten Teil wurden Kleinstädte untersucht, da insbesondere die eigentumslose Bevölkerung von Kleinstädten bisher wenig beachtet worden ist und sich daher in der Literatur nur vereinzelt Hinweise finden lassen.

Ziel ist es, anhand der untersuchten Städte nach Möglichkeit zu generalisierenden Aussagen zu gelangen. Dabei bin ich mir des Paradoxons bewusst, dass zugleich jede Stadt eine individuelle Bevölkerungsgeschichte aufweist, die eine Generalisierung eigentlich unmöglich macht. Neben dem Problem der individuellen Stadtgenese tritt noch erschwerend das Thema der individuellen Überlieferungsgeschichte hinzu. Der erhaltene und für die Analyse nutzbare Quellenbestand weicht von Ort zu Ort hinsichtlich Quantität und Qualität erheblich ab. Von daher wird es bei den Thesen nur um die großen Linien gehen können. Wichtig erscheint es mir aus diesem Grund, immer wieder den Vergleich zu Forschungsergebnissen von Städten aus dem gesamten deutschsprachigen Raum zu suchen. Da auch über Franken hinaus bisher keine flächenhaften Forschungsergebnisse zur eigentumslosen Bevölkerung im deutschsprachigen Raum vorliegen, können auch diese punktuellen Vergleiche nur einzelne Schlaglichter auf die tatsächliche Situation werfen. 


\section{Leben in Gemeinschaft - die räumliche Hierarchie des Wohnens}

In dieser Arbeit wird ein Zeitraum untersucht, der sich vom späten Mittelalter über die gesamte frühe Neuzeit erstreckt. Innerhalb dieser etwa 500 Jahre gab es große gesellschaftliche Umwälzungen, die auch die Lebensform der eigentumslosen Bevölkerung verändert haben. Und doch muss festgestellt werden, dass sich die nachfolgend geschilderte Abstufung, die auch als eine Hierarchie des Wohnens definiert werden kann, im Grundsatz über den gesamten Untersuchungszeitraum erhalten hat. Im folgenden Abschnitt geht es daher nicht um die Frage der politischen und rechtlichen Gliederung der Einwohner, sondern primär um deren räumliche Verortung in dem Wissen, dass sich die verschiedenen Lebensbereiche überschneiden. Diese Arbeit kann und will keine sozialgeschichtlichen Forschungen ersetzen. Eine Anregung zur Diskussion über enge Fächergrenzen hinweg wäre jedoch wünschenswert.

Was ist gemeint, wenn ich von einer Hierarchie des Wohnens spreche? Eine größere Gemeinschaft von Menschen, wie sie sich in einer Stadt noch heute findet und von jeher fand, lebt in räumlichen Strukturen. Die gemeinsame Einheit, der alle angehören, ist im Falle dieser Arbeit die Stadt selbst, sie steht an oberster Stelle. Die Städte wiederum gliedern sich in Viertel. Bei größeren Städten bedurfte es einer weiteren Untergliederung in Straßenabschnitte, die zum Beispiel in Nürnberg als Gassenhauptmannschaften bezeichnet wurden.

Erst jetzt kommen wir in der nächsten Hierarchiestufe zum Einzelanwesen, einer in der Regel besitzrechtlichen Einheit. Ein Anwesen wiederum konnte mit einem oder mehreren Häusern bebaut sein. In den historischen Quellen wird jedoch in der Regel begrifflich nicht klar unterschieden zwischen Anwesen und Haus. Erst Begriffe wie Hinterhaus oder Nebenhaus verdeutlichen, dass auf einem Anwesen mehrere Wohngebäude vorhanden waren. Häufig ist diese Frage jedoch nicht abschließend zu beantworten.

Die dem Haus nachfolgende räumliche Einheit stellen die Räume der Herdgemeinschaft dar, denn in einem Haus kann es mehrere Herdstellen gegeben haben, denen jeweils unterschiedliche Hausbewohner zugeordnet waren. Im weitesten Sinn könnte man diese Bezeichnung mit dem modernen Begriff der Wohnung gleichsetzen. Eine räumliche Abgeschlossenheit zwischen den einzelnen Herdgemeinschaften war jedoch nicht immer vorhanden. 

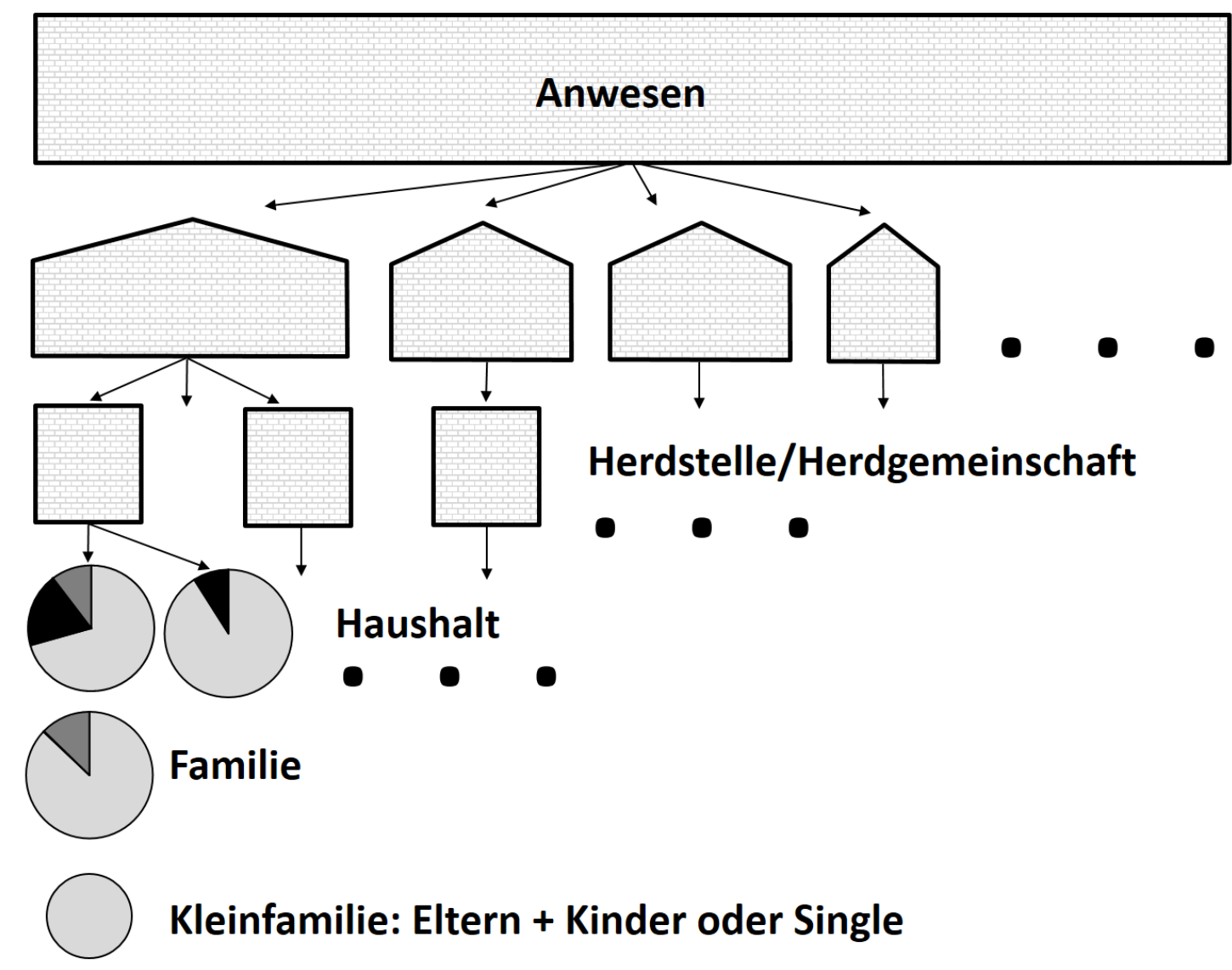

Grafik 1. Die räumliche Hierarchie des Wohnens: hellgrau = Kernfamilie; mittelgrau = Großeltern, Geschwister etc.; schwarz = Gesinde (Thomas Wenderoth 2019)

Ich trenne im Folgenden bewusst zwischen den Begriffen Herdgemeinschaft und Haushalt und ordne zudem den Haushalt als Teileinheit der Herdgemeinschaft unter. Eine Herdgemeinschaft konnte sich aus mehreren der nachfolgend geschilderten Haushalte zusammensetzen, wie Christian Forneck es für Regensburg eindrücklich nachweist. ${ }^{4}$ Einer Herdgemeinschaft stand, wenn überhaupt, lediglich ein Eigentümer vor. Im Falle von Herdgemeinschaften, die sich nur aus Miethaushalten zusammensetzten, konnte bisher nicht abschließend geklärt werden, ob es sich dabei um jeweils einen Hauptmieter mit einem oder mehreren Untermietern oder um gleichberechtigte Miethaushalte handelte.

Als Haushalt möchte ich das Zusammenleben von wirtschaftlich selbstständigen Menschen sowie deren Kindern bezeichnen, die über den gemeinsam genutzten Wohnraum hinaus eine familiäre und/oder ökonomische Einheit bildeten. Ein Haushalt inkludiert also nach dieser Definition sowohl alle Mitglieder einer Familie, die zusammenwohnten, als auch die zugehörigen Mägde, Knechte, Lehrjungen und unverheirateten Gesellen, während verheiratetes Gesinde als eigenständiger Haushalt aufzufassen ist. Im umgekehrten Fall konnte ein Haushalt

\footnotetext{
${ }^{4}$ Vgl. Tabelle 11, Regensburg.
} 
jedoch auch nur eine alleinstehende Person beinhalten. In den Quellen ist in diesen Fällen meist nicht ersichtlich, inwieweit solche Einpersonenhaushalte durch minderjährige Kinder oder Gesinde erweitert waren. Die große Anzahl von armen Witwenhaushalten lässt jedoch den Schluss zu, dass Einpersonenhaushalte auch im eigentlichen Wortsinn einen nennenswerten Anteil unter den Haushalten einnahmen.

Die größte Schwierigkeit bei der Interpretation der Quellen stellt dabei die Unterscheidung zwischen Herdgemeinschaft und Haushalt dar, wenn die in den Quellen genannten Einheiten mehr als eine Person aufweisen, da Untermieter nicht in jedem Fall separat erfasst worden sind. Ich beschränke mich in meiner Untersuchung auf den Haushalt als kleinste Einheit. Eine weitere Differenzierung in Kernfamilie und eigentumslose Haushaltsmitglieder wie Gesinde und Verwandte unterbleibt in der nachfolgenden Analyse.

Die Haupteinheit der Haushalte wiederum sind die Familien als ein Verwandtschaftsverbund. Die Familien unterscheiden sich gegenüber dem Oberbegriff des Haushaltes durch das Fehlen von Gesinde. Der historische Familienbegriff entspricht damit nicht zwangsläufig den heutigen Vorstellungen von Familie. Zur Familie, die in einem Haushalt zusammenlebte, konnten zu den Eltern und Kindern noch Großeltern, Tanten, Onkel, Cousinen, Cousins, Nichten, Neffen etc. hinzukommen. Bei den vielen genannten Möglichkeiten des familiären Zusammenlebens im Untersuchungszeitraum sollte jedoch nicht außer Acht gelassen werden, dass auch damals schon das Zusammenleben als Kern- oder Kleinfamilie nach heutigem Verständnis - bestehend aus Eltern und Kindern - insbesondere bei den eigentumslosen Haushalten weit verbreitet gewesen ist. ${ }^{5}$ Die Kleinfamilie stellt historisch betrachtet jedoch nur die Grundeinheit der größeren Familie dar.

Dass es sowohl in den Quellen als auch in der Literatur zum synonymen Gebrauch und damit zur Vermischung und Verwechslung dieser Begriffe gekommen ist, liegt auf der Hand: Denn in nicht wenigen Fällen wurde ein Anwesen von einer Kleinfamilie allein bewohnt, und das durchaus in der Form der Miete. Eine solche Kleinfamilie war also zugleich die Familie an sich und bildete zudem den Haushalt und die Herdgemeinschaft, die allein für sich in einem Haus auf einem Anwesen lebte. Die Gleichsetzung Anwesen = Kleinfamilie ist also in diesem Fall berechtigt. Das Leben in Gemeinschaft außerhalb der Kleinfamilie begann dann erst auf der Ebene der Gassenhauptmannschaft und des Viertels. In einem solchen Fall ergibt sich folgende Begriffskette: Kleinfamilie $=$ Familie $=$ Haushalt $=$ Herdgemeinschaft $=$ Haus $=$ Anwesen .

Probleme im Verständnis und mitunter ein falsches Geschichtsbild entstehen erst dann, wenn eine Verallgemeinerung erfolgt und die Bewohner der Anwesen

${ }^{5}$ WENDEROTH 2019a, S. 65f. 
zum Beispiel von vornherein als Kleinfamilie charakterisiert werden. Auf diese Weise geht der Blick auf die vielfältigen und hierarchisch abgestuften Formen des Zusammenwohnens verloren. Mit der Einheit des Einzelanwesens und der Differenzierung in sozial abgestufte Verbände beginnt häufig die begriffliche Verwirrung. Zum Verständnis der räumlichen Form des Wohnens erscheint mir die vorgeschlagene Gliederung aber hilfreich. Dies gilt insbesondere im Hinblick auf etwaiges Eigentum, denn ab der Einheit eines Anwesens ist die Frage zu stellen, ob und wenn ja welche Bewohner zur Miete oder im Eigentum wohnten.

Die Beachtung dieser Hierarchie ist auch wichtig, wenn im Folgenden Quellen statistisch ausgewertet werden. So kann nicht ohne Weiteres die Anzahl der Anwesen mit der Anzahl der Häuser gleichgesetzt werden, das Gleiche gilt für die Anzahl der Herdstellen und die Anzahl der Haushalte. Aufgrund von unpräzisen Angaben und der synonymen Verwendung von Begriffen in den Quellen sind auch bei den von mir ausgewerteten Archivunterlagen Verwechslungen in Einzelfällen nicht auszuschließen.

Diese Arbeit konnte daher nur mit dem Mut zur Lücke entstehen. Eine kritische Würdigung der Quellen scheint insbesondere dann geboten, wenn die Wohnund Lebensumstände der untersuchten Bevölkerungsgruppen noch einmal vertieft aus sozial- und wirtschaftsgeschichtlicher Sicht erforscht werden sollen.

Das Thema der (unzureichenden) Quelleninterpretation und der Verwechslung von Begriffen ist noch immanenter bei den statistischen Angaben, die von mir aus der Literatur übernommen worden sind, da zu den an sich schon zumeist indifferenten Quellen noch unterschiedliche Interpretationsmöglichkeiten der Autoren hinzukommen. Eine ausführliche Analyse der von den Autoren diskutierten Quellen und ihrer Rezeption wäre zwar wünschenswert, hätte aber den Rahmen dieser Arbeit gesprengt und muss zukünftigen Forschungen anheimgestellt werden. Vorsorglich muss deshalb darauf hingewiesen werden, dass eine direkte Vergleichbarkeit von in der Literatur genannten bevölkerungsstatistischen Angaben in der Regel nicht gegeben ist. Wenn ich dennoch Zahlen zu eigentumslosen Einwohnern und deren prozentualem Anteil an der Bevölkerung zitiere, sind diese Angaben als Schlaglichter zu verstehen, die eine Tendenz aufzeigen. In der Regel dürfte der Mieteranteil höher gelegen haben, da in den historischen Erfassungen die Eigentümer immer genannt werden, jedoch Mieter oder Mietergruppen fehlen können.

Skepsis scheint auch geboten, wenn in einigen Untersuchungen mit durchschnittlichen Haushaltsstärken von weit über vier Personen operiert wird. Die fränkischen Zahlen vom ausgehenden Mittelalter bis zum Ende der frühen Neuzeit zeigen eindrücklich, dass die Haushalte, durchschnittlich betrachtet, wenig Gesinde und wenig Kinder hatten, die älter als zwölf oder fünfzehn Jahre waren. Auch Familienangehörige außerhalb der Kleinfamilie stellten nicht die Regel dar, 
dies gilt insbesondere für die Großelterngeneration. Bei hohen durchschnittlichen Haushaltsstärken ist immer kritisch zu hinterfragen, ob es sich hier nicht um Herdgemeinschaften gehandelt hat, wie sie oben charakterisiert worden sind, und Mieter/Untermieter bewusst oder unbewusst den Haushalten inkorporiert worden sind. Bei der Berechnung ergeben sich daraus entsprechend größere „Haushaltsstärken“. Insbesondere dieser Umstand verdient bei zukünftigen Forschungen mehr Aufmerksamkeit, will man die Zahl der eigentumslosen Bevölkerung oder konkrete Haushalts- beziehungsweise Familiengrößen ermitteln.

\section{Literatur}

Helmut Bräuer fasst 2003 die Forschungssituation zum Thema der eigentumslosen Bevölkerung (Hausgenossen) treffend zusammen: „Angesichts der quantitativen Dimension der sozialen Gruppe der Hausgenossen ist es erstaunlich, dass es bislang nur unzureichende Auseinandersetzungen mit diesem Thema gibt, ja noch immer mancherlei Missverständnisse kursieren." ${ }^{6}$ Diese Feststellung äußert er im Hinblick auf die Disziplin der Stadthistoriker. An der Situation hat sich bis heute nichts grundlegend geändert. Forschungslücken finden sich sowohl im Hinblick auf prosopographische als auch auf sozialhistorische Fragestellungen.

\section{Sozial- und Wirtschaftsgeschichte}

Wie bereits geäußert, kommen wichtige Impulse in der Forschung aus dem Bereich der Sozialgeschichte, die sich ab den 1960er Jahren verstärkt der Alltagsgeschichte der Mittel- und Unterschichten zugewandt hat. Zu nennen sind hier die Arbeiten Maschkes. ${ }^{7}$ Ansätze für solche Forschungen lassen sich jedoch schon für das 19. Jahrhundert nachweisen, stellvertretend sei hier die Arbeit von Georg Schanz zur "Geschichte der Colonisation und Industrie in Franken aufgeführt“. ${ }^{8}$ Er belegt anhand der Quellen die hohe Zahl der eigentumslosen Haushalte in den Gewerbestädten ab dem 17. Jahrhundert. ${ }^{9}$ Singulär ist ein Aufsatz von Helmut Bräuer aus dem Jahr 2003, der sich bisher als einziger ausschließlich mit der Thematik des Mieterdaseins anhand der „Hausgenossen in den Städten Obersachsens während des Spätmittelalters und der frühen Neuzeit" befasst hat und aus dem ich eingangs zitiert habe. ${ }^{10}$

In seinem Grundlagenwerk „Arme Leute, Bettler und Gauner im Franken des 18.

\footnotetext{
${ }^{6}$ BRÄUER 2003, S. 73.

${ }^{7}$ Vgl. hierzu unter anderem MASCHKE 1967 und MASCHKE 1972.

${ }^{8}$ SCHANZ 1884.

${ }^{9}$ Vgl. Kapitel 7, Exportgewerbestädte Erlangen.

${ }^{10}$ BRÄUER 2003.
} 
Jahrhunderts" schildert Ernst Schubert sehr detailreich anhand vieler Quellen die Lebenssituation der ärmeren Bevölkerung, von der ja auch die überwiegende Mehrheit nicht über Hausbesitz verfügte. ${ }^{11}$ Für die Stadt analysiert er zutreffend: „Nur in Ausnahmefällen lebten all die Arbeiter, Tagelöhner, Gelegenheitsarbeiter in eigenen Katen, zumeist wohnten sie in den Städten zur Miete. "12 Er äußert sich jedoch nicht zu quantitativen Fragen.

Ebenfalls sehr anschaulich schildert Helmut Möller die Lebensumstände der städtischen Kleinbürger im 18. Jahrhundert, bezogen auf Deutschland. Er weist darauf hin, dass „ein nicht unerheblicher Teil der Kleinbürger - und auch der Handwerker unter ihnen - kein eigenes Haus" besaß. ${ }^{13}$ Darüber hinaus führt er auch dezidiert Mieterzahlen zu verschiedenen Kleinstädten an, die jedoch lückenhaft bleiben und einer kritischen Würdigung bedürfen, wenn er davon ausgeht, dass nur ein Viertel bis ein Drittel der Kleinbürger zur Miete wohnten. ${ }^{14}$ Diese Angaben wären eher auf sämtliche Einwohner in den Städten zu beziehen, allein unter den Kleinbürgern ist mit einem höheren Mieteranteil zu rechnen.

Auch das Werk von Ulf Dirlmeier zu Einkommensverhältnissen und Lebenshaltungskosten in oberdeutschen Städten des Mittelalters ist zu nennen. ${ }^{15}$ In einem Kapitel geht er explizit auf die Verbreitung des Mietwohnens ein. ${ }^{16}$ Er kommt zu folgendem Ergebnis: „Die Alltäglichkeit des Mietens und Vermietens von Häusern ist nach diesen allgemeinen Belegen kaum bestreitbar. "17 Anhand der vorliegenden Daten geht er weiterhin davon aus, dass in den oberdeutschen Städten ein großer Teil der Bevölkerung bereits zum Ende des Mittelalters zur Miete lebte. ${ }^{18}$ Neben der Vermietung von Häusern und Wohnungen versucht er auch das Phänomen des Untermietens in Zahlen zu erfassen.

Besonders hervorzuheben sind ebenfalls die Untersuchungen Bernd Roecks zu Augsburg zwischen 1550 und $1650 .{ }^{19}$ Er setzt sich umfassend mit der Lebenssituation der ärmeren Bevölkerungsmehrheit auseinander, die zu 90\% kein Wohneigentum hatten. Unter anderem zeichnet er die topografische Verteilung dieser Menschen nach.

Valentin Groebner befasst sich in seiner Dissertation mit der Lebens- und Wirtschaftssituation der armen Nürnberger Bevölkerung am Ende des Mittelalters, einer Zeit, da Nürnberg in höchster Blüte stand. ${ }^{20}$ Bereits im Titel „Ökonomie ohne

\footnotetext{
${ }^{11}$ Schubert 1990.

12 Ebd., S. 111.

${ }^{13}$ MÖLLER 1969, S. 116.

${ }^{14}$ Ebd.

${ }^{15}$ Vgl. DiRLMEIER 1978.

${ }^{16}$ Ebd., S. 239-260.

${ }^{17}$ Ebd., S. 243.

${ }^{18}$ Ebd.

${ }^{19}$ RoECK 1983 und 1989.

${ }^{20}$ GROEBNER 1993.
} 
Haus. Zum Wirtschaften armer Leute in Nürnberg am Ende des 15. Jahrhunderts" spricht er den Zusammenhang zwischen Eigentumslosigkeit und Armut an.

Umfangreiche sozial- und wirtschaftshistorische Forschungen, die auch die eigentumslosen Einwohner berücksichtigen, liegen für Lübeck vor. Insbesondere die Situation im Mittelalter ist hier umfassend und vorbildlich aufgearbeitet. ${ }^{21}$ Dezidiert im Blick des Forschungsinteresses stehen die eigentumslosen Einwohner in der Dissertation von Monika Fehse zu den Wohnverhältnissen in Dortmund um 1400. ${ }^{22}$ Grundlage ihrer Analyse sind Steuerverzeichnisse von 1396 und 1406 bis 1422 für den Kernbereich der ehemaligen freien Reichsstadt. Sie setzt sich in der Arbeit umfassend mit dem Thema des Mietwohnens auseinander und geht auf Wohnsituationen, Mietverhältnisse, Untermieter, Rendite etc. ein. Zugleich eröffnet sie erstmalig einen Ansatz in der Nachzeichnung der städtischen Sozialtopografie bei dem die Mieter weitestgehend Berücksichtigung finden und kommt dabei zu neuen, sehr differenzierten Ergebnissen.

Abschließend sei noch auf die jüngst erschienene Publikation von Thomas Ertl zu einem Wiener Einwohnerverzeichnis aus dem Jahr 1448 hingewiesen. ${ }^{23}$ Ausgehend von dieser Quelle entfaltet Ertl die Bevölkerungs- und Sozialstruktur Mitte des 15. Jahrhunderts. In dem Verzeichnis sind sowohl die Hausbesitzer als auch die Mieter (Inwohner) für das Widmerviertel gelistet. ${ }^{24}$ Während in der Vorstadt die Eigentümer deutlich in der Mehrzahl waren, kehrt sich die Situation in der Inneren Stadt um, insgesamt ergibt sich ein ausgeglichenes Verhältnis. ${ }^{25}$

Die sozialhistorischen beziehungsweise volkskundlichen Forschungen, die sich mit der Situation eigentumsloser Einwohner auf dem Land auseinandersetzen, wurden von mir an anderer Stelle bereits gewürdigt. Hier sei auf meine Dissertation verwiesen. ${ }^{26}$

Trotz der genannten Bemühungen in der sozialhistorischen Forschung, ist das Wissen um die konkrete Lebenssituation der Eigentumslosen nach wie vor gering. Dies findet auch darin seinen Ausdruck, dass Wolfgang von Hippel in seinem aktuellen Grundlagenwerk zu Armut, Unterschichten und Randgruppen in der frühen Neuzeit an keiner Stelle auf das Thema des Mietwohnens eingeht. ${ }^{27}$

\footnotetext{
${ }^{21}$ Aus der umfangreichen Literatur sei hier stellvertretend auf Aufsätze von Hammel-Kiesow und Ellermeyer verwiesen: ElLERMEYER 1980; HAMMEL 1980, HAMMEL-KIESOW 2005.

${ }^{22}$ FEHSE 2005.

${ }^{23}$ ERTL 2020.

24 Österreichische Nationalbibliothek, Cod. 13.959.

${ }^{25}$ ERTL 2020, S. 44.

${ }^{26}$ WENDEROTH 2019a, S. 32-36.

${ }^{27}$ HIPPEL 2013.
} 


\section{Stadtgeschichte mit einem sozialtopografischen Ansatz}

Sozialtopografische Arbeiten erweisen sich als wertvolle Quellen, um die Städte in ihrer Gesamtheit zu verstehen, sofern auch die unteren Bevölkerungsschichten mit in den Blick genommen werden. Erste sozialtopografische Arbeiten datieren ins 19. Jahrhundert. ${ }^{28}$ Eine der aktuellsten Arbeiten in dieser Richtung ist die Dissertation von Thomas Wozniak zur Sozialtopografie Quedlinburgs im 14. und 16. Jahrhundert aus dem Jahr 2009, publiziert 2013. ${ }^{29}$ In der äußerst verdienstvollen Arbeit bleibt die eigentumslose Bevölkerung jedoch völlig unbeachtet. Gerade eine sozialtopografische Arbeit wird jedoch ohne die Einbeziehung der Eigentumslosen und ihrer Wohnorte ein unzutreffendes Bild einer Stadt zeichnen. ${ }^{30}$ Gleiches gilt für die Dissertation von Martina Reiling zu Freiburg im Breisgau. ${ }^{31}$ Sie verweist lediglich darauf, dass die Mieter des 17. und 18. Jahrhunderts nur durch den Abgleich des Häuserbuches mit den Kirchenbucheinträgen identifiziert werden könnten und erspart sich diese Mühe. ${ }^{32}$

In diesem Zusammenhang ist die Arbeit von Karsten Igel zu Stadtstruktur, Grundbesitz und Sozialstruktur im spätmittelalterlichen Greifswald positiv hervorzuheben. Er benennt die Problematik klar: „Dies bedeutet aber auch, dass ein und dieselbe Hausstätte von Mitgliedern unterschiedlicher sozialer Gruppen (hier grob zwischen Hauseigentümern und Mietern geschieden) in getrennten Haushalten bewohnt werden konnte. Ein Faktor, der bei der Kartierung der meisten Ergebnisse dieser Arbeit, vor allem aber bei der Diskussion der Stadtpläne, immer berücksichtigt werden muss. “ ${ }^{33}$ Folgerichtig widmet er den Mietern auch ein eigenes Kapitel, obwohl in den Stadtbüchern als Hauptquelle seiner Arbeit Mieter nur selten Erwähnung fanden. ${ }^{34}$

\footnotetext{
${ }^{28}$ Ein Abriss der Geschichte der sozialtopografischen Arbeiten findet sich bei DENECKE 1980, S. 161-164.

${ }^{29}$ WOZNIAK 2013.

${ }^{30}$ Vgl. hierzu WENDEROTH 2021, S. 264-272.

${ }^{31}$ REILING 1989.

${ }^{32}$ Ebd., S. 39; diese Untersuchung unterbleibt, folglich fehlt ein wichtiger Teil der Bevölkerung bei der Analyse der Sozialstruktur. Ansonsten geht Reiling nicht mehr auf Mieter ein.

${ }^{33}$ IGEL 2010, S. 163.

${ }^{34}$ Ebd., Kapitel 4.7, S. 253-260.
} 
Ungewöhnlich umfassende Informationen zum Thema der Eigentumslosigkeit enthält hingegen die Arbeit zum Wohnen und Wirtschaften in Oettingen zwischen 1600 und 1800 von Petra Ostenrieder. ${ }^{35}$ Die Ergebnisse dieser Arbeit dürften auf viele kleine Residenzstädte in der frühen Neuzeit übertragbar sein.

\section{Bevölkerungsgeschichte}

Die verstreute Literatur, die basierend auf historischen Quellen, zu fundierten und nachvollziehbaren Aussagen zum Anteil der eigentumslosen Bevölkerung und damit überhaupt erst zu belastbaren Einwohnerzahlen kommt, ist überschaubar. Schwierig ist es hingegen, fundierte heimatgeschichtliche Aufsätze zu dieser Thematik aufzuspüren.

Der geringe Kenntnisstand ist zum einen sicher begründet durch fehlende Quellen, die eine entsprechende Erfassung der Bevölkerung nicht erlauben, zum anderen ist bisher keine flächendeckende, systematische Forschung zur Bevölkerungsgeschichte erfolgt. Eine zusammenfassende Würdigung der Einzelergebnisse unter Berücksichtigung der eigentumslosen Bevölkerung fehlt. ${ }^{36}$ Die vorliegenden Arbeiten können daher lediglich punktuell Erkenntnisse bieten. Die bisher untersuchten Orte folgen keiner übergeordneten Systematik. Wenn Ergebnisse zu wichtigen Städten fehlen, beruht dies nicht immer auf fehlenden Quellen, sondern häufig auf fehlendem Forschungsinteresse.

Auch für die Städte, deren Bevölkerungsgeschichte bereits analysiert worden ist, muss im Hinblick auf die eigentumslose Bevölkerung festgestellt werden, dass diese Fragestellung nicht immer berücksichtigt wurde. Auch hier gilt, dass vermutlich in vielen Fällen die zur Verfügung stehenden und bearbeiteten Quellen keine konkreten Aussagen zulassen. In nicht wenigen Fällen scheint diese Fragestellung aber auch einfach unberücksichtigt geblieben zu sein. Entweder wird die eigentumslose Bevölkerung ganz übergangen und die Bevölkerungszahlen werden nur anhand von Hausbesitzern rekonstruiert, oder aber es werden die Angaben in historischen Bevölkerungszählungen summiert, ohne die in manchen Fällen darin vorhandene Differenzierung zwischen Eigentümern, Mietern etc. zu berücksichtigen. Dies gilt zum Beispiel für Göttingen, dessen Bevölkerungsgeschichte mit den ansonsten herausragenden Dissertationen von Walter Kronshage und Wieland Sachse vorbildlich vom 14. bis zum 19. Jahrhundert geklärt

\footnotetext{
35 OSTENRIEDER 1993.

${ }^{36}$ Erich Keysers Überblickswerk zur Bevölkerungsgeschichte ist stark von der nationalsozialistischen Ideologie geprägt. Er geht - abgesehen von dieser Problematik - aber auch nicht auf die Mietbevölkerung ein; vgl. KEYSER 1938.
} 
ist. ${ }^{37}$ Das verwendete Quellenmaterial hätte hier durchaus Informationen zur eigentumslosen Bevölkerung geboten. ${ }^{38}$

Otto Richter hat sich bereits recht früh, nämlich im Jahr 1880, in einem Aufsatz mit der Bevölkerungsstruktur Dresdens im 15. Jahrhundert befasst, bei dem die Mietbevölkerung zumindest ansatzweise Berücksichtigung fand. ${ }^{39}$

Deutlich detaillierter hat sich Joseph Greving 1904 in seinem Aufsatz zu den Wohnungs- und Besitzverhältnissen im Kölner Kirchspiel St. Kolumba mit der Frage der eigentumslosem Bevölkerung auseinandergesetzt. ${ }^{40}$ Sein Aufsatz ist wegweisend bis heute, nicht nur im Hinblick auf die Ergebnisse, sondern auch auf die Sorgfalt, mit der er das zugegebenermaßen hervorragende Quellenmaterial diskutiert.

Eine ebenfalls sehr sorgfältige Bevölkerungsanalyse hat zum Beispiel Werner Schnyder für die Stadt und Landschaft Zürich in seiner Dissertation erarbeitet. ${ }^{41}$ Allein - auch er differenziert nicht zwischen Eigentümern und Mietern, auch wenn er in Zürich-Stadt Mieter für das frühe 15. Jahrhundert nennt, die in den dortigen Akten mit huswirt, husfrow oder huslüt bezeichnet wurden. ${ }^{42} \mathrm{Als}$ ein wichtiges Ergebnis seiner Arbeit kann die Rekonstruktion der durchschnittlichen Haushaltsgröße in Zürich für das Jahr 1467 gelten. Schnyder errechnet hier 3,58 bis 3,86 Personen. ${ }^{43}$

Als besonders ergiebig zum Thema der eigentumslosen Bevölkerung erweist sich die Dissertation von Christian Forneck zur Regensburger Einwohnerschaft im 15. Jahrhundert. ${ }^{44}$ Als Hauptquelle dienen ihm 45 Einwohnerverzeichnisse, die um 1436 datieren.

In diesem Zusammenhang ist auch die Dissertation von Hannelore Götz hervorzuheben, die zumindest für Würzburg die Miethaushalte und die Bevölkerungszahlen des 16. Jahrhunderts detailliert erfasst und diskutiert hat. ${ }^{45}$

Damit ist bereits die Bevölkerungsgeschichte der frühen Neuzeit berührt. Ein frühes Werk, das das geschilderte Dilemma gut zum Ausdruck bringt, stellt die Dissertation zu den Bevölkerungs- und Vermögensverhältnissen der Stadt Mühlhausen in Thüringen in der Zeit von 1563 bis 1614 dar. ${ }^{46}$ Lediglich in der Literaturdiskussion geht der Autor auf das Thema Besitzlosigkeit in anderen Städten ein,

\footnotetext{
${ }^{37}$ KRONSHAGE 1960; SACHSE 1987.

${ }^{38}$ Kronshage führt selbst aus, dass in den von ihm verwendeten Schoßregistern differenziert ist zwischen Hausbesitzern, Mietern, Mitbewohnern und Untermietern; KRONSHAGE 1960, S. 9.

${ }^{39}$ RiCHTER 1881, S. 273-289.

${ }^{40}$ GREVING 1904.

${ }^{41}$ SCHNYDER 1925.

42 Ebd., S. 47.

${ }^{43}$ Ebd., S. 53f.

${ }^{44}$ FORNECK 2000.

45 GÖTZ 1986.

${ }^{46}$ WEIß 1926.
} 
ohne seine Annahme daraufhin zu hinterfragen, ob alle Einwohner Mühlhausens Immobilieneigentümer waren.

Die Dissertation von Alfred Riedle zur „Wirtschaft und Bevölkerung Heilbronns zur Zeit des Dreißigjährigen Krieges" lässt ebenfalls eine differenzierte Betrachtung vermissen, er begnügt sich mit zumeist vagen Schätzungen. ${ }^{47}$

Ein singuläres Werk stellt hier die Habilitationsschrift von Katrin Keller zu den Kleinstädten in Kursachsen zwischen dem Dreißigjährigen Krieg und der Industrialisierung dar. ${ }^{48}$ Das Besondere an der Arbeit ist die topografische Untersuchung einer ganzen Region mit immerhin 95 Städten. Für die Bevölkerungsgeschichte analysiert Keller die Zeitschnitte 1550, 1700, 1750 und das frühe 19. Jahrhundert. Die breite Datenbasis ermöglicht ihr die Bildung von Stadtgruppen und die Herausarbeitung von allgemein gültigen Tendenzen in Kursachsen, die sicher darüber hinaus zu diskutieren wären. Auf die eigentumslose Bevölkerung geht aber auch sie nur am Rande ein. Immerhin wird diese von ihr ausführlich gewürdigt im Kontext der vier Städte, die sie exemplarisch vertieft.

Besonders hervorzuheben ist für die Neuzeit noch die Arbeit von Konrad Otto Roller zur Einwohnerschaft Durlachs im 18. Jahrhundert. ${ }^{49} \mathrm{Ihm}$ gelingt es, für das Jahr 1762 die Wohnorte der eigentumslosen Haushalte zu klären. Die Arbeit bezieht sich also auf das Ende meines Untersuchungszeitraumes.

\section{Edierte Quellen, Häuserbücher}

Von den edierten Quellen soll besonders auf die Kopfsteuerbeschreibungen des späten 17. Jahrhunderts in den Teilstaaten der Welfen hingewiesen werden, die eine Fülle von Hinweisen zu eigentumslosen Einwohnern enthalten. Sie werden dort als Häuslinge bezeichnet. Zu nennen wären hier die Kopfsteuerbeschreibungen für das Fürstentum Braunschweig-Wolfenbüttel $1678^{50}$ und für das Fürstentum Calenberg-Göttingen und Grubenhagen $1689^{51}$ sowie für die Städte Braunschweig von 1672 und $1687^{52}$ sowie Hannover von 1689. ${ }^{53}$ Eine Auswertung dieser umfangreichen niedersächsischen Verzeichnisse hinsichtlich der eigentumslosen Bevölkerung ist meines Wissens noch nicht erfolgt.

\footnotetext{
${ }^{47}$ RIEDLE 1933.

${ }^{48}$ KELLER 1997.

${ }^{49}$ ROLLER 1907.

${ }^{50}$ MEDEFIND 2000.

${ }^{51}$ BURCHARD/MUNDHENKE 1940-1972.

52 MEDEFIND 2004.

${ }^{53}$ STUDTMANN 1941.
} 
Ferner sei auf die Mainzer Stadtaufnahmen zwischen 1568 und 1785/86 verwiesen, herausgegeben in drei Bänden von Friedrich Schrohe. ${ }^{54}$ Die sozialgeschichtlich bedeutende Quellensammlung liegt gedruckt vor. Es handelt sich dabei um sieben Stadtaufnahmen aus der Zeit zwischen 1568 und 1785. Neben der Beschreibung der Anwesen enthalten die Listen die Namen der Eigentümer und Mieter. Die Aufstellungen von 1568, 1594 und 1657 nennen im Falle, dass der Eigentümer das Haus selbst bewohnt, keine weiteren Mieter für die Anwesen. Ist ein Haus nicht vom Eigentümer bewohnt, wird lediglich ein Mieter genannt. ${ }^{55}$ Die Aufstellungen aus den Jahren $1687^{56}$ und $1747^{57}$ nennen zumeist nur den jeweiligen Eigentümer, ohne der Frage nach dem tatsächlichen Bewohner nachzugehen. Davon abgewichen wird 1687 nur bei stiftischem Besitz. ${ }^{58}$ Die abgabefreien Anwesen sind für das Jahr 1747 zusammengefasst. ${ }^{59}$ Anhand dieser Aufstellung wird der umfangreiche Hausbesitz der kirchlichen Institutionen und damit deren Bedeutung als Vermieter deutlich.

Aussagen $\mathrm{zu}$ weiteren Mitbewohnern enthalten dagegen die Aufnahmen von 1644; bei den hier genannten Mitbewohnern scheint es sich zum überwiegenden Teil um Menschen aus den umliegenden Dörfern zu handeln, die vor der Kriegsgefahr in der Stadt Schutz suchen. ${ }^{60}$ Geschildert wird also eine Ausnahmesituation, die ein Schlaglicht auf die Wohnumstände in Kriegszeiten wirft.

Von besonderem Interesse ist dagegen die unvollständige Stadtaufnahme der Jahre 1785/87. ${ }^{61}$ Hier werden für jedes Haus sowohl die Eigentümer als auch sämtliche Mieter genannt. Die eigentumslose Bevölkerung hat dabei unterschiedliche rechtliche Stellungen: So heißt es bei der Liste der Mieter zu Haus Graben 2: Joachim Müller, schiffknecht, burg.[er]; Michael Aleiter, pumpenmacher, toleriert; Niclas Müller, karcherknecht, beisa $3 .{ }^{62}$ Die Anwesen selbst sind aufgegliedert in einzelne Gebäude samt der Geschosszahl.

Für das eigentliche Untersuchungsgebiet in Franken wichtig sind die beiden von Gerhard Rechter publizierten Reichssteuerregister der Fürstentümer Brandenburg-Ansbach-Kulmbach unterhalb und oberhalb Gebürgs von $1497 .{ }^{63}$ Hier sind

\footnotetext{
54 SCHROHE 1930a, 1930b und 1931.

55 2. Ein behausung daneben samt hoflein, ist aigen Johann Hadamaren, apoteker, bewohnt umb zins Cornelius Kempf; zitiert nach SCHROHE 1930b, S. 91.

${ }^{56}$ Dies wird zum Beispiel daran deutlich, dass Johann Kilian zwei Häuser zu eigen hat, ohne dass ein Mieter für eines des Häuser aufgeführt ist; SCHROHE 1930b, S. 55, Haus 494 und 495.

${ }^{57}$ SCHROHE 1931, S. 1-142.

58457 Das Eckhaus an der großen treppen gegen der Fischpforten, gehört dem stift ad gradus und bewohnt Johann Heckenbroch, krämer; zitiert nach SCHROHE 1930b, S. 51.

${ }^{59}$ SCHROHE 1931, S. $143 f$.

${ }^{60}$ SCHROHE 1931.

${ }^{61}$ Hier sind etwa 1/3 der Häuser erfasst; SCHROHE 1931, S. 145-211.

${ }^{62}$ SCHROHE 1931, S. 145.

${ }^{63}$ RECHTER 1985 und 1988.
} 
im Wesentlichen jedoch nur die Untermieter als Mieter kenntlich gemacht. Die Quelle wird nachfolgend ausführlich gewürdigt.

Nur wenige Informationen zum Thema der Eigentumslosigkeit enthalten dagegen die Grundbücher. Entsprechende Quelleneditionen wie die des Rostocker Grundregisters 1550-1600 können daher unberücksichtigt bleiben. ${ }^{64}$

Eine weitere wichtige Quelle, um eigentumslose Einwohner zu erfassen, sind die historischen Adressbücher. Sie bieten sich vor allem an, um die Bevölkerungsstruktur des 19. Jahrhunderts zu klären. Aus dem weiteren Untersuchungsraum wäre hier das publizierte Verzeichnis zu Bamberg für die Jahre 1804/05 und 1809/10 zu nennen, ${ }^{65}$ eine wichtige Quelle, die insgesamt ebenfalls noch der Auswertung harrt.

\section{Literatur zu eigentumslosen Einwohnern in fränkischen Städten}

Die ortsgeschichtliche Literatur wächst entsprechend der Größe der Stadt und nimmt wie im Falle von Nürnberg einen erheblichen Umfang an. In der Stadtgeschichtsschreibung werden die eigentumslosen Einwohner jedoch nur selten thematisiert. Im Folgenden beschränke ich mich weitgehend auf die von mir verwendete ortsgeschichtliche Literatur zu dem Thema.

Zur Bevölkerungsgeschichte Nürnbergs liegen wichtige Schriften von Paul Sander, Caspar Ott und Rudolf Endres vor, die jedoch in der Zahlendiskussion das Thema der eigentumslosen Bevölkerung unberücksichtigt lassen. ${ }^{66}$ Als eine der wenigen Arbeiten, auf das gesamte deutsche Sprachgebiet bezogen, die sich bis heute explizit mit dem Thema beschäftigt haben, darf die Zulassungsarbeit von Christian Feja mit dem Titel „Mietverhältnisse im vorindustriellen Nürnberg. Eine Fallstudie für das 16. Jahrhundert" nicht unerwähnt bleiben, gleichwohl diese bedauerlicher- oder auch bezeichnenderweise bisher nicht publiziert worden ist. ${ }^{67}$ Grundlage dieser Arbeit sind die Nürnberger Feuerschaulisten von $1561 .^{68}$

\footnotetext{
${ }^{64}$ MÜNCH 2017.

${ }^{65}$ Siehe hierzu FRÄNKEL 1999.

${ }^{66}$ OTT 1907, ENDRES 1970, ENDRES 1990b, SANDER 1902.

${ }^{67}$ FEJA 1989. Eine Kopie der Arbeit sollte im Stadtarchiv Nürnberg und in der Bibliothek des Vereins „Geschichte für Alle e. V. “ in Nürnberg liegen. Eine weitere Kopie befindet sich im Besitz des Autors.

${ }^{68}$ Vgl. hierzu Kapitel 3, Feuerschaulisten.
} 
Für Nürnberg ist darüber hinaus unbedingt noch der Aufsatz von Erich Mulzer über die Häuser am Unschlittplatz anzuführen. ${ }^{69}$ Dort analysiert dieser präzise die Eigentums- und Mieterstruktur exemplarisch für einen Häuserblock.

Ziel vieler lokalhistorischer Forschungen ist die Veröffentlichung eines Häuserbuches, bei dem die ausgewerteten historischen Schriftquellen einem bestimmten Anwesen zugeordnet werden. Wobei es hierfür keine einheitlichen Vorgaben gibt und das Ergebnis stark von der Qualifikation und dem Interesse des jeweiligen Autors abhängt. In der Regel beschränkt sich die Aussage dieser Werke auf die Nachzeichnung der Besitzverläufe. Eigentumslose Einwohner und ihre Wohnorte werden nur in Einzelfällen thematisiert. ${ }^{70}$ Eine Ausnahme stellt ferner das Häuserbuch von Karl Dehm und Gottlob Heckel zu Schwabach dar. ${ }^{71}$ In diesem Buch wird zu jedem Haus die Zahl der Mieter für das Jahr 1799 genannt und auch die Mietthematik für die frühe Neuzeit angesprochen. Gerhard Rechter thematisiert in seinem Häuserbuch zu Uffenheim immerhin die Mieter, kann sie jedoch anhand der zur Verfügung stehenden Quellen nicht den einzelnen Anwesen zuordnen. ${ }^{72}$

Als frühe Publikation, die auch das Thema der eigentumslosen Bevölkerung ab dem späten 17. Jahrhundert anspricht und eine Fülle an entsprechenden Quellen erschließt, ist an dieser Stelle noch einmal die Arbeit „Zur Geschichte der Colonisation und Industrie in Franken“ von Georg Schanz von 1884 zu nennen. ${ }^{73} \mathrm{Ne}-$ ben der Stadt Schwabach liegt ein Hauptschwerpunkt dieser Arbeit bei der Etablierung der Planstadt Erlangen als Gewerbestandort.

Die Bevölkerungsgeschichte Erlangens vor der Barockzeit, bis 1619 ist inklusive der Mieter gut dargestellt in drei kurzen Aufsätzen von Johannes E. Bischoff. ${ }^{74}$

\footnotetext{
${ }^{69}$ MULZER 1981.

${ }^{70}$ Stellvertretend sei hier auf das zehnbändige Häuserbuch der Stadt Coburg von Helmut Wolter verwiesen. Im Hinblick auf die historsiche Zugehörigkeit zu den sächsischen Teilstaaten habe ich auf eine Auswertung und Berücksichtigung der Ergebnisse verzichtet, grundsätzlich erscheint eine solche Auswertung jedoch lohnenend. WOLTER 2001-2012.

${ }^{71}$ DEHM/HECKEL 1970.

72 RECHTER 2003.

73 SCHANZ 1884.

${ }^{74}$ BisCHOFF 1959, 1961a und 1961b.
} 


\section{Bezeichnungen der Eigentumslosen in den Quellen}

Bevor ich auf die für die vorliegende Studie herangezogenen Quellen eingehe, sollen die dort verwendeten Begriffe für die eigentumslose Bevölkerung erläutert werden, da dies die Grundlage zum Verständnis der Quellen darstellt. Einige der verwendeten Begriffe beziehen sich auf den rechtlichen Status der Mietbevölkerung. Sie werden in diesem Kapitel hinsichtlich ihres Gebrauch vorgestellt, im übernächsten Kapitel im Kontext der rechtlichen Situation der eigentumslosen Bevölkerung.

Erschwert wird die Beschäftigung mit den Eigentumslosen und ihrem Anteil an der Bevölkerung durch die nicht einheitliche Verwendung von Bezeichnungen; dies stellt nicht nur ein Problem hinsichtlich der Auswertung von Literatur, sondern auch hinsichtlich der historischen Quellen dar.

Auf die synonyme Verwendung verschiedener Bezeichnungen, mit denen eigentumslose Haushalte in Franken charakterisiert wurden, weist bereits Georg Andreas Will 1780/81 hin: Gewöhnlicher sind die Benennungen, Versprich Mann, Inn Mann, Beständner, Innwohner, Hintersäs. Die Bedeutung aller dieser Wörter ist homogen. Sie bezeichnen dermal ohne Unterschied, eine Person, die mit keinen Gütern, inner Etters oder im Dorf, angesessen ist, sondern nur Bestandsweis oder um Zins darinn wohnet. [...]. Die gleiche Bedeutung obiger Synonyme, bezeuget nicht nur die tägliche Erfahrung, vermög deren, in den Aemter Rechnungen, eines oder mehr derselben, ohne Unterschied für die andern gebraucht wird. ${ }^{75}$

Im Folgenden sollen verschiedene Bezeichnungen und ihr Gebrauch in den Quellen erörtert werden. Dabei fällt auf, dass sich oftmals der genaue Anwendungsbereich dieser Begriffe erst aus dem Kontext erschließt und damit von Situation zu Situation unterschiedlich gewesen sein kann. Auf diese Problematik weist auch Helmut Bräuer für die Mieter in Sachsen hin. ${ }^{76}$ Ein Teil der Bezeichnungen leitet sich vom rechtlichen Status der Einwohner ab; wenn diese Begriffe hier nochmals erläutert werden, dann im Hinblick auf ihre Verwendung in den zeitgenössischen Quellen und nicht um den rechtlichen Status ein weiteres Mal zu erläutern.

\section{Hausgenossen}

Im Hinblick auf den unterschiedlichen Gebrauch dieses und anderer Begriffe ist bei der Auswertung der Quellen entsprechende Vorsicht geboten. Die Begriffsdefinition des Hausgenossen ist nicht zu 100\% deckungsgleich mit unserem heutigen Begriff des Mieters. Mit dem Begriff des Hausgenossen kann in historischen

\footnotetext{
75 WILL 1780/1, S. $72 f$.

${ }^{76}$ BRÄUER 2003, S. 74-77.
} 
Quellen zum Beispiel lediglich eine Teilgruppe innerhalb der gesamten Mieterschaft gemeint sein, in anderen Fällen ist der Begriff ausgeweitet auf Hausgesinde etc. und in einigen mittelalterlichen Quellen handelt es sich dabei primär um einen bischöflichen Dienstmann.

Dieser Begriff scheint wie viele andere Begriffe einer Wandlung zu unterliegen. Nach dem Grimmschen Wörterbuch wurde der Begriff auch im Sinne von Mietsmann verwendet, gleichfalls jedoch für das Gesinde und Amtleute gebraucht. ${ }^{77}$ Letzteres findet seine inhaltliche Bestätigung in den ältesten Nennungen von Hausgenossen in Bamberg. ${ }^{78}$ In Zusammenhang mit dem Domstift lässt sich die Bezeichnung erstmalig 1231 nachweisen: ${ }^{79}$ Als Hausgenossen werden dort bürgerliche Familien geführt, die zur städtischen Oberschicht in Bamberg gehörten. 1301 werden die Ämter des Küchen-, Mehl- und Pfistermeisters von Hausgenossen ausgeübt. Der Status des domstiftischen Hausgenossen hatte unter anderem den Vorteil, von bischöflichen Steuern befreit zu sein. Ob diese Hausgenossen tatsächlich auch in einer Immobilie des Domstiftes zur Miete wohnten, ist bisher nicht geklärt. Diese Hausgenossen rekrutierten sich aus wenigen Bamberger Familien, die zu diesem Amt berechtigt waren. Zu ihren Aufgaben gehörte wohl die Kontrolle der Münzprägung, aber auch die Aufsicht über den städtischen Markt.

In Würzburg waren einige angesehene Einwohner im Mittelalter ebenfalls Hausgenossen des dortigen Domstifts, auch hier handelte es sich nicht primär um die Definition als Bewohner einer Mietunterkunft, sondern um die mit dem Begriff verbundenen einträglichen Ämter. Bereits 1344 heißt es in einem Schiedsvertrag in Würzburg, dass die Bürger der Stadt auch der stiffte zu Wirtzburg hausgenozzen, amptleut, dyner in ihren alten Rechten belassen sollen. ${ }^{80}$ In der zweiten Hälfte des 15. Jahrhunderts schafften es sogar dreizehn der domstiftischen Hausgenossen, Ratsherrn der Bürgerstadt zu werden. ${ }^{81}$

Für die Stadt Würzburg ist der Begriff allerdings auch schon im Jahr 1342 für Mieter im eigentlichen Sinn bezeugt. In den Gesetzen von 1342 heißt es zum Beispiel zum Schwören von falschen Eiden, dass der Hausherr Verantwortung hat für seine Familie, das Gesinde und die Hausgenossen. ${ }^{82}$ Im dortigen „Seldenerbuch “ ${ }^{43}$ finden sich um 1400 bei insgesamt 2.600 Einträgen etwa 500 Einträge mit

\footnotetext{
77 DWB, Bd. 10, Sp. 667.

${ }^{78}$ Vgl. hierzu und im Folgenden SCHIMMELPFENNIG 1964, S. 64-67.

${ }^{79}$ Ebd.

${ }^{80}$ Staatsarchiv Würzburg 803 (B1) Abschrift, zitiert nach HofFMANN 1955, S. 70.

${ }^{81}$ SPRANDEL 2003, S. 12.

82 Universitätsbibliothek München, Codex 731, f 250-250'; gedruckt bei HoFFMANN 1955, S. 67.

831932 veröffentlicht von Georg Meyer-Erlach; MEYER-ERLACH 1932.
} 
der Bezeichnung husgenoß. ${ }^{84} 1577$ wird in den Vorbemerkungen zur Steuererhebung wieder auf die gewünschte Differenzierung, die wohl zwischenzeitlich vernachlässigt worden war, hingewiesen. Für den Hausgenossen sollte nunmehr die Bezeichnung hus verwendet werden. ${ }^{85}$

Vom Wortsinn her ist eine Person angesprochen, die (als Genosse) mit im Haus einer anderen Person lebt. Dies impliziert zugleich eine mehr oder minder stark ausgeprägte Hausgemeinschaft. Die Verwendung dieses Begriffes im Rahmen der Erhebung des Gemeinen Pfennings 1497 im Fürstentum BrandenburgKulmbach-Ansbach legt nahe, dass dort - dem Wortsinn entsprechend - primär Untermieter als Hausgenossen erfasst worden sind, in jedem Fall jedoch nur solche Mieter, die mit im Haus des Eigentümers gelebt haben.

In den überlieferten Huldigungslisten des Markgraftums Brandenburg-Ansbach, die mit der Huldigung 1603 beginnen, ist der Haußgenosse dann der gebräuchlichste Begriff für die Mieter. ${ }^{86}$ Nunmehr wurden unter diesem Begriff alle Hauptmieter erfasst. Die Untermieter mögen nach wie vor auch als Hausgenossen bezeichnet worden sein, für die Huldigungslisten wurden sie jedoch aus anderen Gründen größtenteils nicht aufgeführt. In den Huldigungslisten 1603 heißt es: Folgen die Haußgenossen in der Stadt Feuchtwangen auch Bürger sein. ${ }^{87}$ Daran schließt sich aber keine Auflistung mit Hausgenossen an, die keine Bürger waren. Detaillierter ist diese Personengruppe bei der Erbhuldigung 1723 in Prichsenstadt beschrieben: folgen die Erbgehuldigte Bürger so keine Häußer haben und zu Haußgenoßen sizen. ${ }^{88}$

Zumindest in den von mir untersuchten Gebieten in Franken und den hierfür bekannten Quellen ist mit dem Hausgenossen in der Regel ein Mieter aus der ärmeren Bevölkerungsschicht gemeint und kein Untergebener. Bedienstete, die im Haushalt mitlebten, wurden stattdessen als Ehalten bezeichnet. ${ }^{89}$

\section{Herberger}

Der historische Begriff Herberger/Herbergsmann ist dem Begriff des Mieters im heutigen Sprachgebrauch am verwandtesten, da mit Herberger nur die Wohnsituation als Mieter und nicht zugleich auch eine Rechtssituation angesprochen ist.

\footnotetext{
${ }^{84}$ Vgl. hierzu und im Folgenden: GöTZ 1986, S. 10, Anm. 31 (Stadtarchiv Würzburg R 5467 1/2).

${ }^{85}$ GÖTZ 1986, S. 119-122.

${ }^{86}$ StAN, Fm AN, Brdbg. Lit. Nr. 592ff.

${ }^{87}$ Ebd., Nr. 592, fol. 98'.

${ }^{88}$ Ebd., Nr. 602, fol. 735.

${ }^{89}$ Vgl. u. a. RECHTER 1985, S. 35, Nr. 958: Cunradt Kune, Dorothea sein hausfrau, der schlecht sein hab an auf tawsent gulldin, Hanns, Michel, Anna, Els unnd aber Els, sein ehalten.
} 
Im Deutschen Wörterbuch heißt es: „der mietsmann, der zur miete wohnende, womit in Baiern auf dem platten lande sich gewöhnlich der begriff einer hohen armut verbindet. " ${ }^{90}$ Unklar bleibt jedoch, warum in den Gewerbesiedlungen im Nürnberger Umland die Begriffe Beständner und Herberger zugleich Verwendung fanden. ${ }^{91}$ Regional scheint dieser Begriff vor allem im nordöstlichen Franken geläufig gewesen zu sein. So heißt es in einem Dekret im Fürstentum Brandenburg-Bayreuth im Jahr 1730 zur Unterscheidung, wer wie viel Nachsteuer zu zahlen hätte: „ob ein solcher [...] ein bloser Herberger ohne Besitzung einiger buergerlicher Gueter, oder aber, ob er buergerliche Gueter, es sei nun gleich ein Haus, Garten, Feld oder Wiesen und dergleichen besitze. “92 Belegt ist er aber auch für das Hochstift Bamberg im 17. und 18. Jahrhundert. ${ }^{93}$

\section{Beständner}

Ursprünglich und im juristisch korrekten Sinn handelt es sich bei einem Beständner um einen Pächter, der ein Wirtschaftsgut gepachtet hat, sei es ein Bauernhof, ein Stück Land, ein Gasthof oder ein Hammerwerk. Im dörflichen Bereich der Reichsstadt Nürnberg wurde der Begriff aber bereits früh auf alle Mietverhältnisse übertragen und nicht differenziert verwendet. So sind in den Bauernverzeichnissen ab 1529 unter diesem Begriff alle Bewohner subsumiert, die zur Miete wohnten. ${ }^{94}$ Überwiegend handelte es sich dabei um klassische Mieter, die lediglich ein Quartier, aber kein Land gepachtet hatten.

Spätestens seit Anfang des 17. Jahrhunderts scheint sich der Begriff des Beständners in Franken zu verbreiten. In den Huldigungslisten des Fürstentums Ansbach ist 1603 für eigentumslose Haushaltsvorstände noch der Begriff des Haußgenossen in Gebrauch. Einzig in der Stadt Schwabach werden diese bereits zu diesem frühen Zeitpunkt als Beständner, die keine Häuser haben, beschrieben. ${ }^{95} \mathrm{Zu}$ dem finden sich auch Hinweise auf die synonyme Verwendung der Begriffe Hausgenosse und Beständner, so heißt es in der Zusammenfassung der Huldigungslisten für das Amt Castell 1703: 21 Beständnere und Haußgenoßen. ${ }^{96}$

\footnotetext{
${ }^{90}$ DWB, Bd. 10, Sp. 1063.

${ }^{91}$ WENDEROTH 2019a, S. 190-195.

92 BRUNNER/ELLROd/LONGOLIUS 1747, S. 278.

${ }^{93}$ MORLinghaus 1940.

${ }^{94}$ Vgl. WENDEROTH 2019a, Kapitel I.2.9.1, S. 25; StAN, Rst. N, Bauernverzeichnisse.

${ }^{95}$ StAN, Fm AN, Brdbg. Lit. Nr. 592, fol. 233.

${ }^{96}$ Ebd., Nr. 599, fol. 176.
} 


\section{Inwohner}

Im Amt Roth werden in den Huldigungslisten von 1603 die Mieter dagegen als Inwohner bezeichnet. ${ }^{97}$ Die Bezeichnung des Inwohners ist in den Quellen aber auch schon zuvor gebräuchlich. So lässt sie sich bereits 1476 in einem kaiserlichen Privileg nachweisen; hier wurde sie verwendet für Personen, die in der Reichsstadt Nürnberg arbeiten und wohnen durften, jedoch nicht über das Bürgerrecht verfügten und damit in Nürnberg auch kein Haus erwerben konnten. ${ }^{98}$ In Roth scheint sich der Begriff zu halten, noch 1723 heißt es dort in den Huldigungslisten: An Bürgern, so Innwohner, und keine Häußer haben. ${ }^{99}$ In der abschließenden Zusammenfassung wird die Gruppe aber bezeichnet als Bürger, so in Zinn $\beta$ sizen. ${ }^{100}$

Der Begriff wurde aber auch weniger eindeutig verwendet. In den Huldigungslisten aus dem Jahr 1703 für die Stadt Feuchtwangen findet sich eine sehr differenzierte Zusammenstellung: ${ }^{101}$

Haußsässige Bürger

Haußgenoßen so Bürger

Inwohner, so zwar bürgerliche Häußer haben, aber nicht Bürger sind 4

Stiftsbürger

Stiftl. Haußgenoßen, so Bürger

Haußsäßige Juden

Juden, so Haußgenoßen

Wittben uf Bürgers Häußern

Weiber von Männern verlaßen 1

Wittib, so Haußgenoßin 1

Juden wittib 1

unbesetzte Bürgers Häußer 2

oede Haußplätz $\quad 15$

$\begin{array}{lr}\text { Schutzverwandte oder Pfahlbürger } & 8\end{array}$

In der Liste werden sowohl Haußgenoßen so Bürger als auch Innwohner, die bürgerliche Häuser haben, aber nicht Bürger sindt, erfasst. ${ }^{102}$ Auf den ersten Blick könnte man erwarten, dass die aufgeführten Inwohner über Eigentum verfügt haben,

\footnotetext{
${ }^{97}$ Ebd., Nr. 592, fol. 204-207' . Die Inwohner der Stadt Roth folgen daher erst auf fol. 210-211.

${ }^{98}$ Vgl. SCHUltheiss 1972, S. 191.

${ }^{99}$ StAN, Fm AN, Brdbg. Lit. Nr. 592, fol. 58'.

${ }^{100}$ Ebd., Nr. 592, fol. 60.

101 Ebd., Nr. 599, fol. 201'.

102 Ebd., Nr. 599, fol. 201'.
} 
dem dürfte aber nicht so gewesen sein, denn es handelt sich nachweislich bei drei der vier Personen um städtische Bedienstete: nämlich den Stadtmusket, den Bettelvogt und einen Nachtwächter, also um eine Personengruppe, die gemeinhin in einer Dienst- oder Mietwohnung lebte.

Die gleiche Verwendung des Begriffs ist für Feuchtwangen auch noch für das Jahr 1723 belegt, dort heißt es wiederum in der Huldigungsliste: Innwohner [sic!], die bürgerliche Häußer haben, aber nicht Bürger sind. ${ }^{103}$ Deren Anzahl hat sich jedoch von vier auf nunmehr einen verringert. Der Definition nach dürften sie Schutzgeld bezahlt haben und aus rechtlicher Sicht zur Gruppe der Schutzverwandte oder so genannte Pfahlbürger gehört haben, die die Auflistung 1703 abschließt. ${ }^{104}$

\section{Inquilinus}

Wie bereits aufgezeigt, ist die Bezeichnung der eigentumslosen Bevölkerung in den Quellen sehr unterschiedlich. In manchen Quellen werden sie als inquilinus oder inq. aufgeführt, was so viel bedeutet wie Mieter, Mietsmann. ${ }^{105}$ Gleichzeitig können jedoch auch andere Bezeichnungen für Mieter verwendet worden sein, wie im Falle Coburg: Hier wird in einem Dokument neben dem Begriff inquilinus auch der Begriff Inwohner für einen Mieter verwendet. ${ }^{106}$ Parallel dazu heißt es in den Coburger Dokumenten auch: ist geliehen, was ebenfalls eine Mietsituation vermuten lässt, jedoch für den Eigentumsübergang verwendet wurde. ${ }^{107}$

\section{Schutzverwandte, Verspruchleute, Mundleute}

Einwohner, die nicht den Bürgerstatus in den Städten erwarben, mussten eine Ersatzabgabe leisten, das sogenannte Schutz- oder Verspruchgeld. Georg Andreas Will beschreibt den engen Zusammenhang zwischen dem SchutzverwandtenStatus und dem Mieterdasein, indem er angibt, dass der Schutzverwandte sich die Zugehörigkeit zur Herrschaft seines jeweiligen Vermieters mit dem entsprechenden Schutzgeld erkaufen musste. ${ }^{108}$

Bereits im 17. Jahrhundert taucht in den Ansbacher Akten der Begriff des Schutzverwandten auf. Ernst Kober hat die Situation in Ansbach untersucht und kommt

\footnotetext{
${ }^{103}$ Ebd., Nr. 602, fol. 125.

${ }^{104}$ Ebd., Nr. 599, fol. 201'.

105 Zum Beispiel StadtACoburg, B 111, III, 115, Seelenlisten von 1721.

106 Ebd.

107 WOLTER 2010, S. 23f: Für das Haus Badergasse 5 in Coburg findet sich gleich zweimal der Eintrag: ist geliehen Jobst Raaben. Wolters deutet diese Notizen wie folgt. „Dem Eintrag von 1574 zufolge erwirbt Jobst Raaben die eine Hälfte, im Eintrag von 1588 die andere Hälfte dieses Hauses."

108 WiLl 1781, S. 74.
} 
zu folgendem Ergebnis: „Soweit ersichtlich, wohnten die Schutzverwandten in keinem eigenen Haus, sondern in Miete, manche in dem Gartenhaus eines Ansbacher Bürgers. " 109 Während im 17. Jahrhundert vor allem Frauen und Witwen den Status des Schutzverwandten erhielten, wird die Bezeichnung im 18. Jahrhundert häufiger gebraucht, die Zahlen steigen 1746 bis auf 83 Personen an. Laut den Berufsbezeichnungen gehören die Personen der Unterschicht an, genannt werden: Taglöhner, Gesellen, etc. ${ }^{110}$

Für die Frage nach den eigentumslosen Einwohnern sind die Schutzverwandten von großer Relevanz, da diese Personengruppe zum allergrößten Teil kein Eigentum erwarb, sondern zur Miete wohnte. In einer differenzierenden Anmerkung im Jahr 1686 für die Stadt Roth heißt es in den Huldigungslisten: Schutzverwanthe so auch keine Häußer haben. ${ }^{111}$ Diese Beschreibung bezeugt einerseits den Mieterstatus der Schutzverwandten, andererseits gibt sie auch einen Hinweis darauf, dass Schutzverwandte Häuser besitzen konnten. In einem Eintrag zu Schwabach heißt es im Jahr 1668 zu einem Hausbewohner, der wohl auch Eigentümer, aber kein Bürger war: Hannß Christoph Dürnbacher dieser will nicht Bürger werden. ${ }^{112}$

Hausgenossen oder Beständner, sprich Mieter, konnten hinsichtlich ihres Aufenthaltsstatus sowohl Bürger als auch Schutzverwandte sein. ${ }^{113}$ In Roth findet sich zum Beispiel vor der Auflistung der eigentumslosen Schutzverwandten eine Rubrik mit zwölf Beständnern. ${ }^{114}$ In diesem Fall handelte es sich bei den Beständnern also um Mieter, die das Bürgerrecht besaßen und daher gesondert von den nichtbürgerlichen Mietern (Schutzverwandten) erfasst worden sind. In den Huldigungslisten in Ansbach 1672 findet sich ebenfalls eine Differenzierung: Hier werden in der Einzelaufstellung unter der Überschrift Haußgenoßen 73 Namen und unter der Überschrift SchutzVerwande zwei Namen aufgeführt. ${ }^{115}$

Im 17. Jahrhundert stellen Schutzverwandte in den fränkischen Städten jedoch nur eine kleine Randgruppe gegenüber den Bürgern dar, folgt man den Angaben der Huldigungslisten, wobei auch hier wiederum gilt, dass die verschiedenen Begriffe synonym verwandt worden sind. In Gunzenhausen wurden zum Beispiel 1668 unter der Rubrik Schutzverwandte 23 Haushalte erfasst, eine Rubrik zu

\footnotetext{
${ }^{109}$ KOBER 1956, S. 31.

110 Ebd., S. 28-30.

${ }^{111}$ StAN, Fm AN, Brdbg. Lit. Nr. 596, fol. 464'.

112 StAN, Fm AN, Brdbg. Lit. Nr. 594, fol. 453.

113 11. Haußgenoßen so bürger: und 7. Haußgenoßen welche nit bürger sein, also im Schutz; StAN, Fm AN, Brdbg. Lit. Nr. 595, fol. 21'.

${ }^{114}$ StAN, Fm AN, Brdbg. Lit., Nr. 596, fol. 464'.

115 Ebd., Nr. 595, fol. 378-379.
} 
Hausgenossen oder Beständnern fehlt dagegen in dieser Aufstellung. ${ }^{116} \mathrm{Im}$ Vergleich mit den anderen erfassten Städten ist es jedoch mehr als unwahrscheinlich, dass hier sämtliche Mieter den Status von Schutzverwandten hatten, vielmehr ist davon auszugehen, dass in diesem konkreten Fall alle Mieter, egal ob Bürger oder Schutzverwandte, unter dem Begriff des Schutzverwandten versammelt sind. Denn bereits fünf Jahre später, 1673, werden für Gunzenhausen bei einer erneuten Huldigung fünfzehn Hausgenossen und lediglich drei Schutzverwandte erfasst. ${ }^{117}$ Aus diesem Grund ist bei der statistischen Auswertung, wie so häufig, mit einer großen Fehlertoleranz zu rechnen. Neben Gunzenhausen findet sich im Jahr 1668 nur noch in Schwabach eine größere Anzahl von Schutzverwandten. ${ }^{118}$ Den 23 Schutzverwandten stehen hier allerdings 62 männliche Hausgenossen gegenüber. ${ }^{119} \mathrm{Im}$ Jahr 1673 sind es in Schwabach 43 männliche Hausgenossen und fünfzehn Schutzverwandte, darunter vier Witwen. ${ }^{120}$ Die Schwabacher Huldigungsliste von 1723 ist deutlich differenzierter: Aufgeführt werden 186 Haußgenoßen, oder Bürger so keine aigenthumbl. Häußer haben und fünf Bürger, die außerhalb der Stadt wohnhaft waren. ${ }^{121}$ Bürgerwittiben, so keine aigenthumbl. Häußer haben, werden 24 genannt. ${ }^{122}$ Schutzverwanthe MannsPersonen sind 171 aufgelistet, ${ }^{123}$ weibliche Schutzverwandte $39 .{ }^{124}$ Für sämtliche Schutzverwandten ist kein Hausbesitz angeführt, sodass es sich hier wohl insgesamt um Mieter gehandelt haben muss. In Roth heißt es in den Huldigungslisten 1686: zwölf Haußgenoßen, so bürgerliche pflicht uf sich haben und fünfzehn Schutzverwanthe so auch keine Häußer haben. ${ }^{125}$

Aus den Huldigungslisten für Fürth 1703 wird aber auch ersichtlich, dass Hausgenossen/Beständner nicht immer und automatisch mit dem Begriff des Schutzverwandten gleichgesetzt werden können. In einer Aufstellung zu den Schutzverwandten des Amtes Cadolzburg vom 16. Juli werden 81 zum Amt gehörige Fürther Haushalte als Schutzverwandte geführt. ${ }^{126}$ Hierunter befanden sich aber auch sechs christliche und 22 jüdische Hausbesitzer, neben 25 jüdischen und 28 christlichen Mietern. ${ }^{127}$

\footnotetext{
116 Ebd., Nr. 594, fol. 643.

117 Ebd., Nr. 595, fol. 260 und 263.

118 Ebd., Nr. 594, fol. 473.

119 Ebd., Nr. 594, fol. 457'-459.

${ }^{120}$ Ebd., Nr. 595, fol. 442-444.

${ }^{121}$ Ebd., Nr. 601, fol. 116-120.

122 Ebd., Nr. 601, fol. 121'.

${ }^{123}$ Ebd., Nr. 601, fol. 122'-125'.

${ }^{124}$ Ebd., Nr. 601, fol. 126-127.

${ }^{125}$ Ebd., Nr. 596, fol. 463-463'.

126 Ebd., Nr. 599, fol. 199.

${ }^{127}$ Ebd., Nr. 599, fol. 192-193'.
} 
In der Liste für Ansbach aus demselben Jahr wird wiederum unterschieden zwischen Haußgenoßen, so keine Häußer haben und doch Bürger sind und Schutzverwandten. ${ }^{128}$ Letztere Gruppe besteht zu einem Gutteil aus Tagelöhnern, sodass es sich hier wohl ebenfalls um Mieter, jedoch ohne Bürgerrecht, gehandelt haben dürfte. Abgesehen von der undifferenzierten Verwendung des Begriffs in Fürth im Jahr 1703 kennzeichnet der Begriff des Schutzverwandten in der Regel einen Mieter.

\section{Pfahlbürger}

Während der Begriff bis ins 16. Jahrhundert einen Rechtsstatus zum Ausdruck bringt, wird er später auch stadttopografisch für Bewohner der Vorstädte verwendet, wohl im Hinblick darauf, dass diese zwar außerhalb der Stadtmauer, aber noch innerhalb des durch Pfähle gesicherten Stadtgebietes wohnten. ${ }^{129}$ In einer Definition des 18. Jahrhunderts heißt es: pfahlbuerger. es bedeutet dieses wort [...] diejenigen, welche leibeigen waren und sich in den staedten niederliessen.[...] Werden die Vorstaedtere also genennet, vermuthlich weil sie durch Phaele oder Pallisaden von der Stadt abgesondert wurden. ${ }^{130}$ Im gleichen Text werden die Pfahlbürger aber auch als Hausgenossen genannt und damit als eigentumslos charakterisiert. In letzterem Sinne scheint der Begriff auch in den Huldigungslisten des Fürstentums Ansbach verwendet worden zu sein. Er taucht dort nicht sehr häufig auf, dafür aber früh, nämlich bereits in der ersten Aufstellung von 1603 für den Ort Crailsheim. Dort werden neun Pfaalburger aufgeführt. ${ }^{131}$ Anschließend wird der Begriff in den Huldigungslisten erst wieder 1673 in Wassertrüdingen verwendet, genannt werden acht Pfahlbürger genannt. ${ }^{132}$

Überhaupt scheint es lokale Traditionen bei der Begriffswahl gegeben zu haben, denn in den Huldigungslisten des Jahres 1686 findet sich wiederum nur in Wassertrüdingen die Rubrik Pfahlbürger, diesmal waren es dort sechzehn Männer. ${ }^{133}$ Wie bereits im Falle von Crailsheim 1603 fehlt auch hier eine Rubrik zu Hausgenossen oder Schutzverwandten, sodass davon ausgegangen werden kann, dass der Begriff des Pfahlbürgers synonym für Hausgenossen und Schutzverwandte verwendet worden ist und es sich somit auch um Mieter handelte. Die synonyme Verwendung des Begriffs ist für Feuchtwangen zum Beispiel für das Jahr 1723

\footnotetext{
${ }^{128}$ Ebd., Nr. 599, fol. 368'.

${ }^{129}$ vorstaedter, so eigne haeuser haben, auch die pfal-buerger, so ausser der stadt wohnen, Codex Augustei I 1463, datiert 1612; zitiert nach DRW: (Art.) Pfahlbürger.

130 WIESAND 1762, S. 808.

${ }^{131}$ StAN, Fm AN, Brdbg. Lit. Nr. 592, fol. 46.

132 Ebd., Nr. 595, fol. 570.

${ }^{133}$ Ebd., Nr. 596, fol. 615. Die Rubriken Hausgenossen und Schutzverwandte fehlen.
} 
belegt, dort heißt es wiederum in der Huldigungsliste: Schutzverwandte oder so genannte Pfahlbürger. ${ }^{134}$

\section{Hintersasse}

Als Hintersasse wird derjenige bezeichnet, der einer anderen Person rechtlich nachgeordnet war. In der Hauptverwendung des Wortes wird damit der Untertanenstatus der Landbevölkerung angezeigt, wenn von Hintersassen städtischer Stiftungen oder auch einzelner Bürger gesprochen wird. In den Huldigungslisten für Schwabach 1603 wird diese Gruppe als Spital: Pfründt und Burgbauern bezeichnet. ${ }^{135}$ Es handelt sich dabei um lehensabhängige Bauern.

Diese rechtlich nachgeordnete Stellung gilt aber auch für Mieter, da der Vermieter de jure verantwortlich und haftbar für den Mieter war. ${ }^{136}$ Wohl aufgrund dieses Sachverhaltes wurde der Begriff gelegentlich auch für Mieter verwendet. ${ }^{137}$ Es ist aber keineswegs so, dass es sich bei einem Hintersassen immer um einen Mieter gehandelt hat.

\section{Verwendete Quellen}

Die Quellen zu Bevölkerungszahlen sind bisher selten im Hinblick auf die eigentumslose Bevölkerung ausgewertet worden.

Nachfolgend möchte ich die wichtigsten Quellen würdigen, die für diese Arbeit herangezogen worden sind. Auf den ersten Blick mag die Würdigung einzelner Quellen sehr ausführlich erscheinen, es ist jedoch das Gegenteil der Fall: Die aufgeführten Quellen enthalten sämtlich wertvolle sozial- und regionalhistorische Angaben und waren mit Ausnahme des Reichssteuerregisters und des Nürnberger Grabenbuches noch nicht Gegenstand einer wissenschaftlichen Betrachtung. In meiner Analyse musste ich mich auf die für das Thema der eigentumslosen Haushalte relevanten Aspekte beschränken. Eine monografische Bearbeitung jeder dieser Quellen stellt ein Desiderat dar. Die nachfolgenden Würdigungen sollen daher zumindest einen ersten Überblick zu diesen Quellen ermöglichen und zu einer weiteren kritischen Auseinandersetzung ermutigen.

\footnotetext{
${ }^{134}$ Ebd., Nr. 599, fol. 201'.

135 StAN, Fm AN, Brdbr. Lit. Nr. 592, fol. 233.

136 WENDEROTH 2021, S. 288-290.

137 WENDEROTH 2019a, S. 56.
} 


\section{Wehrdienstlisten}

Hier sind die historischen Verzeichnisse in zwei Gruppen zu unterscheiden: Erstens in die Erfassung der wehrfähigen Männer, entweder zur aktiven Kriegsführung oder zu Verteidigungszwecken. Zweitens in Listen zur Neuerrichtung oder zum Ausbau der Wehranlagen, also einem indirekten Wehrdienst. Hierfür konnten sämtliche arbeitsfähigen Bewohner unabhängig ihres Geschlechtes und Alters herangezogen und damit auch erfasst werden. Letztere Listen sind daher für sozialgeschichtliche Fragestellungen in der Regel deutlich ergiebiger.

Für meine Untersuchungen sind zwei Listen von Interesse, da sie bei entsprechender Interpretation Hinweise zu den eigentumslosen Haushalten im Nürnberg am Anfang des 15. Jahrhunderts geben. Die Quellen wurden bereits 1907 von Caspar Ott in einer kleinen Schrift gewürdigt, ohne jedoch Fragen zu den eigentumslosen Einwohnern zu behandeln. ${ }^{138} \mathrm{Im}$ Rahmen meiner Forschung war es zeitlich nicht möglich, die Listen neu zu bearbeiten. Ich stütze mich bei der statistischen Auswertung daher auf die von Ott ermittelten Angaben. Im Folgenden gehe ich daher nur kurz auf die Beschaffenheit der beiden Quellen ein.

\section{Nürnberger Grabenbuch}

Ursächlich für die Arbeiten an der Stadtbefestigung und damit dem Grabenbau waren die Kriegszüge der Hussiten ab 1419. Die Erfassung der arbeitsfähigen Bevölkerung im grapenbuch selbst geht auf einen Ratserlass vom 16. Oktober 1427 zurück. ${ }^{139}$ Mit der Zählung selbst wurde aber erst am 4. Dezember 1430 begonnen. Erfasst sind alle Personen beiderlei Geschlechts, die älter als zwölf Jahre waren, in ihren jeweiligen Herdgemeinschaften. Namentlich genannt werden dabei jeweils nur die Vorstände der Herdgemeinschaften, nicht jedoch die weiteren arbeitsfähigen Personen in den Herdgemeinschaften. Deren Gesamtzahl ist lediglich in römischen Ziffern angegeben. Dies setzt der Interpretation der Quelle Grenzen.

Da die Möglichkeit bestand, sich durch eine Zahlung dieses gemeinschaftlichen Dienstes zu entledigen, tragen die entsprechenden Listen bereits Wesenszüge der nachfolgend behandelten Kopfsteuerlisten. Als Ablösung vom Frondienst waren $10 \mathrm{dn}$ (Pfennig) pro Person fällig, die Zahlung dieser Summe wurde ebenfalls in den Listen vermerkt. Die Aufnahme erscheint vollständig und hat sich bis heute ohne Verluste erhalten. ${ }^{140}$

\footnotetext{
138 OTT 1907.

139 OTT 1907, S. 30.

${ }^{140}$ StAN, Rst. N, Amts- und Standbücher 284 (Grabenbuch 1430).
} 


\section{Verzeichnis der wehrfähigen Bevölkerung Nürnberg 1431}

Caspar Ott ist es als großes Verdienst anzurechnen, dieses aussagekräftige Verzeichnis gehoben und als Ganzes erschlossen zu haben. Galt das Quellenmaterial doch bis dahin als zerstreut und nur unvollständig überliefert. Zudem konnte er die Zählung der waffenfähigen Bevölkerung auf den Zeitraum vom März bis zum Juli 1431 datieren. ${ }^{141}$ Erfasst wurden nicht nur die waffenfähigen Bewohner der Stadt, sondern auch die Nürnberger Untertanen auf dem Land, was die Dauer der Zählung erklärt. Als wehrfähig galt die männliche Bevölkerung zwischen 18 und 60 Jahren. Die Zählung erfolgte separat für jedes der damaligen sechs Stadtviertel. In den Stadtvierteln selbst bilden die Gassenhauptmannschaften die nächste Gliederungsebene. Die Zählungslisten selbst haben sich zum Teil nur als lose Zettelsammlung erhalten, was nicht verwundert, da die Listen von den Gassenhauptleuten erstellt worden sind. ${ }^{142}$ Ott ermittelt für diese Zeit 94 Gassenhauptmannschaften. Entsprechend gibt es auch Abweichungen in der Art der Überlieferung. Für die Frage nach den eigentumslosen Haushalten ist eine Besonderheit in den Listen von großer Bedeutung: Durch Klammern vor den Namen werden vereinzelt Namenseinträge als zusammengehörig gekennzeichnet. Es konnte bisher jedoch nicht abschließend geklärt werden, ob die so gekennzeichneten Personen in einem Anwesen als Herdgemeinschaft zusammengelebt haben, oder diese bereits über in sich abgeschlossene Wohnungen mit eigenen Herdstellen verfügten. Letztere Situation setzt nicht per se Etagenwohnungen voraus, es könnte sich auch um verschiedene Häuser auf einem Anwesen gehandelt haben. ${ }^{143}$ Auf jeden Fall beinhalten diese Einträge damit auch eigentumslose Haushalte, denn geteiltes Wohneigentum lässt sich für Nürnberg bisher nicht im größeren Umfang nachweisen.

Darüber hinaus folgt auf viele Namenseinträge ein weiterer Name mit anschließendem Zusatz bey im/bey ir: Hans Gundelfinger Els des Bollenden Tochter bey im oder Barb. Winterbergern die Polanin bey ir. ${ }^{144} \mathrm{Ott}$ interpretiert diese Einträge wahlweise als Personen, die sowohl zu einer "Haushaltungsgemeinschaft“ als auch zu einem „Haushalt“ gehören. Mit dem Begriff „Haushaltungsgemeinschaft" spricht er wohl eine Wohnsituation an, die von mir zur besseren Abgrenzung vom Begriff „Haushalt“ als „Herdgemeinschaft“ bezeichnet wird. Aufgrund dieser fehlenden Abgrenzung bleibt er entsprechend diffus in der Verwendung der Begrifflichkeiten.

\footnotetext{
${ }^{141}$ OTT 1907, S. $24 f$.

142 Ebd., S. 29.

${ }^{143}$ Ott interpretiert die mit Klammern zusammengefassten Einträge vorschnell als Häuser. Er erhält aufgrund dieser Annahme eine Gesamtzahl von Häusern, die weit über der Zahl der 1796 vergebenen Hausnummern liegt. Ebd., S. 30.

144 Zitiert nach OTT 1907, S. 30.
} 
Mit allergrößter Wahrscheinlichkeit sind diese Einträge als Untermietverhältnis zu interpretieren, darauf deutet sowohl die Wortwahl bei ihm/bei ihr hin, als auch der Umstand, dass es sich bei den zweiten Namensnennungen überwiegend um Frauen handelt. In der Auswertung der Listen finden diese Einträge jedoch keine Berücksichtigung. Um die Frage nach Untermietverhältnissen sowohl qualitativ als auch quantitativ zu klären, wäre eine erneute Auswertung der überlieferten Listen mehr als wünschenswert.

\section{Kopfsteuerlisten}

Auch im Falle von besonderen finanziellen Lasten für ein Gemeinwesen wurde bereits in der Vergangenheit versucht, diese auf möglichst viele Schultern zu verteilen. Von diesen Sonderabgaben waren daher nicht nur die vermögenden Bevölkerungsschichten mit Eigentum, sondern auch die eigentumslose Bevölkerung betroffen. Sofern sich für diese Abgabeform schriftliche Namenslisten erhalten haben, ermöglichen sie einen Blick auf nahezu die gesamte damalige Bevölkerung. Erfasst sind dort jedoch nicht die aus verschiedenen Gründen von Abgaben befreiten Personen. Für die Sozialgeschichtsforschung und insbesondere auch für die Frage nach den eigentumslosen Haushalten innerhalb der Bevölkerung handelt es sich um eine ganz entscheidende Quellengruppe, lassen sich Eigentumslose doch ansonsten weder in ihrer Gesamtheit noch namentlich nachweisen.

\section{Reichssteuerregister 1497 - Der Gemeine Pfenning}

Eine der frühesten seriellen Quellen zur Bevölkerungsgeschichte und der Sozialstruktur ist das Reichssteuerregister aus dem Jahr 1497, das sich für das Fürstentum Brandenburg-Ansbach-Kulmbach sowie die Reichsstadt Nürnberg und die Reichspflege Weißenburg erhalten hat. ${ }^{145}$ Erfasst sind dort die steuerbaren Herdgemeinschaften. An erster Stelle jeder Herdgemeinschaft steht der Haushaltsvorstand mit Frau beziehungsweise eine alleinstehende Frau, diese waren auch für die Abgabe der Steuer für alle Haushaltsmitglieder verantwortlich. Zu den einzelnen Herdgemeinschaften gehörten zudem noch Bedienstete, Kinder über fünfzehn Jahre, Altenteiler und sonstige Familienangehörige, aber auch Mieter. Gerhard Rechter bezeichnet diese Einheiten als „Rechnungseinheit Haushalt“, ich bezeichne sie als Herdgemeinschaft. ${ }^{146}$ Die Organisation der Steuererhebung

145 Die Verzeichnisse liegen als editierte Quellensammlung vor; vgl. RECHTER 1985, RECHTER 1988 und FLEISCHMANN 1993.

Eine ausführliche Würdigung der Quelle findet sich bei RECHTER 1990.

${ }^{146}$ Vgl. hierzu und im Folgenden RECHTER 1990, S. 72-73. 
durch die Reichsstadt Nürnberg ist von Otto Puchner bereits ausführlich beschrieben worden ${ }^{147}$ und soll daher an dieser Stelle nicht weiter ausgeführt werden.

Insbesondere die Verzeichnisse für das Fürstentum Brandenburg-Ansbach-Kulmbach enthalten vielfache Hinweise auf die eigentumslose Bevölkerung. Diese ist in der Regel durch die Bezeichnung Hausgenosse etc. gekennzeichnet. Bedienstete werden als Knecht, Magd oder sonstige Ehehalten unterschieden, Verwandte durch entsprechende Bezeichnungen charakterisiert.

Aber auch diese Erfassung erfolgte nicht einheitlich. Am augenscheinlichsten weichen einige Verzeichnisse dahingehend ab, dass sie lediglich summarisch die Anzahl der über Fünfzehnjährigen angeben und auf jegliche Differenzierung der Einzelpersonen verzichten. Dies gilt insbesondere für Nürnberg, hier wurden nicht die Häuser, sondern die Herdgemeinschaften veranlagt. ${ }^{148}$ Da außer dem Namen des Haushaltsvorstandes und der Personenanzahl und dem Betrag der Vermögenssteuer keine weiteren Anmerkungen erfolgen, kann das Reichssteuerregister für Nürnberg selbst bei der Identifizierung eigentumsloser Haushalte nicht weiterhelfen.

Summarisch erfasst sind neben der Reichsstadt Nürnberg und ihrem Landgebiet auch die brandenburg-ansbachischen Ämter Lobenhausen ${ }^{149}$ und Thann ${ }^{150}$ und - für diese Untersuchung wichtiger - auch einige Städte, wie zum Beispiel Prichsenstadt, ${ }^{151}$ Baiersdorf, ${ }^{152}$ vor allem aber auch die großen Städte Kitzingen ${ }^{153}$ und Schwabach ${ }^{154}$.

Da in den Listen anscheinend summarisch die Herdgemeinschaften mit ihrem Gesinde/Personal und eventuell auch den Untermietern (Hausgenossen) erfasst sind, nicht jedoch die Anzahl der Häuser, können nur bei entsprechendem Wissen um die Häuserzahl im Jahr 1497, anhand der überzähligen Haushaltungen, zumindest überschlägig weitere eigentumslose Haushalte ermittelt werden. Der Überhang von Herdgemeinschaften gegenüber der Anzahl der Häuser ist ebenfalls den eigentumslosen Haushalten hinzuzufügen. Diese Gruppe lässt sich als selbstständige Hauptmieter, die in einer eigenen Mieteinheit im Haus des Vermieters mitlebten, interpretieren. Die Gruppe der zur Gänze vermieteten Häuser und der darin lebenden eigentumslosen Haushalte lässt sich aber auch auf diese Weise nicht ermitteln: Zumindest für Nürnberg ist für 1564 bekannt, dass eine

\footnotetext{
147 PUCHNER 1975, S. 909-915.

148 FLEISCHMANN 1993, S. XXI.

149 RECHTER 1985, S. 44-45.

150 Ebd., S. 46-48.

${ }^{151}$ Ebd., S. 104-107, Eintrag Nr. 2971-3080.

152 Ebd., S. 244-245, Eintrag Nr. 6090-6160.

153 Ebd., S. 278-301, Eintrag Nr. 6658-7441.

${ }^{154}$ Ebd., S. 434-444, Eintrag Nr. 10223-10687, darunter aber auch viele Haushalte aus den umliegenden Orten.
} 
große Zahl von Häusern komplett vermietet war. In den Reichssteuerlisten finden sich lediglich für die Stadt Roth bei drei Einträgen jeweils am Ende der Namensauflistung der Zusatz alle haußgenoßen, ${ }^{155}$ in Selb im Fichtelgebirge sind sechs weitere Einträge gekennzeichnet, ${ }^{156}$ sodass in diesen Fällen von zur Gänze vermieteten Anwesen ausgegangen werden kann.

Wie in vielen dieser vorstatistischen Quellen verdichten sich damit die Hinweise, dass es sich bei den Personen, die explizit als eigentumslos kenntlich gemacht werden, nur um eine Teilgruppe unter den Mietern gehandelt hat. Insbesondere die öffentlich Bediensteten wären in allen Orten als Mietergruppe zu berücksichtigen - sofern sie nicht als Hausgenossen bezeichnet werden - da sie in jeder größeren Siedlung vorhanden waren. In der Kleinstadt Pegnitz sind sie durch ihre Berufsbezeichnung kenntlich gemacht: Unter den 70 Einträgen sind es hier allein drei Listeneinträge: 4582 Mostel und sein Haußfr(au), arm hirt; 4591 Statknecht und sein haußfraw; 4593 Kirchner und statschreiber, sein haußfraw, ein standt, hab(e)n I son. ${ }^{157}$ Die hochrangigen Beamten fehlen aber auch hier. Der Klerus ist in der Regel nicht erfasst, da dieser die Steuer über den jeweiligen Diözesanbischof zu entrichten hatte. ${ }^{158}$ Allein aus dem Klerus und den hohen Beamten ergäbe sich rechnerisch ein zusätzlicher Mieteranteil von 3\% bis $5 \%$, bezogen auf alle Haushalte.

Eine Besonderheit in der Erfassung stellt die Stadt Hof dar. Allein für die Kernstadt finden sich 775 Einträge. Es scheint so, als ob in diesem Fall alle Haushalte mit einer eigenen Nummer erfasst wären. Bei den Einträgen findet sich nur in seltensten Fällen die Bezeichnung als Hausgenosse. Dass die Erfassung hier anders vonstattenging, legt auch ein Eintrag nahe, aus dem klar die Vermietung eines Hauses hervorgeht: 676 Anna von Hawsch, in Heintz Roten hinter haw $3 .{ }^{159}$ Besagter Heinz Rot findet sich unter der Nummer 642. Für sein Anwesen werden an anderer Stelle - nicht am Ende seines Eintrages! - zwei weitere Miethaushalte genannt: 677 Nickel Erhart; 678 Alt Soffler cu(m) uxo(r)e; im Roten haw $3 .{ }^{160}$ Eine solche Differenzierung und nachvollziehbare Verteilung von Bewohnern eines Anwesens auf mehrere Einträge findet sich ansonsten nicht. Ein weiteres Indiz

\footnotetext{
155 RECHTER 1985, S. 35 f, Eintrag 950, 951 und 983.

${ }^{156}$ Die Liste für Selb ist sehr detailliert, daraus ist zu schließen, dass das Wort Hausgenosse sich auf die davor namentlich genannten Personen, mithin bei den Einträgen 4874, 4875, 4922, 4923, 4924, 4925 auf das ganze Haus bezieht. STAB, Geheimes Hausarchiv Plassenburg, Akten und Bände 5977/1, fol. 28, zitiert nach RECHTER 1988, S. 25.

157 Ebd., fol. 125; zitiert nach RECHTER 1988, S. 124.

158 RECHTER 1990, S. 73.

${ }^{159}$ STAB, Geheimes Hausarchiv Plassenburg, Akten und Bände 5977/1, fol. 133-134, zitiert nach RECHTER 1988, S. 134-135.

160 Ebd.
} 
für diese Vermutung sind die vielen Einpersonenhaushalte unter den 775 Einträgen, die in dieser Massierung sonst nicht auftauchen, darunter auch viele Einträge von alten alleinstehenden Frauen. Zuletzt sei noch auf folgende Einträge verwiesen: 1082 Die alt Steinerin, witbe; 1083 Jung Steinerin; 1084 Sporhans; 1085 Els Sporhansin. ${ }^{161}$ Hier dürfte es sich nicht um vier Haushalte oder gar vier Häuser, sondern wohl eher um nur zwei Haushalte gehandelt haben. $\mathrm{Zu}$ bedenken ist auch, dass sich die Listen zum Gebiet oberhalb Gebürg, also auch zu Hof, nicht im Original, sondern nur in Abschriften erhalten haben. ${ }^{162}$

Berücksichtigt man all diese Anmerkungen, wird im Ergebnis deutlich, dass im Reichssteuerregister in den fränkischen Landstädten am Ende des 15. Jahrhunderts nur wenige eigentumslose Haushalte in unserem heutigen Sinn aufgeführt werden. Der Vergleich mit weiteren Quellen für Wunsiedel ${ }^{163}$ und Erlangen ${ }^{164}$ macht deutlich, dass es sich bei den als Hausgenossen oder alte Frauen bezeichneten Personen nicht um die einzigen Mieter gehandelt haben kann, sondern damit lediglich eine Teilgruppe angesprochen ist.

Bisher ist nicht bekannt, was zu dieser Differenzierung im Rahmen der Erfassung geführt hat. Denkbar ist, dass unter dem Begriff der Hausgenossen lediglich die Eigentumslosen ohne Bürgerrecht notiert sind: Im 17. und 18. Jahrhundert wurden in vielen dieser Orte bei der Erb- und Landeshuldigung die Mieter unterschieden in Hausgenossen/Beständner mit Bürgerrecht und Schutzverwandten/ Pfahlbürger ohne Bürgerrecht. In einigen Fällen scheinen auch hier die Mieter mit Bürgerrecht gar nicht von der Liste der bürgerlichen Eigentümer geschieden, sondern mit diesen als wirkliche Mannschaft gemeinsam erfasst worden zu sein. So treten uns in den Listen für Wassertrüdingen der Jahre $1673,{ }^{165} 1686 / 87^{166}$ nur Pfahlbürger als separat erfasste Mietergruppe entgegen. Noch deutlicher wird die Situation, wenn man die Frauenhaushalte betrachtet, die 1497 ungewöhnlich zahlreich als Hausgenossen erfasst worden sind. In den jüngeren Huldigungslisten finden sich die weiblichen Miethaushalte nämlich überwiegend in der Rubrik der Schutzverwandten erfasst, sie wurden nur selten unter den Hausgenossen gelistet. Besonders deutlich wird dies anhand der Angaben aus dem Jahr 1723 für Gunzenhausen, Merkendorf, Roth, Schwabach (vgl. Tab. Anhang I.8.). ${ }^{167}$ Im Hinblick auf den hohen Anteil von Einpersonen- und Frauenhaushalten, die

\footnotetext{
161 Ebd., fol. 37; zitiert nach RECHTER 1988, S. 34.

162 RECHTER 1988, S. XIV.

${ }^{163}$ Vgl. JÄGER 1987.

${ }^{164}$ Vgl. Kapitel 6.

165 StAN, Fm AN, Brdbg. Lit. Nr. 595, fol. 569-571.

${ }^{166}$ Ebd., Nr. 596, fol. 612-614'.

${ }^{167}$ Ebd., Nr. 596, fol. 485-494. In den Huldigungslisten des Jahres 1686/87 werden zum Beispiel für Schwabach nur 52 männliche Hausgenossenhaushalte genannt, denen 33 männliche und 23 weibliche Haushalte von Schutzverwandten gegenüberstanden.
} 
in den Listen des Reichssteuerregisters zumeist als Hausgenoßen bezeichnet werden, scheinen hierunter vor allem Untermietverhältnisse erfasst worden zu sein, während Hauptmieter, die eine Herdstelle gemietet hatten, nicht eigens gekennzeichnet und damit von den Hauseigentümern unterschieden worden sind. Da die Untermieter aber in den meisten anderen Verzeichnissen gänzlich fehlen, ist ein direkter Vergleich dieser Quelle mit anderen Überlieferungen zum Anteil eigentumsloser Bevölkerung nur in wenigen Fällen möglich.

\section{Türkensteuerregister 1716/17}

Weitere Erhebungen für Kopfsteuern sind mit den sogenannten „Türkensteuern“ für die Jahre 1663/64, 1716/17 und 1739 überliefert. ${ }^{168}$ Bei letzterer Steuer heißt es: der geringste Bürger gab 11/2 fl, ein Kaufdiener 1 fl ein Knecht und eine Magd aber 30 Kreuzer. ${ }^{169}$

Näher betrachtet wird allerdings im Folgenden lediglich die Steuer der Jahre 1716/17, da sich nur für diese Zählung Listen erhalten haben (Abb. Titelseite.): ${ }^{170}$ Im Jahr 1716 wurde von der Reichsstadt Nürnberg beschlossen, die Steuer Anfang des Jahres 1717 einzusammeln. Zur Erhebung wurden sieben Vorgaben definiert. Darin heißt es unter anderem:

1. Alle Bürger und Schutzverwanthe in denen Städtlein, auch die Unterthanen und Beständern in den Dorffschaften, mit Beysetzung was ein jeder so wohl von Höfen und Hütten als walzenden Stücken jährlich an Haupt-Steuer $z u$ geben hat. 2. Wieviel Kinder das das 15te Jahr hingelegt haben und ob die solchen in ihren eigenen Haushaltungen zu ihrer Nahrung gebrauchen, oder ob solche anderwärtig und wo? in Diensten stehen.

3. Wieviel Schreiber, Diener, Gesellen, Handwercks=Pursche, Knecht und Mägde jeder habe, und wie viel er jedem zu Lohn gebe.

5. Sollen auch die Wittwen mit ihren Angehörigen beschrieben werden.

6. Auch diejenigen, die sich den Winkel in ihren verkaufften Häußern vorbehalten und ausgedinget haben.

7. In Summa alle und jede, die in denen Städtlein und Dörffern sich aufhalten, sie mögen seyn weß Condition sie wollen, groß und klein, die das 15te Jahr erreichet haben, Geistlich und weltlich, civil oder Millitar=Bediente, Einspänniger, Jäger, Ampt=Knecht, arm und reich, niemand außgenommen, und sollte er auch nur ein Bettler seyn, mit Beysetzung womit er sich nehren, und was für Handthierungen sie treiben. ${ }^{171}$

\footnotetext{
${ }^{168}$ StadtAN, B11 Nr. 51, S. 46' ; hier wird jedoch die Türkensteuer von 1716/17 nicht erwähnt. 169 Ebd.

170 StAN, Rst. N, Bauernverzeichnisse 7.

${ }^{171}$ Ebd., Einführung ohne fol.
} 
Für die Reichsstadt Nürnberg selbst haben sich die Listen leider nicht erhalten, sondern nur einige Dokumente, die sich mit der Erhebung beschäftigen. ${ }^{172}$ In einer der Ausführungen wird detailliert aufgeführt, wie viel wer zu geben hat. Zum Beispiel wird differenziert zwischen Handwerkern mit Hauseigentum und denen, die keine eigenen Häußer haben, letztere zahlten nur $1 \mathrm{fl} 30 \mathrm{xr}$ bis $2 \mathrm{fl}$, Hausbesitzer jedoch 3 bis 6 fl. ${ }^{173}$

Die Steuererhebung erfolgte dann aber nicht durchgängig in dieser Differenzierung. In Altdorf sind zu Beginn die Hauseigentümer aufgeführt, anschließend folgt eine Auflistung der Beständner, die von der Liste der Wittiben beendet wird. ${ }^{174}$ Der konkrete Wohnort der Mieter (Beständner und Witwen) kann anhand dieser Aufstellung nicht nachvollzogen werden. Lediglich für die Stadt Lauf ist die Aufstellung in sehr differenzierter Form erfolgt. ${ }^{175}$ Dort werden die einzelnen Häuser mit ihren sämtlichen Bewohnern gelistet. Hinter dem Eigentümer folgen dort auch die Mieter. Für die Mieter werden wiederum verschiedene Begriffe verwandt. So heißt es zum Beispiel, bei einem zur Gänze vermieteten Haus:

50. In Leonhard Schmids Häuslein, zahlt

Paulus Reck, [...]knecht ... -20-

1 Tochter 21. Jahr alt ist $z$ u Gründlach in Diensten

Conrad Götz Schutzverwanther ... -20-

Margaretha Haaßin, Rockenspinnerin zahlt ... -3-

Veit Fischer, Maurergesell ... -15-

Catharina [...], Rockensp. .... -3- . ${ }^{176}$

In der Regel bewohnt der Eigentümer das angegebene Haus aber selbst, die Mieter sind dann unter der Überschrift Im Zinnße aufgelistet, ${ }^{177}$ es gibt aber auch die Überschrift Innwohnerin, ${ }^{178}$ seltener Inwohner. ${ }^{179}$ Dabei ist es keineswegs so, dass nur arme Menschen als Inwohner lebten, unter ihnen findet sich auch Herr RegimentsQuatierMeister Hummel zahlt ... -1fl-. ${ }^{180}$

Nur wenige Male taucht die Bezeichnung In Wohnung als Zwischenüberschrift auf, so heißt es unter dem Eintrag 75: In Wohnung Anna Dörin, Lodenspinnerin

\footnotetext{
172 StadtAN, B 11 Nr. 86.

${ }^{173}$ StadtAN, B 11 Nr. 951, pag. 11: Die Einforderung und den Erlang der Türken-Steuer betreffend Anno 1717.

${ }^{174}$ StAN, Rst. N, Bauernverzeichnisse 7, fol. 318-332.

175 Ebd., fol. 402-408'.

${ }^{176}$ Ebd., fol. 403' : Eintrag 50.

177 Ebd., fol. 403' : Eintrag 46.

178 Ebd., fol. 403' : Eintrag 48.

${ }^{179}$ Ebd., fol. 405' : Eintrag 104. Innwohner David Ließ, Taglöhner, zahlt ... -10-.

${ }^{180}$ Ebd., fol. 402: Eintrag 6.
} 
zahlt... -3- ${ }^{181}$ Vom Stand, Beruf und Steuersatz unterscheidet sich diese Mieterin nicht von den zuvor genannten Mieterinnen unter dem Begriff Im Zinnße. In Lauf wurden bei der Zählung zudem auch die Alteigentümer im Winkel erfasst, diese spielen prozentual betrachtet jedoch keine große Rolle. ${ }^{182}$

Nachfolgend wird von mir jedoch exemplarisch lediglich die Mietbevölkerung der Stadt Altdorf detailliert ausgewertet (vgl. Tab. 5 und 6). Weiter unten findet sich eine tabellarische Zusammenstellung der Mietbevölkerung in den vier nürnbergischen Städten Altdorf, Hersbruck, Lauf und Velden (vgl. Tab. 21).

\section{Quartiergeldbeiträge Nürnberg 1798}

Eine weitere Quelle mit Angaben zur Mietbevölkerung liegt mit der schriftlichen Aufstellung der Quartiergeldbeiträge für die Reichsstadt Nürnberg vom Ende des Untersuchungszeitraumes vor. Die Zahlung erfolgte als Ablösung der privaten Haushalte von der Pflicht, ein Soldatenquartier für die durchziehende französische Armee zu stellen, und war nach den jeweiligen finanziellen Leistungsmöglichkeiten gestaffelt. Die Zahlung konnte aber auch durch die Stellung eines Soldatenquartiers vermieden werden, was aber kaum praktiziert wurde, von daher unterscheidet sie sich von den älteren Kopfsteuern: Nur in wenigen Einträgen findet sich folgender Vermerk, wie bei L 180: Hartlein, Scribent, hat 1 Mann in Quartier. ${ }^{183}$ Aufgrund der Naturalleistung war er von dem Beitrag befreit.

Die Berechnung und Erhebung der Quartiergeldbeiträge erfolgt vom 1. Oktober 1797 bis zum 31. Januar 1798. Erfasst sind die einzelnen Haushaltsvorstände in jedem Haus, weitere Haushalts- und Familienmitglieder werden hingegen nicht genannt. Die Ordnung folgt den damaligen Hausnummern, getrennt nach Sebalder und Lorenzer Seite, wobei die Einträge auf der Lorenzer Seite mit L 1578 enden. ${ }^{184}$ Nachdem auch verarmte Personen erfasst sind, die kein Quartiergeld zahlen, ${ }^{185}$ scheinen aber grundsätzlich sämtliche Haushalte hier dokumentiert worden zu sein. Neben dem Namen ist der Beruf angegeben und die individuelle Quartiergeldhöhe. Nach der detaillierten Aufstellung folgt eine Zusammenfassung der Einnahmen, sortiert nach den Gassenhauptmannschaften. Im zweiten

\footnotetext{
${ }^{181}$ Ebd., fol. 405' : Eintrag 75.

182 Ebd., fol. 402, fol. 402' und fol. 406.

${ }^{183}$ Quartiergeldbeiträge 1798, S. 3.

${ }^{184}$ Ebd., S. 68.

185 Ebd., zum Beispiel L162: Staudinger, Schneller, verarmt; Sieder, Schneller, arm; Seizin, arm. Alle drei Haushalte zahlen nichts.
} 
Teil finden sich die Zahlungslisten mit den Ausgaben für die Soldaten vom 1. Oktober 1797 bis 28 . Februar $1798 .{ }^{186}$ Von der folgenden zweiten Quartiergelderhebung liegen die Ergebnisse nur in einer Zusammenfassung vor, sodass keine Informationen zu den Eigentumsverhältnissen daraus ersichtlich werden. ${ }^{187}$

Auffällig ist auf den ersten Blick, dass bei den meisten Häusern nur eine Partei verzeichnet ist. Die Nennung von mehreren Wohnparteien findet sich deutlich seltener. Eine Differenzierung zwischen Eigentümer und Mieter lässt sich anhand der Listen nicht erkennen, etwa an der Höhe des Quartiergeldes oder gar an entsprechenden schriftlichen Hinweisen auf Mietverhältnisse. Auf Eigentumslosigkeit kann daher nur indirekt geschlossen werden, wenn mehrere Parteien im Anwesen verzeichnet sind und das ist - wie gesagt - die Ausnahme. Hausnummern wie S [Sebalder Seite] 823 mit der Nennung von siebzehn Haushalten sind äußerst selten. Eine dichte Belegung der Häuser mit Mietern scheint Ende des 18. Jahrhunderts in Nürnberg nicht weit verbreitet gewesen zu sein. Vielmehr bewegt sich auch bei Häusern, die von mehreren Parteien bewohnt wurden, die Anzahl der Nennungen pro Haus in der Regel zwischen zwei und fünf Haushalten. Die Mieter, die ein ganzes Haus gemietet haben und allein bewohnen, können aufgrund fehlender Angaben zum Eigentumsstatus anhand der Listen nicht ermittelt werden.

Nachweislich sind hier jedoch nicht nur die Eigentümer und Hauptmieter, sondern auch Haushalte, die in einem Untermietverhältnis standen, erfasst. Damit ist immerhin die Anzahl der Haushalte insgesamt zuverlässig überliefert.

${ }^{186}$ Quartiergeldbeiträge 1798, S. 68ff, ab hier ohne Seitennummerierung.

${ }^{187}$ Quartiergeldbeiträge 1798, am Ende des gedruckten Bandes, ohne Seitennummerierung. 


\section{Feuerschaulisten}

Feuerschaulisten erweisen sich ebenfalls als wichtige und aussagekräftige serielle Quellen zum Wohnen im fremden oder eigenen Eigentum. In Nürnberg hat sich konkret die Auflistung der amtlichen Feuerschau aus dem Jahr 1561 erhalten. ${ }^{188}$ Erfasst wurden alle Herdstellen innerhalb der Stadtmauern und mit ihnen die Haushalte. Zudem wurde notiert, ob die Bewohner Eigentümer waren oder zur Miete wohnten und wer in diesem Fall der Eigentümer war. ${ }^{189}$ In den Listen sind Mietsituationen mit dem Vermerk Zins oder einer ähnlichen Beschreibung versehen.

Christian Feja hat diese Quelle ausführlich im Hinblick auf Mietverhältnisse ausgewertet. ${ }^{190}$ Auf seine Ergebnisse wird im Folgenden immer wieder verwiesen werden. Die Erfassung erfolgte getrennt für beide Stadthälften, die Listen in den Foliobänden sind interessanterweise unterschiedlich: Der Band für die Sebalder Seite besteht aus zwei Teilen. Im ersten Teil sind die Haushalte mit den jeweiligen Mängeln in den Feuerstätten aufgelistet, im zweiten Teil folgen die „mängelfreien" Haushalte. Die Erfassung erfolgte hier streng in der Ordnung der Gassenhauptmannschaften. In der Lorenzer Hälfte sind Listen nach Tagestouren notiert, die laut Feja nicht immer Rücksicht auf die Gassenhauptmannschaften genommen haben. ${ }^{191}$

Die Feuerschaulisten ordnen die vermieteten Herdstellen (Wohnungen) leider nicht immer eindeutig einem bestimmten Haus zu, sodass sich aus dieser Quelle nicht generell Hausgrößen oder gar einzelne Hausstrukturen ableiten lassen. In vielen Fällen sind jedoch mehrere Haushalte mit einer Klammer zusammengefasst. Die Sebalder Listen sind generell präziser, dies gilt insbesondere für den ersten Teil mit den ausführlichen Mängelbeschreibungen. Auf der Sebalder Seite scheinen Haushalte, die zusammen in einem Anwesen wohnten, gekennzeichnet worden zu sein, in den Lorenzer Listen finden sich entsprechende Hinweise erst ab fol. 221. Aus vielen dieser Einträge, so zum Beispiel Herrn Hans Diettrich Loffelholz Hauß und seine Zins darInnen, ${ }^{192}$ lassen sich Besitzstrukturen und größere Mietanwesen erschließen (Abb. 1). Es gibt daneben aber auch Einträge wie folgenden: Endres Örttels Zins am Schwabenberg nemblich so, es folgen hierauf acht

\footnotetext{
${ }^{188}$ StadtAN, B1/I, Nr. 29 und 30.

${ }^{189}$ Endres bezieht sich wiederum auf die Ergebnisse der Zulassungsarbeit von Christian Feja. Die Angaben und Zahlen im Folgenden beruhen auf dessen Auswertung. Die Ergebnisse sind nicht veröffentlicht. Eine Kopie der Zulassungsarbeit findet sich jedoch im Stadtarchiv Nürnberg und beim Verein „Geschichte für Alle“; ENDRES 1990b.

${ }^{190}$ Vgl. hierzu und im Folgenden FEJA 1988.

191 FEJA 1988, S. 9f.

${ }^{192}$ StadtAN, B1/I Nr. 30, Teil 2 fol. 36: genannt werden anschließend drei Haushalte.
} 
Haushalte - darunter sieben Leineweber -, die nicht mit einer Klammer zusammengefasst sind, der Eintrag wird stattdessen mit einem horizontalen Strich beendet. ${ }^{193}$ Dieser und ähnliche Einträge zeigen zugleich die Grenzen der Interpretation auf, denn es ist nicht immer ersichtlich, ob die Mieter zusammen in einem Haus, einem Anwesen oder gar verteilt auf verschiedene benachbarte Anwesen wohnten.

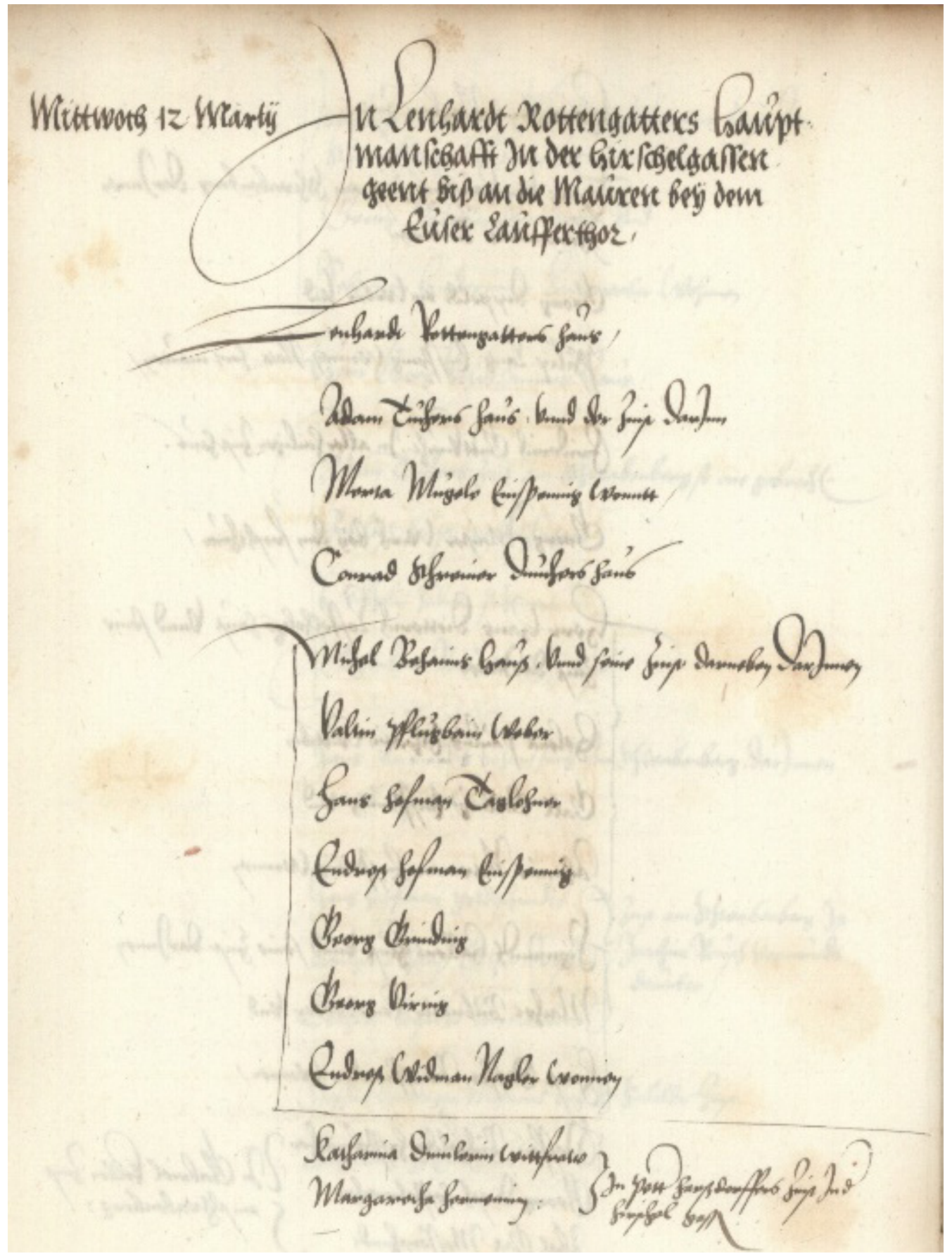

Abb. 1. Nürnberg, Feuerschauliste St. Sebald, 12. März 1561 (StadtAN, B 1/I, Nr. 30, fol. 36')

${ }^{193}$ Ebd., fol. 34-34'. 
Trotz der an sich sehr detaillierten Quelle bleibt eine gewisse Unsicherheit im Hinblick auf die statistische Auswertung bestehen, da es in den einfachsten Wohnungen durchaus üblich gewesen ist, dass sich zwei Haushalte eine Herdstelle teilten. ${ }^{194}$ Solche Mietverhältnisse sind in den Feuerschaulisten nicht erfasst.

Untermieter sind ebenfalls nicht vermerkt. Dies wird daran deutlich, dass bei den vielen aufgeführten Miethaushalten, die eine Einheit für sich bewohnten, keine weiteren Personen genannt werden, Untermieter aber sowohl bei Eigentümer- als auch Miethaushalten Aufnahme fanden. Da zur Identifikation der gelisteten Feuerstellen etwaige Untermieter keine nennenswerte Hilfe gewesen wären, ist ihr Fehlen verständlich.

\section{Huldigungslisten}

Die Erbhuldigung erfolgte als Treueschwur der Untertanen ihrem weltlichen Herrn gegenüber. Anlass war der Herrschaftswechsel, in der Regel nach dem Tod des Vorgängers, beim Amtsantritt des jeweiligen Nachfolgers. Die Erbhuldigung war sowohl in weltlichen als auch geistlichen Territorien ein wichtiger Bestandteil des Herrschaftszeremoniells. Dies kommt unter anderem zum Ausdruck in zeitgenössischen Druckwerken. Stellvertretend seien hier die Historische Beschreibung des Fürstenthums Sachsen Gotha von Friedrich Rudolphi (wohl 1717) genannt, 195 in der der Erbhuldigung ein ganzes Kapitel gewidmet ist sowie der Bericht von Laurentius David Bollhagen aus dem Jahr 1741: Historischer Bericht, wie es Bey der solennen Erb=Huldigung Ihro weyl. Königl. Majestät in Preußen Herrn Friedrich Wilhelm, zu Stettin und Pommern gehalten worden; Ingleichen mit was für Formalitäten sie solenne Erb=Huldigung von Ihro itzt regierenden Königl. Maj. in Preußen, sowohl in Königsberg, Berlin, Magdeburg ec. als auch in Breslau, Liegnitz und Schweidnitz eingenommen worden. ${ }^{196}$

Ein guter Überblick aus historischer Sicht findet sich bei André Holenstein in seinem Werk zum Thema der Huldigung. Von besonderem Interesse ist insbesondere der zweite Teil zur landesherrlichen Huldigung in den Territorien. ${ }^{197}$ Auf die Anfertigung und Archivierung von Huldigungslisten mit den Namen der Huldigenden geht aber auch er nicht ein.

Karin Plodeck schildert ausführlich den Vorgang der Erb- und Landeshuldigung

${ }^{194}$ Zum Beispiel teilten sich zwei Zinswohnungen bei Schloss Schwarzenbruck vor 1727 eine gemeinsame Flurküche; StAN, Rst. N, Waldamt Lorenzi I Nr. 515/12 Pl.1.

195 Rudolphi 1717, Erster Theil, Caput XII: „Von der Erbhuldigung/ mit welcher sich die Landes=Herren ihre Untergebene verpflichtet gemacht/..."; S. 81-97.

196 BOLLHAGEN 1741.

197 Holenstein 1991, insbesondere ab S. 217. 
beim Antritt eines neuen Markgrafen in Ansbach. ${ }^{198}$ Dazu wurden alle Untertanen und Schutzverwandten aus einem Umkreis von etwa einer Viertel-Wegstunde zentral zusammengeholt ${ }^{199}$ Die versammelten Personen traten entsprechend ihrer rechtlichen Situation geordnet vor den Markgrafen oder dessen Stellvertreter, ${ }^{200}$ denn der Markgraf ließ sich dabei zumeist von hochrangigen Amtsträgern vertreten. In einem Protokoll aus dem Jahr 1729 ist die Reihenfolge geschildert. Zuerst kamen die städtischen Honoratioren, dann in Viererreihen die Bürgerschaft von Uffenheim, die Witwen und die Juden. Die Uffenheimer Hausgenossen werden hier nicht eigens erwähnt. Am Ende des Zuges folgten die Unterthanen, auch Hausgenoßen, je 8 in einem Glied vom Lande, [...]. Nach diesen aber gingen der Zollbereither und Amts-Schultheiß Dietlein von Ergersheim und der Schultheiß von Mönchsondheim, denen die gesamte hiesige Ober-Amts eingehörige SchirmsUnterthanen nachfolgten, zuletzt aber die Juden vom Lande, welche den Train beschlossen. ${ }^{201}$ Die Untertanen leisteten einen Schwur, die Schirms-Unterthanen jedoch nur die Hand-Treu. ${ }^{202} 1729$ erhielten die Untertanen im Anschluss an den Schwur erstmals eine Gedächtnismünze: Untertanen im Wert von zwei Groschen, Schutzverwandte im Wert von einem Groschen, entsprechend ihrer unterschiedlichen rechtlichen Bindung. ${ }^{203}$

Anlässlich solcher Huldigungen wurden vom 16. bis zum 18. Jahrhundert die betroffenen Haushaltsvorstände in Listen erfasst; Untertanen fremder Herrschaften, die es vor allem in den gemischtherrschaftlichen Dörfern Frankens gab, fehlen dort selbstverständlich. Zum Teil haben sich auch zusätzliche Listen erhalten mit Namen von Untertanen, die aus dargelegten Gründen nicht persönlich zur Erbhuldigung erscheinen konnten. ${ }^{204}$

Die Erbhuldigung erfolgte jeweils nach dem Tod des vorausgehenden Landesherrn, war der Erbe noch nicht volljährig, trat an seine Stelle eine Vormundschaftsregierung, die die Erbhuldigung entgegennahm. Vormundschaften bestanden im Fürstentum Ansbach eigentlich bei fast allen Huldigungen: 1625 (keine Überlieferung), 1634, 1667, 1686 und 1692 und 1723. So huldigten zum Beispiel im Jahr 1723 die Untertanen der Markgräfin Christiane Charlotte, geborene Prinzessin von Württemberg, als Regentin und Mutter des minderjährigen Wilhelm Friedrich. Für den Übergang der Regierungsgeschäfte an den Erben haben sich hingegen keine Listen erhalten. Eine Ausnahme bildet hier lediglich der

\footnotetext{
198 PLODECK 1971/72, S. 175-194, besonders S. 179-183: Huldigung an Zentren des Landes.

${ }^{199}$ Ebd., Anm. 32.

${ }^{200}$ Ebd., S. 183.

${ }^{201}$ StAN, Fm AN, Brdbg. Lit. Nr. 607, fol. 142 f. zitiert nach: PLODECK 1972, S. 181-182.

${ }^{202}$ StAN, Fm AN, Brdbg. Lit. Nr. 607, fol. 143. zitiert nach: PLODECK 1972, S. 182.

203 PLODECK 1972, S. 183f.

${ }^{204}$ Siehe hier zum Beispiel die Listen zu Mainbernheim 1723: StAN, Fm AN, Brdbg. Lit. Nr. 602, fol. 688-689 und zu Prichsenstadt fol. 692-693.
} 
Regierungsantritt nach Erreichen der Volljährigkeit von Johann Friedrich im Jahre 1672.

\section{Überlieferung}

Da die Huldigungslisten bisher von der Forschung weitgehend unbeachtet geblieben sind, sollen sie an dieser Stelle ausführlicher gewürdigt werden. Lediglich Otto Morlinghaus hat bisher die Huldigungslisten, in diesem Fall des Hochstiftes Bamberg, für einen Aufsatz zur Bevölkerungsgeschichte als Quelle herangezogen. ${ }^{205}$ Die dortigen Huldigungslisten decken die Zeitspanne von 1591 bis 1755 $a b .{ }^{206}$ Hans Mauersberger erwähnt sie ausdrücklich als wichtige Quellengattung zur Ermittlung historischer Bevölkerungszahlen. ${ }^{207}$ Zugleich weist er darauf hin, dass diese Listen nicht unbedingt alle Einwohner erfassen. Auf diesen Umstand habe ich bereits hingewiesen.

Für das Fürstentum Brandenburg-Ansbach haben sich Listen zu den Regierungswechseln 1603, 1634 (39/41), 1667 (68), 1672 (73), 1686, 1692 (95), 1703, 1723 erhalten, ${ }^{208}$ wobei die dem Regierungswechsel folgenden Erbhuldigungen häufig mit zeitlichem Verzug stattfanden, die Jahresangaben dazu sind in Klammern angegeben. Im Anhang (vgl. Tab. Anhang I.1.-I.8.) findet sich eine Zusammenstellung aller in den Huldigungen aufgeführten Gruppen mit der jeweiligen Haushaltsanzahl für jeden Huldigungsvorgang.

In den Listen sind jeweils nur die Haushaltsvorstände und keine weiteren Haushaltsmitglieder erfasst, teilweise ist der Beruf angegeben. Für die ältesten Listen von 1603 sind folgende Unterschiede festzuhalten: Für den größten Ort des Markgraftums, Kitzingen, erfolgt die Aufstellung differenziert nach den Stadtteilen. ${ }^{209}$ Die Hausgenossen finden sich verstreut in diesen Listen genannt. Anschließend folgen Nachträge mit den Einwohnern, die noch nicht gehuldigt haben, ${ }^{210}$ und eine Liste der Witwen. Die eigentumslosen Frauen sind wiederum gekennzeichnet. ${ }^{211}$ Am Ende, nach diesen insgesamt neun Teillisten, folgt die Auflistung der zwanzig öffentlichen Bediensteten; erst die weltlichen, dann die geistlichen. ${ }^{212}$ Für die Stadt Schwabach hat sich im gleichen Zeitraum hingegen nur eine Seite

\footnotetext{
205 Morlinghaus 1940.

206 Ebd., S. 131.

${ }^{207}$ MAUERSBERGER 1960, S. 20f.

${ }^{208}$ Huldigungslisten 1603-1723, StAN, Fm AN, Brand. Lit. Nr. 592ff. Die Zahlen in den Klammern geben den Zeitpunkt der Listenerfassung wieder, diese erfolgte unter Umständen zeitlich verzögert zum Regierungswechsel.

${ }^{209}$ StAN, Fm AN, Brdbg. Lit. Nr. 592, fol. 118-140.

${ }^{210}$ Ebd., Nr. 592, fol. 141-145.

211 Ebd., Nr. 592, fol. 145'-148'.

${ }^{212}$ Ebd., Nr. 592, fol. 149-150.
} 
mit der Zusammenfassung der Gesamtzahlen erhalten, getrennt nach Eigentümer und Beständner, die keine Häuser haben. ${ }^{213}$

Für den Regierungswechsel 1625 fehlen die Listen, ursächlich dürften die Wirren des Dreißigjährigen Krieges sein. Insbesondere bei den frühen Jahrgängen fehlen zudem in den vorhandenen, gebundenen Foliobänden auch verschiedene Ämter und Ortschaften. Dies gilt insbesondere für den Band Nr. 593. Verzeichnet sind hier lediglich zwei Erbhuldigungen für Gunzenhausen und Wassertrüdingen, datiert 1639, und eine aus dem Jahr 1641 für Creglingen. ${ }^{214} \mathrm{Ab}$ dem Jahr 1668 sind die Listen für die fränkischen Gebiete des Fürstentums wohl weitgehend vollständig. Merkendorf erscheint erstmalig 1686 in den gebundenen Listen; Treuchtlingen fällt 1647 an das Fürstentum Ansbach, ${ }^{215}$ findet sich in den gebundenen Listen aber erst ab 1703.

Die einzelnen Bände beinhalten in der Regel nur einen Regierungswechsel. Abweichend davon sind im Band 598 drei Jahre erfasst (1719, 1723 und 1725), allerdings beschränkt sich die Erhebung in diesem Fall auf einige wenige Orte, darunter als einzige Stadt Merkendorf. ${ }^{216}$ Deutlich ausführlicher und umfangreicher als für alle anderen Erbhuldigungen ist die Überlieferung für das Jahr 1723: Anstelle eines Foliobandes ist die archivalische Überlieferung in vier schwere Foliobände geteilt. ${ }^{217}$ In diesen Bänden sind die Orte entsprechend den einzelnen Reiserouten und nicht alphanumerisch in der sonst üblichen Reihenfolge gelistet. Zudem sind hier den Ämtern diverse Exzerpte vorausgegangener Huldigungen ab 1603 beigeheftet. Für die Jahre nach 1723 konnten keine Huldigungslisten mehr aufgefunden werden. Die Huldigung an sich wurde aber bis in preußische Zeit fortgeführt. $^{218}$

\footnotetext{
213 Ebd., Nr. 592, fol. 233.

${ }^{214}$ Ebd., Nr. 593, fol. 217-218, 226-227, 253-255.

215 KeYSER/STOOB 1971, S. 550.
}

216 StAN, Fm AN, Brdbg. Lit. Nr. 598: Huldigungs Acta der Ämter Hailsbronn, Merkendorff, Weyzendorff und Nördlingen, dann der amtlichen versetzten Bayreuthischen Ämter Wind= und Altheim. 1719, 1729 et 1730. 1758. In diesen Heilsbronner Huldigungslisten wird neben den Hausinhabern und Schutzverwandten eine dritte Gruppe ausgeschieden, nämlich die Lehen Leuthe. Unter diesen finden sich auch Personen mit einem Hinweis auf den Herkunfts-/ Wohnort. Worin sich die Lehensleute von den erstgenannten Haus- und Hofinhabern (Würklichen Unterthanen) unterscheiden, wird nicht klar.

${ }^{217}$ StAN, Fm AN, Brdbg. Lit. Nr. 600, 601, 602, 603. Nr. 600 beginnt mit Ansbach und der zweiten Tour zu den Ämtern Gunzenhausen, Wülzburg und Triesdorf. Am Beginn dieses Bandes findet sich eine detaillierte Zusammenfassung des Ablaufs der Erbhuldigung, insbesondere der Reihenfolge, in der die Städte gehuldigt haben.

218 StAN, Ansbacher Archivalien 14703: Acta. Die nach dem erfolgten Regierungs Antritt des Königs von Preußen [...] vorzunehmende allgemeine Landes Huldigung in dem Fürstenthum Ansbach, 1792. 
Die Gliederung erfolgt entsprechend der Ämter, zumeist beginnend mit dem jeweiligen Amtsort. In der Regel folgen die Oberämter in alphanumerischer Reihung aufeinander. So ergibt sich die für die Auswertung relevante Reihenfolge der Städte: Crailsheim, Creglingen, Feuchtwangen, Gunzenhausen, Leutershausen, Langenzenn, Mainbernheim, Merkendorf (ab 1686), Ansbach (Onoldsbach), Prichsenstadt, Roth, Schwabach, Uffenheim, Wassertrüdingen, Windsbach.

Die Gliederung der Listen selbst ist nicht einheitlich. Dies hat die Ursache darin, dass die Listenerstellung in der Obhut der örtlichen Amtleute lag und diese ihre ausgefüllten Listen bei der Regierung abgegeben haben. Dort wurden die individuellen Listen zusammen mit weiterem Schriftverkehr, die Huldigung betreffend, zu den im Staatsarchiv Nürnberg überlieferten Bänden zusammengeführt, ohne dass zuvor eine vereinheitlichende Abschrift erfolgte. Erst beim Zusammenbinden wurden die Folio-Nummern vergeben. Auf die teilweise topografische Unvollständigkeit der Bände wurde bereits hingewiesen.

\section{Gliederung der Verzeichnisse}

Grundsätzlich erfolgt eine Gliederung der Bevölkerung in unterschiedliche Gruppen (Abb. 2); diese kann jedoch nach Jahr und Ort differieren.

Die Listen zu den einzelnen Städten werden angeführt von der Gruppe der Bürger. Gelegentlich findet sich innerhalb dieser ersten Gruppe noch eine Hierarchisierung: An der Spitze stehen in diesen Fällen die geistlichen Bedienten (Pfarrer, Diakon, ...) gefolgt von den weltlichen Staatsbediensteten (Beambte). ${ }^{219}$ Dann folgen die Bürgermeister und Ratsmitglieder, am Ende die Gemeinen, also die bürgerlichen Haushaltsvorstände. In vielen Fällen erfolgt innerhalb dieser Gruppe jedoch keine Differenzierung. Sie werden häufig als (würkliche) Mannschaften bezeichnet, was im Grundsatz dem heutigen Begriff eines Haushalts entspricht. In Kitzingen sind, wie bereits oben erwähnt, die zwanzig öffentlich Bediensteten im Jahr 1603 aus der ersten Gruppe herausgezogen und an das Ende der gesamten Liste gesetzt worden, auf die weltlichen Bediensteten folgen hier die Geistlichen. ${ }^{220}$

Seit dem Jahre 1639 werden die Witwen mit Wohneigentum als eigene Gruppe erfasst, die auf die erste Gruppe folgt.

\footnotetext{
${ }^{219}$ StAN, Fm AN, Brdbg. Lit. Nr. 593, fol. 253: Erbhuldigung 1639 in Wassertrüdingen: Auf den Dechant und den Schulmeister folgen der Kastner und der Vogt.

StAN, Fm AN, Brdbg. Lit. Nr. 594, fol. 244. Mainbernheim 1686, Nr. 596, fol. 383: Stadtschreiber, Cantor und Kirchner, der zugleich Organist war, finden sich in der Gruppe der Rathsverwandten, fol. 383'. In den Listen fehlen aber Hirten, Stadtknechte, Torwärter, etc.

${ }^{220}$ Ebd., Nr. 592, fol. 149-150.
} 
Die nächste Hauptgruppe bilden die Hausgenossen. Gelegentlich ist der Begriff des Hausgenossen ersetzt, zum Beispiel heißt es in Roth im Jahr 1603 Inwoh$n e r,{ }^{221}$ in Schwabach findet sich zur gleichen Zeit stattdessen die Beschreibung: Beständner die keine Häuser haben. ${ }^{222}$ Ganz ähnlich heißt es 1668 in Prichsenstadt: Folgen die Jenigen, welche gleichfalls Bürgerliche Pflicht uff sich haben, Undt im bestand seyen. ${ }^{223}$ Die Differenzierung der Bevölkerungsgruppen verstärkt sich nach dem Ende des Dreißigjährigen Krieges, beginnend mit den Huldigungslisten des Jahres 1668. Nunmehr ist häufig eine separate Gruppe unter der Überschrift Schutzverwandte anzutreffen. Der Begriff kommt aus der Rechtssprache und meint Einwohner, die kein volles Bürgerrecht erworben haben (vgl. Kap. 2).

Diese Ausdifferenzierung gilt gleichermaßen für jüdische Einwohner. Während diese in den frühen Erfassungen häufig unsystematisch, quasi eingestreut, in den Listen genannt werden, finden sie sich ab dem Jahr 1668 zumeist als eigenständige Gruppe unter der Überschrift Juden zusammengefasst.

Zuvor werden sie in der Regel nicht in eigenen Listen erfasst, sondern lediglich hinter dem (Vor-)Namen als Jud bezeichnet. Dass es sich bei einer solch differenzierten Gruppenbildung nicht um eine grundsätzliche Neuerung handelt, wird anhand von Crailsheim deutlich. Dort sind die Juden bereits 1603 als Gruppe zusammengefasst: Am Ende der Namen der Rats und ganzer Burgerschafft der fürstlichen Statt Creilsheim findet sich die Überschrift: Juden so [...] und bürgerliche Häuser besitzen; es folgen dann zwei jüdische Namen. ${ }^{224}$ In dieser Aufstellung erscheint zum ersten und einzigen Mal auch eine Gruppe von Adligen mit acht Einträgen. ${ }^{225}$

Die letzte eigenständig erfasste Gruppe bilden die unbesetzten Hausstellen, als öede Plätz bezeichnet. ${ }^{226}$ Dies ist als eine direkte Folge des Dreißigjährigen Krieges anzusehen und zeigt, dass die Huldigungslisten nicht nur dazu dienten, dass jeder Untertan huldigte, sondern auch um einen Überblick über die wirtschaftliche Situation des Landes zu erhalten. Bis zum Jahr 1723 hatte sich die Bevölkerung weitestgehend wieder erholt. Ab diesem Jahr fehlen in der Regel Einträge zu

\footnotetext{
${ }^{221}$ Ebd., fol. 210.

${ }^{222}$ Ebd., fol. 233.

${ }^{223}$ Ebd., Nr. 594, fol. 418'.

${ }^{224}$ Ebd., Nr. 592, fol. 37-50.

225 Ebd., fol. 47.

${ }^{226}$ Ebd., Nr. 595, fol. 407.
} 
öden Hausplätzen. ${ }^{227}$ Lediglich in Creglingen und Feuchtwangen sind noch jeweils acht öde Plätze erfasst. ${ }^{228}$ Stattdessen wird nun in vielen Orten präzise erfasst, wer wie viele Häuser besitzt. Auffällig ist hier, dass insbesondere im westlichen Franken im Jahr 1723 bereits viele Häuser zwei Eigentümer aufwiesen. ${ }^{229}$

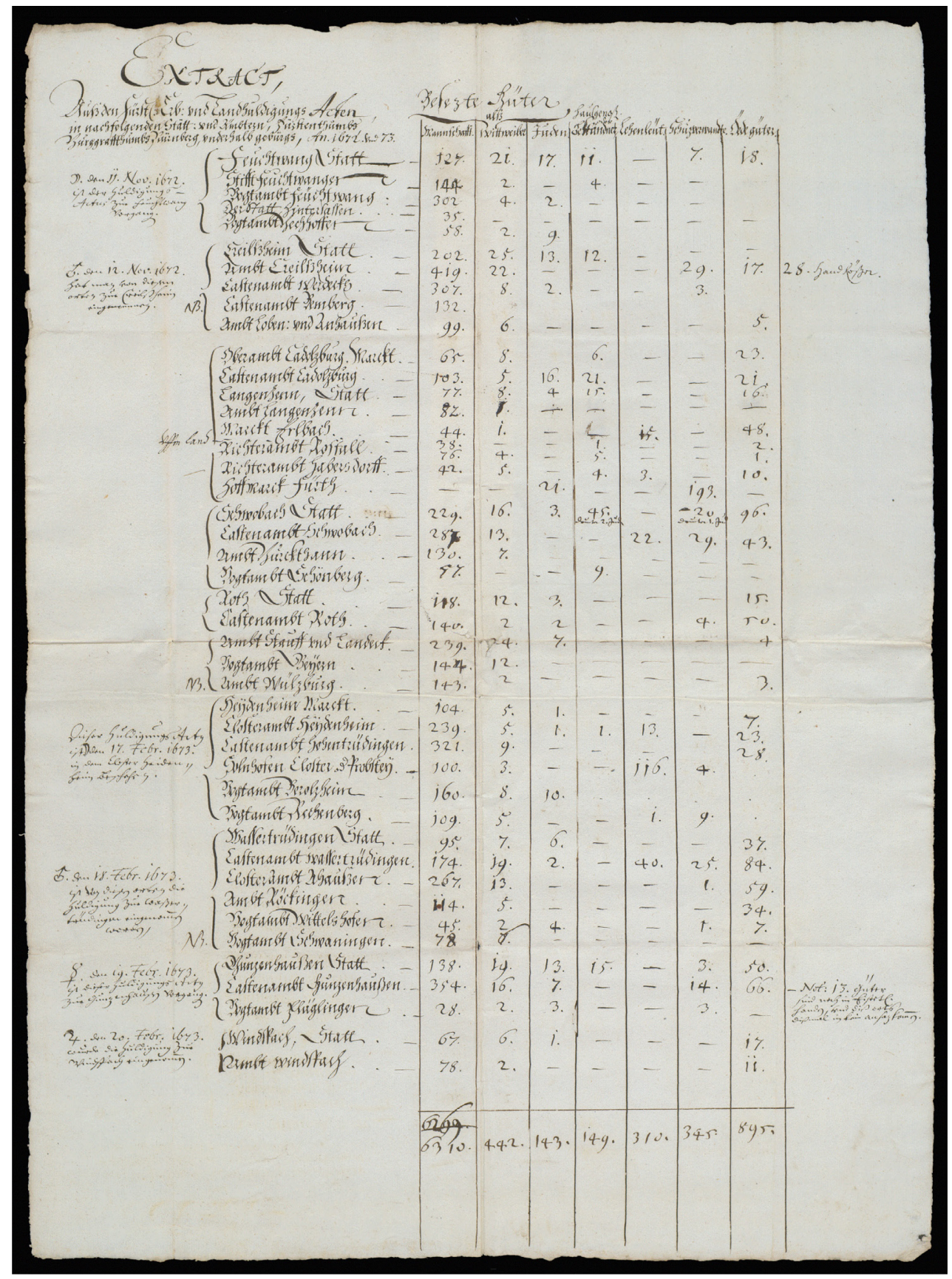

Abb. 2. Extract aus den Fürstl. Erb: und Landhuldigung Acten in nachfolgenden Stätt. und Ämbtern, Fürstenthumbs Burggrafthumbs Nürnberg, underhalb gebürgs, An. 1672. und 73. (StAN, Fm AN, Brdbg. Lit. B 595 fol. 655)

${ }^{227}$ Ebd,. Nr. 600-603.

${ }^{228}$ Ebd., Nr. 602, fol. 126' und 523'.

${ }^{229}$ Vgl. hierzu WENDEROTH 2021, S. 438-440. 


\section{Eigentumslose Haushalte in den Huldigungslisten}

Eine Erfassung der eigentumslosen Haushalte lag nicht in der Absicht der Verfasser; die eigentumslosen Haushalte müssen daher aus den einzelnen Gruppen herauskristallisiert werden.

Die Hauptgruppe bilden die bereits angesprochenen Hausgenossen. Im Jahr 1603 heißt es über diese: Folgen die Haußgenoßen in der Stadt Feuchtwangen auch Bürger sein. ${ }^{230}$ Dabei ist es keineswegs selbstverständlich, dass Hausgenossen Bürgerrechte aufweisen. Charakterisiert werden sie dadurch, dass sie kein Hauseigentum haben und nicht über ihren rechtlichen Status. Unter der Rubrik Hausgenossen sind also erst einmal die Mieter zu verorten und in keinem Fall Eigentümer. In Feuchtwangen wird 1672 explizit unterschieden in zwölf Hausgenossen mit Bürgerrecht, darunter eine Witwe, und sieben Hausgenossen ohne Bürgerrecht. $^{231}$

Letztere Gruppe findet sich primär erfasst unter der Rubrik der Schutzverwandten. So auch in Feuchtwangen nur vier Jahre zuvor: Neben 8 Haußgenossen werden 9 ablösende Schutzverwandte aufgeführt. ${ }^{232}$ Synonym wird ortsweise auch der Begriff des Pfahlbürgers verwandt. ${ }^{233}$ In den Huldigungslisten ab 1668 sind häufig separate Einträge unter der Überschrift Schutzverwandte anzutreffen. Bisher konnte nicht zweifelsfrei geklärt werden, ob es sich dabei in jedem Fall auch um Mieter handelt. Einige Hinweise sprechen jedoch dafür. So finden sich in der detaillierten Aufstellung des Jahres 1668 für die Stadt Roth neun Beständner oder Haußgenoßen sowie fünf Schutzverwanthe. ${ }^{234}$ In der abschließenden Gesamtliste sind diese zusammengefasst als vierzehn Schutzverwandte [sic!]. ${ }^{235}$ Eindeutig ist die Zuordnung der Schutzverwandten in den Huldigungslisten zu den eigentumslosen Bevölkerungsschichten in den Dörfern. So wird zum Beispiel die ländliche Bevölkerung des Stiftsamtes Wülzburg im gleichen Jahr in Besitzende (Höff, Halbhöff, Sölden, so Anspann haben und Sölden, so mit der Handt dienen) sowie in Nichtbesitzende (Hierauff folgen die jetziger Zeit in dem Ampt befindliche Schutzverwanthe und Mundtleuth) unterschieden. ${ }^{236}$ Die Beschreibung im Falle von Roth zwanzig Jahre später, 1686, bringt den Sachverhalt nochmals gut zum Ausdruck: Schutzverwanthe so auch keine Häußer haben. ${ }^{237}$

\footnotetext{
${ }^{230}$ StAN, Fm AN, Brdbg. Lit. Nr. 592, fol. 98’ . Hier ist die Gruppe der Witwen, wie bereits geäußert, nicht extra erfasst.

${ }^{231}$ Ebd., Nr. 595, fol. 235.

232 Ebd., Nr. 594, fol. 646'. 12. Sept: 1686 Feuchtwang Stadt 130 würkliche Häuser mit 8 Haußgenossen; 55. so Handtreu geben; 9 öedt 9. ablösige Schutzverwandte.

${ }^{233}$ Wassertrüdingen 1686: PfahlBürgere. StAN, Fm AN, Brdbg. Lit, Nr. 596, fol. 615.

${ }^{234}$ Ebd., Nr. 594, fol. 433.

${ }^{235}$ Ebd., fol. 642'.

${ }^{236}$ Ebd., fol. 637' und 638.

${ }^{237}$ Roth 1686, StAN, Fm AN, Brdbg. Lit. Nr. 596, fol. 464' .
} 
Gleichzeitig wird deutlich, dass es in den Städten auch Schutzverwandte mit Hauseigentum gegeben haben muss. Zumindest für das Fürstentum Ansbach scheint diese Annahme geradezu zwingend, finden sich doch hier in fast allen Städten jüdische Hauseigentümer, die ebenfalls nur einen SchutzverwandtenStatus und nicht das Bürgerrecht erwerben konnten. Auf die jüdischen Einwohner wird anschließend noch eingegangen werden. Christliche Schutzverwandte mit Hauseigentum lassen sich anhand der Huldigungslisten dagegen nur schwer nachweisen. Für Schwabach heißt es 1668 in der ausführlichen Liste: Schutz Verwandte, und andere Innwohner so nit Bürger. ${ }^{238}$ Aufgelistet werden dreizehn Personen. In der abschließenden Gesamtliste sind unter den 23 Schutzverwandten aber nicht nur die eben genannten dreizehn Personen, sondern auch noch zehn Außwendige und die Jenige, welche steuerbahre güther hier haben, aufgeführt, ${ }^{239}$ dabei handelt es sich keinesfalls um Mieter, sondern um „Investoren“ mit überwiegend auswärtigem Wohnsitz. Allerdings finden sich auch unter der Rubrik „Eigentümer" weitere Personen mit auswärtigem Wohnsitz, so zum Beispiel Simon Model, Jud zu Onolzbach hat ein hauß allhier. ${ }^{240}$ Der Grund, warum auswärtige Eigentümer in zwei getrennten Rubriken auftauchen, ist nicht ersichtlich. In der Gesamtliste sind zudem die Schwabacher Hausgenossen unter der ersten Rubrik, besetzte [Hausstellen], erfasst. ${ }^{241}$ In der detaillierteren Aufstellung sind hingegen 62 Haußgenoßen separat aufgelistet. ${ }^{242}$ All dies sind Hinweise, die belegen, wie uneinheitlich die Begriffe verwendet worden sind. Wichtig, um die Schutzverwandten in den Huldigungslisten zutreffend zu würdigen, erscheint jedoch die Feststellung, dass es sich bei den namentlichen Einträgen weitestgehend um Personen mit geringem Sozialprestige handelt: Tagelöhner und Handwerker. Zudem finden sich christliche Schutzverwandte in nennenswerter Anzahl nur in den gewerblichen Zentren Schwabach und Roth (ab 1686) sowie in der Residenzstadt Ansbach (ab 1703); also in Städten, die sich im Folgenden dynamisch entwickeln und eine vergleichsweise große Anzahl armer Bevölkerung und Mieter aufweisen. Aus diesen Gründen werden die in den Listen genannten Schutzverwandten von mir in der Auswertung den eigentumslosen Haushalten zugerechnet. In den Huldigungslisten vor 1703 sind die Schutzverwandten nur in wenigen Orten separat erfasst.

Von der Gruppe der Schutzverwandten ist es nur ein kleiner Schritt zur Gruppe der Juden, da diese, wie bereits gesagt, in den untersuchten Städten ebenfalls nur unter diesem Status leben durften. Sie werden jedoch in den Huldigungslisten ab

\footnotetext{
${ }^{238}$ StAN, Fm AN, Brdbg. Lit. Nr. 594, fol. 473.

${ }^{239}$ Ebd., fol. 471.

${ }^{240}$ Ebd., fol. 459.

${ }^{241}$ Ebd., fol. 642'.

${ }^{242}$ Ebd., fol. 457'-459.
} 
1668 in aller Regel als eigenständige Bevölkerungsgruppe geführt. Ab dem Jahr 1686 wird in einzelnen Orten zudem noch zwischen jüdischen Hauseigentümern und jüdischen Mietern unterschieden. ${ }^{243}$ In Creglingen werden 1686 vier von 23 Juden (17\%) und in Feuchtwangen drei von dreizehn Juden (23\%) als Hausgenossen bezeichnet, in Leutershausen waren im Jahr 1703 ebenfalls drei von dreizehn Juden Mieter. Für das ausgehende 17. Jahrhundert kann man also von einem Mieteranteil von etwa 20\% unter den städtischen Juden ausgehen.

Auf den ersten Blick eindeutiger scheint die Situation bei der zuerst genannten Gruppe in den Huldigungslisten, den würklichen Mannschaften und Bürgern. Auf den zweiten Blick können sich hier neben den Hauseigentümern aber auch Mieter verbergen, sofern sie über das Bürgerrecht verfügten und der Listenerfasser die Haushalte nicht nach Besitzstand, sondern nach ihrem rechtlichen Status erfasst hat. So wird 1603 in Crailsheim die Bevölkerung nur in zwei Gruppen unterschieden: 346 Bürgern stehen neun Pfaalburger gegenüber. ${ }^{244}$ Es ist zwar nicht ausgeschlossen, dass der Begriff des Pfahlbürgers synonym zum Hausgenossen gebraucht wurde, aber Mieter mit Bürgerrecht fallen eigentlich nicht unter diese Bezeichnung. ${ }^{245}$ Daher ist anzunehmen, dass sich auch in der ersten Gruppe anteilig und fallweise eigentumslose Bürger finden lassen. Ohne eine ausführliche ortsgeschichtliche Forschung, die weitere Quellen mitheranzieht, ${ }^{246}$ können die Mieter in diesen Fällen weder qualitativ noch quantitativ ermittelt werden. Es gibt an anderen Stellen aber auch Erläuterungen zu dieser ersten Gruppe wie die Folgende: Bürger, so ihre aigene Häußer bewohnen, ${ }^{247}$ aus denen klar hervorgeht, dass es sich nur um Eigentümer handelt. Weniger eindeutig, aber wohl doch die gleiche Aussage versteckt sich hinter dem differenzierenden Adjektiv seßhafte Bürger. ${ }^{248}$

Eindeutig scheint die Situation auch dann, wenn - wie bereits geschildert - neben der Gruppe der Hausgenossen noch separat die Gruppe der Schutzverwandten oder Pfahlbürger aufgeführt ist: In diesen Fällen sind in der Gruppe der Hausgenossen die Mieter mit Bürgerrechten zusammengefasst, wohingegen die Mieter ohne Bürgerrecht in der Gruppe der Schutzverwandten zu suchen sind. Bei einer solch starken Differenzierung in den Huldigungslisten darf davon ausgegangen

\footnotetext{
${ }^{243}$ Creglingen: Nr. 596, fol. 154; Feuchtwangen: Nr. 596, fol. 233;

Leutershausen 1703: Nr. 599, fol. 297.

${ }^{244}$ StAN, Fm AN, Brdbg. Lit. Nr. 592, fol. 46.

${ }^{245}$ Vgl. hierzu Kapitel 2, eingeschränkte bürgerliche Rechte, Pfahlbürger.

${ }^{246}$ Unter anderem wären hier die Verspruchgeldlisten, Grundbuch und

Grundsteuerregister anzuführen.

${ }^{247}$ Feuchtwangen 1686: StAN, Fm AN, Brdbg. Lit. Nr. 596, fol. 230-232'.

248 Creglingen 1686: ebd., fol. 148.
} 
werden, dass in der ersten Gruppe tatsächlich nur würkliche Bürger mit Wohneigentum und keine Mieter verzeichnet sind. In vielen Fällen ist die Gliederung in den Huldigungslisten aber weniger stark differenziert.

Die gleiche Problematik besteht in Bezug auf die Gruppe der Witwen. Eine eigene Rubrik unter dem Oberbegriff Witwen, ohne differenzierende Angaben, findet sich in den Huldigungslisten 1639/41. ${ }^{249} 1672$ heißt es dagegen in Crailsheim: Witweiber, welche Häußer [besitzen], ${ }^{250}$ oder Treuchtlingen 1686: Von Wittiben so Häußer und Gütter haben. ${ }^{251}$ Häufig fehlt jedoch eine Angabe zum Besitzstand; es kann also schwerlich davon ausgegangen werden, dass unter dieser Rubrik in allen Fällen nur Eigentümerinnen erfasst worden sind, auch wenn sich nirgends an dieser Stelle der Hinweis auf besitzlose Witwen findet. Nur in wenigen Fällen sind Frauen in der Gruppe der Witwen dezidiert als Mieterinnen ausgewiesen: In Creglingen werden 1695 zwei von vierzehn Witwen mit einem Zusatz als Haußgenoßen bezeichnet. ${ }^{252}$ Für das Jahr 1668 findet sich ein interessanter Eintrag zu Feuchtwangen unter der Überschrift: Hieraufffolgen die wittfrauen in der Statt, welche Bürgerinn sein undt Häußer haben. Es sind neunzehn Frauen verzeichnet, zu den letzten drei Frauen findet sich dann die Anmerkung: diese 3 haben keine Häu$3 e r .{ }^{253}$ Lediglich in diesen beiden Listen werden die Frauenhaushalte differenziert, direkte Rückschlüsse auf andere Listen lassen sich daraus jedoch nicht ableiten. Im Huldigungsjahr 1668 wird zugleich in anderen Städten auf eine eigene Rubrik zu Witwen verzichtet, so in Langenzenn und Schwabach; dort finden sich Witwen unter der Rubrik der Bürger genannt, jedoch nicht unter den Hausgenossen. ${ }^{254}$ In Ansbach fehlen Frauen in diesem Jahr gänzlich in den Huldigungslisten, sowohl unter den 277 Einträgen zu den Eigentümern, als auch in den 87 Einträgen zu den Mietern. ${ }^{255}$

Im Jahre 1603 fehlen weibliche Eigentümerinnen und Mieterinnen in den Huldigungslisten nahezu komplett. Nur in Kitzingen werden sie dort separat, ganz am Ende, genannt. ${ }^{256}$ Ihre Huldigung scheint zu Beginn der Listenüberlieferung rechtlich nicht erforderlich gewesen zu sein, denn in den Listen der Pfahlbürger, Inwohner, Beständner und Hausgenossen tauchen ansonsten nur männliche Namen auf. 257

\footnotetext{
249 Ebd., Nr. 593, fol. 218 (Creglingen 1641), 226' -227 (Gunzenhausen 1639).

250 Ebd., Nr. 595, fol. 128.

${ }^{251}$ Ebd., Nr. 596, fol. 554. Weiter wären noch für dieses Huldigungsjahr anzuführen Creglingen und Gunzenhausen, fol. 148-153 und 163-167.

252 Ebd., Nr. 597, fol. 128.

253 Ebd., Nr. 594, fol. 213'.

${ }^{254}$ Ebd., Nr. 594, fol. 338-339, 452-457.

255 Ebd., fol. 377-382.

256 Ebd., Nr. 592, fol. 145'-148'.

${ }^{257}$ Ebd., fol. 38'-39, 46, 210--211, 270'-271, 318-319'.
} 
Diese Uneinheitlichkeit in der Erstellung der Listen bleibt auch späterhin bestehen. In Roth werden 1723 unter der Rubrik der Frauen sogar ausschließlich die Mieterinnen erfasst: An Weibs Personen, so in Schutz sizen. ${ }^{258}$ Die weiblichen Hauseigentümerinnen sind in diesem Fall bereits in der ersten Gruppe der Eigentümer mit aufgelistet. Der Ablauf der Erbhuldigung für das Amt Roth im Jahr 1723 ist ausführlich dokumentiert. Nachdem die Honoratioren, Stadtbürger und Untertanen gehuldigt hatten, kamen die Schutzverwandten an die Reihe. Hierzu heißt es: 9.) Die Schuzverwanten von der Statt und dem Land, so mann alß weiblichen geschlechts jedoch ein jedes geschlecht besond. ${ }^{259}$ Demnach war vorgegeben, dass sowohl die männlichen als auch die weiblichen Schutzverwandten (so sie einem Haushalt vorstanden) getrennt huldigen mussten. Es finden sich keine Formulierungen in den Protokollen, die auf eine Ausnahme für bestimmte Frauen hindeuten. Die häufig geringe Zahl von aufgeführten Frauen in den Listen der Hausgenossen legt jedoch nahe, dass weibliche Hausgenossen aus unbekannten Gründen andernorts einfach nicht erfasst worden sind und nicht gehuldigt haben. Grundsätzlich bildeten alleinstehende Frauen eine wesentliche Gruppe unter den eigentumslosen Haushalten. ${ }^{260}$ Umso auffälliger ist es, wenn in den Huldigungslisten zwar eine Rubrik zu Frauenhaushalten (Witwen, Wittweiber) vorhanden ist, diese aber weitestgehend nur Eigentümerinnen auflistet, unter der Rubrik der Hausgenossen hingegen nur männliche Namen aufgeführt sind und Frauen dort fehlen. ${ }^{261}$

Um den Anteil der Frauenhaushalte zu ermitteln und damit exakte Zahlen zu den Mieteranteilen zu erhalten, sind die Angaben in den Huldigungslisten nicht ausreichend präzise (vgl. Tab. 2). Alle prozentualen Ermittlungen auf der Basis dieser Listen stellen daher nur einen Näherungswert dar. Es ist anhand der vorliegenden Zahlen nicht möglich, diesen Bevölkerungsanteil qualifiziert zu bestimmen und mit einem festen prozentualen Ansatz zu operieren. Dies gilt sowohl für die Haushalte der Untermieterinnen als auch für die Mieterinnen, die ein Haus oder eine Wohnung gemietet hatten und dort einen eigenständigen Haushalt führten. Für letztere Gruppe gibt es zwar punktuell konkrete Angaben in den Listen, deren

\footnotetext{
258 Ebd., Nr. 601, fol. 58'.

${ }^{259}$ Ebd., fol. 87.

${ }^{260}$ Vgl. WENDEROTH 2019a, S. $72 f$.

${ }^{261}$ Folgende beide Erklärungsversuche möchte ich anführen, auch wenn sie mir nicht plausibel erscheinen. Erstens, sie wurden mehrheitlich bei der Gruppe der Witwen erfasst, auch wenn sich hier die genannten Hinweise auf Hauseigentum finden. Zweitens, sie wurden unter dem Namen ihres verstorbenen Mannes weitergeführt, in diesen Fällen war jedoch der Zusatz „Witwe“ allgemein gebräuchlich, dieser Zusatz fehlt aber in den Listen zu den Hausgenossen. Nach freundlicher Mitteilung von Robert Zöllner ist durchhaus der Fall anzutreffenden, dass die Witwen unter dem Namen ihrer verstorbenen Männer aufgelistet worden sind.
} 
Anteil an den Referenzgruppen (Mieter, Gesamthaushalte) schwankt jedoch zu stark, als dass daraus ein verlässlicher Kennwert abgeleitet werden könnte.

\section{Beamte und öffentlich Bedienstete}

$\mathrm{Zu}$ den „versteckten“ Eigentumslosen können auch die öffentlichen Angestellten gehören. Nur in wenigen Fällen, und dann nur bei den hierarchisch betrachtet untersten Dienstgraden, sind diese innerhalb der Gruppe der Hausgenossen als solche gekennzeichnet und damit identifizierbar, so zum Beispiel in Creglingen 1672. Hier finden sich unter den neun Hausgenossen der Totengräber, der Nachtwächter und der Stadtdiener. ${ }^{262}$ In Uffenheim wird mehrfach ein Torwächter unter den Hausgenossen aufgeführt, ${ }^{263}$ aber eben nicht konsequent: Der zweite Torwächter bleibt gänzlich unerwähnt bei den Hausgenossen, dürfte also innerhalb der Gruppe der Bürger erfasst gewesen sein.

Für die hochrangigen öffentlich Bediensteten gilt das in jedem Fall. Diese sind in vielen der vorliegenden Huldigungslisten überhaupt nicht abgebildet. Dabei haben sie zumeist als Mieter in einer „Dienstwohnung“ gelebt und nur selten im Eigentum oder in einer privaten Mietwohnung. Vermutlich wurden die staatlichen und geistlichen Beamten anderweitig erfasst, standen sie doch in einem herausgehobenen Treueverhältnis zu ihrem Landesherrn. Einträge, wie 1668 in Gunzenhausen mit einer Rubrik für diesen Personenkreis, sind daher die Ausnahme: 1. Geist: Undt Weltliche Herrschafft diener: Auf den Dekan folgen der Diakon, der Rector, der Castner, der Vogt und der Stattschreiber. ${ }^{264}$ Nach den Mitgliedern des Inneren und Äußeren Rats unter Punkt 2. schließt Punkt 3. mit der Überschrift GemeinerStattdiener an, hierunter fallen fünfzehn Personen: ein Organist, ein Schulmeister, zwei Meßner oder Kirchner, ein Türmer, vier Torwachen, zwei Wächter, zwei Hirten, die Hebamme und ein StattKnecht. ${ }^{265}$ In Mainbernheim werden 1686 am Anfang die beiden Geistlichen gelistet, gefolgt von drei herrschaftlichen Beampten. Stadtschreiber, Cantor und Kirchner, der zugleich Organist war, finden sich in der anschließenden Gruppe der Rathsverwandten. ${ }^{266}$ In den Listen fehlen aber Hirten, Stadtknechte, Torwärter, ebenfalls fehlt eine Rubrik der Beständner. Anders verhält es sich dagegen in Merkendorf 1686, hier sind am Schluss der Liste die neun Haußgenoßen aufgeführt, es folgen darauf 3 Thorwarth, 4 Welsche [...] Bürgerl: im bestand und zum Abschluss 2 Huthmänner. ${ }^{267}$ Die einfachen öf-

\footnotetext{
${ }^{262}$ StAN, Fm AN, Brdbg. Lit. Nr. 595, fol. 181.

${ }^{263}$ Ebd., Nr. 596, fol. 553 und Nr. 597, fol. 529'.

${ }^{264}$ Ebd., Nr. 594, fol. 244.

265 Ebd., fol. 244'.

${ }^{266}$ Ebd., Nr. 596, fol. 383f.

${ }^{267}$ Ebd., Nr. 596, fol. 407. Mit Welsche Bürger dürften protestantische Immigranten aus Österreich gemeint gewesen sein, die auch als Exulanten bezeichnet werden.
} 
fentlich Bediensteten sind sinnhaft im Kontext der Mieter aufgelistet. Die höherrangigen öffentlich Bediensteten werden weder genannt noch mittels Status gekennzeichnet, sie scheinen in dieser Aufstellung zu fehlen.

Der Eintrag aller öffentlich Bediensteten unter der Rubrik der Hausgenossen, ihrem Status als Mieter entsprechend, ist bisher nur für Crailsheim im Jahr 1686 nachgewiesen: Eingereiht in dieser Auflistung finden sich auch die städtischen Honoratioren: Hl: [Hochlöblich] Andreas Ley, Hl: Castner Jägers Wittib, Hl: Conrad Schmidt, Hl: Decanus und Hl: Johann Nitzler, Infimus. ${ }^{268}$

\section{Militär}

Für die Erb- und Landeshuldigung hat sich auch der Ablauf für die Militärangehörigen erhalten. ${ }^{269}$ Konkrete Zahlen werden aber nur für die auf der Veste Wülzburg sich befindenden Guarnison, was effective Dienste thun, genannt. ${ }^{270}$ Insgesamt handelt es sich um 63 Männer, davon lediglich 41 Gemeine [Soldaten]. ${ }^{271}$

\section{Würdigung der Huldigungslisten als prosopographische Quelle}

Ein Problem, das immer wieder in der Auswertung der Quellen auftaucht und das bereits ausführlich dargelegt wurde, ist die unterschiedliche Verwendung von Begriffen. Die Huldigungslisten entsprechen aber auch in anderer Hinsicht nicht den Anforderungen an eine heutige statistische Erhebung. So soll die Problematik der unpräzisen Zahlen am Beispiel der Huldigungslisten für Ansbach vom 29. October $a^{\circ} 1672$ erläutert werden: In der detaillierten Namensliste sind zuerst 288 Bürger aufgeführt, es folgen 73 Namen unter der Überschrift Haußgenoßen und zum Schluss 2 Schutzverwande. ${ }^{272} 22$ Bürger, die nicht erscheinen konnten, sind mit einem "o“ gekennzeichnet. In einer Zusammenstellung, die vom gleichen Tag datiert, werden wiederum 288 Bürger genannt (darunter 3. so doppelte Häuser haben), 43. Witwen, so Häußer besitzen, 7. Juden, so Häußer haben, 100. Haußgenoßen und 21. öede Plätz. ${ }^{273}$ Aus welchem Grund in der zweiten Liste deutlich mehr Personen aufgeführt werden, bleibt unklar. Der Hausgenossenanteil an allen christlichen Haushalten erhöht sich damit leicht von $21 \%$ auf $23 \%$.

Trotz dieser vielen Einschränkungen, aufgrund derer die Maßstäbe an eine heutige statistische Erfassung weit verfehlt werden, bilden die Huldigungslisten eine unverzichtbare Quelle für die Frage nach den eigentumslosen Haushalten innerhalb der Bevölkerung. Zum einen existieren für das 17. und 18. Jahrhundert in

\footnotetext{
${ }^{268}$ Ebd., fol. 117-117'.

${ }^{269}$ Ebd., Nr. 600, fol. 354-425.

${ }^{270}$ Ebd., Nr. 600, fol. 405-407'.

${ }^{271}$ Für das Jahr 1818 sind sogar nur 9 Familien (Haushalte) mit insgesamt 36 Personen als

Bewohner der Festung überliefert; Alphabetisches Verzeichnis 1818, S. 105.

${ }^{272}$ StAN, Fm AN, Brdbg. Lit. Nr. 595, fol. 372-379.

273 Ebd., fol. 407.
} 
den hohenzollerischen Territorien Frankens keine weiteren aussagefähigeren seriellen Quellen und zum andern können mit einer gewissen Vorsicht doch Ergebnisse gewonnen werden. Dabei scheint die Anzahl der eigens erfassten Hausgenossen so etwas wie die Kerngruppe der Mietbevölkerung zu bilden, die Schutzverwandten sind tendenziell ebenfalls den Mietern zuzurechnen. Etwaige Nichtmieter innerhalb dieser Gruppen werden überkompensiert durch die versteckten Eigentumslosen innerhalb der Bürgerschaft. Hier sind insbesondere die öffentlich Bediensteten zu nennen, von denen es besonders in den Städten - die ja nahezu alle eine Funktion als Amtssitz hatten - eine nennenswerte Anzahl gab. Vorsicht ist aber dennoch geboten bei dem Vergleich der Orte miteinander und über verschiedene Zeiträume hinweg. Das System der Erfassung war immer stark geprägt von dem jeweiligen Verfasser, was zu Schwierigkeiten in der Kompatibilität führt.

Sehr differenziert ist zum Beispiel die Aufstellung zur Huldigung 1723 für Schwabach. Diese soll daher exemplarisch erläutert werden:

Es werden dort sowohl die Haushaltsvorstände als auch die Häuser gezählt. In Summe ergibt dies 276 Bürger, die in 264 Häusern lebten. ${ }^{274}$ Die Liste wird angeführt von den Mitgliedern des alten Rates, hier findet sich das einzige Mal ein Eintrag für ein Ratsmitglied, Stephan Leichtenschupf, der anscheinend kein Haus besaß. ${ }^{275}$ Für alle anderen 275 Bürger ist Hauseigentum angegeben; darunter einmal drei Häuser bei Georg Fischer, ${ }^{276}$ achtmal zwei Häuser, einmal eineinhalb Häuser und siebenundvierzigmal ein halbes Haus.

Es folgen in der Schwabacher Huldigungsliste von 1723 die 186 Haußgenoßen, oder Bürger so keine aigenthumbl. Häußer haben und fünf Bürger, die außerhalb der Stadt wohnhaft waren. ${ }^{277}$ An bürgerlichen Witwen mit Hausbesitz werden 31 aufgezählt, sechs mit je einem halben Haus, eine mit eineinhalb Häusern und eine mit zwei Häusern. ${ }^{278}$ Bürgerwittiben, so keine aigenthumbl. Häußer haben werden 24 genannt. ${ }^{279}$ Schutzverwanthe MannsPersonen sind 171 aufgelistet, ${ }^{280}$ weibliche Schutzverwandte $39 .{ }^{281}$ Für sämtliche Schutzverwandte ist kein Hausbesitz angeführt, sodass es sich hier wohl insgesamt auch um Mieter gehandelt haben muss. Die jüdischen Einwohner untergliedern sich in 29 Hauseigentümer, davon elf mit nur einem halben Haus, und neun jüdische Mieter, darunter eine Witwe. Hinzu kommen noch neun Verheyrathe Juden, so noch keine Schutzbriefe haben und für

\footnotetext{
${ }^{274}$ Ebd., Nr. 601, fol. 115'

${ }^{275}$ Ebd., fol. 109.

276 Ebd., fol. 114.

277 Ebd., fol. 116-120.

278 Ebd., fol. 120'-121.

${ }^{279}$ Ebd., fol. 121'.

${ }^{280}$ Ebd., fol. 122'-125'.

${ }^{281}$ Ebd., fol. 126-127.
} 
die kein Hausbesitz genannt ist, darunter wiederum eine Witwe. ${ }^{282}$ Insgesamt wurden also 47 jüdische Haushalte erfasst.

Die französische Kolonie bestand 1723 aus 88 Haushaltsvorständen, darunter sechzehn Witwen. ${ }^{283}$ Den Franzosen gehörten jedoch lediglich sieben Häuser, sie wohnten also weit überwiegend zur Miete.

Separat erfasst sind zudem die 23 Inwohner bey der herrschaftlichl. Cottonfabrique außerhalb der Stadtmauern, darunter eine Witwe. ${ }^{284}$

Am Schluss der Liste sind 31 eximierte Personen aufgeführt, denen neun ganze Häuser und ein halbes Haus gehören, 21 dieser Haushalte wohnten demnach nicht im Eigentum. ${ }^{285}$

Insgesamt sind im Jahr 1723 in Schwabach 931 Haushalte und 333 Häuser erfasst.

\section{Frühe statistische Quellen um 1800}

\section{„Seelenliste“ Schwabach, 1799}

Im späten 18. Jahrhundert beginnt die systematische Erfassung der Bevölkerung. Ein frühes Beispiel stellen die erhaltenen Seelenlisten für die Stadt Schwabach dar. Nunmehr wurden alle Personen, die in einem Ort lebten, komplett verzeichnet, darunter auch Kinder unter zwölf Jahren. Aus diesem Grund lassen sich die entsprechenden Verzeichnisse sehr viel exakter mit heutigen Fragestellungen statistisch auswerten. Das Führen der Seelenlisten basiert wohl auf dem „Abriß der Polizeyverfassung des königl. preussischen Fürstentums Ansbach von D. J. D. A. Höck, königl. preussischen Justitzrath und Polizeidirektor in Schwabach und mehrerer gelehrter Gesellschaften Mitgliede. Amberg und Sulzbach 1804.“286 Dort heißt es zu den Aufgaben des Polizeiinspektors: „Er muss eine genaue Liste von allen Einwohnern der Stadt halten, alle Jahr gegen Ende des Monats Oktober eine neue Seelenliste der Stadt mit Hülfe der Viertelmeister von Haus zu Haus mit der größten Treue nach seinem geleisteten Eide aufnehmen und bey seiner Behörde einreichen. “287 Höck gibt im Folgenden genau an, wie die Erfassung zu erfolgen hat.

„In den Seelen=Tabellen müssen [...]

\footnotetext{
282 Ebd., fol. 126-127.

${ }^{283}$ Ebd., fol. 129-131.

${ }^{284}$ Ebd., fol. 127'.

285 Ebd., fol. 132-133.

${ }^{286}$ HÖCK 1804.

${ }^{287}$ Ebd., S. 15f.
} 
3) Die Zahl der Familien in jedem Hause angegeben werden. Hierbei ist zu bemerken, daß alle, welche ihre eigen Haushaltung führen, sie mögen gleichwohl in eines Andern Hause wohnen, oder es mögen ihrer viel oder wenig Personen seyn, eine Familie ausmachen. Hingegen die, welche bei Andern in Diensten sind, oder an deren Tisch gehen, gehören zur Familie desselben, und werden nicht als Familien besonders gezählt. Z. E. Knechte, Mägde, Bedienten, Eltern, welche bei ihren Kindern, denen sie ihre Güter übergeben haben, in die Kost gehen, und keine eigene Haushaltung führen. [...] Wenn Kinder an einem andern Orte, oder auch in einem anderen Hause dienen, oder in Logis sind, so werden sie nur da gezählt, wo sie wohnen."

Die Verordnung fußt vermutlich auf den gesammelten Erfahrungen Höcks in seiner Schwabacher Dienstzeit. Es ist daher nicht verwunderlich, dass sich gerade in Schwabach solche Listen erhalten haben. ${ }^{288}$

\section{„Verzeichniß aller im Rezatkreise enthaltenen Ortschaften“}

Eine weitere wichtige statistische Quelle für das Untersuchungsgebiet Mittelfranken stellt das „Alphabetische Verzeichniß aller im Rezatkreise [...] enthaltenen Ortschaften" dar, das 1818 in Ansbach erschienen ist. Die Bevölkerung des Rezatkreises, der im Wesentlichen dem heutigen Regierungsbezirk Mittelfranken entsprach, ist tabellarisch ortsweise in folgenden Rubriken erfasst: Feuerstellen, Familien, Seelenzahl. Festzustellen ist, dass die Erfassung trotz modernerer Methoden nicht immer einheitlich erfolgte. Die drei Begriffe wurden von einzelnen Erfassungsstellen unterschiedlich interpretiert und angewandt. Damit weist auch dieses Verzeichnis ähnliche Defizite auf wie die älteren Erhebungen. Eine amtliche Vorgabe mit einer exakten Begriffsdefinition, auf die ich mich in der Auswertung beziehen könnte, ist der Schrift nicht beigefügt.

Insbesondere der Begriff „Feuerstelle“ wurde nicht einheitlich aufgefasst. In den von mir ausgewerteten Angaben zu 31 mittelfränkischen Städten lassen sich verschiedene Interpretationsmuster erkennen (vgl. Tab. 23). Vergleicht man die Zahl der in den Listen aufgeführten Feuerstellen mit der Anzahl der in den Uraufnahmen verzeichneten Häuser, ergibt sich folgendes Bild:

In den meisten Fällen deckt sich die Zahl der Feuerstellen in etwa mit der Anzahl der Häuser und halben Häuser. Gewisse Schwankungen lassen sich damit erklären, dass die Uraufnahme zeitlich etwas später erfolgte und damit in der Regel weitere Bauten in überschaubarer Anzahl hinzugekommen waren. Für Weißenburg werden zum Beispiel 543 „Feuerstellen“ gelistet, in der jüngeren Uraufnahme finden sich etwa 500 volle Hausnummern, von denen knapp 50 geteilt

${ }^{288}$ Nachfragen in anderen kommunalen Archiven nach entsprechenden „Seelenlisten“ blieben bisher erfolglos. 
waren, also insgesamt 550 Hausteile. In Herrieden, Lauf, Leutershausen, Scheinfeld und Schwabach scheinen dagegen unter dem Begriff „Feuerstelle“ sämtliche Wohnungen (eigengenutzt und vermietet) innerhalb der jeweiligen Stadt erfasst worden zu sein. Die Anzahl der „Feuerstellen“ liegt hier deutlich über der Anzahl der vorhandenen Anwesen und Teilanwesen, jedoch dicht bei der gelisteten Anzahl der Familien.

Im Falle von Altdorf, wo die Anzahl der „Familien“ die Anzahl der Feuerstellen = Wohnungen übertrifft, liegt die Annahme nahe, dass es sich bei den überzähligen „Familien“ um Haushalte gehandelt hat, die zur Untermiete wohnten und daher keine eigene Feuerstelle hatten. Bei dieser Interpretation errechnet sich für Altdorf ein Wert von 6,6\% Untermieterhaushalten.

In Herrieden, Lauf und Schwabach übersteigt die Anzahl der Wohnungen dagegen leicht die Anzahl der Familien, der Überhang lässt sich durch Leerstände erklären: Bei 312 Wohnungen und 295 Familien errechnet sich für Herrieden eine Leerstandsquote von 5,4\%, für Lauf mit 656 Wohnungen und 631 Familien von 3,2\% und für Schwabach bei 1.979 Feuerstellen $=$ Wohnungen und 1.855 Familien von $1,2 \%$.

Bei drei weiteren Städten, Langenzenn, Windsbach und Pappenheim, deckt sich die angegebene Zahl der „Feuerstellen“ in etwa mit der Anzahl der Hausnummern in den Uraufnahmeblättern. Diese Städte wiesen jedoch darüber hinaus eine beträchtliche Zahl von geteilten Anwesen und Hausnummern auf, sodass die Anzahl der Hauseigentümer deutlich höher lag als die Anzahl der gelisteten „Feuerstellen“.

In Uffenheim wiederum scheinen unter dem Begriff „Feuerstellen“ die Anzahl der Heizmöglichkeiten gezählt worden zu sein, denn die genannten 472 Feuerstellen übersteigen deutlich die Anzahl der gleichzeitig aufgeführten 387 Familien. ${ }^{289}$

Nicht erklärt werden können bisher die Angaben zu Wassertrüdingen, dort sind 492 „Familien“ aufgeführt und 386 Feuerstellen. Gleichzeitig lag die Zahl der Wohnhäuser nach dem Uraufnahmeblatt jedoch nur bei rund 225. Hier entsprechen die „Feuerstellen“ also weder der Anzahl der Eigentümer noch der Anzahl aller Wohnungen, es sei denn, dass in Wassertrüdingen zu diesem Zeitpunkt 116 „Familien“ (23,6\%) zur Untermiete gewohnt haben.

In Velden wiederum wäre zu prüfen, ob der Erfasser den Begriff der „Familie“ abweichend interpretiert hat: Gelistet sind 89 Feuerstellen und 90 „Familien“. Im Uraufnahmeblatt finden sich etwas mehr Anwesen, nämlich 93, davon waren fünfzehn geteilt. Mit der „Feuerstelle“ könnten hier also sehr gut die ungeteilten

${ }^{289}$ Alphabetisches Verzeichnis 1818, S. 92. 
Anwesen gemeint gewesen sein, wie in Langenzenn, Windsbach und Pappenheim. Auffällig ist für Velden darüber hinaus die durchschnittliche Familiengröße von 5,3 Personen. Der Durchschnittswert aller 28 Städte lag im Jahr 1818 bei lediglich 3,83 Personen, also um 1,5 Personen niedriger! Dies legt die Vermutung nahe, dass im Falle von Velden Untermieter als Mitglieder der „Familien“ gezählt wurden. 100 Jahre zuvor lässt sich dort noch ein überdurchschnittlich hoher Mieteranteil von 30\% aller Haushalte nachweisen. ${ }^{290}$ Dass ausgerechnet in Velden die Mietbevölkerung 1818 bereits abgewandert gewesen wäre, ist daher mehr als unwahrscheinlich.

Auch in Greding lag die Anzahl der 149 erfassten Feuerstellen deutlich unterhalb der Anzahl der 189 „Familien“, diese Zahl wiederum entspricht in etwa der Anzahl der Hauseigentümer im Uraufnahmeblatt. Da in Greding sicher keine 40 Häuser (21\%) leer standen, können die überlieferten Zahlen aktuell nicht interpretiert werden.

Trotz der aufgeführten Einschränkungen bilden die in dem Verzeichnis überlieferten Daten eine wichtige Grundlage, um eine Vorstellung vom Anteil der eigentumslosen Bevölkerung in Mittelfranken zu Beginn des 19. Jahrhunderts zu erhalten. Dabei kann die „Familie“ mit jeweils einem Haushalt gleichgesetzt werden. Nachdem in der Regel die Eigentümer in Mittelfranken nur ein Haus (-Teil) besaßen und dieses auch selbst bewohnten, lässt sich der Anteil der Mietbevölkerung einfach errechnen: Der Anteil der Familien, der die Zahl der Eigentümer übersteigt, kann pauschal als Mieteranteil gelten. In den Fällen, wo der Begriff der „Feuerstelle“ nicht mit der Zahl der (Teil-)Anwesen übereinstimmt, wird hilfsweise auf die Angaben in den Uraufnahmeblättern zurückgegriffen, um den Anteil der Eigentümer unter den Familien zu bestimmen. Der so errechnete Mieteranteil dürfte daher etwas unterhalb des tatsächlichen Mietquotienten liegen, da in geringem Umfang Eigentümer auch mehr als ein Haus besaßen. Für Nürnberg lassen sich sehr viel mehr Häuser nachweisen, die zur Gänze vermietet und nicht vom Eigentümer bewohnt wurden. Entsprechend ist hier gegenüber dem so errechneten Wert von einem deutlich höheren Mieteranteil auszugehen. ${ }^{291}$ Für Fürth mag dies auch zutreffen, nur ist hier der Mieteranteil bereits so hoch, dass dieser Effekt keine Rolle mehr spielt.

Schwieriger einzuschätzen ist der Anteil der Altenteiler unter den eigentumslosen Haushalten. Nachdem die durchschnittliche Familiengröße bei den bereits genannten 3,83 Personen lag, dürften hier die Altenteiler nicht mitgerechnet sein, sie sind also in der Gruppe der zuvor ermittelten Miethaushalte zu finden.

${ }^{290}$ StAN, Reichsstadt Nürnberg, Bauernverzeichnisse Nr. 7, fol. Fol. 533' -537.

${ }^{291}$ Vgl. Kapitel 6, Nürnberg. 


\section{Probleme}

Die zuvor besprochenen Quellen, auf die von mir zurückgegriffen wurde, um Aussagen zum Anteil der Miethaushalte zu gewinnen, enthalten in der Regel keine präzisen Angaben. Zum einen sind sie im Hinblick auf ihre Genauigkeit nicht mit heutigen statistischen Erhebungen zu vergleichen, zum anderen standen die eigentumslosen Haushalte nicht im Fokus der jeweiligen Erfassungen.

\section{Vollständigkeit}

Ein grundlegendes Problem der frühen Quellen zu Bevölkerungszahlen und der prosopographischen Forschung ist deren Unvollständigkeit. In der Regel fehlen Personenkreise, deren Erfassung für die ursprüngliche Fragestellung nicht relevant erschien. Aus diesem Grund ist die Frage nach den Bevölkerungsteilen, die nicht erfasst worden sind, ebenso elementar wie die Betrachtung der erfassten Personen. Nur in wenigen Fällen wird es gelingen, anhand weiterer lokalhistorischer Quellen die fehlenden Bevölkerungsteile zu quantifizieren. Dieses Problem soll anhand der Mieterzahlen für Schwabach um 1670 erläutert werden:

In enger zeitlicher Folge liegt hier Datenmaterial vor: In den Huldigungslisten der Jahre 1668 und 1673 sind sowohl Eigentümer als auch Mieter erfasst. ${ }^{292} \mathrm{Als}$ dritte Quelle kann ein Steuerregister von 1671 herangezogen werden. ${ }^{293}$ Für das Jahr 1668 sind 251 Eigentümer, 64 männliche und 23 weibliche Miethaushalte aufgeführt, dies ergibt zusammen 338 Haushalte. Zwei Jahre später, 1673, sind es hingegen 249 Eigentümer, 60 männliche und vier weibliche Miethaushalte, zusammen also nur 313 Haushalte. Die Zahlen in den Huldigungslisten 1673 entsprechen dennoch weitgehend denen der Huldigungsliste von 1668, mit einer signifikanten Ausnahme: Unter den Miethaushalten sind nur vier Schutzverwandtenhaushalte mit weiblichem Haushaltsvorstand erfasst, anstelle von 23 weiblichen Miethaushalten fünf Jahre zuvor. Der direkte Vergleich dieser Zahlen lässt auf unterschiedliche Erfassungskriterien schließen.

Irritierender ist jedoch ein Blick auf die Gesamtzahlen der Haushalte: Im Jahr 1671 waren nach den Angaben des Steuerregisters 402 Haushalte vorhanden. ${ }^{294}$ Im Hinblick auf die deutlich niedrigere Zahl der erfassten Haushalte in den Huldigungslisten drei Jahre zuvor und zwei Jahre später ergeben sich viele Fragen, da es unwahrscheinlich ist, dass die Anzahl der Haushalte in so kurzer Zeit solchen Schwankungen unterlag. Demnach müssen in den Huldigungslisten eine

\footnotetext{
${ }^{292}$ StAN, Fm AN, Brdbg. Lit. Nr. 594, fol. 457' - 478; fol. 642 und Nr. 595, fol. 335-444 und 449-451.

${ }^{293}$ StadtASchwabach Steuerveranlagung von 1671 (Dekanat Schwabach Klasse II Tit. I. Bd.1). Vgl. DEHM/HECKEL 1970, S. 8 und 12.

${ }^{294}$ Die Zahlen sind dem Häuserbuch (DEHM/HECKEL 1970) entnommen, dort S. 8.

Quellenverzeichnis, S. 12.
} 
ganze Anzahl von Haushalten unerwähnt geblieben sein. Gegenüber dem Steuerregister fehlen in den Huldigungslisten 63 beziehungsweise 89 Haushalte. Bezogen auf die 402 Haushalte im Steuerverzeichnis sind dies immerhin 16\% beziehungsweise $22 \%$. In den Huldigungslisten sind die Eigentümer, Hausgenossen, Schutzverwandten und Juden nach Gruppen differenziert. Eine Gruppe, die fehlt, sind die öffentlich Bediensteten, hier ist von 20 bis 30 Haushalten auszugehen. ${ }^{295}$ Darüber hinaus enthalten die Huldigungslisten keine Namen zu sonstigen Eximinierten, die Stärke dieser Gruppe kann entsprechend schwer eingeschätzt werden. Während in der Gruppe der Hausgenossen 1686 nur männliche Namen verzeichnet sind, finden sich in der Gruppe der Schutzverwandten nur Frauen. Im Jahr 1673 sind jedoch bei den Schutzverwandten nur noch vier Frauen, dafür aber zehn christliche Männer genannt. Der starke Rückgang der Frauenhaushalte unter den Schutzverwandten legt die Vermutung nahe, dass die in den Huldigungslisten nicht erfassten Haushalte unter den Haushalten der Schutzverwandten - darunter vermutlich viele mit weiblichem Haushaltsvorstand - zu suchen sind. Diese unbürgerlichen Haushalte lebten wie die Beamten zum allergrößten Teil nicht im Eigentum, sind also den Miethaushalten zuzurechnen. Der sich aus den Angaben der Huldigungslisten ergebende Anteil von Miethaushalten 1668 von $26 \%$ und 1673 von $20 \%$ wäre also deutlich nach oben zu korrigieren auf maximal $42 \%(26 \%+16 \%$ beziehungsweise $20 \%+22 \%)$. Da unter den nicht erfassten Haushalten jedoch auch einige über Hauseigentum verfügt haben könnten, wäre der Anteil der Miethaushalte in entgegengesetzter Richtung um einen unbekannten, aber wohl deutlich geringeren, Faktor zu reduzieren.

\section{Altenteiler}

Ein großes Problem für die Forschung stellt die Frage nach der Situation der Altenteiler dar. ${ }^{296}$ Diese können in den Quellen als eigenständige und eigentumslose Haushalte erfasst oder in anderen Fällen ohne weitere Nennung stillschweigend in den Haushalten der übernehmenden Generation enthalten sein. Das Thema der ungenauen Abgrenzung und Begriffsdefinition ist dabei nicht neu. Bereits Johann Gottlob Klingner geht auf dieses Problem Mitte des 18. Jahrhunderts in seiner Rechtssammlung ein. Im Kapitel mit der Überschrift „Von Hausgenossen “297 unterscheidet er die "würklichen Auszugs=Leuthe“, die aufgrund von Eigentum und Einkommen als Hausgenossen angesehen und die entsprechenden Abgaben leisten sollen, von „denjenigen Eltern und Schwieger= Eltern,

\footnotetext{
${ }^{295}$ In der deutlich kleineren Amtsstadt Gunzenhausen werden im Jahr 166821 (StAN, Fm AN, Brdbg. Lit. Nr. 594, fol. 247) und 167322 öffentlich Bedienstete aufgeführt (StAN, Fm AN, Brdbg. Lit. Nr. 595, fol. 263).

${ }^{296}$ Vgl. hierzu WENDEROTH 2019a, S. 68f.

${ }^{297}$ KLINGNeR 1749, S. 148-149.
} 
welche an ihrer Kinder lohn und brodt seyn", also kein eigenes Einkommen aufweisen. ${ }^{298}$ Von Letzteren soll nach seiner Definition weder „Schutz noch Hausgenossen=Geld" eingefordert werden. Gleiches gilt für erwachsene Kinder und Schwiegerkinder, wenn diese kein eigenes Gewerbe ausüben. ${ }^{299}$ Diese Personen gehören seiner Auffassung nach nicht zu den Hausgenossen. Er verweist dabei auf ein Mandat aus dem Jahr 1709 und einen Rezess aus dem Jahr 1726, dort heißt es: „[...] die gebrechlichen Leute, welche ihr Brod nicht selbst verdienen können, noch anderen ums Lohn arbeiten, sondern von den ihrigen erhalten werden müssen, als Hausgenossen nicht anzusehen, folglich von ihnen einige Dienste oder Schutz=Geld nicht zu verlangen. “300

Der überwiegende Teil der Quellen zur Bevölkerungsanzahl trifft keine Aussage zum Status der Altenteiler. Diese können noch einen Haushalt im eigenen Haus geführt haben, in diesem Fall wurden sie in den Listen wie jeder andere Hausbesitzer erfasst, die nachfolgende Generation wohnte dann entweder in einem anderen Haus oder in Einzelfällen auch mit im Haus des Seniors oder dessen Witwe. ${ }^{301}$ Wohlhabende Senioren lebten zudem auch als (Herren-)Pfründner im Spital, ${ }^{302}$ arme Senioren dagegen in Spitälern, die besser als Armenspitäler zu bezeichnen sind. Ein nicht unerheblicher Teil der Großelterngeneration dürfte aber unter den Rubriken Hausgenossen oder Beständner erfasst worden sein. Unklar ist dabei, ob es sich bei dieser Gruppe auch im heutigen Sinne um Mieter gehandelt hat, da sie eine fremde Wohnung gemietet hatten, oder ob sie weiter im angestammten Haus - nun aber nicht mehr als Eigentümer - lebten. Letztgenannte Personen wären nach heutigem Maßstab wohl kaum als Mieter zu bezeichnen.

Wertvolle Quellen, um den Anteil der Altenteiler an der Gesamtbevölkerung des Untersuchungsgebietes zu ermitteln, stellen sowohl das Reichssteuerregister oberhalb und unterhalb Gebürgs von $1497^{303}$ als auch in Teilen das Türkensteu-

erverzeichnis der Reichsstadt Nürnberg von 1717 dar. ${ }^{304}$ Exemplarisch soll der Fragestellung nach den Altenteilern in der Bevölkerung und ihren Eigentumsverhältnissen anhand des Reichssteuerregisters von 1497 nachgegangen werden.

\footnotetext{
${ }^{298}$ Ebd., S. 148.

${ }^{299}$ Ebd., S. 148-149.

300 Ebd., S. 149 Anm. 429.

${ }^{301}$ StAN, Rst. N, Bauerverzeichnisse Nr. 7, fol. 368: Bei Elias Preß in Hersbruck ist ein Beständner aufgeführt, der als Schwiegersohn bezeichnet wird und die Arbeit des Knechtes übernimmt. ${ }^{302}$ Für Lauf werden 1717 zehn Pfründner im Spital aufgeführt, die nach Beschluss von Abgaben befreit waren. StAN, Rst. N, Bauerverzeichnisse Nr. 7, fol. 406'.

${ }^{303}$ Vgl. RECHTER 1985 und 1988.

${ }^{304}$ StAN, Rst. N, Bauerverzeichnisse Nr. 7.
} 


\begin{tabular}{|c|c|c|c|c|}
\hline 1497 & Herdgemein- & & Altenteiler & \\
\hline Ort & schaften & Spitalbewohner & Herdg. mit Altenteilern & Anteil Herdg. mit Altenteilern \\
\hline Ansbach gesamt & 450 & 6 & 21 & $5 \%$ \\
\hline Arzberg & 69 & & 3 & $4 \%$ \\
\hline Bayreuth & 310 & & 18 & $6 \%$ \\
\hline Berneck & 76 & & 1 & $1 \%$ \\
\hline Crailsheim & 219 & 47 & 10 & $5 \%$ \\
\hline Erlangen & 92 & & 4 & $4 \%$ \\
\hline Feuchtwangen & 151 & 20 & 11 & $7 \%$ \\
\hline Gunzenhausen & 217 & 9 & 5 & $2 \%$ \\
\hline Helmbrechts & 59 & & 8 & $14 \%$ \\
\hline Hof, Vorstadt & 128 & & 3 & $2 \%$ \\
\hline Kulmbach gesamt & 291 & & 9 & $3 \%$ \\
\hline Langenzenn & 112 & & 16 & $14 \%$ \\
\hline Neustadt/Aisch & 286 & 8 & 17 & $6 \%$ \\
\hline Neustadt/Kulm & 101 & & 2 & $2 \%$ \\
\hline Roth & 111 & & 2 & $2 \%$ \\
\hline Schauenstein & 38 & & 1 & $3 \%$ \\
\hline Selb & 73 & & 9 & $12 \%$ \\
\hline Uffenheim & 103 & 8 & 9 & $9 \%$ \\
\hline Wassertrüdingen & 149 & & 9 & $6 \%$ \\
\hline Weißenstadt & 138 & & 16 & $12 \%$ \\
\hline Wunsiedel & 188 & & 7 & $4 \%$ \\
\hline Gesamt & 3.361 & 98 & 181 & $5 \%$ \\
\hline
\end{tabular}

Tabelle 1. Dreigenerationenhaushalte in Städten des Fürstentums Brandenburg-Ansbach-Kulmbach nach dem Reichssteuerregister 1497

(Thomas Wenderoth 2019, nach RECHTER 1985 und 1988)

Aus diesem Grund wurden alle Angaben mit ausgewertet, bei denen verheiratete Kinder zusammen mit einem oder beiden Elternteilen in häuslicher Gemeinschaft lebten. Unberücksichtigt geblieben ist bei dieser Zusammenfassung, ob noch die Eltern- oder schon die Kindergeneration den Haushaltsvorstand stellte, da die Kernfrage darauf abzielt, in welchem Umfang Dreigenerationenhaushalte vertreten waren. ${ }^{305}$ Bei den gelisteten Hausgenossenhaushalten können solche Mehrgenerationenhaushalte in jedem Fall nicht nachgewiesen werden. Hier blieben die Altenteiler entweder bis zum Lebensende Mieter mit einem eigenen Hausstand oder wurden im Armenspital versorgt. Aber auch bei den Eigentümerhaushalten war der Anteil von Dreigenerationehaushalten gering. Der Anteil der Haushalte mit Altenteilern ist in der Tabelle prozentual nur im Hinblick auf die Haushalte mit einer eigenen Listennummer (Herdgemeinschaft) errechnet. Durchschnittlich finden sich in den 21 berücksichtigten Städten des Fürstentums Brandenburg-Ansbach-Kulmbach nur bei $5 \%$ der Listeneinträge Haushalte mit drei Generationen. Zum Vergleich: Christian Forneck kommt für Regensburg auf

${ }^{305}$ Rechter erfasst dagegen sämtliche Haushalte mit Verwandten, RECHTER 1985, S. 660-664, SOwie RECHTER 1988, S. 221-224. 
4\% Haushalte, bei denen im Jahr 1436 Altenteiler mitlebten. ${ }^{306}$ Festzustellen ist, dass diese Zahlen nah bei den fränkischen Angaben liegen.

Nach den Angaben im Reichssteuerregister streuen die Werte zwischen 1\% in Berneck und 14\% in Helmbrechts, beides Zwergstädte in Oberfranken die sich im Hinblick auf einen hohen Mieteranteil ansonsten gleichen. Hier wird bereits deutlich, dass kein Zusammenhang festzustellen ist zwischen dem Anteil an Hausgenossen und dem Anteil von Altenteilern in den Häusern. Ungewöhnlich hoch ist deren Anteil lediglich in Oberfranken - und hier neben Helmbrechts auch in Selb (12\%) und Weißenstadt (12\%), letztere Stadt gehört wiederum zu den Städten mit dem geringsten Hausgenossenanteil (2\%). Interessant erscheint auch die Feststellung, dass das Vorhandensein eines Spitals nicht als Erklärung für den Anteil der Dreigenerationenhaushalte in den betreffenden Städten herangezogen werden kann. Weder ist der Anteil an Dreigenerationenhaushalten noch der an Hausgenossen, bei denen es sich ja häufig um arme alte Witwen handelte, in diesen Städten signifikant geringer. Vermutlich wäre der Anteil der Altenteiler ohne das Vorhandensein eines Spitals in diesen Städten dennoch höher gewesen. Dies gilt insbesondere für Crailsheim, dort sind mit Abstand die meisten Spitalbewohner verzeichnet. Würde man von den 47 Spitalbewohnern 37 den Hausgenossen und zehn den Dreigenerationenhaushalten zuordnen, stiege der Anteil der Altenteiler von 5\% auf $9 \%$ und der der Hausgenossen von 15\% auf 26\% und somit auf einen Spitzenplatz. Insgesamt betrachtet stehen den 175 Altenteilern 98 Pfründner und Spitalinsassen gegenüber.

Für die Barockzeit kann zur Beantwortung dieser Frage das Türkensteuerverzeichnis der Reichsstadt Nürnberg für deren Landgebiet herangezogen werden. Nach einer entsprechenden Vorgabe sollten auch die Menschen erfasst werden, die sich den Winkel in ihren verkaufften Häußern vorbehalten und ausgedinget haben. ${ }^{307}$ Zumindest in einem Teil der erfassten Orte sind die Altenteiler dann auch entsprechend unter der Rubrik Im Winkel, aufgeführt, diese Differenzierung findet sich aber nicht in allen erfassten Orten. In Einzelfällen sind zudem nicht nur Altenteiler als Im Winkel lebend aufgeführt. So wohnten 1717 bei Christoff Rupprecht in Hersbruck gleich vier Frauen im Winkel. ${ }^{308}$ Es ist mehr als unwahrscheinlich, dass es sich hierbei um vier Alteigentümerinnen gehandelt hat, zumindest deuten die Familiennamen keine verwandtschaftlichen Beziehungen an. ${ }^{309}$ Zählt

\footnotetext{
${ }^{306}$ FORNECK 2000, S. 81.

${ }^{307}$ StAN, Rst. N, Bauerverzeichnisse Nr. 7, Einführung ohne fol., dort Punkt 6.

${ }^{308}$ Ebd., Bauerverzeichnisse Nr. 7, fol. 363.

${ }^{309}$ Es handelt sich hier wohl um eine Art privates „Altenheim“, bei dem Menschen einen Versorgungsvertrag mit Personen außerhalb der eigenen Familie schließen. Ähnliches lässt sich seit dem 15. Jh. für Nürnberg selbst archivalisch belegten. Vgl. StadtAN, B 14/II, Nr. E, Bl. 165 (Jahr 1489); Nr. G, Bl. 104' (1492) und Bl. 220 (1493); Nr. H, Bl. 159' und Bl. 213' (1495); Nr. K, Bl. 15 (1496); Nr. L, Bl. 38 (1498), Nr. Q, Bl. 226' (1505); Nr. 7, Bl. 102 und 152 (1515); Nr. 14,
} 
man auch in diesem Fall nur eine der Mieterinnen als Austräglerin, lebten in Hersbruck maximal in fünfzehn Haushalten Altenteiler im Winkel im Sinne von Austrag. ${ }^{310}$ Bezogen auf insgesamt 212 Hauseigentümer ergibt dies einen Anteil von 7,08\%. In der Nachbarstadt Lauf sind es dagegen zur gleichen Zeit nur 2,03\%: In acht von insgesamt 394 Haushalten lebten dort Altenteiler, hinzu kamen noch zehn Pfründner im Spital und drei Spitalbewohner. In der Beschreibung der 225 Miethaushalte in Altdorf findet sich kein einziger Hinweis auf mitlebende Altenteiler.

Für die nürnbergischen Dörfer hatte ich die Zahlen bereits in meiner Dissertation ausgewertet, hier muss Anfang des 18. Jahrhunderts auf den Höfen mit durchschnittlich $12 \%$ Altenteilern gerechnet werden. ${ }^{311}$ Im Fürstentum Ansbach steigt deren Anteil am Ende des 18. Jahrhunderts auf etwa 16\%, bezogen auf Dörfer und Städte. ${ }^{312}$

Generell gilt festzuhalten, dass zum einen der Prozentsatz der Altenteiler im Verlauf der Jahrhunderte zunimmt und zum anderen deren Anteil in den Städten deutlich unter dem Anteil der Austrägler auf dem Land lag.

Die Frage, ob unter den historischen Begriffen Hausgenossen und Beständner auch Austrägler erfasst sein können, muss wohl bejaht werden, sofern diese nicht anderweitig ausgewiesen sind. Petra Ostenrieder kann diesen Sachverhalt für Oettingen im 18. Jahrhundert nachweisen. Dort wurde 1751 eigens eine Bestimmung erlassen, die ein Mindestvermögen von $200 \mathrm{fl}$ für das Ansässigmachen von Austräglern vorsah, da andernfalls zu befürchten wäre, dass die Eltern ihren ganzen Besitz an die Kinder überschreiben und infolgedessen von der öffentlichen Hand ernährt werden müssten. ${ }^{313}$ Tatsächlich lassen sich aber unter den 107 evangelischen Hausgenossen im Jahr 1727 nur sieben Austragshaushalte nachweisen, darunter zwei Austrägler, die ihr Haus an Fremde verkauft, aber ein lebenslanges Wohnrecht vereinbart hatten.

Für die von mir weiter unten ausgewerteten Huldigungslisten kann dies jedoch weitgehend ausgeschlossen werden: Bei den dort als Hausgenossen/Beständner erfassten Personen handelt es sich in aller Regel um Männer mit einer Berufsbezeichnung, also nicht um Austrägler. In der Gruppe der Schutzverwandten dürften sich ebenfalls keine Austragshaushalte finden lassen, da diese Personen kein

Bl. 105 (1520); Nr. 26, Bl. 27 und 120 (1528); Nr. 28, Bl. 198' und Nr. 29, Bl. 82 (1529); Nr. 29, Bl 125' und Nr. 30, Bl. 116' und Bl. 134 (1530); Nr. 41, Bl. 193' (1537).

${ }^{310}$ StAN, Rst. N, Bauerverzeichnisse Nr. 7, fol. 358' -371 . Das Thema ist bisher nicht erforscht. Für die archivalischen Hinweise danke ich Walter Bauernfeind vom Stadtarchiv Nürnberg.

311 WENDEROTH 2019a, Kapitel II.3.4.4., S. 68f., Tab. 5.

312 Ebd.

313 OSTENRIEDER 1993, S. $76 f$. 
Bürgerrecht hatten, entsprechend mobil waren und nicht in Mehrgenerationenhaushalten lebten.

Bisher nicht klären ließ sich die Frage, ob unter den im „Alphabetischen Verzeichnis 1818“ mit der Bezeichnung „Feuerstelle“ charakterisierten Haushalten auch Altenteiler separat als eine „Feuerstelle“ erfasst worden sind.

\section{Familiäre Situation der Mieter}

Eine weitere, bisher nicht zufriedenstellend gelöste Frage betrifft die Haushaltsgröße und die familiäre Situation. Dies gilt insbesondere für die unmündigen Kinder. In den historischen Verzeichnissen sind regelhaft nur Kinder erfasst, die älter als zwölf Jahre waren, in einigen Fällen liegt die Grenze auch erst bei fünfzehn Jahren.

Eine zusammenfassende Schilderung der familiären Situation in allen städtischen Haushalten versucht Heinz Schilling. ${ }^{314}$ Er legt dar, dass die durchschnittliche Haushaltsgröße bei gut vier Personen lag und dass Kinder früh und regelhaft aus dem elterlichen Haushalt ausschieden. Die letzte Feststellung deckt sich mit den archivalischen Quellen für Franken (vgl. Tab. 17).

Die von Schilling angegebene durchschnittliche Haushaltsgröße spiegelt aber wohl eher die Situation der Eigentümer. Für die eigentumslosen Haushalte scheint sie mir als Durchschnittwert zu groß, da sich unter diesen erstens überproportional viele Einpersonenhaushalte befunden haben und zweitens erwachsene Kinder oder Gesinde dort nur selten anzutreffen waren. Zum Beispiel hat es sich bei den im Jahr 1497 als Hausgenossen erfassten (Unter-)Mietern im Wesentlichen um alleinstehende Personen gehandelt. Weiter unten werde ich noch eingehend die Familienstruktur der eigentumslosen Haushalte in Altdorf 1717 analysieren. ${ }^{315}$

${ }^{314}$ SCHILLING/EHRENPREIS 2015, S. $18 \mathrm{f}$.

${ }^{315}$ Vgl. Kap. 5, Strukturen eigentumsloser Haushalte am Beispiel Altdorf 1717. 


\section{Rechtliche Stellung der eigentumslosen Bevölkerung}

Es kann nicht häufig genug betont werden, dass die Einwohner einer Stadt juristisch betrachtet keine Einheit darstellten. Die städtische Bevölkerung bestand eben nicht nur aus Bürgern, sondern kannte eine ganze Reihe abgestufter Teilhabemöglichkeiten. Dies gilt in besonderem Maß für die Gruppe der Eigentumslosen. Während die Hauseigentümer in aller Regel juristisch betrachtet zur Gruppe der Vollbürger gehörten, lebten die eigentumslosen Personen in den unterschiedlichsten rechtlichen Status in den Städten. In den meisten Städten, so auch in Nürnberg, war der Erwerb von Hauseigentum an das Bürgerrecht gebunden, von daher ergibt sich bei den Eigentümern eine rechtlich geschlossene Gruppe. An dieser bürgerlichen Gruppe konnten jedoch auch Mieter mit Bürgerstatus gleichberechtigt teilhaben.

Wenn hingegen verschiedentlich die Gruppe der Stadtbewohner ohne Bürgerrecht thematisiert wird, kann davon ausgegangen werden, dass die dort aufgeführten Personen weit überwiegend, wenn nicht geschlossen, als Mieter in den Städten lebten.

Hans Mauersberger stellt fest, dass es bereits im Mittelalter Stadtbewohner ohne Bürgerrecht gab. ${ }^{316}$ Auf der einen Seite sieht er dort Kleriker und hochgestellte oder vermögende Personen, auf der anderen Seite Mitglieder der Unterschicht. Die erste Gruppe war politisch protegiert und letztendlich durch die städtische Verwaltung auch gut im Blick zu haben, wohingegen die zweite Gruppe eine gesonderte Aufsicht erforderte. Während des Mittelalters versuchten die Städte, diese Gruppe klein zu halten und möglichst alle Einwohner zur Annahme des Bürgerrechtes und den damit verbundenen Pflichten zu bewegen. Mit der fortschreitenden wirtschaftlichen Entwicklung der Städte, insbesondere ab dem 16. Jahrhundert, wurde die Arbeitskraft einer zahlreich zuziehenden Unterschicht, die sich das Bürgerrecht nicht leisten konnte, aber unentbehrlich für die Gewerbe- und Handelszentren. Um diese Menschen rechtlich zu fassen, haben die Städte verschiedene Wege eingeschlagen. Letztendlich ging es ja auch darum, durch Steuereinnahmen sowie Wach-, Wehr- und Grabendienste das städtische Gemeinwesen aufrecht zu erhalten und gegebenenfalls zu schützen. So entwickelten sich nichtbürgerliche Bevölkerungsgruppen, die mit unterschiedlichen Rechten, Pflichten und Bezeichnungen versehen wurden. Zum einen macht sich hier die politische Zersplitterung innerhalb des Hl. Römischen Reiches Deutscher Nation mit einer Vielzahl von Herrschaftsrechten bemerkbar, andererseits gilt es auch zu bedenken, dass innerhalb des hier geschilderten langen geschichtlichen Zeitraumes vom Mittelalter bis zum Ende des 18. Jahrhunderts in den je-

${ }^{316}$ Vgl. hierzu und im Folgenden MAUERSBERGER 1960, S. 128-151. 
weiligen Herrschaftsbereichen selbst Veränderungen stattgefunden haben. So gewann insbesondere ab dem späten 16 . aber vor allem im 18 . Jahrhundert in den Residenzstädten die Gruppe der Hofbediensteten, die nicht Teil der Bürgergemeinde war, rein quantitativ stark an Bedeutung. ${ }^{317}$

Der ungebremste Zuzug neuer Einwohner wurde zu keiner Zeit lange geduldet; sobald das „Maß“ voll war, wurden entsprechende Gegenmaßnahmen ergriffen und der Zuzug erschwert, zumeist bedeutete dies den Nachweis der charakterlichen Eignung - im jeweiligen Wertekontext - und einer materiellen Grundsicherung. So hatte der Rat der Stadt Basel im Jahr 1528 die Einwanderung und Annahme des Bürgerrechtes so leicht gemacht, dass ein erheblicher Zuzug stattfand und sich der Rat bereits 1541 genötigt sah, mit den oben genannten Anforderungen gegenzusteuern, da nun auch die negativen Folgen deutlich geworden waren. $^{318}$

Im Folgenden sollen verschiedene Rechtsstellungen kurz angesprochen werden, die auf die Mieter in der frühen Neuzeit Anwendung fanden, die Begriffe wurden zum Teil auch synonym für Mieter verwendet, da ein enger Zusammenhang zwischen den (geminderten) persönlichen Rechten und Eigentumslosigkeit besteht.

\section{Bürgerrecht}

Über all den weiter unten folgenden städtischen Einwohnern mit geschmälerten Rechten soll nicht vergessen werden, dass uns Menschen mit Bürgerrecht nicht nur als Hauseigentümer begegnen, sondern sehr wohl auch als Mieter innerhalb der Stadt leben konnten. Da Bürgerkinder leicht und häufig mit geringem finanziellem Aufwand das Bürgerrecht erwerben konnten, entwickelt sich in den Städten auch eine Unterschicht, die aufgrund ihrer Armut nicht in der Lage war, Eigentum zu erwerben, aber sehr wohl das Bürgerrecht besaß, ohne realistisch die Chance zu haben, in den Kreis des städtischen Rates aufgenommen zu werden. In Prichsenstadt heißt es in den Erbhuldigungslisten 1723 entsprechend: folgen die Erbgehuldigte Bürger so keine Häußer haben und zu Haußgenoßen sizen. ${ }^{319} \mathrm{Ne}-$ ben der großen Schicht der vermögenslosen Bürger lassen sich aber auch vermögende Bürger als Mieter nachweisen. ${ }^{320}$

\footnotetext{
${ }^{317}$ Vgl. Kapitel 7, Residenzstädte.

318 MAUERSBERGER 1960, S. $130 f$.

${ }^{319}$ StAN, Fm AN, Brdbg. Lit. Nr. 602, fol. 735.

${ }^{320}$ Vgl. WENDEROTH 2021, S. 152-154.
} 


\section{Privilegierte Einwohner}

Privilegierte Einwohner gehörten ökonomisch betrachtet häufig der Oberschicht oder zumindest der Mittelschicht an. Somit war diesen der Erwerb eines Wohnhauses in vielen Fällen grundsätzlich möglich, sofern der Erwerb von Häusern in den jeweiligen Städten nicht ausschließlich an das Bürgerrecht gebunden war. Da die Privilegierung vom Stadtherrn ausging, bewegen sich die privilegierten Einwohner im Spannungsverhältnis zwischen den unterschiedlichen Rechtskreisen des Landesherrn und der Kommune. Mit der Zunahme dieser Bevölkerungsgruppe im 18. Jahrhundert wird zugleich der zunehmende Einfluss der Territorialherrn auf die Selbstverwaltung der Städte deutlich.

Eine häufig kleine, aber wichtige und privilegierte, Bevölkerungsgruppe innerhalb der Städte stellen die Eximierten dar. Diese hatten nicht das städtische Bürgerrecht, sondern waren stattdessen direkt mit dem Landesherrn verbunden. Ulrich Rosseaux definiert diesen Bevölkerungsteil als in der Stadt lebend, aber nicht der städtischen Bürgergemeinde, sondern einem anderen Rechtsverband angehörig. ${ }^{321}$ Eximiert waren zum Beispiel: Adlige, Kleriker, Angehörige des Hofstaates und öffentlich Bedienstete. Häufig wohnten diese in fremdem Eigentum oder in Dienstwohnungen, die im Eigentum der öffentlichen Hand standen und daher nicht bei den bürgerlichen Häusern erfasst sind. Der Erwerb von Häusern war dieser Gruppe nicht grundsätzlich verwehrt und wäre zumindest für einen Teil auch finanziell leistbar gewesen. $\mathrm{Zu}$ den Eximierten gehörten aber auch Soldaten und - wie im Falle von Altdorf und Erlangen - die Mitglieder der Universitätsgemeinden.

Da diese Personen nicht dem städtischen Rechtskreis angehören, tauchen sie in den Bevölkerungs- und Steuerlisten der Stadtgemeinden in der Regel nicht auf. Lediglich in den Huldigungslisten für Schwabach werden am Schluss 31 eximirte Persohnen aufgeführt, denen neun ganze Häuser und ein halbes Haus gehörten; 21 dieser Haushalte wohnten demnach nicht im Eigentum. ${ }^{322}$ Sie bewohnten wohl überwiegend Dienstwohnungen. Festgehalten werden kann, dass diese Bevölkerungsgruppe generell einen hohen Mieteranteil aufwies, im Hinblick auf die Frage nach eigentumslosen Haushalten also unbedingt Berücksichtigung finden muss.

Sie werden auch nicht durchwegs als Eximierte bezeichnet. In der Huldigungsliste für Erlangen aus dem Jahr 1735 ist der Gruppe der deutschen Bürger zum Beispiel neben den öffentlich Bediensteten eine Personengruppe vorangestellt, die als von ihren Mitteln lebende Inwohner und andere privilegirte

\footnotetext{
321 Rosseaux 2006, S. $55 f$.

${ }^{322}$ StAN, Fm AN, Brdbg. Lit., Nr. 601, fol. 132-133.
} 
Persohnen, bezeichnet wird. ${ }^{323}$ Es handelt sich um achtzehn deutsche Frauen und Männer, darunter vielfach Beamte und Beamtenwitwen. Am Beginn der anschließenden Auflistung der französischen Gemeinde wird eine vergleichbare Gruppe als La Noblese, et Autres Personnes Privilegies bezeichnet. ${ }^{324}$ Es handelt sich um fünfzehn hochgestellte Personen: Adlige, Botschafter etc. Auch für diesen Personenkreis muss in Erlangen mit einem hohen Mieteranteil gerechnet werden.

In einer rechtlich verwandten Stellung lebten die Hofschutzverwandten. Diese bildeten in der ersten Hälfte des 18. Jahrhunderts in den Residenzstädten eine große Bevölkerungsgruppe. Es handelt sich bei den Hofschutzverwandten um Hofangehörige der untersten Klasse.

Der Hofschutzverwandte erhielt bei Hof keine Festanstellung, er stammte aus dem Kreis der Bürger, Einwohner und der Judenschaft. ${ }^{325}$ Sie sind nicht zu verwechseln mit den lediglich als Schutzverwandte bezeichneten Einwohnern, zum Beispiel den Schutzverwandten des Ansbacher Hofkastenamtes oder anderer Obrigkeiten. Das Ziel dieser Personengruppe war es, mittelfristig in den vollen Genuss der höfischen Privilegien zu kommen, von daher ist auch ihre Vermögensund Eigentumssituation ganz anders als die der sonstigen Schutzverwandten $\mathrm{zu}$ bewerten: „Es sind Handwerker, meist Bürger oder zumindest Vertreter von Gewerben, die sie zum Erwerb der Bürgerrechte qualifizieren sollten. Vordringlich entstammen sie der Bekleidungs- und Modebranche [...] dazu kommen Kunsthandwerker und Spezialgewerbe [...] die den gehobenen Bedarf des Hofes teilweise zu decken haben.“" 326

Die Hofbauhandwerker sind dagegen Vollbürger des Hofes (nicht der Stadt). Beide, Hofschutzverwandte und Hofbürger, sind vom Zunftzwang befreit und genießen aufgrund geringerer steuerlicher Lasten einen Wettbewerbsvorteil gegenüber den städtischen Bürgern. ${ }^{327}$ Letzteres führt bei einem starken Anstieg dieser Personengruppe im 18. Jahrhundert zwangsläufig zu Konflikten mit der städtischen Verwaltung, der die Einnahmen wegbrechen.

\section{Eingeschränkte bürgerliche Rechte}

Die Einwohner mit eingeschränkten Bürgerrechten bilden keinesfalls eine geschlossene Gruppe. Allen gemeinsam ist aber, dass sie gegenüber den Bürgern nur geschmälerte Rechte besaßen. Dies gilt in gewisser Weise schon für die oben

\footnotetext{
${ }^{323}$ StABA, Markgraftum Brandenburg-Bayreuth, Geheimes Archiv Bayreuth Nr. 235.

${ }^{324}$ Ebd.

${ }^{325}$ Vgl. BAHL 1974, S. 80.

${ }^{326}$ Ebd., S. 81.

${ }^{327}$ Vgl. hierzu und im Folgenden ebd., S. 83f.
} 
erwähnte Gruppe der Privilegierten, wobei hier wohl die Vorteile die Nachteile überwogen und diesen Personen der Erwerb der vollen Bürgerrechte in der Regel wohl jederzeit möglich gewesen wäre.

Als erstes soll hier die Gruppe der Pfahlbürger angesprochen werden, da dieser im Verlauf des Mittelalters seine Bedeutung wechselt. ${ }^{328}$ Ursprünglich wurden als Pfahlbürger die Bürger einer Stadt bezeichnet, die nicht ihren (ständigen) Wohnsitz in der Stadt hatten, aber sich sowohl den Zugang zu den städtischen Marktprivilegien als auch den Schutz der Mauern sichern wollten. Es handelte sich dabei um wohlhabende Dorfbewohner und Ritter, also einen Personenkreis der später als „Privilegierte/Examinierte“ bezeichnet wird. Unklar ist, inwieweit diese Personen überhaupt einen dauerhaften Wohnsitz in der Stadt hatten.

Im Bürgerbuch der Stadt Ansbach, 1488-1562, finden sich zum Beispiel nur drei entsprechende Einträge: Melcher Ziegeldecker 1510, Hanns von Hall 1532 und Wolff Marquart 1561. Für dieses Jahr ist auch die jährliche Zahlung für Pfahlbürger in Höhe von $1 \frac{1}{2} \mathrm{fl}$ Steuer und eine wöchentliche Zahlung von $1 / 2 \mathrm{fl}$ bezeugt, was zusammen fast $30 \mathrm{fl}$ im Jahr ergibt! Ob die so bezeichneten drei Pfahlbürger bereits dauerhaft im Sinne eines Schutzverwandten in der Stadt lebten, ist unbekannt. Es findet hier also ein Wechsel in der Begrifflichkeit statt. Bis zum Ende des Mittelalters wurde diese Rechtskonstellation jedoch zumeist aufgehoben. ${ }^{329}$ An ihre Stelle traten andere Formen der Aufenthaltsgewährung, die in ihrem rechtlichen Umfang nicht dem Bürgerrecht entsprachen.

Spätestens in der Neuzeit wird der Begriff des Pfahlbürgers dann aber synonym für Schutzverwandte gebraucht bzw. durch letzteren abgelöst, dies trifft zum Beispiel für Ansbach im 17. und 18. Jahrhundert zu. ${ }^{330}$ Die rechtlichen Rahmenbedingungen dürfte daher identisch mit denen der Schutzverwandten gewesen sein.

Im Deutschen Rechtswörterbuch findet sich zum Begriff des Schutzverwandten folgende Definition: „Einwohner eines Landes (II 1) oder einer Stadt ohne volles Wohn- oder Bürgerrecht, der gegen Bezahlung eines Schutzgeldes (III) geduldet wird und den Schutz (II) der Obrigkeit genießt; häufig synonym für Schutzjude.“ Folgende synonyme Bezeichnungen werden zudem angegeben: „Beisasse (I 1), Hintersasse (I), Schirmverwandte (II), Schultheißenbürger, Schutzangehörige, Schutzgenosse (III), Schutzleute, Schutzuntertan.“331

\footnotetext{
${ }^{328}$ Vgl. DilCHER 1980, S. 96-97.

${ }^{329}$ In Hagenau wurden nur die auswärtigen Handwerker als „Pfahlbürger“, die Adligen dagegen als „Ausbürger bezeichnet“. Sie lassen sich dort ab 1352 bis ins 17. Jh. archivalisch nachweisen; BURG 1967, S. 93.

${ }^{330}$ Vgl. hierzu und im Folgenden KoBer 1964, S. 9.

${ }^{331}$ DRW: (Art.) Schutzverwandte.
} 
Auffällig ist, dass die anschließend zitierten Rechtsquellen ausschließlich der Neuzeit zwischen 1530 und 1810 entstammen. Bei Georg Stephan Wiesand heißt es 1762: SchutzLueute, SchutzVerwandte: Die zwar an einem Ort den Schutz der Obrigkeit, nicht aber das BürgerRecht geniessen. ${ }^{32}$ Georg Andreas Will beschreibt die rechtliche Bindung und das Prozedere ausführlicher: Sobald der Verspruch Mann, Hintersäs ec. Pflicht gethan, stehet er unter dem Schuz, und zugleich der Gerichtbarkeit seines Mund $=$ und Vogtey $=$ Herrn. An einigen Orten werden ihm besondere Schutzbriefe ausgefertigt, darinn beurkundet wird, daß sie in Schuz, Schirm und Verspruch genommen, und ihnen, wie anderen Unterthanen, Verteidigung zugesichert worden [...]. Es darf sich daher kein Beständner, Hintersäs, nirgend anders zu Recht stellen, nicht Vormund werden, kein Zeugnis geben, als nach beschehener Ledigung von seinem Schuz = oder welches im Lande Franken einerley ist, von seinem Vogtey $=$ Herrn. Er darf nur von ihm Recht geben und nehmen. Er reicht dem Territorial $=$ Herrn, so auf seinem Zins = Verleiher [Vermieter], Steuer, Reiß und Folge hat, seine Steuer, und anders und ist also in allen demienigen unterworfen, dem sein Hausherr unterworfen ist. Zur thätigen Anerkennung seines Schuz = oder Vogteyherrn, und seines ihm schuldigen Gehorsams, gibt er iemen alliährlich, meistens um Martini, einen Mundweck [...]. ${ }^{333}$

Zur Situation auf dem Land führt er dann weiter aus: Ein solcher Beständner, Hintersäs ec. der in ein Dorf ziehen will, muss vor allem die Erlaubnis darzu, bey dem Mund $=$ oder Vogtey $=$ Herrn, unter dessen Gerichtszwang der Vermieter stehet, und unter dessen Schuz und Botmäßigkeit er sich begeben will, erhalten. An einigen Orten muß auch die Gemeine vernommen werden, ob sie nichts wider den Aufziehenden einzuwenden habe, in deren Lade er etwas für Aufzug Geld zu entrichten hat. Bekommt er die Erlaubnis, so hat er von seiner vorherigen Herrschaft ein Zeugnis seines Wohlverhaltens und seiner Entlassung beyzubringen. Dann, wann er aufgezogen ist, wird er von dem neuen Herrn angeherre oder verherre, d. i. es wird ihm die Verspruch = Pflicht vorgelesen, und er gelobt darauf Treue und Gehorsam, mit dem Versprechen, seinem Mundweck, oder Verspruchgeld, und andere Obliegenheiten richtig abzuführen. [...]

Die Dauer dieser beederseitigen Verbindlichkeit, nehmlich des Schuzes und des Gehorsams, hängt von dem kurzen oder langen Aufenthalt des Beständners ab. Meistens an Allerheiligen, wird der Bestand entweder aufgekündet, oder wieder auf ein Jahr verneuert. In letztern Fall giebt er einen Leykauf, der gewonlich 30 kr. beträgt. Wird der Bestand aufgekündet, so zieht er an Lichtmeß aus. Hat sein Locator nichts darwider einzuwenden, so entrichtet er sein Abzug Geld, oder Abzug Henne, und er bekommt von seinem Vogtey $=$ oder Schuz $=$ Herrn einen schriftlichen Abschied, und darinn ein Zeugnis seines Wohlverhaltens, nebst seiner Pflichts Entlassung, unter Vorbehalt der

\footnotetext{
332 WIESAND 1762, S. 980.

333 WILL 1781, S. 74.
} 
gewöhnlichen Nachrechte. Das heist: der Abziehende ist verbunden, bey dem nächsten Ehehaft $=$ Recht, das in dem Dorf seiner letzten Wohnung gehalten wird, bey allenfallsiger Beschwehrung über ihn, noch zu erscheinen, und Red und Antwort zu geben. ${ }^{334}$

In den für Franken untersuchten Quellen findet sich ein Hinweis auf Schutzverwandte erstmalig $1497 \mathrm{im}$ Reichssteuerregister für die Stadt Wunsiedel. ${ }^{335}$ Nur in dieser Stadt wird bei der Auflistung unterschieden zwischen den Bezeichnungen Hausgenosse und incola/incole, der lateinischen Bezeichnung für Menschen, die kein volles Bürgerrecht besitzen. Dass es sich bei den als incola/incole bezeichneten Personen um Mieter handelt, geht nur indirekt aus den Einträgen hervor: Sie stehen immer mit Namen an zweiter Stelle, sodass es sich eben nicht um Eigentümer gehandelt haben dürfte, die an erster Stelle des Eintrages genannt werden. ${ }^{336}$ Von den zehn Nennungen zu Mietern heißt es viermal incola/incole.

Nur die Bürger verfügten über die vollen städtischen Rechte, Schutzverwandte hingegen hatten weder passives noch aktives Wahlrecht, ${ }^{337}$ was im Zeitalter des Feudalismus vielleicht nicht ganz so relevant war. Die fälligen Aufnahmegebühren für die Schutzverwandten waren hingegen deutlich günstiger als die für die Bürger. Nachteilig am Status des Schutzverwandten war jedoch, dass in vielen Orten nur Bürger Häuser erwerben konnten. Die Unterscheidung zwischen Bürgern und Schutzverwandten als Nichtbürger hatte bis ins 19. Jahrhundert hinein Bestand. 338

Im Zuge der Erbhuldigung im Fürstentum Ansbach 1668 ist die Ablösung des Schutzverwandten-Status und die Vollaufnahme in die Bürgerschaft ein großes Thema. Erfasst sind für die meisten Orte auch Personen, die im Zusammenhang mit dem Schutzverwandtenstatus stehen. Folgende Anmerkungen sind zu finden: Sechs Maindörfer: 4. Erbschutzverwandte. ${ }^{339}$ Bemberg: 1 . Christ im Schuz ist ablösig. Lobenhausen: 1. in Erbschutz, 1. ist ablösig. ${ }^{340}$ In der Zusammenfassung zu den südöstlichen Gebieten des Fürstentums Ansbach werden aufgelistet: Besetzte 4410, Schutzverw. 405, Lehenbare 270, Öede 920. ${ }^{341}$ Für die westlichen Gebiete heißt es dagegen: 5628. würkliche Mannschaften, 543. Öede, 115. Im Erbschutz, 142. ablösig, 105. Juden. ${ }^{342}$ Neben der Erfassung der noch immer öd liegenden Haus-

\footnotetext{
${ }^{334}$ WiLL 1781, S. 73-75; vgl hierzu auch Kapitel 2.

${ }^{335}$ StABA, Geheimes Hausarchiv Plassenburg, Akten und Bände 5977/1, fol. 47-50, zitiert nach RECHTER 1988, S. 41-45.

${ }^{336}$ Ebd., S. 41: 1583 Rebin, witbe, Hanns peck, sein hawsfr(au), incole.

${ }^{337}$ Vgl. hierzu und im Folgenden GöHRING 1934, S. 26.

338 Ebd.

${ }^{339}$ StAN, Fm AN, Brdbg. Lit. Nr. 594, fol. 645'.

${ }^{340}$ Ebd., fol. 646.

${ }^{341}$ Ebd., fol. 643.

${ }^{342}$ Ebd., fol. 647.
} 
und Hofstellen als Nachwirkung des Dreißigjährigen Krieges scheint die Reduzierung der Zahl der Schutzverwandten ein wichtiges Ziel gewesen zu sein. Beispielsweise werden in den Huldigungslisten aus dem Jahr 1668 für Feuchtwangen neun ablösige Schutzverwandte erwähnt. ${ }^{343}$ Auch im nachfolgenden Jahrhundert dauerten diese Bemühungen an: Die Obrigkeit versuchte weiterhin, Schutzverwandte zur Annahme des Bürgerrechtes zu bewegen. So war der Markgraf 1754 bemüht, in Schwabach die Anzahl der Schutzverwandten zu reduzieren auf Personen der unteren Sozialschichten. ${ }^{344}$

Auch die Juden lebten im Status des Schutzverwandten. Hans Hubert Hofmann schreibt dazu: „Dabei galten die Juden auch nie als ,Untertanen“ eines Herrn oder Glieder einer Gemeinde, sondern stets als Schutzverwandte, genossen folglich keine jener Vorformen ,staatsbürgerlicher' Rechte, sondern lediglich den unscharf umrissenen persönlichen Sonderstatus im Rahmen ihrer Körperschaft. “345 Ebenso wie das Aufenthalts- und Niederlassungsrecht war für Juden in Franken auch das Recht, Hauseigentum zu erwerben stark reglementiert und nach Zeit und Ort verschieden.

In den Huldigungslisten des Fürstentums Ansbach aus dem Jahr 1668 werden explizit die Juden in Hausbesitzer und Mieter unterschieden. ${ }^{346}$ So heißt es in dem summarischen Eintrag zu Feuchtwangen: 19 Juden als 12. so Häußer, und 7. so keine Häußer haben. ${ }^{347}$ Damit unterscheiden sie sich in der Eigentumsfrage von den christlichen Hausgenossen, für die die Möglichkeit ein Haus zu besitzen, eine Ausnahme darstellte. Bei entsprechendem Wohlstand konnten die christlichen Schutzverwandten jedoch die vollen Bürgerrechte und damit auch Hausbesitz erwerben, ihnen standen also andere Wege offen.

Grundsätzlich war ein Zusammenhang zwischen dem Mieterdasein und fehlendem Bürgerrecht auch in den anderen deutschen Regionen gegeben. So mussten die eigentumslosen Haushaltsvorstände in Zwickau 1564/66 gegenüber dem Rat der Stadt einen Treueeid schwören. ${ }^{348}$ Ursächlich dafür war nicht das Mieterdasein, sondern das fehlende Bürgerrecht. In Chemnitz lag der Fokus des Eides im Jahr 1600 mehr auf dem Fernhalten der Mieter von zünftischen Arbeiten und war

\footnotetext{
${ }^{343}$ Ebd., fol. 213'.

${ }^{344}$ Privilegien des Markgrafen Carl Wilhelm Friedrich vom 10. Juli 1754, Art. VII: „Zur Erhaltung guter Ordnung, dann gemeiner Stadt und Bürgerschafft Nutzen und Aufnehmen, hiermit wohlbedächtig statuieren, daß alle diejenigen, so bürgerliche Gewerb, Handel und Wandel treiben, als blosse Schutz=Verwandten nicht geduldet, sondern gehalten seyn sollen, sich als wirkliche Burgere einzulassen." Zitiert nach FALCKENSTEIN 1756, S. 127.

${ }^{345}$ HOFMANN 1986, S. 2897.

${ }^{346}$ StAN, FM AN, Brdbg. Lit. Nr. 594, fol. 646.

${ }^{347}$ Ebd., Nr. 594, fol. 646'.

348 BRÄUER 2003, S. 83.
} 
zugleich als Schutz der bürgerlichen Handwerker gedacht. ${ }^{349}$ Hier waren die Bemühungen der Obrigkeit, möglichst viele Einwohner als Vollbürger zu gewinnen, schon früh erfolgreich: 1548 werden 629 Miethaushalte genannt, davon besaßen $90 \%$ das Bürgerrecht. Im Jahr 1700 waren dann nahezu alle Mieter, die zur Steuer veranlagt wurden, Bürger. ${ }^{350}$ Abweichend von der Situation in Franken besaßen also bis ins 18. Jahrhundert hinein die Chemnitzer Mieter überwiegend das Bürgerrecht. Im Verlauf des 18. Jahrhunderts, mit dem starken Anwachsen der (armen) Bevölkerung, sollte sich das aber auch in Chemnitz wieder ändern. ${ }^{351}$

Eine Bezeichnung, die wohl ebenfalls weitgehend dem Status des Schutzverwandten entspricht, ist der Begriff des Beisassen. „Unter Beisassen verstand man in Mainz solche Zugewanderten, denen die häusliche Niederlassung und die Ausübung eines Berufes auf bestimmte Zeit und auf Widerruf gestattet wurde.“352 Beisassen durften an sich in der Stadt kein Haus erwerben, wenn sie nicht zuvor Bürgerrechte annahmen, oder eine Konzession vom Landesherrn erhielten. ${ }^{353}$ Es handelte sich also bei den hier angeführten Beisassen in Mainz um Stadtbewohner und Mieter auf Zeit. In Franken wurde der Begriff zuweilen auch anders verwandt, nämlich für Personen im öffentlichen Dienst, die ebenfalls nicht das örtliche Bürgerrecht besaßen und in einer Dienstwohnung oder zur Miete lebten. ${ }^{354}$

\section{Ohne bürgerliche Rechte: Gäste und Illegalität}

Neben den Vollbürgern und den Einwohnern mit eingeschränkten bürgerlichen Rechten lebten in den Städten seit dem Mittelalter auch Personen, die nur einen Gaststatus hatten, der jedoch nichts über die Länge des Aufenthaltes aussagt, sondern nur über die rechtliche Stellung. In einer Regensburger Steuerschatzung aus dem Jahr 1436 werden sie als hospes geführt. ${ }^{355}$ Mit 278 Nennungen lag ihr Anteil deutlich über $10 \%$ aller Haushaltungen. Diese Gäste lebten sämtlich als Mieter in der Stadt. Zum Teil waren sie wirtschaftlich gut situiert, was sich in einer entsprechend großen Haushaltung niederschlug.

Auch das Thema des illegalen Aufenthaltes ist nicht neu. Früh lässt sich dies in Würzburg fassen. Dort wird im 16. Jahrhundert der Begriff Eingeschleifte ${ }^{356}$ verwandt. An sich war das Bürgerrecht bei ehelicher Geburt in Würzburg über einen

\footnotetext{
349 Ebd.

350 Ebd., S. 84.

${ }^{351}$ Ebd., Anm. 62.

352 SCHROHE 1920, S. 171.

353 Ebd., S. 186.

${ }^{354}$ Vgl. hierzu WENDEROTH 2019a, S. 57.

355 FORNECK 2000, S. 24f.

${ }^{356}$ GÖTZ 1986, S. 35-46.
} 
langen Zeitraum einfach zu erhalten und wohl auch nicht an Hausbesitz gebunden. ${ }^{357}$ Erst 1564 wird ein Mindestvermögen von $40 \mathrm{fl}$ und die Zahlung eines Bürgergeldes von $5 \mathrm{fl}$ festgesetzt. ${ }^{358}$ Es fanden sich aber auch schon zuvor eine ganze Zahl illegaler Einwohner in der Stadt: Bereits 1528 sollten diese zur Anzeige gebracht werden. ${ }^{359}$ Die Anordnung blieb aber ohne Konsequenzen. Erst ab 1570 wurde diese Fragestellung ernsthafter verfolgt: So wurden 1575 in 128 Haushalten "verdächtige“ Personen festgestellt, 1580 waren es schon 219. ${ }^{360}$ Es handelte sich dabei um verheiratete Männer, zumeist der Unterschicht, mit Familie und um ledige oder verwitwete Frauen, darunter ehemalige Mägde und Köchinnen des Klerus. Die aufgegriffenen Männer mussten Bürger werden, Mägde wieder in einen Dienst eintreten, ledige Frauen und Mütter wurden dagegen ausgewiesen. Von der Ausweisung betroffen waren jedoch auch ehemalige städtische Bedienstete und andere Personen.

Die Ausweisung von fremden Bettlern aus der Stadt ist auch ein Thema in Augsburg seit der Mitte des 16. Jahrhunderts. ${ }^{361}$ Im Hungerjahr 1572 wurden sage und schreibe 9.589 Menschen aus der Stadt geschafft, die sich dort ohne Erlaubnis aufhielten. Sonst waren es in den Jahren 1583-1621 jährlich zwischen 32 und 74 Personen. ${ }^{362}$

In Nürnberg selbst findet sich in den Feuerschaulisten des Jahres 1561 ein indirekter Hinweis auf einen rechtlosen Einwohner. Dort heißt es über einen Mieter: Wolf Niemandesgenoß, Ein schleiffer. ${ }^{363}$ Er scheint toleriert und integriert gewesen zu sein, denn zumindest an dieser Stelle wird keine Ausweisung verlangt, sondern nur die Herstellung der Feuersicherheit durch seinen Vermieter.

Mit der Bevölkerungszunahme im 18. Jahrhundert scheint sich das Problem grundsätzlich zu verschärfen. Wichtige Hinweise zur Situation der ohne Genehmigung in den Städten lebenden Bevölkerungsteile liegen zum Beispiel für die hessische Residenzstadt Kassel vor. Sie werden dort als Herrenlos Gesinde bezeichnet, eben weil sie in keinem Untertanen- oder Schutzverhältnis zum hessischen Landgrafen standen. ${ }^{364}$ In einer Zählung der Einwohner aus dem Jahr 1723 werden 523 herrenlose Haushalte mit zusammen 1.244 Personen gelistet. ${ }^{365}$ Anhand der durchschnittlichen Haushaltsgröße von 2,38 Personen wird deutlich, dass es

\footnotetext{
357 GöTZ 1986, S. 39-41.

${ }^{358}$ Ebd., S. 45.

${ }^{359}$ Ebd., S. 41.

${ }^{360}$ Vgl. hierzu und im Folgenden GöTZ 1986, S. 42-44, Anm. 169.

${ }^{361}$ ROECK 1989, S. 155-157.

${ }^{362}$ Ebd., S. 156, Schaubild 2 und 5. Statistischer Anhang zur Auswertung der Strafbücher. Es handelt sich dabei statistisch betrachtet um das häufigste Vergehen, noch vor Ehebruch und Unzucht.

${ }^{363}$ StadtAN, B 1/I Nr. 30, fol. 6, N 75.

364 LASCH 1969, S. 83 Anm. 281.

365 Ebd., Anm. 282.
} 
sich hier nicht überwiegend um alleinstehende Personen, sondern um Klein- oder Kernfamilien gehandelt haben muss. Sie ernährten sich als Taglöhner, Heimarbeiter oder in den Manufakturen. Unter ihnen war aber auch ein beträchtlicher Anteil von alten und gebrechlichen Leuten, die schon mehrere Jahrzehnte in der Stadt lebten. ${ }^{366}$ Am überraschendsten ist jedoch eine Gruppe von Personen, die nicht arm waren, sondern von ihren Mitteln lebten, also Vermögen hatten. Warum diese kein Bürgerrecht erworben hatten, bleibt unklar. Insgesamt wurden 3.670 Haushalte erfasst, der Anteil der „Illegalen“-Haushalte lag also bei $14 \% !^{367}$ Dies stimmt überein mit den von Ostenrieder genannten Zahlen aus Oettingen. ${ }^{368}$ Vermutlich hatten sich darüber hinaus etliche „Illegale“ dieser Zählung entzogen, ${ }^{369}$ denn die Erfassung stellte sich als schwierig durchführbar heraus. Eine erste Zählung erbrachte nur 261 Personen, woraufhin die Zählung mit dem oben genannten Resultat wiederholt worden war. ${ }^{370}$ Diese „illegalen“ Einwohner stellten für die Regierungen ein Problem dar: Nachdem ihre Anzahl im 18. Jahrhundert rasch und stark anstieg, konnte man sie nicht mehr ohne Weiteres, wie in den Jahrhunderten zuvor, ausweisen. Auch in der Landgrafschaft Hessen-Kassel war dies bis dahin die gängige Praxis, welche sich in vielen Erlassen manifestierte. ${ }^{371} 1708$ wurden sie daher erstmals für die Steuer erfasst, denn wenn man diese Personen nicht loswerden konnte, dann sollten sie nach Möglichkeit doch Steuern zahlen. Zudem glaubte man, diese Personen nunmehr als Arbeitskräfte für die neu gegründeten Manufakturen zu benötigen. ${ }^{372}$ Die Tolerierung von Einwohnern ohne Bürgerrecht und Schutzstatus führte dann, wie zu erwarten, in den folgenden Jahrzehnten zu einem verstärkten Zuzug mittelloser Arbeitskräfte. Wer von nun an einen dieser herrenlosen Haushalte als Mieter oder Untermieter bei sich aufnahm, musste sich zuvor vergewissern, dass dieser zumindest steuerlich erfasst war, andernfalls drohten 20 bis $30 \mathrm{fl}$ Strafe, was die Einnahme aus einer Jahresmiete überstiegen haben dürfte. ${ }^{373}$

Dass es sich bei den „Geduldeten“ quantitativ um eine bedeutende Bevölkerungsgruppe handeln konnte, belegen ebenfalls Zahlen aus Hamburg: Im Jahr 1759 wurden an Haushaltsvorständen 9.000 Bürger, 4.000 Schutzverwandte und weitere 3.300 Personen erfasst, die weder Bürger waren noch Schutzgeld zahlten. ${ }^{374}$ Der Anteil der letzten Gruppe lag immerhin bei $20 \%$.

\footnotetext{
366 Ebd. S. 89f.

367 Vgl. Tabelle 25.

368 OSTENRIEDER 1993, S. 96.

369 LASCH 1969, S. 90 Anm. 312.

370 Ebd., S. 90.

371 Ebd., Anm. 309.

372 Ebd., S. 91f.

373 Vgl. WENDEROTH 2021, S.201-203.

374 MAUERSBERGER 1960, S. 38.
} 
Einwohner ohne rechtlichen Aufenthaltsstatus konnten zumindest im 18. Jahrhundert auch in kleineren Städten quantitativ stark vertreten sein, wie Zahlen aus der Residenzstadt Oettingen belegen. ${ }^{375}$ Dort waren im Jahr 1762 17\% der erfassten Haushaltsvorstände ohne Schutz, das heißt, sie hatten weder den Bürgernoch den Beisassen- oder Schutzverwandtenstatus, lebten also ohne gesicherten Rechtsstatus mehr oder weniger geduldet in der Stadt. Absolut waren es 121 Personen. Mehrheitlich handelte es sich um Frauenhaushalte, die ja bereits unter den Schutzverwandten häufig einen überdurchschnittlich großen Anteil stellten, es folgen entsprechend der Gruppenstärke Juden, Taglöhner und andere Unselbstständige. All die Genannten waren überwiegend mittellos. Da sie der Herrschaft bekannt waren, muss davon ausgegangen werden, dass sie zumindest passiv geduldet worden waren. Wahrscheinlich wusste man kein Mittel, diese Personen „loszuwerden“.

Überhaupt scheint sich im 18. Jahrhundert ein gewisser Pragmatismus in dieser Frage breitgemacht zu haben. Dies wird unter anderem mit der Bezeichnung Tolerierte deutlich, die sich in Mainz erstmals 1776 nachweisen lässt. Nach Heinrich Schrohe handelte es sich dabei um Personen, die nur für drei bis sechs Monate Aufenthalt in der Stadt suchten. ${ }^{376}$ Die Bezeichnung wurde aber auch für evangelische Einwohner verwendet, die zu dieser Zeit dauerhaft in Mainz lebten, ob ihrer Konfession aber keinen Aufenthaltsstatus in der erzbischöflichen Stadt erlangen konnten. ${ }^{377}$ Die Grenze zwischen illegalem Aufenthalt und Gaststatus verschwimmt bei dieser Personengruppe.

Einen indirekten Hinweis auf das Vorhandensein von illegalen Einwohnern noch im 19. Jahrhundert enthalten die Gebührensätze zur Bürger- und Schutzbürgeraufnahme in Erlangen 1834: ${ }^{378}$ Denn wer bereits ohne Aufenthaltsstatus in Erlangen lebte, musste für den Erwerb des Schutzverwandten-Status lediglich $20 \mathrm{fl} \mathrm{Ge-}$ bühren entrichten, für Auswärtige betrug die Gebühr dagegen 22 bis 25 fl. Interessant ist bei der Gebührenordnung, dass es demnach eine nennenswerte Anzahl von Bewohnern in Erlangen gab, die weder Bürger- noch SchutzverwandtenStatus hatten, letzteren aber erwerben konnten.

In der Realität gab es also neben den Bürgern und Schutzverwandten noch eine dritte Bevölkerungsgruppe ohne amtlichen Status, die sich unerlaubt, illegal und mehr oder weniger geduldet in den Städten aufhielt.

\footnotetext{
375 OSTENRIEDER 1993, S. 96.

${ }^{376}$ SCHROHE 1920, S. 171, Anm. 2.

377 Ebd., S. $184 f$.

${ }^{378}$ Vgl. hierzu und im Folgenden GöHRING 1934, S. 26.
} 


\section{5 Ökonomische und soziale Stellung eigentumsloser Haushalte}

Im Folgenden soll auf die eigentumslosen Haushalte hinsichtlich ihrer wirtschaftlichen und sozialen Stellung eingegangen werden. Mit der Zunahme des Mietwohnens im Verlauf der Neuzeit steigt auch der Kreis der Eigentumslosen. So heißt es 1785 zum Beispiel über Berlin: „Es wohnen die mehrsten Personen und Familien zur Miete, selbst Minister und Generale haben gemietete Zimmer in großen Häusern. Ohnerachtet die Häuser so voller Menschen stecken, so leidet doch die Gesundheit nicht dabei, weil die Häuser nicht zu hoch und die Straßen nicht zu enge seyn. “" ${ }^{379}$ Auch wenn spätestens seit dem 18. und 19. Jahrhundert Mitglieder aus allen gesellschaftlichen Gruppen zur Miete wohnten, lassen sich einige charakteristische Personengruppen definieren, die einen hohen Anteil an Mietern umfassten.

\section{Unterschicht - Handwerker und Taglöhner}

Bei diesen Berufsgruppen handelt es sich um die klassische Mieterschicht. Dies gilt nach Dirlmeier und Schmidt bereits für das späte Mittelalter. ${ }^{380}$ In den Feuerschaulisten in Nürnberg von 1561 finden sich vielfach Angaben zum ausgeübten Beruf: ${ }^{381}$ Die Mehrzahl der dort genannten Mieter (64\%) ging einem handwerklichen Beruf nach, dieser Wert liegt nur leicht über dem Anteil der Handwerkerhaushalte an der Gesamtbevölkerung von $61 \%$. Von den genannten 3.369 Handwerkerhaushalten lebten 2.148 Handwerker mit ihren Familien zur Miete. ${ }^{382}$ Innerhalb der Stadt hatten sich verschiedenste Schwerpunkte entwickelt. Während im Kartäuserviertel die Metallverarbeitung dominierte und 32,7\% der Miethaushalte und 23,9\% der Vermieter in dieser Branche tätig waren, ist es im Egidienviertel am Schwabenberg die Textilproduktion, wobei hier alle 62 Weber zur Miete wohnten und sich unter den Eigentümern keine Textilhandwerker fanden. $\mathrm{Zu}$ den typischen Mieterberufen gehören neben der Metall- und Textilverarbeitung auch die Leder- und Pelzherstellung. Neben den räumlichen Produktionsschwerpunkten innerhalb der Stadt existierten aber auch Bereiche mit einer ausgeprägten Mischstruktur und hohem Mieteranteil (Am Neuen Bau, Vorstadtbereich von Egidien-, Kornmarkt- und Salzmarktviertel). Hier existiert kein

\footnotetext{
${ }^{379}$ KRÖGEN, KARL HeINRICH: Freye Bemerkungen über Berlin, Leipzig und Prag, Original und Kopie, 1785; zitiert nach Gut 1917, Sp. 113f, dort ohne Seitenangabe.

380 DiRLMEIER/SCHMIDT 1998, S. 254.

${ }^{381}$ Vgl. hierzu und im Folgenden ENDRES 1990b. Die Angaben und Zahlen im Folgenden beruhen auf dessen Auswertung, Endres bezieht sich wiederum auf die Ergebnisse der Zulassungsarbeit von Christian Feja 1988.

382 Vgl. FEJA 1988, S. $19 f$.
} 
direkter Zusammenhang zwischen Produktionsstätten und Berufen. Das Barfüßerviertel im Kernstadtbereich von St. Sebald wies erwartungsgemäß einen niedrigen Mieteranteil von lediglich gut $40 \%$ auf. Interessant ist die Feststellung, dass hier 23\% der Mieter zwar mit Namen, aber ohne Berufszugehörigkeit gelistet werden. Dies ist vielleicht als Hinweis zu verstehen, dass diese Mieter zum Teil auch der vermögenden Schicht angehörten.

Für Nürnberg errechnet sich für die Zeit um 1620 ein Anteil der Miethaushalte zwischen $67 \%$ und 79\%! ${ }^{383}$ Michael Diefenbacher beschreibt für diese Zeit die Einkommensstruktur wie folgt: „5 Prozent Angehörige der reichen Oberschicht (Patriziat und Großkaufleute, reiche Handwerker-Unternehmen), 8 bis 10 Prozent Handwerksmeister, ca. 40 Prozent regelmäßig arbeitende Handwerker, Gesellen und Stückwerker, des Weiteren 10 Prozent Knechte und Mägde sowie rund 30 Prozent Taglöhner, Hilfs- und Gelegenheitsarbeiter [...], der Rest waren Arbeitslose, Bettler, Kranke und Sieche. “384 Zumindest die letzten beiden Bevölkerungsgruppen sind sicher als arm bis sehr arm anzusehen und konnten sich in der Regel kein Wohneigentum leisten. Diese beiden Gruppen ergeben zusammen bereits gut 35\% der Gesamtbevölkerung. Knechte und Mägde wohnten im Haushalt des Dienstherrn und hatten entsprechend ebenfalls kein Wohneigentum. Bei dem großen Anteil an Miethaushalten in der Stadt lebten demnach aber auch große Teile der „regelmäßig arbeitenden Handwerker, Gesellen und Stückwerker" als Mieter.

In Uffenheim, Fürstentum Ansbach, werden in den Quellen 1603 und 1668 nur die Namen der Mieter, nicht aber die Berufe genannt. ${ }^{385}$ Entsprechende Angaben finden sich bei den Hausgenossen erst zwischen 1686 und 1732: Bäcker, Büttner, Maurer, Metzger, Hausmetzger, Pfeifer, Schneider, Schmied, Schreiner, Schuhmacher, Wagner, Zimmermann und immer wieder Tagwerker. Hinzu kommen noch Büchsenmacher, Bortenwirker, Buchbinder, Drechsler, Färber, Flaschner, Haubenmacher, Kannengießer, Kürschner, Nagelschmied, Pflasterer, Sattler, Schlosser, Schnitzer, Seckler, Siebmacher, Strohschneider, Turmwächter, Tuscherer, Zinngießer, Zuckerbäcker. Auffallend häufig wird der Beruf des Zeugmachers genannt. Überraschend ist zudem die Bezeichnung Bauer bei einem Hausgenossen!

Für Schwabach liegen quantifizierte Berufsangaben für das Jahr 1739 vor. ${ }^{386}$ Die Stadt ist damals die wirtschaftlich führende Kommune im Fürstentum Ansbach. Dabei waren es vor allem Handwerker im produzierenden Gewerbe, die als Hausgenossen zur Miete wohnten. An erster Stelle, als größte Gruppe, stehen die

\footnotetext{
${ }^{383}$ Vgl. hierzu Kapitel 5, Nürnberg.

384 DiefENBACHER 2000, S. 212.

385 RECHTER 2003, S. 28-30.

386 SCHLÜPFINGER 1977, S. 55-57.
} 
Strumpfwirker mit 113 Hausgenossen bei insgesamt nur 126 Strumpfwirkern, gefolgt von den Bortenwirkern (28 von 43), den Nadlern (27 von 39), den Schuhmachern (22 von 30), den Tuchmachern (17 von 29), den Schneidern (14 von 23) und den Drechslern und Drahtziehern mit jeweils 13 Hausgenossen von 15 Handwerkern.

Die Arbeit im Nahrungsmittelgewerbe scheint dagegen vielfach ein Auskommen gesichert zu haben, das für den Erwerb von Hausbesitz ausreichte. Auffällig ist hier lediglich, dass von den 13 Metzgern 11 zur Miete wohnten. Bei den Tabakmachern, die auch für den Export arbeiteten, verwundert die Anzahl von 15 Hausgenossen unter den 24 Tabakmachern weniger. Dagegen waren von den 45 Bierbrauern 44 Hausbesitzer, zudem beschäftigten diese noch 11 Braumeister, von denen 9 zur Miete wohnten.

In der Liste der Hausgenossen und Schutzverwandten aus dem Jahr 1723 für Windsbach werden ebenfalls die Berufe genannt. ${ }^{387}$ Es finden sich bei den 28 Nennungen sieben Taglöhner, drei Metzger, zwei Ziegler, zwei Schneider, ein Bortenwirker, ein Fischer, ein Maurer, ein Tabakspinner. Auch zwei Honoratioren werden genannt, zum einen Christian Joh. Kreyselmeyer, gewesener Castner, Richter und Vogt allhier und H. Adoph Christoph Benz, Lt.Med. ${ }^{388}$ Zudem sind hier fünf Juden ohne weitere Berufsangabe gelistet, gleiches gilt auch für zwölf Wittweiber, die in einer extra Rubrik am Ende der Liste stehen. Ähnliche Berufsangaben sind für die Mieter in dem nürnbergischen Dorf Leinburg 1615 überliefert, auch hier finden sich überwiegend Handwerker und Taglöhner genannt. ${ }^{389}$

Es war also eine ganze Bandbreite von Handwerksberufen unter den Mietern vertreten. Entsprechend der wirtschaftlichen Struktur der Orte lassen sich auch Unterschiede nachweisen. Uffenheim als kleine Amtsstadt versorgte einen größeren und differenzierteren Markt als Kirchdörfer, in Schwabach wurde darüber hinaus für den Export produziert. So ist es nicht verwunderlich, dass neben den Grundhandwerkern auch einige Spezialhandwerker in den Städten ihr Auskommen suchten und fanden. Die Arbeit, auch in einem Spezialgewerk, scheint aber nicht von vornherein geeignet gewesen, eine gesicherte Existenz zu erlangen, die es ermöglicht hätte, Wohneigentum zu erwerben. Grundsätzlich waren die angegebenen Gewerke, mit Ausnahme der auch als Stückarbeiter charakterisierten Handwerker in der Massenproduktion, durchaus geeignet, um sich sesshaft zu machen. Für das Dasein vieler Handwerker in den Kleinstädten als Mieter spielten sicher die Zunftordnungen mit dem oft mühsamen Weg zum Meistertitel eine

\footnotetext{
${ }^{387}$ Altmann 1992, S. 62.

388 Ebd., S. 62.

389 StAN, Rst. N, Landpflegamt, Pflegamt Lauf, Mappe Nr. 1615/4; vgl. hierzu WeNDEROTH 2019a, S. 47 und 70f.
} 
entscheidende Rolle. Da die Zahl der Meister in den Zunft-Handwerken beschränkt war, mussten die Gesellen schauen, wo sie für wie lang eine Anstellung und damit ein Auskommen fanden. Die damit verbundene wirtschaftliche Unsicherheit und der von vornherein geringere Lohn verschlossen oftmals den Weg zu Eigentum und sozialem Aufstieg.

Das gleiche Phänomen lässt sich auch in anderen Regionen und Städten beobachten, stellvertretend sei auf die Ausführungen Helmut Bräuers für Sachsen verwiesen. ${ }^{390}$

Viele Mieter waren unvorstellbar arm, ihre ökonomische Situation mehr als erbärmlich. Zum überwiegenden Teil gehörten Mieter der ärmsten Bevölkerungsgruppe an. Im Türkensteuerverzeichnis heißt es 1717 für Hiltpoltstein zum Beispiel bei einigen Mieterinnen, dass sie bettelarm seien und daher die Kopfsteuer zu erlassen sei. ${ }^{391}$ In einer Beschreibung aus dem Jahr 1792 über die Unterschichten in Erlangen ist Folgendes zu lesen: Unglaublich und doch war ist, daß in dem sogenannten Polen, wo die meisten dieser Armen wohnen, Familien tagelang hungern müssen und am Abend kein Bett haben, wo sie ihr Haupt hinlegen. ${ }^{392}$ Nicht viel besser war die Lebenssituation von einer nicht geringen Zahl von Menschen, die sich immerhin die Miete leisten konnten, in der Beschreibung heißt es dazu weiter: Fünf oder sechs nackte Kinder in Lumpen gehüllt, wälzen sich bei der grimmigsten Kälte, in einem feuchten Dachstübchen, von Krätze und Aussatz überdeckt, auf Stroh; Erdäpfel in Wasser gekocht sind der armen Kranken einzige Nahrung, und verschmachtend hängt das Kleinste an der mütterlichen Brust, die keine Milch mehr geben kann, weil die Mutter selbst gehungert hat. Schilderungen himmelschreiender Armut und Wohnverhältnisse finden sich auch für andere Regionen. ${ }^{393}$

Jenseits dieser drastischen Schilderungen war die Realität differenzierter, wenn auch die meisten Mieter arm geboren wurden und arm starben. Für einige fränkische Dörfer und die Kleinstadt Uffenheim finden sich Hinweise zur differenzierten Vermögenstruktur der Mieter in meiner Dissertation. Neben einer großen Anzahl von vermögenslosen Mietern aus dem Handwerk gab es auch einige wenige, die geringen Besitz hatten. ${ }^{394}$ In Uffenheim wird in der Aufstellung des Jahres 1723 bei den Mietern zwischen begütert und unbegütert unterschieden. In der Aufstellung aus dem Jahr 1732 fällt diese Kategorie weg, stattdessen ist bei fünf Hausgenossen Feldbesitz angegeben. ${ }^{395}$

\footnotetext{
${ }^{390}$ BRÄUER 2003, S. 86-90.

${ }^{391}$ StAN, Rst. N, Bauerverzeichnisse Nr. 7, fol. 513-513'.

392 Zitiert nach SCHUBERT 1982, S. 36. Mit „Polen“ ist das Viertel um den heutigen Bohlenplatz gemeint. Man beachte die typisch fränkische Lautverschiebung.

${ }^{393}$ Vgl. BRÄUER 1997. Er schildert die Situation in Leipzig anhand der Gassenmeister- und Armenvisitationsberichte.

${ }^{394}$ Vgl. WENDEROTH 2019a, S. 69f.

${ }^{395}$ RECHTER 2003, S. 29-30.
} 
Ergänzend dazu soll an dieser Stelle noch auf die Erb- und Landeshuldigung 1723 für die Amtsstadt Feuchtwangen eingegangen werden. In den zugehörigen Listen wurde auch das Vermögen der Einwohner erfasst. Die 121 Hauseigentümer hatten zusammen ein Vermögen von $3.688 \mathrm{fl} .{ }^{396}$ Dabei schwanken die Vermögenswerte zwischen $1 / 2$ und $165 \mathrm{fl}$. Im Durchschnitt errechnet sich so ein Vermögen von 30,48 fl je Hauseigentümer. Die Vermögenswerte der Hausgenossen lagen deutlich darunter. Diese schwankten zwischen 1 und $4 \mathrm{fl}$ und unterschieden sich somit kaum voneinander. Die Gesamtsumme von $47 \mathrm{fl}$ verteilte sich auf zwanzig männliche Mieterhaushalte, woraus sich ein durchschnittliches Vermögen von 2,35 fl errechnet. ${ }^{397}$

Valentin Groebner befasst sich mit der Armut um 1500 in Nürnberg. ${ }^{398}$ Als Beispiel führt er die Teuerung 1501 an, die die Stadt veranlasste, verbilligtes Notbrot zu backen, das an exakt erfasste 5.002 Personen ausgeteilt wurde. Diese als bedürftig erkannten Mitbewohner erhielten für den Bezug metallene Brotzeichen. ${ }^{399}$ Wichtig ist in diesem Zusammenhang, dass es sich dabei nicht um Bettler, sondern um Handwerker, Tagelöhner und Hausgesinde gehandelt hat. ${ }^{400}$ Bei 28.000 Einwohnern ${ }^{401}$ war der Anteil der Bedürftigen an der Bevölkerung sehr hoch, denn es wurden ja nur die Haushaltsvorstände, nicht die zugehörigen Frauen und Kinder, erfasst, sodass die Zahl der bedürftigen Personen vorsichtig geschätzt zu verdreifachen ist, was gut die Hälfte der Gesamtbevölkerung ergibt. Die Bedürftigen verfügten über kein Vermögen, das es ihnen ermöglicht hätte, eine schwierige wirtschaftliche Phase zu überbrücken, obwohl sie in Lohn standen. Groebner charakterisiert die Nürnberger Armen wie folgt: „Sie besitzen kein Haus, sondern wohnen zur Miete in den Armeleutevierteln am Äußeren Laufer Tor, um die Grasergasse und in den billigen Vorstädten. Sie sind nicht unbedingt Bürger; dafür muss man zwei Stadtwährungsgulden Gebühr und (wenigstens de jure) den Nachweis von 100 oder $200 \mathrm{fl}$ (rh) Vermögen beibringen, und das ist sehr viel Geld. Sie sind gezwungen, ihre Arbeitskraft auf dem städtischen Markt zu verkaufen, aber nach all dem, was wir wissen, ermöglicht ihnen das nicht unbedingt eine gesicherte wirtschaftliche Existenz." ${ }^{402}$

\footnotetext{
${ }^{396}$ StAN, Fm AN, Brdbg. Lit. 603, fol 376-379'.

397 StAN, Fm AN, Brdbg. Lit. 603, fol 380-380'. Vgl. dazu auch die Zahlen für Zwickau 1496: Das durchschnittliche steuerbare Vermögen betrug bei den Eigentümern $495 \mathrm{fl}$, bei den Mietern lediglich 40 fl; BRÄUER 2003, S. 86.

398 GROEBNER 1993.

${ }^{399}$ Ebd., S. 15.

${ }^{400}$ Groebner erläutert dazu, dass unter die 5002 Personen nicht die berufsmäßigen Bettler, fahrende Leute und Prostituierte gezählt wurden. Ebenfalls wurde damit nicht die Bevölkerung der Vorstädte und Vororte erfasst; GROEBNER 1993, S. 16.

${ }^{401}$ DiefenbaCHer 2000, S. 211. Er verweist auf ein Steuerregister von 1497 für die Lorenzer Stadtseite und kommt auf mindestens 28.000 Einwohner.

402 GROEBNER 1993, S. 16-17.
} 
Rainer Stahlschmidt geht ebenfalls davon aus, dass die wirtschaftliche Lage der Stückwerker - gemeint sind Arbeiter, die gewerbliche Waren für einen Verleger im Stücklohn herstellen - schlecht war, zumal im 16. Jahrhundert eine Rezession einsetzte. ${ }^{403}$ Nach Groebners Auffassung handelte es sich bei diesen Leuten um die vermögenslose Mehrheit der Bevölkerung. In jedem Fall betraf es nicht nur eine kleine Minderheit. Armut und Eigentumslosigkeit gehören zusammen. Armut bedingt das Wohnen zur Miete, umgekehrt ist es nicht so.

Hironubo Sakuma hat die Lebensbedingungen der Nürnberger Tuchmacher, Weber, Färber und Bereiter vom 14. bis zum 17. Jahrhundert untersucht. ${ }^{404}$ Vor allem die Meister des Tuchmacherhandwerks gehörten der oberen Mittelschicht oder sogar der Oberschicht der Reichsstadt an, während die Meister der anderen Textilzünfte deutlich ärmer und weniger angesehen waren. ${ }^{405}$ Von 24 Meistern haben sich Nachlassinventare aus der Mitte des 16. Jahrhunderts erhalten. Lediglich vier von ihnen hatten Immobilienbesitz, zwanzig Meister wohnten demnach zur Miete, das sind 83\%. ${ }^{406}$ Die Vermögenslage der Gesellen war im Durchschnitt deutlich schlechter. Von neun verheirateten Gesellen, die einen eigenen Haushalt führten, haben sich ebenfalls Nachlassinventare aus dieser Zeit erhalten. ${ }^{407}$ Diese weisen die Gesellen sämtlich als Mitglieder der Unterschicht mit einem maximalen Gesamtvermögen von $30 \mathrm{fl}$ aus. Wohneigentum war für diese Personengruppe unerschwinglich. Den Gesellen vermögensmäßig gleich schlecht gestellt waren allerdings auch elf der zuvor genannten 24 Meister. Bei den Meistern klaffte die Vermögensspanne eben deutlich weiter auseinander. Relativ ähnlich war allerdings die Grundausstattung mit Möbeln und Kochgerätschaften bei Meistern und Gesellen. Der Vermögensunterschied drückte sich neben Immobilienbesitz und Barvermögen in fehlenden Tuchvorräten, einfacherer Kleidung und günstigem Bettzeug aus. Das fehlende Barvermögen bei den armen Meistern und den Gesellen zeigt zugleich die prekäre finanzielle Lage der meisten Miethaushalte an: Rücklagen für Notsituationen fehlten, der Schritt in die Armut und Unterstützungsbedürftigkeit war klein.

1561 beträgt der Anteil der eigentumslosen Haushalte in Nürnberg bereits gut $60 \% .{ }^{408}$ In jedem Fall belegt diese Zahl eine signifikante Zunahme der Haushalte an sich und des Zinswohnens im Besonderen vom späten Mittelalter zur Neuzeit.

\footnotetext{
${ }^{403}$ Vgl. STAHLSCHMIDT 1971, S. 215-220.

404 SAKUMA 1993.

${ }^{405}$ Ebd., S. 183-188.

${ }^{406}$ Ebd., besonders S. 186-188, Tabelle 27.

${ }^{407}$ Ebd., S. 200-205, Tabelle 27.

${ }^{408}$ ENDRES 1990b; FEJA 1988.
} 


\section{Staats-, Kommunal- und Hofbedienstete}

Eine Gruppe Eigentumsloser, die Erwähnung finden muss, sind die öffentlich Bediensteten. An erster Stelle sind hier die Staatsbediensteten, auch als Beampte bezeichnet, ${ }^{409} \mathrm{zu}$ nennen. Vor gut 100 Jahren mutmaßte Adalbert Gut für Berlin, dass bereits in der Regierungszeit des großen Kurfürsten (1640-1688) die Beamten zur Miete wohnten: „Die besseren ,Beamten', die kein eigenes Vermögen hatten, werden dagegen für den Hausbesitz in der damaligen Zeit weniger in Frage gekommen sein. Für sie war das Mieten einer Wohnung das gegebene, und man wird nicht Fehl gehen, wenn man zum Schluß kommt, daß bei diesen zweistöckigen Häusern das Erdgeschoß von dem Eigentümer, das Obergeschoß von dem Mieter bewohnt wurde. “410 Dass dieses Phänomen weit in die Neuzeit zurückreicht, wird an der fürstbischöflichen Residenzstadt Salzburg deutlich. ${ }^{411}$ Dort hatte die Familie Riß im Jahr 1608 ein Haus in der Pfeifergasse 2 an den fürstlichen Truchseß Hans Hartwig von Haunsberg und den fürstlichen Jägermeister Georg von Greiffensee vermietet, beides hohe und vornehme Mitglieder des Hofstaates.

$\mathrm{Zu}$ dieser Mietergruppe gehörte auch der vielleicht berühmteste Deutsche, Johann Wolfgang von Goethe: Neu im weimarischen Staatsdienst mietete er von 1782 bis 1789 einen Teil des Hauses Am Frauenplan für $36 \mathrm{fl}$ (rh) pro Quartal. ${ }^{412}$ Als Mieter bewohnte er dort lediglich den westlichen Teil von Vorderhaus und Rückgebäude. Die übrigen Bereiche wurden von anderen Parteien genutzt. Erst im Jahr 1792 erwarb Herzog Carl-August von Sachsen-Weimar und Eisenach das Gebäude aus Privatbesitz, überließ es Goethe als Dienstwohnung und schenkte das Anwesen schließlich dem mittlerweile geadelten Minister im Jahr 1794. Zwischenzeitlich bewohnte Goethe von 1789 bis 1792 zusammen mit Christiane Vulpius in Weimar ein Jägerhaus an der Marienstraße, das ihm als Dienstwohnung überlassen worden war.

Im weiteren Sinne gehören zur Gruppe der Beamten auch die Geistlichen, seien es Superintendenten, Pfarrer, Diakone, Kapläne oder Vikare. In den bereits genannten Huldigungslisten wurde diese Personengruppe häufig gar nicht erfasst.

$\mathrm{Zu}$ den Beamten zählen weiterhin auch die Universitätsangehörigen, auf sie wird im nachfolgenden Kapitel ausführlich eingegangen. An zweiter Stelle sind die städtischen oder gemeindlichen Angestellten anzuführen. Die Bandbreite reicht

\footnotetext{
${ }^{409}$ Mainbernheim 1686, StAN, Fm AN, Brdbg. Lit. Nr. 596, fol. 383.

${ }^{410}$ GUT 1917, Sp. 28.

${ }^{411}$ Vgl. hierzu und im Folgenden ZILLNER 1885, S. 285f.

412 Vgl. hierzu und im folgendem Holler/KNEBEL 2011, S.10-14. Das Haus war auch zuvor schon komplett oder in großen Teilen vermietet gewesen.
} 
von Lehrern, die häufig in direktem Zusammenhang mit den Geistlichen genannt werden, da der Kirche in der Regel die Schulaufsicht oblag, über Torwächter bis hin zu den Schweinehirten. In den Huldigungslisten sind auch diese Personen nur in wenigen Ausnahmefällen unter den Hausgenossen erfasst. Die Hirten wurden interessanterweise nicht $\mathrm{zu}$ den bürgerlichen Hausgenossen gerechnet, sondern zu den Schutzverwandten, wenn zwei verschiedene Rubriken geführt worden sind, demnach besaßen sie kein Bürgerrecht und nahmen somit unter den öffentlich Bediensteten nochmals eine Sonderstellung ein. ${ }^{413}$ Ohne eine intensivere lokalhistorische Forschung lassen sich die öffentlich Bediensteten in der Regel nicht in den Einwohnerlisten nachweisen, da dort nicht regelhaft die Berufe angegeben werden. Daher ist bisher auch nicht geklärt, inwiefern und in welchem Umfang diese überhaupt in den Einwohnerlisten erfasst sind.

Dass es sich bei dieser Bevölkerungsgruppe auch quantitativ um einen wichtigen Teil der Einwohnerschaft handelte, soll anhand einiger Zahlen aus den Huldigungslisten des Fürstentums Brandenburg-Ansbach belegt werden:

In den Huldigungslisten für Gunzenhausen sind die öffentlich Bediensteten zweimal in Folge separat erfasst worden, 1673 und 1686. Hier finden sich am Anfang der Liste Beambte und Geistliche und nach den Bürgermeistern folgen die Gemeinen Statt Bediente. ${ }^{414}$

1673 sind 22 öffentlich Angestellte und achtzehn sonstige Miethaushalte genannt. Die Zahl der Eigentümerhaushalte belief sich auf $141 .^{415} \mathrm{Im}$ Jahr 1686 waren es 23 Haushalte von öffentlich Angestellten, denen ebenso viele Haushalte von Hausgenossen gegenüberstanden, Eigentümerhaushalte sind 158 vermerkt. ${ }^{416}$ Rechnet man die öffentlich Angestellten den Hausgenossen als Mieter hinzu, ergibt sich für beide Zählungen ein Anteil der Miethaushalte von $21 \%$ an der christlichen Bevölkerung. Berücksichtigt man diese Gruppe nicht, sondern vergleicht nur die Anzahl der Hausgenossen mit der der Eigentümer, reduziert sich der Anteil der Miethaushalte erheblich, 1673 auf 11\% und 1686 auf 13\%. Bezogen auf sämtliche Haushalte stellten die Haushalte von Beamten und öffentlich Bediensteten im Jahr 1673 immerhin 22 von 180 Haushalten, ${ }^{417}$ also $12 \%$. Im Jahr 1686 waren es 23 von 222 Haushalten, womit die Quote leicht auf $10 \%$ sinkt. Für Mainbernheim werden 1686 zwei Geistliche, drei Beamte, ein Stadtschreiber, ein Schuldiener und ein Kirchner genannt. ${ }^{418}$ In Merkendorf wiederum fehlen

\footnotetext{
${ }^{413}$ StAN, Fm AN, Brdbg. Lit. Nr. 603, fol. 625' $-626^{\prime}$ : III. Schutzverwanthe und Huth=Männer. Merkendorf 1723.

${ }^{414}$ Ebd., Nr. 596, fol. 263-263'.

415 Ebd., fol. 260 und 263. Die 13 jüdischen Haushalte werden hier nicht weiter berücksichtigt, da nicht unterschieden wurde zwischen Eigentümern und Mietern.

${ }^{416}$ Ebd., fol. 263-267. Die nun 18 jüdischen Haushalte werden wiederum nicht berücksichtigt.

${ }^{417}$ Inklusiv der Haushalte der öffentlich Bediensteten und der Juden.

${ }^{418}$ Ebd., fol. 383-383'.
} 
diese, stattdessen sind explizit drei Torwärter und zwei Hirten aufgeführt. ${ }^{419}$ In Schwabach sind in der Huldigungsliste 1695 verstreut in der Rubrik der Bürger sechs Wächter und ein Türmer, durch die Namenszusätze als solche kenntlich gemacht. ${ }^{420}$ Warum diese nicht unter der Rubrik der Hausgenossen erfasst worden sind, ist bisher nicht geklärt, dennoch erscheint es unwahrscheinlich, dass diese Personengruppe zu 100\% über Wohneigentum verfügte. Viel eher dürften sie in den entsprechenden Türmen gewohnt haben.

Für Uffenheim sind anhand der Ämterbeschreibung vom 8. und 9. Mai 1727 allein vierzehn öffentlich Bedienstete überliefert, hinzu kamen noch der Amtmann und der Schreiber. ${ }^{421}$ Die restliche Bevölkerung bestand aus 221 Eigentümern und 30 Mietern samt den unerwähnt gebliebenen Familienangehörigen. Hier stellten die öffentlich Bediensteten immerhin 6\% der Bevölkerung. Berücksichtigt man diese unter den Miethaushalten, würde deren Anteil von 11\% auf 17\% ansteigen.

Der hohe Anteil der öffentlich Bediensteten mag überraschen. Zum Vergleich sollen hier noch Zahlen für die freie Reichsstadt Windsheim aus dem Jahr 1677 und für Nürnberg für das Jahr 1797/98 angeführt werden. In Windsheim waren von 529 Haushaltsvorständen $21(4,0 \%)$ im höheren städtischen Dienst beschäftigt (Akademiker), neun (1,7\%) im mittleren Dienst und $42(8,0 \%)$ im niederen Dienst. ${ }^{422}$ Insgesamt handelte es sich also um fast $14 \%$ der Haushaltsvorstände, nämlich 66 Männer und sechs Frauen. Ein Großteil dieser Gruppe dürfte zur Miete gewohnt haben, entweder in einem städtischen Gebäude ${ }^{423}$ oder bei privaten Vermietern. In Nürnberg ergibt sich bei der Analyse der Sebalder Stadtseite, dass am Ende des 18. Jahrhunderts von 2.754 Haushaltsvorständen 261 (9\%) im öffentlichen Dienst und 74 (3\%) in der Verwaltung beschäftigt waren. ${ }^{424}$

Wichtig erscheint es, festzuhalten, dass alle diese Personen weit überwiegend nicht im eigenen Haus lebten. Einige von ihnen konnten Dienstwohnungen in Anspruch nehmen, andere mussten eine Wohnung anmieten. Ein Hinweis auf die Anmietung eines Privathauses durch Beamte findet sich zum Beispiel für Uffenheim 1695: Adam Strebel, Bierprauer und WeinSchenck. Nota, hat noch ein Haus, im ersten Viertel, worinn aber derzeit hl Stattvogt wohnet. ${ }^{425}$ Dienstwohnungen wurden vor allem für die Geistlichen und für hohe Verwaltungsbeamte zur Verfügung gestellt. Auch die gering entlohnten Bediensteten konnten vielfach mit

${ }^{419}$ Ebd., fol. 408.

${ }^{420}$ Ebd., Nr. 597, fol. 457-463.

${ }^{421}$ Vgl. hierzu und im Folgenden StAN, Fm AN, Ämterbeschreibung Nr. 62, fol. 4-64.

${ }^{422}$ KORNDÖRFER 1971, S. 53. I

${ }^{423}$ Ebd.: Im selben Jahr sind 47 Häuser verzeichnet, die der Reichsstadt gehörten.

${ }^{424}$ Die Auswertung der Quartiergeldlisten für die Sebalder Seite erfolgte durch Frau Sarina Werner im Rahmen einer Seminararbeit an der Universität Bamberg WS 2018/19.

${ }^{425}$ StAN, Fm AN, Brdbg. Lit. Nr. 597, fol. 527. 
Dienstwohnungen rechnen, seien es die Hirten oder Wächter. Nach einer ersten Einschätzung waren es vor allem die mittleren Dienstgrade, die sich auf eigene Faust eine Mietunterkunft suchen mussten.

Eine dritte Gruppe stellen die Hofbediensteten dar. Ähnlich den Universitätsgemeinden gab es auch Hofgemeinden mit Hofschutzverwandten, die ebenfalls nicht der Kommune zugehörig waren und somit nicht dem kommunalen Recht unterstanden. Folgerichtig fehlen auch diese Personen häufig in den städtischen Einwohnerlisten und wurden dort weder als Eigentümer noch als Mieter verzeichnet.

Für den bayreuthischen Witwensitz in Erlangen haben sich Zahlenangaben zu den Bediensteten erhalten, die deren Wohnsituation veranschaulichen: Von den fürstlichen Angestellten wohnten im Jahr 1775 sechs in Dienstunterkünften und vier privat zur Miete. Bei weiteren vier Personen fehlen diesbezügliche Angaben. ${ }^{426}$ Wesentlich umfassender sind die Angaben für das Jahr 1817: Die Markgräfin-Witwe Sophie Caroline Marie von Brandenburg-Bayreuth (1737-1817) unterhielt im 53. und letzten Jahr ihrer Witwenschaft noch immer einen großen Hofstaat mit 45 Hofbediensteten in Erlangen. Von diesen hatten sechzehn Personen eine dienstliche Unterkunft, die anderen 29 mussten sich bei Privatleuten einmieten. ${ }^{427}$

\section{Akademiker}

Daneben gab es eine akademisch gebildete Schicht, von der ebenfalls viele Personen nicht über Wohneigentum verfügten. Zuallererst sind hier die Pfarrer und Priester zu nennen, denen im Rahmen ihres Amtes Wohnungen überlassen worden sind. Auch viele Professoren wohnten in Dienstwohnungen oder zur Miete.

1743 wurde in Erlangen die Universität gegründet. Über den Wohnort der Professoren und weiterer Universitätsmitarbeiter gibt erstmals die Aufstellung von Johann Caspar Müller aus dem Jahr 1751 vollständig Auskunft. ${ }^{428}$ Genannt werden insgesamt 29 Haushalte, darunter fünf Haushalte in Dienstwohnungen, inklusive der Pfarrer der Alt- und Neustadt, sieben Haushalte ohne Hinweise zum Wohnort und 22 Haushalte mit Adressangabe. Auffällig ist, dass hier nicht angegeben wird, ob die betreffenden Personen im eigenen Hause wohnten. Nachdem diese Differenzierung auch bei den anschließend aufgelisteten Honoratioren,

\footnotetext{
${ }^{426}$ FREYESLEBEN 1775, S. 29-30.

427 Vgl. hierzu und im Folgenden ENGELHARDT 2017, S. 36.

${ }^{428}$ MÜLLER 1751/2002, S. 8-11.
} 
Kaufleuten und Handwerkern nicht vorgenommen ist, ${ }^{429}$ scheint der Autor eine Differenzierung nach Miete und Wohneigentum nicht für notwendig erachtet zu haben. Von dem Professor Andreas Elias Rossmann ist aber überliefert, dass er im Jahr 1758 das Richthaus Hauptstraße 20 kaufte. ${ }^{430}$ Auch 1751 wohnte er bereits bei der Französischen Kirche, ${ }^{431}$ ob im gleichen Haus zur Miete oder in einem anderen Haus ist nicht bekannt. Der Hofrat und Professor Johann Justin Schierschmidt erwirbt 1749 das dreigeschossige Eckhaus Hauptstraße 19 und verkauft dafür das kleinere Nachbarhaus Hugenottenplatz 1. 432

Im angeschlossenen Gymnasium unterrichteten neben vier Professoren weitere sechs Männer. ${ }^{433}$ Im letzten Eintrag heißt es „Herr Martin Philipp Seyfert, docirt in der Französischen Sprache, logiert in der Hauptstraße." Dies kann wohl als Hinweis auf ein Untermietverhältnis gedeutet werden.

Unter den Erlanger Universitätsmitgliedern werden 26 Jahre später (1775) jedoch eine ganze Anzahl von Personen genannt, die nunmehr eindeutig und weit überwiegend zur Miete wohnten: ${ }^{434}$ Insgesamt werden 54 Personen angeführt. In sechzehn Fällen wird zum Wohnort keine Aussage getroffen, viermal werden Dienstwohnungen benannt. So zum Beispiel bei folgendem Eintrag: „Gärtner bey dem Horto Botanico Hr. Adam Rümelein, wohnt in dem Botanischen Gartenhause. “435 Zweimal sind Pfarrer vertreten, so der Superintendent Joachim Ehrenfried Pfeiffer, der als Theologieprofessor wirkte und entsprechend in der Superintendentur wohnte. ${ }^{436}$ Doch dort lebte er mit seinem Haushalt nicht allein, denn auch „August Friedrich Pfeiffer, Professor philos. publ. extraord.“ wohnte in der Superintendentur. ${ }^{437}$ Lediglich viermal wird Hausbesitz angezeigt, dagegen siebenundzwanzigmal der Status eines Mieters.

Bei den nachfolgenden Lehrkräften am Gymnasium und den Mitgliedern der Oberamtsverwaltung wird ebenfalls kein eigengenutzter Hausbesitz in Erlangen vermerkt. ${ }^{438}$ Eigens aufgeführt sind die Witwenhaushalte der Honoratioren: ${ }^{439}$ Fünf Witwen wohnten im eigenen Haus, zwölf Witwen zur Miete. Hier war der Eigentümeranteil deutlich höher als bei den aktiven Honoratioren.

\footnotetext{
429 Ebd., ab S. 12.

430 NÜRMBERGER 2015, S. 180f, Anm. 182.

431 MÜller 1751/2002, ab S. 9.

432 NÜRMBERGER 2015, S. 231, Anm. 236.

433 MÜLLER 1751/2002, S. 12.

${ }^{434}$ FREYESLEBEN 1775, S. 8-14.

435 Ebd., S. 14.

436 Ebd., S. 14.

437 Ebd., S. 12.

438 Ebd., S. 15-17.

439 Ebd., S. 31-32.
} 
Olaf Willett nennt noch einmal Zahlen für das späte 18. Jahrhundert. 1810 wohnten sechs von neunzehn Ordinarien im eigenen Haus, anschließend sank dieser Anteil wieder. Häufig investierten die Professoren jedoch deutlich mehr Geld in ihre Bibliotheken und Sammlungen als in Grundeigentum. Insgesamt fällt auf, dass die akademische Schicht in Erlangen größtenteils kein Hauseigentum besaß.

Dass das Mietwohnen von Akademikern in Erlangen keinen Einzelfall darstellte, belegen Zahlen aus Göttingen. Dort wurden zwischen 1733 und 1755 insgesamt 44 ordentliche Professoren und 24 weitere Gelehrte berufen. ${ }^{440}$ Von diesen erwarben lediglich sechzehn eigene Häuser, die übrigen 52 Universitätsmitglieder wohnten zur Miete! Unter den deutschen Universitätsstädten soll noch kurz auf Greifswald eingegangen werden. In der dortigen Universität gab es ebenfalls eine Tradition, bei der den Professoren Amtshäuser zur Verfügung gestellt wurden, in denen sie jedoch auch unterrichteten, ihre Sammlungen aufbewahrten und Studenten gegen Kostgeld beherbergten. ${ }^{441}$ Ein großer Teil der Greifswalder Hochschullehrer erhielt jedoch keine Amtswohnung als Teil der Entlohnung, sondern einen Mietzuschuss und das bereits in der frühen Zeit der Universität. ${ }^{442}$

\section{Studenten}

In Franken gab es zur Barockzeit vier Universitäten: Würzburg (1402, Neugründung 1582), Altdorf (Universität der Reichsstadt Nürnberg, 1622-1809), Bamberg (1647, ab 1773 Volluniversität, ab 1803 nur noch Lyzeum für Theologie und Philosophie) und Erlangen (1743). Die Universitäten bildeten eigene Gemeinden in den Städten. Auch wenn die Studentenzahlen an den genannten Hochschulen im 17. und 18. Jahrhundert überschaubar waren, so stellten sie doch, insbesondere in einer kleinen Stadt, eine wichtige Gruppe innerhalb der eigentumslosen Bevölkerung dar.

\footnotetext{
${ }^{440}$ Vgl. hierzu und im Folgenden BRÜCKNER et al. 1988, S. 166.

${ }^{441}$ SCHÖNROCK 2006, S. 28.

${ }^{442}$ Ebd., S. 26.
} 


\section{Militär}

Mit der Etablierung von stehenden Heeren in den Territorien im 17. Jahrhundert wuchs die Zahl der Soldaten, die dauerhaft beherbergt werden mussten. Besonders stark waren die Auswirkungen auf die Bewohnerstrukturen der Städte in Ländern, die eine starke Militarisierung betrieben. Den Einfluss des Soldatenwohnens auf den Wohnhausbau der Barockzeit schildert Friedrich Mielke anschaulich für Potsdam, eine Stadt, die symbolhaft für den preußischen Militarismus steht und entsprechend stark von militärischer (Miet-)Bevölkerung geprägt war. ${ }^{443}$ Auf Mielkes doppelbändiges Werk sei ausdrücklich verwiesen, da ihm wenig hinzuzufügen ist.

In Göttingen führte die Gründung der Universität dazu, dass die Soldaten verstärkt auf Mietunterkünfte bei der ärmeren Bevölkerung angewiesen waren. Als Mietergruppe hatten sie im Gegensatz zu den Gesellen und Taglöhnern den Vorteil eines regelmäßigen Einkommens aus der Staatskasse. ${ }^{444}$ So gab es im Jahr 1734 allein achtzehn Vermieter, die sich auf das Militär als Mietergruppe spezialisiert hatten und ganze Häuser an Soldaten vermieteten. Ralf Pröve ermittelt, dass dort im Jahr 1740 rund ein Drittel der Militärangehörigen privat eingemietet waren. ${ }^{445}$ Die Auswirkungen, die mit einer Garnison verbunden waren, schildert Fred Kaspar ebenfalls exemplarisch anhand der Situation in Warendorf im Münsterland. ${ }^{446}$ Mit dem Festungsbau ab 1662 wurde dort die Zahl der Soldaten stark auf etwa 500 erhöht, was 10\% der Bevölkerung entsprach. Die Unterbringung erfolgte in Privatquartieren. Im Jahr 1707 regelte der Rat dies folgendermaßen: Soldaten mit Familie wurden bei den wohlhabendsten Bürgern einquartiert, alle übrigen Bürgerhaushalte mussten einen alleinstehenden Soldaten in Quartier nehmen. Wer keinen Soldaten beherbergte, musste eine Abgabe zahlen.

In den untersuchten fränkischen Städten spielte das Militär eine deutlich untergeordnetere Rolle. Immerhin wurden im frühen 18. Jahrhundert auch in Schwabach $^{447}$ und Ansbach ${ }^{448}$ große dreiflüglige Kasernen errichtet. Über das Soldaten-

\footnotetext{
${ }^{443}$ MiELKE 1972, besonders S. 110-128 und S. 168.

${ }^{444}$ Vgl. hierzu und im Folgenden PRÖVE 1995, S. 251f.

${ }^{445}$ Die Regierung zahlte 1740 laut Pröve den 223 privat einquartierten Männern 1.800 Taler als Mietentschädigung, also pro Soldat 78 Taler. Dies erscheint im Hinblick auf die Mieten in Franken recht hoch; Pröve 1995, S. 252.

446 KASPAR 2014, S. 121-151.

4471718 unter Johann Wilhelm von Zocha errichtet; HAUSLADEN 1930, S. 17.

${ }^{448} 1722$ wurde die dortige Infanteriekaserne nach Plänen von Karl Friedrich von Zocha als Dreiflügelanlage mit polygonalem Zentralkirchenbau in der Mittelachse errichtet. Dem Hof vorgelagert war ein niedriger Stalltrakt. Der Abbruch erfolgte 1900-1901; HAUSLADEN 1930, S. 17 und Abb. 7a, 7b.
} 
wohnen in den fränkischen Städten ist bisher nicht geforscht worden. Da die Soldaten als eximierte Bevölkerung in den Huldigungslisten nicht erfasst sind, müssten für diese Fragestellung andere Quellengattungen herangezogen werden. ${ }^{449}$ Neben der Unterbringung in Kasernen dürfte die Einmietung in Privathäusern die häufigste Wohnform dargestellt haben.

Die Anzahl der Soldaten war jedoch in vielen Städten gering, dies gilt auch für die kleineren souveränen Reichsstädte. Ein Hinweis über die Stärke der stationierten Truppen hat sich für die oberhalb von Weißenburg gelegene ansbachische Festung Wülzburg erhalten: Um 1700 waren an einem der wichtigsten militärischen Stützpunkte des Markgraftums lediglich 41 Soldaten stationiert. ${ }^{450}$ Für das Jahr 1818 sind sogar nur neun Familien (Haushalte) mit insgesamt 36 Personen als Bewohner der Festung überliefert. ${ }^{451}$

Auch in der kleinen schwäbischen Residenzstadt Oettingen lebten 1749 nur 23 Soldatenhaushalte, 1775 waren es dann unwesentlich mehr, nämlich 25 Soldatenhaushalte. ${ }^{452}$ Deren durchschnittliche Haushaltsgröße lag 1749 bei 3,82 und 1775 bei 3,44 Personen. Für 1749 lässt sich für zwei Soldatenhaushalte Hausbesitz nachweisen, ${ }^{453}$ überwiegend haben diese wohl aber zur Miete gewohnt. Ihr Anteil an der Bevölkerung war gering, 1772 stellten sie nur knapp $3 \%$ der Haushalte. ${ }^{454}$

Der Effekt, den eine Garnison auf die Stadtstruktur und die Zusammensetzung ihrer (Miet-)Bevölkerung haben konnte, sollte dennoch nicht unterschätzt werden. Für die bayerische Hauptstadt Ingolstadt hat sich eine Bevölkerungsaufstellung von 1762 erhalten. ${ }^{455}$ Gezählt wurden 621 Eigentümerhaushalte und 654 Miethaushalte. Unter den dort aufgelisteten Haushalten fanden sich keine einfachen Soldaten, vermutlich waren diese in Kasernen untergebracht. In der Rubrik der Mieter ist jedoch eine große Anzahl an oberen Rängen, abgedienten Soldaten und Soldatenwitwen verzeichnet. Hinzu kamen noch Haushalte, deren Vorstände ihren Unterhalt direkt bei den Regimentern verdienten, seien es Proviantbäcker, Feldscherer oder auch der Casernenstrohsackfüller Johann Scheich ${ }^{456}$. In Summe waren dies 125 Miethaushalte, nicht eingerechnet die (Miet-)Haushalte, die wirtschaftlich indirekt von den Kasernen profitierten. Dies bedeutet, dass 19\% der Miethaushalte direkt auf die Anwesenheit der Regimenter zurückgehen. Bezogen

\footnotetext{
${ }^{449}$ Für die Erb- und Landeshuldigung hat sich jedoch der Ablauf für die Militärangehörigen erhalten. StAN, Fm AN, Brdbg. Lit., Nr. 600, fol. 354-425.

${ }^{450}$ StAN, Fm AN, Brdbg. Lit., Nr. 600, fol. 405-407'.

${ }^{451}$ Alphabetisches Verzeichnis 1818, S. 105.

452 OSTENRIEDER 1993, S. $95 f$.

${ }^{453}$ Ebd. S. 95.

${ }^{454}$ Ebd., S. 134, Tab. 19. Aufgeführt werden dort insgesamt 888 Haushalte.

${ }^{455}$ Haupt-Beschreibung 1762.

${ }^{456}$ Ebd., Hs.Nro. 199, S. 10.
} 
auf die Gesamtzahl der 1.275 Haushalte entspricht dies einem Anteil von knapp $10 \%$ aller Haushalte. Auch in einem vorangehenden Verzeichnis aus dem Jahr 1675 spielten Militärangehörige bereits quantitativ eine große Rolle an der Bevölkerung von Ingolstadt. Genannt werden dort 73 Soldaten, die nach den Angaben von Hoffmann sämtlich im Eigentum wohnten. Es handelte sich im Hinblick auf den geringen Wert der Häuser von unter $500 \mathrm{fl}$ wohl um kleine Häuser, die zumeist in Gruppen dicht beieinanderstanden. ${ }^{457} 87$ Jahre später fanden sich - wie bereits angedeutet - dagegen keine Soldaten mehr unter den Hauseigentümern. Die Ursachen, die dazu führten, dass die Soldaten nicht mehr länger über Wohneigentum verfügten, sind nicht bekannt. Diese Veränderung könnte jedoch mit der Kasernierung der Soldaten in späterer Zeit zusammenhängen. ${ }^{458}$

\section{Untermieter, Kostgänger, Bettgeher und Schlafbursche}

Wohl zu allen Zeiten lebten eigentumslose Personen auch in Mietverhältnissen, in denen der Mietgegenstand auf ein einzelnes Zimmer oder gar nur das Aufenthaltsrecht in der Stube oder einen Schlafplatz reduziert war. In dieser Arbeit wird der Begriff der „Untermiete“ für sämtliche Mietsituationen dieser Art verwendet, unabhängig davon, ob der Vermieter nun Eigentümer oder selbst nur Mieter war, während im juristischen Sinn lediglich der Mieter eines Mieters als Untermieter bezeichnet wird.

Archivalisch sind Untermieter nur schwer nachzuweisen, da sich selten die entsprechenden Mietverträge erhalten haben. Ein früher Hinweis ist für Köln überliefert: In einer Urkunde aus der Zeit um 1170 wird dem Mieter ein Aftervermietungsrecht eingeräumt, woraufhin dieser ein Drittel des Hauses untervermietet. ${ }^{459}$ In Konstanz scheint die Vermietung von Schlafstellen im frühen 13. Jahrhundert weit verbreitet gewesen zu sein. ${ }^{460}$

Anhand der wenigen seriellen Quellen lässt sich der Status Mieter oder Untermieter ebenfalls kaum klären. Ein sprechendes Beispiel für die rechtliche Hierarchie, auf deren unterster Stufe sich der Untermieter befand, ist zum Beispiel der Schneidergeselle Conrad Fleischmann in Boxdorf, Untertan der Nürnberger Patrizierfamilie von Grundherr. Fleischmann wohnte 1759 (als Untermieter) beim Beständer (Mieter) Paul Haas auf dem Hof des Hans Schober, der wiederum Lehensmann der Familie Grundherr war. ${ }^{461}$ Aus dem Lehensverhältnis des Bauern

\footnotetext{
${ }^{457}$ HofmANn 1965, S. 33.

${ }^{458}$ Haupt-Beschreibung 1762.

${ }^{459}$ SCHULIN 1920, S. 148.

${ }^{460}$ Ebd., S. 180.

${ }^{461}$ StadtAN, E 13/III Nr. A 2168, letztwillige Verfügungen des Schneidergesellen Conrad. Fleischmann zu Boxdorf 1758/59.
} 
ergab sich demnach wohl auch die juristische Abhängigkeit von Mieter und Untermieter von der Familie Grundherr.

Konkrete Zahlen zu Untermietern in Franken konnten bisher nur in wenigen Fällen ermittelt werden, sieht man von den Angaben im Reichssteuerregister $1497 \mathrm{ab}$. Dort sind primär die Untermieter, als Hausgenossen bezeichnet, erfasst, wohingegen die Hauptmieter nicht von den Eigentümern unterschieden sind. Ihr Anteil lag durchschnittlich bei $12 \%$ aller aufgelisteten Haushalte (vgl. Tab. 17). Für das späte 18. Jahrhundert lassen sich zudem für einige reichsstädtische Mietwohnungen in Nürnberg Untermieter nachweisen: In den städtischen Zinsmeisterbüchern sind die Hauptmieter vermerkt und in den Quartiergeldlisten 1797/98 sämtliche Bewohner, sodass eine Differenzierung in Hauptmieter und Untermieter möglich ist. Für die sechs Reihenhäuser auf der Insel Schütt ist die Mietzahlung der sechs Hauptmieter für das Jahr 1798 überliefert, ${ }^{462}$ in den Quartiergeldlisten sind dagegen zwanzig Haushaltsvorstände erfasst. Demnach beherbergten die sechs Hauptmieter vierzehn Untermieter. ${ }^{463}$ Unter den Bewohnern befanden sich vier Einspännige, die anscheinend keine Quartiergeldabgabe entrichten mussten. Weiterhin sind sechs Haushaltsvorstände aufgeführt, die nur geringe Beiträge zahlten, zehn Haushaltsvorstände waren so arm, dass sie ebenfalls von der Abgabe befreit waren. Insgesamt entsteht der Eindruck eines sozial sehr schwachen Milieus. Jeder Hauptmieter hatte durchschnittlich 2,3 Untermieter bei sich wohnen. Die meisten Untermieter dürften alleinstehend gewesen sein, auch wenn nähere Angaben zu Haushaltsangehörigen fehlen. Ein solch hoher Anteil an Untermietern stellte aber in Nürnberg zu diesem Zeitpunkt die Ausnahme dar: In den städtischen Weberhäusern, den Sieben Zeilen, lassen sich anhand der beiden Quellen gleichzeitig keine Untermieter nachweisen. ${ }^{464}$

Da die von mir ausgewerteten seriellen Quellen abgesehen vom Reichssteuerregister kaum Angaben zu Untermietern enthalten, sollen hilfsweise Ergebnisse aus anderen Städten referiert werden. Denn aus dem Fehlen dieser Gruppe in den Verzeichnissen sollte nicht der falsche Schluss gezogen werden, dass es keine Untermieter gegeben hat. Ihr Anteil konnte beträchtlich sein.

\footnotetext{
${ }^{462}$ StadtAN, B 17/I Nr. 83, Eintrag 509-514.

${ }^{463}$ Quartiergeldbeiträge 1798, S. 31, Nr. MDCLXXXXVIIb.

${ }^{464}$ StadtAN, B 17/I Nr. 83, Eintrag 576 ff, wobei anzumerken ist, dass in den Feuerschaulisten Untermieter generell keine Erwähnung finden; Quartiergeldbeiträge 1798, S[ebald] 23, S 1239, S 1241, S 1243, S 1244, S 1246, S 1247, S 1250.
} 


\section{Spätes Mittelalter}

Einen der frühesten quantitativen Nachweise für Untermieter führt Christian Forneck für Regensburg im 15. Jahrhundert. ${ }^{465}$ Er kommt auf 28,2\% der erwachsenen Bevölkerung, die als alleinstehende Inwohner in einem größeren Haushalt mitlebten, ohne zum Gesinde zu gehören. 660 Männern standen 1.092 Frauen, davon explizit 276 Witwen, gegenüber. Insbesondere unter den Haushalten mit weiblichen Haushaltsvorständen fällt der hohe Anteil von Untermieterinnen auf. Insgesamt sind 1.646 ledige Frauen erfasst, ${ }^{466}$ davon lebten Zweidrittel zur Untermiete!

In den Esslinger Steuerlisten aus dem Jahr 1384 lassen sich allein 23\% der Steuerpflichtigen als Untermieter (in domo) charakterisieren, unberücksichtigt ist hierbei noch die große Gruppe derer, die keine Steuern zahlten und sicher $\mathrm{zu}$ noch größeren Teilen auf ein Untermietverhältnis angewiesen waren. ${ }^{467}$ In Freiburg im Breisgau waren 1385 dagegen „nur“ 6,8\% der steuerzahlenden Männer Untermieter. ${ }^{468}$

Auch für Dortmund sind Untermieter im frühen 15. Jahrhundert vielfach genannt. Allerdings konnte Monika Fehse hier keinen absoluten Anteil an der Bevölkerung ermitteln. ${ }^{469}$ Anschaulich wird die dortige Situation anhand von zwei Schaubildern zu den Bewohnern der Straßenabschnitte Tegen Sunte Katerinen und Achterste Kampstraße 1406-1409: ${ }^{470}$ Von den untersuchten sechzehn Anwesen wiesen elf Untermieter auf.

\section{Frühe Neuzeit bis zum Dreißigjährigen Krieg}

Aus der Zeit vor dem Dreißigjährigen Krieg liegen zudem Zahlen aus der hessischen Residenzstadt Kassel vor. Im Jahr 1599 wurden dort 993 Haushaltsvorstände als Hausgesessene (hierunter finden sich Eigentümer und Mieter) und 300 Haushalte als bei andern inne wohnende erfasst. ${ }^{471}$ Laut Manfred Lasch handelte es sich dabei um Personen, die weder Hauseigentümer noch Mieter, sondern im heutigen Sinne Untermieter waren. 1607 standen den 1.039 Hausgesessenen 336 Untermieter gegenüber und im Jahr 1622 waren es $1.153 \mathrm{zu}$ 307. Der Anteil der Untermieter lag demnach bei $21 \%$ bis $24 \%$ aller Haushaltsvorstände. Anders ausgedrückt lebte in jedem dritten Haushalt noch ein Untermieter, mit oder ohne Familie. In diesen Fällen ist davon auszugehen, dass die Küche - häufig wohl

${ }^{465}$ FORNECK 2000, S. 80f. Von den 6.204 Erwachsenen (ohne Juden und Geistlichkeit) lebten 1.752 Personen als ledige „Inwohner meist ohne eigenen Haushalt“.

466 Ebd., S. 27.

467 DiRlMEIER 1978, S. 240.

468 Ebd.

${ }^{469}$ FEHSE 2005, S. 294-299.

470 Ebd., S. 302-305, Abb. 11 und 12.

${ }^{471}$ Vgl. hierzu und im Folgenden LASCH 1969, S. $68 f$. 
auch die Stube und in anderen Fällen auch noch die Kammer - mit familienfremden Personen geteilt wurde.

In Augsburg scheinen die Untermieter vor allem unter dem Begriff der Hausarmen Erwähnung zu finden. Ihr Anteil muss ebenfalls ganz erheblich gewesen sein. ${ }^{472}$ Bernd Roeck führt für das Jahr 1638 durchschnittlich fünf Personen an, die sich eine der damals erfassten Feuerstätten teilten. Dabei kann es sich jedoch nicht um Familien im klassischen Sinn gehandelt haben, denn vier Jahre zuvor lag die durchschnittliche Größe der Haushalte, die zur Steuer veranlagt waren, bei lediglich 2,75 Personen. Die Feuerstätten wurden also noch mit durchschnittlich zwei weiteren Personen geteilt, die nicht als Kinder, Gesinde etc. direkt zur Familie gezählt werden können. In den historischen Augsburger Quellen werden solche Wohnverhältnisse mit der Bezeichnung im ögg charakterisiert, was das Aufenthaltsrecht in einer Ecke/einem Teil der Stube meint, und damit der fränkischen Bezeichnung im Winkel entspricht. In Augsburg scheint es sich dabei jedoch nicht primär um das Aufenthaltsrecht von Altenteilern zu handeln, sondern generell ein Untermietverhältnis anzuzeigen.

Josef Ehmer hat sich mit dem Thema ausführlich beschäftigt. ${ }^{473}$ Sein Fokus liegt auf der Situation im 19. Jahrhundert. Er geht aber auch kurz auf die frühe Neuzeit ein: In Salzburg beherbergten im Jahr 1569 17\% aller Haushalte nichtverwandte Mitbewohner, 1647 sinkt der Anteil auf 13\%, steigt aber anschließend bis 1794 auf $22 \%$ an. ${ }^{474}$

\section{Nach dem Dreißigjährigen Krieg}

Direkt nach dem Dreißigjährigen Krieg scheint der Anteil der Untermieter deutlich zurückzugehen. Ursächlich hierfür dürfte der große Bevölkerungsverlust in vielen Teilen Deutschlands gewesen sein. So wurden zum Beispiel in Lübeck in den Steuerbüchern 1663 etwa 6\% der Haushaltsvorstände als Mitbewohner (Untermieter) gekennzeichnet. ${ }^{475}$ Der Anteil der Lübecker Untermieter, die nachweislich mit in den Buden lebten, betrug laut Scheftel im Jahr 1663 ebenfalls 6\% der Budenhaushalte. ${ }^{476}$

Dass dieser niedrige Wert jedoch nicht ohne Weiteres auf andere Städte und nachfolgende Jahrzehnte übertragbar ist, wird an der Situation in Münster/ Westfalen deutlich: Hugo Heidemann ermittelt dort auf der Grundlage der sehr detaillierten

\footnotetext{
${ }^{472}$ Vgl. hierzu und im Folgenden ROECK 1989, S. 784f.

${ }^{473}$ Vgl. EHMER 1979, S. 132-150. Vgl. hierzu auch WENDEROTH 2019a, S. 82f.

${ }^{474}$ Ebd., S. 135.

475 SCHEFTEL 1988, S. 71.

${ }^{476}$ Ebd., S. 71; der Anteil der (alleinstehenden) Frauenhaushalte an den Gangbewohnern lag mit $15 \%$ deutlich darüber.
} 
Schatzungslisten (Steuerlisten) des Jahres 1685 gleichfalls Personen, die er als Untermieter bezeichnet. ${ }^{477}$ Es handelt sich um exakt 487 Personen, die in etwa 300 Haushalten zusammenlebten. Meistenteils waren es Einzelhaushalte von ledigen oder verwitweten Frauen. Es finden sich in dieser Gruppe lediglich 36 Ehepaare und ein alleinstehender Mann, von daher dürfte die Definition dieser Personen als Untermieter in den allermeisten Fällen zutreffend sein. Bezogen auf die erfassten 6.091 Einwohner Münsters ist ihr Anteil mit 8\% der Bevölkerung gering. ${ }^{478}$ Betrachtet man nur die 1.720 erfassten Haushalte, lag der prozentuale Anteil der Untermieter-Haushalte mit 17\% deutlich höher. ${ }^{479}$ In dieser Gruppe finden sich auch 24 männliche und zehn weibliche Kostgänger. ${ }^{480}$ Das Mieten eines Hausteils scheint in Münster gleichbedeutend mit Untermiete gewesen zu sein. Hauptmieter müssen in Münster wohl ein ganzes Haus gemietet haben, denn die 6.874 Bewohner verteilten sich auf 1.435 Häuser. Die Behausungsziffer lag entsprechend niedrig bei lediglich 4,7 Personen pro Haus.

\section{Frauenhaushalte}

Wie schon mehrfach angemerkt wurde, stellten die Haushalte mit einem weiblichen Haushaltsvorstand eine wichtige Gruppe innerhalb der Mieter und Untermieter dar. In einigen Fällen wie dem Reichssteuerregister von 1497 bilden sie die Hauptgruppe der in historischen Listen erfassten Mieter. Bei anderen Erfassungen bleiben sie dagegen weitgehend unerwähnt, zum Beispiel in den Huldigungslisten des Fürstentums Ansbach und den Feuerschaulisten aus Nürnberg von 1561. Der Grund der Nichterwähnung dürfte in vielen Fällen nicht auf das Geschlecht an sich, sondern vielmehr auf den Umstand zurückzuführen sein, dass es sich um Untermietverhältnisse gehandelt hat. Die männlichen Untermieterhaushalte dürften in diesen Fällen in den Aufstellungen ebenfalls fehlen, nur lässt sich dies schwerer nachweisen. Die archivalische Überlieferung muss also insbesondere im Hinblick auf die erwähnten oder nicht erwähnten Frauenhaushalte kritisch gewürdigt werden, denn in jedem Fall gehörten sie zur gesellschaftlichen Realität vergangener Jahrhunderte. Fehlen Frauenhaushalte unter den aufgeführten Einwohnern und Mietern oder ist ihre Anzahl sehr gering, sollte dies zu einer kritischen Würdigung führen. In diese kritische Analyse wäre dann zugleich die Frage nach fehlenden Angaben zu weiblichen und männlichen Untermietern miteinzuschließen.

\footnotetext{
477 Heidemann 1917, S. 28, Tab. 11. Diese werden in den Quellen als Einwohner/Einwohnerin bezeichnet; ebd. S. 26.

478 Ebd., S. 14, Tab. 1.

${ }^{479}$ Ebd., S. 27.

${ }^{480}$ Ebd., S. 26, Tab. 8.
} 


\section{Spätes Mittelalter}

Die ältesten Zahlen zum Anteil von Frauenhaushalten in Nürnberg finden sich in einer Erfassung der waffenfähigen Männer aus dem Jahr 1431. In der Zählung wurden für vier von acht Vierteln („Am Weinmarkt“, „Am Milchmarkt“, „Auf dem Egidienhof“" und „Am Kornmarkt“) die weiblichen Haushaltsvorstände erfasst. Leider wurde dabei nicht zwischen Miete und Eigentum differenziert. Enthalten sind zudem nicht die Hausgenossinnen-Haushalte, die nachgeordnet, quasi als Untermieterinnen, beim Vermieter wohnten. In diesen Vierteln hatten durchschnittlich 12,1\% der (Eigentümer-)Haushalte einen weiblichen Vorstand. Der prozentuale Anteil der waffenfähigen Söhne und Knechte unterschied sich jedoch nicht von den männlich geführten Haushalten, ${ }^{481}$ auch dies deutet darauf hin, dass es sich zum allergrößten Teil um Haushalte von Eigentümerinnen und nicht um solche von Mieterinnen gehandelt haben muss.

Im Jahr 1497 war der Hausgenosse in Franken eine alleinstehende Frau. Diese provokante These soll gleich zu Beginn erläutert werden, da sie wesentliche Auswirkungen auf das Verständnis der Mietsituation am Ende des Mittelalters in den fränkischen Städten hat.

Von den weiter oben im Zusammenhang mit dem Reichssteuerregister 1497 genannten 21 Städten musste in der folgenden Auswertung lediglich Crailsheim unberücksichtigt bleiben, da hier das Geschlecht der Hausgenossen nicht eindeutig aus den Aufstellungen hervorgeht. Insgesamt sind damit 3.142 Haushalte in zwanzig Orten erfasst. Von den dort nachgewiesenen 375 Hausgenossenhaushalten handelt es sich in 237 Fällen um alleinstehende Frauen! Dies entspricht 63\% der gesamten Miethaushalte. Nur in wenigen Städten liegt ihr Anteil unter 50\%: Selb (43\%), Schauenstein (33\%), Langenzenn (29\%), Roth (26\%). Erlangen mit $0 \%$ fällt deutlich heraus und ist sicher nicht repräsentativ, da dort insgesamt lediglich ein männlicher Hausgenosse verzeichnet ist. Die vier erstgenannten Städte gehören umgekehrt zu den Orten mit den meisten Mietern.

${ }^{481}$ OTT 1907, S. 72. 


\begin{tabular}{|l|c|c|c|r|}
\hline Ort & $\begin{array}{c}\text { Anzahl Einträge } \\
\text { = Eigentümer? }\end{array}$ & Hausgenossen & $\begin{array}{c}\text { davon } \\
\text { Mieterinnen }\end{array}$ & $\begin{array}{c}\text { Anteil alleinstehender } \\
\text { Frauen/Hausgenossen }\end{array}$ \\
\hline Ansbach, gesamt & 450 & 46 & 33 & $\mathbf{7 2 \%}$ \\
\hline Arzberg & 69 & 10 & 8 & $\mathbf{8 0 \%}$ \\
\hline Bayreuth & 310 & 33 & 24 & $\mathbf{7 3 \%}$ \\
\hline Berneck & 76 & 1 & 1 & $\mathbf{1 0 0 \%}$ \\
\hline Erlangen & 92 & 1 & 0 & $\mathbf{0 \%}$ \\
\hline Feuchtwangen & 151 & 37 & 20 & $\mathbf{5 4 \%}$ \\
\hline Gunzenhausen & 217 & 9 & 9 & $\mathbf{1 0 0 \%}$ \\
\hline Helmbrechts & 59 & 13 & 10 & $\mathbf{7 7 \%}$ \\
\hline Hof, Vorstadt & 128 & 37 & 30 & $\mathbf{8 1 \%}$ \\
\hline Kulmbach, gesamt & 291 & 21 & 14 & $\mathbf{6 7 \%}$ \\
\hline Langenzenn & 112 & 17 & 5 & $\mathbf{2 9 \%}$ \\
\hline Neustadt/Aisch & 286 & 22 & 19 & $\mathbf{8 6 \%}$ \\
\hline Neustadt/Kulm & 101 & 12 & 6 & $\mathbf{5 0 \%}$ \\
\hline Roth & 111 & 35 & 9 & $\mathbf{4 0 \%}$ \\
\hline Schauenstein & 38 & 10 & 4 & $\mathbf{6 3 \%}$ \\
\hline Selb & 73 & 21 & 9 & $\mathbf{8 9 \%}$ \\
\hline Uffenheim & 103 & 18 & 11 & $\mathbf{1 0 0 \%}$ \\
\hline Wassertrüdingen & 149 & 18 & 16 & $\mathbf{5 0 \%}$ \\
\hline Weißenstadt & 138 & 3 & 3 & $\mathbf{6 3 \%}$ \\
\hline Wunsiedel & 188 & 12 & 6 & 1497, \\
\hline \hline Gesamt & 3.142 & $\mathbf{3 7 6}$ & $\mathbf{2 3 7}$ & \\
\hline
\end{tabular}

Tabelle 2. Anteil der Frauenhaushalte unter den Hausgenossen (Untermietern) im Jahr 1497, anhand der Angaben des Reichssteuerregisters (Thomas Wenderoth 2019)

In der Regel handelte es sich bei den verzeichneten Mieterinnen um arme, alte, alleinstehende Witwen. Ihre Wohnsituation dürfte entsprechend bescheiden gewesen sein und sich auf ein Untermietverhältnis mit Aufenthaltsrecht in der Stube des Hausherrn beschränkt haben. Von eigenständigen Wohneinheiten für diese Personengruppe kann in der Regel wohl nicht ausgegangen werden. Eine sinnvolle Erklärung für den hohen Frauenanteil steht noch aus, es könnte dies aber als ein weiterer Hinweis darauf gewertet werden, dass in diesem Fall unter dem Begriff Hausgenossen nach unserem heutigen Verständnis primär Untermieter erfasst sind. Flächenhafte Vergleichsdaten aus dem späten Mittelalter fehlen. Allerdings weisen die Angaben aus dem Reichssteuerregister des Fürstbistums Speyer in die gleiche Richtung. Peter-Johannes Schuler stellt fest, dass auch hier der Anteil an Haushalten von alleinstehenden Frauen verhältnismäßig hoch war. ${ }^{482}$ Für einen Vergleich und eine Einordnung der Zahlen bieten sich - als räumlich und zeitlich am nächsten liegend - die Bauernverzeichnisse der Reichsstadt Nürnberg an, die aber nur die ländliche Bevölkerung außerhalb der Städte

482 Vgl. SCHULER 1978, S. 131. 
zwischen 1538 und 1548 erfassen. Die Bauernverzeichnisse wurden von mir bereits im Rahmen meiner Dissertation ausgewertet. ${ }^{483}$ Die Mieter werden in den Bauernverzeichnissen unter dem Begriff Beständner aufgeführt, der alle Mietverhältnisse einschließt. Der Anteil der Frauenhaushalte lässt sich dort also bezogen auf alle Miethaushalte ermitteln: Bei den stadtnahen Orten lag der Anteil der Mieterinnen unter den Miethaushalten im Jahr 1538 bei 26,5\% und 1548 bei 23,3\%. Es ist also ein deutlicher Unterschied zu den Zahlen des Reichssteuerregisters hinsichtlich der Geschlechterverteilung festzustellen: Im Nürnberger Landgebiet waren die männlichen Mieter nachweislich in der Mehrheit, sie stellten drei von vier Miethaushalten. Bezieht man die Angaben zu den weiblichen Miethaushalten auf alle Haushalte (Mieter und Eigentümer), so lag der Anteil der Hausgenossinnen im Reichssteuerregister 1497 in den Städten bei 7\% (236 von 3.517) und damit deutlich unterhalb des Mieterinnenanteils in den Vorstädten um Nürnberg im Jahr 1538 in Höhe von 17\% (84 von 496). Obwohl es mehr Mieterinnen in den vorstädtischen Gebieten Nürnbergs gab, war ihr Anteil an den Miethaushalten deutlich geringer! Vergleicht man dazu die Angaben zu den nürnbergischen Dörfern fern der Stadt, wo der Anteil der Mieterinnenhaushalte an der Gesamtbevölkerung im Jahr 1538 knapp 5\% (24 von 496) beträgt, verschwindet die Differenz zur Stadtgemeinde Nürnberg. Allerdings bleibt sie bestehen, wenn man allein den Anteil der Frauenhaushalte unter den Miethaushalten betrachtet: Dieser lag 1538 bei 15,5\% und 1548 bei 27\%, also im Rahmen der gleichzeitigen Angaben zu den nürnbergischen Vororten, aber deutlich niedriger als die Angaben von $1497 \mathrm{zu}$ den hohenzollerischen Landstädten. Überraschend scheint mir daher weniger der hohe Anteil der weiblichen Miethaushalte in den fränkischen Städten des späten Mittelalters als vielmehr der dazu im Verhältnis geringe Anteil an männlichen Miethaushalten, was ebenfalls die These untermauert, dass die männlichen Hauptmieter im Reichssteuerregister überwiegend nicht als Miethaushalte gekennzeichnet worden sind, sondern in den Angaben zu den Eigentümern enthalten sind, die ja dort nicht explizit als solche charakterisiert werden.

Dass es sich bei der hohen Zahl der im Reichssteuerregister und in den Bauernverzeichnissen genannten weiblichen Mieter nicht um ein Versehen handelt, wird auch deutlich anhand von Zahlen aus Wismar. Dort ist für das Jahr 1475 überliefert, dass 26,2\% der Bewohner von Kellern und Buden Haushalte mit einem weiblichen Haushaltsvorstand waren. Bei Kellern und Buden handelte es sich um die klassischen Mietunterkünfte in den norddeutschen Städten. In den Häusern selbst, die weit überwiegend von Eigentümern bewohnt wurden, lag der Anteil weiblich geführter Haushalte dagegen nur bei $7,8 \%{ }^{484}$ So gesehen muss

\footnotetext{
${ }^{483}$ Vgl. hierzu und im Folgenden Wenderoth 2019a, S. 72f, Tab. 1 und Tab. 6.

${ }^{484}$ MASCHKE 1967, S. 27.
} 
wiederum die geringe Zahl an männlichen Hausgenossen im Reichssteuerregister verwundern. Einen vergleichsweise hohen Anteil von Frauenhaushalten unter den Mietern benennt auch Monika Fehse für Dortmund im Jahr 1406, dort stand jedem vierten Miethaushalt eine Frau vor. ${ }^{485}$ In Regensburg war die Anzahl der weiblichen Miethaushalte im 15. Jahrhundert noch beträchtlicher. Von den weiter oben bereits genannten 2.333 Miethaushalten entfielen allein 1.092 auf weibliche Untermieterinnen, dies waren $47 \%$ aller Miethaushalte, wobei für Regensburg generell ein sehr hoher Anteil von Untermietverhältnissen überliefert ist.

Zusammenfassend darf wohl regelhaft von einem Anteil der Frauenhaushalte von etwa $25 \%$ an den Miethaushalten ausgegangen werden. Sind ausschließlich oder zu einem großen Anteil Untermietverhältnisse erfasst, konnte deren Anteil auch über $50 \%$ liegen. Umgekehrt bedeutet dies auch, dass bei einem höheren Mieterinnenanteil mit einem entsprechend hohen Anteil an Untermietverhältnissen gerechnet werden muss. Im Rahmen zukünftiger Forschungen scheint es zielführend, die Gruppe der armen und alten Witwen als eigenständige soziale Schicht zu begreifen und sie nicht primär unter dem Blickwinkel der Miethaushalte zu betrachten. Diese Bevölkerungsgruppe scheint über die Jahrhunderte hinweg existent gewesen zu sein.

\section{Um 1600}

Die zeitliche Lücke zwischen dem Mittelalter und der Barockzeit kann leider nicht anhand der von mir ausgewerteten Quellen geschlossen werden. Stellvertretend sollen daher Zahlen aus anderen Städten angeführt werden. Bernd Roeck stellt für Augsburg im frühen 17. Jahrhundert fest, dass alleinstehende Frauen häufig zusammen eine Wohnung mieteten. In vielen Fällen dürfte es sich hierbei um Untermietverhältnisse gehandelt haben, die aus der finanziellen Not der Mieterin - aber wohl zum Teil auch der Vermieter - herrührten. Roeck beobachtet zwar auch einige wohlhabende Mieterinnen, aber seiner Kenntnis nach „war der Anteil der Frauen, die extrem ärmlich gelebt haben müssen, im Vergleich zu den Männerhaushalten deutlich überproportional.“486 Er folgert daraus, „daß sehr viele dieser ,Hausarmen', die im ,ögg anderer Familien lebten, alleinstehende Frauen, Witwen waren.“487

\footnotetext{
${ }^{485}$ FEHSE 2005, S. 270-274.

486 ROECK 1989, S. 478.

${ }^{487}$ Ebd., S. $478 f$.
} 
Etwas später, 1663, wurden in Lübeck 15\% der Haushalte in den Gängen als alleinstehende Frauen erfasst. ${ }^{488}$ Die Bewohner der Buden in den Gängen waren zu 99\% Mieter. ${ }^{489}$ Von den dort insgesamt erfassten 186 Frauenhaushalten sind 48 ausdrücklich als „arm“ gekennzeichnet.

\section{Barockzeit}

In den Huldigungslisten des Markgraftums Ansbach sind nur sehr wenige eigentumslose Frauenhaushalte erfasst. Die Quelle scheint in dieser Hinsicht nicht aussagekräftig: Dort werden nur ganz vereinzelt weibliche Miethaushalte aufgeführt und dann zum Teil nicht unter der Rubrik der Hausgenossen, wo sich häufig nur männliche Namen finden, sondern unter der Rubrik der hausbesitzenden Witwen, wo sie eigentlich nicht hingehören. Neben den wenigen explizit aufgeführten Mieterinnen dürfte es eine große Zahl von Mieterinnen gegeben haben, die im Rahmen der Huldigungen nicht erfasst worden sind und wohl auch nicht gehuldigt haben. ${ }^{490}$ Ein wichtiger Hinweis für die Frage nach den Frauenhaushalten im Fürstentum Brandenburg-Ansbach während der Barockzeit findet sich in der Huldigungsliste für Creglingen 1672, dort heißt es: Wittweiber bey der Statt Creglingen, die mit Herd: undt Feuerstätt. ${ }^{491}$ Demnach sind in den Listen der Wittweiber nur die Frauen erfasst, die einen eigenständigen Haushalt führen, unabhängig ob als Eigentümerin oder Mieterin. Frauen, die zur Untermiete wohnten, sind nicht aufgeführt, vermutlich hatte der Hausherr für diese rechtlich einzustehen. Zugleich liefert dieser Hinweis auch eine Erklärung dafür, warum so wenige Mieterinnen namentlich aufgeführt wurden, denn das Wohnen zur Untermiete dürfte für einen Großteil der Mieterinnen die obligatorische Lebensform gewesen sein.

Anders scheint auch der extreme Unterschied zwischen dem Anteil der weiblichen Miethaushalte in den nürnbergischen Landstädten und den ansbachischen Städten nicht erklärbar. Wie für die Nürnberger Feuerschaulisten von 1561 muss auch in den meisten ansbachischen Huldigungslisten mit einem deutlich größeren Anteil an weiblichen (Unter-)Mieterinnen und damit einem insgesamt deutlich höheren Anteil an eigentumslosen Haushalten gerechnet werden, ohne dass dieser sich exakt bestimmen ließe. Die wenigen greifbaren Zahlen sind in Tabelle 2 zusammengefasst und den Zahlen aus dem Nürnberger Land gegenübergestellt.

\footnotetext{
${ }^{488}$ SCHEFTEL 1988, S. 71.

${ }^{489}$ Ebd., S. 73.

${ }^{490}$ Die Ursachen für die Situation konnten bisher nicht geklärt werden.

${ }^{491}$ StAN, Fm AN, Brdbg. Lit. Nr. 595, fol 180.
} 
Der direkte Vergleich zwischen dem Anteil der Mieterinnenhaushalte in der markgräflichen Stadt Schwabach 1703 und den Nürnberger Landstädten Altdorf und Velden 1716 zeigt deutliche Parallelen und bestätigt damit die Zahlen aus den Nürnberger Landstädten. Zugleich wird noch einmal deutlich, dass die Mieterinnenhaushalte ansonsten in den Huldigungslisten zu großen Teilen nicht erfasst sein dürften. Letzteres gilt vermutlich auch für Schwabach im Jahr 1723; auch wenn diese Erfassung ansonsten äußerst differenziert ist, verwundert der prozentual geringe Mieterinnenanteil. Dieser liegt deutlich unterhalb der Schwabacher Angaben für das Jahr 1703. Ob dieser Einbruch nur an der explosionsartigen Zunahme der männlichen Miethaushalte von 73 auf 482 lag oder im Jahr 1723 ein Teil der weiblichen Miethaushalte nicht gelistet wurde, ist bisher nicht geklärt.

\begin{tabular}{|l|c|c|c|r|r|r|}
\hline Ort und Jahr & Eigentümer & Eigentümerin & Mieter & Mieterin & $\begin{array}{c}\text { Anteil Mieterinnen } \\
\text { an allen Mietern }\end{array}$ & $\begin{array}{r}\text { Anteil Mieterinnen } \\
\text { an den Haushalten }\end{array}$ \\
\hline $\begin{array}{l}\text { Feuchtwangen } \\
\text { 1668 }\end{array}$ & 130 & 16 & 17 & 3 & $\mathbf{1 5 \%}$ & $\mathbf{2 \%}$ \\
\hline $\begin{array}{l}\text { Mainbernheim } \\
\text { 1673 }\end{array}$ & 125 & 18 & 13 & 5 & $\mathbf{2 8 \%}$ & $\mathbf{3} \%$ \\
\hline Ansbach 1673 & 288 & 43 & 73 & 27 & $\mathbf{2 7 \%}$ & $\mathbf{6 \%}$ \\
\hline Schwabach 1703 & 356 & & 73 & 58 & $\mathbf{4 4 \%}$ & $\mathbf{2 7 \%}$ \\
\hline Schwabach 1723 & 321 & 31 & 482 & 82 & $\mathbf{1 5 \%}$ & $\mathbf{2 2 \%}$ \\
\hline Altdorf 1716 & \multicolumn{2}{|c|}{289} & 133 & 92 & $\mathbf{4 1 \%}$ & $\mathbf{2 9 \%}$ \\
\hline Velden 1716 & \multicolumn{2}{|c|}{78} & 9 & 25 & & \\
\hline
\end{tabular}

Tabelle 3. Anteil der weiblichen Miethaushalte in ausgewählten Städten Frankens. Zusammenstellung von Angaben aus den Huldigungslisten des Fürstentums Ansbach und dem Türkensteuerverzeichnis der Reichsstadt Nürnberg (Thomas Wenderoth 2019)

Die These, dass weibliche Mieterinnen oftmals nicht erfasst worden sind, scheint sich auch für die Huldigungslisten des Jahres 1686/87 in Roth zu bestätigen: Während in der Rubrik der praesenten haußsäßig Bürger 115 Mann und 9 Wittweiber genannt sind, fehlen Frauen bei den zwölf Hausgenossen und den fünfzehn Schutzverwandten, die sämtlich Mieter waren. ${ }^{492}$ Unter den Eigentümern waren immerhin 7\% weiblich. Etwas differenzierter ist zur selben Zeit die Situation in Schwabach, zwar sind auch hier unter der Rubrik der Haußgenossen lediglich 52 Männer erfasst, unter der Rubrik Schutzverwanthe oder sogenannte Pfahlbürger sieht es aber anders aus: Von 56 Einträgen sind nur 33 männlich, 23 Einträge beziehen sich auf Frauen. ${ }^{493}$ Von diesen 23 Frauen sind lediglich zwei Frauen mit einem Zusatz als Witwe bezeichnet. In der Zusammenfassung am Schluss der

492 Erfassung im Jahr 1686/87. StAN, Fm AN, Brdbg. Lit. Nr. 596, fol. 463-464.

${ }^{493}$ StAN, Fm AN, Brdbg. Lit. Nr. 596, fol. 492'-494. 
Liste werden sie insgesamt als alte Weiber bezeichnet. Unter den 271 Hausbesitzern finden sich neunzehn Witwen. ${ }^{494}$

Aus anderen Quellen zu anderen Orten lassen sich ebenfalls für die Barockzeit hohe Anteile an weiblichen Miethaushalten nachweisen: Wichtige Informationen zum Anteil der Mieterinnen enthält zum Beispiel das Türkensteuerregister 1716/17 für die Stadt Altdorf (Tab. 21). ${ }^{495}$ Dort sind 289 Eigentümerhaushalte sowohl mit männlichem als auch weiblichem Haushaltsvorstand erfasst, diesen standen 133 männliche Miethaushalte und 92 Haushalte von Witwen gegenüber. Im Hinblick auf die aufgeführten Witwen unter den Eigentümern handelt es sich bei den zuletzt genannten Frauen um Mieterinnen. Bezogen auf die Gesamtzahl der Haushalte - die neunzehn Haushalte der öffentlich Angestellten sollen hier außer Betracht bleiben - nahmen die Haushalte der Mieterinnen immerhin einen Anteil von 22\% ein. Bezogen nur auf die Miethaushalte waren 41\% der Miethaushalte weiblich geführt. In der deutlich kleineren Stadt Velden mit insgesamt 118 Haushalten - ohne die sechs Haushalte von öffentlich Bediensteten - lag der Anteil der Mieterinnenhaushalte bei 29\%, bezogen auf alle Haushalte und bei 74\% innerhalb der Gruppe der Miethaushalte. ${ }^{496}$

Die Zahlen aus den nürnbergischen Landstädten werden durch Forschungen zu anderen Orten betätigt. Ein hoher Anteil von Mieterinnen lässt sich zum Beispiel auch für Chemnitz 1728 feststellen. ${ }^{497}$ Erfasst wurden 392 steuerpflichtige Hausgenossenhaushalte. Bei 37,6\% der genannten Haushalte handelte es sich um ledige Frauen, bei weiteren 17,9\% um Witwen mit bis zu fünf Kindern. Frauenhaushalte stellten also mit 55,5\% die Mehrheit bei den Miethaushalten. ${ }^{498}$ An dieser Stelle möchte ich noch einmal auf die Untersuchungsergebnisse Petra Ostenrieders für die schwäbische Residenzstadt Oettingen zurückkommen: Für die Zeit 1612/14 sind 41 Eigentümerhaushalte mit weiblichem Haushaltsvorstand überliefert und 58 Haushalte von Mieterinnen. ${ }^{499} \mathrm{Im}$ Jahr 1762 wurden 199 weibliche Haushalte erfasst, von denen 103 über Hausbesitz verfügten, ${ }^{500} 96$ Haushalte mit weiblichem Haushaltsvorstand wohnten demnach zur Miete. Der Anteil der zur Miete Wohnenden war also unter den Frauen besonders hoch und lag mit 44\%

\footnotetext{
${ }^{494}$ Ebd., Nr. 596, fol. 485-491.

${ }^{495}$ StAN, Reichsstadt N, Bauernverzeichnisse Nr. 7, fol. 318-326.

${ }^{496}$ Ebd., Bauernverzeichnisse Nr. 7, fol. 533' -537 .

${ }^{497}$ BRÄUER 2003, S. 79.

${ }^{498}$ Der Anteil der ledigen Männer betrug 4,4\%, der der kinderlosen Paare 13\%

und der der Familien mit zwei Elternteilen 27,1\%.

${ }^{499}$ OSTENRIEDER 1993, S. 124, Tab. 17.

${ }^{500}$ Ebd., S.122 und 126.
} 
fast bei der Hälfte dieser Gruppe. Insgesamt ermittelt Ostenrieder 737 Haushalte für das Jahr 1762, davon 518 Eigentümerhaushalte. ${ }^{501}$

\begin{tabular}{|l|c|r|c|r|r|r|}
\hline Ort und Jahr & Eigentümer & Eigentümerin & Mieter & Mieterin & $\begin{array}{c}\text { Anteil Mieterinnen } \\
\text { an allen Mietern }\end{array}$ & $\begin{array}{c}\text { Anteil Mieterinnen } \\
\text { an den Haushalten }\end{array}$ \\
\hline Oettingen 1612/14 & 299 & 41 & 144 & 58 & $\mathbf{2 9 \%}$ & $\mathbf{1 1 \%}$ \\
\hline Oettingen 1762 & 415 & 103 & 123 & 96 & $\mathbf{4 4 \%}$ & $\mathbf{1 3 \%}$ \\
\hline
\end{tabular}

Tabelle 4. Anteil der weiblichen Miethaushalte in Oettingen

(nach OSTENRIEDER 1993, Thomas Wenderoth 2019)

Im Jahr 1612 war der Anteil der Mieterinnen unter allen Miethaushalten mit 29\% deutlich geringer, wohingegen der Anteil der Mieterinnen bezogen auf alle Haushalte mit 11\% kaum Abweichungen aufweist zum Jahr 1762, der dann bei 13\% lag. Der Anteil und auch die absolute Zahl an weiblichen Miethaushalten ist also in diesen 150 Jahren deutlich stärker gestiegen als die Zahlen in der männlichen Vergleichsgruppe, die absolut betrachtet sogar kleiner geworden ist, denn der Anteil aller Miethaushalte (männlich und weiblich) sinkt in dieser Zeit von 37\% auf $30 \%$.

Eine eindeutige Sprache sprechen auch die von mir im Rahmen meiner Dissertation ausgewerteten Listen zur dörflichen Bevölkerung im Nürnberger Landgebiet. ${ }^{502}$ Den eigentumslosen Haushalten standen dort im 16. Jahrhundert zu über $20 \%$ Frauen vor. In der Barockzeit sank deren Anteil zwar, war aber mit fast 15\% immer noch nennenswert. Damit muss endgültig festgestellt werden, dass die Huldigungslisten zur Klärung dieses Sachverhaltes in der Regel nicht zu Rate gezogen werden können.

\section{Strukturen eigentumsloser Haushalte am Beispiel Altdorf 1717}

Einen selten differenzierten Einblick in die Familienstruktur der Miethaushalte enthält das Türkensteuerregister 1716/17 für die nürnbergische Stadt Altdorf, Sitz eines Pflegamtes und der Nürnberger Universität. Im Folgenden sollen die dort enthaltenen Angaben zur Familienstruktur der Mieter insgesamt besprochen werden, da diese einen außergewöhnlich detaillierten Einblick in die Zusammensetzung und soziale Struktur der eigentumslosen Haushalte ermöglichen. ${ }^{503}$ Vermutlich können die Altdorfer Angaben auf die Situation in anderen deutschen

\footnotetext{
501 Ebd., S. 134, Tab. 19.

502 WENDEROTH 2019, S. 72-74.

${ }^{503}$ Im genannten Türkensteuerverzeichnis (StAN, Rst. N, Bauernverzeichnisse 7) findet sich eine solch differenzierte Aufstellung nicht für die anderen drei Städte: Hersbruck, Lauf und Velden. Vergleichbar detailliert sind zum Beispiel die niedersächsischen Kopfsteuerverzeichnisse des späten 17. Jh., allerdings ist dort der Anteil der Miethaushalte deutlich geringer, vgl. BURCHARD/MUNDHENKE 1940-1971 und MEDEFIND 2000.
} 
Städten des 18. Jahrhunderts übertragen werden. In diesen Listen finden sich neben den grundlegenden Angaben zu den Haushaltsvorständen, ihrem Geschlecht und Beruf, auch ergänzende Angaben zu den Kindern und zum Gesinde. ${ }^{504}$ Die Kinder wiederum sind sowohl nach Geschlecht als auch nach ihrem Status unterschieden, ob sie noch mit im Haushalt oder schon außer Haus lebten. Das Gesinde lässt sich anhand der Angaben in drei Gruppen gliedern: Lehrjungen, Gesellen und als weibliches Pendant die Mägde.

Altdorf war mit 533 Haushalten zu diesem Zeitpunkt eine mittelgroße Landstadt. Als Mieter erfasst sind im Türkensteuerverzeichnis allein 225 Haushalte, denen nur wenig mehr Eigentümerhaushalte gegenüberstanden, nämlich 289. Die neunzehn Haushalte der öffentlich Bediensteten bleiben im Folgenden unberücksichtigt, sie werden überwiegend als Mieter in Dienstwohnungen gewohnt haben. Von den zuvor genannten Miethaushalten hatten wiederum 59\% einen männlichen und $41 \%$ einen weiblichen Haushaltsvorstand. Kinder wurden in diesem Kopfsteuerverzeichnis erst ab einem Alter von fünfzehn Jahren erfasst. ${ }^{505}$ In Altdorf sind dafür aber auch die Kinder gelistet worden, die nicht mehr bei ihren Eltern wohnten. Von den 225 Miethaushalten hatten 92 Haushalte Kinder über fünfzehn Jahren ( $=41 \%)$. Bei den männlichen Miethaushalten waren es 57 von 133 (= 43\%), bei den weiblichen Miethaushalten 35 von 92 (= 38\%) Haushalte. Es liegt also in diesem Punkt kein signifikanter Unterschied vor. Absolut erfasst sind 148 Kinder über fünfzehn Jahren, darunter aber auch verheiratete "Kinder" von 40 Jahren und älter. Die Ursache für die differenzierte Erfassung der Kinder liegt sicher auch darin begründet, zu ermitteln, ob Angehörige vorhanden waren, die finanziell Verantwortung übernehmen konnten.

\begin{tabular}{|l|c|c|c|c|c|c|c|c|}
\hline & $\begin{array}{c}\text { HH } \\
\text { gesamt }\end{array}$ & $\begin{array}{c}\text { davon HH } \\
\text { mit Kindern }\end{array}$ & 1 Sohn & 2 Söhne & 1 Tochter & Töchter & $\begin{array}{c}\text { 1 Sohn + } \\
\text { 1 Tochter }\end{array}$ & $\begin{array}{c}\text { 1 Sohn + } \\
\text { 2 Töchter }\end{array}$ \\
\hline männliche HH & 133 & 28 & 13 & 1 & 12 & 0 & 1 & 1 \\
\hline weibliche HH & $\mathbf{9 2}$ & 22 & 8 & 2 & 11 & 1 & 0 & 0 \\
\hline \hline Gesamt & 225 & $\mathbf{5 0}$ & 21 & $\mathbf{3}$ & $\mathbf{2 3}$ & $\mathbf{1}$ & $\mathbf{1}$ & $\mathbf{1}$ \\
\hline
\end{tabular}

Tabelle 5. Altdorf 1717, Miethaushalte, sortiert nach Geschlecht und Anzahl der Kinder, berücksichtigt sind hier nur die Kinder über 15 Jahre, die noch im elterlichen Haushalt mitleben (Thomas Wenderoth 2020)

Von diesen 148 Nachkommen lebten noch 57 Kinder bei Ihren Eltern (= 39\%), 81 Kinder waren bereits aus dem Elternhaus ausgezogen $(=61 \%)$. Der Prozentsatz der zu Hause gebliebenen Mädchen ist mit 35\% (28 von 80 Mädchen) etwas geringer als der der Jungen mit $43 \%$ (29 von 68 Jungen). Immerhin kommen die 92 Haushalte mit Kindern durchschnittlich auf 1,56 Kinder über fünfzehn Jahre. Bezogen auf alle 225 Miethaushalte sind es jedoch nur 0,65 Kinder. Ermittelt man

${ }^{504}$ StAN, Rst. N, Bauernverzeichnisse 7, fol. 327-332.

${ }^{505}$ WENDEROTH 2019a, S. $25 f$. 
die Zahlen im Hinblick auf die Haushaltsgrößen, sind nur die 57 Kinder relevant, die noch zuhause lebten. Hier ergibt sich ein Wert von 0,25 Kindern über fünfzehn Jahren pro Miethaushalt. Studiert man die einzelnen Einträge, wird deutlich, dass in der Regel maximal ein Kind bei den Eltern blieb. Lediglich in sechs Miethaushalten sind mehrere ältere Kinder belegt: So hat der Zimmermann Hanns Christoph Müller einen Sohn und eine Tochter bei sich, ${ }^{506}$ der Taglöhner Hanns Ziegler sogar einen Sohn und zwei Töchter, ${ }^{507}$ der Metzger Hans Kellermann zwei Söhne ${ }^{508}$ und die Witwe des Veit Gräbner ebenfalls zwei Söhne. ${ }^{509}$ Dass in den Mieterfamilien Kinder das Elternhaus schnell verlassen haben, führt zu der für diese Wohnsituation charakteristischen geringen Haushaltsgröße, die zumeist nur aus dem Elternpaar und - bei entsprechendem Alter der Eltern - aus jüngeren Kindern bestand.

Nachfolgend sollen noch einmal die Familien differenziert betrachtet werden, bei denen die Kinder von über fünfzehn Jahren noch mit im Haushalt lebten: Diese Situation traf insgesamt nur auf 50 der 225 Miethaushalte zu (22\%), wobei der Anteil bei den Frauenhaushalten mit 25\% etwas höher lag. Der Unterschied zu den Miethaushalten mit männlichem Vorstand (20\%) ist jedoch nicht signifikant. In 43 Familien und damit in den meisten Fällen (86\%) lebte nur ein Kind mit im Miethaushalt, auch hier ist kein Unterschied zwischen den Geschlechtern sowohl des Haushaltsvorstandes als auch der Kinder festzustellen. Von den 225 erfassten Haushalten hatte lediglich der Taglöhner Hanns Ziegler das Maximum von drei Kindern bei sich wohnen. ${ }^{510}$

Schwierig ist es, die Anzahl der Kinder unter fünfzehn Jahren zu ermitteln. Gerhard Rechter schlägt an anderer Stelle einen Wert von maximal 1,5 Kindern pro Haushalt vor. ${ }^{511}$ Für Altdorf würden sich daraus weitere 337 Kinder errechnen. Dieser Wert erscheint mir aber für die Miethaushalte zu hoch. Denn die 225 Miethaushalte in Altdorf hatten nach Ausweis des Verzeichnisses zusammengenommen nur 141 Kinder zwischen fünfzehn und etwa 45 Jahren. Rein rechnerisch ergeben sich bezogen auf eine Zeitspanne von fünfzehn Jahren lediglich 75 Kinder. Bedenkt man die erhöhte Mortalität im Kleinkindalter, so wäre maximal von 150 Kindern unter fünfzehn Jahren in den Miethaushalten Altdorfs auszugehen. Rechnerisch käme man so auf einen Wert von 0,67 Kindern unter fünfzehn Jahren pro Miethaushalt. Bei den Frauenhaushalten ergibt sich daraus eine durchschnittliche Haushaltsgröße von 1,94 Personen. ${ }^{512}$ Bei den mietenden

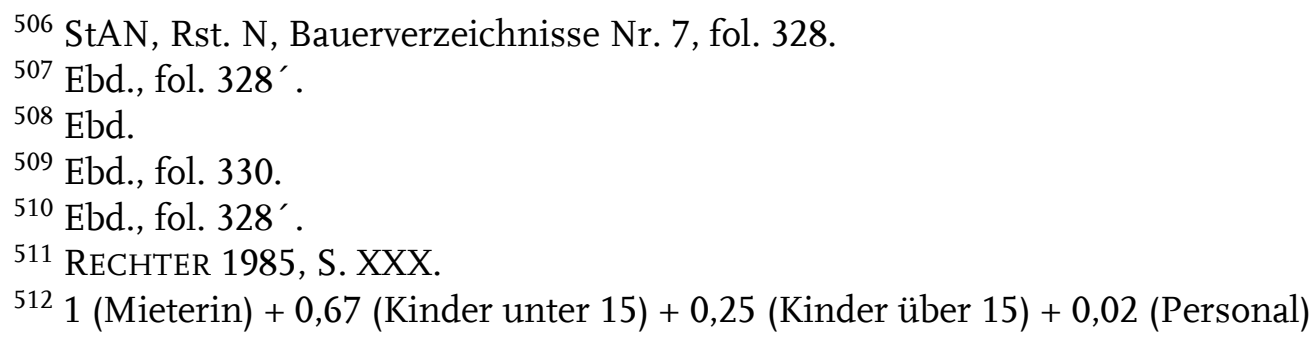


Männern ist die Bestimmung der Haushaltsgröße schwieriger, da nicht angegeben wird, ob diese verheiratet waren und ob die Ehefrau gegebenenfalls noch am Leben war. Rechnet man hier großzügig mit $90 \%$ Ehepaaren, ergibt sich eine durchschnittliche Haushaltsgröße von 2,92 Personen. ${ }^{513}$ Diese Werte dürften in anderen Städten kaum übertroffen worden sein, da sich unter den Altdorfer Miethaushalten eben viele Familien und nicht nur alleinstehende alte Menschen befunden haben. Auch die Berufsstruktur mit einer großen Zahl von Handwerkern zeugt eher von einer wirtschaftlich gefestigten Mieterschaft. ${ }^{514}$

\begin{tabular}{|l|c|c|c|c|c|c|c|c|c|c|}
\hline Altdorf 1717 & \multicolumn{4}{|c|}{ Mieter } & \multicolumn{2}{|c|}{ Anzahl Söhne } & \multicolumn{2}{|c|}{ Anzahl Töchter } & \multicolumn{2}{c|}{ Anzahl Gesinde } \\
\hline & $\begin{array}{c}\text { Miethaushalte, } \\
\text { gesamt }\end{array}$ & $\begin{array}{c}\text { HH ohne } \\
\text { Kinder/ } \\
\text { Gesinde }\end{array}$ & $\begin{array}{c}\text { HH mit } \\
\text { Kindern/ } \\
\text { Gesinde }\end{array}$ & $\begin{array}{c}\text { mit im } \\
\text { Haus }\end{array}$ & $\begin{array}{c}\text { außer } \\
\text { Haus }\end{array}$ & $\begin{array}{c}\text { mit im } \\
\text { Haus }\end{array}$ & $\begin{array}{c}\text { außer } \\
\text { Haus }\end{array}$ & Lehrling & Geselle & Magd \\
\hline männliche HH & $\mathbf{1 3 3}$ & 76 & 57 & 17 & 24 & 15 & 32 & 3 & 8 & 3 \\
\hline weibliche HH & $\mathbf{9 2}$ & 57 & 35 & 12 & 15 & 13 & 20 & 0 & 0 & 2 \\
\hline Gesamt & $\mathbf{2 2 5}$ & $\mathbf{1 3 3}$ & $\mathbf{9 2}$ & $\mathbf{2 9}$ & $\mathbf{3 9}$ & $\mathbf{2 8}$ & $\mathbf{5 2}$ & $\mathbf{3}$ & $\mathbf{8}$ & $\mathbf{5}$ \\
\hline
\end{tabular}

Tabelle 6. Altdorf 1717, Mieter und die Anzahl ihrer Kinder, berücksichtigt sind alle aufgeführten Kinder, auch erwachsene Kinder, die nicht mehr im Elternhaus leben (Thomas Wenderoth 2020)

Gesinde war in den unterschichtigen Miethaushalten in der Regel nicht anzutreffen. Von den insgesamt 225 Miethaushalten hatten nur fünfzehn Haushalte Personal $(=6,7 \%)$. Darunter finden sich sieben Haushalte, die zusätzlich erwachsene Kinder zuhause hatten, für die anderen acht Haushalte werden keine Kinder genannt. Absolut sind drei Lehrjungen, acht Gesellen und fünf Mägde erfasst, also sechzehn Bedienstete. Nur in dreizehn der 133 Miethaushalte mit männlichem Haushaltsvorstand fand sich Gesinde, unter diesen dreizehn Haushalten hatte lediglich der kinderlose Büttner Johann Georg Jakob zwei Hilfskräfte bei sich im Haushalt: einen Lehrling und einen Gesellen, dafür jedoch keine erwachsenen Kinder. ${ }^{515}$ Es scheint so, dass Lehrjungen oder Gesellen in der Regel nur dann bei den eigentumslosen Handwerkern eingestellt worden waren, wenn keine oder nicht ausreichend viele Söhne über fünfzehn Jahren im Haushalt lebten, um die anfallenden Arbeiten zu erledigen. Zwei der drei aufgeführten Mägde finden sich entsprechend gleichfalls in kinderlosen Haushalten, darunter auch die beiden Mägde in den Witwenhaushalten.

Die Größe der Miethaushalte in Altdorf verändert sich aufgrund der Bediensteten kaum: Auf jeden dieser Haushalte kamen rechnerisch im Durchschnitt nur 0,07 Bedienstete! Bei den Witwenhaushalten sind es sogar noch weniger, da hier

\footnotetext{
513 1,9 (Mieter mit Frau) + 0,67 (Kinder unter 15) + 0,25 (Kinder über 15) + 0,1 (14 Dienstleute bei 133 Haushalten)

${ }^{514}$ Die Mieter waren zumindest nicht überwiegend auf Almosen angewiesen. Armut verhinderte bekanntlich die Familiengründung. Eine ausführliche Analyse der Angaben in den Türkensteuerregistern auch zu Eigentümerfamilien und zu anderen Orten wäre wünschenswert, muss jedoch zukünftigen Forschungen überlassen bleiben.

515 StAN, Rst. N, Bauerverzeichnisse Nr. 7, fol. 328'.
} 
lediglich in zwei Fällen Dienstmägde genannt werden. ${ }^{516}$ Der Personalanteil beträgt hier durchschnittlich nur 0,02 Personen.

Zudem werden für keinen der 225 Miethaushalte Altenteiler oder entfernte Verwandte als Haushaltsmitglieder genannt. Bei der Erfassung der bereits ausgezogenen Kinder handelt es sich diesbezüglich ebenfalls um eine wichtige Information: Zum einen wird damit die Beobachtung bestätigt, dass in eigentumslosen Haushalten Kinder früh das Elternhaus verließen, und zum anderen, dass altgewordene Mieter entsprechend nicht als Altenteiler mit im Haushalt der Kinder wohnten. Wichtig ist zudem die Feststellung, dass es sich bei den erfassten alleinlebenden Mietern nicht grundsätzlich um kinderlose Einzelpersonen gehandelt hat, sondern dass diese in nicht wenigen Fällen ebenfalls Kinder hatten, die zum Zeitpunkt der Erfassung jedoch außer Haus lebten. Trotzdem ist die überwiegende Mehrheit der Mieter (gut 60\%) in dieser Aufstellung als kinderlos ausgewiesen.

\section{Einfluss der Stadtgröße auf den Anteil eigentumsloser Haushalte}

Der Anteil der Miethaushalte konnte je nach Stadt und Zeitschicht sehr unterschiedlich ausfallen, dennoch lassen sich gemeinsame Tendenzen und Entwicklungen feststellen. Um diese aufzuzeigen, habe ich die zu untersuchenden Städte in verschiedene Kategorien eingeordnet, die eine Unterscheidung in Stadttypen ermöglichen. Dieser Gliederung liegt erst einmal die These zugrunde, dass ein direkter Zusammenhang zwischen Stadtkategorie und der Höhe des Mieteranteils besteht. Um der historischen Entwicklung Rechnung zu tragen, werden zudem verschiedene Zeitschichten behandelt und dargestellt. Grob ist zu unterscheiden in die mittelalterliche Situation vor 1500, in die Zeit bis zum Dreißigjährigen Krieg und die anschließende Zeit des späten 17. und 18. Jahrhunderts mit der Konsolidierung der Territorialstaaten und der endgültigen Ausbildung von Haupt- und Residenzstädten. Am Ende des betrachteten Zeitabschnitts sind verstärkt Bemühungen greifbar, die jeweilige Bevölkerung statistisch präzise zu erfassen. Anhand der nun grundsätzlich besseren Quellenlage wird die Situation und der Anteil der Mietbevölkerung um 1800 dargestellt. Dies erscheint mir zudem wichtig, um die bevölkerungshistorische Ausgangslage für die Industrialisierung Deutschlands im 19. Jahrhundert zu fassen. Für die von mir untersuchten Städte in Franken lassen sich anhand der Überlieferung flächenhaft nur für drei der vier genannten Zeitabschnitte Aussagen treffen. Für das 16. Jahrhundert

${ }^{516}$ Ebd., fol. 330: Hanns Wolff Hübners Wittib, fol. 331: Leonhard Reuters Wittib. 
fehlen aussagekräftige Quellen, hier ist es nur möglich, für einzelne Städte Zahlen zu ermitteln. Für die Situation des ausgehenden Mittelalters kann auf das Reichssteuerregister von 1497, für die Barockzeit auf die Huldigungslisten des Fürstentums Ansbach und für das frühe 19. Jahrhundert auf das Alphabetische Verzeichnis für den Rezatkreis von 1818 zurückgegriffen werden.

Bei der Definition der Stadtkategorien folge ich verschiedenen Ordnungsprinzipien. Grundsätzlich scheint die Größe einer Stadt relevant zu sein für ihre Bevölkerungsstruktur, daher wird im Folgenden unterschieden in Großstadt, Mittelstadt und Kleinstadt. Bei der Gliederung nach Stadtgrößen kann der Zeithorizont mit einem grundsätzlichen Bevölkerungswachstum nicht unberücksichtigt bleiben. Dieser Situation wird Rechnung getragen, indem sich die Grenzwerte für die Einordnung als Großstadt verschieben: Für die Zeit vor 1500 können Städte ab 10.000 Einwohnern oder 2.500 Haushalten als Großstadt gelten. ${ }^{517}$ Anschließend, bis etwa 1700, wird dieser Grenzwert von mir auf 15.000 Einwohner oder 3.500 Haushalte angehoben. Für das 18. Jahrhundert erscheint ein Schwellenwert von 20.000 Einwohnern oder 5.000 Haushalten angemessen. ${ }^{518}$ Anders verhält es sich bei der Gruppe der mittelgroßen Städte, die hier als Mittelstädte bezeichnet werden. Sowohl der Übergang zur Kleinstadt als auch zur Großstadt bleibt in gewisser Weise fließend. Die Bedeutung einer Stadt misst sich nicht anhand der letzten Dezimale der Bevölkerungszahl. Als unterer Grenzwert wird eine Bevölkerungsgröße von 3.500 Einwohnern oder 800 Haushalten definiert. Ich orientiere mich dabei an der Preußischen Städteordnung des Freiherrn von Stein aus dem Jahr 1808. ${ }^{519}$ Man könnte hier aber auch einen zeitlich gestuften Korridor von 2.000 bis 5.000 Einwohner eröffnen. ${ }^{520}$ Der Wert von 3.500 Einwohnern wird von mir jedoch über den gesamten Untersuchungszeitraum beibehalten. Den Städten dieser Größe kam, unabhängig von ihrer wirtschaftlichen oder politischen Stellung, stets auch eine überregionale Bedeutung allein aufgrund ihrer Größe zu. Es er-

\footnotetext{
${ }^{517}$ Vgl. HiRSCHMANN 2016, S. 19-21. Dort sind eine Reihe von mittelalterlichen Großstädten mit Einwohnerzahlen angegeben.

${ }^{518}$ Hildegard Ditt legt den Zeitpunkt für den Sprung von 15.000 auf 20.000 Einwohner als Kriterium an eine Großstadt erst auf das Jahr 1720. Da viele deutsche Städte erst gegen Ende des 17. Jhs. wieder an die Einwohnerzahlen vor dem Dreißigjährigen Krieg anknüpfen können und anschließend darüber hinauswachsen, ist auch ein späterer Zeitpunkt nachvollziehbar. In den wenigsten Fällen wird dies jedoch aufgrund der geschilderten Situation zu einer abweichenden Zuordnung führen; DITT 1979, S. 121-124.

${ }^{519}$ Ebd.

${ }^{520}$ Heinz Schilling zieht die Grenze für eine Mittelstadt sehr viel niedriger, bei 2.000 Einwohnern. Zugleich listet er die frühneuzeitlichen Großstädte im Deutschen Reich auf, ebenfalls mit dem Schwellenwert von 10.000 Einwohnern; SCHILLING/EHRENPREIS 2015, S. 2-17, sowie Tabelle 1.5. Otto Brunner bezeichnet eine Stadt ab 2.000 Einwohnern als kleine Mittelstadt und ab 3.000 Einwohnern als große Mittelstadt; BRUNNER 1948, S. 42.
} 
scheint mir müßig zu diskutieren, ob der Schwellenwert bereits am Ende des Untersuchungszeitraumes auf 5.000 oder mehr Einwohner angehoben werden müsste. Für eine Diskussion der Mittelstadt im weiteren Verlauf des 19. Jahrhunderts wären diese Werte allemal zu niedrig gewählt.

Die Städte mit weniger als 3.500 Einwohnern werden in der Gruppe der Kleinstädte zusammengefasst. Schlaglichtartig wird zudem die Situation in Erlangen als Kleinst- oder Zwergstadt betrachtet, um feststellen zu können, ob Kleinststädte eine grundsätzlich abweichende Mieterstruktur aufweisen. Denn trotz der Zusammenfassung unter den Begriff der Kleinstadt bestehen doch erhebliche Unterschiede, ob solch eine Stadt 300 oder 3.000 Einwohner hatte. Zwischen den Extremwerten liegt ein Multiplikationsfaktor bis zum Zehnfachen der Einwohnerschaft. Die Streuung ist in den Gruppen der Groß- und Mittelstädte deutlich geringer: Bei den deutschen Großstädten setzten sich allein Wien und Berlin im 18. Jahrhundert deutlich von den anderen Großstädten hinsichtlich ihrer Einwohnerzahlen ab.

\section{Großstädte}

\section{Entwicklung des Mietwohnungsbaus vom späten Mittelalter bis zum Dreißigjährigen Krieg}

Die großen Städte des Mittelalters im Heiligen Römischen Reich Deutscher Nation waren u. a. Köln, Prag, Nürnberg, Augsburg und Frankfurt sowie die Hansestädte Lübeck, Danzig, Hamburg und Bremen. Aufgrund ihrer für die damalige Zeit großen Bevölkerungszahlen war auch der zahlenmäßige Anteil der armen und damit häufig auch eigentumslosen Bevölkerung entsprechend hoch. Durch die Attraktivität der Zentren gab es in diesen Städten aber ebenfalls eine nennenswerte Anzahl von Haushalten aus der Mittel- und Oberschicht, die aus verschiedenen Motiven heraus mobil sein wollten und (erst einmal) nicht über Bürgerrecht und Hausbesitz verfügten. Die verwaltungsmäßig gut organisierten und weitgehend unabhängigen Städte haben unterschiedliche Lösungen der Wohnungsfürsorge gefunden. Für die Bedeutung einer Stadt war nicht nur die Höhe der Einwohnerzahlen relevant; hohe Mieteranteile unter der Bevölkerung waren und sind noch immer charakteristisch für große Städte, fanden sich aber aus verschiedenen Gründen auch bei manch kleiner Stadt, wie weiter unten noch zu zeigen sein wird.

Nürnberg ist bis heute die Metropole Frankens geblieben und kann seit dem Mittelalter durchwegs als Großstadt angesehen werden. Sie steht daher im Zentrum meiner Betrachtungen. Aufgrund einer komplett unterschiedlichen Quellenlange zu den anderen untersuchten fränkischen Städten ist ein direkter Vergleich mit den kleineren fränkischen Städten schwierig. 


\section{Nürnberg}

\section{Einleitung zur Stadtgenese}

Nürnberg entstand aus mehreren Siedlungskernen, auf die hier nicht näher eingegangen werden soll. Wichtig ist, dass die Stadt sich ab dem 12. Jahrhundert beidseitig des Flusses Pegnitz entwickelt, der die Stadt in zwei etwa gleich große Hälften teilt. Die Stadtseiten sind nach den jeweiligen Hauptkirchen St. Sebald und St. Lorenz benannt. St. Sebald liegt nördlich und St. Lorenz südlich der Pegnitz. Bis ins 15. Jahrhundert überwog die Sebalder Seite gegenüber der Lorenzer Seite hinsichtlich Bebauungsdichte und Bevölkerungszahlen. Hier war auch das wirtschaftliche Zentrum der Stadt lokalisiert, was sich mit der Anlage des Hauptmarktes nach 1349 niederschlug. Die ältere Befestigungsanlage bestand bis Anfang des 14. Jahrhunderts aus zwei getrennten Mauerringen für die beiden Stadthälften. ${ }^{521}$ Erst um 1325 wurden die Befestigungsanlagen vereinigt. Bald darauf begann man einen neuen Befestigungsring zu errichten und damit das Stadtgebiet im Osten, Süden und Westen deutlich zu erweitern. Zumindest die hohe Stadtmauer mit den Türmen dürfte Ende des 14. Jahrhunderts fertig gestellt gewesen sein. Der Verlauf der älteren Stadtmauer lässt sich in der Straßenführung noch gut nachvollziehen. Die Stadt nutzte den frei gewordenen städtischen Baugrund im Bereich der älteren Befestigung, um im 15. Jahrhundert die notwendigen öffentlichen Gebäude zu errichten. Insbesondere westlich der St. Lorenzkirche finden sich diese großen Baukörper wie Perlen an einer Schnur aufgereiht auf dem Areal der älteren Befestigungsanlage. Im Osten hinter der Egidienkirche diente die Fläche ab 1489 zum Bau von 21 städtischen Mietshäusern zur Ansiedlung von Barchentwebern.

Die Stadt war in Viertel aufgeteilt, auch hier spiegelt sich das Wachstum der Stadt im 14. und 15. Jahrhundert wider: Bis Ende des 14. Jahrhunderts waren es lediglich fünf Viertel. Von den älteren Vierteln liegen vier auf der Sebalder Seite: Milchmarkt, Weinmarkt, Salzmarkt und Egidien, während die Lorenzer Seite ursprünglich ungeteilt war und spätestens 1408 in zwei Viertel geteilt war: Kornmarkt und Barfüßer. Mit dem weiteren Stadtwachstum im 15. Jahrhundert werden dann auch auf der Lorenzer Seite vier Viertel gebildet, durch Teilung entstehen das Elisabeth- und das Kartäuserviertel. ${ }^{522}$ Zudem wurde seit dem Bau der letzten Stadtmauer im 14. Jahrhundert eine Unterscheidung der Viertel in die Gebiete innerhalb der älteren Befestigung (intra muros) und die Gebiete zwischen alter und neuer Stadtmauer (extra muros) vorgenommen. Lediglich beim Milchmarktviertel

\footnotetext{
${ }^{521}$ Vgl. hierzu und im Folgenden WEINGÄRTNER 2018, S. 25.

522 Puchner bezeichnet den nicht abgetrennten Teil des Kornmarktviertels als Frauenbrüderviertel; PUCHNER 1975.
} 
unterblieb eine solche Differenzierung, da dieses Viertel kaum Erweiterungsflächen aufwies. Der Bereich zwischen den beiden Stadtmauern wird auch gelegentlich als Vorstadt bezeichnet, obwohl er seit 1400 innerhalb der Befestigung lag. Hier klingt noch an, dass das Gebiet in Teilen zuvor schon bebaut war. Vor der letzten Stadtmauer entwickeln sich dann erneut vorstädtische Siedlungsstrukturen, nur bei diesen kann im heutigen Sinn von Vorstädten gesprochen werden. ${ }^{523}$ Die Viertel wiederum gliederten sich in Gassenhauptmannschaften, womit übersichtliche Einheiten vorhanden waren. Die Gliederung der Stadt in Viertel und Gassenhauptmannschaften diente der Lokalisierung der einzelnen Bewohner; mit der Zugehörigkeit zu einer Gassenhauptmannschaft und einem Viertel waren bürgerliche Pflichten verbunden, wie die Verteidigung eines definierten Stadtmauerabschnittes,

\section{Die eigentumslose Bevölkerung in Nürnberg bis ins späte 15. Jahrhundert}

Für die mittelalterliche Zeit kommt Caspar Ott das Verdienst zu, die Bevölkerung Nürnbergs innerhalb der Stadtmauer als erster wissenschaftlich, unter Zuhilfenahme verschiedener Quellen, analysiert zu haben. Für diese Zeit liegen zum Anteil der eigentumslosen Haushalte nur indirekt Angaben vor.

Grundsätzlich kann für die erste Hälfte des 15. Jahrhunderts für Nürnberg anhand der Dresdner Zahlen von einem Mieteranteil von mindestens 30\% ausgegangen werden, $40 \%$ erscheinen jedoch als unterer Wert realistischer. Da der Mieteranteil im Mittelalter in allen großen Städten mit dem Anwachsen der Bevölkerung ebenfalls zunimmt, scheint ein Anteil im späten 15. Jahrhundert von $50 \%$ und in der ersten Hälfte des 16 . Jahrhunderts von $60 \%$ für Nürnberg keinesfalls zu hoch angesetzt, belegen lassen sich diese höheren Zahlen bisher allerdings nicht, wie weiter unten ausgeführt wird.

Caspar Ott verbindet die Daten des Grabenbuches aus dem Jahr 1430 mit der Erfassung der wehrfähigen Bevölkerung zwischen achtzehn und 60 Jahren aus dem Jahr 1431. ${ }^{524}$ Aus den Angaben des Grabenbuches ergeben sich 15.499 weltliche Personen über zwölf Jahre, als waffenfähig werden 7.146 Männer geführt. Ott errechnet dazu noch die Zahl der Kinder, der Juden und der Geistlichen und kommt dann für das Jahr 1431 auf insgesamt 22.797 Einwohner. ${ }^{525}$

Das Grabenbuch aus dem Jahr 1430 nennt die Zahl der Bewohner pro Zähleinheit. ${ }^{526}$ Während Ott davon ausgeht, dass mit der Zähleinheit die Einzelhaushalte unter ihrem Haushaltsvorstand erfasst sind, werde ich die Einheiten neutral als

\footnotetext{
${ }^{523}$ Vgl. Kapitel 7, Vorstädte.

524 OTT 1907.

525 Vgl. hierzu ebd., S. 44.

${ }^{526}$ Vgl. hierzu und im Folgenden OTT, S. 30f.
} 
Herdgemeinschaften definieren. In römischen Ziffern steht im Grabenbuch hinter dem Namen des jeweiligen Vorstandes die Zahl der weiteren Angehörigen über zwölf Jahre, erfasst sind beide Geschlechter. In der Zusammenfassung sieht dies wie folgt aus: ${ }^{527}$

\begin{tabular}{|l|c|c|c|c|c|c|c|c|c|c|c|c|}
\hline Personen pro Einheit & $\mathbf{1}$ & $\mathbf{2}$ & $\mathbf{3}$ & $\mathbf{4}$ & $\mathbf{5}$ & $\mathbf{6}$ & $\mathbf{7}$ & $\mathbf{8}$ & $\mathbf{9}$ & $\mathbf{1 0}$ & $\mathbf{5 1 5}$ & $\mathbf{5} 22$ \\
\hline Anzahl der Einheiten & $\mathbf{7 9}$ & $\mathbf{6 5 6}$ & $\mathbf{7 1 7}$ & $\mathbf{7 2 8}$ & $\mathbf{5 4 4}$ & $\mathbf{3 5 5}$ & 184 & 134 & $\mathbf{6 2}$ & $\mathbf{4 6}$ & $\mathbf{4 9}$ & 11 \\
\hline Anteil an allen Einheiten & $\mathbf{2 \%}$ & $\mathbf{1 8 \%}$ & $\mathbf{2 0 \%}$ & $\mathbf{2 0 \%}$ & $\mathbf{1 5 \%}$ & $\mathbf{1 0 \%}$ & $\mathbf{5 \%}$ & $\mathbf{4 \%}$ & $\mathbf{2 \%}$ & $\mathbf{1 \%}$ & $\mathbf{1 \%}$ & $\mathbf{0 , 3} \%$ \\
\hline
\end{tabular}

Tabelle 7. Nürnberg, Anzahl der im Grabenbuch 1430 angegebenen Bewohner pro Einheit (Thomas Wenderoth 2019 nach OTT 1907)

Demnach handelte es sich zum Großteil (87\%) um kleine Herdgemeinschaften mit lediglich 1-6 Bewohnern über zwölf Jahren. Insbesondere die Einheiten mit 1-3 Erwachsenen in der Herdgemeinschaft (42\%) können wohl zu einem größeren Teil als Kleinfamilien charakterisiert werden, die in Einzelfällen durch Untermieter ergänzt wurden. Für die Einheiten mit 4-6 Erwachsenen (45\%) dürfte die Wohnform als Kleinfamilie dagegen nur noch zum Teil gelten. Für 12\% der Einheiten lässt sich eine Zahl von 7-10 Bewohnern über zwölf Jahren nachweisen. Nur in Einzelfällen, nämlich in 58 Einheiten, wohnten zwischen 11 und 22 Personen. Große Herdgemeinschaften waren - wenn überhaupt - nur in Einzelfällen vertreten, denn zur Gruppe der 58 stark bevölkerten Einheiten gehören sicher auch große patrizische Anwesen mit einer entsprechenden Anzahl von Knechten und Mägden und eben der Spitalhof mit zwanzig über zwölfjährigen Personen.

Ott errechnet pro Einheit einen hohen Durchschnittswert von 4,34 Personen über zwölf Jahre beziehungsweise mit Kindern von 5,41 Personen. Für diesen im Vergleich ungewöhnlich hohen Wert führt Ott die wirtschaftliche Stärke der Stadt in dieser Zeit an, die sich in einer sehr hohen Zahl an Knechten (Gesellen) anhand der waffenfähigen Männer belegen lässt. ${ }^{528}$ Die hohe Anzahl der waffenfähigen Männer ist unbestritten von ihm nachgewiesen. Nur wissen wir nicht, ob es sich dabei um abhängige Gesellen und Mitarbeiter im Haushalt der Meister oder eben um Gesellen gehandelt hat, die zur (Unter-)Miete wohnten und andernorts einer Arbeit nachgingen.

Als direkter Vergleich zu den Angaben des Grabenbuches eignet sich die weiter unten aufgeführte Zählung aus Regensburg aus dem Jahr 1436 (vgl. Tab. 12). Dort sind ebenfalls alle erwachsenen Bewohner ab dem 14. Lebensjahr erfasst und den Häusern zugeordnet. Die durchschnittlich 4,46 erwachsenen Bewohner pro Haus in Regensburg liegen nah an den 4,34 Über-Zwölfjährigen in Nürnberg, die von Ott als Angehörige einer Familie aufgefasst werden. Auch die Verteilung der Personen auf die Häuser ist sehr ähnlich: In Regensburg wurden $42 \%$ der Häuser

527 Ebd. S. 82.

${ }^{528}$ Ebd., S. 56f. 
von 1-3 Erwachsenen, 36\% von 4-6 Erwachsenen, 16\% von 7-9 Erwachsenen und 6\% von 11-28 Erwachsenen bewohnt. Während der Anteil der von 1-3 Erwachsenen bewohnten Häusern mit $42 \%$ identisch ist, lassen sich in Regensburg weniger mittelgroße Häuser für 4-6 Erwachsene, dafür aber mehr große Häuser nachweisen. Für Regensburg ist darüber hinaus ein Anteil der eigentumslosen Haushalte von über $77 \%$ nachgewiesen, allein $45 \%$ der Häuser waren komplett vermietet und nicht vom Eigentümer bewohnt, der hohe Mieteranteil ergibt sich aber vor allem aus der hohen Zahl von über 1.752 alleinstehenden Untermietern.

Die Regensburger Zahlen machen deutlich, dass für Nürnberg die Ottsche Definition der Zähleinheit als ein Haushalt nicht zutrifft und dem offeneren Begriff der Herdgemeinschaft der Vorzug zu geben ist, denn es muss in jedem Fall mit einem nennenswerten Anteil von (alleinstehenden) Untermietern innerhalb dieser Einheiten gerechnet werden, die ihr Auskommen außerhalb der Kernfamilie fanden und auch verwandtschaftlich nicht mit dieser verbunden waren. Ob diese Herdgemeinschaften jeweils ein Haus für sich allein bewohnt haben oder sich in anderen Fällen auf mehrere Gebäude verteilten, muss offenbleiben.

Während das Grabenbuch von 1430 nur Informationen zur Größe der Herdgemeinschaften und damit der Gesamteinwohnerzahl enthält, ist in der Auflistung zu den waffenfähigen Männern aus dem Jahr 1431 durch Klammern gekennzeichnet, wenn mehrere Haushaltsvorstände in einer Wohneinheit zusammenlebten. ${ }^{529}$ Aus der Quelle selbst ist nicht ersichtlich, ob es sich bei diesen angedeuteten Einheiten um eine abgeschlossene Wohnung, ein Hausteil, ein Haus oder gar ein komplettes Anwesen handelt. Aber allein die Information zum Zusammenleben mehrere Haushaltsvorstände in einer Einheit ist wichtig im Hinblick auf die Frage nach eigentumslosen Haushalten, denn als Eigentümer kommt lediglich ein Haushaltsvorstand innerhalb dieser Herdgemeinschaften in Frage. Aus der Differenz zwischen der Anzahl der Wohneinheiten und der Anzahl der Haushaltsvorstände solcher Herdgemeinschaften errechnet sich automatisch auch ein Mindestanteil an eigentumslosen Herdgemeinschaften. Einschränkend muss angemerkt werden, dass sich in den Listen zu zwei der damals insgesamt sechs Viertel („Am Salzmarkt“ und „Bei den Barfüßern“) Hinweise auf von mehreren Herdgemeinschaften gemeinsam genutzte Häuser nicht finden. Ott gewinnt für diese beiden Stadtviertel die Zahlen aus Vergleichen und kommt für die Gesamtstadt auf insgesamt 3.569 Häuser, die Anzahl der Herdgemeinschaften gibt er mit 4.142 an. ${ }^{530}$ Durchschnittlich errechnen sich somit 1,16 Herdgemeinschaften pro Haus. ${ }^{531}$ Diese Zahl stellt nur einen Näherungswert dar, da Ott

${ }^{529}$ Ebd., S. 30.

${ }^{530}$ Ebd., S. 55 und S. 66. Hinzu kamen noch 41 Priesterhaushalte und etwa 30 jüdische Haushalte in eigenen Häusern, woraus sich eine Gesamtzahl von 4.213 Haushalten errechnet.

${ }^{531}$ Ebd., S. 76. 
verschiedentlich Angaben ergänzen musste. Die sich daraus ergebende Unschärfe ändert jedoch nichts an der grundsätzlichen Aussage.

Allerdings zeichnet sich eine klare Unterscheidung innerhalb der sechs Stadtviertel ab. Die drei östlichen Stadtviertel kommen auf etwa eineinviertel Herdgemeinschaften pro Einheit: Egidienhof $(1,29)$, Salzmarkt $(1,24)$ und auf der Lorenzer Seite Barfüßer $(1,25)$. Die drei westlichen Stadtviertel weisen dagegen fast nur „Einzeleinheiten“ auf: Weinmarkt $(1,082)$, Milchmarkt $(1,00)$ und auf der Lorenzer Seite Kornmarkt $(1,06)$. Caspar Ott hat diese Angaben für drei Stadtviertel detailliert ausgewertet, dabei übernimmt er auch die Untergliederung der Stadtviertel in das ältere Kerngebiet (intra muros) und das Gebiet der spätmittelalterlichen Stadterweiterung (extra muros). ${ }^{532}$

\begin{tabular}{|l|r|r|r|r|r|r|}
\hline Nürnberg, Viertel 1431 & leer & 1 HG & 2 HG & 3 HG & 4 HG & 5 HG \\
\hline Weinmarkt, intra muros & - & 381 & 3 & - & 1 & \\
\hline Weinmarkt, extra muros & - & 44 & 1 & - & - & - \\
\hline Egidienhof, intra muros & 1 & 279 & 32 & 11 & 2 & 1 \\
\hline Egidienhof, extra muros & - & 124 & 44 & 14 & 5 & 1 \\
\hline Kornmarkt, intra muros & - & 416 & 20 & 6 & 1 & \\
\hline Kornmarkt, extra muros & - & 453 & 16 & 2 & 1 & $\mathbf{1}$ \\
\hline \hline Summe & $\mathbf{1}$ & $\mathbf{1 . 6 9 7}$ & $\mathbf{1 1 6}$ & $\mathbf{3 3}$ & $\mathbf{1 0}$ & $\mathbf{( 0 , 5 \% )}$ \\
& $\mathbf{( 0 , 0 5 \% )}$ & $\mathbf{( 9 1 , 3 4 \% )}$ & $\mathbf{( 6 , 2 4 \% )}$ & $\mathbf{( 1 , 7 8 \% )}$ & $\mathbf{( 0 , 5 4 \% )}$ & $\mathbf{( 0 , 0 5 \% )}$ \\
\hline
\end{tabular}

Tabelle 8. Nürnberg, Anzahl der Herdgemeinschaften (HG) pro Wohneinheit im Jahr 1431 in drei von sechs Stadtteilen (Thomas Wenderoth 2019 nach OTT 1907)

Anhand seiner Interpretation erhält er 3.569 „Häuser“ (darunter 76 Wohnungen in der Stadtbefestigung) zuzüglich der Häuser der Juden, Priester und Klöster. Diese Zahl erscheint sehr hoch: Er selbst gibt an, dass im Jahr 1796 in Nürnberg nur 3.292 Häuser gezählt worden sind, ${ }^{533}$ allerdings erhielten die Wohnungen in der Stadtbefestigung und andere kommunale Gebäude Ende des 18. Jahrhunderts keine Hausnummern. ${ }^{534}$

Ott begründet den Rückgang der Häuserzahlen mit dem wirtschaftlichen Niedergang der Stadt im 18. Jahrhundert, lässt dabei jedoch drei wichtige Punkte außer Acht: Erstens waren die Einwohnerzahlen - absolut betrachtet - mit denen von 1430/31 vergleichbar. Zweitens sank die durchschnittliche Personenzahl zwischen 1430 von 5,41 Personen pro Zähleinheit auf 3,83 Personen im Jahr 1806, beziehungsweise stieg die Anzahl der Zähleinheiten stark an von 4.213 auf 6.717, wonach auch die Zahl der Wohnungen gestiegen sein müsste. Drittens, und das erscheint mir ausschlaggebend, befand sich die Stadt im frühen 15. Jahrhundert

\footnotetext{
532 Ebd., S. 77-79.

533 Ebd., S. 82.

${ }^{534}$ Vgl. hierzu Uraufnahme, URL: http://geoportal.bayern.de/bayernatlas-klassik.
} 
noch im Ausbau. Insbesondere die mit der Stadterweiterung neu geschaffenen Viertel extra muros dürften bei weitem noch nicht vollständig bebaut gewesen sein. In Summe können die 3.569 Einträge nicht direkt verglichen werden mit der Vergabe der 3.292 Hausnummern im Jahr 1796, die ja der Identifizierung von Anwesen dienten. Besonders deutlich wird das Problem, wenn man nur die Zahlen für die Sebalder Seite miteinander vergleicht. Für das dortige Weinmarktviertel ermittelt Ott 430 „Häuser“ im Jahr 1430, 1796 werden jedoch nur 304 Hausnummern vergeben. ${ }^{535}$ Beim Milchmarktviertel sind es $306 \mathrm{zu} 244 .{ }^{536}$ Als Interpretation bieten sich verschiedene Möglichkeiten an, drei davon seien genannt: Erstens, die Listenerfasser haben 1431 nicht sämtliche Haushaltsvorstände, die in einem Anwesen zusammenwohnten, mit Klammern markiert. Zweitens, die mit einer Klammer verbundenen Haushaltsvorstände lebten zusammen in einer Wohnung; Haushaltsvorstände die mit ihren Herdgemeinschaften zusammen in einem Haus, aber in getrennten Wohnungen lebten, sind demgegenüber nicht gesondert markiert und unterschieden von den Herdgemeinschaften, die allein in einem Haus lebten. Drittens, es wurden korrekt sämtliche Häuser erfasst, aber nicht unterschieden in Haupthäuser und Nebenhäuser, sodass ein Anwesen mit mehreren Wohngebäuden mehrfach in der Liste auftaucht, während im Jahr 1796 lediglich eine Hausnummer vergeben wurde.

Grundsätzlich können Wohnungen, Häuser oder Anwesen, die von mehreren Parteien bewohnt waren, als ein eindeutiger Hinweis auf eine Mietsituation gedeutet werden, da es bisher in Nürnberg keine Indizien im größeren Umfang für geteiltes Eigentum gibt. Nicht ermittelbar sind auf diese Weise aber Mieter, die ohne Vermieter in einem Haus oder Nebenhaus wohnten. Bereits Dirlmeier weist darauf hin, dass die Anzahl der nur von einem Haushalt bewohnten Häuser nichts über die Eigentumsverhältnisse an denselben aussagt, vielmehr ist davon auszugehen, dass auch diese häufig von Mietern bewohnt wurden. ${ }^{537}$

Festzuhalten ist, dass der Mieteranteil, der sich aus den mit Klammern zusammengefassten Herdgemeinschaften errechnen lässt, äußerst gering ist. Auf diese Weise erhält man nur etwa 5\% eigentumslose Herdgemeinschaften. Dieser Anteil liegt bereits am unteren Rand des für andere Städte und Zeiten überlieferten Anteils an Untermietern. ${ }^{538}$

Erfolgversprechender erscheint es daher, die Herdgemeinschaften des Jahres 1430 auf die weiter oben für das Jahr 1796 angegebenen Hausnummern zu beziehen. Dies gilt insbesondere für das Milchmarkt- und Weinmarktviertel, die beide schon im frühen 15. Jahrhundert dicht bebaut gewesen sein dürften. Für

535 OTT 1907, S. 76 und Quartiergeldbeiträge 1798, S. 1-6.

536 OTT 1907, S. 76 und Quartiergeldbeiträge 1798, S. 6-11.

537 DIRLMEIER 1978, S. 241f.

${ }^{538}$ Vgl. hierzu die nachfolgend geschilderte Situation in Hamburg und Erfurt. 
das Weinmarktviertel errechnen sich auf dieser Grundlage 126 überzählige Häuser/Einheiten (430 zu 304) und für das Milchmarktviertel 62 (306 zu 244). ${ }^{539}$ Setzt man wiederum diese überzähligen Häuser/Einheiten mit eigentumslosen Herdgemeinschaften gleich, ergibt sich für das Weinmarktviertel ein Anteil eigentumsloser Herdgemeinschaften von 29\% und für das Milchmarktviertel von $20 \%$. Auch in diesem Fall wäre dem Anteil eigentumsloser Haushalte noch ein nicht bestimmbarer Anteil komplett vermieteter Anwesen zuzuschlagen. So deuten einzelne Archivale darauf hin, dass die Nürnberger Patrizier im Mittelalter über eine Vielzahl vermieteter Häuser verfügten. ${ }^{540}$ Allein Margarete Behaim zählt im Jahr 1512 über zwölf Häuser zu ihrem Eigentum. ${ }^{541}$ Der Anteil der eigentumslosen Haushalte dürfte somit noch einmal deutlich über dem Anteil der überzähligen Häuser/Herdgemeinschaften gelegen haben. Hinzu kommen noch die zusätzlichen Haushalte in den Herdgemeinschaften, die in den Listen der waffenfähigen Männer mit dem Zusatz bei ihm/bei ihr zumindest teilweise miterfasst worden sind. Hier dürfte es sich uneingeschränkt um eigentumslose Haushalte gehandelt haben, die wohl überwiegend in Untermiete lebten. Eine statistische Auswertung dieser Einträge ist, wie gesagt, bisher noch nicht erfolgt.

Auffallend ist eine soziale Differenzierung zwischen der Kernstadt und dem Bereich der letzten Stadterweiterung, die sich anhand der topografischen Verteilung der insgesamt 7.146 Waffenfähigen im Jahr 1431 nachvollziehen lässt. ${ }^{542}$ Besonders differenziert wurden die Zahlen für das Barfüßerviertel um St. Lorenz erhoben. Anhand dieser Zahlen sollen die Unterschiede verdeutlicht werden: Während in dem älteren Stadtkerngebiet des Viertels (intra muros) 44,52\% der Haushalte einen waffenfähigen Knecht hatten, waren es in dem jünger besiedelten Stadtrandgebiet (extra muros) nur 15,38\%, ${ }^{543}$ ein Anzeichen dafür, dass hier die Haushalte im Wesentlichen keine Bediensteten hatten. Zum Sozialgefüge der Familien ist es auch wichtig festzustellen, dass im gesamten Barfüßerviertel nur in 4,17\% der Haushalte waffenfähige Söhne erfasst wurden. Diese Zahl belegt einmal mehr, dass die meisten Haushalte nicht aus mehreren Generationen bestanden und die Söhne - vor allem der ärmeren Bevölkerungsschichten - bereits früh den elterlichen Haushalt verließen. ${ }^{544}$ Anhand der von Ott ermittelten Zahlen der waffenfähigen Männer ${ }^{545}$ lässt sich für das Barfüßerviertel zudem noch nachweisen, dass neben den Söhnen und Knechten, die zu einem Kernhaushalt gehörten,

\footnotetext{
${ }^{539}$ Die mit Klammern gekennzeichneten Haushalte sind in diesen Vierteln äußerst gering und können daher vernachlässigt werden.

${ }^{540}$ WENDEROTH 2021, S. 227-229.

${ }^{541}$ KAMANN 1886, S. 119-121.

542 OTT 1907, S. 66.

543 Ebd., S. 67.

${ }^{544}$ Ebd., 70.

${ }^{545}$ Ebd., 63.
} 
weitere männliche Mitbewohner in den Herdgemeinschaften lebten. Bei diesen Mitbewohnern handelt es sich weder um Familienmitglieder (Söhne) noch um Mitglieder eines Haushaltes als Wirtschaftseinheit (Knechte).

\begin{tabular}{|l|c|c|c|}
\hline Nürnberg, Barfüßerviertel 1431 & intra muros & extra muros & gesamt \\
\hline Herdgemeinschaften gesamt & 411 & 572 & 983 \\
\hline davon mit männlichem Haushaltsvorstand (88\%) & 362 & 503 & 865 \\
\hline $\begin{array}{c}\text { davon waffenfähige männliche } \\
\text { Haushaltsvorstände unter 60 Jahren (95\%) }\end{array}$ & 344 & 488 & 832 \\
\hline waffenfähige Söhne & 34 & 13 & 47 \\
\hline waffenfähige Knechte & 319 & 164 & 483 \\
\hline $\begin{array}{l}\text { waffenfähige Mitbewohner in den } \\
\text { Herdgemeinschaften = männliche Mieter }\end{array}$ & 76 & 87 & 163 \\
\hline \hline Waffenfähige insgesamt, 18-60 Jahre & 773 & 752 & 1.525 \\
\hline
\end{tabular}

Tabelle 9. Nürnberg, männliche Mitglieder einer Herdegemeinschaft im Barfüßerviertel 1431 (Thomas Wenderoth 2019)

Die Anzahl der Herdgemeinschaften im Barfüßerviertel konnte Ott sehr genau ermitteln. ${ }^{546}$ Nicht differenziert wurde in den Quellen hingegen zwischen Herdgemeinschaften, denen ein Mann oder eine Frau vorstand. Hier muss hilfsweise auf die Angaben aus den vier Stadtvierteln der Sebalder Seite zurückgegriffen werden: Durchschnittlich fanden sich dort $12 \%$ weiblich geführte Herdgemeinschaften, diese sind in Abzug zu bringen. ${ }^{547}$ Zudem wurde von mir geschätzt, dass $5 \%$ der männlichen Haushaltungsvorstände als nicht waffenfähig eingestuft wurden, sei es aufgrund von Alter oder Krankheit. Bei der so ermittelten Zahl der waffenfähigen männlichen Haushaltsvorstände ergibt sich also eine gewisse Ungenauigkeit. Die waffenfähigen Söhne und Knechte sind dagegen exakt bestimmt. ${ }^{548}$ Zieht man von der Gesamtzahl der Waffenfähigen die männlichen Haushaltsvorstände, die Söhne und Knechte ab, erhält man eine Zahl von waffenfähigen Männern, die in den Herdgemeinschaften mitlebten, aber weder im ökonomischen noch im verwandtschaftlichen Sinn zum Haushalt dazugehörten, sondern eigene Haushalte bildeten. In dem Gebiet intra muros waren es 76, extra muros 87 Personen.

\footnotetext{
546 Ebd., 66f.

547 Ebd., S. 70-72. Die Werte in den einzelnen Vierteln schwanken stark: Weinmarkt 21\%, Milchmarkt 4\%, Egidienhof 14\%, Kornmarkt 10\%, daraus errechnet sich ein Durchschnittswert von $12 \%$.

${ }^{548}$ Ebd., S. 66-68.
} 
Zusammenfassend für das Barfüßerviertel ergibt sich daraus folgende Situation: ${ }^{549}$ In den von Ott ermittelten 788 Wohneinheiten lebten 983 Herdgemeinschaften mit weiteren 163 Männern, die nicht zu den Kernhaushalten dazugehörten und als (Unter-)Mieter charakterisiert werden können. Allein hieraus ergibt sich ein Verhältnis von 1.146 Haushaltsvorständen zu 788 Wohneinheiten, mithin ein Überhang von absolut 358 Haushaltsvorständen, was einem Anteil von $31 \%$ entspricht. Eine entsprechend hohe Zahl von Haushalten alleinstehender Frauen, die ebenfalls nicht zur Kernfamilie gehörten, kann vorausgesetzt werden.

Zudem wird anhand dieser Zahlen deutlich, dass die von Ott ermittelte durchschnittliche Haushaltsgröße von 4,34 Personen über zwölf Jahren weder mit der Größe einer Kernfamilie noch mit der Größe der um Gesinde erweiterten Familie identisch ist. Bringt man allein die 163 männlichen „Untermieter“ in Abzug, reduziert sich die durchschnittliche Größe der um Gesinde erweiterten Familie auf 4,17 Personen über zwölf Jahren. Geht man davon aus, dass ein Teil der „Untermieter" verheiratet war und auch alleinstehende fremde Frauen mit im Haushalt lebten, lag die durchschnittliche Haushaltsgröße (Familie mit Gesinde) bei unter vier Personen.

Die geschilderte Wohnsituation änderte sich auch in den folgenden Jahrzehnten nicht, wenn man die Zahlen für das Jahr 1478 heranzieht: Es sind nunmehr 4.348 Herdstellen, sprich Herdgemeinschaften, überliefert. ${ }^{550}$ Der Zuwachs beträgt lediglich 222 Herdstellen oder 5,4\%. Da die Bebauung in den ehemaligen Vorstädten in diesen 50 Jahren gleichzeitig verdichtet wurde und einige (Neben-)Häuser hinzugekommen sein dürften, kann davon ausgegangen werden, dass sich die für eine Herdgemeinschaft zur Verfügung stehende Wohnfläche nicht verändert hat.

\section{Die Einwohner Nürnbergs am Ende des Mittelalters}

In der Erfassung des Gemeinen Pfennings (Reichssteuerregister) im Jahr 1497 haben sich detaillierte Angaben zu den über fünfzehnjährigen Mitgliedern aller Herdgemeinschaften auf der Lorenzer Stadtseite überliefert. ${ }^{551} \mathrm{Im}$ Folgenden sind die Größen der Herdgemeinschaften nach den Ergebnissen Otto Puchners aufgeführt. ${ }^{552}$ Insgesamt ermittelt Puchner 8.508 Einwohner über fünfzehn Jahren in 2.593 „Haushaltungen“ und somit durchschnittlich 3,25 Personen pro Herdgemeinschaft. Den Anteil der Kinder unter den Einwohnern berechnet er

\footnotetext{
${ }^{549}$ In die Zahlen sind die Anstalten nicht eingerechnet.

${ }^{550}$ MÜLLNER 1623/1984, S. 44.

${ }^{551}$ Vgl. hierzu und im Folgenden PUCHNER 1975, S. 916-928.

StadtAFrankfurt (Institut für Stadtgeschichte) H.06.03 2.781.

552 PUCHNER 1975, S. 925.
} 
anhand verschiedener Quellen auf 30\%. ${ }^{553}$ Daraus ergibt sich eine durchschnittliche Personenanzahl von 4,69 pro Einheit. Insgesamt kommt er allein für die Lorenzer Stadtseite auf 12.701 Einwohner zuzüglich 93 Geistlicher.

\begin{tabular}{|l|c|c|c|c|c|c|c|c|c|c|c|c|}
\hline Personen pro Einheit & $\mathbf{1}$ & $\mathbf{2}$ & $\mathbf{3}$ & $\mathbf{4}$ & $\mathbf{5}$ & $\mathbf{6}$ & $\mathbf{7}$ & $\mathbf{8}$ & $\mathbf{9}$ & $\mathbf{1 0}$ & $\mathbf{5 1 5}$ & $\mathbf{5} \mathbf{2 6}$ \\
\hline Anzahl der Einheiten & 227 & $\mathbf{9 0 5}$ & 590 & 370 & 228 & 122 & $\mathbf{6 6}$ & 43 & 19 & 10 & $\mathbf{8}$ & 5 \\
\hline Anteil an allen Einheiten & $\mathbf{9 \%}$ & $\mathbf{3 5 \%}$ & $\mathbf{2 3 \%}$ & $\mathbf{1 4 \%}$ & $\mathbf{9 \%}$ & $\mathbf{5 \%}$ & $\mathbf{3 \%}$ & $\mathbf{2 \%}$ & $\mathbf{1 \%}$ & $\mathbf{0 , 4 \%}$ & $\mathbf{0 , 3 \%}$ & $\mathbf{0 , 2 \%}$ \\
\hline
\end{tabular}

Tabelle 10. Nürnberg, Lorenzer Seite, Anzahl der im Reichssteuerregister 1497 angegebenen Personen, die über 15 Jahre alt sind, pro Herdgemeinschaft

(Thomas Wenderoth 2019 nach PUCHNER 1975, Tab. VI)

Im Großteil der Herdgemeinschaften lebten lediglich zwei bis drei Personen über fünfzehn Jahren (58\%), es dürfte sich hier in vielen Fällen um „klassische“ Kleinfamilien, bestehend aus Eltern mit Kindern ohne Personal, gehandelt haben. Das gilt wohl auch für die Einheiten mir vier Personen (14\%), wobei hier neben älteren Kindern sicher auch schon Mägde, Gesellen und Untermieter anzusetzen wären. Große Herdgemeinschaften mit sechs bis acht Erwachsenen fanden sich in $10 \%$ der Fälle, also etwa so häufig wie die Kleinhaushalte mit lediglich einer erwachsenen Person (9\%). Wirklich große Herdgemeinschaften mit mehr als acht über fünfzehnjährigen Mitgliedern bildeten dagegen mit unter $2 \%$ eine verschwindend geringe Minderheit. Durchschnittlich ergab sich für die Herdgemeinschaften eine Stärke von gut drei Personen zuzüglich der Kinder. Diese schwankte 1497, topografisch betrachtet, stark: ${ }^{554}$ Im Bereich innerhalb der älteren Stadtbefestigung lag sie bei 3,63 Personen und in den Gebieten der Stadterweiterung bei 3,00 Personen. Die Extreme finden sich dabei im Kartäuserviertel mit 4,00 Personen intra muros und 2,90 Personen extra muros.

Für die Zeit um 1500 darf angenommen werden, dass die innerstädtischen Flächen nunmehr weitgehend bebaut waren und die Anzahl der Parzellen nahezu identisch war mit den im Jahr 1796 erfassten 1.578 Anwesen auf der Lorenzer Seite. ${ }^{555}$ Für den Ausbauprozess im Bereich der Stadterweiterungsflächen im 15. Jahrhundert liefert Otto Pucher ebenfalls wichtige Hinweise: ${ }^{556} \mathrm{Im}$ Jahr 1431 gab es auf der Lorenzer Seite extra muros 1.067 Einheiten, die er als „Haushaltungen" bezeichnet, ich aber im Folgenden wieder unter dem Begriff der Herdgemeinschaft behandeln möchte. Im Jahr 1497 waren es dagegen bereits 1.568 Herdgemeinschaften was eine Steigerung um $50 \%$ bedeutet. Gleichzeitig nahm

\footnotetext{
553 Ebd., S. $927 f$.

${ }^{554}$ Vgl. hierzu und im Folgenden PUCHNER1975, S. 926.

555 Zumindest muss die Reichsstadt bereits 1489 auf den schwierigen Baugrund des älteren Stadtgrabens zurückgreifen, um die Zinshäuser für die schwäbischen Weber errichten zu können. Dies ist als Indiz zu werten, dass nicht mehr viele freien Flächen für eine Bebauung zur Verfügung standen.

556 PUCHNER 1975, S. 927.
} 
die Zahl der Herdgemeinschaften in der Kernstadt nur um 15\% zu. Den anzunehmenden 1.578 Anwesen standen nunmehr die bereits genannten 2.593 Herdgemeinschaften gegenüber. Vereinfacht ausgedrückt bedeutet dies, dass rund 1.000 Herdgemeinschaften mit in einem anderen Anwesen wohnten, was ein Mietverhältnis nahelegt. Wiederum nicht erfasst werden können auf diese Weise Mieter, die ein Anwesen allein bewohnt haben. Immerhin ergibt sich aus der Verbindung der Zahlen aber ein Anteil der Miethaushalte von minimal 40\% auf der Lorenzer Seite für das Jahr 1497. Pro Anwesen errechnet sich zugleich eine Belegungsziffer von 1,64 Herdgemeinschaften oder 8,05 Personen.

Otto Puchner selbst bezeichnet die angeführten Einheiten als „Haushaltungen“, ein Blick auf den Anteil der Einheiten mit weiblichem Vorstand zeigt jedoch, dass die Einheit von mir korrekt im Sinne einer Herdgemeinschaft interpretiert wurde. Nach seiner Aufstellung lag der Anteil von Einheiten mit weiblichem Vorstand im Jahr 1497 lediglich bei 10,3\%. ${ }^{557}$ Die Zählung der Nürnberger Landbevölkerung im Jahr 1538 ergab für zehn stadtnahe Orte einen Mieteranteil von 63,9\%. Von den dort 1538 angeführten vorstädtischen Miethaushalten hatten 26,5\% einen weiblichen Haushaltsvorstand, bezogen auf alle Haushalte waren es dort 17\%. ${ }^{558}$ Für die nahegelegene Stadt Schwabach ist für das Jahr 1530 mit 20\% ebenfalls ein höherer Frauenanteil unter den Miethaushalten belegt. ${ }^{59}$ Der Unterschied zu den 10,3\% in den städtischen „Haushaltungen“ Nürnbergs 1497 erscheint signifikant. Ein Erklärungsmodell wäre, dass in den Nürnberger Einheiten in der Zählung 1497 die Untermieterinnen und Untermieter mit einbezogen waren und nicht separat erfasst worden sind, der von Puchner verwendete Begriff der „Haushaltung“ also nicht mit dem Begriff des Haushalts verwechselt werden sollte.

Vergleicht man die durchschnittliche Anzahl der Erwachsenen pro Zähleinheit in den Jahren 1431/32 und 1497, so fällt eine große Differenz auf: Pucher errechnet für das Jahr 1497 exakt 3,25 über Fünfzehnjährige pro Einheit, Ott kommt für das Jahr 1430/31 auf eine durchschnittliche Größe von 4,34 über zwölfjährigen Personen. Es wurde bereits anhand von Vergleichszahlen dargelegt, dass es sich in beiden Zählungen bei den Einheiten um Herdgemeinschaften handeln muss, also um Haushalte, bestehend aus Kleinfamilien, Verwandten und Gesinde, die um Mieter erweitert sind. Da die Größe der Kleinfamilie und auch die der Haushalte (Familie und Gesinde) wohl kaum in einem solch großen Maß Schwankun-

\footnotetext{
${ }^{557}$ Ebd., Tab. I-IV: Barfüßerviertel 106 Frauenhaushalte von 711 Haushalten insgesamt, Kartäuserviertel 49 von 622, Frauenbrüderviertel 54 von 589, Elisabethviertel 59 von 671.

558 Vgl. WENDEROTH 2019, Tabelle 1, S. 44.

${ }^{559}$ Vgl. StAN, Fm AN, Salbücher Nr. 92, fol. 81-121. Eine Transkription findet sich bei: SCHLÜPFINGER 1977, S. 76-82.
} 
gen unterworfen war, muss die Differenz in der Anzahl der erwachsenen Personen vor allem außerhalb der Familienmitglieder und des Gesindes zu suchen sein.

Die stark gesunkene Durchschnittsgröße der Einheiten im Jahr 1497 legt also den Schluss nahe, dass in diesen 70 Jahren die Verselbstständigung der eigentumslosen Haushalte weit fortgeschritten ist. Der Anteil der an einem Herd lebenden familienfremden Personen scheint stark zurückgegangen zu sein: Neben der Kernfamilie und dem Gesinde wohnten am Ende des 15. Jahrhunderts wohl überwiegend nur noch alleinstehende fremde Personen mit in der Wohneinheit. Zeitgleich kann mit einer Zunahme des Mietwohnungsbaus gerechnet werden. Auf den Anwesen entstehen zusätzliche separate Wohneinheiten. Die Anzahl in Nürnberg steigt von 1,25 Herdgemeinschaften pro Gebäude im Jahr 1431 auf 1,64 Herdgemeinschaften pro Anwesen 1497. Allerdings sind die Zahlen aufgrund der unterschiedlichen Bezugsgrößen (Gebäude/Anwesen) nicht direkt miteinander vergleichbar.

\section{Die eigentumslose Bevölkerung im Jahr 1561}

In den von Christian Feja ausgewerteten Feuerschaulisten sind insgesamt 5.487 Haushalte erfasst worden. ${ }^{500}$ Nicht erwähnt werden dort die Untermieter, da diese keinen eigenen Herd nutzten. Nach der Analyse Fejas wurden zu diesem Zeitpunkt etwa 1.500 Häuser allein vom Eigentümer bewohnt, sind also als klassisches Bürger- oder Handwerkerhaus zu bezeichnen. In rund 500 Häusern wohnten neben dem Eigentümer noch Hauptmieter mit eigenem Herd, weitere 1.500 Häuser waren komplett vermietet. Nach Feja verfügten 2.144 Haushalte über Wohneigentum, 3.343 Haushalte lebten in Miete, dies entspricht einem Anteil von $61 \%$ der erfassten Haushalte. ${ }^{561}$ Hinzuzurechnen ist eine unbekannte Zahl von Haushalten, die zur Untermiete lebten. Ihr Anteil dürfte zwischen 10\% und $20 \%$ aller Haushalte gelegen haben. ${ }^{562}$ Daraus ergibt sich ein entsprechend höherer Anteil von über $70 \%$ Miethaushalten an der Bevölkerung.

Schon bald nach dieser Zählung wurde Nürnberg von einer großen Pestepidemie heimgesucht, der zwischen Dezember 1561 und Mai 1563 insgesamt 9.034 Menschen zum Opfer fielen. ${ }^{563}$ Dieses Ereignis zeigt auf, welch großen Schwankungen die Bevölkerungsentwicklung und damit auch die Belegungsdichte der Häuser unterworfen waren. Die nachfolgenden Zahlen beweisen jedoch die weiter oben geäußerte These, dass insgesamt betrachtet für die zweite Hälfte des

\footnotetext{
${ }^{560}$ FEJA 1988, S. 20.

561 Ebd., S. 39.

562 Vgl. WENDEROTH 2021, S. $165 f$.

563 PORZELT 2000, S. 38-40; darin enthalten sind auch die Opfer in Wöhrd und im Pestspital St. Sebastian. In seuchenfreien Jahren wurden dagegen lediglich 1.400-1.800 Todesfälle verzeichnet (PORZELT 2000, S. 35).
} 
16. Jahrhunderts und den Beginn des 17. Jahrhunderts ein starkes Bevölkerungswachstum festzustellen ist.

Für das Jahr 1622 werden exakt 10.069 Herdgemeinschaften überliefert, vier Jahre später wurde die Bevölkerung Nürnbergs mit 39.128 Personen erfasst. ${ }^{564}$ Aus der Zusammenschau der beiden Angaben ergibt sich eine durchschnittliche Größe von 3,89 Personen. ${ }^{565}$ Bemerkenswert ist die starke Zunahme bei der Anzahl der Herdgemeinschaften und damit auch der Haushalte seit 1561. Die Unterschiede zu den Ergebnissen der Zählungen 1497 und 1561 in Anzahl und Größe der Einheiten könnten zumindest zum Teil auch in einer unterschiedlichen Erfassung derjenigen Haushalte, die zur Untermiete wohnten, liegen.

Da sich die Anzahl der Hausstellen in diesem Zeitraum innerhalb der Stadtmauer kaum verändert hatte, muss im Hinblick auf die gestiegenen Haushalts- und Bevölkerungszahlen eine starke bauliche Verdichtung bei den Anwesen selbst stattgefunden haben: Sei es die Überbauung von Hofbereichen oder die Aufstockung beziehungsweise der Ausbau der Dachgeschosse zu Wohnzwecken. Damit einher geht sicher auch die Zunahme der Miethaushalte.

Geht man für das frühe 17. Jahrhundert weiterhin von 3.300 Anwesen aus, wie im Jahr 1796, ergibt sich allein daraus ein Überhang von 6.769 Herdgemeinschaften, mithin ein Mieteranteil von 67\%. Jedes Anwesen beherbergte im Durchschnitt drei Parteien. Wird zudem berücksichtigt, dass ein nicht unerheblicher Teil der Anwesen zur Gänze vermietet gewesen ist, erhöht sich die Zahl der eigentumslosen Haushalte entsprechend. Für das Jahr 1561 ist bekannt, dass nur 2.144 Haushalte über Wohneigentum verfügten, ${ }^{566}$ was überwiegend mit dem Besitz eines ganzen Hauses gleichgesetzt werden kann. Hält man diese Zahl an Eigentümern aufrecht, ergibt sich rein rechnerisch sogar ein Mieteranteil von fast $79 \%$ !

\section{Situation am Ende des 18. Jahrhunderts}

Bisher sind für den Zeitraum zwischen 1626 und 1798 keine Bevölkerungszahlen zu Nürnberg bekannt geworden. Mit dem Abzug der im Dreißigjährigen Krieg aus dem Umland geflüchteten Menschen dürfte die Bevölkerung erheblich zurückgegangen sein. Hinzu kamen noch die hohen Verluste durch die Pest.

Erst mit den Listen zu den Quartiergeldbeiträgen 1798 liegt wieder eine wichtige Quelle zur Wohnsituation zum Ende der frühen Neuzeit vor. Es wird dort zwar

\footnotetext{
564 DiEFEnBACHER 2000, S. 211.

565 Endres übersieht die Ergebnisse dieser Zählungen und geht stattdessen von einem Anstieg der Kopfzahl pro Haushalt bis 1620 aus, ohne diese Annahme nachvollziehbar zu begründen; ENDRES 1970, S. 248.

566 ENDRES 1990b; FEJA 1988.
} 
nicht der Status der Bewohner - Eigentümer, Mieter oder Untermieter - angegeben, einzeln erfasst sind aber sämtliche Haushalte, die in einem Anwesen wohnten. Bei den Anwesen mit mehr als einem Haushalt kann davon ausgegangen werden, dass es sich hier auch um eine Mietsituation gehandelt haben muss, da geteiltes Eigentum in Nürnberg weiterhin keine nennenswerte Rolle spielte. $\mathrm{Zu}$ den so ermittelten Miethaushalten wäre zum einen noch ein unbekannter prozentualer Anteil von Anwesen hinzuzurechnen, die nur von einem Miethaushalt bewohnt worden sind, und zum anderen ein unbekannter Prozentsatz von den erstgenannten Haushalten in den Etagenwohnhäusern. Denn sowohl Einparteien- als auch Etagenwohnhäuser konnten komplett fremdbewohnt gewesen sein. Für die Sebalder Seite wurden die Einträge aufgeschlüsselt, sodass einige verallgemeinernde Aussagen zur Eigentumssituation möglich werden: Insgesamt sind dort 1.838 Gebäude unter 1.705 Hausnummern erfasst. Davon waren $99 \mathrm{Ge}$ bäude $(5,4 \%)$ unbewohnt. Die überlieferten 2.754 Haushalte verteilten sich demnach auf 1.739 Häuser. $65 \%$ der Gebäude waren nur von einer Partei bewohnt, $30 \%$ von zwei oder drei Parteien. Nur in 5\% der Häuser fanden sich zwischen vier und zehn Haushalte.

\begin{tabular}{|l|c|c|c|c|c|c|c|c|c|c|}
\hline Anzahl Haushalte/Haus & $\mathbf{1} \mathrm{HH}$ & $\mathbf{2} \mathrm{HH}$ & $\mathbf{3} \mathrm{HH}$ & $\mathbf{4} \mathrm{HH}$ & $\mathbf{5 ~ H H}$ & $\mathbf{6 ~ H H}$ & $\mathbf{7 ~ H H}$ & $8 \mathrm{HH}$ & $\mathbf{9} \mathrm{HH}$ & $\mathbf{1 0} \mathrm{HH}$ \\
\hline Anzahl Häuser & 1.123 & 374 & 148 & 46 & 27 & 11 & 3 & 2 & 1 & 2 \\
\hline Anteil der Haushalte & $\mathbf{6 5 \%}$ & $\mathbf{2 2 \%}$ & $\mathbf{9 \%}$ & $\mathbf{1 4 \%}$ & $\mathbf{3 \%}$ & $<1 \%$ & $<1 \%$ & $<1 \%$ & $<1 \%$ & $<1 \%$ \\
\hline
\end{tabular}

Tabelle 11. Nürnberg, Sebalder Seite, Haushaltsanzahl (HH) pro Haus 1797/98

(Quartiergeldbeiträge 1798, Auswertung Sarina Werner 2019)

Von der Gesamtbevölkerung lebte mehr als die Hälfte der Haushalte (59\%) in einem Haus mit mindestens zwei Parteien. Allein daraus ergibt sich ein Minimum an eigentumslosen Haushalten in Höhe von $30 \%$. Zu den Haushalten in Mehrparteienhäusern zählten mehrheitlich die Handwerker (59\%), die Verwaltungsangehörigen (66\%), die Erwerbslosen (79\%) und die Witwen (72\%). Es waren aber nicht nur die Ärmsten der Stadtbevölkerung, die sich ein Haus teilten. Auch von den 197 vermögendsten Haushalten, die den höchsten Quartiergeldbeitrag leisten mussten, lebten 84 (42,6\%) nicht allein in einem Haus.

Anhand der Zahlen wird auch deutlich, dass am Ende des 18. Jahrhunderts nur noch jedes zehnte Haus von drei und mehr Parteien bewohnt war. Die Belegungsziffer war mit 1,58 Haushalten pro Haus extrem niedrig, vergleicht man dies mit den Zahlen in Fürth, wo im Jahr 1818 viermal so viele Haushalte, nämlich 6,09, auf ein Haus kamen. In Ansbach waren es sogar 7,41, in dem beschaulich wirkenden Erlangen mit einer nur punktuell dreigeschossigen Bebauung 2,73 Haushalte und in Schwabach ebenfalls 3,37 (vgl. Tab. 23). Man kann Nürnberg zu diesem Zeitpunkt zugespitzt auch als eine „entvölkerte“ Stadt bezeichnen. 


\section{Eigentumslose Haushalte in anderen Großstädten}

Um die oben genannten Zahlen zum Anteil eigentumsloser Haushalte besser einordnen zu können, erscheint neben der Gegenüberstellung mit den Gruppen der fränkischen Mittel- und Kleinstädte, die weiter unten erfolgen wird, auch ein direkter Vergleich mit anderen Großstädten sinnvoll. Im Hinblick auf die gering ausgeprägte Forschung zu diesem Thema, werde ich für verschiedene Zeitabschnitte auf verschiedene Städte im deutschsprachigen Raum eingehen.

\section{Mittelalter}

Köln gehört sicher zu den bedeutendsten deutschen Großstädten des Mittelalters. Im Hinblick auf die Geschichte des Mietwohnens nimmt sie ebenfalls eine wichtige Stellung ein, da sich für den kernstädtischen Bereich des Kirchspiels St. Kolumba wertvolle Quellen erhalten haben, die Aufschlüsse über das Mietwohnen seit dem 13. Jahrhundert ermöglichen. ${ }^{567}$

Für das Jahr 1487 sind für dieses Kirchspiel 885 Häuser erfasst, lediglich 161 Häuser wurden von den Eigentümern selbst bewohnt, für 661 Häuser ist eine Vermietung nachgewiesen. ${ }^{58}$ Der Anteil der eigengenutzten Häuser lag also bei lediglich $18 \%$, der Anteil der Mietshäuser bei mindestens 75\%. Dass sich Eigentumsverhältnisse im Lauf der Zeit ändern konnten und Zahlen nicht ohne Weiteres auf andere Zeitschnitte übertragen werden können, wird anhand der Vergleichszahlen für das gleiche Kirchspiel 100 Jahre später deutlich. Die Anzahl der Häuser war 1589 absolut auf 949 gestiegen, 305 Häuser (32\%) waren nunmehr vom Eigentümer selbst bewohnt und mindestens 585 (62\%) vermietet. ${ }^{569}$ In diesen Zahlen sind Untermietverhältnisse nicht eingerechnet, ebenfalls unberücksichtigt sind dort Situationen mit mehr als einem Mieter im Haus, sodass der Anteil der eigentumslosen Haushalte an der Bevölkerung des Kirchspiels entsprechend höher lag. Aus der Kölner Aufstellung des Jahres 1589 geht jedoch auch hervor, dass die meisten der dortigen Häuser nur von einer Partei bewohnt wurden. Lediglich bei zwölf der 949 Häuser sind zwei Parteien vermerkt. Unklar ist jedoch, inwieweit Untermieter in dieser Liste erfasst worden sind.

Regensburg kann bis zum Ende des Mittelalters noch zu den Großstädten gezählt werden. Christian Forneck geht von 10.000 Einwohnern im 15. Jahrhundert aus. ${ }^{570}$ Die wirtschaftliche Entwicklung stagniert dann aber, sodass Regensburg

\footnotetext{
567 GREVING 1904.

568 Ebd., S. 33.

${ }^{569}$ Ebd.

${ }^{570}$ FORNECK 2000, S. 21-24. Erfasst sind in dem Steuerregister von 1436 exakt 6.024 Einwohner ohne Kinder, Juden und Geistliche. Die Anzahl der Kinder schätzt er auf maximal 3.700, was vermutlich zu hoch gegriffen ist.
} 
im Verlauf dieses Jahrhunderts den Anschluss an die großen deutschen Metropolen, darunter die aufsteigenden Hansestädte, verlor. Äußeres Zeichen dieser Entwicklung war der kurzzeitige Verzicht auf Reichsunmittelbarkeit und die Huldigung der Stadt an den bayerischen Herzog 1486.

Aus diesem Grund sollen hier nur die mittelalterlichen Zahlen zur eigentumslosen Bevölkerung näher betrachtet werden: Für Regensburg lassen sich für das Jahr 1436 etwa 690 Anwesen nachweisen, die von ihren Eigentümern selbst bewohnt worden sind. ${ }^{571}$ Diesen standen 413 Häuser in privatem Besitz gegenüber, die komplett fremdvermietet waren, hinzu kamen weitere 168 Anwesen im Besitz der Stadt oder geistlicher Institutionen, die zum Großteil ebenfalls vermietet waren. Demnach waren 46\% der bewohnten Anwesen zur Gänze vermietet. Darüber hinaus sind noch 85 unbesetzte/leerstehende Anwesen überliefert.

Der Anteil der Miethaushalte lag jedoch höher, da bei dieser Zahl keine zusätzlichen Mietparteien in den Häusern berücksichtigt worden sind. Forneck schreibt dazu: „Das Wohnhaus als Idealtypus, in dem eine einzige Familie mit ihren Kindern, dem Gesinde und weiteren Verwandten wohnte und dem häuslichem Erwerbsbetrieb angegliedert war, ist in Regensburg im 15. Jahrhundert ein verhältnismäßig seltenes Phänomen. Vielmehr fanden sich eine ganze Reihe von Gebäuden, in denen mehrere gleichrangige Familien aus teilweise ganz unterschiedlichen Berufsgruppen unter einem gemeinsamen Dach wohnten und womöglich auch arbeiteten. Es gab eine Reihe von Anwesen mit beträchtlichen Einwohnerzahlen, darunter zwei mit nicht weniger als 26 Hausgenossen. “ 572

Forneck ermittelt allein 1.752 Haushalte von alleinstehenden Personen, die überwiegend zur Untermiete wohnten. ${ }^{573}$ Zusammen mit den 581 fremdvermieteten Anwesen ergibt dies ein Minimum an 2.333 Miethaushalten, denen lediglich 690 Eigentümerhaushalte gegenüberstanden. Der Anteil der Miethaushalte lag demnach bei über 77\%! Das Mietwohnen stellte also die Regel dar, insbesondere in der Form der Untermiete.

Auch wenn es einzelne Häuser mit vielen Bewohnern, darunter auch Mietparteien, gegeben hat, so handelte es sich bei der Bausubstanz doch im Wesentlichen um Gebäude, die jeweils nur von wenigen Personen bewohnt wurden. Pro Haus ermittelt Forneck durchschnittlich 4,46 erwachsene Bewohner. ${ }^{574}$ Er führt 1.271 Wohnhäuser an, von denen 528 (41,54\%) von einem bis drei Erwachsenen (Personen ab einem Alter von vierzehn Jahren) ${ }^{575}$ bewohnt wurden. Es folgen 461

\footnotetext{
${ }^{571}$ Ebd. S. $119 f$.

572 FORNECK 2000, S. 77.

573 Vgl. Hierzu und im Folgenden FORNECK 2000, S. 80f. Von den 6.204 Erwachsenen (ohne Juden und Geistlichkeit) lebten 1.752 Personen als ledige „Inwohner meist ohne eigenen Haushalt".

${ }^{574}$ FORNECK 2000, S. $77 f$.

575 Ebd., S. 13.
} 
Häuser (36,27\%) mit vier bis sechs Erwachsenen. 206 Häuser (16,21\%) wurden von sieben bis zehn Erwachsenen, 59 (4,64\%) von elf bis fünfzehn und siebzehn $(1,34 \%)$ von sechzehn bis sechsundzwanzig Erwachsenen bewohnt.

\begin{tabular}{|l|c|c|c|c|c|c|c|c|c|c|c|c|c|}
\hline Erwachsene/Haus & 1 & 2 & 3 & 4 & 5 & 6 & 7 & 8 & 9 & 9 & 10 & $11-15$ & $16-26$ \\
\hline Anzahl & 98 & 218 & 212 & 195 & 159 & 107 & 76 & 56 & 48 & 48 & 26 & 59 & 17 \\
\hline Anteil & $8 \%$ & $17 \%$ & $17 \%$ & $15 \%$ & $13 \%$ & $8 \%$ & $6 \%$ & $4 \%$ & $4 \%$ & $4 \%$ & $2 \%$ & $5 \%$ & $1 \%$ \\
\hline
\end{tabular}

Tabelle 12. Regensburg, Belegungsdichte der Wohnhäuser 1436 (FORNECK 2000, Tab. 8)

Das Einwohnerverzeichnis von 1436 gibt darüber hinaus auch die Anzahl Stuben in jedem Haus an. ${ }^{576}$ Als Stube wird der beheizbare Aufenthaltsraum, also das Kernelement eines Hauses oder einer Wohnung, bezeichnet. Um von einer eigenständigen Wohneinheit zu sprechen, ist mindestens eine Stube erforderlich, in repräsentativen Wohnungen können dem Bewohner aber auch mehrere Stuben zur Verfügung gestanden haben, sodass aus dem Vorhandensein von mehreren Stuben in einem Haus nicht von vornherein auf die entsprechende Anzahl von getrennten Wohneinheiten geschlossen werden kann. ${ }^{577}$ Zur Veranschaulichung soll hier die Wohnsituation in einem großen Anwesen mit sechs Stuben wiedergeben werden: Das Haus stand in der Brückenstraße und gehörte dem Ratsherrn Lienhart Notscherf, der nicht selbst dort wohnte, sondern es komplett an diverse Schuster vermietet hatte. ${ }^{578}$ Mieter der ersten Stube waren zwei Schuster mit Ehefrauen und einem beziehungsweise zwei Dienern (Gesellen). In der zweiten Stube wohnte ein Ehepaar mit Diener, in der dritten und sechsten Stube je ein Ehepaar mit zwei Dienern. Die vierte Stube war an einen Schuster mit Ehefrau, Diener und Magd vermietet. In der fünften Stube wohnte wiederum ein Schuster mit Frau und Diener, hinzu kamen aber noch seine Mutter und eine Witwe. Ob solche Anwesen auch über mehrere Küchenstandorte verfügten oder die Haushalte sich eine Küche teilen mussten, ist bisher nicht geklärt. Die Zahlen deuten aber darauf hin, dass Häuser mit mehreren in sich abgeschlossenen Wohnungen wohl nicht oder nur kaum verbreitet waren. Die überwiegende Masse der Häuser, nämlich 1.002 (79\%), hatten nur eine Stube, waren also im heutigen Sinn Einparteienhäuser, auch wenn sie von mehreren Parteien gemeinsam bewohnt wurden. Kombiniert man die Angaben zu den Stuben mit den Angaben zur durchschnittlichen Bewohnerzahl der Häuser, so dürfte im Standardfall ein Regensburger Haus von einer Kleinfamilie - als Eigentümer oder Mieter - und ein bis zwei alleinstehenden (Unter) -Mietern bewohnt gewesen sein.

\footnotetext{
576 Vgl. hierzu und im Folgenden FORNECK 2000, S. 81-83.

577 Hier irrt Forneck, wenn er annimmt, dass eine Familie nur eine Stube bewohnt hat, S. 81.

578 FORNECK 2000, S. 82 und S. 236

(Quelle: Bayerisches Hauptstaatsarchiv München, RRLit 441, fol. 264).
} 
Für Hamburg sind frühe Angaben wiederum nur für einzelne Kirchspiele überliefert, dennoch werfen diese ein wichtiges Schlaglicht auf die dortige Situation. ${ }^{579}$ So wurden im Kirchspiel St. Nikolai im Jahr 1442 insgesamt 885 Wohnparteien gezählt. Aufgeführt sind 506 Wohnungen, in denen neben den Besitzern noch 293 Mieter wohnten. Die restlichen 86 Haushalte dürften als Mieter in Kellerwohnungen gelebt haben, daraus errechnet sich ein Mieteranteil von $43 \%$ aller Haushalte. Bereits 1462 lassen sich archivalisch zudem Mietshäuser für mehrere Parteien nachweisen. Anscheinend war aber auch das Zusammenleben mehrerer Haushalte als Herdgemeinschaften in einer „Wohnung“ noch weit verbreitet, wie die oben angeführten 293 Mietereinträge nahelegen.

\section{Frühe Neuzeit bis Mitte des 17. Jahrhunderts}

Während sich die Anzahl der eigentumslosen Haushalte in Hamburg im Mittelalter bereits im unteren Bereich des zu Erwartenden bewegt, fallen die überlieferten Zahlen für das 16. Jahrhundert noch einmal leicht ab: Exakt 100 Jahre später haben sich erneut Zahlen zum Kirchspiel St. Nikolai überliefert. Im Jahr 1542 werden 693 Hauseigentümer, 305 Mieter wohl in Vorderhäusern, weitere 106 Kellerbewohner, 25 Budenbewohner und neun Bewohner in den Obergeschossen von Buden (Säle) genannt, bei denen es sich ebenfalls um eigentumslose Einwohner gehandelt haben dürfte. Insgesamt errechnet sich daraus ein leicht gesunkener Anteil der Miethaushalte von 39\%. Der Wert ist gegenüber den für Nürnberg 1561 ermittelten Zahlen sehr gering.

Dieser relativ niedrige Wert korrespondiert jedoch mit der Überlieferung für Erfurt. Bis zur Zeit des Dreißigjährigen Krieges kann Erfurt noch zu den Großstädten gezählt werden. Für das Jahr 1569 errechnet Aloys Loffing 3.992 weltliche Haushalte, von denen 2.559 auch als Hausbesitzer gekennzeichnet waren. ${ }^{500}$ Also lebten 36\% dieser Haushalte nicht im Eigentum. Im Verhältnis zu anderen, weiter oben behandelten Großstädten erscheint der Anteil der Miethaushalte insgesamt betrachtet sehr gering.

Im 17. Jahrhundert erlebte die Stadt einen starken Bevölkerungsrückgang. Für das Jahr 1692 sind nur noch 2.752 Haushalte mit zusammen 11.231 Einwohnern überliefert, daraus errechnet sich eine Haushaltsgröße von 4,08 Personen. ${ }^{581}$ Der Anteil der als eigentumslos erfassten Haushalte lag nunmehr bei bescheidenen 24\%. Der Mieteranteil stieg auch im 18. Jahrhundert nur unwesentlich auf 28\% im Jahr 1774 an. ${ }^{582}$ Dieser niedrige Wert überrascht, hatte Erfurt doch nach wie vor eine überörtliche Bedeutung und mit dem Waidhandel auch eine lukrative ökonomische Basis. Diese hohe Eigentumsquote könnte jedoch auch direkt mit

\footnotetext{
${ }^{579}$ Vgl. hierzu und im Folgenden MAUERSBERGER 1960, S. 30-48.

${ }^{580}$ LOFFING 1911, S. $92 \mathrm{f}$.

581 BLAHA 1989, S. 154f.

582 Ebd., S. 170.
} 
dem Schrumpfen der Bevölkerung und einem damit verbundenen Leerstand sowie Preisverfall bei den Immobilien einhergehen. Hinzu kommt die hohe Eigentumsquote bereits im 16. Jahrhundert.

Das Beispiel Erfurt zeigt, dass eine Verallgemeinerung problematisch ist und die Lebens- und Wohnsituationen von Stadt zu Stadt sehr unterschiedlich gewesen sein können. Während sich in sehr vielen Großstädten bereits vor dem 17./18. Jahrhundert ein hoher Anteil von eigentumslosen Haushalten belegen lässt, weichen die Zahlen in Hamburg und vor allem in Erfurt von dieser Tendenz $\mathrm{ab}$, wobei sich in Hamburg die Situation mit dem Stadtwachstum ab dem 16. Jahrhundert der geschilderten mittelalterlichen Situation in anderen Großstädtenin anpasst.

Vielleicht drückt sich in den niedrigen Zahlen zu Hamburg und Erfurt aber auch die im 16. Jahrhundert geringere wirtschaftliche Bedeutung gegenüber Nürnberg und den im folgenden behandelten Städten Augsburg und Lübeck aus.

Bernd Roeck schildert für Augsburg anschaulich die Probleme, die sich im Zusammenhang mit der Rekonstruktion historischer Bevölkerungszahlen ergeben. ${ }^{583}$ Entsprechend schwer ist es auch, den jeweils exakten prozentualen Anteil der eigentumslosen Haushalte erfassen zu wollen. Auch wenn exakte Prozentanteile für Augsburg daher nicht angegeben werden, nennt Roeck doch einige absolute Zahlen, die ein Schlaglicht auf die Wohnsituation dieser Großstadt werfen. Im Jahr 1622 wurden 6.410 reichsstädtische Haushalte erfasst, die subventioniertes Brot erhalten wollten. Diese „armen“ Haushalte entsprechen in etwa 60\% der damaligen Bevölkerung. ${ }^{584}$ Wie nicht anders zu erwarten, handelt es sich dabei weitestgehend um Mieter, nur 520 dieser Haushalte $(8,3 \%)$ verfügten über Wohneigentum. ${ }^{555}$ An anderer Stelle nennt er eine Zahl von etwa 10.000 Haushalten, die vor dem Dreißigjährigen Krieg Steuern zahlten. ${ }^{586}$ Für das Jahr 1618 wird die Anzahl der Häuser mit 2.524 für die gesamte Stadt angegeben. Das bedeutet, dass die wohlhabenderen vierzig Prozent der Bevölkerung ebenfalls in beträchtlichem Umfang zur Miete wohnten. Während der Mietanteil bei den erfassten ärmeren Haushalten nachweislich bei $91,8 \%$ lag, kann er für die wohlhabenderen Haushalte nur geschätzt werden, es dürfte sich um 3.500 bis 4.500 Haushalte gehandelt haben, auf die rund 2.000 Häuser entfielen. Der Mieteranteil unter diesen Haushalten lag also zwischen $43 \%$ und 56\%; vermutlich aber im oberen Bereich, da eine gewisse Eigentumskonzentration bei der Stadt selbst, den wohlhabenderen Bürgern, Patriziern und Stiftungen angenommen werden muss. Zusammenge-

\footnotetext{
583 RoECK 1989, S. 301-308.

584 Ebd., S. 515.

585 Ebd., S. 556, Anhang I.

586 Ebd., S. 304.
} 
nommen scheint der Mieteranteil daher mindesten bei 75\% der Haushalte gelegen zu haben!

Die erheblichen Bevölkerungsverluste im Dreißigjährigen Krieg werden deutlich, wenn man sich die exakte Zählung der Einwohner Augsburgs aus dem Jahr 1635 vor Augen führt: Inklusiv der Frauen, Kinder, Bediensteten und Fremden wurden nur noch 16.422 Menschen gezählt, die in 5.800 Steuerhaushalten lebten. ${ }^{587}$ Daraus errechnet sich eine durchschnittliche Haushaltsgröße von lediglich 2,8 Personen.

Eine deutliche Sprache zum Thema des Wohneigentums sprechen auch die Zahlen, die Roeck zur Belegung der einzelnen Häuser angibt. ${ }^{588}$

\begin{tabular}{|l|c|c|c|c|c|c|}
\hline $\begin{array}{l}\text { Zahl der Haushalte } \\
\text { pro Haus }\end{array}$ & \multicolumn{2}{|c|}{$\begin{array}{c}\text { Zahl der Häuser } \\
1550\end{array}$} & \multicolumn{2}{c|}{$\begin{array}{c}\text { Zahl der Häuser } \\
1618\end{array}$} & \multicolumn{2}{c|}{$\begin{array}{c}\text { Zahl der Häuser } \\
1646\end{array}$} \\
\hline 1 & 705 & $30 \%$ & 678 & $27 \%$ & 893 & $45 \%$ \\
\hline $2-3$ & 935 & $40 \%$ & 823 & $33 \%$ & 729 & $37 \%$ \\
\hline $4-5$ & 377 & $16 \%$ & 531 & $21 \%$ & 257 & $13 \%$ \\
\hline $6-10$ & 268 & $12 \%$ & 370 & $15 \%$ & 102 & $5 \%$ \\
\hline $11-15$ & 38 & $2 \%$ & 87 & $4 \%$ & 16 & $1 \%$ \\
\hline $16-70$ & 23 & $1 \%$ & 35 & $1 \%$ & 2 & \\
\hline $\begin{array}{l}\text { Summe der erfassten } \\
\text { Häuser }\end{array}$ & 2.346 & & 2.524 & & 1.999 & \\
\hline
\end{tabular}

Tabelle 13. Augsburg, Belegung der Häuser mit Haushalten 1550-1646 (nach RoECK 1989, Tab. 61 und Tab. 111, S. 493 und 887)

Ein Haus nur mit der eigenen Familie zu bewohnen, war im Augsburg der Neuzeit vor allem ein Privileg von (reichen) Eigentümern. Aber auch diese hatten überwiegend Mietparteien bei sich wohnen. Die Zunahme der Bevölkerung zwischen 1550 und 1618 spiegelt sich vor allem in der Zunahme von Häusern mit mehr als vier Parteien wider, wohingegen die Zahl der von einem Haushalt allein oder mit bis zu drei Haushalten bewohnten Häuser sowohl absolut als auch prozentual zurückging. Für das Jahr 1610 führt Roeck insgesamt 9.653 Miethaushalte an. Stellt man diese den 2.524 Häusern des Jahres 1618 gegenüber - eine Zahl, die man vereinfacht mit einem Maximalwert an Eigentümern gleichsetzen kann - wird deutlich, dass der Anteil der Miethaushalte direkt vor dem Dreißigjährigen Krieg bei $80 \%$ zu suchen ist. Der hohe Bevölkerungsverlust im Dreißigjährigen Krieg führt zu einer deutlichen Entspannung der Wohnungssituation. Die Zahl der bewohnten Häuser sinkt signifikant, doch noch auffälliger ist die nunmehr geringere Belegungsdichte der bewohnten Häuser: Fast die Hälfte aller Häuser $(45 \%)$ wurde nur noch von einer Partei allein bewohnt, vermutlich in vielen Fällen

${ }^{587}$ Ebd., S. $775 f$.

${ }^{588}$ Vgl. hierzu und im Folgenden RoECK 1983, S. 492 f. 
auch von Mietern. Lediglich in 19\% der Häuser lebten mehr als drei Familien. Hinweise liegen zudem für eine Binnenwanderung aus den „schlechten“ Wohngegenden in bessere Viertel vor. ${ }^{589}$ So änderte sich die Belegungszahl der Häuser nur in der vornehmen Oberstadt nicht, ungeachtet der großen Bevölkerungsverluste, damit darf für dieses Quartier mit einem gleichbleibenden Anteil an eigentumslosen Bewohnern gerechnet werden. In allen anderen Vierteln sank die Belegungszahl dagegen deutlich. $\mathrm{Zu}$ vermuten ist, dass aber auch hier der Anteil der eigentumslosen Bevölkerung auf einem hohen Niveau blieb.

Für Lübeck lässt sich der Anteil der eigentumslosen Bevölkerung erst etwas später ermitteln. Überraschend erscheint auf den ersten Blick die hohe Anzahl an vermieteten Häusern: Margrit Christensen geht für die Jahre 1663/64 von einem Bestand von 3.200 Häusern aus. ${ }^{590}$ In den Steuerbüchern wurden 2.067 Anwesen taxiert. Die übrigen 1.133 Häuser gehörten besitzrechtlich zu den erfassten 2.067 Anwesen und waren vermietet. Es handelte sich dabei überwiegend um Kleinhäuser am Rande der Stadt und in Querstraßen, hierunter fallen noch nicht die vielen Gangbuden in den Hinterhöfen, die von vornherein zusammen mit dem Hauptanwesen erfasst worden waren. Neben den 1.133 zugehörigen Kleinhäusern wurden auch 687 taxierte Hauptanwesen (33\%) allein von Mietern bewohnt. Insgesamt betrachtet war die Mehrzahl der Anwesen zur Gänze vermietet (57\%). Lediglich $40 \%$ der Häuser wurden von ihren Eigentümern selbst bewohnt. Weitere $3 \%$ der Hausstellen waren unbesetzt oder lagen vor der Stadtmauer. Der Anteil der vermieteten Häuser und damit der eigentumslosen Haushalte steigt noch einmal deutlich, wenn man die Gangbuden ebenfalls als separate Wohnhäuser auffasst und mitzählt: Weitere 3.222 Miethaushalte haben in solchen Gangbuden oder mit in den Haupthäusern gewohnt. Insgesamt errechnet Christensen auf Grundlage dieser Auswertungen einen Anteil von eigentumslosen Haushalten in Höhe von $79 \%$ !

Christensen geht auch für die Zeit vor 1500 davon aus, dass lediglich 50\% der Anwesen von den Eigentümern bewohnt, die andere Hälfte aber vermietet war. ${ }^{591}$ Demnach waren bereits im Mittelalter die eigentumslosen Haushalte in Lübeck in der Mehrzahl.

\section{Die großstädtische Wohnsituation in der Barockzeit}

Während einige der älteren Großstädte nach dem Dreißigiährigen Krieg wirtschaftlich stagnierten und ihre ehemalige Größe nicht mehr erreichten, wie Nürnberg, Augsburg und Erfurt, blühten andere Städte auf. So steigt zum Beispiel die Bedeutung Leipzigs als Handelszentrum nach dem Dreißigiährigen

\footnotetext{
${ }^{589}$ Ebd. S. 888, Anm. 294; Roeck weist darauf hin, dass die Frage nur mit sehr hohem Aufwand zu klären wäre.

${ }^{590}$ Vgl. hierzu und im Folgenden CHRISTENSEN 2006, S. 69 und 112f, Karte 12.

${ }^{591}$ CHRISTENSEN 2006, S.111.
} 
Krieg und damit auch die Einwohnerzahl. Katrin Keller nennt für das Jahr 1699 21.969 Einwohner in 1.094 Häusern und für das Jahr 181135.382 Einwohner in 1.359 Häusern. ${ }^{592}$ Die Behausungsziffer stieg von 19,8 auf 26,0 Personen pro Haus! Der Mieteranteil dürfte demnach bei $80 \%$ bis $90 \%$ gelegen haben.

Rudolf Stein nennt für Breslau, seit dem Mittelalter eine der größten und bedeutendsten deutschen Städte, für das Jahr 17432.103 selbstständige Häuser ${ }^{593}$ und für das Jahr 177058.215 Einwohner. ${ }^{594}$ Vereinfacht errechnen sich daraus pro Haus 27,7 Bewohner oder sieben Haushalte! ${ }^{595}$ Vergleicht man diese Zahlen mit den Nürnberger Zahlen wird deutlich, welche Spannweite sich in der Gruppe der Großstädte hinsichtlich der Wohnsituation und dem Anteil der Miethaushalte auftat. Helmut Möller gibt für das 18. Jahrhundert folgende Behausungsziffern an (Bewohner/Haus): ${ }^{596}$

$\begin{array}{ll}\text { Potsdam (1. H. 18. Jh.) } & 10,2 \\ \text { Potsdam (2. H. 18. Jh.) } & 13,4 \\ \text { Augsburg } & 12,3 \\ \text { Stettin } & 14,0 \\ \text { Berlin } & 14,5 \\ \text { Frankfurt } & 15-16 \\ \text { Linz } & 15,4 \\ \text { Hannover } & 16,7\end{array}$

Während sich die Wohnsituation in den stagnierenden Großstädten deutlich entspannte, verschlechterte sich die Situation in den emporstrebenden Städten der Barockzeit dramatisch, denn eine durchschnittliche (!) Belegung der Häuser mit 20 bis 26 Personen bedeutet, dass in Leipzig fünf bis sieben Familien in einem Haus gewohnt haben! In Augsburg waren es zur Boomzeit am Beginn des 17. Jahrhunderts wohl ebenfalls noch fünf Familien gewesen. ${ }^{597}$ Eine Dichte, die wohl in Nürnberg zu keiner Zeit - abgesehen von Kriegssituationen - herrschte, denn zur Zeit der größten Blüte ergab im Jahr 1622 eine amtliche Zählung exakt 10.069 Haushaltungen. Bei angenommenen 3.283 Hausstellen ${ }^{598}$ errechnet sich

\footnotetext{
592 KELLER 1997, Tabelle 1 und 7.

${ }^{593}$ STEIN 1966, S. 86: „Als nach der Besitzergreifung 1743 die Häuser nummeriert wurden, ergaben sich zwischen Oder und Stadtgraben 2.103 selbstständig bewohnte Häuser.“

${ }^{594}$ Ebd., S. 80.

${ }^{595}$ Möller gibt hingegen nur eine Behausungsziffer von 20,0 Bewohner pro Haus für das 18. Jh. an, ohne einen konkreten Zeitraum zu nennen; MöLLER 1969, S. 120.

596 Ebd.

${ }^{597}$ Gerechnet wird hier mit den für 1610 genannten 9.653 Miethaushalte und den 2.524 Häusern des Jahres 1618. Auf etwa 12.000 Haushalte kamen 2.524 Häuser.

${ }^{598}$ Vgl. Quartiergeldbeiträge 1798.
} 
überschlägig eine Belegungsziffer von 3,3 Haushalten beziehungsweise eine Behausungsziffer von 12 bis 13 Personen als Maximalwert für Nürnberg. Um die Wohnverhältnisse tatsächlich vergleichen zu können, müssten zudem noch die Parzellengrößen und die Bebauungsdichte miteinander verglichen werden, dabei lässt sich insbesondere die Bebauungsdichte vergangener Jahrhunderte nicht ohne Weiteres detailliert ermitteln. Vielleicht erklären diese Zahlen aber auch, warum sich im Dreißigjährigen Krieg in Nürnberg noch Platz fand zur Aufnahme der österreichischen Exulanten und für die Bevölkerung, die aus dem Umland hinter die schützenden Mauern floh.

In der Neuzeit führten die veränderten Handelswege zu einem relativen Bedeutungsverlust Nürnbergs und anderer Großstädte und verliehen anderen Städten einen Wachstumsschub. Zudem ist für Deutschland vor allem bei den Residenzstädten der größeren Territorialstaaten eine erhebliche Zunahme an Bevölkerung zu beobachten. Diese wurden damit auch zu neuen Zentren der eigentumslosen Bevölkerung. ${ }^{599}$

\section{Mittelstädte}

\section{Ehemalige fränkische Reichsstädte}

Neben Nürnberg gab es in Franken weitere Reichsstädte, die jedoch niemals die Bedeutung und Größe Nürnbergs erreicht haben. Aufgrund ihrer politischen und wirtschaftlichen Bedeutung und zumindest teilweise auch der Bevölkerungszahlen können sie als Mittelstädte angesprochen werden. Windsheim als kleinste der vier Städte hat im Jahr 1818 lediglich 2.873 Einwohner, rein von den Einwohnerzahlen her betrachtet handelt es sich streng genommen nur noch um eine große Kleinstadt. ${ }^{600}$ Weißenburg liegt im Hinblick auf die Bevölkerungszahlen ebenfalls im Grenzbereich zwischen Kleinstadt und Mittelstadt.

Die Bevölkerungsentwicklung dieser Städte wurde bisher nicht systematisch untersucht. Daher kann die Entwicklung der eigentumslosen Bevölkerung seit dem Mittelalter hier nicht dargestellt werden. Es finden sich in der Literatur lediglich verstreut Einzelhinweise. ${ }^{601}$ Stellvertretend sollen die wenigen Angaben für Din-

\footnotetext{
${ }^{599}$ Vgl. hierzu auch MoLs 1971, S. $58 f$.

${ }^{600}$ In den Zahlen zu Rothenburg sind die Einöden und Mühlen außerhalb der Stadt einbezogen.

${ }^{601}$ So für Windsheim: Für das Jahr 1675 nennt M. A. Pastorius 506 Haushaltungen, darunter 51 Unbürger und 26 Ratsbürger. Die Unbürger sind sicher als Mieter zu werten; vgl. dazu KEYSER/STOOB 1971, S. 88. Korndörfer erfasst für das Jahr 1677529 Haushalte, denen 371 private und 47 städtische Wohnhäuser zur Verfügung standen, dabei sind jedoch 70 Häuser als ganz böse, das heißt ganz baufällig bezeichnet (vgl. KORNDÖRFER 1971, S. 53 und 57). 158 Haushalte
} 
kelsbühl angeführt werden: Für das Jahr 1468 sind 1.034 Steuerpflichtige und insgesamt 855 Herdstätten überliefert. ${ }^{602} 1491$ waren dort 704 Haushalte erfasst. Um 1610 werden 800 Bürger und 400 Pfahlbürger genannt. ${ }^{603}$ Da letztere über kein Eigentum verfügten und sicher auch einige Bürger zur Miete wohnten, kann für das frühe 17. Jahrhundert von mindestens $40 \%$ Miethaushalten ausgegangen werden. Bis zum Jahr 1750 reduziert sich die Anzahl der Pfahlbürger auf 96 gegenüber 702 Bürgerhaushalten. In der Uraufnahme von 1827 sind etwa 730 Häuser ${ }^{604}$ verzeichnet, in denen 5.202 Einwohner lebten. ${ }^{605}$ Aufgrund der schlechten Forschungslage ist vor 1818 kein direkter Vergleich dieser Städte untereinander oder mit anderen Städten möglich.

Erst mit dem Alphabetischen Verzeichnis aus dem Jahr 1818 lassen sich Zahlen zu Miethaushalten in Dinkelsbühl, Rothenburg ob der Tauber, Windsheim und Weißenburg für das Ende des Untersuchungszeitraumes gewinnen (vgl. Tab. 15). ${ }^{606}$ Für Dinkelsbühl werden dort 1.008 Feuerstellen, 1.428 Familien und 4.991 Seelen genannt. Die Haushaltsgröße war mit 3,50 Personen sehr klein. Bei gut 700 Häusern lebten demnach Anfang des 19. Jahrhunderts durchschnittlich zwei Haushalte in jedem Haus. Mit einem rechnerischen Anteil von dann 49\% Miethaushalten lag Dinkelsbühl an der Spitze der ehemaligen Reichsstädte.

Rothenburg brachte es immerhin auf 5.302 Einwohner. Dennoch sollen die vier Städte im Folgenden als eine Gruppe behandelt werden.

Gegenüber den ehemals fürstlichen Mittelstädten fällt der deutlich geringere Mieteranteil auf. Auch die Bevölkerungszahlen blieben deutlich hinter denen von Ansbach, Fürth, Erlangen und Schwabach zurück. Im Hinblick auf die Größe übertrafen die ehemaligen Reichsstädte zwar die fränkischen Kleinstädte zu Beginn des 19. Jahrhunderts deutlich (vgl. Tab. 23). Dies hatte sich jedoch insgesamt betrachtet nicht in höheren Mieterzahlen ausgewirkt: Wider Erwarten weichen die Mieterzahlen der ehemaligen Reichsstädte im Jahr 1818 nicht signifikant von denen der umliegenden Kleinstädte ab. Sie schwankten zwischen 30\% in Windsheim und $49 \%$ in Dinkelsbühl. Mit 38\% für Rothenburg und $42 \%$ für Weißenburg ergibt sich ein Durchschnittswert von 41\% Miethaushalten. Auffällig ist aber auch hier die unterschiedliche Belegungsziffer. Am höchsten war diese mit 1,96

(30\%) hatten demnach kein eigenes privates Haus, diese dürften vereinfacht dem Anteil der Miethaushalte gleichzusetzen sein.

${ }^{602}$ Vgl. hierzu und im Folgenden KeYSER/STOOB 1971, S. 150.

${ }^{603}$ Ebd.

${ }^{604}$ http://geoportal.bayern.de/bayernatlas-klassik, Uraufnahme Dinkelsbühl

(Aufruf 04.09.2018).

${ }^{605}$ KeYSER/STOOB 1971, S. 151.

${ }^{606}$ Die Zahlen sind nur überschlägig ermittelt, da nicht zwischen öffentlichen Wohnungen und Wohnungen in Privatbesitz unterschieden wurde, geteilte Häuser konnten ebenfalls nicht im Detail berücksichtigt werden. Erfasst ist hierbei nicht die ehemalige Reichsstadt Schweinfurt in Unterfranken. 
Familien pro Haus in Dinkelsbühl und 1,87 in Weißenburg. Die Behausungsziffer der vier ehemaligen Reichsstädte lag zwischen 5,4 und 6,8 Personen pro Haus. Im direkten Vergleich mit den Mieteranteilen und der Behausungsziffer der mittelfränkischen Kleinstädte dieser Zeit liegen die ehemaligen Reichsstädte am unteren Ende. Nur im Gebiet des ehemaligen Hochstiftes Eichstätt wiesen die Kleinstädte nochmals einen deutlich geringeren Anteil an Miethaushalten auf.

Vorsichtig ausgedrückt spiegeln die Bevölkerungs- und Mieterzahlen den Bedeutungsverlust der ehemaligen freien Reichsstädte wider, auch wenn sie absolut betrachtet noch mehr Einwohner aufwiesen als die umliegenden Kleinstädte. In ihrer Sozialstruktur und der Wohnsituation unterschieden sie sich kaum noch, man könnte sie daher bereits mit dem Ende des alten Reiches auch als große Kleinstädte ansprechen.

\section{Hohenzollerische Städte in Mittelfranken}

Kitzingen gehörte nur von 1443 bis 1629 zum Fürstentum Brandenburg-Ansbach und war zu dieser Zeit die größte Stadt im Herrschaftsgebiet. Sie stand größenmäßig an der unteren Schwelle zu einer Mittelstadt. ${ }^{607}$ Ingrid Bátori hat die Zahl der Eigentümer und Mieterhaushalte für das 16. Jahrhundert in Kitzingen ermittelt. ${ }^{608}$

Insgesamt betrachtet nahm die Zahl der Haushalte während des Untersuchungszeitraumes von 85 Jahren um 11\% zu. Die Zahl der versteuerten Häuser stieg dagegen lediglich von 573 auf 582, also um 3,9\%. Das Bevölkerungswachstum war verbunden mit einem prozentualen Anstieg der eigentumslosen Haushalte von 36,2\% auf 39,4\%. Auf der anderen Seite war zugleich ein Rückgang bei den Mehrfachhausbesitzern zu verzeichnen. Dies galt ebenfalls für die Eigentümer eines Hausanteils. Teileigentum lässt sich bereits für 1515 nachweisen, der Anteil war, bezogen auf die Gesamtzahl der Häuser, jedoch gering und halbierte sich zwischen 1536 und 1546. Insgesamt scheint sich in diesem Jahrzehnt die Besitzstruktur der Stadt am nachhaltigsten verändert zu haben, lässt man die unvollständigen und damit nicht korrekten Zahlen von 1566 unberücksichtigt. Miethaushalte spielten während des gesamten 16. Jahrhunderts für Kitzingen eine bedeutende Rolle, ihr Anteil schwankte zwischen 36,2\% und 42,3\%.

\footnotetext{
${ }^{607}$ KEMMETER, ERNST/MAIERHÖFER, HANSJOSEPH UND ISOLDE: (Art.) Kitzingen, in: KEYSER/STOOB 1971, S. 296-300.

${ }^{608}$ BÁTORI/WEYRAUCH 1982, S. 110-113, Tab. 7.
} 


\begin{tabular}{|c|c|c|c|c|c|c|c|c|c|c|c|}
\hline \multirow[b]{2}{*}{ Jahr } & \multirow{2}{*}{\begin{tabular}{|c|} 
Gesamtzahl \\
$\mathrm{HH}$
\end{tabular}} & \multicolumn{2}{|c|}{$>2$ Häuser } & \multicolumn{2}{|c|}{$>1$ Haus } & \multicolumn{2}{|c|}{1 Haus } & \multicolumn{2}{|c|}{$<1$ Haus } & \multicolumn{2}{|c|}{ kein Haus } \\
\hline & & $\mathrm{HH}$ & & $\mathrm{HH}$ & & $\mathrm{HH}$ & & $\mathrm{HH}$ & & $\mathrm{HH}$ & Mieter \\
\hline 1515 & 769 & 20 & $2,6 \%$ & 82 & $10,7 \%$ & 373 & $48,5 \%$ & 36 & $4,7 \%$ & 278 & $36,2 \%$ \\
\hline 1520 & 729 & 27 & 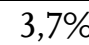 & 83 & $11,4 \%$ & 36 & $49,5 \%$ & 37 & $5,1 \%$ & 248 & $4,0 \%$ \\
\hline 1530 & 752 & 18 & $2,4 \%$ & 85 & $11,3 \%$ & 353 & $46,9 \%$ & 41 & $5,5 \%$ & 273 & $36,3 \%$ \\
\hline 1536 & 730 & 18 & $2,5 \%$ & 79 & $10,8 \%$ & 374 & $51,2 \%$ & 38 & $5,2 \%$ & 239 & $32,7 \%$ \\
\hline 1546 & 844 & 15 & $1,8 \%$ & 62 & $7,3 \%$ & 425 & $50,4 \%$ & 24 & $2,8 \%$ & 333 & $39,5 \%$ \\
\hline 1566 & 815 & 9 & $1,1 \%$ & 42 & $5,2 \%$ & 422 & $51,8 \%$ & 0 & $0,7 \%$ & 345 & $42,3 \%$ \\
\hline 1590 & 852 & 13 & $1,5 \%$ & 64 & $7,5 \%$ & 431 & $50,6 \%$ & 21 & $2,5 \%$ & 336 & $39,4 \%$ \\
\hline
\end{tabular}

Tabelle 14. Kitzingen, Veränderungen der Hausbesitzeranteile (HH = Haushalte) im 16. Jahrhundert. Die Zahlen für 1566 sind unvollständig

(nach BÁTORI/WEYRAUCH 1982, Thomas Wenderoth 2019)

Der Bevölkerungshöhepunkt war schon vor dem Ende des 16. Jahrhunderts erreicht, wie die nachfolgenden Zahlen belegen: Im Jahr 1603 hatte Kitzingen nach den Angaben in den Huldigungslisten nur noch 520 haussässige Bürger. ${ }^{609}$ Für den größten Ort des Markgraftums ist die Aufstellung mehrfach differenziert: $\mathrm{Zu}$ Beginn sind die Bewohner der inneren Stadt angeführt, gefolgt von den Bewohnern im ersten Theil der Vorstadt, den Bewohnern im anderen Theil der Vorstadt und den Bewohnern im Vorort Etwashausen. ${ }^{610}$ Die Hausgenossen finden sich verstreut in diesen Listen genannt, die einzelnen Listen folgen wohl einer topografischen Ordnung, ohne dass diese erklärt ist. An manchen Stellen in der Liste folgen die Nennungen von Hausgenossen sehr rasch aufeinander, an anderen Stellen nur vereinzelt. In einer zweiten Runde sind dann für jedes der vier Quartiere die Bewohner gelistet, die noch nicht gehuldigt haben. ${ }^{611}$ Daran schließt sich eine Liste mit den Witwen an, die Mieterinnen unter diesen sind wiederum mit dem Wort Hausgenossin gekennzeichnet. Insgesamt betrachtet wohnten in der Stadt 219 Hausgenossen und zwanzig öffentlich Bedienstete, die auch als eigentumslos anzusehen sind, dies entspricht einem Anteil von 30,6\% an allen Haushalten.

Diese Zahlen liegen erstaunlich nahe an den bereits für das Jahr 1429 genannten 720 Familien, ${ }^{612}$ jedoch deutlich unter den Zahlen von 1590.

${ }^{609}$ Die folgenden Angaben sind den Huldigungslisten von 1603 entnommen: StAN, Fm AN, Brdbg. Lit. Nr. 592, fol. 118-150. Von den Eigentümerhaushalten hatten 50 Haushalte eine Witwe als Vorstand.

${ }^{610}$ StAN, Fm AN, Brdbg. Lit. Nr. 592, fol. 118-140.

${ }^{611}$ Ebd., Nr. 592, fol. 141-145.

${ }^{612}$ Vgl. hierzu und im Folgenden KEMMETER, ERNST/MAIERHÖFER, HANSJOSEPH UND ISOLDE: (Art.) Kitzingen, in: KeYSER/STOов 1971, S. 296-300, hier S. 297. Für die nachfolgenden beiden Jahrhunderte werden dort folgende Zahlen angegeben: 1521: 554 Bürger; 1534: 538 Bürger; 1549: 630 Bürger; 1590: 652 Bürger; 1651: 481 Bürger. Der Bevölkerungshöhepunkt scheint damit schon Ende des 16. Jhs. erreicht gewesen zu sein. Die Zahlen liegen insgesamt betrachtet deutlich unterhalb der von Bátori ermittelten Anzahl an Haushalten. 
Anhand der Einwohnerzahlen von 1818 können für den mittelfränkischen Bereich neben den behandelten ehemaligen Reichsstädten die fürstlich hohenzollerischen Städte Ansbach, Erlangen, Fürth und Schwabach zu den Mittelstädten gezählt werden, Kitzingen war ja 1629 an das Hochstift Würzburg zurückgefallen.

Der Aufstieg von einer Kleinstadt zu einer Mittelstadt erfolgt bei diesen vier Städten allerdings erst im 18. Jahrhundert. Fürth wird sogar erst 1818 vom Marktort zur Stadt erhoben. Dagegen erleben die vier Reichsstädte nach 1600 einen Bedeutungsverlust, der sich unter anderem in einem nunmehr geringeren Mietbevölkerungsanteil gegenüber den vier fürstlichen Mittelstädten ausdrückt.

\begin{tabular}{|c|c|c|c|c|c|c|c|c|}
\hline \multirow[t]{2}{*}{ Stadt } & \multirow{2}{*}{$\begin{array}{l}\text { Häuser } \\
\text { /Feuer } \\
\text { stellen }\end{array}$} & \multirow{2}{*}{\begin{tabular}{|c} 
Eigen- \\
tums- \\
teile
\end{tabular}} & \multirow[t]{2}{*}{ Familien } & \multirow[t]{2}{*}{ Seelenzahl } & \multicolumn{4}{|c|}{ Auswertung } \\
\hline & & & & & $\begin{array}{c}\text { Familien } \\
\text { größe }\end{array}$ & $\begin{array}{c}\text { Behausungs } \\
\text { ziffer }\end{array}$ & $\begin{array}{c}\text { Familien/ } \\
\text { Haus }\end{array}$ & $\begin{array}{c}\text { Mieter } \\
\%\end{array}$ \\
\hline Nürnberg & 3.283 & & 6.593 & 26.382 & 4,00 & 8,0 & 2,01 & - \\
\hline Summe & 2.375 & 3.066 & 10.807 & 40.509 & 3,75 & 17,1 & 4,55 & $72 \%$ \\
\hline Fürth & 550 & 573 & 3.347 & 12.942 & 3,87 & 23,5 & 6,09 & $83 \%$ \\
\hline Ansbach & 455 & 1.066 & 3.370 & 11.320 & 3,36 & 24,9 & 7,41 & $68 \%$ \\
\hline Erlangen & 820 & 867 & 2.235 & 8.826 & 3,95 & 10,8 & 2,73 & $61 \%$ \\
\hline Schwabach & 550 & 560 & 1.855 & 7.421 & 4,00 & 13,5 & 3,37 & $70 \%$ \\
\hline Summe Reichsstädte & 2.599 & 2.623 & 4.435 & 16.866 & 3,80 & 6,5 & 1,71 & $41 \%$ \\
\hline Dinkelsbühl & 730 & 730 & 1.428 & 4.991 & 3,50 & 6,8 & 1,96 & $49 \%$ \\
\hline Rothenburg, incl. Einöden & 839 & 803 & 1.289 & 5.302 & 4,11 & 6,3 & 1,54 & $38 \%$ \\
\hline Weißenburg & 500 & 543 & 933 & 3.700 & 3,97 & 7,4 & 1,87 & $42 \%$ \\
\hline Windsheim & 530 & 547 & 785 & 2.873 & 3,66 & 5,4 & 1,48 & $30 \%$ \\
\hline Summe Mittelstädte & 4.974 & 5.689 & 15.242 & 57.375 & 3,76 & 11,5 & 3,06 & $66 \%$ \\
\hline
\end{tabular}

Tabelle 15. Einwohnerzahlen und Wohnsituation in den mittelfränkischen Mittelstädten 1818 (Quelle: Alphabetisches Verzeichnis des Rezatkreises und Uraufnahme)

Ansbach, Erlangen, Fürth und Schwabach weisen ab dem 18. Jahrhundert dann aber entsprechend hohe Mieteranteile auf. Diese lagen bei den vier Städten im Jahr 1818 im Mittel bei 72\%! Auf jedes Haus kamen durchschnittlich 17,1 Bewohner oder 4,55 Familien. Für das Stadtwachstum allgemein und den hohen Anteil an Mietbevölkerung im Besonderen war in Ansbach die Residenzfunktion ausschlaggebend. ${ }^{613}$ Die Entwicklung in Erlangen, Fürth und Schwabach ist vor allem auf den hohen Anteil an Exportgewerbe zurückzuführen. In Erlangen kommen als weitere Effekte die Funktion als Nebenresidenz in der 1. Hälfte des 18. Jahrhunderts sowie, zeitlich anschließend, die Universitätsgründung hinzu. Die Situation Ansbachs wird im Kapitel zu den Residenzstädten behandelt.

${ }^{613}$ Auch mit der Abdankung des letzten Markgrafen Alexander 1791 blieb Ansbach zentraler Verwaltungssitz der nun folgenden auswärtigen Landesherren. 
Ausführliche Darstellungen zur Bevölkerungsentwicklung in Erlangen und Schwabach finden sich im Kapitel zu den Exportgewerbestädten.

Auffällig ist neben den hohen Mieteranteilen auch die hohe Behausungsziffer, dies gilt insbesondere für Fürth und Ansbach, wo ein Haus im Jahr 1818 durchschnittlich von 23,5 beziehungsweise 25 Personen bewohnt wurde. Die Belegungssituation in Ansbach und Fürth darf wohl als sehr kritisch gelten, betrachtet man die in weiten Teilen kleinteilige Parzellen- und Baustruktur dieser Städte. Die gleichzeitige Situation in der Großstadt Nürnberg mit acht Personen pro Haus wirkt da geradezu entspannt und fast kleinstädtisch.

Insgesamt betrachtet kommen diese vier Mittelstädte auf eine Behausungsziffer von 17,1 Personen oder 4,55 Haushalte pro Haus/Anwesen. Dieser Wert liegt weit oberhalb dem der kursächsischen Vergleichsgruppe (vgl. Tab. 16), die nachfolgend geschildert wird.

\section{Mittelstädte außerhalb Frankens}

Für das späte Mittelalter fehlen für die vier ehemaligen mittelfränkischen Reichsstädte die zu dieser Zeit die Gruppe der Mittelstädte bilden, entsprechende Forschungen zur Bevölkerungsstruktur. Stellvertretend soll daher auf die Situation in sächsischen Mittelstädten verwiesen werden:

Yves Hofmann und Uwe Richter haben sich ausführlich mit der Wohnsituation in Freiberg/Sachsen auseinandergesetzt. ${ }^{614}$ Bereits im Stadtrecht aus der Zeit um 1300 sind dort Hausgenossen belegt. Im Steuerregister von 1499 finden sich folgende Angaben: ${ }^{615}$ Im Petriviertel kamen auf 218 Hausbesitzer 203 Hausgenossen (48\%), im Nikolaiviertel auf 155 Eigentümer 119 Hausgenossen (43\%), im Domviertel $110 \mathrm{zu} 81$ (42\%) und im Jakobiviertel waren es 79 Eigentümer und 47 Hausgenossen (37\%). In den Vorstädten wurden 84 Anwesen mit 50 Hausgenossen (37\%) erfasst. Insgesamt ergibt sich daraus ein Zahlenverhältnis von 646 Eigentümerhaushalten zu 450 Hausgenossen, in Freiberg lebten also gegen Ende des 15. Jahrhunderts $41 \%$ der Haushalte zur Miete. Im 16. Jahrhundert scheint jedoch der Anteil der Miethaushalte rückläufig gewesen zu sein: Für das Jahr 1611 sind 980 Gebäude überliefert, zwei Jahre später werden jedoch nur 245 Hausgenossen erfasst, was einem Anteil von lediglich $20 \%$ entspricht. ${ }^{616}$ Nachdem sich für das Jahr 1699 wieder ein Anteil von weit über 50\% Miethaushalten errechnet (vgl. Tab. 16), müssen die Angaben zu 1611 kritisch hinterfragt werden, eventuell

\footnotetext{
${ }^{614}$ Vgl. hierzu und im Folgenden: HofFMANN/RiCHTER 2012, S. 220-223.

${ }^{615}$ Ebd., S. 221; Hoffmann und Richter versuchen eine begriffliche Trennung zwischen Hausgenossen, im engeren Sinne als Fremdmieter verstanden, und Inwohnern als Gruppe aus Fremdmietern, Familienangehörigen und Bediensteten.

${ }^{616}$ Ebd. S. 222.
} 
sind dort nicht alle Mieter und vor allem keine Untermieter erfasst. Die Zahlen für 1699 werden durch eine Erfassung aus dem Jahr 1681 bestätigt: In dieser sind 773 Hausgenossen in der Innenstadt und 317 Hausgenossen in den Vorstädten angegeben. Bezogen auf die für das Jahr 1699 aufgeführten 827 Häuser errechnet sich überschlägig ein Mieteranteil von 56\%.

Im ausgehenden 15. Jahrhundert, 1496, wurden in Zwickau 570 Hausbesitzer und 343 steuerzahlende Hausgenossen verzeichnet, von der letzteren Gruppe standen 41 Personen im verwandtschaftlichen Verhältnis mit den Hausbesitzern, sodass im engeren Sinn nur 302 Miethaushalte vorhanden waren. Dies entspricht etwa einem Anteil von 35\%. Unter Hinzuziehung der Personen, die keine Steuer zahlten, muss der Wert jedoch auf 40\%-45\% erhöht werden. ${ }^{617}$ Für Chemnitz ermittelt Helmut Bräuer für das Jahr 1557 sogar ein Verhältnis von 558 Hausbesitzerhaushalten zu 747 Miethaushalten, was einem Mieteranteil von 57\% entspricht. ${ }^{618} 140$ Jahre später errechnet sich exakt der gleiche Wert. ${ }^{619}$

Auch für Görlitz sind frühe Zahlen zum Anteil der Mietbevölkerung überliefert: ${ }^{620}$ Während der Mieteranteil 1426 in der Innenstadt bei 38,9\% lag, waren es in der Vorstadt nur 18,9\%. Das Bevölkerungswachstum des 15. Jahrhunderts zeichnete sich in Görlitz vor allem durch eine Zunahme der Mietverhältnisse in der Innenstadt aus, 1472 lag der Mieteranteil hier bereits bei 47,4\%, während er in den Vorstädten leicht auf 15,8\% sank. Die Vorstadt wies - wie allgemein üblich - eine deutlich kleinteiligere Bebauungsstruktur mit einem hohen Anteil von kleinen Häusern auf, die nur von einem Haushalt bewohnt wurden. Der Erwerb eines solchen Hauses war damit für eine viel größere Anzahl von Haushalten möglich. Diese Ausgangssituation muss sich aber schon bald geändert haben, denn im Jahr 1528 wohnten neben den 470 Eigentümern in den Vorstädten 476 Hausgenossen. ${ }^{621}$ Mit einem Anteil der Miethaushalte von gut $50 \%$ in der Vorstadt war sowohl der Anteil der Eigentümerhaushalte in der Vorstadt als auch der Mieteranteil in der Innenstadt überflügelt, denn zur gleichen Zeit sind für die Innenstadt lediglich 317 Hausgenossen bei 436 Eigentümern überliefert, was einem Anteil der Miethaushalte von $42 \%$ entspricht. Die Mieter wohnten weit überwiegend als inquilini (Einlieger) mit im Haus eines Bürgers ${ }^{622}$ und gehörten zu $85 \%$ zur Gruppe der ärmsten Stadtbewohner. Nur 15\% dieser Mieter zählt Jacob zu den „Kleinund Mittelbürgern“. Zusammenfassend lässt sich feststellen, dass die vier großen

\footnotetext{
617 BRÄUER 2003, S. $77 f$.

${ }^{618}$ Ebd. S. 78.

${ }^{619}$ Vgl. Tabelle 2.4., Sachsen Mittelstädte.

${ }^{620}$ MASCHKE 1967, S. 22, Anmerkung 129. Die Zahlen beruhen wohl im Wesentlichen auf einem Aufsatz von Richard Jecht 1896 zu den Geschossbüchern der Stadt.

${ }^{621}$ JAСОВ 1972, S. 23.

${ }^{622}$ Ebd.
} 
sächsischen Städte des ausgehenden Mittelalters, Chemnitz, Freiberg, Görlitz und Zwickau, in dieser Epoche zwischen 35\% und 47\% Miethaushalte aufwiesen.

Für die ehemalige freie Reichsstadt Dortmund sind Zahlen aus dem frühen 15. Jahrhundert überliefert. Allerdings beziehen sich diese nur auf den zentral gelegenen Bereich der Borgbauerschaft, die etwa ein Drittel der Stadt einnahm. Absolut ermittelt Monika Fehse unter den steuerzahlenden Einwohnern für das Jahr 1406150 Haushalte im eigenen Haus und 299 Miethaushalte. ${ }^{623}$ Sie geht daher insgesamt von 70\% Miethaushalten und nur 30\% Eigentümerhaushalten aus. ${ }^{624}$ Damit gleicht die Situation in Dortmund sehr viel stärker der Situation in Regensburg. Der Anteil der eigentumslosen Bevölkerung liegt deutlich über deren Anteil im fränkischen Kitzingen und in den sächsischen Mittelstädten. Wobei sich für all diese Städte keine solch frühen Zahlen überliefert haben. Auch wenn es sich um eine singuläre Überlieferung handelt, wird an der Situation in Dortmund deutlich, dass Eigentumslosigkeit schon um 1400 prägend für einen großen Bevölkerungsanteil gewesen sein kann.

Für die frühe Neuzeit bietet die Untersuchung von Katrin Keller zu kursächsischen Städten eine gute Vergleichsbasis zu den oben diskutierten acht fränkischen Mittelstädten. ${ }^{625}$ Sie analysiert die Bevölkerungsentwicklung anhand von drei Zeitschnitten: 1550, 1699 und 1811. Für die beiden letzten Zeitschnitte lässt sich der Anteil der eigentumslosen Haushalte ermitteln. Da für diesen Zeitraum auch flächige Daten für mittelfränkische Städte vorliegen, ist ein direkter Vergleich möglich. Unter den von ihr erfassten Datensätzen zu 95 Städten können ebenfalls acht Städte ermittelt werden, deren Einwohnerzahl ab 1550 über 3.500 lag. Hinzu kommen noch die beiden größten Städte Dresden und Leipzig. Sie werden in der nachfolgenden Tabelle bei den Mittelstädten jedoch nicht berücksichtigt, da beide 1699 bereits knapp über 20.000 Einwohner zählten. 1550 gehörte Dresden mit 7.693 Einwohnern und Leipzig mit 8.481 Einwohnern jedoch noch in diese Städtegruppe. Vor 1500 wies Dresden maximal 734 Haushaltungen (1489) auf, darf also zu diesem Zeitpunkt noch als Kleinstadt gelten. Die Entwicklung in Dresden wird ausführlich im Kapitel zu den Residenzstädten geschildert.

Die Werte zum Anteil der Miethaushalte sind für die Tabelle sehr überschlägig und pauschal ermittelt, der tatsächliche Anteil dürfte höher gelegen haben. In zwei Fällen kann direkt nachgewiesen werden, dass diese überschlägige Ermittlung aber vom Grundsatz her richtige Ergebnisse liefert. ${ }^{626}$ Während Leipzig und Dresden, wie bereits ausgeführt, nach dem Dreißigjährigen Krieg zu Großstädten

\footnotetext{
${ }^{623}$ Ebd. S. 265, Anm. 2.

${ }^{624}$ FEHSE 2005, S. 267 und Karte 10, S. $396 f$.

${ }^{625}$ KELLER 1997.

${ }^{626}$ Dies trifft für Freiberg 1699 und Chemnitz $1811 \mathrm{zu}$.
} 
mutierten, sinken Meißen, Pirna, Torgau und Zwickau aufgrund starker Bevölkerungsverluste auf das Niveau von größeren Kleinstädten. Der Wohndruck auf die bestehenden Häuser war hier entsprechend gering, was sich in der niedrigen Behausungsziffer im Jahr 1699 von lediglich 4,3 bis 5,9 Bewohnern pro Haus zeigt. Die gleiche Entwicklung, wenn auch in abgeschwächter Form, fand in Annaberg statt.

\begin{tabular}{|l|c|c|c|c|c|c|c|c|c|}
\hline Jahr & $\mathbf{1 5 5 0}$ & \multicolumn{9}{|c|}{1699} & \multicolumn{3}{c|}{1811} \\
\hline Stadt & Einwohner & Einwohner & Häuser & $\begin{array}{c}\text { Behausungs } \\
\text {-ziffer }\end{array}$ & $\begin{array}{c}\text { Miethaus } \\
\text {-halte }\end{array}$ & Einwohner & Häuser & $\begin{array}{c}\text { Behausungs } \\
\text {-ziffer }\end{array}$ & $\begin{array}{c}\text { Miethaus } \\
\text {-halte }\end{array}$ \\
\hline Annaberg & 5.411 & 3.391 & 575 & 6 & $27 \%$ & 4.643 & 593 & 7,8 & $45 \%$ \\
\hline Chemnitz & 3.901 & 4.873 & 484 & 10 & $57 \%$ & 13.193 & 916 & 14,4 & $70 \%$ \\
\hline Freiberg & 7.359 & 8.200 & 827 & 10 & $57 \%$ & 8.167 & 911 & 9,0 & $52 \%$ \\
\hline Meißen & 3.125 & 2.331 & 294 & 8 & $46 \%$ & 4.484 & 450 & 10,0 & $57 \%$ \\
\hline Pirna & 3.538 & 1.819 & 423 & 4 & $0 \%$ & 4.122 & 448 & 14,4 & $53 \%$ \\
\hline Torgau & 3.500 & 2.806 & 583 & 5 & $11 \%$ & 4.811 & 563 & 8,5 & $50 \%$ \\
\hline Wittenberg & 3.000 & 5.000 & 455 & 11 & $61 \%$ & 5.361 & 322 & 16,6 & $74 \%$ \\
\hline Zwickau & 7.031 & 2.968 & 635 & 5 & $8 \%$ & 4.380 & 770 & 5,7 & $24 \%$ \\
\hline Gesamt & $\mathbf{3 6 . 8 6 5}$ & $\mathbf{3 1 . 3 8 8}$ & $\mathbf{4 . 2 7 6}$ & $\mathbf{7}$ & $\mathbf{4 1 \%}$ & $\mathbf{4 9 . 1 6 1}$ & $\mathbf{4 . 9 7 3}$ & $\mathbf{9 , 9}$ & $\mathbf{5 7 \%}$ \\
\hline
\end{tabular}

Tabelle 16. Einwohnerzahlen zu den kursächsischen Mittelstädten 1550-1811 (KELLER 1997, Tab. 1, 7 und 11). Erfasst sind nur die Städte, die vor 1811 bereits 3.500 Einwohner aufwiesen. Der Anteil der Miethaushalte wurde von mir nur überschlägig ermittelt, in der Annahme, dass auf jedes Haus genau ein Eigentümer kam und alle übrigen Haushalte zur Miete wohnten. Als Haushaltsgröße dient der von Keller ermittelte Durchschnittswert von 4,3 Personen.

(Thomas Wenderoth 2019)

Chemnitz, Freiberg und Wittenberg wiesen dagegen eine deutlich höhere Behausungsziffer auf, diese lag 1699 zwischen 9,9 und 11,0 Bewohnern pro Haus und 1811 zwischen 9,0 und 16,6. Da die anderen fünf Mittelstädte im 18. Jahrhundert wieder deutlich wuchsen und nach 1800 sämtlich über 4.000 Einwohner zählten, ergibt sich für alle acht Mittelstädte 1811 ein durchschnittlicher Mieteranteil von $57 \%$ beziehungsweise eine Behausungsziffer von 9,9. Die sächsischen Ackerbürgerstädte kommen im Vergleich 1811 durchschnittlich nur auf eine Behausungsziffer von 6,1. ${ }^{627}$ Der Durchschnittswert aller 95 Städte lag im Jahr 1699 bei 5,3 und 1811 bei 7,3. Hinsichtlich der Behausungsziffer werden die kursächsischen Mittelstädte ihrem Namen gerecht und nehmen eine vermittelnde Rolle zwischen den Klein- und Großstädten ein.

Was die Belegung der Häuser und den Anteil der eigentumslosen Haushalte betrifft, bleiben die kursächsischen Mittelstädte zu Beginn des 19. Jahrhunderts jedoch deutlich hinter den mittelfränkischen Mittelstädten zurück. Für die Zeit um 1700 fehlen für die ehemaligen Reichsstädte leider Zahlen, sodass die Situation nicht in Gänze vergleichbar ist. Vermutlich dürften die Zahlen der mittelfränkischen und kursächsischen Mittelstädte aber nah beieinander gelegen haben. Die sächsischen Städte hatten durchschnittlich 41\% Miethaushalte und 7,3 Bewohner

${ }^{627}$ KeLLER 1997, Tabelle 10. 
pro Haus. In den fränkischen Städten lag der Mieteranteil 1703 zum Beispiel in Schwabach bei $41 \%$ und in Ansbach bei 37\%. ${ }^{628}$

Abschließend soll der Blick wieder zum Ende des Untersuchungszeitraumes wandern. Für das Jahr 1762 hat sich eine Haupt-Beschreibung Ingolstadts erhalten, in der sämtliche Haushalte erfasst sind. ${ }^{629}$ Unterschieden sind diese in zwei Rubriken: Haus=Eigenthümer und Innwohner. ${ }^{630}$ Diese stehen zudem in Spalten nebeneinander, sodass ersichtlich ist, welche Mieter bei welchem Eigentümer wohnten. Aufgeführt sind insgesamt 782 Hausnummern, geteilte Häuser spielten keine Rolle, nur in sechs Fällen sind halbe Hausnummern vergeben, sodass insgesamt 788 Anwesen verzeichnet sind.

Private Eigentümerhaushalte lassen sich insgesamt 621 ermitteln, denen insgesamt 654 Mieterhaushalte gegenüberstanden, damit errechnet sich ein Verhältnis von 49\%: 51\% zugunsten der Miethaushalte. Unter den Miethaushalten sind hier auch die Untermieter erfasst, denn es finden sich sehr viele Nennungen von alleinstehenden Frauen, die in einigen Fällen auch zu zweit oder dritt bei einem Vermieter wohnten. Von daher dürfte der Bevölkerungsanteil mit Hauseigentum im Hinblick auf die zahlenmäßig kleineren Miethaushalte deutlich über $50 \%$ gelegen haben. Aufgeführt unter der Rubrik der Inwohner sind in fünf Fällen auch Austrägler, diese wurden bei den Miethaushalten von mir jedoch nicht berücksichtigt. Im Hinblick auf die geringe Zahl würden sie das Ergebnis nicht verändern. Unter den Mietern werden aber nicht nur die Ärmsten der Armen genannt, sondern auch Bürgermeister und vor allem hochrangige Offiziere mit Adelsprädikat. Auf der anderen Seite gab es eine sehr große Anzahl von Taglöhnern auch unter den Eigentümern. Anzumerken ist noch, dass von den insgesamt 654 Miethaushalten 155 in öffentlichen Gebäuden wohnten, dies sind immerhin 24\% der Miethaushalte.

In einem älteren Verzeichnis aus dem Jahr 1675 werden 113 Mieter genannt, denen 643 Besitzer gegenüberstanden. ${ }^{631}$ Hieraus errechnet sich ein Mieteranteil von $15 \%$. Tatsächlich dürfte dieser aber um einiges höher gelegen haben, da es sich bei den dort genannten Besitzern nicht nur um Privatpersonen gehandelt hat, sondern auch kirchliches und städtisches Eigentum verzeichnet ist. Unter den Mietern sind wiederum nur sechs Arme ohne Berufsangaben erfasst. Hieraus

${ }^{628}$ StAN, Fm AN, Brdbg. Lit. Nr. 599 fol. 362-368' und 445-459. Zumindest in Ansbach wäre noch eine unbekannte Anzahl Miethaushalte von alleinstehenden Frauen hinzuzurechnen.

${ }^{629}$ Haupt-Beschreibung zufolge des gnädigst erlassenen Generalmandats und beigefügter Instruktion d. d. 11. Mai anno 1762 über die dermalen vorhandenen Wohnungen, Gebäude und Häuser, dann deren sonderbaren Inwohner nebst betreffenden Herdstätten, churfürstliche und bürgerliche beiderseitigen Jurisdiktionen in der churfürstlichen Hauptstadt Ingolstadt verfaßt den 15. Dezember anno 1762 Haupt-Beschreibung 1762.

${ }^{630}$ Vgl. Haupt-Beschreibung 1762.

${ }^{631}$ Vgl. hierzu und im Folgenden HofmANn 1965, S. 38. 
ist zu schließen, dass die große Gruppe der Untermieter(-innen) nicht enthalten ist. Die Zahlen von 1675 können also nicht direkt verglichen werden mit den sehr viel detaillierteren Angaben des Jahres 1762. Eine nennenswerte Zunahme der Mietbevölkerung ist dennoch herauszulesen. Damit verläuft die Entwicklung in Ingolstadt parallel zur Entwicklung in den ab dem Ende des 17. Jahrhunderts aufstrebenden mittelfränkischen Mittelstädten Ansbach, Erlangen und Schwabach.

\section{Kleinstädte}

Unter diesem Begriff sollen die kleinen Städte mit weniger als 3.500 Einwohnern verstanden werden. Dies entspricht etwa einer Zahl von 800 Haushalten. Die Städtelandschaft in Franken war geprägt durch eine Vielzahl dieser kleineren Städte; neben „unbedeutenden“ Landstädten finden sich in dieser Gruppe auch Städte, die beschränkte zentrale Funktionen übernahmen oder im Falle von Ansbach und Bayreuth sogar Hauptstädte größerer Territorien waren und bezogen auf ihre Einwohnerzahlen erst spät zu Mittelstädten anwuchsen.

Serielles Quellenmaterial zu einer größeren Zahl von Städten hat sich für Mittelfranken vom späten 15. Jahrhundert bis ins frühe 19. Jahrhundert erhalten. Die Situation dieser Städte im Hinblick auf die eigentumslose Bevölkerung soll anhand der vorliegenden Quellen chronologisch getrennt in drei Zeitphasen diskutiert werden.

Für das späte 15. Jahrhundert liegt als wertvolle Quelle das Reichssteuerregister für die markgräflich brandenburgischen Städte vor (vgl. Tab. 17).

Für das 17. und 18. Jahrhundert enthalten die Listen zur Erb- und Landeshuldigung eindeutige Hinweise auf den Anteil der Mietbevölkerung. Diese wurden von mir exemplarisch für das Fürstentum Brandenburg-Ansbach ausgewertet (vgl. Tab. Anhang I.1.-I.8.). Erschlossen ist damit der Zeitraum zwischen 1603 und 1723. Die Zahlen zu den ansbachischen Städten können für das 18. Jahrhundert anhand des Türkensteuerverzeichnisses der Reichsstadt Nürnberg von 1716/17 mit den Zahlen zu den vier nürnbergischen Landstädten (vgl. Tab. 21) verglichen werden.

Nach dem eigentlichen Ende des Untersuchungszeitraums wurde im Jahr 1818 die gesamte Bevölkerung des damaligen Rezatkreises, der in etwa dem heutigen Regierungsbezirk Mittelfranken entspricht, ortsweise erfasst. In Ermangelung anderer Quellen soll anhand dieses Verzeichnisses die Eigentumssituation um 1800 diskutiert werden (vgl. Tab. 23). 


\section{Mieteranteil in fränkischen Kleinstädten 1497}

Grundlegend für die Auswertung ist die Transkription der Listen des Reichssteuerregisters durch Gerhard Rechter. ${ }^{632}$ Eine Überprüfung der Zahlenangaben in den zugehörigen statistischen Zusammenfassungen hat jedoch viele Ungereimtheiten aufgezeigt, sodass ich mich entschlossen habe, für diese Untersuchung die Auszählung neu vorzunehmen.

Rechter bezieht die Gesamtzahl der Haushaltungen der Hausgenossen in seiner Tabelle auf die Herdgemeinschaften. Statistisch betrachtet führt das zu einem höheren Mieteranteil. Um die Zahlen vergleichbar zu halten zu den Tabellen aus späterer Zeit, habe ich mich demgegenüber entschlossen, die Zahl der Miethaushalte auf die Gesamtzahl aller Haushalte (Eigentümer und Mieter) zu beziehen. Rechter berücksichtigt zudem bei seiner Ermittlung des prozentualen Anteils auch nicht, ob bei dem betreffenden Vermieter ein, zwei oder mehr Miethaushalte lebten, sondern fasst die Gesamtzahl der Mieter zu einem Haushalt zusammen. Auch hier habe ich versucht, stattdessen die Anzahl der Miethaushalte an sich zu erfassen.

Relevant wird dies eigentlich erst, wenn mehr als ein Mieter im Haus genannt wird. Auch wenn es sich um zwei oder mehr alleinstehende Personen handelt, kann es bedeuten, dass diese einen gemeinsamen Haushalt führen. Nachweisbar ist dies jedoch nicht. Da sie rechtlich eigenständig waren, werden sie von mir auch als selbstständige (Einpersonen-)Haushalte geführt. Ehepaare oder noch größere Miethaushalte stellen die Ausnahme dar. Ehepaare sind zudem überwiegend als solche kenntlich gemacht, wie zum Beispiel im Eintrag Nr. 5799: Anna Reuchin, Appolonia, Barbara, ir dochter [Eigentümerhaushalt], Jorg Koch, Margaret, sein hawsfraw, sein ir hawsgenossen, Hanns Johenlein, Margaret, sein hawsfraw, sein auch hawsgenossen. ${ }^{633}$ Bei der oben genannten Anna Reuchin wohnten neben den beiden Töchtern Apollonia und Barbara zwei Ehepaare zur Miete: Jorg und Margaret Koch sowie Hanns und Margaret Johenlein. Ob zu den beiden Mieterpaaren noch kleine Kinder gehörten, ist nicht überliefert. Nicht abschließend zu klären ist darüber hinaus die Frage, ob die beiden Miethaushalte über getrennte Wohneinheiten verfügten oder in einer Herdgemeinschaft, eventuell sogar zusammen mit der Vermieterin, gelebt haben.

${ }^{632}$ RECHTER 1985 und 1988.

633 Zitiert nach RECHTER 1985, S. 225. 


\begin{tabular}{|c|c|c|c|c|c|c|c|c|c|c|c|c|}
\hline 1497 & Haus & halte, Anzo & & Personen & & Anzal & 1 HG/ & lerd & mei & cha & & Anteil \\
\hline Ort & $\begin{array}{c}\text { Herdgemein- } \\
\text { schaften }\end{array}$ & \begin{tabular}{|c|}
$\begin{array}{c}\text { Haus- } \\
\text { genossen }\end{array}$ \\
\end{tabular} & \begin{tabular}{|c|}
$\begin{array}{c}\text { Haushalte } \\
\text { gesamt }\end{array}$ \\
\end{tabular} & & 1 & 2 & 3 & 4 & 5 & 6 & 7 & $\begin{array}{c}\text { HG/ } \\
\text { Haushalte }\end{array}$ \\
\hline Ansbach gesamt & 450 & 46 & 46 & 1.240 & 41 & 6 & 1 & & & & & $100 \%$ \\
\hline Arzberg & 69 & 10 & 79 & 179 & 9 & 1 & & & & & & $13 \%$ \\
\hline Bayreuth & 310 & 33 & 343 & 891 & 21 & 4 & 2 & & & & & $10 \%$ \\
\hline Berneck & 76 & 1 & 77 & & 1 & & & & & & & $1 \%$ \\
\hline Crailsheim & 219 & 38 & 257 & 658 & 14 & 9 & 3 & 1 & 1 & & & $15 \%$ \\
\hline Erlangen & 92 & 1 & 93 & 212 & 1 & & & & & & & $1 \%$ \\
\hline Feuchtwangen & 151 & 37 & 188 & 442 & 13 & 14 & 3 & & & & & $20 \%$ \\
\hline Gunzenhausen & 217 & 9 & 226 & 548 & 9 & & & & & & & $4 \%$ \\
\hline Helmbrechts & 59 & 13 & 72 & 205 & 5 & 4 & 1 & & & & & $18 \%$ \\
\hline Hof, Vorstadt & 128 & 37 & 165 & & 24 & 9 & & 1 & & & & $22 \%$ \\
\hline Kulmbach gesamt & 291 & 21 & 312 & & 17 & 2 & & & & & & $7 \%$ \\
\hline Langenzenn & 112 & 17 & 129 & 324 & 6 & 9 & 2 & 1 & & & & $13 \%$ \\
\hline Neustadt/Aisch & 286 & 22 & 308 & 1.192 & 17 & 3 & & & & & & $7 \%$ \\
\hline Neustadt/Kulm & 101 & 12 & 113 & 267 & 11 & 1 & & & & & & $11 \%$ \\
\hline Roth & 111 & 35 & 146 & 339 & 8 & 7 & 2 & 2 & 1 & 1 & 1 & $24 \%$ \\
\hline Schauenstein & 38 & 9 & 47 & & 6 & 2 & & & & & & $19 \%$ \\
\hline Selb & 73 & 21 & 94 & & 9 & 10 & & & & & & $22 \%$ \\
\hline Uffenheim & 103 & 18 & 121 & 325 & 6 & 2 & 3 & & & & & $15 \%$ \\
\hline Wassertrüdingen & 149 & 18 & 167 & 395 & 17 & 1 & & & & & & $11 \%$ \\
\hline Weißenstadt & 138 & 3 & 141 & 343 & 2 & & & & & & & $2 \%$ \\
\hline Wunsiedel & 188 & 12 & 200 & 469 & 8 & 3 & & & & & & $6 \%$ \\
\hline Gesamt & 2.911 & 413 & 3.324 & & 245 & 87 & 17 & 5 & 2 & 1 & 1 & $12 \%$ \\
\hline
\end{tabular}

Tabelle 17. Hausgenossenhaushalte (= HG; = „Untermieter“) in Städten des Fürstentums Brandenburg-Ansbach-Kulmbach, nach dem Reichssteuerregister 1497 (Thomas Wenderoth 2019, nach RECHTER 1985 und 1988)

Auch 1497 ist der Begriff Hausgenosse sehr unterschiedlich verwandt worden. In Uffenheim werden zum Beispiel eine ganze Reihe von Angehörigen, Eltern und Kindern mit Familie als Hausgenossen bezeichnet, ${ }^{634}$ der darüberhinausgehende Großelternanteil in Uffenheim liegt mit drei Haushalten (= 3\%) entsprechend unter dem Durchschnittswert. Addiert man die sechs Altenteiler dazu, die selbst oder deren Kinder zugleich als Hausgenossen bezeichnet werden, kommt man auf einen Anteil von 9\% der Haushalte mit Altenteilern, einem Wert im oberen Bereich. Rechnet man die Familienangehörigen in Uffenheim, die explizit auch als Hausgenossen bezeichnet werden, jedoch zu den Hausgenossen, würde der Anteil der Hausgenossenhaushalte auf 19\% (24 Haushalte) steigen. ${ }^{635}$ Dieser Fall, bei dem Familienangehörige zugleich als Hausgenossen bezeichnet werden beziehungsweise Hausgenossen als Familienangehörige, ist aber lediglich in Uffenheim zu beobachten. Aus diesem Grund werden Eltern, Söhne und Töchter in

\footnotetext{
${ }^{634}$ Ebd. S. 97: 2717: Niclaus Gunther, sein sone und hausgenos; 2718: Enndris Lorß, sein aiden und hausgeno $\beta$, Barbara, sein hausfrau; 2729: Hanns Loe $\beta$, sein sone, hausgeno $\beta$, Cecilia, sein hausfrau, Fritz, sein sone, Anna, sein tochter. 2753: Conntz Vttenhofer, sein vatter, hausgenoß; 2785: Peter Peyel, sein aiden, hausgenoß; 2821: Fritz Stieber [...], Oswalt Koberle, sein aiden, haußgenoß, Anna, sein hausfrau, Hein (rich) Stieber, sein sone, haußgenos, Els sein hausfrau.

${ }^{635}$ Im Haushalt von Fritz Stieber lebten sowohl sein Schwiegersohn als auch sein Sohn mit ihren Familien; vgl. RECHTER 1995, S. 97.
} 
der Auswertung nicht als Hausgenossen im Sinne von Mietern gewertet, Schwäger jedoch schon. ${ }^{636}$

Inwieweit in den Listen tatsächlich alle Mieter unter dem Begriff des Hausgenossen erfasst wurden, muss dahingestellt bleiben. Auffällig ist zum Beispiel das Fehlen von Ehepaaren unter den Hausgenossen in Neustadt an der Aisch. Vermutlich verbergen sich unter einer ganzen Reihe von Haupteinträgen (Herdgemeinschaften) weitere Mieter, als solche eindeutig identifiziert werden konnten jedoch nur der Spitalmeister und seine Frau. ${ }^{637}$

Festzuhalten gilt: Anhand des Reichssteuerregisters lassen sich nicht die Mieter herausfinden, die ein ganzes Anwesen oder zumindest eine Herdstelle (Wohnung/Nebenhaus) gemietet hatten und nicht mit dem Eigentümer in einer Herdgemeinschaft lebten, da diese wohl überwiegend nicht als Hausgenossen etc. bezeichnet wurden.

Nachfolgend soll diese Gruppe exemplarisch anhand weiterer Quellen für die Stadt Wunsiedel ermittelt werden. Dort werden zum Beispiel im Jahr 1485 in einem Steuerregister 193 Hausbesitzer und 1492 im Wehrmusterungsregister 182 Hausbesitzer (?) genannt. ${ }^{638} \mathrm{Im}$ Jahr der Erhebung des Reichspfennigs, 1497, sind nur 178 Hausstellen überliefert, von denen zehn keinen Besitzer hatten und aufgrund wirtschaftlicher Schwierigkeiten wohl leer standen. 1509 waren sogar nur noch 159 Häuser bewohnt. Im Reichssteuerregister von 1497 sind dagegen insgesamt 188 Herdgemeinschaften aufgeführt. ${ }^{639}$ Daraus errechnet sich eine Anzahl von mindestens zwanzig Herdgemeinschaften, die nicht im Eigentum gewohnt haben können, da zur gleichen Zeit nur 168 bewohnte Häuser überliefert sind. Zudem lässt sich eine weitere Herdgemeinschaft bzw. ein Haushalt näher fassen: Es handelt sich um einen Mann namens Beber, der mit seiner Frau einen Stadtturm bewohnte. ${ }^{640}$ Weitere „versteckte“ Mieter sind anzunehmen. Bei den 188 Herdgemeinschaften sind nur elf Haushalte inkludiert, die als hawsgenossen/incola bezeichnet werden. ${ }^{641}$ Würde man allein diese statistisch als Mieter erfassen, läge der Anteil der Miethaushalte bei $6 \% .{ }^{642}$ Dieser muss jedoch deutlich höher gelegen haben, berücksichtigt man die zwanzig „überzähligen“ Herdgemeinschaften. Im Falle von Wunsiedel würde der Anteil der Mieter von 6\% auf $16 \%$ ansteigen, ohne dass die Geistlichen und sonstigen Personen, die nicht genannt werden, Berücksichtigung finden.

\footnotetext{
${ }^{636}$ Ebd.: 2779: Michel Schneiderin, hausgenos, Hanns Tuscherer, swager, hausgenos.

${ }^{637}$ Eintrag 11083, fol. 360' ; zitiert nach RECHTER 1985, S. 456.

${ }^{638}$ Vgl. hierzu und im Folgenden JÄGER 1987, S. 285f. Bei dem Wehrmusterregister ist nicht eindeutig geklärt, ob es sich bei den Einträgen tatsächlich um Hausbesitzer handelt.

${ }^{639}$ RECHTER 1985, S. 41-45.

${ }^{640}$ Ebd., S. 43.

${ }^{641}$ Darunter 8 alleinstehende Frauen, 2 ledige Männer und 2 Ehepaare; ebd., S. 41-45.

${ }^{642} 11$ hawsgenossen/incola bezogen auf insgesamt 199 Haushalte (188+11).
} 
Für die Stadt Neustadt am Kulm wiederum tritt bei der Auswertung als Problem auf, dass die Hausgenossen ohne Vor- und Familiennamen genannt sind. Es heißt dort: haußgenossin, ein haußgenossin, ein haußgenoß oder haußgenossen. ${ }^{643}$ Bei der letzten Form ist nicht ersichtlich, ob es sich um eine Maskulin-, Singular- oder Pluralform handelt. Bei der Auswertung habe ich mich dafür entschieden, die so Bezeichneten als männliche Mieter zu interpretieren, der prozentuale Anteil der Hausgenossinnen-Haushalte ist hier mit 36\% entsprechend gering.

Für Hof gilt die Feststellung in noch viel stärkerem Maß, dass die überlieferten Aufstellungen nicht immer ausreichend präzise sind, um die Anzahl der Miethaushalte in Erfahrung zu bringen: Während der Anteil der Miethaushalte in der Vorstadt bei 22\% liegt, lassen sich anhand der Überlieferung in der Innenstadt nur 1\% Miethaushalte nachweisen! Ursächlich hierfür könnte in diesem Fall auch die Bequemlichkeit des Schreibers gewesen sein, da die Listen des Oberlandes sich nur in Abschriften erhalten haben. ${ }^{644}$ Nicht auszuschließen ist, dass der Schreiber Zusätze wie Hausgenosse etc. einfach beim Abschreiben weggelassen hat. Diese These wird dadurch untermauert, dass sich am Anfang der Liste noch zweimal ein sehr detaillierter Hinweis auf Mietsituationen findet, in diesem Fall sogar mit Angaben zum Vermieter und zur konkreten Wohnsituation im Hinterhaus, ${ }^{645}$ in den folgenden Einträgen aber jegliche Differenzierung fehlt. Die Hofer Innenstadt wurde daher von mir nicht weiter betrachtet.

In Ansbach fällt beim Reichssteuerregister die vielfache Nennung alter Frauen auf, als stellvertretend können folgende Einträge gelten: 572 Hermann Peßmann, sein weib unnd ein alts Frewlein; 588 Langhanns, sein weib unnd die alt Pegnin. ${ }^{646}$ Unklar bleibt hierbei, ob ein verwandtschaftliches Verhältnis zwischen den Bewohnern bestanden hat oder nicht. Da alleinstehende Frauen im ausgehenden Mittelalter in den Listen des Reichssteuerregisters die größte fassbare Mietergruppe bildeten, in Ansbach ansonsten aber erstaunlich wenig Mieter (Hausgenossen) verzeichnet sind, wäre die absolute Zahl der Mieter in einzelnen Städten ebenfalls zu erweitern. Die Gruppe der als Hausgenossen in den Listen bezeichneten Personen wird dabei ergänzt von Einzelpersonen, die ich dieser Gruppe zurechnen möchte. Folgende Einträge sind für diese Gruppe charakteristisch: 5735 Heintz Kadisch, Barb, sein tochter, alt Tischnerin, Heintz, sein Sohn. ${ }^{647}$ Die als alt Tischnerin bezeichnete Frau gehört der charakteristischen Mietergruppe der

\footnotetext{
${ }^{643}$ StABA, Geheimes Hausarchiv Plassenburg, Akten und Bände 5977/1, fol. 13; zitiert nach RECHTER 1988, S. 14-16.

${ }^{644}$ RECHTER 1988, S. XIV.

${ }^{645}$ StABA, Geheimes Hausarchiv Plassenburg, Akten und Bände 5977/1, fol. 133-134, zitiert nach RECHTER 1988, S. 134-135.

${ }^{646}$ RECHTER 1985, S. 26.

${ }^{647}$ StABA, Geheimes Hausarchiv Plassenburg, Akten und Bände 5977/1, fol. 158' , zitiert nach Rechter 1988, S. 156.
} 
alten alleinstehenden Frauen an. Sie wird zumindest nicht durch einen Zusatz als Familienmitglied oder Gesinde bestimmt. Nicht auszuschließen ist jedoch, dass es sich um eine ausgediente Magd handelt, die im Haushalt ihres ehemaligen Dienstherrn ihr „Gnadenbrot“ erhält. Was im Einzelfall zutreffen kann, gilt jedoch sicher nicht allgemein. Zum Beispiel sind in der Vorstadt von Hof in Oberfranken für 128 Anwesen 30 alleinstehende Frauen überliefert, die hier auch sämtlich als Hausgenossinnen bezeichnet sind, diesen standen lediglich zwei Mägde im aktiven Dienst gegenüber. Aber auch wenn es sich bei einem Teil der alleinstehenden Frauen um ehemalige Mägde gehandelt hätte, die eventuell keine Miete zahlten, blieben es doch familienfremde Personen, von daher ist die Zuordnung in die Gruppe der Hausgenossen nicht grundsätzlich verkehrt. Auch Frauen, die nicht als alt bezeichnet sind, wurden von mir dieser Mietergruppe zugeordnet, sofern aus dem Kontext abzuleiten ist, dass sie nicht direkt zur Familie gehören, zum Beispiel in den Fällen, wo sie einen anderen Nachnamen tragen. ${ }^{648}$ Das Gleiche gilt für alleinstehende Männer. ${ }^{649} \mathrm{Im}$ Übrigen sind die alleinstehenden Frauen, die in einem „fremden“ Haushalt mitleben, in der Tabelle noch einmal separat erfasst, da sie die größte Gruppe unter den Hausgenossen bilden und vermutlich nicht in unserem heutigen Sinn eine eigene Wohnung für sich allein bewohnt haben.

Im Hinblick auf die Abweichungen in den Einzelaufstellungen konnten nur zwanzig hohenzollerische Städte herangezogen werden, deren Angaben miteinander vergleichbar erscheinen. Es sind dies in alphabetischer Reihenfolge: Ansbach, Arzberg, Bayreuth, Berneck, Crailsheim, Erlangen, Feuchtwangen, Gunzenhausen, Helmbrechts, Kulmbach, Langenzenn, Neustadt an der Aisch, Neustadt am Kulm, Roth, Schauenstein, Selb, Uffenheim, Wassertrüdingen, Weißenstadt, Wunsiedel. Zusätzlich wurde von der Stadt Hof die Vorstadt, die als Altstadt bezeichnet wird, in die Betrachtung miteinbezogen, zum einen ist sie relativ groß, zum anderen lässt sie Rückschlüsse auf die Gesamtstadt zu.

\section{Quantitative Verteilung der Mieterzahlen 1497}

In den untersuchten 21 Städten liegt der Anteil der Haushalte von Hausgenossen zwischen $24 \%$ und $1 \%$ bezogen auf alle Haushalte (vgl. Tab. 17). An der Spitze stehen Roth (24\%), gefolgt von Selb und Helmbrechts (22\%), es schließen an Feuchtwangen (20\%) und Schauenstein (19\%), alles kleine Städte; einzig Feuchtwangen liegt hinsichtlich der Größe mit 151 Hauseinträgen im Mittelfeld der

${ }^{648}$ Zum Beispiel Katharina Pethmul: 5744 Berbingin, Steffan, ir sun, Barb, Martsch, ir tochter, Katherina Pethm[u]lin; ebd., fol. 158', zitiert nach RECHTER 1988, S. 156.

${ }^{649}$ Zum Beispiel Hans Preunlein: 5649 Fritz Kursner, sein weib, Hans Preunlein; ebd., fol. 157', zitiert nach RECHTER 1988, S. 154. 
Städte. Weit oben beim Anteil der Hausgenossen liegt ebenfalls die Hofer Vorstadt (22\%). Bei den Schlusslichtern in der Liste handelt es sich ebenfalls um sehr kleine Städte: Erlangen und Berneck (1\%) sowie Weißenstadt (2\%). Erstaunlich ist, dass hier direkt die Gruppe der größten Kleinstädte anschließt: Gunzenhausen (4\%), Wunsiedel (6\%), Kulmbach und Neustadt an der Aisch (7\%), Ansbach (9\%) sowie Bayreuth (10\%). Der durchschnittliche Anteil der Hausgenossen liegt, bezogen auf alle untersuchten Städte, bei 11\%, unter den insgesamt 3.361 Listeneinträgen befinden sich 415 Einträge mit Hausgenossen. Das Mittelfeld der Städte bewegt sich leicht darüber und weist zwischen 11\% und 15\% Miethaushalte auf. Diese Städte gehören den größeren Kleinstädten an, einzig Arzberg mit nur 69 Hauseinträgen und Crailsheim mit 219 Hauseinträgen fallen hinsichtlich der Stadtgröße etwas aus dem Rahmen.

Hohe Mieterzahlen stellen in der Regel ein Indiz für eine prosperierende Wirtschaft mit einem hohen Anteil an frühindustriellem Gewerbe dar. ${ }^{650}$ Für Roth mag dies uneingeschränkt gelten, stand die Stadt doch auch in späteren Jahrhunderten im Sog der nahen Wirtschaftsmetropole Nürnberg, die früh Teile der Produktion ins Umland auslagerte. Die drei oberfränkischen Zwergstädte Selb, Schauenstein und Helmbrechts bilden eine weitere Gruppe; hier wäre zu prüfen, inwieweit der Bergbau und die nachfolgende Metallverarbeitung Ende des 15. Jahrhunderts dort die wirtschaftliche Situation geprägt haben. ${ }^{651}$ Eine Erklärung für den verhältnismäßig hohen Hausgenossenanteil in Feuchtwangen fehlt bisher, in den Städten vergleichbarer Größe und Bedeutung im Fürstentum wurden im gleichen Zeitraum deutlich weniger Hausgenossen erfasst.

\section{Stadtgröße und Mieteranteil 1497}

Ein Zusammenhang zwischen der Größe einer Kleinstadt und dem überlieferten prozentualen Anteil an Hausgenossen im Jahr 1497 ist in keiner Weise zu erkennen (vgl. Tab. 17).

Die großen Städte wie Ansbach und Bayreuth, Kulmbach und Neustadt an der Aisch hatten max. 10\% Hausgenossen und liegen damit unterhalb des Durchschnittswertes von 11\%. Für Kitzingen und die Gesamtstadt Hof, als die beiden damals größten Städte des Fürstentums, liegen leider für 1497 keine Zahlen zu den Hausgenossen vor. Spätere Zahlen deuten aber darauf hin, dass neben der Kleinstadt Roth vor allem Schwabach seit Anbeginn über einen hohen Mieteranteil verfügte. Für das Jahr 1530 überliefert das Salbuch für Schwabach 303 Häuser und zusätzlich 144 Beständer und 40 Witwen (ohne Hauseigentum), was einem Mieteranteil von 37\% entspricht! ${ }^{652}$ Erstaunlich ist zudem, dass die Zwergstädte

\footnotetext{
${ }^{650}$ WENDEROTH 2019a, S. 49-51.

${ }^{651}$ Vgl. KÖHL 1913, S. 29-32.

${ }^{652}$ StAN, Fm AN Salbücher Nr. 92, fol. 109-121. Vgl. auch DEHM/HECKEL 1970, S. 7; WOLFGANG DIPPERT: (Art.) Bevölkerungszahlen, in: HistORISCHES STADTLEXIKON SCHWABACH 2008, S. 77.
} 
in Oberfranken, wie bereits erwähnt, durch verhältnismäßig hohe Mieteranteile auffallen. Die Größe einer Stadt hatte also am Ende des Mittelalters keine unmittelbare Auswirkung auf den Mieteranteil, betrachtet man lediglich die hier analysierte Gruppe der kleineren Städte.

\section{Hausgenossenhaushalte 1497: Frauen und alleinstehende Männer}

Auffällig ist bei genauerer Betrachtung der Zahlen der hohe Anteil an Hausgenossenhaushalten mit weiblichem Haushaltsvorstand. Da ich die Einträge aus denen das Geschlecht nicht eindeutig hervorgeht, den männlichen Hausgenossenhaushalten zugerechnet habe, dürfte der tatsächliche Anteil der weiblichen Hausgenossenhaushalte sogar etwas höher gelegen haben.

\begin{tabular}{|c|c|c|c|c|c|c|c|c|c|}
\hline 1497 & & Haus & shalte, & Anzahl & & Hausgeno & ssenanteil & Aufteilung $\mathrm{H}$ & ausgenossen \\
\hline Ort & $\begin{array}{c}\text { Herdgemein- } \\
\text { schaften }\end{array}$ & \begin{tabular}{|l|} 
HG \\
\end{tabular} & \begin{tabular}{|c|} 
davon \\
HG \\
weibl.
\end{tabular} & \begin{tabular}{|c|} 
davon \\
HG- \\
Ehepaare
\end{tabular} & \begin{tabular}{|c|} 
Haushalte \\
gesamt
\end{tabular} & $\begin{array}{c}\text { HG/ } \\
\text { alle } \\
\text { Haushalte }\end{array}$ & \begin{tabular}{|c|} 
HG \\
weibl./ alle \\
Haushalte
\end{tabular} & \begin{tabular}{|c|} 
alleinst. \\
Frauen/HG
\end{tabular} & $\begin{array}{c}\text { Ehepaare/ } \\
\text { HG }\end{array}$ \\
\hline Ansbach gesamt & 450 & 46 & 33 & 3 & 496 & $9 \%$ & $7 \%$ & $72 \%$ & $7 \%$ \\
\hline Arzberg & 69 & 10 & 8 & 1 & 79 & $13 \%$ & $10 \%$ & $80 \%$ & $10 \%$ \\
\hline Bayreuth & 310 & 33 & 24 & 2 & 343 & $10 \%$ & $7 \%$ & $73 \%$ & $8 \%$ \\
\hline Berneck & 76 & 1 & 1 & 0 & 77 & $1 \%$ & $1 \%$ & $100 \%$ & $0 \%$ \\
\hline Crailsheim & 219 & & - & - & 219 & $15 \%$ & & & \\
\hline Erlangen & 92 & 1 & 0 & 0 & 93 & $1 \%$ & $0 \%$ & $0 \%$ & $0 \%$ \\
\hline Feuchtwangen & 151 & 37 & 20 & 12 & 188 & $20 \%$ & $11 \%$ & $54 \%$ & $60 \%$ \\
\hline Gunzenhausen & 217 & 9 & 9 & 0 & 226 & $4 \%$ & $4 \%$ & $100 \%$ & $0 \%$ \\
\hline Helmbrechts & 59 & 13 & 10 & 3 & 72 & $18 \%$ & $14 \%$ & $77 \%$ & $30 \%$ \\
\hline Hof, Vorstadt & 128 & 37 & 30 & 3 & 165 & $22 \%$ & $18 \%$ & $81 \%$ & $8 \%$ \\
\hline Kulmbach gesamt & 291 & 21 & 14 & 0 & 312 & $7 \%$ & $4 \%$ & $67 \%$ & $0 \%$ \\
\hline Langenzenn & 112 & 17 & 5 & 2 & 129 & $13 \%$ & $4 \%$ & $29 \%$ & $12 \%$ \\
\hline Neustadt/Aisch & 286 & 22 & 20 & 2 & 308 & $7 \%$ & $6 \%$ & $91 \%$ & $10 \%$ \\
\hline Neustadt/Kulm & 101 & 12 & 6 & 0 & 113 & $11 \%$ & $5 \%$ & $50 \%$ & $0 \%$ \\
\hline Roth & 111 & 35 & 9 & 23 & 146 & $24 \%$ & $6 \%$ & $26 \%$ & $66 \%$ \\
\hline \begin{tabular}{|l|} 
Schauenstein \\
\end{tabular} & 38 & 9 & 4 & 3 & 47 & $19 \%$ & $9 \%$ & $44 \%$ & $33 \%$ \\
\hline Selb & 73 & 21 & 11 & 5 & 94 & $22 \%$ & $12 \%$ & $52 \%$ & $24 \%$ \\
\hline Uffenheim & 103 & 18 & 13 & 2 & 121 & $15 \%$ & $11 \%$ & $72 \%$ & $11 \%$ \\
\hline Wassertrüdingen & 149 & 18 & 16 & 0 & 167 & $11 \%$ & $10 \%$ & $89 \%$ & $0 \%$ \\
\hline Weißenstadt & 138 & 3 & 3 & 0 & 141 & $2 \%$ & $2 \%$ & $100 \%$ & $0 \%$ \\
\hline Wunsiedel & 188 & 12 & 6 & 2 & 200 & $6 \%$ & $3 \%$ & $50 \%$ & $17 \%$ \\
\hline Gesamt & 3.361 & 375 & 242 & 63 & 3.736 & $10 \%$ & $6 \%$ & $65 \%$ & $17 \%$ \\
\hline
\end{tabular}

Tabelle 18. Hausgenossen (HG) - alleinstehende Frauen und Ehepaare (Familien) - in Städten des Fürstentums Brandenburg-Ansbach-Kulmbach, nach dem Reichssteuerregister 1497 (Thomas Wenderoth 2019, nach RECHTER 1985 und 1988)

Für die untersuchten 21 Städte lassen sich 413 Hausgenossenhaushalte ermitteln, von denen 242 Haushalte einen weiblichen Haushaltsvorstand hatten, dies entspricht 59\%. Lässt man Crailsheim unberücksichtigt, da dort keine Geschlechterdifferenzierung erkennbar ist, erhöht sich der prozentuale Anteil sogar auf $65 \%$ ! Hinzu kommt noch eine große Anzahl von wohl alleinstehenden männlichen Hausgenossen. Ehepaare mit oder ohne Kinder stellen dagegen nur eine Minder- 
heit unter den aufgeführten Hausgenossenhaushalten. Nur bei 17\% der Hausgenossenhaushalte handelt es sich um Ehepaare. Auch hier ist das Problem gegeben, dass in den Einträgen nicht immer ersichtlich ist, ob es sich um ein Ehepaar oder zwei Einpersonenhaushalte handelt, wenn es wie im folgenden Eintrag heißt: zwen hawsgenossen. ${ }^{653}$ Berücksichtigt habe ich in der Tabelle jedoch nur Einträge, bei denen es sich offensichtlich um ein Ehepaar handelt. In einigen wenigen Fällen lassen sich auch Hausgenossinnen mit erwachsenen Kindern nachweisen, inwieweit die Haushalte Kinder unter fünfzehn Jahren beherbergten, lässt sich nicht klären.

Aufgrund des hohen Anteils von alleinstehenden Mietern und vor allem alleinstehenden Frauen dürfte es sich bei den im Rahmen des Reichssteuerregisters erfassten Hausgenossen weitgehend um Untermieter gehandelt haben. Die Zahlen können also in der Regel nicht mit den sonstigen Zahlen zu eigentumslosen Haushalten verglichen werden.

\section{Mieteranteil in fränkischen Kleinstädten der Barockzeit}

Für das Ansbacher Territorium haben sich Huldigungslisten aus der Zeit zwischen 1603 bis 1723 erhalten, diese wurden auf die Fragestellung nach der eigentumslosen Bevölkerung ausgewertet. Eine tabellarische Zusammenfassung findet sich im Anhang. Aufgeführt sind dort fünfzehn Städte. Allerdings ist die Überlieferung für die erste Hälfte des 17. Jahrhunderts sehr lückenhaft. Die zeitliche Abfolge der Listen ist stellenweise sehr eng (1603, 1639/41, 1668, 1673, $1686 / 87,1695,1703,1723)$ und spiegelt den häufigen Regentenwechsel in dieser Zeit wider.

Für das Nürnberger Territorium ist dagegen als serielle Quelle nur das Türkensteuerverzeichnis aus dem Jahr 1716/17 überliefert, sodass für diese vier Städte keine engmaschige zeitliche Entwicklung der Mietbevölkerung nachvollzogen werden kann. Dafür enthält das Türkensteuerverzeichnis deutlich detailliertere Angaben zur erfassten Bevölkerung.

\section{Die brandenburg-ansbachischen Kleinstädte 1603 bis 1723}

Grundlage für die Analyse sind die Huldigungslisten des Fürstentums, sofern sie sich im Staatsarchiv Nürnberg erhalten haben. Die Ergebnisse sind in Tabelle 19 zusammengefasst. Folgende Einschränkungen sind beim Lesen unbedingt zu berücksichtigen:

Die Prozentangaben sind anhand der Zahlen in den Huldigungslisten ermittelt. Juden wurden nur bei den Mietern berücksichtigt, wenn eine Differenzierung in

${ }^{653}$ Langenzenn 1497, Eintrag 2184; RECHTER 1985, S. 78. 
Eigentümer und Mieter im Rahmen der Erfassung erfolgte. Öffentlich Bedienstete wurden ebenfalls nur dann den Mietern zugerechnet, wenn sie als solche kenntlich gemacht wurden. Weibliche Miethaushalte fehlen häufig in den Listen, Untermieter(innen) scheinen nicht erfasst zu sein. Aus allen diesen Gründen liegen die angegebenen Prozentsätze sicher mehr oder weniger stark unterhalb der realen Situation und geben lediglich ein Minimum an Mietbevölkerung wieder. Erfasst sind in acht Zeitabschnitten insgesamt gut 20.000 Einträge. Im Anhang finden sich die detaillierten Zusammenfassungen zu den einzelnen Erb- und Landes-huldigungen mit Quellennachweisen.

Im Hinblick auf ihre bescheidene Stadtgröße können alle ansbachischen Städte bis 1723 weiterhin als Kleinstädte gelten und behandelt werden. Es sind dies in alphabetischer Reihenfolge: Crailsheim, Creglingen, Feuchtwangen, Gunzenhausen, Leutershausen, Langenzenn, Mainbernheim, Merkendorf, Ansbach (Onoldsbach), Prichsenstadt, Roth, Schwabach, Uffenheim, Wassertrüdingen und Windsbach.

Die größten Städte, Ansbach und Schwabach, wiesen 1723 lediglich 768 beziehungsweise 747 Haushalte auf. An dieser Stelle muss noch einmal betont werden, dass allem Anschein nach alleinstehende Mieterinnen nur selten erfasst wurden und daher die Zahlen zwar den größten Teil der Miet-Haushalte wiedergeben, aber eben nicht als vollständig betrachtet werden können. Dies ändert allerdings grundsätzlich nichts an der bescheidenen Größe der Städte. Die kleinsten Städte kamen lediglich auf gut 100 Haushaltungen. 1603 hatte Langenzenn 106 Haushalte, 120 Jahre später bildete Merkendorf mit 135 Haushalten das Schlusslicht. Einen Tiefpunkt bildete sicher der Dreißigjährige Krieg mit den Kampfhandlungen um Nürnberg vor allem in den Jahren 1632 bis 1634. Die Kriegsfolgen sind deutlich in den Bevölkerungszahlen der Landstädte nachvollziehbar: In Wassertrüdingen wurden 1639 nur noch 60 Haushalte gezählt, in Gunzenhausen waren es 96 und in Creglingen zwei Jahre später immerhin 117. Überraschend im Hinblick auf die Frage nach der Mietbevölkerung erscheint die Feststellung, dass der Anteil der Miethaushalte nicht merklich zurückgegangen ist. Für Creglingen ergab die Zählung $8 \%$ und für Gunzenhausen $20 \%$, Werte, die sich bis zum Jahr 1723 kaum verändern. Eine Erklärung für dieses Phänomen könnte darin liegen, dass es überlebenden Familien nicht möglich war, in diesen Jahren wieder ein Haus zu errichten, beziehungsweise dass nicht nur der Verlust an Bevölkerung, sondern auch der Verlust an Häusern gravierend war. Denn in den folgenden Jahren, mit dem beginnenden Wiederaufbau, sinkt der Anteil der Miethaushalte deutlich. Die Voraussetzungen zum Erwerb einer Immobilie waren im Hinblick auf die vielen öden Hausstellen günstig. 


\begin{tabular}{|c|c|c|c|c|c|c|c|c|}
\hline Jahr & 1603 & $\begin{array}{c}1639 \\
/ 41\end{array}$ & 1668 & 1673 & $\begin{array}{c}1686 \\
/ 87\end{array}$ & 1695 & 1703 & 1723 \\
\hline Crailsheim, Mieter in \% & $3 \%$ & k. A. & $8 \%$ & $5 \%$ & $11 \%$ & $6 \%$ & $10 \%$ & $14 \%$ \\
\hline Miethaushalte, Anzahl & 9 & & 18 & 12 & 28 & 15 & 28 & 50 \\
\hline alle Haushalte, Anzahl & 355 & & 238 & 250 & 254 & 255 & 281 & 360 \\
\hline $\begin{array}{l}\text { Eigentümerhaushalte und öde } \\
\text { Plätze }\end{array}$ & 346 & & 220 & 238 & 226 & 240 & 253 & 310 \\
\hline $\begin{array}{l}\text { öde, unbesetzte Hausstellen, } \\
\text { Anzahl }\end{array}$ & & & k. A. & k. A. & k. A. & k. A. & k. A. & \\
\hline Creglingen, Mieter \% & k. A. & $8 \%$ & $9 \%$ & $6 \%$ & $4 \%$ & $7 \%$ & $5 \%$ & $9 \%$ \\
\hline Miethaushalte, Anzahl & & 19 & 13 & 9 & 7 & 11 & 8 & 17 \\
\hline alle Haushalte, Anzahl & & 117 & 147 & 151 & 160 & 148 & 157 & 190 \\
\hline $\begin{array}{l}\text { Eigentümerhaushalte und öde } \\
\text { Plätze }\end{array}$ & & 108 & 126 & 142 & 153 & 137 & 149 & 181 \\
\hline $\begin{array}{l}\text { öde, unbesetzte Hausstellen, } \\
\text { Anzahl }\end{array}$ & & & k. A. & k. A. & k. A. & k. A. & k. A. & 8 \\
\hline Feuchtwangen, Mieter in \% & $18 \%$ & k. A. & $15 \%$ & $11 \%$ & $6 \%$ & $5 \%$ & $15 \%$ & $15 \%$ \\
\hline Miethaushalte, Anzahl & 35 & & 27 & 20 & 10 & 8 & 29 & 36 \\
\hline alle Haushalte, Anzahl & 198 & & 185 & 176 & 169 & 176 & 200 & 240 \\
\hline $\begin{array}{l}\text { Eigentümerhaushalte und öde } \\
\text { Plätze }\end{array}$ & 163 & & 167 & 174 & 170 & 191 & 188 & 212 \\
\hline $\begin{array}{l}\text { öde, unbesetzte Hausstellen, } \\
\text { Anzahl }\end{array}$ & & & 9 & 18 & 11 & 23 & 17 & 8 \\
\hline Gunzenhausen, Mieter \% & k. A. & $20 \%$ & $20 \%$ & $21 \%$ & $21 \%$ & $10 \%$ & $8 \%$ & $16 \%$ \\
\hline Miethaushalte, Anzahl & & 19 & 36 & 40 & 46 & 21 & 16 & 43 \\
\hline alle Haushalte, Anzahl & & 96 & 177 & 194 & 222 & 209 & 211 & 277 \\
\hline $\begin{array}{l}\text { Eigentümerhaushalte und öde } \\
\text { Plätze }\end{array}$ & & 77 & 193 & 204 & 218 & 188 & 195 & 234 \\
\hline $\begin{array}{l}\text { öde, unbesetzte Hausstellen, } \\
\text { Anzahl }\end{array}$ & & k. A. & 52 & 50 & 42 & k. A. & k. A. & k. A. \\
\hline Leutershausen, Mieter in \% & k. A. & k. A. & $7 \%$ & $6 \%$ & $5 \%$ & $13 \%$ & $12 \%$ & $13 \%$ \\
\hline Miethaushalte, Anzahl & & & 7 & 6 & 5 & 14 & 13 & 21 \\
\hline alle Haushalte, Anzahl & & & 102 & 106 & 103 & 104 & 105 & 162 \\
\hline $\begin{array}{l}\text { Eigentümerhaushalte und öde } \\
\text { Plätze }\end{array}$ & & & 95 & 100 & 98 & 128 & 92 & 141 \\
\hline $\begin{array}{l}\text { öde, unbesetzte Hausstellen, } \\
\text { Anzahl }\end{array}$ & & & k. A. & k. A. & k. A. & 38 & k. A. & k.A. \\
\hline Langenzenn, Mieter in \% & k. A. & k. A. & $7 \%$ & $14 \%$ & $11 \%$ & $16 \%$ & $24 \%$ & $31 \%$ \\
\hline Miethaushalte, Anzahl & & & 6 & 15 & 13 & 19 & 35 & 51 \\
\hline alle Haushalte, Anzahl & 106 & & 84 & 104 & 118 & 120 & 144 & 165 \\
\hline $\begin{array}{l}\text { Eigentümerhaushalte und öde } \\
\text { Plätze }\end{array}$ & & & 99 & 89 & 105 & 101 & 109 & 114 \\
\hline $\begin{array}{l}\text { öde, unbesetzte Hausstellen, } \\
\text { Anzahl }\end{array}$ & & & 21 & k. A. & k. A. & k.A. & k. A. & k. A. \\
\hline
\end{tabular}




\begin{tabular}{|c|c|c|c|c|c|c|c|c|}
\hline Mainbernheim, Mieter in \% & k. A. & k. A. & $8 \%$ & $13 \%$ & $4 \%$ & $9 \%$ & $12 \%$ & $12 \%$ \\
\hline Miethaushalte, Anzahl & & & 12 & 23 & 8 & 17 & 24 & 32 \\
\hline alle Haushalte, Anzahl & & & 150 & 172 & 198 & 191 & 200 & 272 \\
\hline $\begin{array}{l}\text { Eigentümerhaushalte und öde } \\
\text { Plätze }\end{array}$ & & & 177 & 149 & 190 & 197 & 176 & 240 \\
\hline $\begin{array}{l}\text { öde, unbesetzte Hausstellen, } \\
\text { Anzahl }\end{array}$ & & & 39 & k. A. & k. A. & 23 & k. A. & k.A. \\
\hline Merkendorf, Mieter \% & k. A. & k. A. & k. A. & k. A. & $17 \%$ & $15 \%$ & k. A. & $25 \%$ \\
\hline Miethaushalte, Anzahl & & & & & 18 & 15 & & 34 \\
\hline alle Haushalte, Anzahl & & & & & 108 & 100 & & 135 \\
\hline $\begin{array}{l}\text { Eigentümerhaushalte und öde } \\
\text { Plätze }\end{array}$ & & & & & 92 & 88 & & 101 \\
\hline $\begin{array}{l}\text { öde, unbesetzte Hausstellen, } \\
\text { Anzahl }\end{array}$ & & & & & 2 & 3 & & k.A. \\
\hline Ansbach, Mieter in \% & k. A. & k. A. & $25 \%$ & $23 \%$ & 0 & $28 \%$ & $37 \%$ & $46 \%$ \\
\hline Miethaushalte, Anzahl & & & 87 & 103 & k.a. & 132 & 168 & 353 \\
\hline alle Haushalte, Anzahl & & & 354 & 441 & 464 & 478 & 457 & 768 \\
\hline $\begin{array}{l}\text { Eigentümerhaushalte und öde } \\
\text { Plätze }\end{array}$ & & & 267 & 359 & 464 & 346 & 289 & 415 \\
\hline $\begin{array}{l}\text { öde, unbesetzte Hausstellen, } \\
\text { Anzahl }\end{array}$ & & & k. A. & 21 & k. A. & k. A. & k. A. & k. A. \\
\hline Prichsenstadt, Mieter in \% & k. A. & k. A. & $4 \%$ & k. A. & $11 \%$ & 0 & $4 \%$ & $11 \%$ \\
\hline Miethaushalte, Anzahl & & & 3 & & 11 & 0 & 4 & 15 \\
\hline alle Haushalte, Anzahl & & & 72 & & 103 & 101 & 112 & 137 \\
\hline $\begin{array}{l}\text { Eigentümerhaushalte und öde } \\
\text { Plätze }\end{array}$ & & & 69 & & 92 & 101 & 108 & 122 \\
\hline $\begin{array}{l}\text { öde, unbesetzte Hausstellen, } \\
\text { Anzahl }\end{array}$ & & & k. A. & & k. A. & k. A. & k. A. & k. A. \\
\hline Roth, Mieter in \% & $22 \%$ & k. A. & $9 \%$ & $7 \%$ & $18 \%$ & $8 \%$ & $19 \%$ & $27 \%$ \\
\hline Miethaushalte, Anzahl & 39 & & 13 & 8 & 27 & 10 & 27 & 62 \\
\hline alle Haushalte, Anzahl & 181 & & 137 & 121 & 151 & 124 & 143 & 226 \\
\hline $\begin{array}{l}\text { Eigentümerhaushalte und öde } \\
\text { Plätze }\end{array}$ & 142 & & 142 & 128 & 139 & 130 & 116 & 164 \\
\hline $\begin{array}{l}\text { öde, unbesetzte Hausstellen, } \\
\text { Anzahl }\end{array}$ & & & 18 & 15 & 15 & 16 & k. A. & k. A. \\
\hline Schwabach, Mieter in \% & $23 \%$ & k. A. & $26 \%$ & $20 \%$ & $28 \%$ & $35 \%$ & $41 \%$ & $59 \%$ \\
\hline Miethaushalte, Anzahl & 97 & & 87 & 64 & 108 & 150 & 201 & 440 \\
\hline alle Haushalte, Anzahl & 415 & & 338 & 313 & 379 & 424 & 490 & 747 \\
\hline $\begin{array}{l}\text { Eigentümerhaushalte und öde } \\
\text { Plätze }\end{array}$ & 318 & & 352 & 345 & 335 & 315 & 322 & 307 \\
\hline $\begin{array}{l}\text { öde, unbesetzte Hausstellen, } \\
\text { Anzahl }\end{array}$ & & & 101 & 96 & 64 & 41 & 33 & k. A. \\
\hline Uffenheim, Mieter in \% & $13 \%$ & k. A. & $2 \%$ & $6 \%$ & $13 \%$ & $13 \%$ & $15 \%$ & $15 \%$ \\
\hline Miethaushalte Anzahl & 20 & & 3 & 9 & 20 & 21 & 27 & 30 \\
\hline Alle Haushalte, Anzahl & 160 & & 126 & 141 & 151 & 158 & 180 & 205 \\
\hline
\end{tabular}




\begin{tabular}{|c|c|c|c|c|c|c|c|c|}
\hline $\begin{array}{l}\text { Eigentümerhaushalte und öde } \\
\text { Plätze }\end{array}$ & 140 & & 123 & 132 & 131 & 150 & 155 & 175 \\
\hline $\begin{array}{l}\text { Öde, unbesetzte Hausstellen, } \\
\text { Anzahl }\end{array}$ & & & k. A. & k. A. & k. A. & 13 & 2 & k. A. \\
\hline Wassertrüdingen, Mieter in \% & $23 \%$ & $\mathbf{0}$ & $9 \%$ & $8 \%$ & $10 \%$ & $6 \%$ & $8 \%$ & $8 \%$ \\
\hline Miethaushalte, Anzahl & 48 & 0 & 8 & 8 & 16 & 9 & 14 & 20 \\
\hline alle Haushalte, Anzahl & 205 & 60 & 65 & 106 & 157 & 153 & 174 & 263 \\
\hline $\begin{array}{l}\text { Eigentümerhaushalte und öde } \\
\text { Plätze }\end{array}$ & 157 & 60 & 134 & 137 & 164 & 144 & 160 & 243 \\
\hline $\begin{array}{l}\text { öde, unbesetzte Hausstellen, } \\
\text { Anzahl }\end{array}$ & & & 57 & 39 & 24 & k.A. & k. A. & k. A. \\
\hline pach, Mieter in \% & k. A. & A. & $9 \%$ & $7 \%$ & $9 \%$ & $5 \%$ & 0 & $19 \%$ \\
\hline shalte, Anzahl & & & 6 & 5 & 7 & 4 & 0 & 27 \\
\hline shalte, Anzahl & & & 68 & 74 & 75 & 82 & 81 & 141 \\
\hline $\begin{array}{l}\text { Eigentümerhaushalte und öde } \\
\text { Plätze }\end{array}$ & & & 81 & 86 & 68 & 83 & 81 & 114 \\
\hline $\begin{array}{l}\text { öde, unbesetzte Hausstellen, } \\
\text { Anzahl }\end{array}$ & & & 19 & 17 & k. A. & 5 & k. A. & k. A. \\
\hline tzahl, Miete & $15 \%$ & $14 \%$ & $15 \%$ & $14 \%$ & $12 \%$ & $16 \%$ & $20 \%$ & $29 \%$ \\
\hline $\begin{array}{l}\text { Summe städtische Miethaus- } \\
\text { halte }\end{array}$ & 248 & 38 & 326 & 322 & 324 & 446 & 594 & 1.231 \\
\hline $\begin{array}{l}\text { aller städtischen } \\
\text { llte }\end{array}$ & 1.620 & 73 & 2.243 & 2.349 & 812 & .823 & 935 & 4.288 \\
\hline $\begin{array}{l}\text { Summe aller erfassten Haus- } \\
\text { stellen }\end{array}$ & 1.266 & 245 & 2.245 & 2.283 & 2.645 & 2.539 & 2.393 & 3.073 \\
\hline $\begin{array}{l}\text { Sum } \\
\text { len }\end{array}$ & A. & k.A. & 316 & 235 & 158 & 111 & 19 & 16 \\
\hline
\end{tabular}

Tabelle 19. Anteil der Miethaushalte in den ansbachischen Städten anhand der Angaben in denHuldigungslisten 1603-1723 (Thomas Wenderoth 2019) 
Der Kurvenverlauf in Grafik 2 wirkt auf den ersten Blick chaotisch. Dort sind nur die Werte für acht ansbachische Städte enthalten, die insgesamt betrachtet einen geringen Mieteranteil aufwiesen, der die Zwanzigprozentmarke kaum überstieg. Gunzenhausen weist die Besonderheit auf, dass hier dreimal in Folge die Beamten und öffentlich Bediensteten separat als Hausgenossen aufgeführt worden sind, während sie in den meisten Zählungen fehlen oder zum Teil „unkorrekt“ zusammen mit den Eigentümern miterfasst worden sind. Aus diesem Grund wurde der Kurvenverlauf zu den Miethaushalten einmal mit und einmal ohne Berücksichtigung der Beamten als Mieter wiedergegeben. Anhand der abweichenden Gunzenhausener Kurve wird deutlich, welchen Einfluss die Berücksichtigung dieser Bevölkerungsgruppe auf den Anteil der Miethaushalte insgesamt hat.

Doch zurück zu den Auswirkungen des Dreißigjährigen Krieges auf die (Miet-) Bevölkerung. Dieser stellt sich deutlich als Zäsur dar. Lediglich in Crailsheim wurden 1603 weniger Miethaushalte erfasst als 1668, wobei die absoluten Zahlen hier immer gering waren; 1603 waren es neun Miethaushalte, was einem Anteil von 3\% an allen Haushalten entsprach, 1668 lag der Anteil mit achtzehn Miethaushalten dann bei $8 \%$. Am auffälligsten ist die Zäsur in Wassertrüdingen, das 1603 einen vergleichsweise hohen Mieteranteil von $23 \%$ aufwies, der sich dann in der Zeit von 1668 bis 1723 auf deutlich niedrigerem Niveau zwischen 6\% und $10 \%$ einpendelte.

Für Creglingen sind keine Zahlen für das Jahr 1603 überliefert. Nach dem Dreißigjährigen Krieg ist aber auch für diese Stadt eine schwer deutbare Kurve festzustellen, die zwischen $4 \%$ und $9 \%$ pendelte. Der geringste Anteil an Miethaushalten ist erst für das Jahr 1686/87 verzeichnet, also 50 Jahre nach dem Höhepunkt der Kampfhandlungen. Dies trifft gleichfalls für Mainbernheim und Leutershausen zu, während der Tiefpunkt in Feuchtwangen und Crailsheim 1692 und für Gunzenhausen gar erst 1703 erreicht ist.

Spätestens ab 1703 hat sich die Miet-Bevölkerung allgemein konsolidiert, entweder ihr Anteil verharrte oder wuchs leicht. In Uffenheim lag die Zäsur sogar vor dem Jahr 1668, seit diesem Zeitpunkt stieg der Anteil der Mietbevölkerung kontinuierlich an und erreichte 1686/87 mit 13\% wieder den Wert des Jahres 1603.

Die Tiefpunkte in den Kurven markieren eine zeitliche Situation, in der es Menschen aus den unteren Vermögensklassen gelang, aufgrund des enormen Bevölkerungsverlustes zu günstigen Konditionen Eigentum zu erwerben. Bis zu diesem Zeitpunkt war die wirtschaftliche Situation auch für diese Bevölkerungsgruppe noch zu problematisch, um ein Haus zu kaufen. Mit der wachsenden Gesamtbevölkerung ab dem späten 17. Jahrhundert nahm dann die Konkurrenz am 
Immobilienmarkt zu, mit der Folge einer wiederum wachsenden Mietbevölkerung. Diese Situation wird an den folgenden ansbachischen Städten sehr viel deutlicher ablesbar.

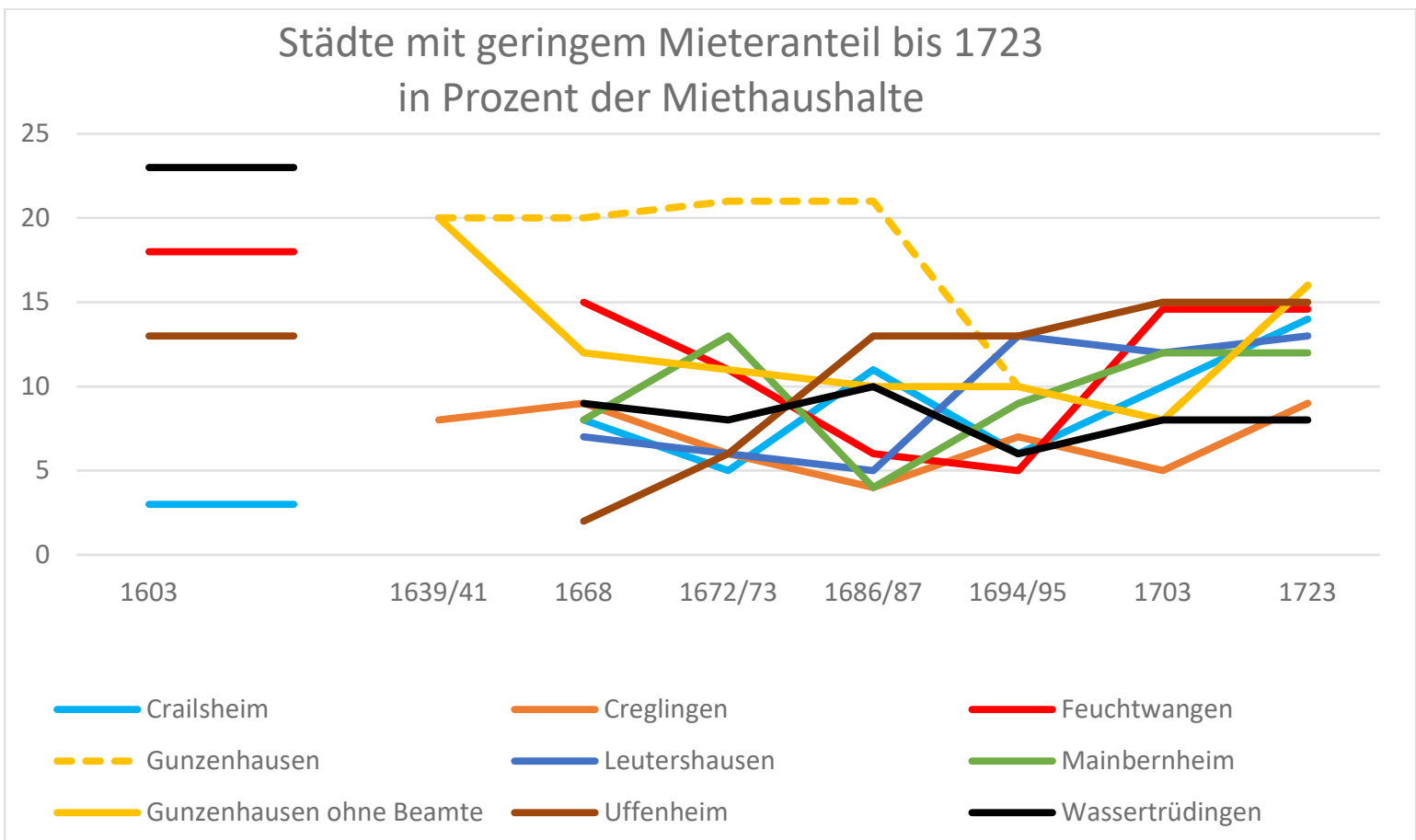

Grafik 2. Städte im Fürstentum Brandenburg-Ansbach 1603-1723 mit geringem Mieteranteil nach dem Dreißigjährigen Krieg (Thomas Wenderoth 2019)

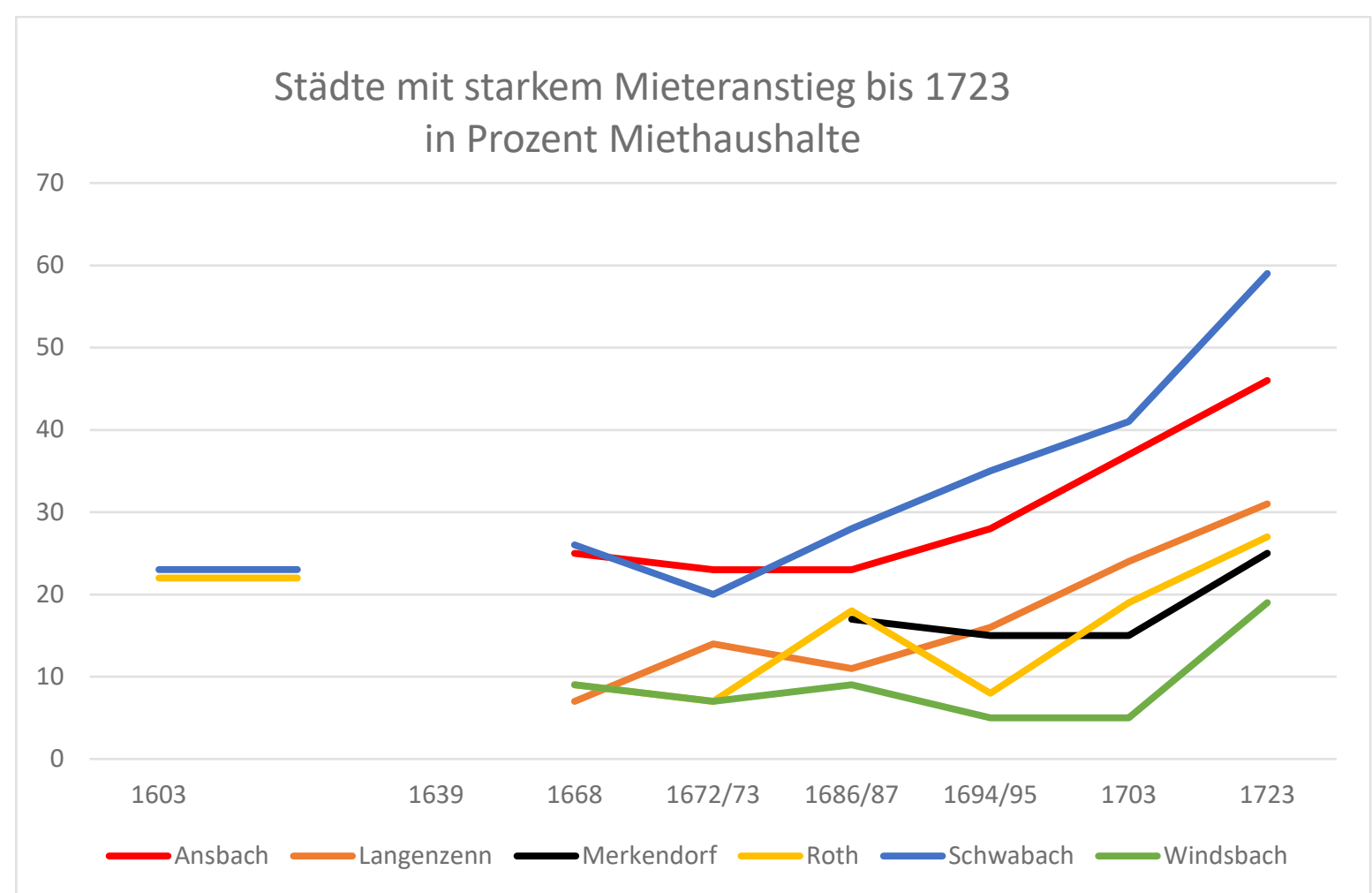

Grafik 3. Städte im Fürstentum Brandenburg-Ansbach 1603-1723 mit starkem Mieteranstieg ab dem letzten Drittel des 17. Jhs. (Thomas Wenderoth 2019) 
In Grafik 3 ist die Entwicklung des Anteils von Miethaushalten für sechs Städte wiedergegeben. Gemeinsam ist diesen Städten allein ein deutlich stärkerer Anstieg der Mietbevölkerung ab dem Ende des 17. Jahrhunderts gegenüber den zuvor diskutierten acht Städten. Bei Langenzenn, Merkendorf und Windsbach handelt es sich um ungemein kleine Landstädte mit maximal 165 Haushaltungen im Jahr 1723. Ansbach als Residenzstadt gehörte immer zu den größten Städten des Territoriums. Eine Abkoppelung Ansbachs hinsichtlich der Mieterstruktur von den anderen Landstädten zeichnet sich aber überraschenderweise erst ab dem Ende des 17. Jahrhunderts ab. Mit einem Anteil von bis dahin 23\% Miethaushalten lag die Stadt zwar im oberen Bereich der betrachteten Städte, aber doch noch innerhalb eines gemeinsamen Korridors von $5 \%$ bis $25 \%$.

Erst jetzt scheint sich die Residenzfunktion vollumfänglich auf die Eigentumsstruktur ausgewirkt zu haben. Dieses Phänomen wird im Zusammenhang mit den Residenzstädten noch eingehender diskutiert. ${ }^{654} \mathrm{Am}$ signifikantesten ist der Anstieg der Kurve für Schwabach. Die Zäsur brachte hier nicht erst der Dreißigjährige Krieg. Bereits 1603 war der Anteil der Miethaushalte von 39\% im Jahr 1530 deutlich auf $23 \%$ zurückgegangen. 1668 liegt er mit 26\% sogar leicht darüber, um 1672/73 den Tiefpunkt mit $20 \%$ zu erreichen. Nachfolgend steigt dann der Mietanteil kontinuierlich stark an: 1703 auf 41\% und um 1723 auf bemerkenswerte 59\%. Die Ursache für diese im Fürstentum ungewöhnliche Bevölkerungsstruktur dürfte in der grundsätzlich auf das Exportgewerbe orientierten Wirtschaftsstruktur der Stadt liegen. ${ }^{655}$ In abgeschwächter Form trifft dies auch auf die deutlich kleinere Nachbarstadt Roth zu. ${ }^{656}$ Bisher nicht zu erklären ist der deutliche Anstieg der Miethaushalte in Langenzenn im 18. Jahrhundert auf 31\% im Jahr 1723, gehörte diese Stadt doch mit lediglich 165 Haushalten zu den kleinsten des Fürstentums und war auch nicht Sitz eines Oberamtes. ${ }^{657}$

Die Stadtgröße spielt bei den untersuchten fünfzehn Städten keine nachvollziehbare Rolle im Hinblick auf den Anteil der Miethaushalte. Letztendlich handelt es sich bei allen Städten mit Ausnahme von Ansbach und Schwabach um kleine bis sehr kleine Landstädte mit einem überschaubaren Anteil an Miethaushalten, der ohne die Haushalte der alleinstehenden Mieterinnen und der Beamten selten den Anteil von $15 \%$ an den Gesamthaushalten übersteigt.

Im Folgenden sind die Zahlen der kleinen bis sehr kleinen Landstädte zusammengefasst. Dabei wurden Ansbach und Schwabach wegen ihrer eigenständigen

\footnotetext{
${ }^{654}$ Vgl. Kapitel 7.

${ }^{655}$ Vgl. hierzu DipPERT 2015, S. 170-182.

${ }^{656}$ Auch hier lässt sich spätestens seit 1569 exportorientiertes Metallgewerbe nachweisen. Vgl. PEUSCHEL 2015, S. 32.

${ }^{657} 1720$ gab es einen großen Stadtbrandt. Ein nennenswerter Teil der ehemaligen Eigentümer könnte 1723 daher noch interimsweise zur Miete gewohnt haben. Freundlicher Hinweis von Robert Zöllner.
} 
Entwicklung sowie Merkendorf und Prichsenstadt wegen der lückenhaften Überlieferung nicht mitberücksichtigt.

\begin{tabular}{|l|c|c|c|c|c|c|c|c|}
\hline Jahr & 1603 & $1639 / 41$ & 1668 & 1673 & $1686 / 87$ & 1695 & 1703 & 1723 \\
\hline Anzahl der erfassten Städte & 6 & 3 & 11 & 11 & 11 & 11 & 11 & 11 \\
\hline Gesamtzahl, Mieter in \% & $12 \%$ & $14 \%$ & $10 \%$ & $10 \%$ & $11 \%$ & $9 \%$ & $11 \%$ & $16 \%$ \\
\hline Summe erfasster Miethaushalte & 151 & 38 & 149 & 155 & 187 & 149 & 222 & 389 \\
\hline Summe Eigentümerhaushalte & 1.144 & 235 & 1.366 & 1.440 & 1.572 & 1.571 & 1.659 & 2.112 \\
\hline Summe aller erfassten Haushalte & 1.295 & 273 & 1.515 & 1.595 & 1.759 & 1.720 & 1.876 & 2.501 \\
\hline Summe unbesetzter Hausstellen & k.A. & k.A. & 215 & 139 & 92 & 67 & 19 & 16 \\
\hline
\end{tabular}

Tabelle 20. Durchschnittlicher Mieteranteil der kleinen bis sehr kleinen Landstädte im Fürstentum Ansbach 1603-1723 (Thomas Wenderoth 2019)

Die Zahlen vor dem Dreißigjährigen Krieg sind insgesamt sehr lückenhaft, da für das Jahr 1603 nur für sechs der elf und 1639/41 gar nur für drei Städte Angaben vorliegen. Gut vergleichbar sind die Zahlen ab dem Jahr 1668. Es ist eine kontinuierliche Zunahme der Hausbesitzer von 1.366 auf 2.112 festzustellen. Gleichzeitig war die Zahl der erfassten öden Hausstellen rückläufig. Die Anzahl der Miethaushalte nahm ebenfalls kontinuierlich zu, von absolut 149 auf $389 \mathrm{im}$ Jahr 1723. Bis zum Jahr 1703 sind jeweils rund 10\% der Haushalte als Miethaushalte erfasst, der Rückgang 1692 auf 9\% könnte auch in der Art und Weise der Erfassung begründet sein, er ist in jedem Fall nicht so signifikant, dass er einer Erklärung bedürfte. Auffällig ist dagegen der prozentuale Anstieg der Miethaushalte im Jahr 1723 auf dann durchschnittlich 16\%: Im 18. Jahrhundert führt das Bevölkerungswachstum vor allem zu einem Anstieg der Mietbevölkerung im Fürstentum. Dieses Phänomen wird anhand der Bevölkerungszahlen des frühen 19. Jahrhunderts noch deutlicher.

Vorsichtig geschätzt darf man bei diesen elf untersuchten kleinen Landstädten von zusätzlich 5-10\% mietenden Beamtenhaushalten (bezogen auf alle Haushalte) und ebenso vielen Haushalten von weiblichen Mieterinnen, die in den Huldigungslisten nicht erfasst worden sind, ausgehen. Daraus ergibt sich, bei aller Vorsicht, ein Mieteranteil von durchschnittlich 20\% bis um 1700. 


\section{Die nürnbergischen Kleinstädte im 17. und 18. Jahrhundert}

Auch in den Landstädten Nürnbergs ist selbstverständlich mit einer eigentumslosen Bevölkerung zu rechnen. 1620 wird in Hersbruck ein Stadtgerichtsbeschluss erlassen, in welchem die Anzahl der Haushalte pro Haus reglementiert wurde. ${ }^{658}$ Anzunehmen ist daher eine Häufung von Mietern in einzelnen Häusern. Ernst Wiedemann geht in seinem Häuserbuch leider nicht auf die eigentumslosen Haushalte ein. Dennoch bieten die Zahlen, die er ermittelt, Anhaltspunkte. Für das Jahr 1691 erfasst er 285 verheiratete Männer, ${ }^{659}$ dies dürfte in etwa der Anzahl der Haushaltungen entsprechen, pauschal kann man noch 30 Haushaltungen von alleinstehenden Witwern und Witwen hinzuzählen. Die Zahl der Wohnhäuser gibt er für das 17. Jahrhundert mit 200 an. ${ }^{660}$ Daraus lässt sich ableiten, dass am Ende des 17. Jahrhunderts mindestens 1/3 der Haushalte nicht in einem eigenen Haus wohnte. Auch in der Nürnberger Landstadt Hersbruck lebte demnach zu dieser Zeit eine große Bevölkerungsschicht als Mieter. Die oben genannten Schätzungen beruhen auf Analogieschlüssen. Sie werden aber vollumfänglich durch die nun nachfolgenden Zahlen aus einer Erfassung des Jahres 1716/17 bestätigt. Damit stellte sich die Situation der Mietbevölkerung in den vier nürnbergischen Kleinstädten deutlich unterschiedlich zu den überwiegend geringen Mieteranteilen in den ansbachischen Städten dar. Ein direkter Vergleich ist jedoch allein für die Zeit um 1720 möglich, da sich als serielle Quelle für die vier nürnbergischen Kleinstädte Altdorf, Hersbruck, Lauf und Velden nur die Türkensteuerverzeichnisse von 1716/17 erhalten haben.

Die Entwicklung der (eigentumslosen) Bevölkerung im 16. und 17. Jahrhundert kann bisher für diese Städte nicht genau bestimmt werden. In der vorausgehenden Tabelle sind die Ergebnisse zusammengeführt.

In Lauf wurden die Bewohner der vorstädtischen Hammerwerke nicht erfasst, neben wenigen Eigentümern lebten hier 84 Arbeiter zur Miete. ${ }^{661}$ In Altdorf wiederum liegen keine Angaben für die Universitätsangehörigen vor, da diese juristisch eine eigene Gemeinde darstellten. Aufgrund dieser Auslassungen sind die Zahlen der vier Kleinstädte grundsätzlich besser miteinander vergleichbar, da die Sondereffekte Gewerbebetriebe und Universität entfallen, die den Mieteranteil in Lauf und Altdorf deutlich nach oben verschieben würden.

\footnotetext{
${ }^{658}$ WIEDEMANN 1963, S. 51.

${ }^{659}$ Ebd., S. 46.

${ }^{660}$ Ebd., S. 22

${ }^{661}$ Vgl. WENDEROTH 2019a, S. 192-194;

dort erfolgt eine ausführliche Auflistung der einzelnen Betriebe.
} 


\begin{tabular}{|l|c|c|c|c|c|r|}
\hline Ort & $\begin{array}{c}\text { Eigentümer } \\
\text { mit Haus }\end{array}$ & $\begin{array}{c}\text { öffentlicher } \\
\text { Dienst }\end{array}$ & Mieter & $\begin{array}{c}\text { Mieter } \\
\text { gesamt }\end{array}$ & $\begin{array}{c}\text { im } \\
\text { Winkel }\end{array}$ & $\begin{array}{c}\% \text { Mieter- } \\
\text { haushalte }\end{array}$ \\
\hline Altdorf, Kernstadt & 283 & 19 & 225 & 244 & & $46 \%$ \\
\hline Vorstadt & 6 & 0 & & 0 & & $0 \%$ \\
\hline \hline Altdorf, gesamt & 289 & 19 & 225 & 244 & & $46 \%$ \\
\hline Lauf, Kernstadt & 130 & 14 & 82 & 96 & 3 & $42 \%$ \\
\hline Oberes Tor, Vorstadt & 40 & 2 & 35 & 37 & 3 & $48 \%$ \\
\hline Unteres Tor, Vorstadt & 25 & 2 & 28 & 30 & 1 & $55 \%$ \\
\hline Wassertor, Vorstadt & 20 & 3 & 13 & 16 & 1 & $44 \%$ \\
\hline \hline Lauf, gesamt & 215 & 21 & 158 & 179 & 8 & $45 \%$ \\
\hline Hersbruck & 212 & 35 & 103 & 138 & 18 & $39 \%$ \\
\hline Velden & 78 & $\mathbf{6}$ & 34 & 40 & & $34 \%$ \\
\hline \hline Alle 4 Städte & 794 & 81 & 520 & 601 & 26 & $43 \%$ \\
\hline
\end{tabular}

Tabelle 21. Anteil der Miethaushalte in den Nürnberger Landstädten 1716/17. Für Lauf wurden die Bewohner der Hammerwerke nicht berücksichtigt (StAN, Rst. N, Bauernverzeichnisse Nr. 7, fol. 318-326, 357'-372' , 402-412, 533'-537; Thomas Wenderoth 2019)

Unter dem modernen Begriff der Mieter sind verschiedene zeitgenössische Gruppen zusammengefasst: Beständner, Wittiben ohne Eigentum, Schutzverwandte und öffentlich Bedienstete. Besonders differenziert ist die Auflistung in Lauf erfolgt. ${ }^{662}$ Die Mieter werden unterschieden in drei Gruppen: Im Zinnße, In Wohnung und Innwohner, ohne dass die begriffliche Differenzierung erklärt ist. Den Begriff Innwohner darf man hier vielleicht als ein Untermietverhältnis interpretieren, genannt sind immerhin 44 Personen. Schutzverwandte werden ebenfalls nahezu ausschließlich für die Laufer Vorstädte aufgeführt. Beim Oberen Tor waren es fünf, beim Unteren Tor neun Schutzverwandte.

Alle vier Städte hatten zusammengenommen durchschnittlich $43 \%$ Miethaushalte. Altdorf und Lauf lagen leicht darüber, Hersbruck mit 39\% etwas unter dem Mittelwert. Für Altdorf sind 533 Haushalte erfasst, hinzuzurechnen wären ungefähr noch 150 Haushalte von Universitätsangestellten und Studenten. ${ }^{663}$ In Lauf sind 394 Haushalte erfasst, zuzüglich der 84 Miethaushalte in den Hammerwerken ergeben sich 478 Haushalte. In Hersbruck hatte die Reichsstadt Nürnberg Militär stationiert, ${ }^{664}$ die Anzahl der Soldaten ist jedoch bisher nicht erforscht, dürfte aber die Bevölkerungsanzahl über die erfassten 350 Haushalte nicht wesentlich in die Höhe getrieben haben. Damit waren diese drei Städte um einiges

\footnotetext{
${ }^{662}$ Für Lauf werden genannt: 21 öffentlich Bedienstete, 10 Pfründner im Spital, 3 Spitalbewohner, 76 Haushalte Im Zinnße, 11 Haushalte In Wohnung, 44 Innwohner, 8 Altenteiler Im Winkel, 15 Schutzverwandte. StAN, Rst. N, Bauernverzeichnis Nr. 7, fol. 402-412.

${ }^{663}$ Vgl. hierzu Kapitel 7, Universitätsstädte.

${ }^{664} 1798 / 99$ wurde außerhalb der Stadt an der Amberger Straße eine Kaserne erbaut. Denkmalnummer D-5-74-132-12. http://geoportal.bayern.de/bayernatlas-klassik (Aufruf 17.3.2019).
} 
größer als die zuvor angeführten brandenburg-ansbachischen Landstädte und entsprachen hinsichtlich ihrer Bevölkerungszahl eher den Städten Ansbach und Schwabach. Mit diesen gemeinsam hatten sie auch einen hohen Anteil an Miethaushalten.

Ansbach als Residenzstadt verzeichnete im Jahr 1723 46\% und Schwabach als Exportgewerbestandort 59\% Miethaushalte. In Lauf lag der Anteil der Miethaushalte unter Einbeziehung der Miethaushalte bei den Hammerwerken im Jahr $1716 / 17$ bei $55 \%$, Altdorf kam zusammen mit der Universitätsgemeinde auf etwa 57\% Miethaushalte. Sondereffekte hinsichtlich der Mietbevölkerung, die bisher nicht berücksichtigt wurden, sind allerdings für Hersbruck und Velden nicht auszumachen. Insbesondere Velden mit lediglich 78 Eigentümern überrascht als Kleinststadt mit einem Mieteranteil von 34\%. Lässt man bei der Berechnung jedoch die öffentlich Bediensteten und die Frauen unberücksichtigt, entfielen auf 78 Eigentümerhaushalte nur noch neun Miethaushalte mit männlichem Haushaltsvorstand, was rein rechnerisch zu einem Mieteranteil von 10,3\% führt, einem Wert, der voll übereinstimmt mit den kleinstädtischen Mieteranteilen in den Huldigungslisten des Fürstentums Ansbach. Allerdings erscheint die Anzahl von 25 weiblichen Miethaushalten in Velden außergewöhnlich hoch und nicht ohne Weiteres übertragbar auf die ansbachischen Städte.

Zusammenfassend bleibt festzustellen, dass im Nürnberger Territorium der Anteil der Mietbevölkerung deutlich über dem des Fürstentums Ansbach lag. Auf den ebenfalls ungewöhnlich hohen Mieteranteil innerhalb der dörflichen Bevölkerung des reichsstädtischen Territoriums in der frühen Neuzeit bin ich schon im Rahmen meiner Dissertation eingegangen. ${ }^{665}$ Als Ursachen können mehrere Gründe in Frage kommen, die nachfolgend als Thesen angeführt werden:

Das Nürnberger Territorium war insgesamt betrachtet wesentlich weniger betroffen von Zerstörungen und Bevölkerungsverlusten im Dreißigjährigen Krieg. Es gab nur geringere Bevölkerungsverluste auszugleichen. Die Wirtschaftskraft der Metropole Nürnberg wirkte sich ebenfalls positiv auf die Einkommensmöglichkeiten der Bewohner der nürnbergischen Landstädte und Dörfer aus. Vor allem war es leichter möglich, seinen Lebensunterhalt auch ohne Immobilien- und Grundbesitz zu erwirtschaften.

Während in einigen Städten des Fürstentums Ansbach das Bevölkerungswachstum im 18. Jahrhundert durch Eigentums- und Hausteilungen kompensiert worden ist, wurde diese Option allem Anschein nach von der reichsstädtischen Verwaltung unterbunden. Zumindest bleiben Hausteilungen in den nürnbergischen Städten eine große Ausnahme.

665 WENDEROTH 2019a, S. 41-49. 


\section{Die Landstädte des Fürstbistums Bamberg in der frühen Neuzeit}

Zum weiteren Vergleich sollen noch die Zahlen zu einigen Kleinstädten im Hochstift Bamberg herangezogen werden. Die Bevölkerungszahlen für das Fürstbistum Bamberg im Zeitalter des Absolutismus wurden bereits 1940 von Otto Morlinghaus untersucht. ${ }^{666} \mathrm{Im}$ Folgenden stütze ich mich auf dessen Ergebnisse. Grundsätzlich behandelt er den Zeitraum zwischen 1558 und 1811. Auf die Angaben aus den Huldigungslisten stützt er sich für die Jahre 1609, 1623, 1653, 1672, 1716, 1731 und 1750, ${ }^{667}$ differenziert dort aber nicht zwischen den einzelnen Gruppen. Lediglich für das Jahr 1676/77 bezieht er sich auf eine Bevölkerungsaufnahme, die sehr detailliert Haushaltsvorstände, Kinder, Dienstboten und eigentumslose Haushalte (Herberger) unterscheidet. ${ }^{668}$ Allerdings sind dort nur die Zahlen für die Ämter Kronach, Wallenfels, Teuschnitz, Fürth und Stadtsteinach überliefert.

\begin{tabular}{|l|c|c|c|c|r|}
\hline Stadt & Haushalte & Kinder & Dienstboten & Mieter & Anteil Miethaushalte \\
\hline Kronach & 382 & 932 & 170 & 50 & $12 \%$ \\
\hline Kupferberg & 70 & 131 & 38 & 8 & $10 \%$ \\
\hline Stadtsteinach & 108 & 235 & 54 & 14 & $11 \%$ \\
\hline Wallenfels & 123 & 295 & 31 & 18 & $13 \%$ \\
\hline \hline 4 Städte & $\mathbf{6 8 3}$ & $\mathbf{1 . 5 9 3}$ & $\mathbf{2 9 3}$ & $\mathbf{9 0}$ & $\mathbf{1 2 \%}$ \\
\hline $\begin{array}{l}\text { Dörfliche Bevölke- } \\
\text { rung in den Ämtern }\end{array}$ & $\mathbf{1 . 4 8 7}$ & $\mathbf{3 . 4 6 4}$ & $\mathbf{5 8 3}$ & $\mathbf{1 7 6}$ & $\mathbf{1 1 \%}$ \\
\hline
\end{tabular}

Tabelle 22. Prozentuale Anteil der Miethaushalte in den Gebirgsämtern des Hochstiftes Bamberg 1676/77 (nach MoRLingHAUs 1940, S. 46-66; Thomas Wenderoth 2019)

Festzuhalten bleibt, dass in den Gebirgsämtern im heutigen Oberfranken der Anteil an Miethaushalten nach dem Dreißigjährigen Krieg gering war. In den vier Städten schwankte er zwischen $10 \%$ und $13 \%$ und unterscheidet sich damit nicht von dem Mieteranteil in den umliegenden Dörfern, der bei durchschnittlich 11\% lag. Selbst eine in Größe und Bedeutung ansehnliche Stadt wie Kronach bewegte sich innerhalb dieses Rahmens. Vergleicht man diese Zahlen mit den Angaben der Huldigungslisten 1672/73 des Fürstentums Ansbach (vgl. Tab. Anhang I.4.), fällt ein ebenfalls niedriger Anteil an Miethaushalten auf: Für die dortigen Städte errechnet sich ein Durchschnittswert von 14\%, allerdings schlagen hier die beiden größeren Städte Ansbach (23\%) und Schwabach (20\%) stärker zu Buche.

\footnotetext{
${ }^{666}$ Morlinghaus 1940.

${ }^{667}$ Ebd. S. 35f.

${ }^{668}$ Ebd. S. 25; StABA, Hochstift Bamberg Hofkammer, Akten und Bände 1581. Morlinghaus datiert die dort festgehaltene Zählung fälschlicherweise auf 1674. Für die Klärung der aktuellen Archivsignatur und die korrekte Datierung danke ich Johannes Haslauer, Staatsarchiv Bamberg.
} 
Ohne diese beiden Städte liegt der Durchschnittswert an Miethaushalten ebenfalls nur bei $10 \%{ }^{669}$ Hinsichtlich des Anteils an eigentumslosen Haushalten gleichen sich demnach die Ansbacher und Bamberger Kleinstädte Ende des 17. Jahrhunderts.

\section{Mieteranteil in fränkischen Landstädten um 1800}

Als Grundlage für die nachfolgende Analyse dient die Schrift „Alphabetisches Verzeichniß aller im Rezatkreise nach seiner durch die neueste Organisation erfolgten Constituirung enthaltenen Ortschaften: mit Angabe a. der Steuer-Distrikte, b. Gerichts-Bezirke, c. Rentämter, in welchen sie liegen, dann mehrerer anderer statistischen Notizen. Ansbach 1818. “670 In die Auswertung einbezogen wurden von mir insgesamt 33 Städte aus dem heutigen Regierungsbezirk Mittelfranken. Lässt man Nürnberg und die vier großen Städte weg, ${ }^{671}$ bleiben 28 Kleinstädte für die Auswertung übrig.

Diese Städte habe ich entsprechend ihrer territorialen Zugehörigkeit in Gruppen zusammengefasst.

\section{Die nürnbergischen Kleinstädte 1818}

Die ehemaligen Nürnberger Landstädte Altdorf, Lauf und Hersbruck kommen durchschnittlich auf 46\% Mieter. Hersbruck liegt mit 38\% wieder deutlich hinter Lauf und Altdorf. ${ }^{672}$ Velden bleibt hier unberücksichtigt, da die Zahlen nicht kongruent sind mit den übrigen Daten. Aus den Türkensteuerlisten von 1716/17 ist bekannt, dass Velden bereits 100 Jahre zuvor einen Mieteranteil von 30\% aufwies.

\footnotetext{
${ }^{669}$ Es sei noch einmal darauf hingewiesen, dass in sämtlichen Zahlen die öffentlich Bediensteten und die alleinstehenden Frauen fehlen dürften und auch sonst mit einer unpräzisen Erfassung gerechnet werden muss. In der Tendenz zeigen die Zahlen aber einen Gleichklang zwischen dem Ansbacher und Bamberger Territorium auf sehr niedrigem Niveau an.

${ }^{670}$ Vgl. Kapitel 3, frühe statistische Quellen.

${ }^{671}$ Fürth wird erst im Jahr 1818 zur Stadt erhoben, hat aber seit dem Ende des 17. Jhs. aufgrund seiner Größe städtischen Charakter. Diese Städte werden weiter oben unter dem Punkt Mittelstädte diskutiert.

${ }^{672}$ Für 1804 sind in Hersbruck dagegen 381 verheiratete Männer erfasst (WIEDEMANN 1963, S. 55). Hinzu kommen noch die Haushalte der Witwen, sodass als unterster Zahl von 420 Haushalten für das Jahr 1804 auszugehen ist. Die Zahl der Wohnhäuser steigt weniger stark auf lediglich 228 an. 1804 leben demnach durchschnittlich 1,84 Haushalte in einem Anwesen. Damit wächst der Prozentsatz der Haushalte, die nicht in einem eigenen Haus leben, auf über $40 \%$ an. Die genaue Zahl der Mietbevölkerung lässt sich anhand dieser Zahlen nicht ermitteln, da in den Zahlen zu den Verheirateten auch Altenteiler miterfasst sind. Vereinzelt muss auch von Häusern ausgegangen werden, die mehreren Haushalten gemeinsam gehören. Demgegenüber stehen Häuser, die zur Gänze fremdvermietet sind. Grundsätzlich decken sich diese Werte mit den Angaben im Alphabetischen Verzeichniß.
} 
Es gibt keinen Anhaltspunkt dafür, dass gerade in dieser Stadt, die mit einem hohen Mieteranteil in der Barockzeit gestartet ist, sämtliche Mieter in den nachfolgenden 100 Jahren verschwunden sein sollten. Am Beispiel Veldens wird ersichtlich, wie notwendig es ist, die überlieferten Zahlen im Kontext anderer Orte und im Kontext der Ortsgeschichte zu interpretieren. Nimmt man für Velden auch für das Jahr 1818 einen Mieteranteil von $30 \%$ an, würde der durchschnittliche Mieteranteil in den vier nürnbergischen Landstädten von $46 \%$ auf dann $44 \%$ aller Haushalte sinken.

\section{Die hohenzollerischen Kleinstädte 1818}

Die untersuchten Kleinstädte der ehemaligen Fürstentümer Ansbach und Bayreuth bilden mit zehn Städten die größte Untersuchungsgruppe. Sie liegen nunmehr mit durchschnittlich 41\% Miethaushalten nur wenig unterhalb der Zahlen für die Nürnberger Landstädte. Betrachtet man die Belegungsziffer der Häuser in den hohenzollerischen Städten, muss man sogar feststellen, dass diese mit knapp zwei Familien pro Haus deutlich dichter besetzt waren als die Häuser in den nürnbergischen Städten: Im Nürnberger Gebiet teilten sich nur 1,81 Haushalte ein Haus. Hier wirkt sich die Aufsplitterung des Hausbesitzes auf mehrere Eigentümer aus, die im 18. Jahrhundert charakteristisch war für viele brandenburgansbachische Städte. Am deutlichsten ist dieser Effekt bei den Städten Feuchtwangen, Leutershausen und Uffenheim zu erkennen. Alle drei Städte wiesen einen verhältnismäßig geringen Mieteranteil auf. Feuchtwangen hatte nur einen Anteil von 31\% an Miethaushalten, dennoch teilten sich hier 2,01 Familien ein Haus. In Leutershausen waren es 33\% Miethaushalte und 1,79 Familien pro Haus und in Uffenheim kamen bei 25\% Miethaushalten 1,73 Familien auf jedes Haus. In Merkendorf hingegen, wo es zu keinen Hausteilungen gekommen war, fanden sich durchschnittlich lediglich 1,35 Familien in einem Haus, dies entspricht dem 25\% Anteil der Mieter an den dortigen Haushalten. 


\begin{tabular}{|c|c|c|c|c|c|c|c|c|c|c|c|c|c|c|c|c|c|c|c|c|c|c|c|}
\hline \multirow{3}{*}{ 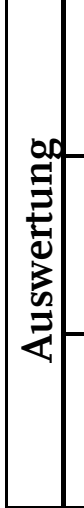 } & 离 & $\begin{array}{l}\text { ১̊ } \\
. \Xi\end{array}$ & \begin{tabular}{|l|}
\multirow{2}{*}{} \\
के \\
\end{tabular} & 犬̊ & \begin{tabular}{|l|}
0 \\
$\infty$ \\
$\infty$
\end{tabular} & $\begin{array}{l}\text { ○̊ } \\
\text { o్ర }\end{array}$ & \begin{tabular}{|l|}
$\frac{0}{6}$ \\
\end{tabular} & ㅇํㅇ & ○े & \begin{tabular}{|l|}
\multirow{2}{*}{} \\
$\stackrel{5}{+}$
\end{tabular} & $\begin{array}{l}\text { ○े } \\
\infty \\
m\end{array}$ & ○े & $\stackrel{\circ}{\circ}$ & \begin{tabular}{|l|}
$\circ$ \\
\\
\end{tabular} & \begin{tabular}{l|}
$\circ$ \\
$m$ \\
\end{tabular} & 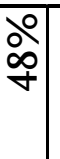 & ○ें & 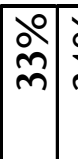 & \begin{tabular}{l|l} 
১̊ \\
$\stackrel{\sim}{+}$
\end{tabular} & 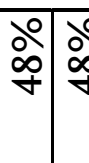 & 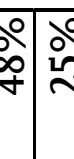 & 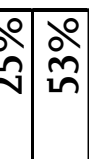 & ○े \\
\hline & 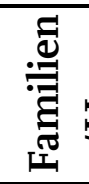 & 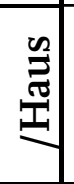 & $\left|\begin{array}{l}-5 \\
0 \\
0\end{array}\right|$ & 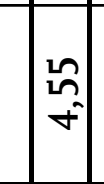 & $\left|\begin{array}{l}8 \\
0 \\
0\end{array}\right|$ & $\begin{array}{l}\vec{y} \\
\mathrm{~J}\end{array}$ & $\stackrel{m}{\hat{N}}$ & $\hat{m}$ & $\begin{array}{l}\infty \\
-1 \\
-1\end{array}$ & $\begin{array}{l}\stackrel{\Omega}{\sigma} \\
-1\end{array}$ & ஸิ & Әे & 命 & Әे & $\begin{array}{l}5 \\
0 \\
0\end{array}$ & $\begin{array}{c}0 \\
\text { - } \\
\sim\end{array}$ & $\left|\begin{array}{c}m \\
⿱ \\
v \\
v\end{array}\right|$ & $\begin{array}{l}\Omega \\
\\
-i\end{array}$ & $\stackrel{n}{m}=$ & \begin{tabular}{l|l}
8 & ㄴ \\
\multirow{2}{*}{} & r
\end{tabular} & 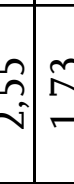 & 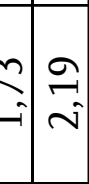 & 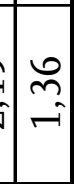 \\
\hline & 音 & 告 & $\begin{array}{l}8 \\
8 \\
+\end{array}$ & \begin{tabular}{|}
$n$ \\
$\hat{m}$ \\
$m$
\end{tabular} & $\left|\begin{array}{l}\infty \\
\infty \\
m\end{array}\right|$ & \begin{tabular}{|c}
$\overrightarrow{0}$ \\
$m$ \\
$m$ \\
$m$
\end{tabular} \mid & $\mid$ & 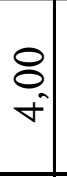 & $\begin{array}{l}\hat{n} \\
\hat{m} \\
m\end{array}$ & $\begin{array}{c}6 \\
m \\
m\end{array} \mid$ & $\begin{array}{l}\infty \\
\check{a} \\
\text { m. }\end{array}$ & $\begin{array}{lll} & \\
\text { mे|l } & \end{array}$ & 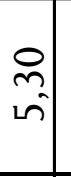 & $\left|\begin{array}{c}n \\
\mathbf{n}\end{array}\right|$ & $\begin{array}{l}\infty \\
0 \\
m\end{array}$ & $\begin{array}{c}\tilde{n} \\
\text { ñ }\end{array}$ & $\begin{array}{c}0 \\
\text { n. } \\
\text { n' }\end{array}$ & $\begin{array}{c}2 \\
m \\
m\end{array} \mid$ & 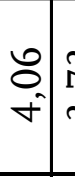 & $\begin{array}{l}m \\
\tilde{n} \\
\tilde{m}\end{array}$ & \begin{tabular}{l|l}
$\infty$ & $\infty$ \\
$\infty$ & \\
$n^{-1}$ & $\nabla$
\end{tabular} & 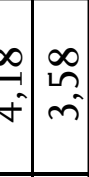 & $\begin{array}{l}8 \\
8 \\
4\end{array}$ \\
\hline & $\frac{\dot{d}}{\stackrel{d}{d}}$ & $\frac{\bar{T}}{\sqrt[7]{3}}$ & 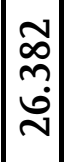 & $\begin{array}{l}\text { 을 } \\
\text { ํํㄹ } \\
\dot{+}\end{array}$ & 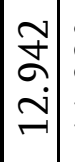 & 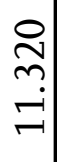 & $\left|\begin{array}{c}0 \\
\infty \\
\infty \\
\infty\end{array}\right|$ & $\underset{\sim}{\stackrel{+}{+}}$ & ڤે. & $\left|\begin{array}{c}0 \\
0 \\
-1 \\
ن \\
ن\end{array}\right|$ & 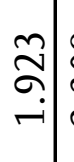 & 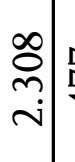 & $\stackrel{尺}{\hat{\sigma}}$ & 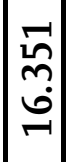 & 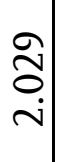 & $\begin{array}{c}\tilde{\sigma} \\
\underset{\sim}{\sim}\end{array}$ & $\begin{array}{c}2 \\
\infty \\
+ \\
-i\end{array}$ & 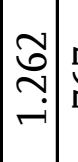 & సิ & 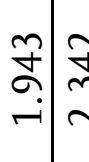 & 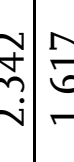 & 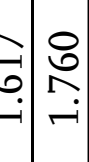 & å \\
\hline & & |⿹弋龴 & กิ๊ & \begin{tabular}{|c|}
1 \\
0 \\
$\infty$ \\
0 \\
0 \\
-1
\end{tabular} & $\mid \begin{array}{c}\tilde{y} \\
m \\
m\end{array}$ & $\begin{array}{l}\stackrel{R}{\Omega} \\
m \\
m\end{array}$ & 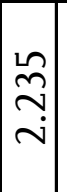 & $\begin{array}{l}\text { Lी } \\
\text { م. } \\
-i \\
-\end{array}$ & 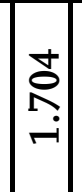 & ำ & $\begin{array}{c}\infty \\
\dot{+} \\
+\end{array}$ & శెర & ㅇ & 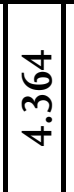 & กิ & స్ర & 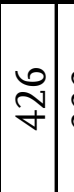 & $\begin{array}{l}n \\
\tilde{m}\end{array}$ & $\stackrel{\infty}{\infty}$ & సี & 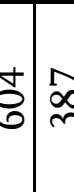 & 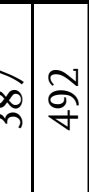 & $\hat{\sim}$ \\
\hline \multirow{3}{*}{ 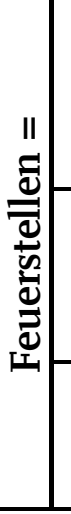 } & & 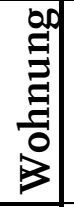 & & & & & & 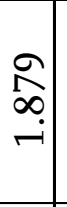 & & 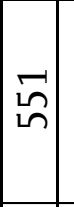 & & ํํํ & & & & & & 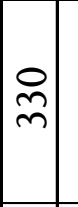 & & & & $\begin{array}{l}\mathrm{v} \\
\dot{f}\end{array}$ & \\
\hline & & 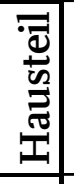 & $\mid \begin{array}{c}\dot{y} \\
\dot{y} \\
\dot{r}\end{array}$ & $\begin{array}{l}0 \\
\text { ஸे } \\
\text { ㄱ. }\end{array}$ & กิ & 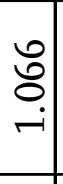 & $\begin{array}{l}1 \\
\infty\end{array}$ & & Әे & & ๙ે & & $\infty$ & $\begin{array}{c}\tilde{m} \\
\dot{f} \\
-\end{array} \mid$ & $\begin{array}{c}\infty \\
\infty \\
m\end{array}$ & $\underset{\approx}{\approx}$ & & & $\stackrel{m}{ \pm}$ & $\stackrel{\nabla}{\sim}$ & $\frac{ \pm}{n}$ & & \\
\hline & 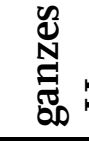 & 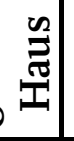 & & & & & & & & & & & & & & & $\stackrel{\sim}{\sim}$ & & & & & & $\stackrel{+}{ \pm}$ \\
\hline \multirow{2}{*}{ 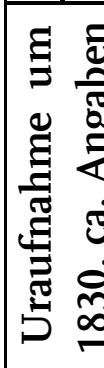 } & & 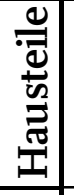 & & ํㅜㅁ & & ำ & & 운 & సิ & 음 & $\frac{1 n}{m}$ & 하 & $\begin{array}{l}\infty \\
0 \\
-1\end{array}$ & 岂 & & & $\stackrel{\operatorname{Ln}}{\stackrel{\sim}{\sim}}$ & $\stackrel{\mathbb{N}}{\sim}$ & & & & 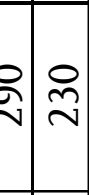 & $\stackrel{\text { n }}{\sim}$ \\
\hline & & 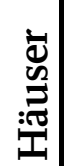 & $\left|\begin{array}{c}m \\
\infty \\
\sim \\
m\end{array}\right|$ & $\left|\begin{array}{c}n \\
\hat{m} \\
\sim\end{array}\right|$ & 은 & ำ & 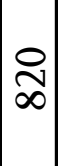 & ํํำ & ঙั & 엄 & $\stackrel{\ln }{m}$ & 吾 & $\tilde{\sigma}$ & $\left|\begin{array}{l}\tilde{\sigma} \\
\stackrel{\sim}{\nu}\end{array}\right|$ & $\stackrel{\llcorner}{\curvearrowright}$ & ঐे & $\stackrel{n}{\curvearrowright}$ & $\underset{\text { D }}{\infty}$ & P) & చ్రి & $\stackrel{\sim}{\sim}$ & 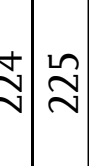 & $\stackrel{ \pm}{\sim}$ \\
\hline \multirow[t]{2}{*}{. } & 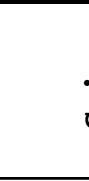 & 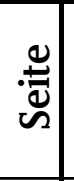 & 5 & & 㸙 & ( & $\approx$ & $\tilde{\infty}$ & 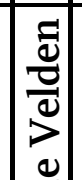 & \begin{tabular}{|l|} 
\\
\end{tabular} & P & กูก & $\widehat{\widehat{\sigma}}$ & 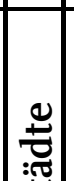 & $\stackrel{\text { นิ }}{\sim}$ & ষे & กิ & जी & 㐫 & \begin{tabular}{l|l}
\multirow{\sigma}{*}{} & $\stackrel{1}{ }$
\end{tabular} & $\hat{~} \approx$ & নী & 孛 \\
\hline & & $\begin{array}{l}+\vec{T} \\
\stackrel{\vec{t}}{\Delta}\end{array}$ & 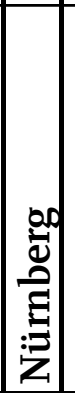 & 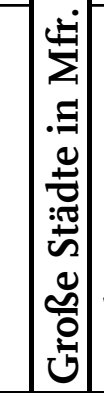 & 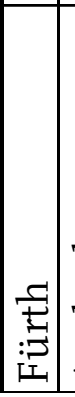 & 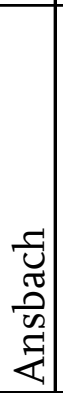 & 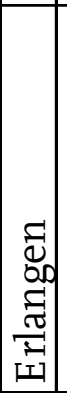 & 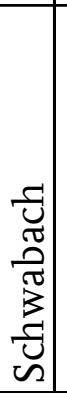 & 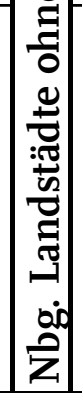 & 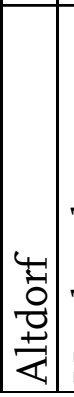 & 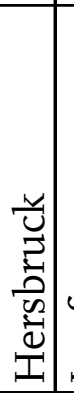 & 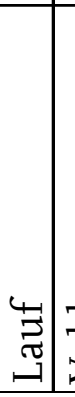 & 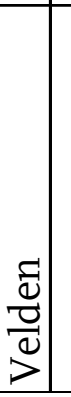 & 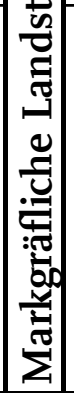 & 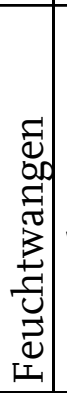 & 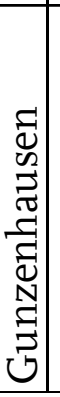 & 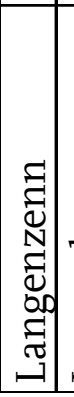 & 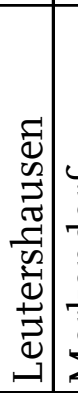 & 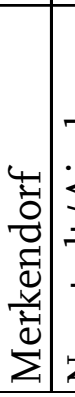 & 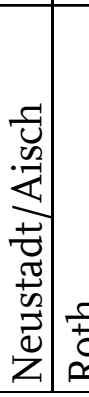 & 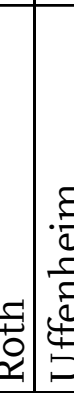 & 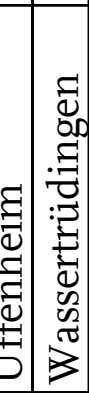 & 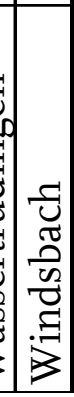 \\
\hline
\end{tabular}




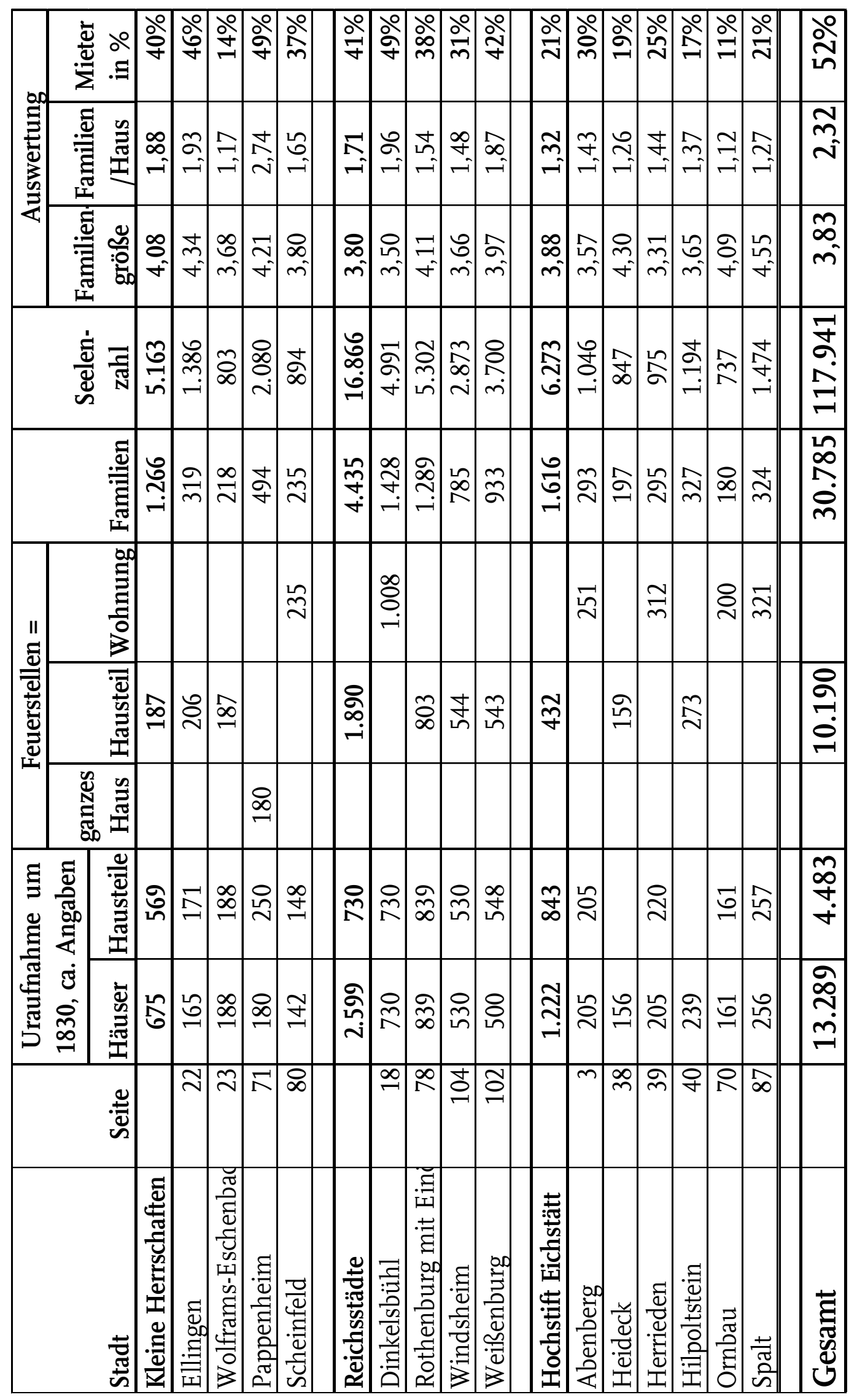

Tabelle 23. Mieteranteil mittelfränkischer Städte im Jahr 1818, ermittelt anhand der Daten aus dem Alphabetischen Verzeichnis des Rezatkreises (Thomas Wenderoth 2019) 


\section{Städte der kleinen Herrschaften 1818}

Im Hinblick auf den prozentualen Anteil der Miethaushalte folgt dicht hinter den hohenzollerischen Städten die Gruppe der Städte in den kleinen fränkischen Standesherrschaften. Es sind dies die Städte Ellingen und Wolframs-Eschenbach $^{673}$ der Deutschordensballei Franken, Pappenheim als Residenzstadt der gleichnamigen Grafschaft und Scheinfeld als Zentralort der gefürsteten Herrschaft Schwarzenberg.

Mit Ausnahme von Wolframs-Eschenbach hatten diese Städte bis zum Ende des Alten Reiches Haupt- und Residenzortfunktionen zu erfüllen. Der Unterschied zu anderen Städten wird ersichtlich, wenn man die Mieterzahlen mit denen der Deutschordensstadt Wolframs-Eschenbach vergleicht. Bei einem Gesamtdurchschnitt von $40 \%$ dieser Städtegruppe weicht der Mieteranteil in WolframsEschenbach mit lediglich 14\% deutlich ab. Ausschlaggebend für den Mieteranteil einer Stadt ist also nicht ausschließlich die jeweilige Herrschaftssituation, sondern vielmehr die politische und wirtschaftliche Funktion.

\section{Kleinstädte im ehemaligen Hochstift Eichstätt 1818}

Der geringste Mieteranteil unter den untersuchten Stadtgruppen findet sich bei den sechs katholischen Landstädten des aufgelösten Hochstiftes Eichstätt. Durchschnittlich kamen diese Orte 1818 auf einen Anteil von lediglich 21\% Familien, die nicht im Eigentum wohnten. Die Werte streuen zwischen 11\% in Ornbau und $30 \%$ in Abenberg. Diese Städte waren allesamt sehr klein mit durchschnittlich gerade einmal 1.046 Einwohnern pro Stadt. Doch auch hier lässt sich kein $\mathrm{Zu}-$ sammenhang zwischen Stadtgröße und Mieteranteil erkennen. In Abenberg lebten exakt 1.046 Menschen, davon wohnten 30\% der Familien nicht im Eigentum. Im größeren Spalt waren dagegen nur 21\% der Familien auf fremdes Wohneigentum angewiesen.

\section{Gemeinsamkeiten und Unterschiede im Jahr 1818}

Nach der Übernahme der fränkischen Herrschaften in das neue Königreich Bayern ändert sich die lokale Wohnsituation nicht sofort. Die Angaben im Alphabetischen Verzeichnis spiegeln vielmehr noch die Situation am Ende des Alten Reiches wider. Von den untersuchten 33 Städten setzten sich die fünf größten Städte sowohl im Hinblick auf ihre Größe als auch im Mieteranteil deutlich ab von den 28 übrigen Orten, die mit ihren maximal 5.302 Einwohnern zurecht als Landstädte zu charakterisieren sind.

${ }^{673}$ Bis 1917 hieß die Stadt Eschenbach, sie wurde erst in diesem Jahr zu Ehren des Minnesängers aus dem Geschlecht der Eschenbach in Wolframs-Eschenbach umbenannt. 


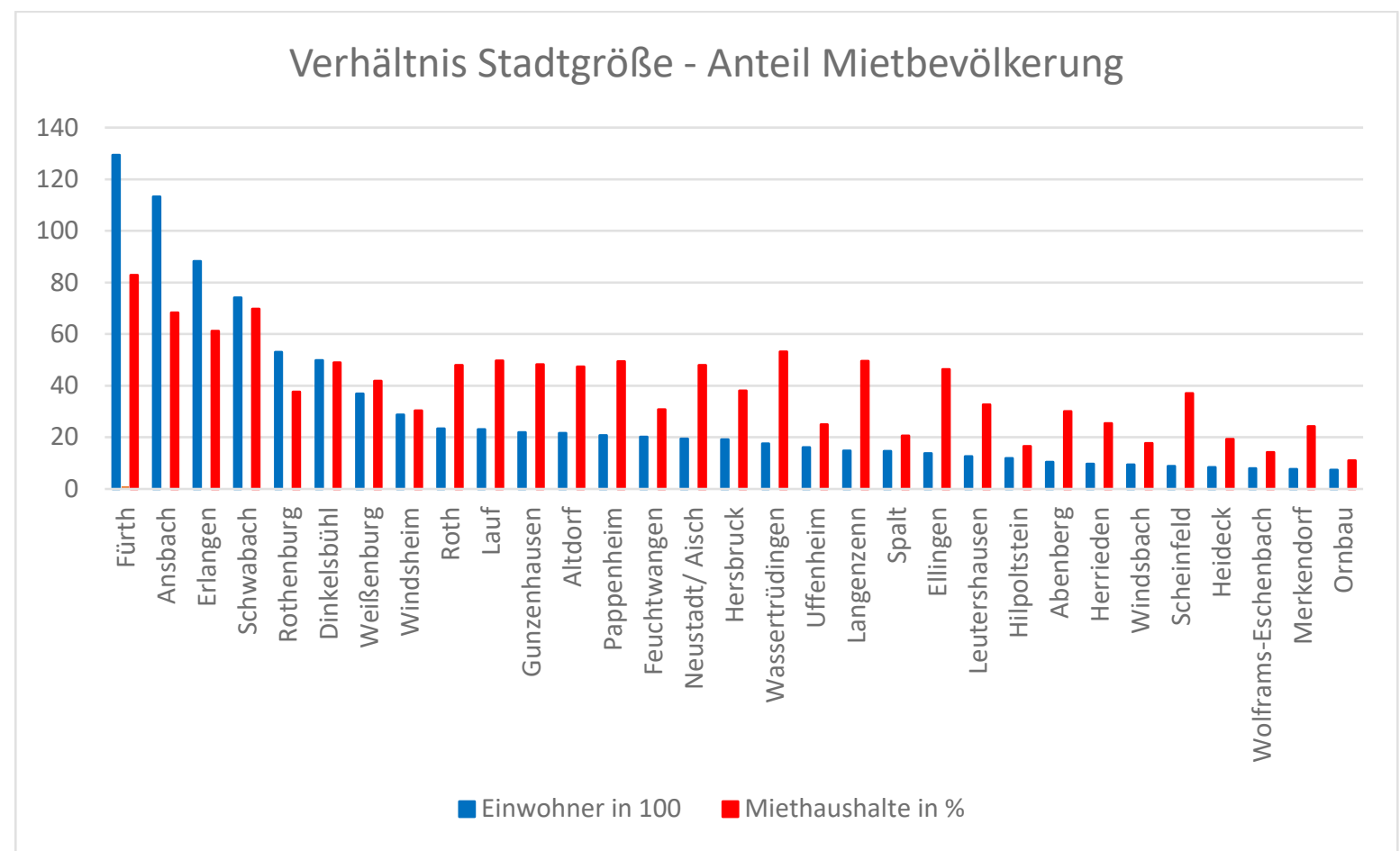

Grafik 4. Mitelfranken, Verhältnis Stadtgröße und prozentualer Anteil der Mietbevölkerung anhand der Angaben des Alphabetischen Verzeichnisses 1818 (Thomas Wenderoth 2019)

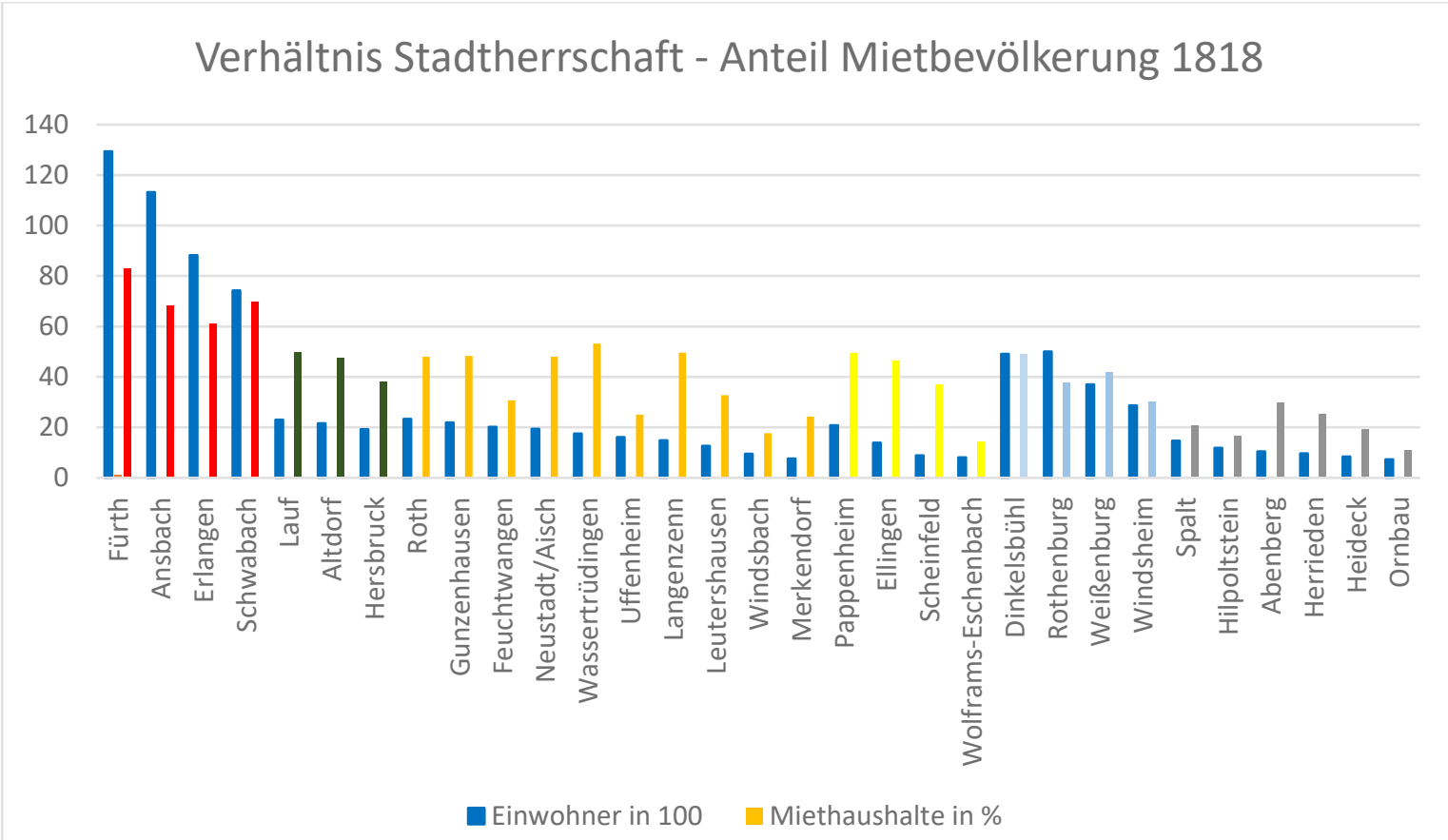

Grafik 5. Mittelfranken, Gliederung der Städte in Gruppen, 1818. blau = Einwohner in 100, rot $=$ Anteil der Miethaushalte in den großen Gewerbestädten in \%; grün = Anteil der Miethaushalte in den ehem. nürnbergischen Landstädten; ocker = Anteil der Miethaushalte in den ansbachischen Landstädten; gelb = Anteil der Miethaushalte in den Städten ehem. kleiner Herrschaften; hellblau = Anteil der Miethaushalte in den ehem. freien Reichsstädten; grau = Anteil der Miethaushalte in den ehem. Landstädten des Hochstiftes Eichstätt (Thomas Wenderoth 2019) 
Für die Gruppe dieser 28 Landstädte muss festgestellt werden, dass die Stadtgröße nur einen geringen Einfluss auf den Anteil der eigentumslosen Haushalte hatte. Im Diagramm sind 31 Städte, absteigend nach ihren Einwohnerzahlen, aufgeführt. ${ }^{674}$ Der Einwohnerzahl ist der prozentuale Anteil der Miethaushalte in jeder Stadt als roter Balken gegenübergestellt.

Der Anteil der Mietbevölkerung verläuft sehr viel unregelmäßiger. Grundsätzlich nimmt er zwar mit der Größe der Städte ab, unterliegt dabei jedoch stärkeren Schwankungen. Festzuhalten bleibt eine leichte Delle im Bereich der vier ehemaligen Reichsstädte, die größenmäßig an der Spitze der Landstädte stehen. Des Weiteren kristallisiert sich eine Gruppe von dreizehn Städten heraus, beginnend bei Roth mit 2.342 Einwohnern und 48\% Miethaushalten und endend bei Ellingen mit 1.386 Einwohnern und 46\% Miethaushalten. Diese Gruppe startet mit einheitlichen Mieterzahlen, kennt aber im zweiten Teil auch heftige Abweichungen der Mieterzahlen nach unten. Die letzte Gruppe, bestehend aus zehn kleinen Städten, erreicht nur in Ausnahmen (Scheinfeld 894 Einwohner, 37\% Miethaushalte) die hohen Mietanteile der größeren Landstädte, darüber hinaus ist jedoch kein Zusammenhang zwischen Stadtgröße und Mieteranteil zu erkennen.

Erfolgversprechender erscheint es, die Voraussetzungen für die unterschiedliche Höhe der Mieteranteile in den territorialen, politischen und ökonomischen Rahmenbedingungen zu suchen. Dafür wurden in der Grafik 5 die Städte, wie zu Beginn, nach ihren territorialen Zugehörigkeiten erfasst. Herausgelöst aus dieser territorialen Zuordnung wurden lediglich die vier mittelgroßen Städte: Fürth, Ansbach, Erlangen und Schwabach. Ansbach nahm als ehemalige Residenzstadt eine Sonderrolle ein, die sich in den $68 \%$ Miethaushalten widerspiegelt. Fürth, Erlangen und Schwabach waren wichtige Gewerbezentren mit einem hohen Anteil an eigentumslosen Arbeitern. Ebenfalls stark gewerblich geprägt waren Lauf und Roth mit 50\% beziehungsweise $48 \%$ eigentumslosen Haushalten. Diese sind jedoch in den zugehörigen territorialen Gruppen belassen worden, da sich hier jeweils Städte mit vergleichbar hohem Mieteranteil finden lassen, bei denen keine entsprechend starke gewerbliche Ausrichtung nachweisbar ist. Damit wird zugleich deutlich, dass die ökonomische Situation ebenfalls nicht allein den Mieteranteil bestimmt hat.

Innerhalb der territorialen Zugehörigkeit sind die Städte entsprechend ihren Einwohnerzahlen geordnet. Besonders gleichmäßig ist der Mieteranteil in den drei erfassten nürnbergischen Städten (grün). Deutliche Schwankungen sind für die hohenzollerischen Landstädte festzustellen (ocker): Fünf von zehn Städten zeichnen sich durch einen hohen Anteil an Mietbevölkerung aus, der um die 50\% lag (Wassertrüdingen 53\%, Langenzenn 50\%, Gunzenhausen, Neustadt an der Aisch

${ }^{674}$ Nürnberg und Velden bleiben unberücksichtigt. 
und Roth je 48\%). Die anderen fünf Städte setzen sich mit einem geringeren Anteil von $18 \%$ bis $33 \%$ Miethaushalten deutlich ab. Für die Differenzierung in zwei Gruppen liegen keine offensichtlichen Gründe vor. Allerdings sind unter der Gruppe mit den geringen Mietanteilen auch die drei kleinsten Städte des Markgraftums Ansbach: Leutershausen, Merkendorf und Windsbach, wobei Windsbach ebenso wie Feuchtwangen und Uffenheim jeweils Sitz eines Oberamtes waren und damit in ihrer politischen und administrativen Bedeutung den Städten in der ersten Gruppe nicht nachstanden.

Große Unterschiede finden sich auch in der Gruppe der vier ehemaligen freien Reichsstädte (hellblau).

Die eichstättischen Städte (grau) weisen allesamt nur geringe Einwohner- und Mieterzahlen auf, dies gilt insbesondere im Vergleich mit den vier ehemaligen Reichsstädten. Aber auch hier schwanken die Anteile der eigentumslosen Bevölkerung deutlich, wenn auch auf niedrigerem Niveau.

Festzuhalten bleibt, dass neben einigen ansbachischen Städten vor allem die Städte des ehemaligen Hochstiftes Eichstätt geringe Mieteranteile zu Beginn des 19. Jahrhunderts aufweisen.

\section{Entwicklung zwischen 1497 und 1700 am Beispiel Erlangens}

Die Entwicklung der Mietsituation in einer Kleinststadt vom ausgehenden 15. Jahrhundert bis zum Ende des 17. Jahrhunderts soll im Folgenden am Beispiel Erlangens dargestellt werden. Die kleinststädtische Situation in Erlangen ändert sich schlagartig im Jahr 1686 mit der Gründung der benachbarten Neustadt Erlangen. Diese brachte bald nach ihrer Gründung als prosperierende Exportgewerbestadt erhebliche wirtschaftliche und soziale Veränderung auch für die Altstadt. Ab 1700 ist daher die Altstadt Erlangen nicht mehr als Kleinststadt zu betrachten, sondern als Teil der nunmehr gewerblichen geprägten Doppelstadt Erlangen. Zudem soll an diesem Beispiel verdeutlicht werden, dass bei genauerer Analyse - sprich dem Lesen zwischen den Zeilen - erheblich mehr eigentumslose Haushalte ausfindig gemacht werden können als auf den ersten Blick wahrgenommen worden sind. Die oben angeführten Zahlen sind daher nur mit Vorsicht zu gebrauchen, da sie mitunter große Lücken aufweisen. Ein Vergleich innerhalb der Gruppe erscheint aber grundsätzlich legitim, da auch bei einer anzunehmenden Individualität in der Erfassung der einzelnen Städte diese doch Teil einer Gesamterfassung mit identischer Zielsetzung sind.

Für Erlangen sind die Quellen zur Bevölkerungsstruktur bereits vorbildlich erschlossen. Auch wenn bei den Veröffentlichungen bisher zu keinem Zeitpunkt die Eigentumslosen im Fokus standen, finden sich dort wichtige Informationen. 
Die ältesten Hinweise sind im Reichssteuerregister von 1497 enthalten: ${ }^{675} \mathrm{Ge}$ nannt werden 92 steuerpflichtige Personen (Ehefrauen, Kinder und Gesinde sind inbegriffen, Kinder unter fünfzehn Jahren bleiben unerwähnt). Nur ein einziges Mal ist ein Haushaltsvorstand mit dem Begriff Hausgeno $\beta$ als Mieter gekennzeichnet. Nicht als Mieter erwähnt werden die öffentlich Bediensteten. Dabei wird es sicher bereits Torwächter, einen Bader und einen Pfarrer gegeben haben, die letztendlich auch zur Gruppe der Mieter zu zählen wären. ${ }^{676}$ Es muss also davon ausgegangen werden, dass mindestens drei weitere Haushalte von den genannten 92 Haushalten kein Wohneigentum besaßen. Dennoch stellten die Eigentumslosen am Ende des Mittelalters in Erlangen eine große Ausnahme dar. Insgesamt werden 212 Einwohner über fünfzehn Jahren genannt. Im Urbar von 1528 sind dagegen nur die Grundstückseigentümer und damit die Hausbesitzer erfasst worden. ${ }^{677}$ Gelistet sind dort 82 Hausstellen mit ihren Eigentümern sowie das Pfarrund das Badhaus. Sollte die Anzahl der Hausstellen zwischen 1497 und 1528 nicht wesentlich von 95 ( 92 Haustellen zuzüglich Pfarrhaus, Badhaus und Türmerwohnung) auf 84 abgenommen haben, muss wohl der Anteil der eigentumslosen Haushalte 1497 um ein Beträchtliches höher gelegen haben: Vereinfacht wären den 95 Haushalten 82 Eigentümer gegenüberzustellen, woraus sich ein Anteil von 14\% Miethaushalten errechnet.

Mit dem Besitzverzeichnis (Urbar) aus dem Jahr 1555-57 verhält es sich ähnlich wie mit dem Reichssteuerregister; Mieter, die mit im Haus des Eigentümers wohnten, waren für die Zwecke des Urbars nicht wichtig und blieben daher unerwähnt. Lediglich der Eintrag Hansen Luffts Behausung, wo er hinlesst, verweist auf ein vermietetes Haus in der nördlichen Vorstadt. ${ }^{678}$ Ansonsten sind 87 abgabepflichtige Eigentümer inklusive des Vermieters Hans Luft genannt, darunter aber auch der Pfarrer und mindestens eine Eigentümerin, die nur Land besaß. ${ }^{679}$

1616 hat Johannes Heilig, der Pfarrer der Altstadt, eine Liste aller Einwohner verfasst, die eine quantitative Aussage zum Mieteranteil ermöglicht. ${ }^{680}$ Insgesamt notierte er 104 Anwesen mit den Nummern 301-404. Von diesen waren zwei unbewohnt. ${ }^{681}$ Weitere neun Anwesen gehörten wohl der öffentlichen Hand, auch wenn dies aus der Aufstellung nicht ersichtlich ist: So wird bei Heilig der Bader Hans Helmreich ohne weiteren Zusatz in der Liste geführt, erst aus der amtlichen Liste von 1619 (siehe unten) geht hervor, dass er kein Bürger, sondern Beständner

\footnotetext{
675 RECHTER 1985, S. 346-348, BISCHOFF 1959, S. 113-114.

${ }^{676}$ Die einzelnen Personen sind nicht mit Berufsbezeichnungen charakterisiert, daher ist weder die genaue Zahl bekannt noch ob diese im Reichssteuerregister überhaupt komplett erfasst sind.

677 BISCHOFF 1959, S. 115-116.

678 BisCHOFF 1961a, S. 31.

${ }^{679}$ Eintrag 211a: Gertraud Peerin; BISCHOFf 1961a, S. 28.

${ }^{680}$ BISCHOFF 1961, S. 49-52.

${ }^{681}$ Ebd.: Nr. 378, Katzenturm und 403, Ziegelhütte.
} 
(Mieter) war. Zur Gruppe der Mieter hinzuzuzählen sind daher sicher noch der Bader, der Stadtknecht - Das Untere thorhau $\beta$, darauf der Stattknecht wonet. Christoff Leutenbacher ${ }^{682}-$, der Pfarrer im Pfarrhof, ${ }^{683}$ der Kantor im Obergeschoss und eine weitere Mieterin im Erdgeschoss des Schulhauses, ${ }^{684}$ ein Mieter im Frühmesshaus (1624 wohnte hier der Sohn des Pfarrers als Mieter) ${ }^{685}$ der Wildmeister im Wildmeisterhaus, ${ }^{686}$ der Stadtschreiber in einem Hinterhaus, ${ }^{687}$ der Richter im Fürstenhaus ${ }^{688}$ sowie Niclauß Edelmann, der im Seelhaus wohnte und vermutlich keine Miete zahlte, weil er zu arm gewesen sein dürfte. ${ }^{69}$ Nach Abzug dieser Hausstellen könnte es sich demnach maximal bei 93 Einträgen um private Anwesen gehandelt haben, die vom Eigentümer bewohnt wurden. Von diesen Häusern werden 1616 vier Häuser genannt, in denen insgesamt sieben Mieter wohnten: Georg Löhners Bestendtner, Georg Linck und Hieronimus Batz. ${ }^{690}$ Johann Heiligen, Pfarrern zu Statt Erlang, erkaufftes Haus darinnen zum bestandt [...] itzo Hieronymus Zahn, ein tuechmacher. ${ }^{691}$ Hans Vischer, Reiser, samt drei Bestendtnern, Veit Wagner, Barthel Henffling, Hans Hoffman. ${ }^{692}$ Conrad Kollöffel, und ein Bestendtnern Wolff Maisenbuech. ${ }^{693}$ In zwei Häusern lebte zudem noch der Eigentümer. ${ }^{694}$ Daraus ergibt sich eine Zahl von 91 privaten Eigentümerhaushalten und siebzehn Miethaushalten. Bei weiteren acht Privathäusern sind jeweils zwei Namen aufgeführt, zum Beispiel: Margaretha Wagnerin, alt Hascherin. ${ }^{695}$ Die zweiten Namen können ebenfalls als mietende Mitbewohner interpretiert werden, sodass sich insgesamt eine Zahl von 25 Miethaushalten ergibt. Daraus errechnet sich an Anteil von gut $20 \%$ eigentumsloser Haushalte.

Für das Jahr 1619 hat sich eine weitere Aufstellung erhalten: Verzeichnis und Specification der Burger zu Erlang mit ihrem Namen, samt den Handwerkern und Hausgenossen, daselbst, Actum 11. August 1619. ${ }^{696}$ Genannt werden 84 Bürger sowie elf Beständner und Hausgenossen. Warum in der Überschrift die beiden Bezeichnun-

\footnotetext{
682 Zitiert nach Bischoff 1961b, S. 52, Nr. 374.

${ }^{683}$ Ebd., S. 50, Nr. 325.

${ }^{684}$ Ebd., S. 50, Nr. 327.

${ }^{685}$ Ebd., S. 50, Nr. 328.

${ }^{686}$ Ebd., S. 50, Nr. 338.

${ }^{687}$ Ebd., S. 51, Nr. 348.

${ }^{688}$ Ebd., S. 51, Nr. 350.

${ }^{689}$ Ebd., S. 50, Nr. 314; NÜRNBERGER 2003, S. 205.

${ }^{690}$ Zitiert nach BISCHOFF 1961b, S. 50, Nr. 332.

${ }^{691}$ Ebd., S. 51, Nr. 57.

${ }^{692}$ Ebd., S. 51, Nr. 361.

${ }^{693}$ Ebd., S. 51, Nr. 370.

${ }^{694}$ Ebd., S. 51, Nr. 361 und 370.

${ }^{695}$ Ebd., S. 51-53, Nr. 402; sowie folgende Nummern:

404., 307., 322., 323., 336., 358., 359., 362., 393.

696 Zitiert nach BISCHOFF 1961b, S. 53.
} 
gen Beständner und Hausgenossen parallel verwandt wurden, ist nicht ersichtlich. Aus diesen Zahlen errechnet sich ein Mieteranteil von 12\%; wobei hier noch die Haushalte in einer Dienstwohnung hinzuzuzählen sind, denn die öffentlich Bediensteten fehlen gänzlich in dieser Aufstellung: Pfarrer, Kantor, Stadtknecht, Wildmeister, Stadtschreiber. Damit erhöht sich der Mieteranteil auf 16\%.

Vergleicht man die Angaben von 1616 und 1619, fällt ein deutlicher Unterschied auf. Während 1616 insgesamt 116 Haushalte verzeichnet sind, wurden 1619 nur 95 Haushalte erwähnt. In jedem Fall dürfte die Anzahl der Hausstellen und Eigentümer konstant geblieben sein. Da 1619 lediglich 84 Bürger (Eigentümer) erfasst worden sind und diese Zahl mit den Angaben von 1528 korrespondiert, kann sie auch für die Erhebung des Jahres 1616 in Ansatz gebracht werden. Demnach lag der Anteil der Miethaushalte 1616 nicht nur bei 20\%, sondern wohl eher bei 27\%. Alleinstehende Frauenhaushalte als klassische Mietergruppe fehlen in den Listen 1616 und 1619 zudem weitgehend.

Diese Modellrechnungen zeigen zugleich auf, wie schwierig es ist, Mieteranteile auf der Grundlage historischer Quellen exakt zu ermitteln. Auf den ersten (oberflächlichen) Blick scheinen Miethaushalte in der Kleinststadt Erlangen keine Rolle gespielt zu haben, erst aus der Zusammenschau verschiedener Quellen kehrt sich das gewonnene Bild ins Gegenteil um. Vermutlich muss bereits am Ende des 15. Jahrhunderts mit einem prozentualen Anteil von 20\% eigentumslosen Haushalten gerechnet werden, der sich kurz vor dem Dreißigjährigen Krieg auf fast $30 \%$ erhöht hatte.

\section{Zusammenfassung: Bevölkerungsstruktur fränkischer Kleinstädte}

Zahlen vornehmlich des 18. und frühen 19. Jahrhunderts zu den beiden größten Herrschaftsgebieten in Franken, den Markgraftümern Brandenburg-Bayreuth und Brandenburg-Ansbach, wurden bereits in meiner Dissertation diskutiert. ${ }^{697}$ Doch wie stellt sich die Situation der Miethaushalte in Franken im Verlauf der frühen Neuzeit insgesamt dar? Bisher konnten nur folgende sieben Städte ermittelt werden, für die belastbare Zahlen über einen langen Zeitraum vorhanden sind: Ansbach, Gunzenhausen, Langenzenn, Roth, Schwabach, Uffenheim und Wassertrüdingen.

${ }^{697}$ WeNDEROTH 2019a, Kapitel II.3.4, S. 65-69. 


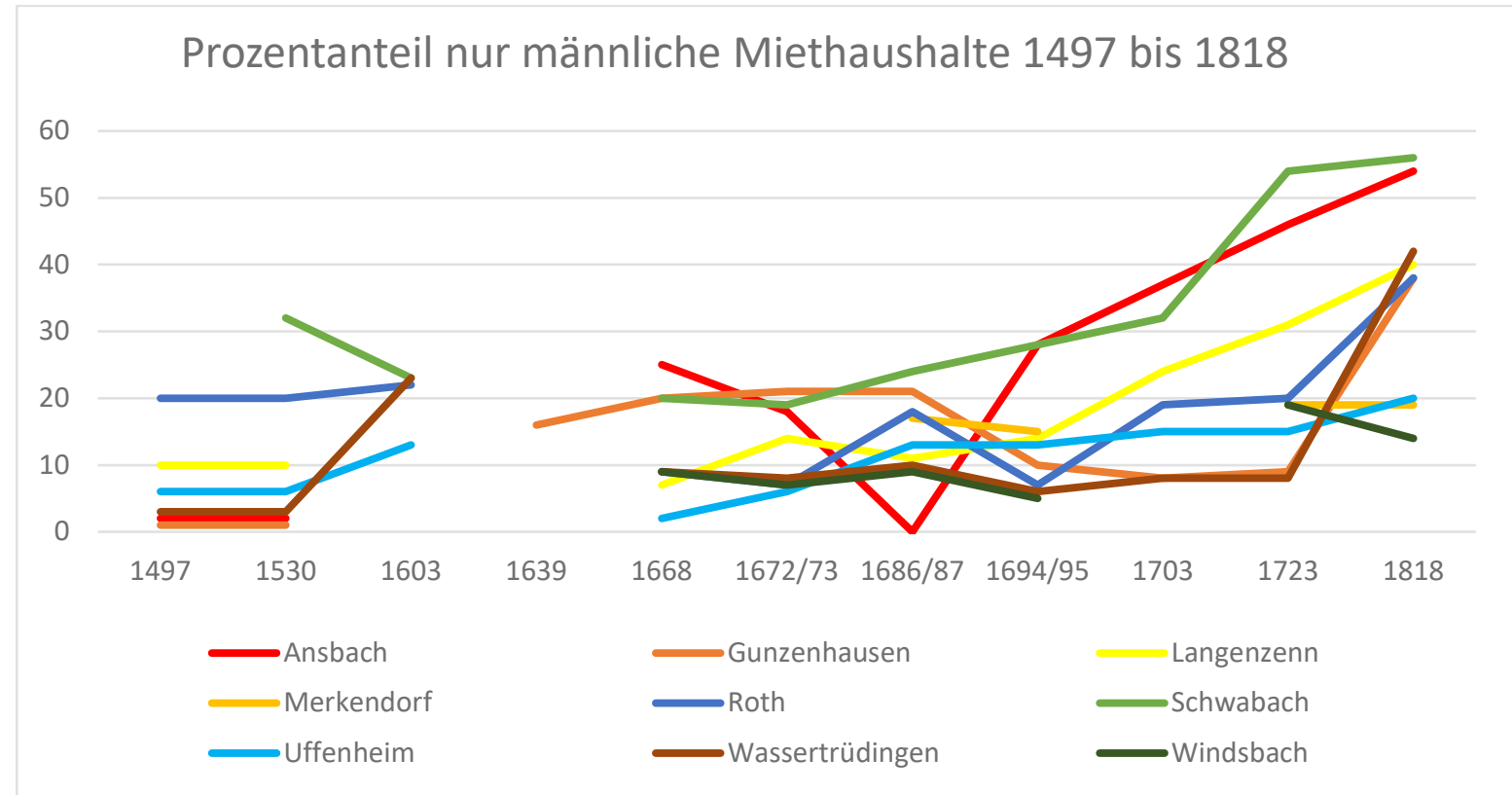

Grafik 6. Anteil der in den Quellen erfassten Miethaushalte in sieben fränkischen Städten 14071818 (Thomas Wenderoth 2019)

In dem Diagramm wurden bewusst nur die männlichen Miethaushalte erfasst, um eine bessere Vergleichbarkeit der Zahlenwerte zu erhalten. Weibliche Haushaltsvorstände sind unter den Mietern in den Huldigungslisten (1603-1723) nur unzureichend enthalten. Auffällig ist der geringe Anteil männlicher Miethaushalte im Reichssteuerregister 1497, dies gilt besonders im Hinblick auf Ansbach, Gunzenhausen und Wassertrüdingen. Hier wären weitere ortsgeschichtliche Forschungen notwendig, um die Angaben des Reichssteuerregisters zu verifizieren.

Für das Jahr 1818 lassen sich nur die Gesamtzahlen der Mieter errechnen, hier wurde als pauschaler Ansatz ein Anteil von 20\% an weiblichen Mietern abgezogen, um eine Vergleichbarkeit herzustellen. In Ansbach führt die Residenzfunktion erst ab 1700 zu einem großen Anstieg der Mietbevölkerung. Schwabach und Roth weisen zu Beginn der Neuzeit einen relativ hohen Anteil an Miethaushalten im Vergleich zu anderen ansbachischen Städten auf. In Schwabach sinkt der Mieteranteil bereits vor dem Dreißigjährigen Krieg, in Roth erst nach dem Krieg. Es folgt allgemein eine Phase der Stagnation. Erst ab den 1670er Jahren nimmt die Mietbevölkerung wieder zu, in Schwabach kontinuierlich, in Roth mit einem Einbruch um 1690. Letzterer könnte aber auch durch eine unpräzise Erfassung in den Huldigungslisten verursacht sein.

Insgesamt betrachtet nimmt der Anteil der Miethaushalte bis zum Ende des Alten Reiches deutlich zu. Insbesondere ist für das 18. Jahrhundert eine starke Dynamik festzustellen. Ganz an der Spitze stehen Ansbach und Schwabach, wobei in Schwabach bereits 1723 der Mieteranteil so hoch lag, dass der entsprechende Prozentsatz in den nachfolgenden 100 Jahren nur noch gering ansteigen konnte, die 
Kurve flacht hier entsprechend ab. Abweichend davon nimmt in Wassertrüdingen, Roth und Gunzenhausen der Mieteranteil erst nach 1723 signifikant zu. In Ansbach und Langenzenn beginnt dieser Prozess dagegen bereits in den 1680er Jahren. Uffenheim wiederum steht stellvertretend für eine Reihe von Landstädten, deren Mieteranteil zwar wächst, aber eben nicht mit der gleichen Dynamik wie im Falle der anderen sechs Städte. Die Situation in Windsbach ist gleichfalls von einer relativ niedrigen Zahl an männlichen eigentumslosen Haushalten geprägt, deren Anteil sinkt sogar noch nach 1723.

\section{Kleinstädte außerhalb Frankens}

\section{Kursachsen, Westfalen, Hessen, Brandenburg, Baden, Württemberg}

Festzustellen bleibt, dass in vielen kleineren Städten der Anteil der Miethaushalte bis ins 18. Jahrhundert hinein überschaubar geblieben ist. Für das untersuchte Gebiet bezeugt dagegen die Stadt Schwabach, dass es schon zu Beginn des 16. Jahrhunderts Kleinstädte mit einem beachtlichen Anteil an Mietbevölkerung gab, es handelt sich dabei aber nicht um einen Einzelfall. Für die sächsische Stadt Plauen liegen Zahlen aus dem Jahr 1551 vor: Nachgewiesen sind für die Altstadt 158 Eigentümer und 154 Mieter (Inwohner), für die Neustadt 85 Eigentümer und 63 Mieter. Der Anteil der eigentumslosen Haushalte lag für die Altstadt also mindestens bei $51 \%$ und für die Neustadt bei $43 \% .{ }^{698}$

Nachfolgend soll daher die Situation in Franken flächenhaft mit der Situation in Kursachsen verglichen werden. Einen Überblick zur Entwicklung von 95 kursächsischen Städten während der frühen Neuzeit bietet die Habilitationsschrift von Katrin Keller. Sie arbeitet drei Stadttypen heraus: Ackerbürgerstadt, Bergstadt und Exportgewerbestadt. ${ }^{69}$ Die meisten der bisher erwähnten mittelfränkischen Städte wären wohl ebenfalls dem Typus Ackerbürgerstadt zuzuordnen. In Kursachsen teilt Keller lediglich sechzehn Städte in diese Gruppe ein (KeLler 1997, Tabelle 3), sie weisen im Jahr 1699 minimal 74 und maximal 248 Haushalte auf, entsprechen also auch im Hinblick auf ihre Größe den fränkischen Landstädten.

Als fränkische Exportgewerbestädte können Erlangen, Fürth, Lauf, Nürnberg, Roth und Schwabach gelten. In Kursachsen sind in diese Gruppe 25 Städte eingeordnet (KELLER 1997, Tabelle 4); mit durchschnittlich 1.517 Einwohnern sind sie deutlich größer als die Ackerbürgerstädte. Dies trifft auch auf die sechs genannten fränkischen Städte zu.

698 BLASCHKE 1957, S. 350.
${ }^{699}$ KELLER 1997, S. 51-63. 
Konkrete Angaben zu Miethaushalten finden sich jedoch nur für vier der behandelten 95 kursächsischen Städte. Ansonsten kann der Anteil der Mietbevölkerung nur grobmaßstäblich anhand der Behausungsziffer nachverfolgt werden. Mit der Behausungsziffer gibt Keller an, wie viele Personen in der betreffenden Stadt durchschnittlich in einem Haus gewohnt haben. Ihren Betrachtungen legt sie generell eine Haushaltsgröße von 4,3 Personen zugrunde. ${ }^{700}$ Diesen Wert hat sie auf der Grundlage von exakten Erhebungen in sechzehn Städten im Jahr 1699 ermittelt. Der Wert dient ihr auch als Multiplikator, um aus den angegebenen Haushaltsvorständen die Einwohnerzahlen zu rekonstruieren. Vergleicht man die kursächsische Behausungsziffer der Jahre 1699 (KeLLER 1997, Tabelle 1) und 1811 (KELLER 1997, Tabelle 7), so stieg die durchschnittliche Behausungsziffer aller 95 Städte von 5,3 auf 7,3 Personen pro Haus an. Nur in ganz wenigen Kleinstädten ist ein Rückgang der Behausungsziffer und damit der Mietbevölkerung zu verzeichnen (Bischofswerda, Geithain, Gräfenhainichen, Hohnstein, Jessen, Niemegk, Radeberg, Senftenberg). In diesen acht Orten wuchs lediglich die Zahl und der Anteil der Eigentümer. In allen betrachteten Ackerbürgerstädten stieg hingegen die Behausungsziffer auf einen Wert von 6,1 im Jahr 1811 (KeLLER 1997, Tabelle 10). Bei der zugrunde gelegten Haushaltsgröße von 4,3 Personen mussten sich also durchschnittlich 1,42 Haushalte ein Haus teilen, der Anteil der Miethaushalte kann daher überschlägig mit 30\% angenommen werden. ${ }^{701} 1699$ lag die Behausungsziffer der Ackerbürgerstädte noch bei 4,6 Personen, woraus sich ein minimaler Anteil der Miethaushalte von lediglich 6,5\% errechnet.

Anhand des Alphabetischen Verzeichnisses ist ein Vergleich der sächsischen Städte mit der Situation in Franken im Jahr 1818 möglich (vgl. Tab. 23). Die 26 fränkischen Landstädte (ohne Nürnberg, Fürth, Ansbach, Erlangen, Schwabach, Velden und Dinkelsbühl) weisen durchschnittlich einen Anteil von 35\% an Miethaushalten auf, die durchschnittliche Haushaltsstärke lag jedoch nur bei 3,82 Personen pro Haushalt.

Die kursächsischen Zahlen des Jahres 1699 wiederum sind am besten zu vergleichen mit den Angaben aus den Ansbacher Huldigungslisten 1692/95 und 1703 (vgl. Tab. Anhang I.6 und I.7.). Der durchschnittliche Anteil an Miethaushalten lag bei den ansbachischen Landstädten (ohne Schwabach und Ansbach) 1692/95 bei $9 \%$ und 1703 bei 10\%. Damit wiesen die mittelfränkischen Landstädte einen etwas höheren Mieteranteil auf als die kursächsischen Ackerbürgerstädte. Bezieht man in die Betrachtung die Exportgewerbestädte und Bergstädte mit ein, würde sich die Situation umkehren.

\footnotetext{
${ }^{700}$ KELLER 1997, S. 43f.

${ }^{701}$ Für die überschlägige Berechnung wird die Annahme zugrunde gelegt, dass jeder Eigentümer ein ganzes Haus in Besitz hatte und dieses auch bewohnte. Geteiltes Hauseigentum und öffentliche Wohnbauten bleiben unberücksichtigt.
} 
Wie bereits erwähnt, gibt Keller für vier Städte konkrete Zahlen zu den Miethaushalten wieder, die Mieter wurden dort ebenfalls als Hausgenossen bezeichnet. ${ }^{702}$ In der Ackerbürgerstadt Schildau stieg der Anteil der Miethaushalte von 9\% $(1701)^{703}$ auf 26\% (1806). In der kleinen Bergstadt Aue wächst die Mietbevölkerung hingegen von hohen $47 \%$ (1701) nur in geringem Maß auf 49\% (1806), was bei dieser extremen Ausgangssituation auch nicht weiter verwundert. Die Parallelen zu Schwabach sind offensichtlich. Die Stadt Frohburg definiert Keller als Exportgewerbestadt, der geringe Anteil an Miethaushalten von 12\% (1688) und 26\% (1753) lässt diese Zuordnung erst einmal nicht vermuten. Für Delitzsch sind lediglich Mieterzahlen für das Jahr 1826 überliefert: Der Anteil der Miethaushalte lag bei $24 \%$.

Ein geringer Anteil an Miethaushalten lässt sich auch für zwei norddeutsche Landstädte nachweisen: Für die Stadt Mölln hat sich Frank Braun mit dortigen Mietern, die als Inste bezeichnet werden, befasst. ${ }^{704}$ Er ermittelt anhand der Steuerregister der Jahre 1587 und 1649 rund 11\% Mieter, nach dem Kontributionsregister 1660 lag deren Anteil bei knapp 15\% (47 Mieter von 315 Steuerpflichtigen). ${ }^{705}$ Gegen Ende des 17. Jahrhunderts sinkt die Zahl der erfassten Miethaushalte und lässt sich erst wieder anhand einer Zählung aus dem Jahr 1740 exakt nachvollziehen. ${ }^{706}$ Erfasst wurden 264 Haushaltsvorstände, darunter nur 23 Miethaushalte, was lediglich einem Anteil von $8,3 \%$ entsprach. Diese wiederum unterschieden sich in fünf Mieter, die mit im Haus des Vermieters lebten, und achtzehn Mieter, die ein ganzes Haus gemietet hatten.

Für die Stadt Blomberg in Lippe sind erstmals 1776 konkrete Angaben zum Anteil der Mietbevölkerung möglich. ${ }^{707}$ Zum damaligen Zeitpunkt wurden 60 Miethaushalte gezählt, davon hatten sechzehn ein ganzes Haus gemietet, der größere Teil von 44 Haushalten lebte dagegen im Haus des Vermieters. Für das Jahr 1624 werden 308 Wohnhäuser genannt, woraus sich eine ungefähre Anzahl von 290 Eigentümern herleiten lässt, der Anteil der Miethaushalte lag in Blomberg demnach gegen Ende des 18. Jahrhunderts noch unter 20\%. Die Mieter gehörten der ärmsten Bevölkerungsschicht an, dies gilt zum Großteil ebenfalls für die Vermieter. Daraus ergab sich für die Mieter eine Wohnlage am Rand der Stadt.

Auch für die Städte der Grafschaft Mark ist ein ähnlich niedriger Mieteranteil anzunehmen: Helmut Möller gibt für das Jahr 1787 an, dass die 24 Städte eine durchschnittliche Behausungsziffer von 4,9 Personen pro Haus aufwiesen. Bei

\footnotetext{
702 Keller 1997, S. 121.

${ }^{703}$ Für das Jahr 1699 gibt Keller (Tabelle 1) eine Behausungsziffer von 5,0 an, woraus sich bei einer Haushaltsstärke von 4,3 ein Mieteranteil von 14\% errechnet.

704 BRAUN 1991.

${ }^{705}$ Ebd., S. 28.

706 Ebd., S. 29 und 32.

${ }^{707}$ STIEWE 1996, S. 234f, Karte 19.
} 
einer Haushaltsgröße von etwa vier Personen kann der Mieteranteil dort nicht viel über $20 \%$ gelegen haben. ${ }^{708}$ Vergleichbare Zahlen $(5,0)$ sind für sieben hessenkasselsche Landstädte belegt, darunter Hersfeld mit 3.642 Einwohnern als größte dieser sieben Städte. Interessant ist dabei der Vergleich mit den Dörfern, die zumindest in Hessen um die Mitte des 18. Jahrhunderts einen vergleichbar hohen Anteil an Miethaushalten von 17,5\% aufwiesen. ${ }^{709}$

Mölln und Blomberg sowie die Städte der Landgrafschaft Hessen-Kassel und der Grafschaft Mark sind daher, bezogen auf die Situation im 18. Jahrhundert, am ehesten mit den eichstättischen Landstädten zu vergleichen, in den übrigen mittelfränkischen Landstädten war das Mietwohnen zu diesem Zeitpunkt dagegen bereits deutlich stärker verbreitet.

Helmut Möller gibt darüber hinaus für weitere Städte Behausungsziffern wieder, die wertvolle Rückschlüsse auf die Wohnsituation an sich und indirekt auch auf den Mieteranteil ermöglichen. ${ }^{710}$ In Durlach lag der Wert im Jahr 1766 bei 8,2 Personen pro Haus, für weitere fünf Städte im Schwarzwald nennt er einen Durchschnittswert von 7,0 für das Jahr 1769.

Ein Anwachsen der Mieterzahlen, wie es für die brandenburg-ansbachischen Städte im 18. Jahrhundert belegt ist, lässt sich anhand seiner Angaben jedoch nur für sieben Städte in der Mark Brandenburg nachweisen: Während die Behausungsziffer dort in der 1. Hälfte des 18. Jahrhunderts bei lediglich 5,2 lag, stieg diese in der 2. Hälfte auf einen Wert von 7,7 an.

${ }^{708}$ Vgl. hierzu und im Folgenden MöLLER 1969, S. 120.

${ }^{709}$ VITS 1993, S. 161-213; zusammengefasst bei WENDEROTH 2019a, S. 55.

${ }^{710}$ MÖLLER 1969, S. 120. 


\section{Einfluss der Stadtfunktion auf den Anteil eigentumsloser Haushalte}

Bei der Gliederung der Städte nach ihrer Größe stand der quantitative Aspekt der Bevölkerungszahl im Vordergrund. Im Folgenden soll nunmehr der Frage nachgegangen werden, ob nicht andere Faktoren ebenfalls großen Einfluss auf die Eigentumsstruktur der Stadtbevölkerung hatten. Lässt sich ein Zusammenhang zwischen der spezifischen Funktion einer Stadt und dem Anteil eigentumsloser Haushalte herstellen? Städte nach Funktionen zu ordnen ist nicht ganz einfach, da eine Stadt grundsätzlich viele Funktionen zu übernehmen hat. Wenn ich im Folgenden Städte nach Funktionen gliedere, so beziehe ich mich auf Funktionen, die den Charakter einer Stadt dominiert haben und sich aus einer politischen oder wirtschaftlichen Aufgabe ergeben.

Ausgehend von der mittelfränkischen Situation, die im Zentrum dieser Untersuchung steht, sollen daher die Stadttypen der Residenzstadt, der Exportgewerbestadt und der Universitätsstadt untersucht werden. Die in diesem Zusammenhang noch anzuführende Kategorie der Ackerbürgerstadt ist in Franken weitgehend identisch mit dem Typus der Kleinstadt und bildet dort die Hauptuntergruppe. Auf eine separate Behandlung des Stadttyps Ackerbürgerstadt wird aus diesem Grund verzichtet. Zudem erscheint der Begriff emotional zu aufgeladen und verleitet zu Fehldeutungen. Ein weiterer wichtiger Stadttyp wäre zudem die Garnisonsstadt, die an dieser Stelle aber ebenfalls nicht separat behandelt wird, da die ausgewerteten Quellen zum Umfang der militärischen Bevölkerung nur unzureichende Informationen enthalten.

Nachdem die Definition der Stadttypen verschiedenen Kriterien folgt, gibt es selbstverständlich auch Überschneidungen und Veränderungen: So war die Kleinstadt Schwabach zeitweise eine bedeutende Exportgewerbestadt, der damit verbundene Bevölkerungsanstieg führte wiederum dazu, dass sie am Ende des Untersuchungszeitraums nicht mehr zu den Kleinstädten, sondern zu den Mittelstädten zu zählen ist. Erlangen wiederum kann in der ersten Hälfte des 18. Jahrhunderts zugleich unter den Kategorien Exportgewerbestadt und Residenzstadt betrachtet werden. Seit 1743 war sie zudem Universitätsstadt.

In einem abschließenden Kapitel soll zudem die Situation in den Vorstädten außerhalb der Stadtbefestigung untersucht werden. 


\section{Residenzstädte}

Die Residenzstadt ist gekennzeichnet durch die Hofhaltung, die insbesondere seit der Barockzeit eine große Zahl an Hofbediensteten und bei gleichzeitiger Hauptstadtfunktion auch von Verwaltungskräften nach sich zog. Der Hof selbst und in seinem Gefolge der Adel veränderten zudem die Konsumstruktur einer Stadt, dies ging zusammen mit einer stärkeren Nachfrage nach Luxusgütern und Dienstleistungen. Eng damit in Zusammenhang standen die entsprechenden Einkommensmöglichkeiten auch für eigentumslose Haushalte. Die Hofhaltung in einer Stadt führte daher zu einer Erhöhung der Einwohnerschaft, wobei die Beamten, Hofdiener etc. weniger zur Gruppe der Eigentümer, sondern in großem Umfang zur Gruppe der Mieter gehörten und sich daher in Folge der Mieteranteil eines Ortes deutlich erhöhte. In der Regel gehörten die Hofangehörigen nicht der bürgerlichen Stadtgemeinde an, sondern bildeten eine eigene juristische „Hofgemeinde“. Aus diesem Grund fehlen in den Listen der Stadtgemeinden häufig Personengruppen, die im großen Umfang zu den Mietern gezählt werden müssen.

$\mathrm{Zu}$ Beginn wird die Situation anhand einiger ausgewählter fränkischer Residenzstädte beleuchtet. Anschließend wird der Blick geweitet auf Residenzstädte, die zu Großstädten mutieren. Zum Schluss geht der Blick zurück zu den Kleinresidenzen und damit auch wieder nach Franken.

\section{Franken}

Für die Stadt Bayreuth ist dieser Umstand bereits für das frühe 17. Jahrhundert belegt, als der neue Markgraf Christian die Stadt als Residenzort nach einem verheerenden Stadtbrand 1605 beibehält. Der Rat der Stadt Bayreuth war von der Wahl des Markgrafen nicht begeistert, vermutlich fürchteten die Ratsherren, zukünftig ihre bürgerliche Unabhängigkeit zu verlieren. ${ }^{711}$ Als Gegenargument wurde von städtischer Seite darauf verwiesen, dass es in der Stadt an geeigneten Quartieren zur Unterbringung der Hofgesellschaft fehle. Karl Hartmann schreibt: „Der Plan kommt zur Durchführung; was nicht im Schloß Quartier fand, wird in Bürgerhäusern untergebracht, und schon im nächsten Jahr teilt der Fürst dem Rat die vorgebrachten Beschwerden mit, daß die Bayreuther viel $\mathrm{zu}$ hohe Mieten von Hofangestellten verlangen. “712

Die frühesten Hinweise zum Anteil der Mietbevölkerung in Ansbach finden sich im Reichssteuerregister 1497. Dort sind 451 Haupteinträge und 63 Hausgenos-

\footnotetext{
711 HARTMANN 1949, S. 107.

712 EBD.
} 
sen aufgeführt. Der Anteil der Hausgenossen entsprach etwa $12 \%$ der aufgeführten Haushalte. ${ }^{713}$ Die Stadt unterscheidet sich damit kaum von den anderen Städten des Markgraftums. Wie bereits ausgeführt, dürfte es sich bei den als Hausgenossen bezeichneten Personen jedoch nur um die Untermieter handeln, denn diese $12 \%$ liegen zum Beispiel deutlich unter dem Wert der Residenzstadt Dresden aus dem Jahr 1489. ${ }^{714}$

Anhand der Huldigungslisten kann für die Residenzstadt Ansbach gut die Bevölkerungsentwicklung in der bürgerlichen Stadtgemeinde zwischen 1668 und 1723 nachvollzogen werden. Grundsätzlich ist innerhalb der Bürgergemeinde ein Anstieg der Miethaushalte in diesen 55 Jahren festzustellen. Dieser fällt besonders deutlich für den Zeitraum ab 1703 auf.

\section{Ansbach, Miethaushalte nach den Huldigungslisten 1668-}

1723

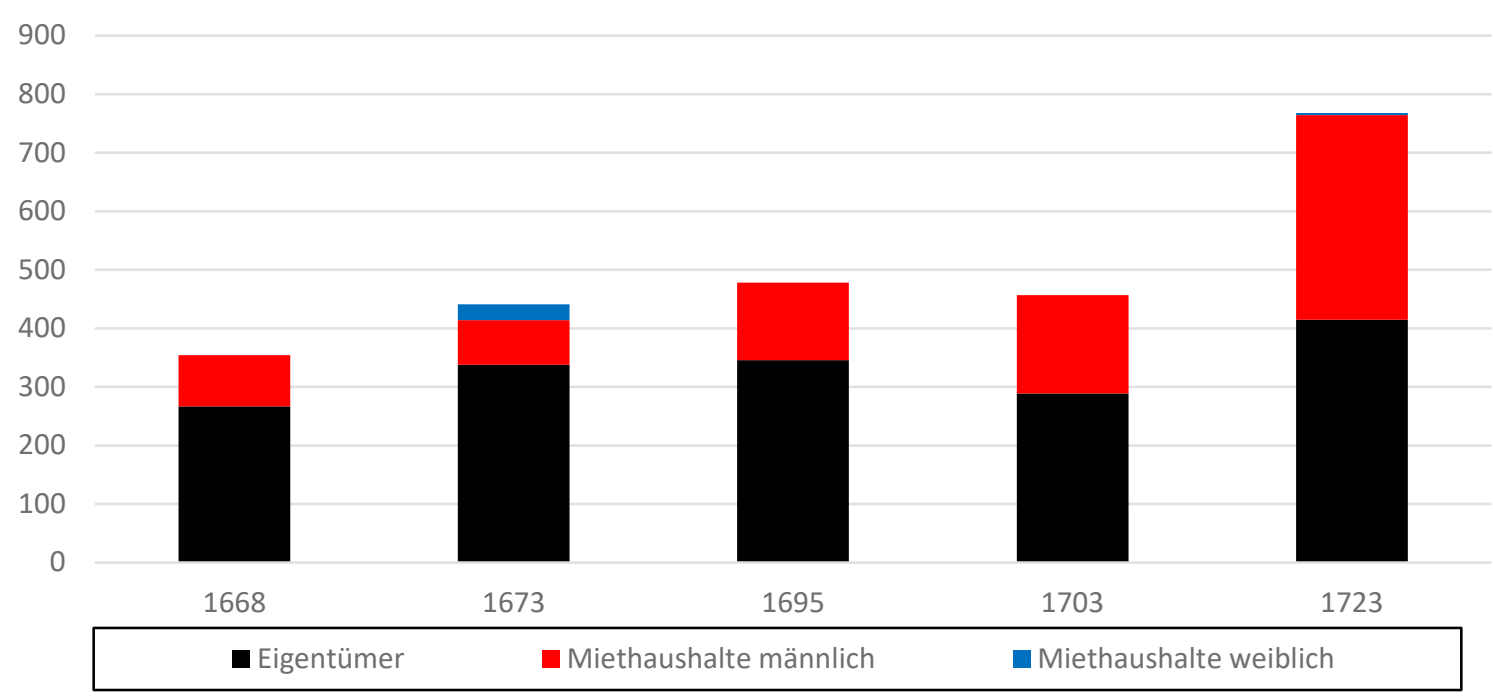

Grafik 7. Ansbach, Anzahl der Haushalte 1668-1723 (nur Bürgergemeinde), nach den Angaben aus den Huldigungslisten (Thomas Wenderoth 2019)

Der Anteil der Miethaushalte dürfte jedoch höher gelegen haben, denn lediglich in der Erfassung des Jahres 1673 sind nennenswert Miethaushalte mit weiblichem Haushaltsvorstand erfasst, diese machen ein Viertel der dort genannten Miethaushalte aus. ${ }^{715}$

\footnotetext{
${ }^{713}$ RECHTER 1985, S. 24-33.

${ }^{714}$ Vgl. im Folgenden den Abschnitt zur Residenzstadt Dresden.

715295 männlich sowie 43 weiblich geführten Eigentümerhaushalten standen 76 männlich geführte und 27 weiblich geführte Miethaushalte gegenüber (StAN, Fm AN, Brdb. Lit. Nr. 595, fol. 372-379 und 407). Zu den männlichen Mietern wurden 1 Jude und 2 Schutzverwandte hinzugezählt, sodass sich ein Anteil von 26\% weiblichen Miethaushalten bezogen nur auf die Mieterhaushalte und von 6,5\% bezogen auf alle Haushalte errechnet.
} 
Dass in den anderen Huldigungslisten nicht alle Haushalte erfasst worden sind, wird auch im Vergleich mit einer Bevölkerungszählung aus dem Jahr 1713 deutlich. Bei dieser Zählung fehlen ebenfalls die Hofangehörigen, das Militär sowie die Stiftsuntertanen. Die dort aufgeführten 3.986 Einwohner ${ }^{716}$ lebten in 908 Haushalten. ${ }^{717}$ Damit wurden für diese Zählung 140 Haushalte mehr erfasst als bei der Huldigung zehn Jahre später, 1723 sind in toto nur 768 Haushalte überliefert. Ergänzt man die Huldigungsliste noch um geschätzte, aber nicht aufgeführte 112 weiblichen Miethaushalte (25\%), würde sich die Differenz auf nur noch 28 Haushalte reduzieren. Bei aller statistischen Ungenauigkeit solcher Zählungen kann man für die Zeit ab 1700 daher von 50\% Miethaushalten innerhalb der Ansbacher Bürgergemeinde ausgehen.

Die Bevölkerungszählung von 1713 enthält darüber hinaus wichtige Informationen zur Bevölkerungsstruktur: Die Kernfamilie mit Kindern umfasste im Schnitt 3,5 Personen. ${ }^{718}$ Nur in 48 Haushalten gab es Großeltern, das entspricht einem Anteil von 5,2\% aller Haushalte. Für weitere 102 Haushalte sind andere dort mitlebende Familienangehörige verzeichnet. ${ }^{719}$ Die gesamte durchschnittliche Haushaltsgröße mit Verwandten und Gesinde betrug 4,37 Personen. ${ }^{720}$ Wobei das Gesinde mit insgesamt 633 Personen $^{721}$ eine relativ große Gruppe darstellte. Bei der Anzahl des Gesindes findet sich auch eine deutliche Zunahme gegenüber 1497.

Aber nach wie vor stellten die Haushalte mit zwei bis fünf Personen die Regel dar. In Ansbach sind dies im Jahr 1713 insgesamt 63,4\% der Haushalte. Immerhin gab es aber auch $9 \%$ Einpersonenhaushalte. ${ }^{722}$ Dass Miethaushalte eine beträchtliche Rolle in Ansbach gespielt haben, belegt ein Schreiben von Markgräfin Christiane Charlotte vom 08.03.1726: „[...] wie der Regularität und dem Embellissement dieser fürstlichen Residenz sehr im Wege stehe, [...]. daß nicht allein die Häuser vielfach vertheilet, darinnen eine Menge allerhand Kleinere Zinnße eingerichtet, und die Höfe zur größeren Feuers Gefahr gänzlich verbauet werden, sondern auch in denen Vorstädten viel neue schlechte Gebäude und Hütten in allerhand zerstreuten Orten zum Vorschein kommen, [...]. ${ }^{723}$ Negativ beurteilt wird hier die Kleinteiligkeit der Stadt, die bedingt war durch Hausteilungen und die Einrichtung vieler (kleiner) Mietwohnungen.

\footnotetext{
716 BAHL 1974, S. 217.

${ }^{717}$ Ebd., S. 230.

718 Ebd., S. 229.

${ }^{719}$ Ebd., S. 229.

${ }^{720}$ Ebd., S. 230.

${ }^{721}$ Ebd., S. 217.

722 Ebd., S. 230.

${ }^{723}$ StadtAAN, AM 403, pag. 11, Schreiben vom 8.3.1726; zitiert nach PIRSON 1961, S. 129.
} 
Bahl ermittelt als Gesamteinwohnerzahl insgesamt 6.700 Personen für das Jahr $1713,{ }^{724}$ daraus errechnen sich mindestens 1.500 Haushaltungen. Da die vorstädtische Bebauung mit der Neuen Auslag erst nach 1740 richtig Fahrt aufnimmt, dürfte die Anzahl der Häuser nicht über 500 gelegen haben, ${ }^{725}$ woraus sich wiederum drei Haushaltungen pro Wohngebäude ergeben würden. Der Anteil der Miethaushalte dürfte insgesamt betrachtet, unter Berücksichtigung der Hofgemeinde, der Stiftsgemeinde und des Militärs, sicher bei über $60 \%$ gelegen haben. Der Einfluss des Hofes auf die Bevölkerungsstruktur, insbesondere auch auf den hohen Anteil an Mietbevölkerung, wird auch daran deutlich, dass im Jahr 1734 über 30\% der Ansbacher Haushalte zum fürstlichen Hofstaat und zu den Beamten gehörten. ${ }^{726}$

An dieser Situation ändert sich im Verlauf des 18. Jahrhunderts nur noch wenig. Die Bevölkerung wächst weiter, dies gilt insbesondere für die Mietbevölkerung: 1818 werden 1.066 Feuerstellen genannt sowie 3.370 Familien mit 11.320 Seelen. ${ }^{727}$ In der Uraufnahme sind etwa 455 Hausnummern vergeben. ${ }^{728}$ Rein rechnerisch entfallen somit 7,4 Familien beziehungsweise fast 25 Personen auf ein Haus und 3,16 Familien auf eine „Feuerstelle“. Die Bezeichnung „Feuerstelle“ kann hier nicht ein ganzes Haus meinen, aber wohl auch nicht die Gesamtanzahl der Wohnungen, wie im Falle von Schwabach, denn dann hätten in jeder Wohnung durchschnittlich 10,62 Personen oder gut drei Familien zusammengelebt; beides Werte, die nicht mit den Vergleichszahlen in Mittelfranken zusammengehen würden. Die einzig plausible Erklärung für die 1.066 Feuerstellen besteht darin, diese Zahl als Anzahl der Hauseigentumsanteile (Eigentümer) zu interpretieren. Erinnert sei hier an das bereits eingangs erwähnte Zitat der Markgräfin Christiane Charlotte aus dem Jahr 1726, die von ihr angeprangerten Zustände hatten sich in den folgenden 100 Jahren wohl keinesfalls gebessert. Die Familienstärke lag bei lediglich 3,36 Personen und damit am unteren Rand: Der Durchschnittswert von 21 mittelfränkischen Städten im Jahr 1818 lag etwas höher bei 3,81 Personen pro Familie. Demnach ist mit relativ vielen Kleinsthaushalten mit ein oder zwei Personen zu rechnen. Vermutlich wurden in der Rubrik Familien auch die Soldatenhaushalte erfasst. Bei dieser Bevölkerungsgruppe war es die Regel, dass sich mehrere alleinstehende Soldaten eine Unterkunft in der Kaserne oder auch in einem Privathaushalt teilten. Aus den so interpretierten Zahlen

\footnotetext{
724 BAHL 1974, S. 213.

${ }^{725}$ Die Zahl bezieht sich überschlägig auf die im Urkataster angegebenen Hausstellen ohne die barocke Vorstadtbebauung.

${ }^{726}$ RousSEAUX 2006, S. 32, ohne Quellengabe.

${ }^{727}$ Alphabetisches Verzeichnis 1818, S. 6.

728 http://geoportal.bayern.de/bayernatlas-klassik, Uraufnahme Ansbach (Aufruf 04.09.2018). Darunter auch für das Rathaus, die Orangerie, Casernen, Kirchen etc.
} 
ergibt sich für die ehemalige Residenzstadt Ansbach im Jahr 1818 ein Mieteranteil von $68 \%$ aller Haushalte und dies 27 Jahre nach Ende der Residenzfunktion 1791 !

Festzustellen ist, dass sich im Falle von Ansbach die Bevölkerungsstruktur der Bürgerstadt im ausgehenden 17. Jahrhundert stark verändert hatte. Die Herausbildung einer absolutistischen Residenzstadt war verbunden mit der Stärkung und Konzentration der Zentralgewalt in Ansbach. Zusammen mit der stark auf Repräsentation bedachten barocken Hofhaltung führte dies durch den Zuzug von Staats- und Hofbediensteten sowie von Militärangehörigen zum Anstieg der Bevölkerung. Überproportional wuchs dabei die Anzahl der Miethaushalte. Dieser Prozess scheint bereits im frühen 18. Jahrhundert abgeschlossen gewesen zu sein. Zumindest änderte sich seit dieser Zeit das Verhältnis zwischen Eigentümer- und Miethaushalten nicht mehr maßgeblich, auch wenn die Gesamtanzahl der Bevölkerung weiter anstieg.

Der Einfluss der Hofhaltung auf die städtische Wohnbevölkerung im Fürstentum Brandenburg-Bayreuth - und auch darüber hinaus - wirkte sich auch auf die Nebenresidenzen aus, hier sicher in abgeschwächter Form. Den Zenit als Aufenthaltsort des Hofes hatte Erlangen in der zweiten Hälfte des 18. Jahrhunderts längst überschritten. Die Stadt diente aber von 1763 bis 1819 als Witwensitz der letzten Markgräfin von Brandenburg-Bayreuth, Sophie Caroline Marie. Das Fürstentum selbst wurde seit diesem Zeitpunkt von Ansbach aus mitregiert, Erlangen spielte also innerhalb der beiden nunmehr vereinigten Fürstentümer politisch nur noch eine unbedeutende Nebenrolle. Anhand der folgenden Zahlen wird aufgezeigt, dass bereits die Hofhaltung eine große Anzahl eigentumsloser Haushalte beinhaltet.

Leonhard Freyesleben gibt für das Jahr 1775 die Einwohnerschaft Erlangens wieder: Die Markgräfinwitwe hatte zu diesem Zeitpunkt 31 Bediente, ihr Oberhofmeister von Künsberg, der im Erdgeschoss des Schlosses wohnte, 22 Bediente. Darunter befanden sich aber viele Mägde und Knechte, die alleinstehend waren und keinen eigenen Haushalt führten. ${ }^{729}$ Dennoch blieb sicher eine nennenswerte Zahl von Familien übrig, die in einer privaten Mietwohnung untergebracht waren. In der Aufstellung Freyeslebens folgt nach den Hofbediensteten der Adel: Achtzehn adlige Haushalte sind mit einem eigenen Haus verzeichnet, bei dreizehn adligen Haushalten ist nur der jeweilige Wohnort in Erlangen angegeben, was in dem Fall ein Mietobjekt meint. Auffällig ist der große Anteil an Frauen unter dem Adel, dies dürfte mit dem Status Erlangens als Witwenresidenz zusammenhängen. Kurz vor dem Tode der Markgräfinwitwe im Jahr 1817 werden

${ }^{729}$ Vgl. hierzu und im Folgenden FREYESLEBEN 1775, S. 4-7. 
insgesamt 45 Hofbedienstete genannt, von denen sechzehn eine dienstliche Unterkunft hatten und sich 29 Bedienstete ein privates (Miet-)Quartier suchen mussten. ${ }^{730}$

\section{Kleine fränkische Residenzen}

Anhand des Alphabetischen Verzeichnis von 1818 lassen sich für die vergleichbar kleinen mittelfränkischen Residenzstädte Ellingen und Pappenheim immerhin 46\% beziehungsweise 49\% Miethaushalte nachgewiesen. Der Wert liegt damit deutlich über dem Vergleichswert für fünfzehn fränkische Landstädte in Höhe von 35\%. Mit in dieser Gruppe kann auch noch Scheinfeld betrachtet werden, als ehemaliger Hauptort der gefürsteten Grafschaft Schwarzenberg. Der Mieteranteil in Scheinfeld lag mit 37\% etwa in der Mitte zwischen den Werten für Oettingen, die weiter unten aufgeführt werden, und Pappenheim. Dies verwundert auch nicht, da die Fürsten Schwarzenberg hauptsächlich in Böhmen und Österreich residierten und ihr Stammschloss oberhalb von Scheinfeld nur selten aufsuchten. In Scheinfeld war es also weniger der Hofstaat als vielmehr die fürstliche und später die bayerische Verwaltung, die die Stadtbevölkerung prägte.

\begin{tabular}{|l|r|c|c|}
\hline Stadt & Miethaushalte & $\begin{array}{c}\text { Belegungsziffer } \\
\text { Haushalte/Haus }\end{array}$ & $\begin{array}{c}\text { Behausungsziffer } \\
\text { Bewohner/Haus }\end{array}$ \\
\hline Ansbach & $68 \%$ & 7,41 & 24,88 \\
\hline Ellingen & $46 \%$ & 1,93 & 8,4 \\
\hline Pappenheim & $49 \%$ & 2,74 & 11,56 \\
\hline Scheinfeld & $37 \%$ & 1,65 & 6,3 \\
\hline \hline$\varnothing$ 4 Städte & $70 \%$ & 3,43 & 13,34 \\
\hline $\begin{array}{l}\varnothing 15 \text { fränkische } \\
\text { Landstädte }\end{array}$ & $35 \%$ & 1,62 & 6,14 \\
\hline
\end{tabular}

Tabelle 24. Anteil Miethaushalte in mittelfränkischen Residenzstädten 1818

(Thomas Wenderoth 2019)

Vergleicht man die einzelnen Werte mit dem Durchschnittswert der fünfzehn fränkischen Ackerbürgerstädte, ${ }^{731}$ wird deutlich, dass sich die Situation in Scheinfeld kaum von der einer Ackerbürgerstadt unterschieden hat. Erst bei einer Eingrenzung der Vergleichsgruppe auf die acht sehr kleinen Landstädte mit weniger als 1.200 Einwohnern ${ }^{732}$ wird auch die abweichende Bevölkerungsstruktur Scheinfelds mit seinen lediglich 894 Einwohnern deutlich (vgl. Grafik 4), wiesen

${ }^{730}$ Vgl. hierzu und im Folgenden ENGELHARDT 2017, S. 36.; WENDEROTH 2019a, S. 65.

${ }^{731}$ Abenberg, Feuchtwangen, Gunzenhausen, Heideck, Herrieden, Hilpoltstein, Leutershausen, Merkendorf, Neustadt/Aisch, Ornbau, Spalt, Uffenheim, Wassertrüdingen, Windsbach, Wolframs-Eschenbach.

${ }^{732}$ Hilpoltstein, Abenberg, Herrieden, Windsbach, Heideck, Wolframs-Eschenbach, Merkendorf, Ornbau. 
diese doch durchschnittlich nur 22\% Miethaushalte auf.

Signifikant wird der Unterschied aber auch im Hinblick auf Ansbach. Dort handelte es sich eben nicht um eine Kleinresidenz, sondern um die ehemalige Hauptstadt eines mittelgroßen Territoriums.

\section{Residenzstädte außerhalb Frankens}

Der Zusammenhang zwischen einer Residenzfunktion und einem überdurchschnittlich hohen Anteil an eigentumslosen Haushalten ist kein fränkisches Phänomen, sondern allgemeingültig.

Für Dresden lässt sich der Anteil der eigentumslosen Haushalte relativ lückenlos vom 15. bis ins 18. Jahrhundert nachvollziehen und auch hier ist die Auswirkung der Residenz auf den Anteil der Mietbevölkerung signifikant. Die Dresdner Zahlen zeigen zudem, wie die Residenzfunktion den Aufstieg von einer großen Kleinstadt im späten Mittelalter zur Mittelstadt nach der Etablierung als dauerhafte Residenz der Wettiner im Jahr 1485 und zur Großstadt im Verlauf des 17. Jahrhunderts nach sich zieht.

Otto Richter hat die Bevölkerungsstruktur für das 15. Jahrhundert anhand von Steuerbüchern eingehend erforscht. ${ }^{733}$ Dresden war zu dieser Zeit sicher keine unbedeutende Landstadt, sondern zählte mit den Vorstädten und Altdresden zu den großen Kleinstädten, größenmäßig an der Schwelle zur Mittelstadt. ${ }^{734} \mathrm{Im}$ Jahr 1396 sind erstmals 657 Steuerpflichtige (Kernstadt) erfasst worden, die anschließende Entwicklung verlief alles andere als kontinuierlich: Nach einem Rückgang werden 1431 zunächst 694 Steuerpflichtige genannt, die Zahl sinkt dann im Jahr 1453 auf 544 ab, um 1489 mit 734 Steuerzahlern einen erneuten Höchststand zu erreichen. ${ }^{735}$ Ein Stadtbrand im Jahr 1491 unterbricht die Aufwärtsbewegung, die mit dem Sitz als ständige Residenz ab 1485 befördert wurde. ${ }^{736}$ In den Steuerregistern wurden die eigentumslosen Haushalte seit 1424 gekennzeichnet. ${ }^{737}$ Dieser Umstand erlaubt Richter eine genaue Analyse, einschränkend sei erwähnt, dass nur Mieter mit Bürgerrecht erfasst worden sind. Die Zahl der nichtbürgerlichen Einwohner war ihm zufolge gering: ${ }^{738}$ Genannt werden für das Jahr 145231 Lohnarbeiter und 52 Lohnarbeiterinnen ohne Bürgerrecht, die eine Lohnsteuer zahlen mussten. ${ }^{739}$

\footnotetext{
733 RICHTER 1881, S. 273-289.

734 Ebd., S. 282. Zusammen mit den Vorstädten errechnet Richter für das Jahr 1489 4.739 Einwohner.

${ }^{735}$ Ebd., S. 275, Tab. I.

${ }^{736}$ Ebd., S. 277.

${ }^{737}$ Zuerst durch das Zeichen II vor dem Namen und ab 1444 durch das Wort ibidem; ebd., S. 276.

${ }^{738}$ Ebd., 278.

${ }^{739}$ Ebd., Anm. 10.
} 
Richter selbst gibt dann im Folgenden leider nur die Zahl der Häuser und der bürgerlichen Steuerzahler an. Daher lässt sich der Anteil der Miethaushalte nur überschlägig ermitteln, indem rechnerisch jedem Haus ein Steuerzahler als Eigentümer zugeordnet wird. Der Überhang an Steuerzahlern wird als Miethaushalt interpretiert. ${ }^{70}$ Aus den Angaben Richters ergeben sich rechnerisch folgende Werte: Der Anteil der Miethaushalte unter den Bürgerhaushalten steigt zwischen den Jahren 1440 und 1489 von 22\% auf 36\%. Berücksichtigt man bei der Berechnung zudem die erwähnten 83 nichtbürgerlichen Lohnarbeiter, so steigt der Anteil der Miethaushalte generell um etwa 10 Prozentpunkte, denn im Jahr 1453 standen den 83 Lohnarbeitern des Jahres 1452 lediglich 125 bürgerliche Miethaushalte gegenüber. Zur Ermittlung der Einwohnerzahlen mag diese Gruppe nicht besonders relevant sein, um jedoch den Anteil an eigentumslosen Haushalten $\mathrm{zu}$ ermitteln, ist sie von entscheidender Bedeutung und keinesfalls zu vernachlässigen. Konkret erhöht sich dadurch der Anteil der Miethaushalte im Jahr 1453 von $23 \%$ auf $33 \%$. Auffällig ist zudem ein besonders hoher Mieteranteil im Jahr 1431 von 39\%, nur bezogen auf die Bürgerhaushalte; als Ursache gibt Richter den vorübergehenden Zuzug von Bürgern aus den unbefestigten Vorstädten während der Hussitenkriege an. ${ }^{741}$

Als Haushaltsgröße ermittelt Richter aufgrund der Angaben für etwa ein Drittel des Stadtgebietes einen Durchschnittswert von 5,07 Personen bei den Eigentümerfamilien und 2,14 bei den Mietern, jeweils ohne minderjährige Kinder. ${ }^{742}$ Durchschnittlich kamen dort 7,2 Erwachsene auf ein Haus. Kleine Zinshäuslein für nur eine Partei scheinen eher selten gewesen zu sein: Im Jahr 1453 sind für dieses Drittel der Stadt dreizehn Häuser belegt, die von maximal drei Erwachsenen bewohnt worden sind. Aus dem Vorhandensein von 154 Miethaushalten kann gefolgert werden, dass in Dresden die Miethaushalte meistenteils nicht allein für sich wohnten, sondern mit im Haus eines Eigentümers. ${ }^{743} \mathrm{Ob}$ in einer separaten Wohnung oder wie in Regensburg zu großen Anteilen als Untermieter, lässt sich anhand der Zahlen nicht klären.

Matthias Meinhard hat den Prozess der Residenzbildung in Dresden im 15. und 16. Jahrhundert umfangreich untersucht, in diesem Zusammenhang geht er auch auf die Mietbevölkerung ein. ${ }^{744}$ Nach Richter lag im Jahr 1477, vor der dauerhaften Etablierung des Hofes in Dresden 1485, der Anteil der Miethaushalte bei $29 \%$ in der Innenstadt, 1489 dann bei 36\%. ${ }^{745}$ Meinhard ermittelt hingegen für

\footnotetext{
${ }^{740}$ Eine nochmalige Durchsicht der Quellen würde hier zu exakteren Zahlen führen.

${ }^{741}$ Ebd., S. 277.

742 Ebd., S. 280.

${ }^{743}$ Ebd., S. 281, Tab. IV.

744 Meinhard 2009, S. 212-217.

${ }^{745}$ RiChTER 1881, S. 275, Tab. I.
} 
das Jahr 1488 nur 29\% Miethaushalte. ${ }^{746}$ Auf die schwierige Interpretation der Quellen und die zumeist lückenhaften Listen wurde bereits mehrfach hingewiesen, von daher müssen diese Werte unvermittelt nebeneinander stehen bleiben. Die vorhergehenden und nun folgenden Werte beziehen sich immer nur auf die Innenstadt und den Hauptbevölkerungsteil, der den Bürgereid geleistet hatte. Die nichtbürgerlichen Einwohner, die in noch stärkerem Maße, wenn nicht ausschließlich, eigentumslos waren, bleiben unberücksichtigt. Bis zum Jahre 1506 ist ein deutlicher Anstieg der Miethaushalte auf 40\% zu verzeichnen. 1550 lebten 43\% und 1577 gar 44\% bürgerliche Miethaushalte in der Innenstadt: ${ }^{747}$ Auf die wachsende (Miet-)Bevölkerung hatte die Hofhaltung beträchtlichen Einfluss. Im Jahr 1603 hatte die Innenstadt bereits 7.829 Einwohner, zusammen mit den Vorstädten und Altendresden ergab sich eine Bevölkerungszahl von 14.739. ${ }^{78}$ Meinhard fasst zusammen, „dass die Residenzbildung in Dresden dazu geführt hat, dass 1603 mit 27,2\% allein innerhalb der Festungsmauern bereits ein großer Anteil der männlichen Bevölkerung unmittelbar dem Adel und Hofgesinde angehörte. Nur der kleinere Teil dieser Gruppe besaß Haus und Grund in der Stadt, die Mehrzahl lebte hingegen zur Miete in Dresden. ${ }^{\text {“749 }}$ Er ermittelt exakt 443 Mieter unter den Adligen und Hofbediensteten, was einem Anteil von 18,7\% aller erwachsenen Männer der Innenstadt entspricht! Meinhard kommt zudem zu dem Ergebnis, dass ab dem 16. Jahrhundert die Hofangehörigen nun überwiegend das Bürgerrecht der Stadt erwarben, höfische Gesellschaft und Stadtgesellschaft verschränkten sich also in Dresden in der frühen Neuzeit ineinander. ${ }^{750}$

Der Aufstieg zur Großstadt dürfte dann nach dem Dreißigiährigen Krieg erfolgt sein. Laut Katrin Keller hatte Dresden 21.298 Einwohner im Jahr 1699. Durchschnittlich lebten zu diesem Zeitpunkt in jedem Haus 11,1 Personen, bei der von ihr angenommenen Haushaltsgröße von 4,3 Personen ${ }^{751}$ entspricht dies 2,6 Haushalten in jedem Haus. Die Haushaltsgröße dürfte jedoch in dieser großstädtischen Situation mit vielen eigentumslosen Haushalten geringer gelegen haben, sodass von weit über $60 \%$ Miethaushalten auszugehen ist. Mit dem weiteren Anwachsen der Stadt im 18. Jahrhundert auf 46.754 Einwohner im Jahr 1811 stieg der Anteil der Mietbevölkerung überproportional. Rechnerisch kamen nunmehr 20,6 Einwohner auf jedes Haus, was mindestens 4,8 Haushalten pro Haus oder $80 \%$ Miethaushalten entspricht!

\footnotetext{
${ }^{746}$ Vgl. hierzu und im Folgenden MEINHARD 2009, S. 215, Tab. III./4.1.

${ }^{747}$ In den Vorstädten waren es 1488 25\%, 1506 40\%, 1550 56\% und 1577 40\%.

${ }^{748}$ MeInHARD 2009, S. 143-147, Tab. III./1.4./1 und III./1.4./1.

${ }^{749}$ Ebd., S. 146.

${ }^{750}$ Ebd., S. 146.

${ }^{751}$ KeLLER 1997, Tab. 1.
} 
In Dresden lässt sich somit der Einfluss der Hofhaltung auf die Zunahme der eigentumslosen Bevölkerung schon für das 16. Jahrhundert belegen.

Ein solches Anwachsen der Mietbevölkerung in der frühen Neuzeit konnte Elisabeth Lichtenberger auch für Wien nachweisen. Sie schildert eindrücklich die Auswirkungen, die die schrittweise Rückverlegung der habsburgischen Hofhaltung nach Wien ab dem frühen 16. Jahrhundert für die Bevölkerungsstruktur der Stadt nach sich zog. ${ }^{752}$ Eng mit der Hofhaltung verbunden war eine große Bevölkerungsgruppe aus Hofbediensteten und Adel, die den Wohnungsmarkt veränderte. Um diesen Menschen Wohnraum zu verschaffen, wurde 1563 eine Aufnahme der Bürgerhäuser durchgeführt, bei der sämtliche Bewohner, also auch die Miethaushalte, erfasst worden sind. Für das Jahr 1563 standen laut Lichtenberger 876 Hausbesitzern 1.999 Mieter gegenüber, der Anteil der Miethaushalte betrug also bereits 72,5\%. ${ }^{753}$ Sie kommt zum Schluss, dass 50\% der Mieterschaft durch die Residenzfunktion bedingt war. ${ }^{754}$ Unter den niederen Hofleuten lag der Mietanteil sogar bei 94,5\%, nur die wenigsten Mitglieder der unteren Hofchargen konnten sich Wohneigentum leisten; aber auch in der Gruppe der Beamten und Universitätslehrer fanden sich 77,9\% Mieter, beim Adel waren es immerhin noch 66,9\%: Selbst in dieser privilegierten Bevölkerungsgruppe bewohnten nur ein Drittel ein eigenes Haus und das häufig wohl nicht allein, sondern zusammen mit Mietparteien.

Am Ende dieses Prozesses stand die zwangsweise Einquartierung von Hofbediensteten und die Verdrängung bisheriger Mietergruppen in die Vorstädte. Die Hofquartierpflicht wurde erst ab 1640 gelockert, um Anreize für einen verstärkten Wohnungsbau zu schaffen, der dringend erforderlich war, und 1781 gänzlich abgeschafft. ${ }^{755}$ Damit war der Mietwohnungsbau in der Innenstadt auch für die Bürger wieder finanziell attraktiv geworden. Entsprechend veränderte sich die Baustruktur und Bebauungsdichte Wiens nachhaltig: Zwischen 1660 und 1730 wurden rund 400 Häuser verändert und aufgestockt. Die durchschnittliche Geschosszahl der Häuser in der Innenstadt stieg daraufhin in Jahrhundertschritten von einer Zwei- bis Dreigeschossigkeit im 16. Jahrhundert hin zu Vier- und Fünfgeschossigkeit Ende des 18. Jahrhunderts. Zugleich stieg aber auch die Zahl der Einquartierungen von 672 auf 1.200 Haushalte zwischen 1644 und 1709. Der größere Anteil der Hofbevölkerung musste sich jedoch auf dem freien Markt eine (Miet-)Unterkunft besorgen, denn der Anteil des Hofes an der Stadtbevölkerung stieg von 35\% im Jahr 1563 auf etwa 60\% im Jahr 1730 an! Zu dieser Zeit waren

752 Vgl. hierzu und im Folgenden LICHTENBERGER 1973, S. 303-315.

${ }^{753}$ LICHTENBERGER 1973, S. 305, Tabelle 2. Nicht bekannt ist der Bevölkerungsanteil der Geistlichen, der Nichtsesshaften, der Dirnen und des Gesindes. Inwieweit Untermietverhältnisse erfasst sind, wäre noch zu prüfen.

${ }^{754}$ Ebd., S. 306.

${ }^{755}$ Vgl. hierzu und im Folgenden WINKELBAUER 2018, S. 283-285. 
etwa 2.500 Personen unmittelbar im Hofdienst beschäftigt! ${ }^{756}$ Auch Ulrich Rosseaux geht davon aus, dass $60 \%$ aller Haushalte vom Hof und der Staatsverwaltung abhängig waren. ${ }^{757}$

Die hessische Residenzstadt kann hinsichtlich ihrer Größe und Bedeutung nicht mit Wien und Dresden mithalten, gleichwohl handelte es sich um eine der wichtigen mitteldeutschen Residenzstädte, die aufgrund eines residenzstadtbedingten kontinuierlichen Wachstums um 1730 wohl gut 15.000 Einwohner zählte. ${ }^{758}$ Damit war Kassel deutlich größer als die fränkische Residenzstadt Ansbach, die im folgenden geschilderte barockzeitliche Situation weist dennoch viele Parallelen auf. Die Zahlen zur Hof- und Mietbevölkerung in Kassel sind für das 18. Jahrhundert sehr präzise ermittelt, sie geben einen guten Einblick in die Bevölkerungsstruktur. Im Jahre 1724 ist dort die männliche Bevölkerung über zwölf Jahre erfasst worden. 678 Eigentümern standen 1.610 Mieter gegenüber, der Anteil der eigentumslosen Haushalte lag demnach bei über $70 \% .{ }^{759}$ Darüber hinaus wurden 696 Söhne, 506 Gesellen, 278 Lehrjungen und 229 Diener und Knechte gezählt.

Überraschend mag die Feststellung klingen, dass es sich bei Kassel rein quantitativ betrachtet nicht mehr um eine Bürgerstadt gehandelt hat, denn der größere Teil der Bevölkerung (61\%) hatte kein Bürgerrecht: 1723 wurden in Kassel lediglich 1.463 Handwerksleuten so Bürger seynd erfasst. Diesen standen 773 höhere und niedere fürstliche Bediente und 715 Handwerksleute so keine Bürger seynd - die aber wohl einen Schutzbrief hatten -, gegenüber. ${ }^{760}$ Mit in die Betrachtung einbezogen werden müssen aber auch die Militärangehörigen, deren Zahl 1731 mit 277 angegeben ist, ${ }^{761}$ und die herrenlosen Haushalte, von denen im Jahr 1724 immerhin 523 erfasst worden sind. ${ }^{762}$ Die Aufsplitterung der Bevölkerung ist wichtig in Bezug auf die Fragestellung nach dem Anteil der eigentumslosen Haushalte, da sich das Hauseigentum bei den Bürgern konzentrierte und andere

\footnotetext{
${ }^{756}$ LICHTENBERGER 1973, S. 312. Mit den Familienangehörigen ergibt dies etwa 10.000 Personen, was $25 \%$ der Gesamtbevölkerung entspricht. Hinzu kamen noch die Bewohner, deren Einkommen als Dienstleister oder Produzent nur mittelbar vom Hof abhingen, sowie diejenigen, die wiederum die Hofbeschäftigten versorgten.

${ }^{757}$ Rosseaux 2006, S. 32, ohne Quellenangabe.

${ }^{758}$ LASCH 1964/65, S. 356. Für das Jahr 1731 liegt eine noch exaktere Zählung vor. Diese ermöglicht einen Blick auf die Wohnverhältnisse: In 1.334 Häusern lebten 15.555 Personen, was einer Behausungsziffer von 11,66 Bewohnern pro Haus entsprach. ${ }^{758}$ In der Oberneustadt, die 1689 zur Aufnahme der Hugenotten neu angelegt worden war, wohnten sogar 1.808 Einwohner in lediglich 194 Häusern, hier lag die Behausungsziffer mit einem Wert von 17,38 also deutlich höher. Parallelen zur Situation in der Erlanger Neustadt gegen Ende des 17. Jahrhunderts sind unübersehbar.

${ }^{759}$ LASCH 1969, S. 85.

760 Ebd., S. 88.

761 Ebd., S. 86.

762 Ebd., S. 90.
} 
Gruppen nur in geringem Maß über Immobilien verfügten. Die nachfolgende Tabelle lässt also schon den hohen Anteil an Mietbevölkerung erahnen.

\begin{tabular}{|l|r|r|r|r|r|r|}
\hline Rechtsstellung & Bürger & $\begin{array}{r}\text { Schutz- } \\
\text { verwandte }\end{array}$ & Beamte & Militär & Herrenlose & Gesamt \\
\hline $\begin{array}{l}\text { Anzahl der } \\
\text { Haushalte }\end{array}$ & 1.463 & 715 & 773 & 277 & 523 & 3.751 \\
\hline Anteil in \% & $39 \%$ & $19 \%$ & $21 \%$ & $7 \%$ & $14 \%$ & $100 \%$ \\
\hline
\end{tabular}

Tabelle 25. Kassel, rechtlicher Status der Haushalte um 1723, Anzahl Militär 1731, Herrenlose 1724 (Thomas Wenderoth 2019)

Stellt man die so gewonnene Gesamtanzahl der Haushalte den bereits oben erwähnten 678 männlichen Eigentümerhaushalten gegenüber und erhöht diese Zahl noch um geschätzte 70 Eigentümerhaushalte mit weiblichem Haushaltsvorstand auf 750 Eigentümerhaushalte, errechnet sich ein Anteil an Miethaushalten von $80 \%$ für die Zeit um 1725! Dieser Wert entspricht der oben geschilderten Situation in Dresden im Jahr 1811. Der hohe Anteil der Hofbedienten mit 21\% wird ebenfalls anhand dieser Zählung deutlich.

Die größeren Residenzstädte,Ansbach kann durchaus zu dieser Gruppe hinzugerechnet werden, weisen sämtlich einen starken Bevölkerungsanstieg im 18. Jahrhundert auf. Entweder verharrte der Anteil der eigentumslosen Bevölkerung auf einem bereits sehr hohen Niveau oder stieg noch einmal deutlich an.

Abschließend soll der Blick noch einmal auf die Situation in den kleinen Residenzstädten gerichtet werden. Diese Städte bildeten in Mittel- und Süddeutschland, rein quantitativ betrachtet, eine große Gruppe. Vorbildlich erforscht ist die Situation für Oettingen in der frühen Neuzeit. Seit dem frühen 15. Jahrhundert war die Stadtherrschaft geteilt zwischen den gräflichen Linien Oettingen-Oettingen und Oettingen-Wallerstein. Die Trennung wurde verstärkt durch die Einführung der Reformation im oettingen-oettingischen Herrschaftsbereich und bestand daher auch nach dem Aussterben der protestantischen Linie 1731 fort. ${ }^{763}$ Die Ergebnisse von Petra Ostenrieders Dissertation zum Thema des Mietwohnens sollen im Folgenden kurz zusammengefasst werden: ${ }^{764}$

Bereits bei der Herrschaftsteilung im Jahr 1414 wurden neben 88 Hausbesitzern auch elf Hausgenossen aufgeführt, was immerhin 11\% der Haushalte entspricht. ${ }^{765} 1575$ waren es dann auf evangelischer Seite schon 32 Miethaushalte, 1694 bereits 43, 1609 nunmehr 75 und 1634 sogar 94. Mit dem Dreißigjährigen Krieg sank deren Anzahl eindrücklich: Im Jahr 1654/55 wurden auf evangelischer

763 OSTENRIEDER 1993, S. 11.

764 OSTENRIEDER 1993.

${ }^{765}$ Vgl. hierzu und im Folgenden ebd., S. 74f. 
Seite nur noch 28 Miethaushalte gezählt, denen 117 evangelische Hausbesitzer gegenüberstanden, was einem Anteil von 19,3\% entsprach. Ab diesem Zeitpunkt lassen sich die Zahlen für beide Stadtherrschaften nachvollziehen. Der Anteil der Miethaushalte steigt zwischen $1654 / 55$ von insgesamt $17,9 \%$ auf $26,1 \%$ bis $1801 / 03$ an.

Damit reicht Oettingen, was den Anteil der Mietbevölkerung betrifft, bei weitem nicht an die zuvor behandelten größeren Residenzstädte heran. Dies gilt insbesondere für das 18. Jahrhundert. Nach Ostenrieder stellte in Oettingen das Hofpersonal auch nur für den Bereich der Innenstadt eine nennenswerte Mietergruppe dar. 1701 wurden allein für die evangelische Linie Oettingen-Oettingen 86 Personen im Hofdienst und in der Verwaltung gezählt, nur für elf dieser Personen lässt sich Hausbesitz nachweisen. ${ }^{766}$ Der hohe Anteil dieser Bevölkerungsgruppe wird deutlich, wenn man bedenkt, dass inklusiv der elf Hofbediensteten nur 194 evangelische Hauseigentümer und 47 Hausgenossen gezählt wurden. ${ }^{767}$ Da die Hofangehörigen auch unter den Mietern in den bürgerlichen Häusern keine große Rolle spielten, dürften sie überwiegend in Dienstwohnungen untergekommen sein. Immerhin ist überliefert, dass 1771 allein in dem dann verwaisten „evangelischen“ Schloss sechzehn Haushalte von Hofangehörigen wohnten, die auch für die Instandhaltung der Räume verantwortlich waren. ${ }^{768}$ Die verhältnismäßig niedrigen Zahlen in Oettingen müssen im Zusammenhang mit der Tradition von Hausteilungen gesehen werden. 1778/80 bestand in Oettingen fast $50 \%$ des Immobilienbesitzes aus Teileigentum an einem Anwesen. Die Bevölkerungsvermehrung - auch durch den Hofstaat - wurde hier wohl auf diese Art und Weise kompensiert. ${ }^{769}$

\section{Universitätsstädte}

Die Zahl der Universitätsstädte war insgesamt betrachtet überschaubar. Ulrich Rousseaux nennt mit den Gründungen des 18. Jahrhunderts 43 deutschsprachige Universitäten. ${ }^{770}$ Die Spannweite ihrer Größe könnte unterschiedlicher kaum sein. Auf der einen Seite standen mit Paris und Prag europäische Metropolen, auf der anderen Seite fanden sich unter den Universitätsstädten auch Mittelstädte und Kleinstädte wie Altdorf oder Dillingen. Da die Universitäten eine eigene Rechtsverfassung hatten, standen die Universitätsgemeinden außerhalb der städtischen Bürgergemeinden und deren Jurisdiktion. In den Universitätsstädten gab

\footnotetext{
766 Ebd., S. 92.

${ }^{767}$ Ebd., Tab. 9, S. 74.

${ }^{768}$ Ebd., S. 68f.

${ }^{769}$ Vgl. WENDEROTH 2021, S. $441 f$.

770 Rousseaux 2006, S. 38, Tab. 5.
} 
es damit mindestens zwei große Rechtskreise, wenn die Städte nicht darüber hinaus weitere eigenständige Bevölkerungsgruppen wie den Hofstaat oder Militär beherbergten. Kennzeichnend für diese Städte ist in jedem Fall ein hoher Mieteranteil auch in den Häusern der Stadtbürger, denn bei den Universitätsgemeinden handelte es sich um Personalgemeinden und weniger um topografische Gemeinden. Neben den Studenten waren auch viele Professoren und andere Mitarbeiter auf Mietwohnungen angewiesen. An dieser Stelle soll nur die bereits genannte Erfassung der Bevölkerung Wiens aus dem Jahr 1563 stellvertretend angeführt werden: Von den 43 Doktoren und Magistern wohnten 60,5\% zur Miete. ${ }^{771}$ Der Mieteranteil unter den Studenten lag nahe bei 100\%.

Im Untersuchungsgebiet finden sich mit Altdorf und Erlangen gleich zwei Universitätsstädte. Ich werde mich nachfolgend auf Altdorf konzentrieren. Der Einfluss der Universität auf die Eigentumsstruktur in Erlangen ist weniger eindeutig, da dort die Bevölkerungsstruktur durch die Funktion als Exportgewerbestadt dominiert wurde.

Altdorf darf abgesehen von der Universität getrost als kleine Ackerbürgerstadt bezeichnet werden. ${ }^{772}$ Die Stadt liegt etwa $25 \mathrm{~km}$ südöstlich von Nürnberg. Im Jahr 1504 gelangte Altdorf mit dem Umland in Folge des Landshuter Erbfolgekrieges unter die Herrschaft der Reichsstadt Nürnberg. Von da an war die Stadt Sitz eines nürnbergischen Pflegamtes. Einschneidend für die Stadt war darüber hinaus 1575 die Gründung des Nürnberger Gymnasiums in Altdorf, das 1578 zur Akademie und schließlich 1622 zur Universität erhoben wurde. Für die Ansiedelung dieser Bildungseinrichtung waren auch andere Orte im Nürnberger Landgebiet in Betracht gezogen worden. Im Jahr 1567 heißt es dazu in einem Brief: In dem Städtlein Altdorf hat es der Burgerschaft halber, da die Kostjungen möchten untergebracht werden, nit geringere Gelegenheit dann zu Herspruck, sondern viel besser, und sind die Häuser besser dazu erbauet. ${ }^{73}$ Von Interesse dabei ist, dass die privaten Unterkunftsmöglichkeiten für die eigentumslose Bevölkerungsgruppe der Schüler/Studenten ein wichtiges Auswahlkriterium darstellten.

Die Universität gab der städtischen Wirtschaft sicher wichtige Impulse. Ein florierendes Handwerk und Gewerbe, das über den regionalen Markt hinaus Bedeutung erlangte, ist nicht bekannt, ${ }^{774}$ mit Ausnahme der Salzburger Migranten, die 1735 als Schnitzer und Schachtelmacher angesiedelt wurden. ${ }^{775}$ Maßgebliche

771 LiCHTENBERGER 1973, S. 305, Tabelle 2. Die Hochschullehrer stellten 1,5\% aller erfassten Haushalte. Die Gruppe der Studenten, die um einiges höher gelegen hat, ist bei Elisabeth Lichtenberger leider nicht aufgeführt.

772 Vgl. hierzu und im Folgenden RECKNAGEL 2009, S. 7-12.

773 Zitiert nach RECKNAGEL 1998, S. 10; dort ohne Quellenangabe.

774 RECKNAGEL 2001, S. 39, WILL 1796, S. $207 f$.

775 WILL 1796, S. 211-213. Es handelte sich um 14 Familien, die für den Nürnberger Export produzierten und in Mietwohnungen untergebracht waren. 
Einnahmequelle der Bewohner war die Landwirtschaft. Georg Andreas Will schreibt 1796: „Die Burgerschaft nährt sich, außer dem, daß sie mancherlei Gewinn und Vortheil von der Universität zieht, im übrigen größtentheils von ihrem vorzüglichen Feld = und besonders Hopfenbau, vom Bierbräuen, vom Bier= und Hopfen=Handel, von verschiedenen Gewerben und Handwerken und von der Viehzucht. Diese mit der Mastung ist sehr vorzüglich, und werden allein 300. Stück Kühe in zwo Heerden zu dem obern und untern Thor, ausgetrieben. Der Ochsen, Schweine und Schafe nicht zu gedenken.“776

Mit der Gründung des Gymnasiums im Jahr 1575, der späteren Universität, spielte diese Bildungseinrichtung eine bedeutende Rolle in der Stadt, was sich stark auf die Zusammensetzung der Bevölkerung auswirkte: Zur Zeit ihrer größten Blüte sind für die Studienjahre 1620/21 (222), 1621/22 (144) und 1622/23 (187) in Summe 553 Neueinschreibungen verzeichnet. ${ }^{777}$ Hans Recknagel geht daher von einer maximalen Anzahl von 600 Studenten zu Beginn des Dreißigjährigen Krieges aus. ${ }^{778}$ Bei rund 320 Anwesen kamen damit rein rechnerisch zwei Studenten auf jedes Haus.

Im Türkensteuerregister der Reichsstadt Nürnberg aus dem Jahre 1716/17 ist die Bevölkerung der Bürgerstadt detailliert erfasst (vgl. Tab. 21). ${ }^{779}$ Die Mitglieder der Universitätsgemeinde, Professoren, Studenten und sonstige Universitäts-angehörige, sind dort nicht erfasst. Gelistet sind im Türkensteuerregister 289 Eigentümerhaushalte, davon sechs außerhalb der Stadtmauer. Diesen standen insgesamt 225 Haushalte von Hausgenossen und neunzehn Haushalte von öffentlich Bediensteten gegenüber. Betrachtet man lediglich die eigentumslosen Haushalte (Hausgenossen, Beamte) und Eigentümer der Stadtgemeinde, kommt man bereits auf einen Mieteranteil von $46 \%$ der Haushalte.

Der Einfluss der Universität auf die Eigentumsstruktur Altdorfs wird deutlich, wenn nachfolgend die eigentumslosen Haushalte der Universitätsgemeinde quantitativ erfasst werden. Im Studienjahr 1714/15 waren laut Elias von Steinmeier 64 Studenten neu eingeschrieben, im folgenden Studienjahr 75 Studenten,

\footnotetext{
776 WILL 1796, S. 207.

777 STEINMEYER 1912, S. 161-179.

${ }^{778}$ Mir erscheint diese Zahl zu hoch gegriffen, da ja nicht alle Studenten drei oder gar vier Jahre an der gleichen Universität verweilten. Horst Claus Recktenwald nennt für das Studienjahr 1636/37 gar 450 Studenten, leider ebenfalls ohne Quellenangabe; RECKTENWALD 1990, S. 30. In den drei zugehörigen Jahrgängen lassen sich lediglich 270 Einschreibungen feststellen; vgl. STEINMEYER 1912, 1. Bd., S. 226-235.

${ }^{779}$ StAN, Rst. N, Bauernverzeichnisse Nr. 7, fol. 318-326.
} 
im Jahr 1716/17 dagegen nur 50. ${ }^{780}$ Überschlägig kann davon ausgegangen werden, dass 1716/17 etwa 190 Studenten in Altdorf gelebt haben. ${ }^{781}$

Hinzu kamen noch die „akademischen Bürger und Universitätsverwandte“, 782 darunter auch die Universitätsprofessoren - etwa fünfzehn an der Zahl - und deren Familien bzw. Witwen sowie weitere Bedienstete, deren Anzahl aber schwankte. Ferner zählten der Notar, die Buchdrucker, Perückenmacher etc. zu den Universitätsverwandten. In Summe sind zum Beispiel für das Jahr 1731 vierzehn Professoren und 24 weitere Universitätsmitglieder beziehungsweise Bedienstete aufgeführt. ${ }^{783}$ Rechnet man diese Personengruppen den Mietern der bürgerlichen Stadtgemeinde hinzu, kamen auf 289 Eigentümerhaushalte etwa 470 Haushalte, die in fremdem Eigentum lebten. ${ }^{784}$ Der an sich schon hohe Anteil eigentumsloser Haushalte steigt bei Berücksichtigung der Universitätsangehörigen von 46\% auf $62 \%$. Damit sind in Altdorf Werte erreicht, die weit über denen anderer Kleinstädte liegen. Dies gilt sogar für die direkte Vergleichsgruppe der Nürnberger Landstädte. Velden kommt zeitgleich „lediglich“ auf einen Anteil von 34\% und Hersbruck auf 39\%. ${ }^{785}$ Für Lauf an der Pegnitz lassen sich die noch höheren Werte durch das Exportgewerbe erklären. Vermutlich dürfte daher in Altdorf der an sich schon hohe Anteil von eigentumslosen Haushalten ebenfalls auf das Vorhandensein der Universität zurückzuführen sein, denn Exportgewerbe ist nicht in nennenswerten Umfang überliefert. Die Kaufkraft der Universitätsangehörigen war ein wichtiger Faktor für die kleinstädtische Ökonomie und kann daher in seinen Auswirkungen auf die Verdienstmöglichkeiten eigentumsloser Haushalte mit dem Exportgewerbe verglichen werden.

Mit der Schließung der Universität 1809 und dem Fehlen der Universitätsgemeinde sinkt der Anteil der eigentumslosen Haushalte zwar insgesamt betrachtet, bleibt aber auf einem relativ hohen Niveau. Für das Jahr 1818 sind 551 Feuerstellen, 590 Familien und 2.116 Einwohner überliefert. ${ }^{786}$ Daraus errechnet sich eine durchschnittliche Familiengröße von 3,59 Personen, inklusive der Kinder. Die Zahl der Anwesen (Häuser) ist bisher für das Jahr 1818 nicht exakt ermittelt.

\footnotetext{
780 STEINMEYER 1912, S. 503-509.

${ }^{781}$ Nach Recknagel ist davon auszugehen, dass die Zahl der Studenten drei- bis viermal über der Zahl der Neueinschreibungen lag; RECKNAGEL 1998, S. 33. Ich habe für die Ermittlung der Studentenzahlen die Einschreibungen der relevanten Studienjahre 1714/15 bis 1716/17 addiert (169) und auf 190 aufgerundet. Maria Rosa di Simone weist allgemein daraufhin, dass in den Matrikeln nicht nur Studenten eingetragen sind und konkrete Studentenzahlen daraus nur mit großer Vorsicht abgeleitet werden könnten; Di SiMONE 1996, S. 236.

782 WILL 1795, S. 147.

783 ZOBEL 1731.

784225 Hausgenossen, 19 öffentlich Bedienstete, 38 Universitätsmitglieder, 190 Studenten.

${ }^{785}$ Vgl. Tab. 21.

${ }^{786}$ Alphabetisches Verzeichnis 1818, S. 4.
} 
Man kann jedoch von rund 310 Wohngebäuden zu diesem Zeitpunkt ausgehen. ${ }^{787}$ Bei den im Alphabetischen Verzeichnis für den Rezatkreis angegeben 551 „Feuerstellen“ handelt es sich also nicht um die Anzahl der Wohngebäude. Vermutlich sind damit die Wohnungen gemeint. Dies bedeutet wiederum, dass 39 Haushalte von den angegebenen 590 Familien keine eigene Feuerstelle (Wohnung) hatten, sondern zur Untermiete wohnten. Mindestens 240 Haushalte verfügten zwar über eine eigene Feuerstelle, besaßen aber kein eigenes Haus. Die bestehenden 310 Häuser wiederum dürften in der Regel von den Eigentümern selbst bewohnt gewesen sein, da nur in ganz wenigen Ausnahmen ein Altdorfer Bürger mehrere Häuser in Besitz hatte. ${ }^{788}$ Überschlägig kann daher für das Jahr 1818 von 310 Haushalten ausgegangen werden, die in der eigenen Immobilie lebten, ${ }^{789}$ diesen standen 280 Miethaushalte gegenüber. Dies bedeutet, dass Anfang des 19. Jahrhunderts - nach Schließung der Universität - in Altdorf weiterhin knapp die Hälfte aller Haushalte zur Miete wohnten. Im Vergleich mit der Situation der Bürgergemeinde 1717 steigt der Anteil der eigentumslosen Haushalte von $46 \%$ auf $47 \%$ leicht an.

\section{Exportgewerbestädte}

Den Begriff und die Definition der Exportgewerbestadt übernehme ich von Katrin Keller. ${ }^{790}$ Da ein gewerblicher Anteil für jede Art von Stadt charakteristisch ist, und sei es nur die Produktion von Gütern für die Stadt und den unmittelbaren Umkreis der Stadt, erscheint eine Präzisierung mit dem Begriff Exportgewerbe zielführend. Entsprechend dem Anteil der für den Export produzierten Waren boten diese Städte mehr oder weniger Menschen eine Einkommensmöglichkeit, dies gilt insbesondere für Menschen ohne Haus- und Grundbesitz. Entsprechend waren diese Städte durch einen vergleichsweisen hohen Anteil an Miethaushalten geprägt. Dabei werden die fränkischen Ergebnisse mit den Angaben zu Kursachsen verglichen.

\footnotetext{
787 Recknagel hat sämtliche Anwesen im Häuserbuch erfasst; RECKNAGEL 2009. Insbesondere bei der vorstädtischen Bebauung, die nach 1800 einsetzt, ist bei vielen Gebäuden kein Erbauungsjahr angeben. Für das Haus mit der alten Haus-Nr. 297 ist allerdings der Erwerb des Bauplatzes für das Jahr 1820 überliefert; RECKNAGEL 2009, S. 216. Es folgen jedoch noch vereinzelt Anwesen, die bereits zuvor Bestand hatten.

${ }^{788}$ Vgl. hierzu das Register der Hausbesitzer bei RECKNAGEL 2009, S. 231-264.

${ }^{789}$ Unter den 310 Anwesen befinden sich auch einige öffentliche Gebäude: das Rathaus (Hs. Nr. 1), das Spital (Hs. Nr. 243), das Pflegschloss (Hs. Nr. 42), das Rentamt (Hs. Nr. 43), die ehemaligen Universitätsgebäude (Hs. Nr. 50a und b) mit Wohnungen sowie kirchliche Bauten Hs. Nr. 95-99; RECKNAGEL 2009, S. 103-105.

${ }^{790}$ KELLER 1997, S. 56-58 und 88-93.
} 


\section{Franken}

Seit dem Mittelalter nahm Nürnberg eine wichtige Stellung unter den deutschen Städten ein. Basis des wirtschaftlichen Erfolges war nicht nur der Handel mit Waren, sondern auch die Produktion von Fertigwaren in der Stadt und im Umland für den Exportmarkt. ${ }^{791}$ Lange Zeit stellte das Metallgewerbe einen Schwerpunkt dar. Die Situation Nürnbergs soll an dieser Stelle aber nicht weiter thematisiert werden, auf die einschlägige Literatur wird verwiesen. ${ }^{792}$ Die Bedeutung der Wirtschaftskraft Nürnbergs darf nicht hoch genug für die Entwicklung in Franken eingeschätzt werden. Ohne die Stellung der Stadt als Warenumschlagplatz und Wohnort innovativer und geschäftstüchtiger Investoren lässt sich die auf Exportgewerbe ausgerichtete Wirtschaftsstruktur vieler Städte und Dörfer im Umkreis nicht erklären. Hektor Amman weist auf die enge wirtschaftliche Verflechtung des Nürnberger Metallgewerbes mit dem Umland bereits während des Mittelalters hin und nennt neben den Vorstädten Wöhrd und Gostenhof auch Lauf. ${ }^{793}$ Für Schwabach und Roth als brandenburg-ansbachische Städte sieht er dagegen gewisse Selbstständigkeitsbestrebungen bei der Produktion, auch wenn man sich der führenden Stellung Nürnbergs nicht entziehen konnte. Letzteres gilt noch stärker für die Marktorte Wendelstein, Kornburg und Fürth. „Im Mittelalter können wir zwar in all diesen Städten und Märkten allerhand Metallgewerbe feststellen, die sich jedoch vollkommen an Nürnberg anpaßten."794

Wie „Satelliten“ umringen in Franken die Exportgewerbestädte Nürnberg. Zuallererst seien hier die zwei Mittelstädte Erlangen und Schwabach genannt, sowie Lauf an der Pegnitz als wichtiger Hammerwerksstandort. Als weitere Städte wären anzuführen Roth und Langenzenn, wobei die Wirtschaftsstruktur von Langenzenn bisher nicht näher untersucht worden ist. Erst ab dem Jahr 1728 lässt sich hier die Strumpfwirkerei in größerem Umfang nachweisen, die Rotgerber erreichten gar erst 1739 die Erlaubnis zur Errichtung einer örtlichen Zunft. ${ }^{795}$

Die dörflichen Gewerbesiedlungen im Umkreis Nürnbergs verdankten ihre Entwicklung dem gleichen Grund. Sie wurden von mir bereits im Rahmen meiner Dissertation hinsichtlich Bevölkerung und Mietwohnungsbau ausführlich behandelt. ${ }^{796}$ Aus der Gruppe dieser dörflichen „Satelliten“ entwickelte sich Fürth zu einer Großsiedlung, die dann mit über 10.000 Einwohnern erst im Jahr 1818 of-

\footnotetext{
${ }^{791}$ Vgl. hierzu WIEST 1968.

792 Ebd., sowie MAYER/THYROFF 2014, S. 11-73.

${ }^{793}$ AMMANN 1968, S. 13.

794 Ebd.

795 EINFALT 1910, S. 79-81.

${ }^{796}$ Vgl. WENDEROTH 2019a, S. 189-211.
} 
fiziell zur Stadt erhoben wurde. Im Hinblick auf ihre Bevölkerungsgröße, Wirtschaftskraft, Bevölkerungsdichte und ihren Mieteranteil übertraf sie bereits seit spätestens 1700 die meisten fränkischen Landstädte. Aufgrund der fehlenden Stadtrechte vor 1800 bleibt sie aber in dieser Untersuchung für den Zeitraum unberücksichtigt.

\section{Schwabach}

Die Stadt Schwabach liegt $15 \mathrm{~km}$ südwestlich der Metropole an dem gleichnamigen kleinen Fluss, der die Stadt von West nach Ost durchfließt und diese in eine nördliche und südliche Stadthälfte teilt. In der südlichen Stadthälfte, als dem wirtschaftlichen Zentrum, erfolgte zwischen 1299 und 1302 die Gründung und Anlage eines Marktes. ${ }^{797}$ Die Siedlung erhielt im 14. Jahrhundert Stadtrechte (erstmalige Nennung 1371), das Stadtgebiet wurde auf ältere bestehende dörfliche Siedlungsbereiche westlich des Marktes und auch nördlich der Schwabach erweitert. Seit dem Mittelalter gehörte die Stadt zum hohenzollerischen Fürstentum Brandenburg-Ansbach-Kulmbach und wurde daher auch schon im Kontext der Quellen mehrfach erwähnt. Schwabach war Sitz eines Oberamtes.

Seit dem späten Mittelalter lässt sich im Vergleich zu anderen Kleinstädten Frankens eine überdurchschnittlich rege Gewerbetätigkeit festzustellen. ${ }^{798}$

$\mathrm{Zu}$ Beginn des 15. Jahrhunderts war das Stadtgebiet noch sehr locker bebaut. ${ }^{799}$ In der nördlichen Stadthälfte verdoppelte sich die Anzahl der Anwesen nach 1410 von 98 auf $196 .{ }^{800}$ In der südlichen Stadthälfte entstanden dagegen vielfach stattliche Neubauten anstelle älterer Anwesen. Diese Entwicklung ist noch heute im Hausbestand ablesbar. Ein Großteil der Anwesen stammt in ihrem baulichen Kern aus dem späten 15. oder frühen 16. Jahrhundert. Im erhaltenen Gebäudebestand zeichnet sich insbesondere eine rege Bautätigkeit im ersten Drittel des 16. Jahrhunderts ab. Dies deutet auf eine prosperierende wirtschaftliche Entwicklung und die Anlage weiterer neuer Hausstellen nach 1500 hin. Einen tiefen Einschnitt innerhalb der Stadtgeschichte stellte die Eroberung und Plünderung der Stadt im Jahr 1632 dar. ${ }^{801}$ Nach den Aussagen v. Falkensteins war die Stadt aufgrund von Plünderungen und Seuchen weitgehend entvölkert. ${ }^{802}$ Von Falkenstein mag dabei übertrieben haben, nachweislich waren aber im Jahr 1650 noch

\footnotetext{
${ }^{797}$ Vgl. hierzu und im Folgenden SCHLÜPFINGER 1994, S. 1-5.

798 Vgl. SCHLÜPFINGER 1994, S. 18-28.

${ }^{799}$ SCHLÜPFINGER 1977, S. $13 f$.

${ }^{800}$ Ebd., S. 14.

${ }^{801}$ DEHM/HECKEL, S. 7f.

${ }^{802}$ SCHANZ 1884, S. 261.
} 
80 Häuser zerstört. Der anschließende Wiederaufbau und vor allem der wirtschaftliche Aufschwung im 18. Jahrhundert führten zur zweiten prägenden Bauphase der Stadt.

Auf die frühe wirtschaftliche Prosperität der Stadt, die ja die Grundlage für die Ansiedlung eigentumsloser Schichten bildet, deutet bereits der Bau einer Schleifund Walkmühle mit Eisenhammer am Hödlertor zwischen 1450 und 1453 hin. ${ }^{803}$ Der hohe Mieteranteil zu Beginn des 16. Jahrhunderts weist ebenfalls auf eine ausgeprägte Gewerbestruktur in der Stadt hin. Äußerlich sichtbar wird dies zum Beispiel in der Gründung einer Schwabacher Eisenhandelsgesellschaft 1522.804 In diesem Kontext sind auch die 49 Meister zu sehen, die im Jahr 1549 in Schwabach Messerklingen für den Export herstellten. ${ }^{805}$ In Anlehnung an die Nürnberger Tuchindustrie wurden zudem nach denselben Gütevorschriften und Prüfungsmaßnahmen sowie zu gleichen Preisen Stoffe produziert. ${ }^{806}$ Spätestens mit den Zerstörungen des Dreißigjährigen Krieges erlebte die Wirtschaft der Stadt einen maximalen Einbruch, die gewerbliche Produktion dürfte stark gelitten haben, Untersuchungen zu dieser Fragestellung liegen jedoch bisher nicht vor. Erstaunlicherweise wurde bereits kurze Zeit später, 1651, eine Nadlerverordnung erlassen. Die Produktion von Näh- und Stecknadeln entwickelte sich in den nachfolgenden 100 Jahren stetig: ${ }^{807} 1787$ wurden jährlich an die 200.000.000 Nadeln in Schwabach hergestellt. Eine erste Gold- und Silberdrahtzugfabrik ließ der Markgraf Johann Friedrich 1680, also bereits vor der Ansiedlung der Hugenotten, einrichten, dieser war jedoch kein großer Erfolg beschieden. ${ }^{808}$ Einen zusätzlichen wirtschaftlichen Impuls erlebte die Stadt mit der Ansiedlung von etwa 500 französischen Glaubensflüchtlingen, nachdem deren Ansässigmachung in der Residenzstadt Ansbach 1685/86 gescheitert war. ${ }^{809}$ Ein gewichtiger Grund für die endgültige Niederlassung der Hugenotten in Schwabach stellte die wirtschaftsstrategisch bessere Lage in unmittelbarer Nähe zum Handelszentrum Nürnberg und an der Handelsroute Nürnberg - Augsburg dar. ${ }^{810}$ Mit dieser Entscheidung wurde Schwabach in den nachfolgenden Jahrzehnten zum wirtschaftlichen Zentrum des Fürstentums. ${ }^{811}$ Bedeutende neue Wirtschaftszweige entstanden mit der

${ }^{803}$ Dies kann als Beleg dafür gelten, dass Schwabach schon im 15. Jh. zur Wirtschaftsregion Nürnberg gehörte.

${ }^{804}$ DIPPERT 2015, S. 170.

${ }^{805}$ STAHLSCHMIDT 1970, S. 51.

${ }^{806}$ AMMANN 1968, S. 12.

${ }^{807}$ SCHANZ 1884, S. 300-308.

808 DIPPERT 2015, S. 177.

${ }^{809}$ SCHANZ 1884, S. 262-265 und 269.

${ }^{810}$ Ebd., S. 266.

811 Die Franzosen richteten unter anderem eine Gobelinmanufaktur ein, die jedoch nicht lange Bestand hatte; WENDEROTH 2019a, S. 216-218, Abb. 153a, b. 
Strumpfwirkerei ${ }^{812}$ und der Bortenwirkerei. ${ }^{813}$ Doch auch unabhängig von den Hugenotten entwickelten sich verschiedene Gewerbezweige in der Stadt. Ganz auf den Export ausgerichtet war die Gründung einer Kattunfabrik durch den Landesherrn 1716 östlich vor der Altstadt. Diese beschäftigte nach erfolgreicher Privatisierung Ende des 18. Jahrhunderts 500 bis 700 Arbeiter! ${ }^{814}$ Die wirtschaftliche Bedeutung, die Schwabach als Exportgewerbestadt im 18. Jahrhundert erlangte, soll mit einigen Zahlen verdeutlicht werden: 1794 lebten nahezu die Hälfte aller Arbeiter des Fürstentums in Schwabach. Hier wurden 36\% aller Waren des Landes und $47 \%$ der Exportwaren produziert. ${ }^{815}$

Der wirtschaftliche Aufschwung der Stadt zu einem bedeutenden Zentrum des Exportgewerbes zeigt sich sehr deutlich anhand der nachfolgenden Zahlen zur Bevölkerungsentwicklung Schwabachs. Die Zunahme der Produktion wirkt sich direkt in einer entsprechenden Zunahme der eigentumslosen Bevölkerung aus. Dies gilt sowohl mit Blick auf die absoluten Zahlen als auch für den prozentualen Anteil an der Gesamtbevölkerung. Die Auswirkungen von Industrialisierungsprozessen auf die Stadtgesellschaft lassen sich in Schwabach daher bereits im 18. Jahrhundert feststellen.

Schwabach entwickelte sich erst recht spät im Verlauf des 15. Jahrhunderts zu einer ansehnlichen Stadt. Für das Jahr 1410 sind lediglich 204 Hausstellen belegt. 816

Im Register des Gemeinen Pfennings 1497 wurden in der Stadt bereits 362 Haushaltungen mit 1.049 Personen über fünfzehn Jahren erfasst. ${ }^{817}$ Leider sind in der Aufstellung für Schwabach nicht die Hausgenossen gekennzeichnet, wie dies bei anderen Orten der Fall ist. Immerhin lassen sich die Haushaltsgrößen anhand der Angaben nachvollziehen.

\begin{tabular}{|l|c|c|c|c|c|c|c|c|c|c|c|}
\hline $\begin{array}{l}\text { Personen pro } \\
\text { Haushalt }\end{array}$ & $\mathbf{1}$ & $\mathbf{2}$ & $\mathbf{3}$ & $\mathbf{4}$ & $\mathbf{5}$ & $\mathbf{6}$ & $\mathbf{7}$ & $\mathbf{8}$ & $\mathbf{9}$ & $\mathbf{1 0}$ & $\mathbf{1 1}$ \\
\hline Anzahl Haushalte & 26 & 162 & 83 & 51 & 21 & 9 & 3 & 2 & 1 & 3 & 1 \\
\hline Prozentanteil & $\mathbf{7} \%$ & $\mathbf{4 5 \%}$ & $\mathbf{2 3} \%$ & $\mathbf{1 4 \%}$ & $\mathbf{6 \%}$ & $\mathbf{2 , 5 \%}$ & $\mathbf{0 , 8} \%$ & $\mathbf{0 , 6} \%$ & $\mathbf{0 , 3} \%$ & $\mathbf{0 , 8} \%$ & $\mathbf{0 , 3} \%$ \\
\hline
\end{tabular}

Tabelle 26. Schwabach, Haushaltsgrößen 1497, erfasst sind Personen über 15 Jahre (Thomas Wenderoth 2020)

Die häufigste Lebensform war die der Kleinfamilie: Drei Viertel der Haushalte hatten maximal drei Mitglieder über fünfzehn Jahren. Weitere 22,5\% der Haushalte hatten zwischen vier und sechs Personen. Die Zahl der größeren Haushalte

\footnotetext{
${ }^{812}$ SCHANZ 1884, S. 271.

${ }^{813}$ SCHANZ 1884, S. 277.

${ }^{814}$ DIPPERT 2015, S. $175 f$.

${ }^{815}$ Ebd., S. 182.

816 DEHM/HECKEL 1970, S. 7.

817 SCHLÜPFINGER 1977, S. 20f.
} 
war mit absolut zehn verschwindend gering. Insbesondere unter den Kleinhaushalten dürften sich bereits eine große Anzahl Mieter verbergen. Gut 30 Jahre später führt das Salbuch von 1530 exakt 303 Anwesen auf. Diese wurden jedoch nicht allein von den Eigentümern, sondern auch von 194 Hausgenossen mitbewohnt, die dort namentlich aufgezählt sind. ${ }^{818}$ Genannt werden 144 männliche Mieter und 39 Witwen. Zusammen ergibt dies 497 Haushaltungen. Der Anteil der eigentumslosen Haushalte lag demnach im Jahr 1530 bereits bei 39\%. Der Anteil der namentlich genannten Witwen unter den Mietern betrug 20\%. Dies ist die früheste Angabe zum Mieteranteil, die aber zugleich belegt, dass Schwabach eine lange Tradition als Wohnort für Mieter aufweist.

\section{Schwabach, Mieterhaushalte nach den Huldigungslisten}

\section{3-1723}

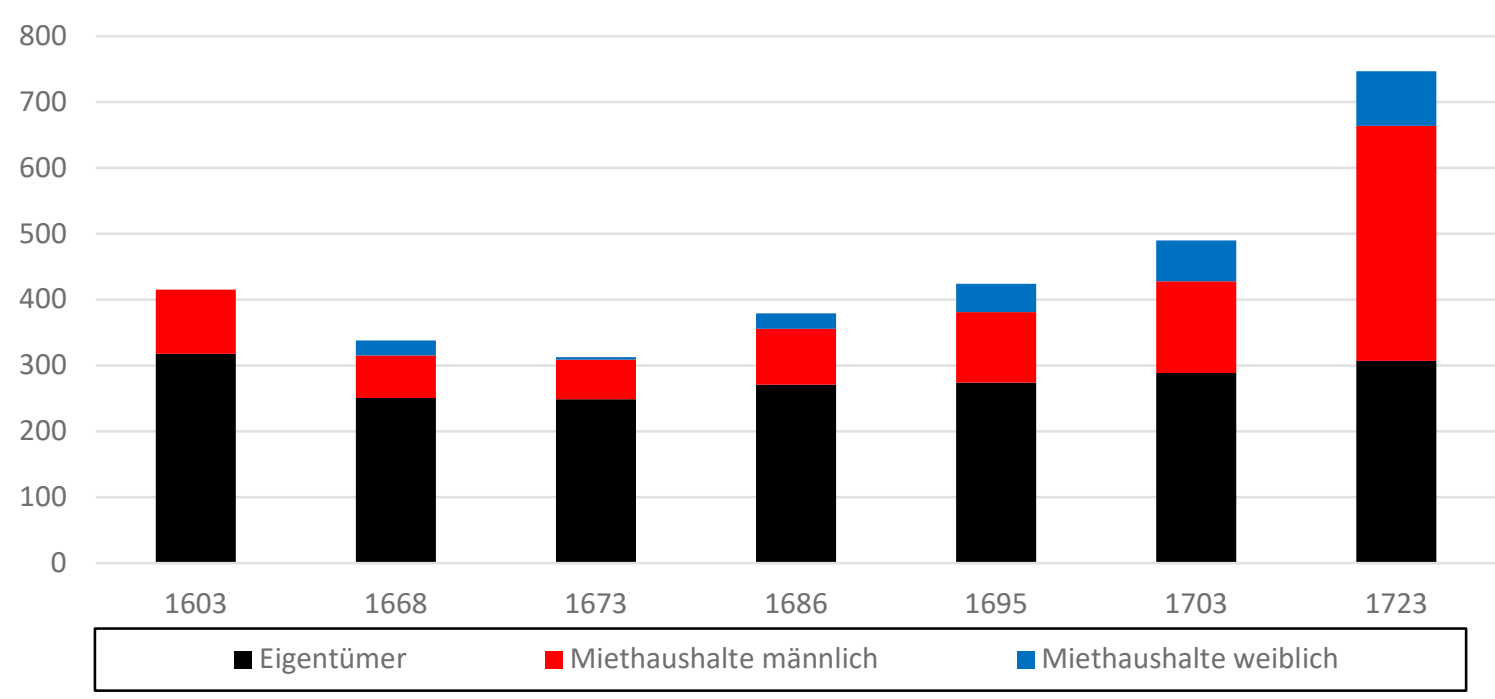

Grafik 8. Schwabach, Mieteranteil nach den Angaben in den Huldigungslisten. In den Jahren 1603 und 1673 fällt der geringe Anteil weiblicher Miethaushalte (blau) auf, vermutlich sind diese nicht vollständig erfasst worden. (Thomas Wenderoth 2019)

In der heimatgeschichtlichen Forschung sind bisher die Bevölkerungszählungen der Huldigungslisten des Fürstentums Ansbach unbeachtet geblieben. Erstmalig liegen hier für das Jahr 1603 Zahlen vor: ${ }^{819}$ Erfasst wurden 318 Eigentümer und 97 Miethaushalte mit männlichem Haushaltsvorstand, eine unbekannte Zahl von (Unter-)Mieterinnen muss gedanklich ergänzt werden. Während die Zahl der Eigentümer gegenüber 1530 leicht angestiegen ist, hat die Zahl der männlichen Miethaushalte von 144 auf 97, also um 50\%, abgenommen. Diese Feststellung ist

${ }^{818}$ StAN, Fm AN Salbücher Nr. 92, fol. 81-121.

Eine Transkription findet sich bei: SCHLÜPFINGER 1977, S. 76-82.

${ }^{819}$ StAN, Fm AN, Brdbg. Lit. Nr. 592. 
bemerkenswert, da sie einen Hinweis darauf gibt, dass nicht erst der Dreißigjährige Krieg zu einer Reduktion der Bevölkerung geführt hat, sondern ein erster Abschwung bereits im 16. Jahrhundert stattgefunden haben muss. Anfang des 17. Jahrhunderts ist wiederum ein wirtschaftlicher Aufschwung anzunehmen, denn 1623 werden 393 Hausbesitzer genannt, zu den Eigentumslosen fehlt leider die entsprechende Angabe. ${ }^{820}$

Noch vor den eigentlichen Kampfhandlungen sank die Zahl der Eigentümer bis zum Jahr 1628 bereits auf 325, also um 17\%. ${ }^{821}$ Gleichwohl bedeutete der nachfolgende Krieg für Schwabach einen markanten Einschnitt: 1650 waren nur mehr 270 Häuser bewohnt, in ihnen lebten 341 Haushalte. ${ }^{822}$ Der Überhang an Haushalten gegenüber der Summe der Häuser beträgt 71, hinzuzurechnen wären noch etwa zwanzig Häuser, die zur Gänze an öffentlich Bedienstete oder andere Mieter vergeben waren, 90 Miethaushalte können daher als minimaler Anteil gelten, der dementsprechend bei mindestens 26\% lag. Für das Jahr 1668 geben die Huldigungslisten erneut einen Anhaltspunkt zur Bevölkerungsentwicklung: ${ }^{823}$ Aufgeführt sind 251 Eigentümer sowie 64 männliche und 23 weibliche Miethaushalte. Dies ergibt zusammen 338 Haushalte, die Zahl liegt sehr dicht an den Angaben für das Jahr 1650, auch der Anteil der Mietbevölkerung bleibt unverändert bei $26 \%$, tatsächlich könnte aber die Bevölkerung bereits leicht gewachsen gewesen sein, denn in den Huldigungslisten fehlen unter anderem die Haushalte der öffentlich Bediensteten. Die Zahlen in den Huldigungslisten 1673 entsprechen denen der Huldigungsliste von 1668, mit einer signifikanten Ausnahme: Unter den Miethaushalten sind nur noch vier Schutzverwandtenhaushalte mit weiblichem Haushaltsvorstand erfasst anstelle von 23 fünf Jahre zuvor. Insgesamt wurden 313 Haushalte gezählt. ${ }^{824}$

Im Jahr 1671 waren nach den Angaben eines Steuerregisters 325 Häuser mit 402 Haushalten vorhanden. ${ }^{825}$ Die Differenz zwischen den 250 Eigentümern 1668/73 und den 325 Häusern kann mit den 101 öden Hausstellen erklärt werden, die in den Huldigungslisten 1668 aufgeführt sind. ${ }^{826}$ Von daher dürfte die Zahl der Eigentümerhaushalte tatsächlich um 250 gelegen haben und es dürften deutlich mehr Mieterhaushalte als in den Huldigungslisten überliefert vorhanden gewesen sein. Wie bereits angemerkt, fehlen in den Huldigungslisten die öffentlich Bediensteten, hier ist von 20 bis 30 Haushalten auszugehen, und die Untermieter,

\footnotetext{
${ }^{820}$ SCHLÜPFINGER 1977, S. 31.

821 Ebd.

822 DEHM/HECKEL 1970, S. 8.

${ }^{823}$ StAN, Fm AN, Brdbg. Lit. Nr. 594, fol. 457' - 478; fol. 642.

${ }^{824}$ Ebd., Nr. 595, fol. 335-444 und 449-451.

${ }^{825}$ Die Zahlen sind dem Häuserbuch von Dehm/Heckel entnommen;

DEHM/HECKEL 1970, S. 8 und Quellenverzeichnis, S. 12.

${ }^{826}$ StAN, Fm AN, Brdbg. Lit. Nr. 594, fol. 478 und fol. 642.
} 
vor allem die Frauen, sodass um 1670 auf 250 Eigentümerhaushalte 152 Haushalte kamen, die nicht im eigenen Haus wohnten, der Mieteranteil lag also deutlich höher, bei etwa $38 \%$.

Die weitere Entwicklung kann anhand der barockzeitlichen Huldigungslisten 1686, 1695, 1703 und 1723 gut nachvollzogen werden. Trotz der oben diskutierten Unvollständigkeit in den Angaben der Huldigungslisten lässt sich ein kontinuierlicher Anstieg der Bevölkerung nachvollziehen, dabei nimmt der Anteil der erfassten Miethaushalte überproportional von $28 \%$ über $35 \%$ und $41 \%$ auf schlussendlich 59\% im Jahr $1723 \mathrm{zu}^{827}$

Für das Jahr 1703 sind 356 Raths- und Bürgerspersonen (Haushalte) aufgeführt. In einer Anmerkung zu den Eigentümern heißt es dazu: Darunter aber ein und anderer Burger mehr als einmahl angesezet, nach der Anzahl der Häußer, so er hat, und die Wittweiber haben mehrerntheils keine eigene Häußer mehr, sondern sazen Bey andern im Bestand. ${ }^{828}$ Unter den Bürgern sind ebenfalls 25 Juden aufgeführt. ${ }^{829}$ Eine Hauptgruppe der Mieter findet sich hier unter der Bezeichnung des Schutzverwanthen: Genannt werden 73 Männer und 58 Frauen, so Wittib als ledige Muether. ${ }^{830}$ Am Anfang dieser Gruppe werden zwei Pfarrerswitwen aus Roth und Katzwang aufgeführt.

In der Schwabacher Huldigungsliste von 1723 werden hingegen 186 Haußgenoßen, oder Bürger so keine aigenthumbl. Häußer haben genannt. ${ }^{831}$ An bürgerlichen Witwen mit Hausbesitz werden 31 aufgezählt, sechs mit je einem halben Haus, eine mit eineinhalb Häusern und eine mit zwei Häusern. ${ }^{832}$ Bürgerwittiben, so keine aigenthumbl. Häußer haben werden 24 genannt. ${ }^{833}$ Schutzverwanthe MannsPersonen sind 171 aufgelistet, ${ }^{834}$ weibliche Schutzverwandte $39 .{ }^{835}$ Für sämtliche Schutzverwandte ist kein Hausbesitz angeführt, sodass es sich hier wohl insgesamt auch um Mieter gehandelt haben muss. Die jüdischen Einwohner, untergliedern sich in 29 Hauseigentümer (davon elf mit nur einem halben Haus), neun jüdische Mieter (darunter eine Witwe) und weitere neun Verheyrathe Juden, so noch keine Schutzbriefe haben (darunter wieder eine Witwe), für die kein Hausbesitz genannt ist. ${ }^{836}$ Separat aufgeführt sind zudem die 23 Inwohner bey der herrschaftlichl. Cottonfabrique, darunter eine Witwe. ${ }^{837}$ Am Schluss sind 31 eximirte

\footnotetext{
${ }^{827}$ Ebd., Nr. 596.

${ }^{828}$ Ebd., Nr. 599, fol. 453.

${ }^{829}$ Ebd., fol. 457'.

${ }^{830}$ Ebd., fol. 457'.

${ }^{831}$ Ebd., Nr. 601, fol. 116-120.

${ }^{832}$ Ebd., fol. 120'-121.

${ }^{833}$ Ebd., fol. 121'.

${ }^{834}$ Ebd., fol. 122'-125'.

${ }^{835}$ Ebd., fol. 126-127.

${ }^{836}$ Ebd., fol. 126-127.

${ }^{837}$ Ebd., fol. 127'.
} 
Persohnen aufgeführt, denen neun ganze Häuser und ein halbes Haus gehören, 21 dieser Haushalte wohnten demnach nicht im Eigentum. ${ }^{838}$ Insgesamt sind 916 Haushalte und 332,5 private Häuser erfasst, hinzu kommen zehn bis zwanzig Dienstwohnungen. Dies bedeutet, dass im Jahr 1723 durchschnittlich 2,7 Haushalte in einem Anwesen lebten. In der Regel wohnte der Eigentümer mit im Haus, nur in dreizehn Fällen waren ganze oder halbe Häuser komplett fremdvermietet, das sind gerade einmal 4\% der Anwesen.

Für das Jahr 1716 findet sich außerhalb der Huldigungslisten eine Notiz zu den Hugenotten: Ab 1686 wuchs die Kolonie der französischen Flüchtlinge auf dann 103 Haushalte mit 494 Personen an. Von diesen 103 Familien erwarben lediglich sechs Familien Hauseigentum. ${ }^{839}$ Die übrigen $94 \%$ wohnten demnach zur Miete. Die Hugenotten dürften entsprechend eine große Mietergruppe innerhalb der städtischen Gesellschaft gestellt haben. Die Schaffung des nötigen Mietraums musste dabei nicht in einem Zug erfolgen, da das Anwachsen der Kolonie sich über 30 Jahre erstreckte. Die französische Bevölkerung nahm aber in den nachfolgenden Jahrzehnten wieder stark ab, bereits sieben Jahre später bestand die französische Kolonie nur noch aus 88 Haushalten, darunter sechzehn Witwen. Der Hausbesitz hatte sich kaum geändert: Den Franzosen gehörten 1723 anstelle von sechs nunmehr sieben Häuser. ${ }^{840}$

Für 1739 hat Heinrich Schlüpfinger die Handwerker und Gewerbetreibenden erfasst, ${ }^{841}$ die den allergrößten Teil der städtischen Bevölkerung ausmachten. Von den dort genannten 674 Haushalten wohnten 380 zur Miete. Das sind 56\% dieser Haushalte. In der Aufstellung fehlen unter anderem die Spitalbewohner, Bettler, Taglöhner sowie die öffentlich Bediensteten wie Pfarrer etc.

1748 wurden 420 Hausstellen gezählt und zwei Jahre später darin 844 Haushaltungen. Demnach ist zur Mitte des 18. Jahrhunderts hin weiter eine starke Zunahme der Bevölkerung festzustellen, der Anteil der Miethaushalte lag kontinuierlich über $50 \%{ }^{842}$ Für die Judenschaft gibt Schlüpfinger genaue Zahlen für diesen Zeitraum an: ${ }^{843} 28$ Familien besaßen ein eigenes Haus, neunzehn Familien wohnten als Hausgenossen zur Miete, das waren $40 \%$ der jüdischen Haushalte. Demnach war in Schwabach der Mieteranteil unter den Juden etwas geringer als innerhalb der christlichen Bevölkerung.

\footnotetext{
${ }^{838}$ Ebd., fol. 132-133.

${ }^{839}$ DISTLER 1986, S. 15.

${ }^{840}$ StAN, Fm AN, Brdbg. Lit. Nr. 601, fol. 129-131.

${ }^{841}$ SCHLÜPFINGER 1977, S. 55-57.

${ }^{842}$ Alle Zahlen sind dem Häuserbuch von Dehm/Heckel entnommen;

DEHM/HECKEL 1970, S. 8 und Quellenverzeichnis, S. 12.

${ }^{843}$ SCHLÜPFINGER 1986, S. 2.
} 


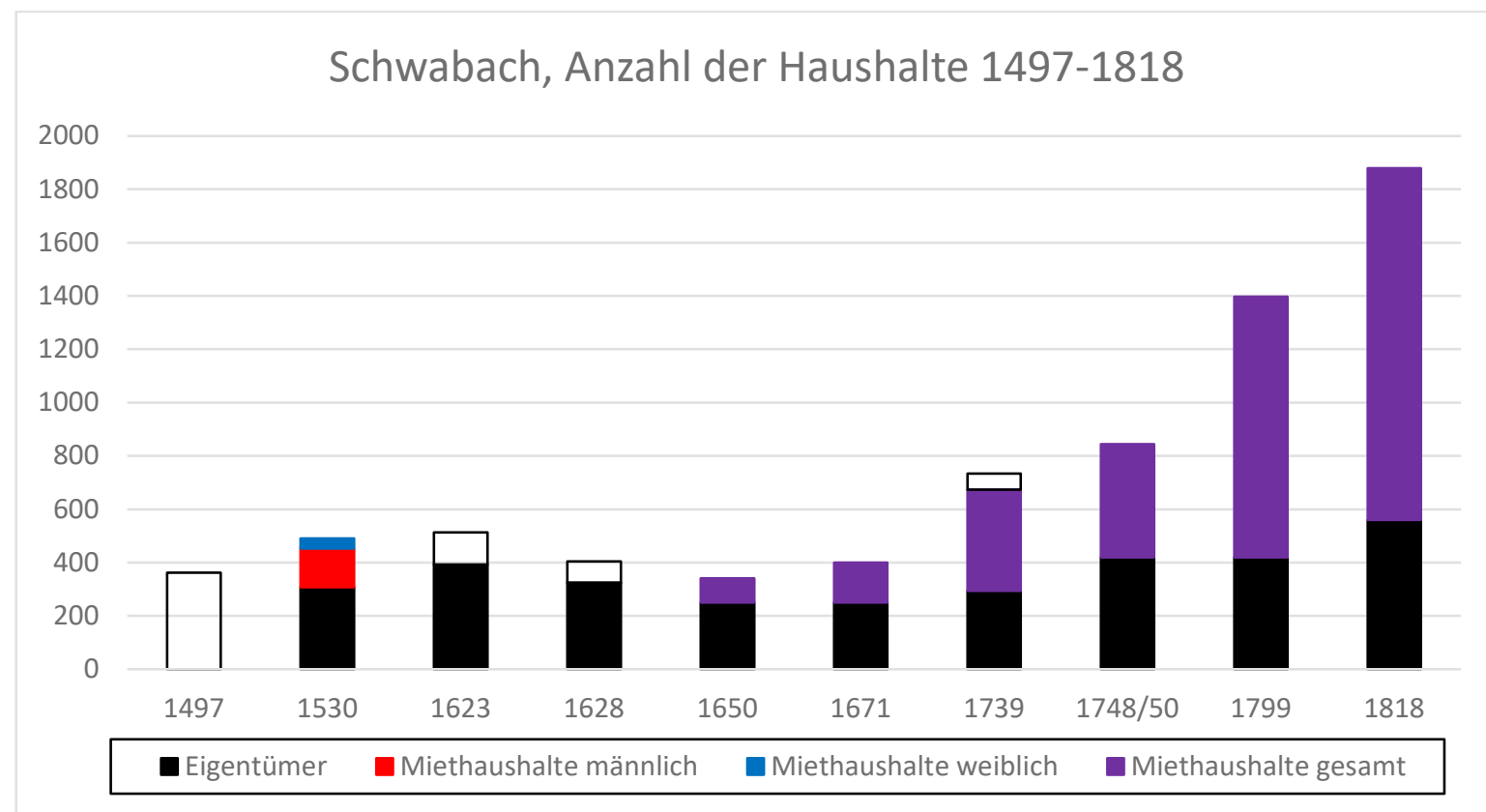

Grafik 9. Schwabach, Anzahl der Haushalte 1497-1818, nach verschiedenen Quellen; weiß = eine Differenzierung in die einzelnen Gruppen ist nicht möglich, bzw. Angaben zu (weiblichen) Miethaushalten fehlen in den Quellen. ${ }^{844}$ (Thomas Wenderoth 2019)

Wiederum 51 Jahre später lässt sich die Situation für Schwabach anhand der „Seelenlisten“ von 1799 sehr exakt ermitteln. ${ }^{845}$ Den Listen zufolge war Schwabach zu diesem Zeitpunkt eine Stadt der Mieter. Die eigentumslosen Haushalte in der Stadt machten $75 \%$ aller Haushalte aus. ${ }^{846}$ Insgesamt wurden die Häuser durchschnittlich von 3,8 Familien bewohnt. ${ }^{847}$

Überraschend erscheint, dass sich zwar der Anteil der Haushalte zwischen 1748 und 1799 fast verdoppelt, die Zahl der Hausstellen aber nur unwesentlich zugenommen hat. Das Bevölkerungswachstum wurde also durch eine Verdichtung des Wohnens kompensiert.

${ }^{844}$ Die Angaben für das Jahr 1497 differenzieren nicht zwischen Eigentümern und Mietern und sind eventuell auch nicht vollständig. 1623 und 1628 fehlen die Angaben zu Mietern komplett, ihr Anteil ist geschätzt (weiß). 1739 fehlen Angaben zu den weiblichen Miethaushalten, ihr Anteil ist ebenfalls als Schätzung angegeben (weiß).

${ }^{845}$ StadtASC, Signatur III-25.

846 SCHLÜPFINGER 1977, S. 60-63.

${ }^{847}$ Die Ergebnisse wurden der Öffentlichkeit am 03.06.2016 bei der bayerischen Hausforschertagung in Schwabach von Herrn Leonhard Salzer unter dem Titel „Untersuchungen zur Wohnstruktur Schwabachs im ausgehenden 18. Jahrhundert" vorgestellt. Salzer erfasst mit 426 Häusern aber lediglich den Großteil der Häuser in der Kernstadt. Die nachfolgenden prozentualen Angaben beziehen sich auf diese 426 Gebäude, die Einzelergebnisse erscheinen daher ausreichend belastbar.

Heinrich Schlüpfinger kommt in seinem Aufsatz von 1977 dagegen auf 464 Häuser in der Kernstadt und 79 Häuser vor den Stadttoren. Während sich Salzer nur auf die Kernstadt bezieht, erfasst Schlüpfinger auch die vorstädtische Bebauung sowie die öffentlichen Bauten wie Spital, Rathaus, Kirchturm etc.; SCHLÜPFINGER 1977. 
Für das Jahr 1818 liegt ebenfalls eine Erfassung vor, die die zuvor genannten Zahlen grundsätzlich bestätigt: Aufgeführt sind 1.879 Feuerstellen (gemeint sind in diesem Fall wohl Wohnungen/Herdstellen und nicht Häuser) und 1.855 Familien mit zusammen 7.421 Einwohnern. ${ }^{848}$

Die Entwicklung der Miethaushalte habe ich mit Hilfe von zwei Diagrammen dargestellt (Grafik 8 und 9). Trotz mancher Unschärfe und unvollständigen Angaben in den Huldigungslisten ist die Tendenz in beiden Tabellen eindeutig. Der Anteil der Miethaushalte ist im Verhältnis zu anderen mittelfränkischen Kleinstädten über die gesamte Neuzeit hinweg sehr hoch. Insgesamt betrachtet weist Schwabach schon sehr früh (1530) mit fast $40 \%$ einen großen Anteil von eigentumslosen Haushalten auf. Aufgrund eines Einbruchs in der wirtschaftlichen Entwicklung im 16. Jahrhundert scheint auch der Anteil der Mieter erst einmal gesunken zu sein. Die anschließende Phase der Erholung bis 1623 wird durch den Krieg zunichtegemacht. Um 1670 kann wiederum von knapp 40\% Miethaushalten ausgegangen werden. Ab 1686 erlebte die Stadt einen merklichen Aufschwung, in dessen Folge der Anteil der Miethaushalte stark anstieg auf über 59\% 1723 und 75\% Ende des 18. Jahrhunderts.

\section{Erlangen}

Die heutige Stadt Erlangen entstand aus dem Zusammenschluss von zwei eigenständigen Stadtgemeinden im Jahr 1812. Erlangen befand sich seit dem Mittelalter unter der Herrschaft der fränkischen Hohenzollern. Etwa 1.000 Meter südlich der Kleinstadt Erlangen gründete Markgraf Christian Ernst von BrandenburgBayreuth im Jahr 1686 eine neue Stadt, um Hugenotten anzusiedeln. Ausschlaggebend für die Ortswahl dürfte die unmittelbare Nähe zum Handelszentrum Nürnberg und die Lage an einer Fernstraße gewesen sein. ${ }^{849}$ Die neue Stadt wird ab $1701 \mathrm{ihm} \mathrm{zu}$ Ehren Christian-Erlang genannt. Geläufiger bleibt jedoch die Bezeichnung Neustadt Erlangen.

Die mittelalterliche Siedlung, im Folgenden als Altstadt Erlangen bezeichnet, erhebt sich strategisch günstig auf einem Geländesporn am Zusammenfluss von Schwabach und Regnitz. Bis ins 18. Jahrhundert hinein ist diese Siedlung als Ackerbürgerstadt zu charakterisieren, die kaum über 100 Haushalte zählte. Mit der Gründung der gewerblich orientierten Neustadt setzte auch hier ein enormer wirtschaftlicher Aufschwung an. Wohl ab 1696 entwickelte sich auch die Altstadt Erlangen zu einer gewerblich geprägten Stadt mit einem ebenfalls beachtlichen

\footnotetext{
${ }^{848}$ Alphabetisches Verzeichnis 1818, S. 83.

${ }^{849}$ WENDEROTH 2019c, S. $59 f$.
} 
flächenmäßigen Wachstum nach Süden und Osten zur Neustadt hin. ${ }^{850}$ So wurden im Jahr 1752 für die Altstadt Erlangen bereits 211 Häuser gezählt. Neben einer Vergrößerung des Stadtgebiets lässt sich auch eine deutliche Zunahme der Mietbevölkerung in der Altstadt nachweisen.

Der Aufbau der Neustadt erfolgte dagegen erst einmal schleppender. In den ersten drei Jahren nach der Stadtgründung wurden 42 Häuser mit etwa 170 Wohnungen errichtet. ${ }^{851}$ Danach geriet der Stadtausbau ins Stocken, bis 1698 kamen nur 30 weitere Häuser hinzu. ${ }^{852}$ Von diesen Häusern standen 40 im Eigentum von Franzosen und $34 \mathrm{im}$ Eigentum von Deutschen. ${ }^{853}$ In den folgenden 50 Jahren erlebte die Stadt dann einen starken Aufschwung. Bis Anfang 1700 waren es 105 Häuser. ${ }^{854} \mathrm{Im}$ Jahr 1723 wurden in Christian-Erlang 251 Häuser gezählt und 455 Häuser im Jahr $1752 .{ }^{855}$ Anschließend ging der Ausbau nur noch langsam voran: 1787 waren in Christian-Erlang 500 Häuser vorhanden.

\section{Erlangen - Altstadt}

Der Kontrast in Bezug auf die Einwohnerstruktur einer agrarisch geprägten Kleinstadt im Gegensatz zu einer Gewerbestadt wird am Beispiel Erlangens signifikant (vgl. Tab. 27 und 28). In der älteren Kleinstadt spielten Mieter bis zur Ankunft der Hugenotten eine untergeordnete Rolle im Sozialgefüge der Stadt. Am Beginn der Neuzeit sind es vor allem öffentlich Bedienstete, die die Gruppe der Mieter dominieren. Bis zum Beginn des Dreißigjährigen Krieges wuchs der Mieteranteil auf gut $20 \%$ an. Mit dem großen Bevölkerungsverlust durch den Krieg dürfte der Anteil der Miethaushalte erst einmal rückläufig gewesen sein und auf einem niedrigen Niveau, vergleichbar dem der Landstädte im Fürstentum Ansbach, verharrt haben. Für das Jahr 1662 wurden anlässlich der Erbhuldigung nur noch 58 Haushaltsvorstände erfasst, eine Differenzierung in Eigentümer und Mieter erfolgte jedoch nicht! ${ }^{856}$

${ }^{850}$ SCHANZ 1884, S. 58. Er geht davon aus, dass sich die Bevölkerung bezogen auf die Mitte des 17. Jhs. im Jahr 1698 verdreifacht hatte.

${ }^{851}$ WendeROTH 2012/13, S. 83; MEMMERT 1955, Abb. 1: Jahrweise Kartierung der Wohnbauten zwischen 1686 und 1689.

852 JАКОВ 1986, S. 66.

${ }^{853}$ SCHANZ 1884, S. 55.

854 SCHANZ 1884, S. 55, Anm. 4.

${ }^{855}$ Vgl. hierzu und im Folgenden Schanz 1884, S. 86f.

856 StABA, Markgraftum Brandenburg-Bayreuth, Geheimes Archiv Bayreuth Nr. 142, ohne Folierung, Erb- und Landeshuldigung 1662. 


\begin{tabular}{|l|c|c|c|c|c|c|c|}
\hline Jahr & Häuser & Eigentümer & Mieter & Einwohner & $\begin{array}{c}\text { HH-Größe } \\
\text { über 15 Jahre }\end{array}$ & $\begin{array}{c}\text { Mieter } \\
\%\end{array}$ & $\begin{array}{c}\text { Haushalte } \\
\text { pro Haus }\end{array}$ \\
\hline 1497 & 85 & 82 & 13 & 212 & 2,3 & $14 \%$ & 1,12 \\
\hline 1616 & & 91 & 25 & & & $22 \%$ & \\
\hline 1619 & & 84 & 16 & & & $16 \%$ & \\
\hline 1662 & & \multicolumn{2}{|c|}{58} & & & $49 \%$ & 1,85 \\
\hline 1712 & 109 & 104 & 99 & & & $50 \%$ & \\
\hline 1735 & & 165 & 168 & & & $55 \%$ & 2,25 \\
\hline um 1752 & 211 & 211 & 263 & 2.038 & $\begin{array}{c}4,3 \\
\text { incl. Kinder }\end{array}$ & & \\
\hline
\end{tabular}

Tabelle 27. Altstadt Erlangen, Anteil der Miethaushalte 1497-1752; die aus Archivalien direkt übernommenen Zahlen sind „normal“ ausgeführt, die interpretierten Zahlen kursiv.

(Thomas Wenderoth 2019)

Die Werte zum Anteil der Miethaushalte vor dem Dreißigjährigen Krieg basieren auf verschiedenen Quellen, die jedoch die öffentlich Bediensteten, die in der Regel nicht im Privateigentum wohnten, nicht berücksichtigen. Die Anzahl der explizit als Mieter ausgewiesenen Personen ist in diesen Auflistungen sehr gering, erst mit den ergänzten öffentlichen Haushalten und dem Abgleich zu den in den Salbüchern aufgeführten Zahlen zu Eigentümern ergibt sich eine nennenswerte Gruppe an Mietern innerhalb der Einwohnerschaft. ${ }^{857}$

Einschneidend auf die Altstadt wirkt sich 1686 die Gründung der Neustadt aus. Nachdem die Hugenotten zuerst in der Altstadt untergebracht waren, muss auch hier ab 1685 mit einem stark angestiegenen Mieteranteil gerechnet werden. Konkrete Zahlen liegen jedoch lediglich für die Neustadt für das Jahr 1698 vor.

${ }^{857}$ Die Quellen sind ausführlich diskutiert in Kapitel 6 unter der Überschrift: Die Entwicklung zwischen 1497 und 1700 am Beispiel Erlangens. 
Im Rahmen der Erbhuldigung 1712 wurde dann die Bevölkerung beider Teilstädte erfasst. ${ }^{858}$ Aufgeführt sind am Anfang der Liste zur Altstadt der Pfarrer, der Richter, zwei Bürgermeister, ein Stadt- und Gerichtsschreiber, sieben Ratsmitglieder, vier Viertelmeister, ein Wildmeister sowie der Cantor. Von diesen Personen dürften lediglich die Bürgermeister, Ratsmitglieder und Viertelmeister in einem eigenen Haus gelebt haben, sodass von den genannten achtzehn Haushalten fünf zur Miete wohnten. Dann folgen die 93 Bürger und Unterthanen so eigene Häußer haben, darunter dreizehn Witwen und je ein Mann und eine Witwe, die in Christian-Erlang lebten. Insgesamt errechnen sich daraus 104 Eigentümerhaushalte. Als Bürger so keine Häußer haben sind sechzehn Männer erfasst, hinzu kommen 54 Beständner oder Haußgenossen und 24 Wittweiber und theils ledige Weibspersohnen. Mit den öffentlich Bediensteten ergeben sich 99 Miethaushalte. Der Anteil der eigentumslosen Haushalte lag also mit 49\% nahe bei der Hälfte aller Haushalte.

Für die nachfolgende Erbhuldigung des Jahres 1735 finden sich abermals detaillierte Zahlen. ${ }^{859}$ Die Anzahl der öffentlichen Personen hatte sich um vier auf dann 22 erhöht (3. Bürgermeister, Steuereinnehmer, Adjunctus, Gegenschreiber, Ziegler, drei statt vier Viertelmeister). Von diesen 22 Personen können wiederum neun als Mieter gerechnet werden. An bürgerlichen Eigentümern werden darüber hinaus 152 Personen gelistet, an Mietern 44 Bürger und Bürgers Witwen ohne Häußer, 98 Beständner und Schutzverwanthe, 17 Wittweiber und ledige Personen. Demnach standen 165 Eigentümern 168 Mieterhaushalte gegenüber.

\section{Erlangen - Neustadt}

Eine Zählung vom 23. Juli 1698 enthält die frühesten Informationen zum Anteil der Miethaushalte in der Neustadt. ${ }^{860}$ Diese wurde bereits 1884 von Georg Schanz veröffentlicht. ${ }^{861}$ Die Erfassung erfolgte getrennt für die französische und die deutsche Gemeinde. Wiedergegeben sind dort jeweils die Bewohner der Häuser vor Ankunft der neuen Flüchtlinge, also ein Stand um das Jahr 1696. Jeweils als Ergänzung in eigenen Listen, wiederum getrennt in Deutsche und Franzosen, folgen dann die ab 1696 neu hinzugekommenen Einwohner. Bei der französi-

\footnotetext{
${ }^{858}$ Ebd., Geheimes Archiv Bayreuth Nr. 194, ohne Folierung, Erb- und Landeshuldigung, 4. Juli 1712.

${ }^{859}$ Ebd., Geheimes Archiv Bayreuth Nr. 235, ohne Folierung, Erb- und Landeshuldigung, 19. Oktober 1712.

860 StadtAER, Rudelsche Chronik, fol. 88.

${ }^{861}$ SCHANZ 1884, Anlage 39, S. 87-95.
} 
schen Gemeinde ist in dieser ergänzenden Liste eine große Manufaktur angeführt. Zudem werden hier am Ende der Aufstellung noch vier Gerbereien westlich außerhalb der Stadt genannt. ${ }^{862}$

In der nachfolgenden Tabelle sind daher die beiden Zeitschnitte 1696 und 1698 getrennt angegeben. ${ }^{863}$ Der Anteil der Miethaushalte steigt leicht von 79\% auf 82\%. Nach einer Notiz von Schwarz und Bever vom 8. Januar 1700 zur Zählung der Einwohner lebten zu diesem Zeitpunkt 1.508 Personen in 395 Familien (Haushalten) in der Neustadt, die sich auf 105 Häusern verteilten. ${ }^{864}$ Daraus ergibt sich eine Familiengröße von 3,82 Personen. Die Häuser waren zu diesem Zeitpunkt sehr dicht belegt mit durchschnittlich 3,76 Familien pro Haus. Der starke Anstieg in der Anzahl der Häuser deutet auf eine neuerliche Bauwelle hin, könnte aber auch zu einem nicht unerheblichen Teil durch Hausteilungen verursacht sein. Diese zusätzlichen Häuser wurden vermutlich überwiegend vom Eigentümer selbst bewohnt, sodass der Anteil der Miethaushalte leicht gesunken sein und bei etwa $75 \%-80 \%$ gelegen haben dürfte. Sehr detailliert erfolgte wiederum die Erfassung der Einwohner im Rahmen der Erbhuldigung am 4. Juli 1712. ${ }^{865}$ Zur deutschen Gemeinde gehörten 273 Miethaushalte und 118 Eigentümerhaushalte. Zu den Miethaushalten sind noch 36 öffentliche Personen (acht Adlige, achtzehn Bewohner der Ritterakademie, zwei Pfarrer und acht Fremde) hinzuzurechnen, zu den Eigentümern kamen lediglich noch vier Adlige hinzu, sodass sich ein Mieteranteil von $72 \%$ errechnet. Für die französische Gemeinde werden 168 Miethaushalte (Locataires) und 50 Eigentümerhaushalte (Proprietaires) genannt, im Sinne von Eigentümern müssen noch die separat erfassten sieben Mitglieder des conseil de ville gewertet werden. Insgesamt ergibt sich gegenüber der deutschen Gemeinde ein leicht höherer Mieteranteil von $75 \%$. Nur elf Jahre später, 1723, entstand wiederum eine detaillierte Erfassung sämtlicher Haushalte. ${ }^{866}$ Nunmehr lebten nur noch Zweidrittel der Haushalte zur Miete, in diesem Punkt bestand auch keine nennenswerte Differenz zwischen der französischen und der deutschen Gemeinde. Für die Erbhuldigung des Jahres 1735 wurden nochmals sämtliche Einwohner erfasst. Da bei der deutschen Gemeinde nicht zwischen Bürgern mit und ohne Hausbesitz unterschieden wurde, lässt sich der Anteil der eigentumslosen Haushalte nicht mehr feststellen.

\footnotetext{
${ }^{862}$ Es dürfte sich um die Anwesen Gerberei 4, 10/11, 13 und 14 handeln, die wohl 1687 errichtet worden sind. Vgl. hierzu NÜRMBERGER 2015, S. 409-412.

${ }^{863}$ Eine Überprüfung der Zahlenangaben hat zu einer deutlichen Reduzierung der dort angegebenen Einwohnerzahlen geführt. Ursächlich dürften Additionsfehler von 1698 gewesen sein.

${ }^{864}$ SCHANZ 1884, S. 55, Anm. 4: Er bezieht sich dabei auf eine Notiz im Stadtarchiv Erlangen ohne Angabe der Quelle.

${ }^{865}$ StABA, Markgraftum Brandenburg-Bayreuth, Geheimes Archiv Bayreuth Nr. 195.

${ }^{866}$ SCHANZ 1884, S. 65-70, Tabelle 82, Anhang, S. 155-159.
} 
Bei der französischen Gemeinde lag er aber weiterhin bei zwei Drittel der Haushalte (67\%). Die nachfolgenden Überlieferungen zu der Einwohnerschaft der Erlanger Neustadt sind weniger präzise, sodass sich die Mieteranteile nicht exakt ermitteln lassen: Für die Zeit um 1752 wurden 455 Häuser und 5.901 Einwohner gezählt. ${ }^{867}$

\begin{tabular}{|c|c|c|c|c|c|c|}
\hline Jahr & Häuser & \begin{tabular}{|l} 
Eigentü- \\
mer
\end{tabular} & Mieter & $\begin{array}{l}\text { Einwohner } \\
\text { mit Kindern }\end{array}$ & $\begin{array}{c}\text { HH- } \\
\text { Größe } \\
\text { mit Kin- } \\
\text { dern } \\
\end{array}$ & $\begin{array}{l}\text { Mie- } \\
\text { ter/ } \\
\text { Anteil }\end{array}$ \\
\hline $1696^{868}$ & 74 & 56 & 206 & 1.171 & 4,46 & $79 \%$ \\
\hline $1698^{869}$ & 79 & 58 & 257 & 1.314 & 4,17 & $82 \%$ \\
\hline $1700^{870}$ & 105 & \multicolumn{2}{|c|}{395} & 1.508 & 3,82 & \\
\hline $1712^{871}$ & & 179 & 477 & & & $73 \%$ \\
\hline $1712 \mathrm{dt}$. & & 122 & 309 & & & $72 \%$ \\
\hline 1712 frz. & & 57 & 168 & & & $75 \%$ \\
\hline $1723^{872}$ & 254 & 251 & 489 & 3.182 & 4,3 & $66 \%$ \\
\hline $1723 \mathrm{dt}$. & & 174 & 343 & 2154 & & $66 \%$ \\
\hline 1723 frz. & & 77 & 146 & 1028 & & $65 \%$ \\
\hline $1735 \mathrm{dt}^{873}$ & & \multicolumn{2}{|c|}{715} & & & \\
\hline 1735 frz. & & 81 & 162 & & & $67 \%$ \\
\hline $1752^{874}$ & 455 & ca. 455 & 917 & 5.901 & $4,3^{875}$ & $67 \%$ \\
\hline $1787^{876}$ & 500 & & & 5.629 & & \\
\hline \multicolumn{7}{|l|}{ Gesamtstadt } \\
\hline $1818^{877}$ & 830 & ca. 830 & 1.405 & 8.826 & 3,95 & $63 \%$ \\
\hline
\end{tabular}

Tabelle 28. Neustadt Erlangen, Anteil der Miethaushalte 1696-1818 (Thomas Wenderoth 2019)

Setzt man die Zahl der Häuser mit der Anzahl der Eigentümerhaushalte gleich und nimmt eine durchschnittliche Familiengröße von 4,3 Personen an, wie im

${ }^{867}$ Ebd., S. 86.

${ }^{868}$ SCHANZ 1884, Anlage 39, S. 87-95.

${ }^{869}$ Ebd.

${ }^{870}$ SCHANZ 1884, S. 55, Anm. 4: Er bezieht sich dabei auf eine Notiz im Stadtarchiv Erlangen ohne Angabe der Quelle.

871 StABA, Markgraftum Brandenburg-Bayreuth, Geheimes Archiv Bayreuth Nr. 194, ohne Folierung, Erb- und Landeshuldigung, 4. Juli 1712.

${ }^{872}$ SCHANZ 1884, Tabelle 82, Anhang, S. 155-159.

873 Ebd.; Geheimes Archiv Bayreuth Nr. 235, ohne Folierung, Erb- und Landeshuldigung,

25. Oktober 1735.

874 SCHANZ 1884, S. 86.

${ }^{875}$ Bei dieser Zahl handelt es sich um eine Annahme, sie bezieht sich auf den ermittelten Wert des Jahres 1723.

${ }^{876}$ SCHANZ 1884, S. 199f, Statistik von Christian=Erlang vom 22. December 1787, aufgestellt von Bürgermeistern und Rath.

877 Alphabetisches Verzeichnis 1818, S. 23. 
Jahr 1723, errechnet sich wiederum ein Mieteranteil von 67\%. Geht man von einer kleineren Durchschnittsfamilie von nur 4,0 Personen aus, wie im Jahr 1818, ergibt sich ein leicht höherer Mieteranteil von 69\%. Bei aller Vorsicht gegenüber solchen Hochrechnungen kann doch davon ausgegangen werden, dass sich der Mieteranteil gegenüber den ersten Jahrzehnten des 18. Jahrhunderts nicht wesentlich verändert hat.

Für das Jahr 1787 zählte der Rat 5.629 Einwohner und 500 Häuser. ${ }^{878}$ Zugleich wurde die Zahl der Professionisten und Taglöhner mit 646 beziehungsweise 89 erfasst. Zusammen ergibt dies 735 Haushaltsvorstände. Unklar ist, welche Haushaltsvorstände und Bevölkerungsgruppen unberücksichtigt geblieben sind, denn eine durchschnittliche Haushaltsgröße von 7,66 Personen sprengt den üblichen Rahmen, zudem waren im Jahr 1735 bereits 958 Haushalte erfasst worden. Mit Sicherheit fehlen die Frauenhaushalte, evtl. aber auch insgesamt die Gruppe der Nichtbürger (Schutzverwandte).

Am Ende des Untersuchungszeitraumes liegt noch einmal eine statistische Erhebung aus dem Jahr 1818 vor, diese bezieht sich auf die Gesamtstadt, also Neustadt und Altstadt zusammen. ${ }^{879}$ Erfasst wurden 830 Feuerstellen (Häuser), 2.235 Haushalte und 8.826 Einwohner. Rechnerisch ergeben sich eine Familiengröße von 3,91 Personen und eine Behausungsziffer von 10,08 Personen pro Anwesen, denn mit dem Wort „Feuerstelle“ muss in diesem Fall das Anwesen bezeichnet sein: Im Urkataster aus dem Jahr 1822 sind etwa 830 Wohnanwesen erfasst. 880 Die Anzahl der Eigentümerhaushalte ist im Falle von Erlangen nahezu identisch mit der Anzahl der Anwesen, da geteiltes Eigentum oder mehrfacher Hausbesitz kaum verbreitet war. Unter Zugrundelegung dieser Annahme bewegte sich der Mieteranteil im frühen 19. Jahrhundert bei $63 \%$.

Zusammenfassend kann festgestellt werden, dass in der Gründungsphase der Mieteranteil in der Neustadt mit etwa $80 \%$ sehr hoch lag, dieser reduzierte sich in den ersten beiden Jahrzehnten des 18. Jahrhunderts auf einen Zweidrittelanteil. In den nachfolgenden 100 Jahren änderte sich das Verhältnis Eigentümer Mieter nicht mehr wesentlich, auch wenn die Stadt kontinuierlich wuchs.

\footnotetext{
${ }^{878}$ Ebd., S. 199f, Statistik von Christian=Erlang vom 22. December 1787, aufgestellt von Bürgermeistern und Rath.

${ }^{879}$ Alphabetisches Verzeichnis 1818, S. 23.

${ }^{880}$ Erlangen im Jahr 1822, Lithografie von Joseph Päringer; UBER, H60/J 237. Die fortlaufende Nummerierung endet mit Nummer 824 bei der Wöhrmühle, es gibt jedoch gelegentlich Nummern mit a, b, c ... . Universitätsgebäude, wie das Theater etc., sind nicht mitnummeriert.
} 


\section{Vergleich zwischen Exportgewerbe- und Ackerbürgerstädten}

Bis zum Dreißigjährigen Krieg ging die Initiative für Gewerbeansiedlungen primär von Nürnberg und den dortigen Kaufleuten aus. Im Zeitalter des Merkantilismus führte nunmehr eine verstärkte Wirtschaftspolitik der an Nürnberg angrenzenden Territorialherren zu gewerblichen Impulsen und zur Gründung von Manufakturen in den umliegenden Orten. Dabei wurde bewusst die Nähe zum wirtschaftlichen Zentrum Nürnberg gesucht und sich zunutze gemacht. ${ }^{881}$

Die Auswirkungen des Exportgewerbes auf die Eigentumsstruktur der Stadtbevölkerung lässt sich für die Barockzeit anhand der Zahlen in den Huldigungslisten des Fürstentums Ansbach nachvollziehen.

\begin{tabular}{|l|r|r|r|r|}
\hline Orte & $\mathbf{1 7 0 3}$ & 1712 & 1717 & $\mathbf{1 7 2 3}$ \\
\hline Erlangen, bayreuthisch & & $73 \%$ & & \\
\hline Lauf, nürnbergisch & & & $55 \%$ & \\
\hline Langenzenn, ansbachisch & $\mathbf{2 4 \%}$ & & & $31 \%$ \\
\hline Roth, ansbachisch & $19 \%$ & & & $27 \%$ \\
\hline Schwabach, ansbachisch & $41 \%$ & & & $59 \%$ \\
\hline $\mathbf{1 0}$ ansbachische Landstädte & $\mathbf{1 0 \%}$ & & & $\mathbf{1 3 \%}$ \\
\hline
\end{tabular}

Tabelle 29. Anteil der Miethaushalte in den Exportgewerbestädten Mittelfrankens Anfang des 18. Jhs. im Vergleich mit zehn ansbachischen Landstädten (Thomas Wenderoth 2019)

Den drei ansbachischen Städten Langenzenn, Roth und Schwabach wird eine Gruppe aus zehn ansbachischen Ackerbürgerstädten (Crailsheim, Creglingen, Feuchtwangen, Gunzenhausen, Leutershausen, Mainbernheim, Prichsenstadt, Uffenheim, Wassertrüdingen, Windsbach) zum Vergleich gegenübergestellt: 882 Die drei Exportgewerbestädte weisen dabei einen signifikant höheren Anteil von Miethaushalten auf, dies gilt sowohl für das Jahr 1703 als auch für das Jahr 1723. Bei den Exportgewerbestädten bewegt sich der Anteil zwischen 19\% und 59\%, der Durchschnittswert der Ackerbürgerstädte liegt im Jahr 1703 bei 10\% und steigt auf 13\% im Jahr 1723. Ebenfalls sehr hoch liegt der Mieteranteil in Erlangen im Jahr 1712 mit 73\% und in Lauf im Jahr 1717 mit 55\%. Setzt man die Laufer Zahlen allerdings in Bezug zu den zur gleichen Zeit erhobenen Zahlen der drei anderen nürnbergischen Landstädte, fällt der Unterschied deutlich geringer aus, denn diese Vergleichsgruppe weist ebenfalls einen hohen Mieteranteil von 42\% der Haushalte auf. 883

\footnotetext{
${ }^{881}$ REUTER 1961.

${ }^{882}$ StAN, Fm AN, Brdbg. Lit. Nr. 599 und 600-603.

${ }^{883}$ StAN, Rst. N, Bauernverzeichnisse Nr. 7, fol. 318-326, 357'-372' , 402-412, 533' -537. Für Lauf wurden zudem die 84 Mieter der Hammerwerke berücksichtigt. Nicht miterfasst sind die Mitglieder der Universitätsgemeinde in Altdorf. Der insgesamt höhere Wert bei den nürnbergischen Städten geht zu einem gewissen Teil auch auf die dort miterfassten Untermieter zurück.
} 
Für das Ende des Untersuchungszeitraums kann ein Vergleich anhand der Zahlen im Alphabetischen Verzeichnis von 1818 erfolgen:

Den sechs mittelfränkischen Exportgewerbestädten ist der Durchschnittswert von fünfzehn kleinen fränkischen Ackerbürgerstädten (Abenberg, Feuchtwangen, Gunzenhausen, Heideck, Herrieden, Hilpoltstein, Leutershausen, Merkendorf, Neustadt a. d. Aisch, Ornbau, Spalt, Uffenheim, Wassertrüdingen, Windsbach, Wolframs-Eschenbach) gegenübergestellt. Während die Exportgewerbestädte einen Anteil von 50\% bis $83 \%$ an Miethaushalten aufweisen, liegt dieser bei den Ackerbürgerstädten durchschnittlich bei $35 \%$.

Aufgrund der exakten Angaben lässt sich nunmehr auch die Belegungsdichte in den Häusern ermitteln. Die Belegungsziffer mit 3,43 (Haushalte/Haus) und die Behausungsziffer mit 13,34 (Bewohner/Haus) liegt bei den sechs Exportgewerbestädten durchschnittlich betrachtet ebenfalls doppelt so hoch wie bei der Vergleichsgruppe. Als extrem ist die Situation in Fürth anzusprechen.

\begin{tabular}{|l|r|c|c|}
\hline Orte & Miethaushalte & $\begin{array}{c}\text { Belegungsziffer } \\
\text { Haushalte/Haus }\end{array}$ & $\begin{array}{c}\text { Behausungsziffer } \\
\text { Personen/Haus }\end{array}$ \\
\hline Erlangen & $61 \%$ & 2,73 & 10,76 \\
\hline Fürth & $83 \%$ & 6,09 & 23,53 \\
\hline Lauf & $50 \%$ & 1,99 & 7,26 \\
\hline Langenzenn & $50 \%$ & 2,43 & 8,51 \\
\hline Roth & $48 \%$ & 2,55 & 9,88 \\
\hline Schwabach & $70 \%$ & 3,37 & 13,49 \\
\hline \hline 6 Städte & $70 \%$ & 3,43 & 13,34 \\
\hline Ø 15 fränkische \\
Landstädte
\end{tabular}

Tabelle 30. Anteil der Miethaushalte in den Exportgewerbestädten Mittelfrankens 1818 (Alphabetisches Verzeichnis 1818; Thomas Wenderoth 2019)

\section{Exportgewerbestädte in Kursachsen und Westfalen}

Zum überregionalen Vergleich sollen wiederum die Zahlen dienen, die Katrin Keller für die Exportgewerbestädte Kursachsens ermittelt hat. Ausschlaggebend für die von ihr vorgenommene Einordnung in diese Gruppe ist der jeweils hohe Anteil von Handwerkern beziehungsweise Gewerbebeschäftigten. ${ }^{884}$ Eine vergleichbar differenzierte Analyse wirtschaftshistorischer Daten wurde von mir

884 KELLER 1997, S. 88-93. 
nicht vorgenommen. ${ }^{885}$ Auf diese Art und Weise hat Keller 25 Exportgewerbestädte für das Jahr 1699 und 27 Exportgewerbestädte für 1811 identifiziert. ${ }^{886} \mathrm{Da}-$ von finden sich lediglich fünfzehn Städte in beiden Erhebungen genannt. ${ }^{887} \mathrm{Im}$ Jahr 1699 lag die Einwohnerzahl der Exportgewerbestädte zwischen 280 und 4.873. Im Jahr 1811 war die kleinste aufgeführte Stadt Zöbitz mit 930 Einwohnern. Der Mittelwert der Einwohner stieg von 1.517 auf 2.642 an, die Behausungsziffer lag 1811 bei 7,3 und damit über dem Wert der Ackerbürgerstädte in Kursachsen von 6,1. Bei der von Möller angenommenen Haushaltsstärke von 4,3 Personen ${ }^{888}$ teilten sich in den Gewerbestädten 1811 1,7 Haushalte ein Haus, während in den Ackerbürgerstädten lediglich 1,4 Haushalte auf ein Haus kamen. Etwas deutlicher wird der Unterschied, vergleicht man den Anteil der Miethaushalte. Bei den Exportgewerbestädten lag dieser über 40\%, bei den Ackerbürgerstädten bei knapp 30\%. ${ }^{889}$ Diese Zahlen bestätigen damit den Einfluss der Wirtschaftsstruktur auf die Behausungsziffer und den Mieteranteil in den Städten. Bei den von mir identifizierten fränkischen Exportgewerbestädten fällt die Differenz zu den übrigen Landstädten jedoch deutlich größer aus.

Die Allgemeingültigkeit dieser Feststellung soll noch mit Forschungsergebnissen Fred Kaspars für Westfalen untermauert werden. Kaspar zeigt eindrücklich die Bedeutung eines florierenden Exportgewerbes für die Ansässigmachung von Miethaushalten anhand der Bevölkerungsstruktur Warendorfs auf: ${ }^{890} 1589$ existierten dort 158 kleine Mietreihenhäuser (Gademe) und 498 Häuser. Grob vereinfacht kann man davon ausgehen, dass in den Häusern je ein Eigentümerhaushalt und in den Gademen je ein Miethaushalt lebten. Hinzu kam dann noch eine unbekannte Zahl an Untermietern. 70 Jahre später, 1659, war die Zahl der Gademe auf 526 angestiegen, denen nur noch 402 Häuser gegenüberstanden. Die Zahl der Mieter und Mietshäuser hatte sich verdreifacht. Der Mieteranteil lag mindesten bei $58 \%$. Ursächlich war eine wirtschaftliche Blüte des Textilhandwerkes in Warendorf. Fred Kaspar drückt dies so aus: „Es kann kein Zweifel daran bestehen, daß die Zahl der zu einer bestimmten Zeit vorhandenen Gademe deutlich etwas über die wirtschaftlichen Verhältnisse einer Stadt aussagt. Ein überdurchschnitt-

\footnotetext{
${ }^{885}$ Es erscheint jedoch lohnend, die fränkischen Landstädte mit hohen Mieteranteilen im 18. Jh., namentlich Langenzenn, Roth und Wassertrüdigen, noch einmal zu betrachten. Hohe Mieterzahlen sind dann 1818 auch für Gunzenhausen und Neustadt/Aisch belegt.

${ }^{886}$ KELLER 1997, Tabellen 4 und 8.

${ }^{887}$ Es sind dies die die Städte Roßwein, Oederan, Stollberg, Hartha, Buchholz, Frankenberg, Sebnitz, Neustadt, Zschopau, Mittweida, Chemnitz, Schellenberg, Grimma, Werdau und Zöbitz. ${ }^{888}$ KELLER 1997, S. 43f.

${ }^{889}$ Die Werte können wiederum nur vereinfacht und überschlägig ermittelt werden unter der Annahme, dass jedes Haus nur einen Eigentümer hatte und dieser mit im Haus lebte. ${ }^{890}$ Vgl. hierzu und im Folgenden KASPAR 2002, S. $137 f$.
} 
lich hoher Bestand an Gademen innerhalb der Bebauung scheint ein Zeichen dafür zu sein, daß es ein florierendes Gewerbe in dem entsprechenden Ort gibt, welches eine größere Zahl von Arbeitern benötigte; freundlicher ausgedrückt: Die wirtschaftlichen Verhältnisse gaben einer breiten Unterschicht Brot." ${ }^{\text {“91 }}$

\section{Vorstädte}

Vorstädte waren seit jeher Bestandteil städtischer Siedlungen. Bei der Definition der Vorstadt steht mehr die geografische Situation als die Funktion im Vordergrund. Die Funktion ist vielleicht am umfassendsten mit der der Stadterweiterungsfläche zu beschreiben: Die Vorstadt als Reservefläche für all das, was in der Kernstadt keinen Platz findet. Unklar ist, ob diese geografische Funktion ebenfalls in einem Kontext steht zum Anteil der eigentumslosen Bevölkerung. Folgende Fragen scheinen eine separate Untersuchung zu rechtfertigen: Lassen sich allgemeingültige Merkmale in der Bevölkerungs- und Eigentumsstruktur in den vorstädtischen Siedlungsbereichen nachweisen? Oder ist die vorstädtische Bevölkerung jeweils nur als individuelles Pendant zur Bevölkerung innerhalb der Mauern einer spezifischen Stadt zu verstehen? Letztendlich führen diese Fragen zu einer Kernfrage: Unterscheidet sich die Bevölkerungsstruktur in der Vorstadt von der Situation in der Kernstadt?

Vorstädte, die bereits im Verlauf des Mittelalters in die Stadtbefestigungen eingeschlossen wurden, sollen hier nicht thematisiert werden. Dies trifft zum Beispiel für Nürnberg und Kitzingen zu. In beiden Städten wurde der Mauerring vergrößert und ehemalige Vorstädte miteinbezogen. Auch in der Neuzeit wurden dort die äußeren Viertel noch als Vorstadt bezeichnet, obwohl sie längst Teil der befestigten Stadt waren. Im Folgenden soll es um Vorstädte und Vororte gehen, die außerhalb der Befestigung lagen und deren Entwicklung sich nur im Hinblick auf die Kernstadt erklärt. Dabei gilt es zu klären, ob sich anhand der Quellen eine spezifische Eigentümerstruktur nachweisen lässt, die von der Kernstadt abweicht.

891 KASPAR 2002, S. 145. 


\section{Nürnberger Vororte im Bann der Großstadt}

Eigentumslose Haushalte fanden sich seit dem Mittelalter nicht nur in der Stadt selbst, sondern auch unmittelbar vor den Stadtmauern. Ein früher Hinweis datiert auf 1432: Damals verkauften Heintz und Anna Plankensteiner ihre Ziegelhütte mitsamt den zugehörigen Zinshäusern vor dem Frauentor an die Reichsstadt Nürnberg. ${ }^{892}$ Exakte Zahlen zu den vorstädtischen Miethaushalten sind in den Nürnberger Bauernverzeichnissen des 16. Jahrhunderts und dem Türkensteuerregister der Reichsstadt von 1716/17 enthalten. ${ }^{893} \mathrm{Im}$ Folgenden sind die Einwohnerzahlen von fünf Siedlungen aufgelistet, die unmittelbar südlich der Stadtmauer liegen. Auf die rechtliche Stellung dieser einzelnen Siedlungen soll hier nicht näher eingegangen werden. Sie lagen allesamt im reichsstädtischen Territorium und waren auf die Stadt bezogen. In den Quellen wird, juristisch betrachtet, zumeist neben Wöhrd nur Gostenhof als Vorstadt angeführt, wobei Wöhrd als ummauerter Marktfleck eine deutlich größere Eigenständigkeit aufwies als die angeführten fünf Siedlungen.

\begin{tabular}{|l|c|c|r|c|c|c|c|c|r|}
\hline & \multicolumn{6}{|c|}{ Bauernverzeichnis 2, 1538 } & \multicolumn{2}{|c|}{ Bauernverzeichnis 6, 1548 } & \multicolumn{2}{c|}{ Türkensteuer 1716/17 } \\
\hline Vororte & $\begin{array}{c}\text { Eigen } \\
\text { tümer }\end{array}$ & Mieter & $\begin{array}{c}\text { Mieter } \\
\text { haushalte }\end{array}$ & $\begin{array}{c}\text { Eigen } \\
\text { tümer }\end{array}$ & Mieter & $\begin{array}{c}\text { Mieter } \\
\text { haushalte }\end{array}$ & $\begin{array}{c}\text { Eigen } \\
\text { tümer }\end{array}$ & Mieter & $\begin{array}{c}\text { Mieter } \\
\text { haushalte }\end{array}$ \\
\hline Glockenhof & 0 & 16 & $100 \%$ & 1 & 12 & $92 \%$ & 1 & 12 & $92 \%$ \\
\hline Gostenhof & 16 & 16 & $50 \%$ & 22 & 20 & $48 \%$ & 20 & 61 & $75 \%$ \\
\hline Steinbühl & 19 & 63 & $77 \%$ & 12 & 82 & $87 \%$ & 20 & 92 & $82 \%$ \\
\hline Tafelhof & 8 & 58 & $88 \%$ & 1 & 72 & $99 \%$ & 20 & 74 & $79 \%$ \\
\hline Untergalgenhof & 6 & 86 & $93 \%$ & 4 & 82 & $95 \%$ & 20 & 54 & $73 \%$ \\
\hline Gesamtzahlen & 49 & 239 & $83 \%$ & 40 & 268 & $87 \%$ & 81 & 293 & $78 \%$ \\
\hline
\end{tabular}

Tabelle 31. Mieterzahlen in den Nürnberger Vororten 1538, 1548 und 1716/17

(WENDEROTH 2019a, Tabelle 1, S. 42)

Die Werte für die einzelnen Orte schwanken zwischen $48 \%$ und 99\%. Durchschnittlich betrachtet wohnten dort weit über Zweidrittel der Haushalte zur Miete. Interessant erscheint, dass die Werte im 16. Jahrhundert über denen des frühen 18. Jahrhunderts liegen. Es handelt sich beim Mietwohnen in Vorstädten also nicht um ein junges Phänomen.

Zum direkten Vergleich mit der Kernstadt bieten sich nur die Feuerschaulisten von 1561 an, die etwas später datieren als die Bauernverzeichnisse. Anhand der Feuerschaulisten konnte belegt werden, dass unter Hinzurechnung der Untermieter mindestens etwa $70 \%$ Haushalte über kein Wohneigentum verfügten. In den Nürnberger Vorstädten ist der Anteil der eigentumslosen Haushalte demgegenüber noch einmal erhöht, in den untersuchten fünf Siedlungen lag er 1548 bei 87\%. Ursächlich hierfür dürfte vermutlich das weitgehende Fehlen einer über Hausbesitz verfügenden Mittelschicht sein.

${ }^{892}$ StAN, Rst. N, Waldamt Sebaldi I Nr. 822, Urkunde vom 2. April 1432.

${ }^{893}$ Vgl. zu den Mietzahlen im Umfeld der Reichsstadt auch WENDEROTH 2019a, S. 48. 


\section{Unbefestigte Vorstädte auf dem Land}

Nicht nur die Großstadt kennt frühe vorstädtische Siedlungen, vielfach finden sich diese seit dem Mittelalter auch in den Landstädten. Der Mieteranteil an der dortigen Bevölkerung ist bisher nicht untersucht worden. In den weiter oben angeführten Huldigungslisten des Fürstentums Ansbach finden sich die Vorstädte zumindest teilweise separat erfasst (vgl. Tab. Anhang I.1.-I.8.). Dies trifft zu für Crailsheim, Leutershausen, Mainbernheim, Merkendorf, Roth, Uffenheim, Wassertrüdingen und Windsbach. Erfasst sind dort unter der Rubrik Vorstadt lediglich Eigentümer und in keinem Fall Mieter. Bei der Erfassung der Mieter wurde wohl nicht zwischen dem Wohnort in der Kernstadt oder Vorstadt unterschieden. Von daher verwundert es auch nicht, wenn sich in den genannten Orten die Trennung in Kernstadt und Vorstadt nicht durchgängig in den Huldigungslisten niederschlägt. Eine Kontinuität ist hier nur für Crailsheim ab dem Jahr 1668 feststellbar. Für die anderen sieben Städte fehlen Angaben zu Vorstädten gänzlich.

In den von mir darüber hinaus untersuchten Quellen findet sich lediglich im Türkensteuerregister 1716/17 für die nürnbergische Stadt Lauf an der Pegnitz eine entsprechend aussagefähige Differenzierung in Kernstadt und Vorstädte (vgl. Tab. 21). ${ }^{894}$ Dabei überflügeln die drei aufgeführten Vorstädte die Kernstadt in Bezug auf den Anteil der Miethaushalte: In der Kernstadt lag dieser bei 42\%, in den Vorstädten vor dem Oberen Tor bei $48 \%$, vor dem Unteren Tor bei $55 \%$ und vor dem Wassertor bei $44 \%$. Zu den Miethaushalten hinzugezählt wurden hier wie in den übrigen Fällen auch - die Personen, die als Schutzverwandte bezeichnet sind. Auffällig ist dabei, dass sich Schutzverwandte nur in zwei Fällen für die Kernstadt, aber in neun Fällen in der Vorstadt vor dem Unteren Tor und in fünf Fällen in der Vorstadt vor dem Oberen Tor nachweisen lassen. Nicht aufgeführt ist in der Tabelle die vorstädtische Gewerbesiedlung entlang der Pegnitz, da diese hinsichtlich ihrer Wirtschafts- und Sozialstruktur mit den hier zu thematisierenden Vorstädten im ländlichen Kontext nicht vergleichbar ist. Die extrem hohen Mieterzahlen in diesem Siedlungsbereich erklären sich nicht durch die räumliche Anbindung an die Stadt Lauf, sondern allein aus dem Vorhandensein der Hammerwerke und sonstiger Produktionsstätten am Fluss. Dementsprechend wurde diese Gewerbesiedlung von mir bereits im Kontext der ländlichen Gewerbesiedlungen und deren Mietsituation im Rahmen meiner Dissertation besprochen. ${ }^{895}$ Nicht auszuschließen ist aber, dass die relativ hohen Mieterzahlen in der Laufer Kernstadt und den drei genannten Vorstädten ursächlich auf die Gewerbebetriebe am Fluss zurückgehen. Die Laufer Zahlen können daher nicht ohne Weiteres auf andere Kleinstädte übertragen werden. Dies gilt sowohl für die absoluten Zahlen

\footnotetext{
${ }^{894}$ StAN, Rst. N, Bauernverzeichnisse Nr. 7, fol. 402-412. Die ebenfalls aufgeführte Vorstadt in Altdorf besteht nur aus wenigen Häusern und ist daher nicht repräsentativ; ebd., fol. 326. ${ }^{895}$ WENDEROTH 2019a, S. 191-196.
} 
als auch für die Miettopografie mit einer erhöhten Mieterkonzentration in den Vorstädten.

Um die eingangs gestellte Frage nach abweichenden Eigentumsstrukturen zwischen Kernstadt und Vorstadt zu beantworten, sind die bisherigen Angaben nicht ausreichend und zu indifferent. Die wenigen ermittelbaren Zahlen deuten eher darauf hin, dass die Eigentumsstruktur der Vorstädte der Situation in den Kernstädten folgt. Dies bedeutet aber auch, dass für die Identifizierung der eigentumslosen Haushalte in den Vorstädten die Situation der Kernstadt mit in den Blick genommen werden muss und umgekehrt. 


\section{Resümee}

\section{Quellenkritik}

Grundsätzlich ist festzustellen, dass die eigentumslosen Haushalte in den prosopographischen Quellen unterschiedlich erfasst worden sind. Die Angaben bedürfen daher immer einer intensiven kritischen Würdigung, um den Anteil der eigentumslosen Bevölkerung richtig interpretieren zu können. Fehlen die Mieter ganz oder teilweise in den Quellen, so darf daraus keinesfalls vorschnell geschlossen werden, dass diese nicht existierten. Wichtig ist zudem die Klärung der verwendeten Bezeichnungen, da diese zum einen von Ort zu Ort abweichen und zum anderen auch unterschiedliche Sachverhalte wiedergeben können. Weiterhin kann auch die Bedeutung der Bezeichnung einer zeitlichen Wandlung unterliegen.

Mit der flächenhaften Untersuchung zu den fränkischen Städten ist eindeutig belegt, dass jede Stadt seit dem Mittelalter unabhängig von ihrer Größe eigentumslose Haushalte in ihren Mauern hatte. Für eine exakte Klärung reicht es dabei nicht aus, einzelne Verzeichnisse heranzuziehen. Anhand von Erlangen und Wunsiedel konnte ich zeigen, dass ein großer Anteil der eigentumslosen Haushalte erst unter Berücksichtigung weiterer, häufig singulär erhaltener, Quellen nachweisbar ist.

Da der Anteil der Miethaushalte sowohl regional als auch im Hinblick auf die Zeitebene stark schwankt, konnte im Rahmen dieser Arbeit kein allgemeingültiger Wert zum Anteil der Mietbevölkerung ermittelt werden.

\section{Bevölkerungsgeschichtliche Ergebnisse}

\section{Familien/Haushaltsgrößen}

Anhand der grundlegenden Bevölkerungsstrukturen, wie dem prozentualen Anteil von Eigentümer- und Mieterhaushalten, der Behausungsziffer und den Haushaltsgrößen, konnten allgemeine Aussagen zum jeweiligen Anteil der eigentumslosen Bevölkerung getroffen werden. Umgekehrt scheint der Blick auf die eigentumslosen Haushalte auch die Diskussion in der Bevölkerungsgeschichte befruchten zu können: Auffällig ist die große Spannbreite der bisher in der Literatur ermittelten Haushaltsgrößen, diese schwankt in den seriösen Arbeiten zwischen drei und fünf Personen. Eine Fragestellung, die zukünftig noch stärker bedacht und überprüft werden sollte, ist dabei der Begriff des „Haushaltes“. Auf die Familie angewandt, lassen sich in den im Rahmen dieser Arbeit untersuchten Quellen selten mehr als vier Personen nachweisen. Wenn sich dennoch aus manchen 
Quellentexten Haushaltsstärken von durchschnittlich fünf und mehr Personen ergeben, so wäre hier zu prüfen, ob in diesen „Haushalten“ nicht Untermieter eingeschlossen sind, die in anderen Quellen als eigene „Haushalte“ gezählt werden und auch wirtschaftlich unabhängig vom Vermieter waren. Ich habe für das Zusammenleben mit der erweiterten Familie und Untermietern weiter oben den Begriff der Herdgemeinschaft eingeführt. Diese zum Teil personenstarken Herdgemeinschaften haben nichts gemein mit dem Begriff des „Haushaltes“ oder des „Ganzen Hauses“, da sie nicht Teil einer gemeinsamen Ökonomie waren, sondern eher unter den heutigen Begriff einer Zweck-WG mit getrennter Kassenführung fallen. Je nach Zweck der historischen Zählung können die Untermieter entsprechend als eigener Haushalt erfasst oder auch in der Herdgemeinschaft des Hauptmieters/Eigentümers inkludiert sein, ohne dass dies angegeben worden wäre. Allein die Vernachlässigung dieser Fragestellung kann bei der Ermittlung der durchschnittlichen Haushaltsgröße eine Verschiebung von 0,5 bis 1 Person bedeuten. Eine sorgfältige Analyse der Quellen, insbesondere im Hinblick auf Frauen- und Einpersonenhaushalte sowie die Identifizierung der Untermieter scheint vonnöten, um hier zukünftig eine bessere Vergleichbarkeit der Angaben zu gewährleisten. Es bleibt abzuwarten, ob sich die Spannbreite der zu ermittelnden durchschnittlichen Haushaltsgrößen nicht aufgrund einer solchen Differenzierung verringern würde.

Ein weiteres wichtiges Ergebnis ist die in vielen Fällen nachgewiesene deutlich geringere Haushaltsgröße der eigentumslosen Haushalte gegenüber den Eigentümerhaushalten. Häufig werden Bevölkerungszahlen mithilfe der Anzahl der Haushalte rekonstruiert und dabei eine Durchschnittsgröße für die Haushalte angenommen. Im Hinblick auf die deutlichen Unterschiede zwischen Eigentümerund Nichteigentümer-Haushalten erscheint es daher problematisch mit einem allgemeinen Durchschnittswert zu operieren. Zuverlässigere Ergebnisse sind dagegen zu erzielen, wenn zum einen zwischen Herdgemeinschaften und Haushalten und zum anderen zwischen Eigentümer- und Nichteigentümer-Haushalten unterschieden wird. 


\section{Differenzierung der Städte}

\section{Anteil der eigentumslosen Haushalte}

In den vorausgehenden Kapiteln habe ich aufzeigen können, dass eigentumslose Haushalte Teil jeder städtischen Siedlungs- und Sozialstruktur waren. Dabei lag der Anteil der Miethaushalte an der Bevölkerung unterschiedlich hoch. Ich habe bewusst darauf verzichtet, die bereits genannten Zahlen in einer Tabelle zusammenzuführen, da die Werte auf unterschiedliche Art und Weise ermittelt worden sind, teilweise Untermieter beinhalten, teilweise wohl aber auch nennenswerte Lücken aufweisen und aus diesem Grund nicht direkt miteinander vergleichbar sind.

Auch wenn in jeder Stadt eigentumslose Haushalte vorhanden waren, so lassen sich doch Merkmale festhalten, die einen Einfluss auf den prozentualen Anteil der eigentumslosen Haushalte hatten. Im Ergebnis sind dies drei Merkmale: Größe, Funktion und Zeithorizont.

\section{Stadtgröße}

Die Größe einer Stadt ist sicher nicht das alleinige Kriterium für einen hohen Anteil eigentumsloser Haushalte. Gleichzeitig ist festzustellen, dass die Stadtgröße in engem Zusammenhang mit ihrer politischen und wirtschaftlichen Funktion steht und entsprechend auch mit dem Mieteranteil. Eine Großstadt wäre nicht zu einer Großstadt angewachsen, wenn dort nur für den lokalen Markt Handel getrieben und produziert worden wäre. Dies gilt in abgeschwächter Form auch für die Mittelstädte.

Für die Großstädte lassen sich historisch Mieteranteile von 30\% bis $80 \%$ feststellen. In dieser Spannweite bewegten sich wohl auch die Mittelstädte und dennoch scheint der durchschnittliche Mietanteil entsprechend der geringeren Stadtgröße etwas niedriger gelegen, jedoch selten die 30\%-Marke unterschritten zu haben. Andererseits lassen sich für die Kleinstädte vereinzelt extrem hohe Anteile an Mietbevölkerung feststellen, wenn die Stadt Sonderfunktionen übernommen hat, auf die ich weiter unten noch eingehen werde. Bei einer stark agrarisch geprägten Wirtschaftsstruktur konnte der Mieteranteil auch bei nur gut 10\% der Haushalte gelegen haben. 


\section{Stadtfunktion}

Neben der Größe einer Stadt als Einflussfaktor konnte nachgewiesen werden, dass Sonderfunktionen einen noch deutlich größeren Einfluss auf den Anteil der eigentumslosen Bevölkerung in einer Stadt hatten.

Als erstes wäre die Residenz- und damit häufig verbunden auch die Hauptstadtfunktion zu nennen. Insbesondere im Falle von großen Residenzstädten entwickelte sich eine charakteristische Bevölkerungsstruktur, die im hohen Maß aus Miethaushalten bestand. Diese Tradition wurde dann von den zentralen Verwaltungsorten fortgeführt, unabhängig davon, ob deren ehemalige Residenzfunktion, wie in Ansbach, erloschen war. Wichtig erscheint auch die Feststellung, dass es sich bei solchen Residenz- und Verwaltungsorten nicht per se um beschauliche Beamtenstädte mit einer behäbigen und wohlsituierten Bevölkerung gehandelt hat, auch wenn es insbesondere in diesen Orten eine gutsituierte Mieterschaft gegeben hat. Die hohe Behausungsziffer in Ansbach 1818 mit durchschnittlich (!) 25 Personen in jedem Anwesen lässt vielmehr auf eine überwiegend prekäre Wohnsituation schließen. ${ }^{896}$ Zur gleichen Zeit lag diese in Dresden mit 20,6 Personen ebenfalls sehr hoch.

Auch wenn die kleineren Residenzorte in den entsprechend kleineren Territorien keine so extremen Werte aufweisen, konnte für diese ebenfalls ein erheblicher Anteil an eigentumslosen Haushalten belegt werden, die direkt im Zusammenhang mit der Residenzfunktion stehen. Durch den direkten Vergleich der kleinen fränkischen Residenzorte mit der Gruppe der kleinen Landstädte ohne Residenzfunktion ist zudem nachgewiesen, dass der Anteil der eigentumslosen Haushalte in den Residenzen deutlich höher lag und damit spätestens ab 1700 als ein wichtiges Charakteristikum des Typus Residenzstadt gelten muss.

Bei der Funktion als Universitätsstadt scheint die Situation umgekehrt: Je kleiner die Stadt war, umso deutlicher veränderte die Anwesenheit einer Universitätsgemeinde die dortige Einwohnerstruktur. Da die akademischen und studentischen Haushalte weitestgehend über kein Wohneigentum verfügten, stieg der Anteil der eigentumslosen Haushalte gerade in Kleinstädten sprunghaft an. In großen und mittelgroßen Städten fallen die Auswirkungen aufgrund eines von vornherein höheren Anteils eigentumsloser Haushalte deutlich geringer aus. Gleiches gilt auch im Falle, dass die Stadt weitere Sonderfunktionen hatte. Hier wäre die Stationierung von Militär oder ein starkes Exportgewerbe zu nennen.

Insbesondere der Einfluss eines starken Exportgewerbes auf die Höhe der eigentumslosen Bevölkerung konnte anhand der mittelfränkischen Städte nachgewie-

${ }^{896}$ Der erhaltene Baubestand in Ansbach wirkt keinesfalls großstädtisch, sondern ist überwiegend zwei- und dreigeschossig. 
sen werden. Die fränkischen Ergebnisse korrespondieren dabei mit den Forschungsergebnissen zu westfälischen und kursächsischen Städten, auch wenn der Anteil der eigentumslosen Haushalte in kursächsischen Exportgewerbestädten deutlich niedriger lag. Nicht geklärt werden konnte in diesem Zusammenhang, warum die Differenz so groß ausfällt. Eine umfassendere Forschung aus wirtschaftshistorischer Perspektive zur Auswirkung der Wirtschaftsstruktur auf die Eigentumsstruktur erscheint lohnend.

Als Ergebnis kann festgehalten werden, dass Städte die eine oder mehrere dieser Sonderfunktionen übernommen hatten, einen überdurchschnittlich hohen Anteil eigentumsloser Haushalte aufwiesen.

Insbesondere im weiten Feld der Kleinstädte war die spezifische Größe einer Stadt irrelevant für den Mieteranteil, hier hing alles an der jeweiligen Wirtschaftsstruktur, wohingegen Mittel- und Großstädte bereits aufgrund ihrer Größe einen entsprechend großen „Binnenmarkt“ für Waren und Dienstleitungen boten und damit ein Auskommen für eigentumslose Bevölkerungsschichten.

\section{Zeitlicher Horizont}

Neben der Größe und Prägung der jeweiligen Stadt stellt auch die Zeitebene einen wichtigen Parameter für den Anteil an Miethaushalten dar.

Problematisch bleibt, dass für viele Städte keine fortlaufenden Daten vorhanden sind, die miteinander vergleichbar wären. Daher kommt den Huldigungslisten eine besondere Bedeutung zu. Für die Zeit vor dem Dreißigjährigen Krieg gilt diese Problematik in noch weit größerem Maß. Zumindest thesenhaft zeichnet sich ab, dass vor dem Krieg der Anteil der Miethaushalte deutlich höher lag als in den nachfolgenden 50 Jahren. Vielerorts scheint der Bevölkerungsverlust, der sich in drastisch gesunkenen Mieterzahlen niederschlägt, erst gegen Ende des 17. Jahrhunderts oder gar im 18. Jahrhundert kompensiert worden zu sein.

Für das späte Mittelalter liegen primär Zahlen zu großen Städten vor, hier zeichnet sich teilweise ein erstaunlich hoher Mieteranteil ab. Interessanterweise scheint das Bevölkerungswachstum des 16. Jahrhunderts nicht unbedingt zu einer Zunahme des Mieteranteils geführt zu haben, wie dies für das 18. und 19. Jahrhundert zu beobachten ist. Zumindest deuten die Kölner Zahlen einen qualitativen Rückgang der Mietbevölkerung an.

In einer vertiefenden Untersuchung wäre zu klären, ob eine prosperierende Wirtschaft, die ja eine Grundvoraussetzung für das materielle Überleben der Mieterschicht war, jenseits des quantitativen Anwachsens der Miethaushalte zwingend auch zu einem qualitativen Wachstum an Miethaushalten, sprich einem höheren Mieteranteil, geführt hat. Für das 16. Jahrhundert gibt es zumindest Anzeichen dafür, dass eine florierende Ökonomie zu einem Bevölkerungswachstum führt und gleichzeitig der Anteil der Hausbesitzer wächst. Für die Mietsituation in den Kleinstädten lässt sich trotz der Angaben im Reichssteuerregister keine abschlie- 
ßende Aussage zum Umfang des Hausbesitzes im 15. und 16. Jahrhundert treffen, da sich die dortigen Hinweise zu Miethaushalten vor allem auf Untermietverhältnisse beziehen. Festzustellen bleibt, dass Untermietverhältnisse eine lange Tradition haben und eine weitverbreitete Wohnform über den gesamten Untersuchungszeitraum und darüber hinaus darstellen.

Es zeichnet sich nicht nur ein absoluter, sondern auch ein prozentualer Rückgang der eigentumslosen Bevölkerung als Auswirkung von Katastrophen und den damit verbundenen generellen Bevölkerungsverlusten ab: Der Anteil der Mietbevölkerung geht überproportional stark zurück.

Mit dem Anwachsen der Gesamtbevölkerung vom späten 17. bis zum 19. Jahrhundert ist dann umgekehrt ein überproportionaler Anstieg der Mietbevölkerung festzustellen. Eindeutig nachgewiesen werden konnte eine generelle Zunahme der eigentumslosen Haushalte im 18. Jahrhundert, welches sich im 19. Jahrhundert fortsetzte. Von dieser Entwicklung betroffen scheinen sämtliche Städte gewesen zu sein. Zumindest konnte keine Stagnation oder gar gegenläufige Bewegung für diesen Zeitraum nachgewiesen werden. Insbesondere das Bevölkerungswachstum des späten 18. Jahrhunderts führt zu einer exponentiellen Steigerung der eigentumslosen Bevölkerung mit dramatischen sozialen Folgen. In dem von mir hier behandelten Forschungsrahmen zeichnet sich diese Entwicklung in extrem hohen Behausungsziffern zu Beginn des 19. Jahrhunderts ab. Wenn in Ansbach 1818 durchschnittlich 25 Menschen und in Fürth über 23 Menschen in einem Haus lebten, muss drangvolle Enge geherrscht haben, denn in die Statistik sind auch kleine eingeschossige Häuser mit eingeflossen!

\section{Sozialgeschichtliche Ergebnisse}

\section{Einwohnerschaft}

Die städtische Gesellschaft setzte sich rechtlich und politisch aus verschiedenen Gruppen zusammen, von denen die Bürger nur eine Gruppe repräsentieren. Neben Einwohnern mit eingeschränkten Rechten, gab es auch in früher Zeit bereits Einwohner ohne jegliche Rechte, die sich illegal in den Städten aufhielten. Die Zahlen, insbesondere des 18. Jahrhunderts, zeigen eindrücklich, dass die Begriffe der „bürgerlichen Stadt“ und auch des „Bürgerhauses“ problematisch sind. Historisch betrachtet verfügten bei weiten nicht alle Einwohner über das Bürgerrecht und waren mitnichten Bürger. Das gleiche gilt für die Häuser, die Charakteristik vieler dieser Gebäude, die einer Mietnutzung dienten, wird nur unzutreffend mit dem Begriff „Bürgerhaus“ wiedergegeben. 


\section{Untermieter}

Anhand der Zahlen aus dem 15. bis 18. Jahrhundert wird deutlich, dass das Phänomen der Untermiete eine lange Tradition aufweist, ja vielleicht sogar die erste Entwicklungsstufe des Mietwohnens darstellt. Die zukünftige Diskussion zu Untermietern, Kostgängern und Schlafburschen sollte sich also keineswegs auf die Zeit der industriellen Revolution im 19. Jahrhundert beschränken, denn diese ist nicht ursächlich für die Entstehung dieser Wohnform. Die oben ausgeführten Angaben widerlegen damit auch Helmut Bräuers These, dass das Wohnen zur Untermiete sich erst im 18. Jahrhundert als neue Kategorie am untersten Ende in der Hierarchie der Hausgenossen herausgebildet habe. ${ }^{897}$

$\mathrm{Zu}$ großen Teilen handelte es sich bei den Untermietern um die Ärmsten der Armen, was den hohen Frauenanteil erklärt. Daneben existierte jedoch auch eine Gruppe von Untermietern, die durchaus finanziell in der Lage gewesen wären, eine eigenständige Wohneinheit zu mieten. Hierzu sei auf meine Dissertation verwiesen. ${ }^{898}$ Ergänzend möchte ich an dieser Stelle noch auf die Lebenssituation der Studenten hinweisen, die in vielen Fällen auch unter dem Begriff der Untermiete $\mathrm{zu}$ fassen ist.

\section{Frauen}

Auch wenn die eigentumslosen Haushalte mit weiblichem Haushaltsvorstand in vielen historischen Verzeichnissen fehlen, konnte anhand zweier Verzeichnisse festgestellt werden, dass diese insbesondere unter den eigentumslosen Haushalten überproportional stark vertreten waren. Zumeist handelte es sich um Witwen, in einigen Fällen wohl aber auch um ledige und alleinstehende Frauen. Während unter den Eigentümerhaushalten der Anteil mit weiblichem Haushaltsvorstand etwa $5 \%$ bis $10 \%$ betrug, lag dieser bei den eigentumslosen städtischen Haushalten in der Regel über 15\% und nicht selten darüber bei bis zu 30\%. Überproportional stark vertreten waren alleinstehende Frauen zudem in der Gruppe der Untermieter, wobei die Gruppe wohl von vornherein zutreffender in der weiblichen Form als Untermieterinnen bezeichnet werden kann. Für 1497 konnte ihr Anteil mit $67 \%$ ermittelt werden.

Die Situation der Frauenhaushalte wurde hier, im Rahmen meiner Fragestellung, nur gestreift. Es erscheint aber lohnend, die verwendeten Quellen noch einmal gezielt im Hinblick auf geschlechtsspezifische Merkmale zu analysieren.

\section{Fiktion vom "Ganzen Haus"}

Dem Begriff des „Ganzen Hauses“ liegt die Annahme zugrunde, dass bis weit ins 18. Jahrhundert hinein nicht die Kleinfamilie, sondern eine Hausgemeinschaft

\footnotetext{
897 BRÄUER 2003, S. 91.

${ }^{898}$ WENDEROTH 2019a, S. $82 f$.
} 
unter Führung des zumeist männlichen Hauseigentümers die kleinste und auch bestimmende gesellschaftliche Gruppe bildete. Erstmals wird der Begriff in dem Buch „Naturgeschichte des deutschen Volkes“ aus dem Jahr 1855 gebraucht. ${ }^{899}$ Wilhelm Heinrich Riehl bringt diese Gedankengänge mit der genannten Schrift in die Volkskunde und die sozialhistorische Forschung ein. ${ }^{900}$ Otto Brunner prägt dann den Begriff im 20. Jahrhundert erneut: Das „Ganze Haus“ fasst er als Lebensform auf, die seiner Auffassung nach ursächlich für die Unterschiede zwischen der frühneuzeitlichen und neuzeitlichen Ökonomie ist. ${ }^{901}$

Die in der vorliegenden Arbeit ausgewerteten Quellen zur Bevölkerungsgeschichte zeigen dagegen ein anderes Bild: Es existierten zwar Herdgemeinschaften, bei denen sich mehrere Haushalte den Wohnraum und hier vor allem die Herdstelle teilten, also zusammen in einem Haus oder einer "Wohnung“ lebten, die kleinste und maßgebliche Einheit bildete jedoch der Haushalt, bestehend aus den Familienangehörigen und dem Gesinde. Zu bedenken ist weiterhin, dass die allermeisten Haushalte seit dem ausgehenden Mittelalter lediglich aus einer Kleinfamilie bestanden, die maximal zwei Generationen umfasste. Das gilt insbesondere für Miethaushalte, aber auch für Eigentümerhaushalte. Größere Haushalte mit drei Generationen, verheirateten Geschwisterpaaren und Bediensteten, auf die sich der Begriff des „Ganzen Hauses“ bezieht, spielten prozentual betrachtet nur eine untergeordnete Rolle. Dies gilt nicht nur für den städtischen Bereich, sondern in Franken auch für den bäuerlichen Kontext. ${ }^{902}$

Da zumindest in den größeren Städten häufig sämtliche Bewohner einer Herdgemeinschaft Mieter waren, standen diese auch nicht zwangsläufig unter der Führung des Hauseigentümers, wenn dieser an einem anderen Ort wohnte.

Die Kleinfamilie und eine auf deren Bedürfnisse zugeschnittene (Klein-)Wohnung setzen sich als Lebensform und Lebensort in Franken bereits seit dem ausgehenden Mittelalter durch. Sie sind eben nicht erst eine Entwicklung des 19. Jahrhunderts, wie Riehl und Brunner vermutet haben. Dennoch entspricht die Kleinfamilie und ihre Wohnsituation sicher nicht dem heutigen Verständnis von Kleinfamilie. Zum einen lässt sich für den Untersuchungszeitraum ein nennenswerter Anteil von Untermietern feststellen, zum anderen sind Knechte, Mägde, Lehrjungen und ledige Handwerksgesellen selbstverständlicher Teil der Haushalte, auch wenn diese sich absolut betrachtet nur in geringer Personenzahl und in verhältnismäßig wenigen eigentumslosen Haushalten nachweisen lassen.

\footnotetext{
${ }^{899}$ RIEHL 1854, S. 147-162.

900 Ebd. S. 164.

${ }^{901}$ Vgl. BRUNNER 1966.

902 WENDEROTH 2019a.
} 


\section{Ausblick}

Die genaue Analyse der fränkischen Quellen hat gezeigt, wie wichtig es für die prosopographische Forschung ist, zwischen Herdgemeinschaften, Haushalten und Familien zu unterscheiden, um eine Vergleichbarkeit der Ergebnisse über einen langen Zeitraum und Stadtgrenzen hinweg zu ermöglichen.

Gleiches gilt für die Unterscheidung zwischen Miethaushalten in eigenen Wohnungen und Untermietern. Zum einen verändern sich im Lauf der Zeit die Bedeutung der verwendeten Begrifflichkeiten, zum andern muss in jedem Fall geklärt werden, welche der beiden Mietergruppen in der Quelle angesprochen wird, beziehungsweise ob beide Mietergruppen in den Verzeichnissen aufgeführt sind.

Für die Vergleichbarkeit der Angaben und Diskussion der Situation eigentumsloser Haushalte haben sich die seriellen Quellen, wie sie sich in Franken zumindest ein Stück weit erhalten haben, als äußerst wertvoll erwiesen. Da die eigentumslose Bevölkerung in den Archivalien häufig nur zwischen den Zeilen zu identifizieren ist, und auch dort nicht immer vollständig, wird ein Vergleich anhand unterschiedlicher Archivaliengruppen in vielen Fällen zu einem befriedigenden Ergebnis führen.

Die flächenhafte Überlieferung in Franken aus vorstatistischer Zeit hat hier außergewöhnliche Möglichkeiten eröffnet. Es bleibt zu hoffen, dass weitere regionale Untersuchungen folgen. Insbesondere die bisher kaum beachtete Quellengattung der Huldigungslisten scheint auch außerhalb Frankens noch weitreichende prosopographische und sozialhistorische Forschungsmöglichkeiten $\mathrm{zu}$ bieten. 


\section{Anhang}

\section{Unveröffentlichte Quellen}

\section{Nürnberg, Bibliothek Geschichte für alle e.V.:}

FEJA 1988 - FEJA, CHRISTIAN: Mietverhältnisse im vorindustriellen Nürnberg. Eine Fallstudie für das 16. Jahrhundert, Zulassungsarbeit, Erlangen 1988

\section{Staatsarchiv Bamberg}

StABA, Geheimes Hausarchiv Plassenburg, Akten und Bände 5977/1 (Verzeichnis des Gemeinen Pfennings, 1495)

StABA, Hochstift Bamberg, Hofkammer, Akten und Bände 1581

(Protokoll über die Zählung der Haushalte, Kinder, Dienstboten und Herberger in sämtlichen Gebirgsämtern, 1676/77)

StABA, Markgraftum Brandenburg-Kulmbach-Bayreuth, Hofkammer 1772 (Der Neubau der Stadt Erlangen, 1690)

StABA, Markgraftum Brandenburg-Kulmbach-Bayreuth, Geheimes Archiv Bayreuth 5574

(Fasciculi Erlanger actorum - Bd. 7: Veränderungen bei den Hugenotten, 1698)

StABA, Markgraftum Brandenburg-Bayreuth, Geheimes Archiv Bayreuth

Nr. 142 (Huldigungsliste 1662)

StABA, Markgraftum Brandenburg-Bayreuth, Geheimes Archiv Bayreuth

Nr. 194 (Huldigungsliste 1712)

StABA, Markgraftum Brandenburg-Bayreuth, Geheimes Archiv Bayreuth Nr. 235 (Huldigungsliste 1735)

StABA, Markgraftum Brandenburg-Bayreuth, Geheimes Archiv Bayreuth Nr. 5568 (Erlangen, Liste der ankommenden Hugenotten 1686)

StABA, Markgraftum Brandenburg-Bayreuth, Geheimes Archiv Bayreuth Nr. 5574 (Einwohnerliste Erlangen 1698)

\section{Staatsarchiv Nürnberg}

StAN, Fm AN, Ämterbeschreibung Nr. 62 (Uffenheim 1727)

StAN, Fm AN, Ansbacher Archivalien 3908, pag. 379

StAN, Fm AN, Ansbacher Archivalien 3926, fol. 62, 29.5.1733

StAN, Fm AN, Ansbacher Archivalien 14703 (Acta. Die nach dem erfolgten Regierungs Antritt des Königs von Preußen [...] vorzunehmende allgemeine Landes Huldigung in dem Fürstenthum Ansbach, 1792)

StAN, Fm AN, Brdbg. Lit. Nr. 592-607 (Landes - und Erbhuldigung)

StAN, Fm AN, Salbücher Nr. 92, fol. 81-121 (Schwabach 1530)

StAN, Grundakt Erlangen

StAN, Libri Conservatorii, 136 (16.02.1616)

StAN, Rst. N, Amts- und Standbücher 284 (Grabenbuch 1430) 
StAN, Rst. N, Ämterbeschreibung, 2241-2327 (Pflegamt Lauf Rechnungen, Verspruchgeldlisten der Laufer Beständner 1560/61 bis 1809)

StAN, Rst. N, Ämterrechnungen VII

StAN, Rst. N, Bauernverzeichnisse Nr. 2 und 6,

StAN, Rst. N, Bauernverzeichnisse Nr. 7 (Türkensteuerregister von 1716/17)

\section{Stadtarchiv Ansbach}

StadtAAN AM 403, pag. 11 (Schreiben vom 8. März 1726)

StadtAAN, AM 263 (Einwohnerlisten Ansbach 1713)

StadtAAN, AM 921 (Einwohnerlisten Ansbach 1665)

\section{Stadtarchiv Augsburg}

StadtAA, Chroniken 27a

StadtAA, Proviantamt Nr. 195

\section{Stadtarchiv Coburg}

StadtACoburg, B 111, III, 115

\section{Stadtarchiv Erlangen}

StadtAER, Rudelsche Chronik

StadtAER, IV.B.14, 76

StadtAER, IV.B.14 (Stadtplan von Friedrich Christian Rudel mit Rekonstruktion der Bauherren, um 1790)

\section{Universitätsbibliothek Erlangen}

UBER, H60/J 237 (Erlangen im Jahr 1822, Lithografie von Joseph Päringer) UBER, Ms. B 117/118 (Reinhardsche Chronik)

\section{Stadtarchiv Nürnberg}

StadtAN, B 1/I Nr. 7, fol. 22' (Brunnenbuch 1479)

StadtAN, B 1/I Nr. 29 und 30 (Feuerschau 1561 St. Sebald und St. Lorenz)

StadtAN, B 1/II Nr. 1837 ( Verzeichniß aller Zinnsheuser und wohnungen, so Heinrich Greffen Becker allhier inn und außer der Stadt zuständig)

StadtAN, B 11 Nr. 86

StadtAN, B 11 Nr. 951, pag. 11 (Die Einforderung und den Erlang der Türken-Steuer betreffend Anno 1717)

StadtAN, B 14/II, Nr. E, Bl. 165 (Jahr 1489)

StadtAN, B 14/II Nr. G, Bl. 104' (1492) und Bl. 220 (1493)

StadtAN, B 14/II Nr. H, Bl. 159' und Bl. 213' (1495)

StadtAN, B 14/II Nr. K, Bl. 15 (1496)

StadtAN, B 14/II Nr. L, Bl. 38 (1498),

StadtAN, B 14/II Nr. Q, Bl. 226' (1505)

StadtAN, B 14/II Nr. 7, Bl. 102 und 152 (1515)

StadtAN, B 14/II Nr. 14, Bl. 105 (1520)

StadtAN, B 14/II Nr. 26, Bl. 27 und 120 (1528) 
StadtAN, B 14/II Nr. 28, Bl. 198'

StadtAN, B 14/II Nr. 29, Bl. 82 (1529)

StadtAN, B 14/II Nr. 29, Bl 125'

StadtAN, B 14/II Nr. 30, Bl. 116' und Bl. 134 (1530)

StadtAN, B 14/II Nr. 41, Bl. 193' (1537)

StadtAN, B 17/I Nr. 83

StadtAN, E 13/III Nr. A 2168 (Letztwillige Verfügungen des Schneidergesellen Conrad Fleischmann zu Boxdorf 1758/59)

Stadtarchiv Schwabach

StadtASC, III-25 (Seelenlisten)

\section{Gedruckte Quellen}

BURCHARD/MUNDHENKE 1940-1971 - BuRCHARD, MAX/MUNDHENKE HERBERT (Bearb.): Die Kopfsteuerbeschreibung der Fürstentümer Calenberg-Göttingen und Grubenhagen von 1689, 13. Bde., Hildesheim 1940-1971

DeHM/HeCKel 1970 - DeHM, KARL/HeCKel, GotTlob: Häusergeschichte der Altstadt Schwabach - mit einem Verzeichnis der Hausbesitzer, Schwabach 1970

Der Stat Nurmberg verneute Reformation 1564 - Der Stat Nurmberg verneute Reformation, Nürnberg 1564

URL Permalink: https://digi.ub.uni-heidelberg.de/diglit/drwNuernbergRef1564/

FRÄNKEL 1999 - FRÄNKEL, HEINZ F. (Bearb.): Adressbuch der Stadt Bamberg für die Jahre 1803/05 sowie nur für Mietleute für 1809/1810, Bamberg 1999

Haupt-Beschreibung 1762 - Haupt-Beschreibung zufolge des gnädigst erlassenen Generalmandats und beigefügter Instruktion d. d. 11. Mai anno 1762 über die dermalen vorhandenen Wohnungen, Gebäude und Häuser, dann deren sonderbaren Inwohner nebst betreffenden Herdstätten, churfürstliche und bürgerliche beiderseitigen Jurisdiktionen in der churfürstlichen Hauptstadt Ingolstadt verfaßt den 15. Dezember anno 1762, in: Sammelblatt des Historischen Vereins in und für Ingolstadt 26, Ingolstadt 1901, S. 1-40

URL: http://daten.digitale-sammlungen.de/bsb00005160/image_3/

Historische Kommission bei der Bayerischen Akademie der Wissenschaften 1862

- Historische Kommission bei der Bayerischen Akademie der Wissenschaften (Hrsg.): Die Chroniken der fränkischen Städte, Nürnberg 1, Leipzig 1862

Historische Kommission bei der Bayerischen Akademie der Wissenschaften 1874

- Historische Kommission bei der Bayerischen Akademie der Wissenschaften (Hrsg.): Die Chroniken der fränkischen Städte, Nürnberg 5, Leipzig 1874 
Hoffmann 1955 - Hoffmann, HeRmann (Hrsg.): Würzburger Polizeisätze. Gebote und Ordnungen des Mittelalters 1125-1495. Ausgewählte Texte, Würzburg 1955

KAMMAN 1886 - KAMANN, JOHANN (Bearb.): Aus den Nürnberger Haushaltungsund Rechnungsbüchern des 15. und 16. Jahrhunderts, in MVGN 6, Nürnberg 1886, S. 57-122

MEDEFInd 2000 - MEDEFIND, HeinRICH (Bearb.): Die Kopfsteuerbeschreibung des Fürstentums Braunschweig-Wolfenbüttel von 1678, Hannover 2000

Medefind 2004 - MEdefind, HeInRICH (Bearb.): Die Kopfsteuerbeschreibungen der Stadt Braunschweig von 1672 und 1687, Hannover 2004

MÜLLNER 1623/1984 - MÜLLNER, JOHANNES: Die Annalen der Reichsstadt Nürnberg von 1623 /3: Von 1470-1544, Nürnberg 1984

MÜNCH 2017 - MÜNCH, ERNST (Hrsg.): Das Rostocker Grundregister 1550-1600. Bearbeitet auf der Grundlage der Hausbücher, Schwerin 2017

Quartiergeldbeiträge 1798 - Berechnung der von hiesig Löbl. Burgerschaft vom 1sten October 1797 bis zum 31sten Januar geleisteten Quartier-Geld-Beyträge und deren in beygefügter Liste enthaltenen Verwendung zu beliebiger Einsicht des hiesig gesammten Publikums an das Licht gestellt und zum Druck befördert von der Deputation zur Einquartierung, Nürnberg 1798 (StadtAN, Bz E 34).

RECHTER 1985 - RECHTER, GERHARD (Bearb.): Das Reichssteuerregister von 1497 des Fürstentums Brandenburg-Ansbach-Kulmbach unterhalb Gebürgs, Nürnberg 1985

RECHTER 1988 - RECHTER, GERHARD (Bearb.): Das Reichssteuerregister von 1497 des Fürstentums Brandenburg-Ansbach-Kulmbach oberhalb Gebürgs, Nürnberg 1988

RECHTER 2003 - RECHTER, GERHARD (Bearb.): Uffenheim Häusergeschichte 1530 bis 1945, Nürnberg 2003

RECKNAGEL 2009 - RECKNAGEL, HANS UND ERIKA: Häuserchronik der Altdorfer Altstadt. Bearbeitet und ergänzt nach einem Manuskript von Dr. Fritz Tischer (+) (Schriftenreihe Altnürnberger Landschaft 51), Neuhaus a. d. Pegnitz 2009

SCHROHE 1920 - SCHROHE, HeInRICH (Hrsg.): Die Stadt Mainz unter Kurfürstlicher Verwaltung (1462-1792) (Beiträge zur Geschichte der Stadt Mainz 5), Mainz 1920

SCHROHE 1930a - SCHROHE, HeINRICH (Hrsg.): Die Mainzer Stadtaufnahmen von 1568 und 1594 (Beiträge zur Geschichte der Stadt Mainz 6), Mainz 1930

SCHROHE 1930b - SCHroHe, HeInRICH (Hrsg.): Die Mainzer Stadtaufnahmen von 1657 und 1687 (Beiträge zur Geschichte der Stadt Mainz 7), Mainz 1930 
SCHROHE 1931 - SCHROHE, HeInRICH (Hrsg.): Die Mainzer Stadtaufnahmen von 1747 und 1785/86 (Beiträge zur Geschichte der Stadt Mainz 8), Mainz 1931

STEINMEYER 1912 - STEINMEYER, ElIAS V. (Hrsg.): Die Matrikel der Universität Altdorf 1. und 2. Teil (Veröffentlichungen der Gesellschaft für Fränkische Geschichte, 4. Reihe, Band 1), Würzburg 1912

STUDTMANN 1941 - STUdTMANn, JoACHIM (Bearb.): Die Kopfsteuerbeschreibungen der Alt- und Neustadt Hannover sowie der Fürstlichen Kanzlei von 1689, Hannover 1941

WiEDEMANN 1963 - WiEDEMANN, ERNST: Hersbrucker Häuserbuch (Freie Schriftenreihe der Gesellschaft für Familienforschung in Franken 15), Neustadt/Aisch 1963

Wolter 2001-2012 - Wolter, Helmut: Das Häuserbuch der Stadt Coburg 14001945, 10 Bde., Hildburghausen 2001-2012

\section{Lexika}

DWB - Deutsches Wörterbuch, hrsg. von Jakob Grimm und Wilhelm Grimm. Online-Publikation Universität Trier.

URL: http://woerterbuchnetz.de/cgi-in/WBNetz/wbgui_py?sigle=DWB

DRW - Deutsches Rechtswörterbuch. Online-Publikation Heidelberger Akademie der Wissenschaften. URL: http://drw-www.adw.uni-heidelberg.de/drw/info/

ERLANGER STADTLEXIKON 2002 - Erlanger Stadtlexikon, hrsg. von Christoph Friederich, Bertold Frhr. v. Haller und Andreas Jakob (Hrsg.), Nürnberg 2002

HistorisCHeS STADTLEXIKON SCHWABACH 2008 - Historisches Stadtlexikon, hrsg. von Eugen Schröder, Neustadt a. d. Aisch 2008

STADTLEXIKON NÜRnBerg 2000 - Stadtlexikon Nürnberg, hrsg. von Michael Diefenbacher, Michael und Rudolf Endres, 2. verb. Aufl., Nürnberg 2000

Online-Publikation Stadtarchiv Nürnberg.URL: https://online-service2.nuernberg.de/stadtarchiv/dokwechs.FAU?sid=03AA29831\&dm=2 


\section{Literatur}

Alphabetisches Verzeichnis 1818 - Alphabetisches Verzeichni $\beta$ aller im Rezatkreise nach seiner durch die neueste Organisation erfolgten Constituirung enthaltenen Ortschaften: mit Angabe a. der Steuer-Distrikte, b. Gerichts-Bezirke, c. Rentämter, in welchen sie liegen, dann mehrerer anderer statistischen Notizen, Ansbach 1818

Altmann 1992 - AltMAnN, HeRBeRT: Verzeichnis der Bürger der Oberamtsstadt Windsbach in der Markgrafschaft Ansbach. II. Teil: Die Zeit von 1675-1794, 2. Auflage, Windsbach 1992

Ammann 1968 - Ammann, HeKTOR: Nürnbergs industrielle Leistung im Spätmittelalter, in: Lütge, Friedrich (Hrsg.): Wirtschaftliche und soziale Probleme der gewerblichen Entwicklung im 15.-16. und 19. Jahrhundert (Bericht über die zweite Arbeitstagung der Gesellschaft für Sozial- und Wirtschaftsgeschichte in Würzburg 8.-10. März 1965), Stuttgart 1968

ANDERMANN/Ehmer 1990 - ANDERMANn, KuRT /EHMER, HeRMANn (Hrsg.): Bevölkerungsstatistik an der Wende vom Mittelalter zur Neuzeit. Quellen und methodische Probleme im überregionalen Vergleich, Sigmaringen 1990

ARNOLD 1966 - ARNOLD, DiETER: Die wirtschaftliche und soziale Entwicklung der Stadt Rinteln im 17. und 18. Jahrhundert (Schaumburger Studien 16), Rinteln 1966 BAHL 1974 - BAHL, HERMS: Ansbach. Strukturanalyse einer Residenz vom Ende des Dreißigjährigen Krieges bis zur Mitte des 18. Jahrhunderts; Verfassung, Verwaltung, Bevölkerung und Wirtschaft, Ansbach 1974

BÁTORI/WEYRAUCH 1982 - BÁTORI, INGRID/WEYRAUCH, ERDMANN: Die bürgerliche Elite der Stadt Kitzingen. Studien zur Sozial- und Wirtschaftsgeschichte einer landesherrlichen Stadt im 16. Jahrhundert (Spätmittelalter und Frühe Neuzeit. Tübinger Beiträge zur Geschichtsforschung 11), Stuttgart 1982

BEDAL 1993 - BEDAL, KONRAD: Historische Hausforschung (Quellen und Materialien zur Hausforschung in Bayern 6/Schriften und Kataloge des Fränkischen Freilandmuseums des Bezirks Mittelfranken in Bad Windsheim 18), 2. überarbeitete und ergänzte Aufl., Bad Windsheim 1993

Beyerle/MAUerer 1908 - BeYerle, KonRAD/MAURer, ANTON: Konstanzer Häuserbücher. Festschrift zur Jahrhundertfeier der Vereinigung der Stadt Konstanz mit dem Hause Baden, Zweiter Band, Geschichtliche Ortsbeschreibung, Heidelberg 1908

BISCHOFF 1959 - BisCHOFF, JOHANNES E.: Erlangens Einwohner 1495 und 1528. Eine Studie über Familiennamen und Bevölkerungswechsel, in: Erlanger Bausteine zur fränkischen Heimatforschung 6, Erlangen 1961, S. 113-118 
BISCHOFF 1961a - BISCHOFF, JOHANNES E.: Erlangens Einwohner im neu ermittelten Urbar 1555-1557. Eine Studie über Familiennamen und Bevölkerungswechsel, in: Erlanger Bausteine zur fränkischen Heimatforschung 8, Heft 1 und 2, Erlangen 1961, S. 26-33

BISCHOFF 1963 - BISCHOFF, JOHANNES E.: Erlangens Einwohner in den Jahren 1616 und 1619. Eine Studie über Familiennamen und Bevölkerungswechsel, in: Erlanger Bausteine zur fränkischen Heimatforschung 10, Heft 2/3, Erlangen 1963, S. 57-94

BISCHOFF 1986 - BISCHOFF, JOHANNES E.: „Hugenotten-Orte“ in Franken neben Erlangen, in: FRIEDERICH 1986, S. 53-58

BlAHA 1989 - BlaHA, WALTER: Kurmainzische absolutistische Herrschaft von 1664 bis 1789, in: Gutsche, Willibald: Geschichte der Stadt Erfurt, 2. bearb. Aufl., Weimar 1989

BLASCHKE 1957 - BlaSCHKe, KARLHEINZ: Historisches Ortsverzeichnis von Sachsen, Leipzig 1957

BLENDINGER 1940 - BLENDINGER, FRIEDRICH: Bevölkerungsgeschichte einer deutschen Reichsstadt im Zeitalter der Glaubenskämpfe. Die Bevölkerungsbewegung in der ehemaligen Reichsstadt Weißenburg am Nordgau von rund 1580 bis 1720, Leipzig 1940

BÖCKEL 1988 - BÖCKEL, ANNEMARIA: 500 Jahre Sieben Zeilen in Nürnberg, Nürnberg 1988

BollHagen 1741 - BollHagen, LAURENTIUs DAVID: Historischer Bericht, wie es Bey der solennen Erb=Huldigung Ihro weyl. Königl. Majestät in Preußen Herrn Friedrich Wilhelm, zu Stettin und Pommern gehalten worden; Ingleichen mit was für Formalitäten sie solenne Erb=Huldigung von Ihro itzt regierenden Königl. Maj. in Preußen, sowohl in Königsberg, Berlin, Magdeburg ec. als auch in Breslau, Liegnitz und Schweidnitz eingenommen worden, Frankfurt und Leipzig 1741

BRÄUER 1997 - BRÄUER, HeLMUT: Der Leipziger Rat und die Bettler. Quellen und Analysen zu Bettlern und Bettelwesen in der Messestadt bis ins 18. Jh., Leipzig 1997

BRÄUER 2003 - BRÄUER, HELMUT: Hausgenossen in Städten Obersachsens während des Spätmittelalters und der frühen Neuzeit, in: Bräuer, Helmut/Jaritz, Gerhard/Sonnleitner, Käthe (Hrsg.): Viatori per urbes castraque (Festschrift für Herwig Ebner zum 75. Geburtstag), Graz 2003, S. 73-95

BRÜCKNER et al. 1988 - BRÜCKNER, CAROLA/MÖHLE, SYlVIA/PRÖVE, RALF/ROSCHMANN, JOACHIM: Vom Fremden zum Bürger: Zuwanderung in Göttingen 1700-1755, 
in: Wellenreuther, Hermann (Hrsg.): Göttingen 1690-1755. Studien zur Sozialgeschichte einer Stadt (Göttinger Universitätsschriften Serie A, 9), Göttingen 1988, S. 88-174

Brunner 1948 - BRunner, OtTo: Die geschichtliche Stellung der Städte Krems und Stein, Krems 1948

BRUNNER 1966 - BRUNNER, OTTO: Das „ganze Haus“ und die alteuropäische „Ökonomik“, in: Ferdinand Oeter (Hrsg.): Familie und Gesellschaft, Tübingen 1966, S. $23-56$

BRUNNER 1968 - BRUNnER, OTTO: Neue Wege der Verfassungs- und Sozialgeschichte, Göttingen 1968

Brunner/EllRod/Longolius 1747 - BRunner, Johann CASPar/Ellrod, PHiLipp ANDReas/LONGolius, PAUl Daniel: Corpvs Constitvtionvm BrandenbvrgicoCvlmbacensivm, Oder Vollständige Sammlung Der Vornehmsten so wohl allgemeinen als besondern in dem Marggrafthume Brandenburg-Culmbach in Ecclesiasticis und Politicis Theils einzeln gedruckten, Theils noch nicht gedruckten Landes-Ordnungen und Gesetze: Welche auf Hochfürstlichen gnädigsten Special-Befehl aus den Archiven und Registraturen colligirt, und dem Publico mitgetheilet werden sollen, Bayreuth 1747

BURG 1967 - BURG, ANDRÉ-MARCEL: Die unteren Volksschichten in Hagenau, in: Maschke, Erich/Sydow, Jürgen: Gesellschaftliche Unterschichten in den Südwestdeutschen Städten (Veröffentlichungen der Kommission für geschichtliche Landeskunde in Baden-Württemberg, Reihe B, Forschungen 41), Stuttgart 1967, S. $90-100$

CRAMER 1984/85 - CRAMER, JOHANNES: Schwabach - Lohr am Main - Hersbruck: Zur Sozialtopographie dreier kleiner Städte in Franken im 17. Jahrhundert, in: Mitteilungen der fränkischen Geographischen Gesellschaft 31/32, Erlangen 1984/85, S. $503-515$

CzOK 1979 - CZOK, KARL: Vorstädte. Zu ihrer Entstehung, Wirtschaft und Sozialentwicklung in der älteren deutschen Stadtgeschichte, Berlin 1979

CHRISTENSEN 2006 - CHRISTENSEN, MARGRIT: Kleinhäuser in Lübeck. Zur Bau- und Sozialstruktur der Hansestadt - Die Stadt der Handwerker und Gewerbetreibenden (Häuser und Höfe in Lübeck 5), Neumünster 2006

DECKER 2006 - DECKER, CHRISTINE: Wismar 1665: eine Stadtgesellschaft im Spiegel des Türkensteuerregisters (Kleine Stadtgeschichte 3), Berlin 2006

DENECKE 1980 - DENECKE, DIETRICH: Sozialtopografische und sozialräumliche Gliederung der spätmittelalterlichen Stadt. Problemstellungen, Methoden und Betrach- 
tungsweisen der historischen Wirtschafts- und Sozialtopografie, in: Fleckenstein, Josef/Stackmann, Karl (Hrsg.): Über Bürger, Stadt und städtische Literatur im Spätmittelalter. Bericht über Kolloquien der Kommission zur Erforschung der Kultur des Spätmittelalters 1975-1977 (Abhandlungen der Akademie der Wissenschaften in Göttingen 121), Göttingen 1980, S. 161-202

DiefenbaCHER 2000 - DiefEnbaCHer, Michael: Massenproduktion und Spezialisierung. Das Handwerk in der Reichsstadt Nürnberg, in: Kaufhold, Karl Heinrich/Reinighaus Wilfried (Hrsg.): Stadt und Handwerk in Mittelalter und früher Neuzeit, Köln/Weimar/Wien 2000, S. 211-228

DILCHER 1980 - DILCHER, GERHARD: Zum Bürgerbegriff im späten Mittelalter in: Fleckenstein, Josef/Stackmann, Karl (Hrsg.): Über Bürger, Stadt und städtische Literatur im Spätmittelalter. Bericht über Kolloquien der Kommission zur Erforschung der Kultur des Spätmittelalters 1975-1977 (Abhandlungen der Akademie der Wissenschaften in Göttingen 121), Göttingen 1980, S. 59-105

DIPPERT 2015 - DIPPERT, WOLFGANG: Landesherrliche Wirtschaftsförderung in Schwabach, in: Wüst, Wolfgang (Hrsg.): Regionale Wirtschafts- und Industriegeschichte in kleinstädtisch-ländlicher Umgebung (Mikro und Makro - vergleichende Regionalstudien 1), Erlangen 2015, S. 167-182

DIRLMEIER 1978 - DIRLMEIER, UlF: Untersuchungen zu Einkommensverhältnissen und Lebenshaltungskosten in oberdeutschen Städten des Spätmittelalters (Mitte 14. bis Anfang 16. Jahrhundert), Heidelberg 1978

DirlmeIER 1998 - DiRlmeIER, UlF (Hrsg.): Geschichte des Wohnens 2, 500-1800: Hausen, Wohnen, Residieren, Stuttgart 1998

DirLMEIER/SCHMIDT 1998 - DirLMEIER, UlF/SCHMIDT, FRITZ: Geschichte des Wohnens im Spätmittelalter, in: DIRLMEIER 1998, S. 231-346

Di Simone 1996 -Di Simone, MARIA Rosa: Die Zulassung zur Universität, in: Rüegg, Walter (Hrsg.): Geschichte der Universität Europas, 2. Von der Reformation zur Französischen Revolution (1500-1800), München 1996, S. 235-262

DISTLER 1986 - DISTLER, UlRICH: Die Geschichte der Hugenotten und der reformierten Gemeinde in Schwabach, Schwabach 1986

DITT 1979 - DITT, HILDEGARD: Ältere Bevölkerungs- und sozialstatistische Quellen, in: Ehbrecht, Wilfried (Hrsg.): Voraussetzungen und Methoden geschichtlicher Städteforschung (Städteforschung 7), Köln/Wien 1979, S. 111-138

DOMSTA 1971 - DOMSTA, HANS J.: Patrizischer Haus- und Rentenbesitz im mittelalterlichen Köln, in: Jahrbuch des Kölnischen Geschichtsvereins e.V. 43, Köln 1971, S. 192-264 
EHMER 1979 - EHMER, JOSEF: Wohnen ohne Wohnung. Zur sozialen Stellung von Untermietern und Bettgehern, in: NIETHAMMER 1979, S. 132-150

Einfalt 1910 - Einfalt, Johannes: Die Geschichte der Stadt, des Klosters und der Pfarrei Langenzenn, Ansbach 1910

ELLERMEYER 1980 - ELLERMEYER, JÜRGEN: Grundeigentum, Arbeits- und Wohnverhältnisse. Bemerkungen zur Sozialgeschichte spätmittelalterlicher-frühneuzeitlicher Städte, in: Lübecker Schriften zur Archäologie und Kulturgeschichte 4/1980, S. 71-95

ENDRES 1970 - ENDRES, RUDOLF: Zur Einwohnerzahl und Bevölkerungsstruktur Nürnbergs im 15./16. Jahrhundert, in: Mitteilungen des Vereins für Geschichte der Stadt Nürnberg 57, 1970, S. 242-272

ENDRES 1971a - ENDRES, RUDOLF: Das Ende des Fränkischen Reichskreises, in: Spindler, Max: Handbuch der Bayerischen Geschichte 3, 1. Teilbd. Franken, Schwaben, Oberpfalz bis zum Ausgang des 18. Jahrhunderts, München 1971, S. 245-248

ENDRES 1971b - ENDRES, RUdOLF: Sozialstruktur Nürnbergs, in: Pfeiffer, Gerhard (Hrsg.): Nürnberg, Geschichte einer europäischen Stadt, Nürnberg 1971, S. 194-199

ENDRES 1979 - ENDRES, RUdolF: Der Dreißigjährige Krieg, in: SPINDLER 1979, S. $223-230$

ENDRES 1982 - ENDRES, RUdOLF: Die Folgen des 30igjährigen Krieges in Franken, in: Kellenbanz, Hermann/Schmitt, Jürgen/Schneider, Eberhard (Hrsg.): Wirtschaftsentwicklung und Umweltbeeinflussung (Beiträge zur Wirtschafts- und Sozialgeschichte 20), Wiesbaden 1982, S. 125-144

ENDRES 1990a - ENDRES, RUdOlF: Die Folgen des 30jährigen Krieges in Franken, in: Mitteilungen der fränkischen geographischen Gesellschaft 35/36, 1990, S. $351-367$

ENDRES 1990b - ENDRES, RUdOLF: Das Handwerk in Nürnberg im ausgehenden Mittelalter, in: Ders. (Hrsg.): Nürnberg und Bern. Zwei Reichsstädte und ihre Landgebiete, Erlangen 1990, S. 49-79

EngElHARDT 2017 - EngelHARDT, THOMAS: Die Altersresidenz der Erlanger Markgräfin im Palais Stutterheim, in: Mitteilungen des Heimat- und Geschichtsvereins Erlangen e. V., 50. Jg., Nr. 2, Erlangen 2017, S. 33-42

ENGELSING 1973 - ENGELSING, Rolf: Zur Sozialgeschichte deutscher Mittel- und Unterschichten (Kritische Studien zur Geschichtswissenschaft 4), Göttingen 1973 
ERTL 2019 - ERTL, THOMAS: Wien 1448. Steuerwesen und Wohnverhältnisse in einer spätmittelalterlichen Stadt, Wien/Köln/Weimar 2019

FALCKenstein 1756 - FalCKenstein, Johann HeinRich von: Chronicon Svabacense, Oder: Ausführliche Beschreibung der Hoch-Fürstl. Brandenburg-Onolzbachischen Haupt-, Münz- und Lege-Stadt Schwabach: worinnen von derselben Ursprung, Alterthum, unterschiedlichen Beherrschern und Regenten, Civil- und Kirchen-Staat, Anwachs und Aufnahme, Privilegien, Glücks- und Unglücks-Fällen, auch andere Denkund Merkwürdigkeiten, ausführlich gehandelt wird, Schwabach 1756

FEHSE 2005 - FEHSE, MONIKA: Dortmund um 1400. Hausbesitz, Wohnverhältnisse und Arbeitsstätten in der spätmittelalterlichen Stadt (Dortmunder Mittelalter-Forschungen 4), Bielefeld 2005

FleisChMANn 1985 - FleisChMANn, Peter: Das Bauhandwerk in Nürnberg vom 14. bis zum 18. Jahrhundert, Nürnberg 1985

Fleischmann 1993 - FleisChMAnN, PeteR: Das Reichssteuerregister von 1497 der Reichsstadt Nürnberg (und der Reichspflege Weißenburg), Nürnberg 1993

FORNECK 2000 - FORNECK, CHRISTIAN: Die Regensburger Einwohnerschaft im 15. Jahrhundert; Studien zur Bevölkerungsstruktur und Sozialtopographie einer deutschen Großstadt des Spätmittelalters (Regensburger Studien 3), Regensburg 2000

FOUQUET 1998 - FOUQUET, GERHARD: „Annäherungen“: Große Städte - Kleine Häuser. Wohnen und Lebensformen der Menschen im ausgehenden Mittelalter (circa 14701600), in: DIRLMEIER 1998, S. 347-501

FRÄNKEL 1999 - FRÄNKEL, HeINZ F.: Adressbuch der Stadt Bamberg für die Jahre 1804/1805 sowie - nur für Mietleute - für 1809/1810. 2. Alphabetisch, Bamberg 1999

FREYESLEBEN 1775 - FREYESLEBen, LeONHARD: Das ietztlebende Erlangen, Erlangen 1775

FuHRMANn et al. 2008 - FuHRMANn, BERND/METELING, WENCKe/RAJKAY, BARBARA/WEIPERT, MATTHIAS: Geschichte des Wohnens vom Mittelalter bis heute, Darmstadt 2008

GöHRING, LUDWIG: Vor 100 Jahren. 1. Bürger und Schutzverwandter, in: Erlanger Heimatblätter. Unterhaltungsblatt zum Erlanger Tagblatt, Nr. 7, 28. März 1934, S. 26

GÖTZ 1986 - GÖTZ, HANNELORE: Würzburg im 16. Jahrhundert. Bürgerliche Vermögen und städtische Führungsschichten zwischen Bauernkrieg und fürstlichem Absolutismus (Veröffentlichungen des Stadtarchivs Würzburg 2), Würzburg 1986 
GREVING 1904 - GREVING, JOSEPH: Wohnungs- und Besitzverhältnisse der einzelnen Bevölkerungsklassen im Kirchenspiel Sankt Kolumba vom 13. bis 16. Jahrhundert (Historischer Verein für den Niederrhein, Annalen 78), Bonn 1904

GroebneR1993 - Groebner, VAlentin: Ökonomie ohne Haus. Zum Wirtschaften armer Leute in Nürnberg am Ende des 15. Jahrhunderts, Göttingen 1993

HAMmel 1980 - HAMmel, RolF: Sozial- und wirtschaftsgeschichtliche Untersuchungen zum Grundeigentum in Lübeck im 14. Jahrhundert. Ein Zwischenbericht, in: Lübecker Schriften zur Archäologie und Kulturgeschichte 4/1980, S. 31-65

HAMMEL-Kiesow 2005 - HAMMEL-KIESOW, Rolf: Die Entstehung des sozialräumlichen Gefüges der mittelalterlichen Großstadt Lübeck, in: Meinhardt, Matthias/Ranft, Andreas (Hrsg.): Die Sozialstruktur und Sozialtopographie vorindustrieller Städte. Beiträge eines Workshops am Institut für Geschichte der Martin-LutherUniversität Halle-Wittenberg am 27. und 28. Januar 2000 (Hallische Beiträge zur Geschichte des Mittelalters und der Frühen Neuzeit 1), Berlin 2005, S. 139-203

HARTMANN 1949- HARTMANN, KARL: Geschichte der Stadt Bayreuth in der Markgrafenzeit, Bayreuth 1949

Helczmanovski 1973 - HelCZMANOvski, HeImold (Hrsg.): Beiträge zur Bevölkerungs- und Sozialgeschichte Österreichs, Wien 1973

Heidemann 1917 - Heidemann, Hugo: Bevölkerungszahl und berufliche Gliederung Münsters i. W. am Ende des 17. Jahrhunderts (Münstersche Beiträge zur Geschichtsforschung 49), Münster 1917

HIPPEL 2013 - HIPPEL, WOLFGANG VON: Armut Unterschichten und Randgruppen in der frühen Neuzeit (Enzyklopädie deutscher Geschichte 34), 2., aktualisierte und um einen Nachtrag erweiterte Auflage, München 2013

Hirschmann 2016 - Hirschmann, Frank G.: Die Stadt im Mittelalter (Enzyklopädie deutscher Geschichte 84), 2., aktualisierte und erweiterte Auflage, Berlin/Boston 2016

HöCK 1804 - HöCK, JoHANn DANIEl AlbReCHT: Abriß der Polizeyverfassung des königl. preußischen Fürstenthums Ansbach, Amberg 1804

Hoffmann/Richter 2011 - Hoffmann, Yves/RiCHTER, Uwe: Der Hausbau Freibergs in der Spätgotik und Renaissance (1484-1632), in: Klein, Ulrich/Sturm, Albrecht (Hrsg.): Wandel im Wohnbau zwischen Gotik und Barock. Die sächsischböhmische Entwicklung im überregionalen Vergleich (Jahrbuch für Hausforschung 53), Marburg 2011, S. 139-192 
HOFFMANN/Richter 2012 - HOFFMANN, YVes/Richter, Uwe: Entstehung und Blüte der Stadt Freiberg. Die bauliche Entwicklung der Bergstadt vom 12. bis zum Ende des 17. Jahrhunderts, Halle 2012

Hofmann 1965 - Hofmann, SIEGFRIED: Die soziale Struktur der Bevölkerung Ingolstadts im ausgehenden 17. Jahrhundert, in: Sammelblatt des Historischen Vereins in und für Ingolstadt 74, 1965, S. 15-53,

http://daten.digitale-sammlungen.de/bsb00005247/image_16

HOLENSTEIN 1991 - HolENSTEIN ANDRÉ: Die Huldigung der Untertanen. Rechtskultur und Herrschaftsordnung (800-1800) (Quellen und Forschungen zur Agrargeschichte 36), Stuttgart/New York 1991

HORSCH 1805 - HORSCH, PHILIPP JOSEPH: Versuch einer Topographie der Stadt Würzburg in Beziehung auf den allgemeinen Gesundheitszustand und die dahin zielenden Anstalten, Arnstadt/Rudolstadt 1805

IGEL 2010 - IGEL, KARSTEN: Zwischen Bürgerhaus und Frauenhaus. Stadtgestalt, Grundbesitz und Sozialstruktur im spätmittelalterlichen Greifswald (Städteforschung 71), Köln 2010

JÄGER 1987 - JÄGER, ELISABETH: Wunsiedel 1163-1560, I. Band einer Geschichte der Burg und der Stadt Wunsiedel, Wunsiedel 1987

JаKов 1986 - JАKOB, ANDREAS: Die Neustadt Erlangen, Planung und Entstehung (Erlanger Bausteine zur fränkischen Heimatforschung, Sonderbd. 33), Erlangen 1986

JANTKe/Hilger 1965 - JANTKe, CARL/Hilger, DieTrich (Bearb. und Hrsg.): Die Eigentumslosen. Der Deutsche Pauperismus und die Emanzipationskrise in der Darstellung und Deutung der zeitgenössischen Literatur, Freiburg/München 1965

KASPAR 2002 - KASPAR, FRED: Unterschichtenwohnen in Westfalens Städten zur frühen Neuzeit - insbesondere am Beispiel der Stadt Warendorf, in: Westfälische Zeitschrift - Zeitschrift für vaterländische Geschichte und Altertumskunde 152, Münster 2002, S. 133-152

KASPAR 2014 - KASPAR, FRED: Wohnungsnot in der Garnison und städtische Miethäuser um 1700. Von der Wedemhove zur Bühlstraße in Warendorf, in: Ders. (Hrsg.): Kleine Häuser in großen Reihen (Einblicke, Schriften der Stiftung kleines Bürgerhaus 3), Petersberg 2014, S. 121-151

KASTNER 1988 - KASTNER, SABINE: Bürgerliches Wohnen und Bauen in Göttingen, in: Wellenreuther, Hermann (Hrsg.): Göttingen 1690-1755. Studien zur Sozialgeschichte einer Stadt (Göttinger Universitätsschriften Serie A, 9), Göttingen 1988, S. $175-251$ 
KeLlENBENZ 1986 - KeLlENBENZ, HeRMANN (Hrsg.): Europäische Wirtschafts- und Sozialgeschichte vom ausgehenden Mittelalter bis zur Mitte des 17. Jahrhunderts, Handbuch der europäischen Wirtschafts- und Sozialgeschichte 3, Stuttgart 1986

KeLleR 1997 - KelleR, KATRIN: Kleinstädte zwischen Dreißigjährigem Krieg und Industrialisierung. Das Beispiel Kursachsen, Habil. Universität Leipzig 1997

KEYSER 1938 - KEYSER, ERICH: Bevölkerungsgeschichte Deutschlands, Naumburg 1938

KeYser/Stoob 1971 - KeYSER, ERICH/StOOb, Heinz (Hrsg.): Bayerisches Städtebuch, Teil 1, Stuttgart u. a. 1971

KLINGNER 1749 - KLINGNER, JoHANn GotTLOB: Sammlungen zum Dorf- und Bauren-Rechte, Leipzig 1749

KOBER 1956 - Kober, ERNST: Pfahlbürger und Schutzverwandte in Ansbach, in: Jahrbuch des Historischen Vereins für Mittelfranken Nr. 76, Ansbach 1956, S. 21-32

KoBer 1964 - Kober, ERnST: Das älteste Bürgerbuch der Stadt Ansbach 1488-1562, Ansbach 1964

KÖHL 1913 - KÖHL, OSKAR: Zur Geschichte des Bergbaues im vormaligen Fürstentume Kulmbach-Bayreuth mit besonderer Berücksichtigung der zum Frankenwald gehörigen Gebiete, eine kulturgeschichtliche Studie, Hof 1913

KÖNIG 1785 - KÖNIG, JOHANN CHRISTOPH: Akademisches Lehrbuch für studirende Jünglinge aus allen Fakultäten, Nürnberg 1785

KRAMER 1961 - KRAMER, KARL-SigiSMUND: Volksleben im Fürstentum Ansbach und seinen Nachbargebieten (1500-1800). Eine Volkskunde aufgrund archivalischer Quellen, Würzburg 1961

KRONSHAGE 1960 - Kronshage, WALTER: Die Bevölkerung Göttingens. Ein demografischer Beitrag zur Sozial- und Wirtschaftsgeschichte vom 14. bis zum 17. Jahrhundert, Göttingen 1960

KRUG, 2006 - KRUG, RAPHAEL MATTHIAS: Es ist doch zem Jungsten ein end daran. Die Augsburger Steuerbü̈her im Spatmittelalter, Augsburg 2006, https://opus.bibliothek.uni-augsburg.de/opus4/frontdoor/index/index/docId/473

LASCH, 1964/65 - LASCH, MANFRED: Die Kasseler Einwohnerverzeichnisse der Jahre 1731 und 1751, in: Zeitschrift des Vereins für hessische Geschichte und Landeskunde 75/76, 1964/65, S. 349-415

LASCH 1969 - LASCH, MANFRED: Untersuchungen über Bevölkerung und Wirtschaft der Landgrafschaft Hessen-Kassel und der Stadt Kassel vom 30jährigen Krieg bis zum 
Tode Landgraf Karls 1730 (Hessische Forschungen zur geschichtlichen Landesund Volkskunde 9), Kassel 1969

LEONHARDI 1797 - LEONHARDI, FRIEDRICH GOTTLOB: Erdbeschreibung der Fränkischen Fürstenthümer Bayreuth und Anspach, Halle 1797

LOCHNER 1857 - LOCHNER, WOLFGANG K.: Die Einwohnerzahl der ehemaligen Reichsstadt Nürnberg, Nürnberg 1857

LOFFING 1911 - LOFFING, ALOYS: Die soziale und wirtschaftliche Gliederung der Bevölkerung Erfurts in der zweiten Hälfte des 16. Jahrhunderts, Diss. Universität Münster 1911

LICHTENBERGER 1973 - LICHTENBERGER, ELISABETH: Von der mittelalterlichen Bürgerstadt zur City. Sozialstatistische Querschnittsanalysen am Wiener Beispiel, in: HELCZMANOVSKI 1973, S. 297-331

LisSOK/LiCHTNAU 2006 - LisSOK, Michael/LichtNAU, BeRnFried (Hrsg.): Das steinerne Antlitz der Alma Mater. Die Bauten der Universität Greifswald 1456-2006, Berlin 2006

MASCHKE 1967- MASCHKE, ERICH: Die Unterschichten der mittelalterlichen Städte Deutschlands, in: Ders./Sydow, Jürgen (Hrsg.): Gesellschaftliche Unterschichten in den Südwestdeutschen Städten (Veröffentlichungen der Kommission für geschichtliche Landeskunde in Baden-Württemberg, Reihe B, Forschungen 41), Stuttgart 1967, S. 1-74

MASCHKE 1972 - MASCHKE, ERICH: Städtische Mittelschichten (Veröffentlichungen der Kommission für geschichtliche Landeskunde in Baden-Württemberg, Reihe B, Forschungen 69), Stuttgart 1972

MAUERSBERGER 1960 - MAUERSBERger, HANS: Wirtschafts- und Sozialgeschichte zentraleuropäischer Städte in neuerer Zeit. Dargestellt an den Beispielen von Basel, Frankfurt a. M, Hamburg, Hannover, München, Göttingen 1960

MAYER/THYroff 2014 - MAYer, WolfGANG/ThYroff, Frank: Zwischen Pfeffer und Hightech, ein Streifzug durch die Nürnberger Wirtschaftsgeschichte, Nürnberg 2014

MeInHARDT 2009 - MeInHARDT, MATTHIAS: Dresden im Wandel. Raum und Bevölkerung der Stadt im Residenzbildungsprozess des 15. und 16. Jahrhunderts (Hallische Beiträge zur Geschichte des Mittelalters und der Frühen Neuzeit 4), Berlin 2009

Meinhardt/RANFT 2000 - MeinhardT, MATTHias/RAnFT, ANDREAS (Hrsg.): Die Sozialstruktur und Sozialtopographie vorindustrieller Städte. Beiträge eines Workshops am Institut für Geschichte der Martin-Luther-Universität Halle-Wittenberg 
am 27. und 28. Januar 2000 (Hallische Beiträge zur Geschichte des Mittelalters und der Frühen Neuzeit 1), Berlin 2005

MeYer-ERlaCh 1932 - Meyer-ERlach, GeOrg: Das Seldener Buch (Mitteilungen der Zentralstelle für deutsche Personen- und Familiengeschichte 48), 1932

MielKe 1972 - MielKe, FRIEDRICH: Das Bürgerhaus in Potsdam (Das deutsche Bürgerhaus 15), Tübingen 1972

MITTERAUER 1973 - MITTERAUER, MICHAEL: Zur Familienstruktur in ländlichen Gebieten Österreichs im 17. Jahrhundert, in: HELCZMANOVSKI 1973, S. 167-222

MÖLLER 1969 - MÖLLER, HeLMUT: Die kleinbürgerliche Familie im 18. Jahrhundert. Verhalten und Gruppenkultur, Berlin 1969

MOLS 1971 - Mols, Roger: Die Bevölkerung im 16. und 17. Jahrhundert, in: Cipolla, Carlo Maria/Borchardt, Knut (Hrsg.): Bevölkerungsgeschichte Europas. Mittelalter bis Neuzeit, München 1971, S. 58-122

MoRlinghaus 1940 - MoRlinghaus, OTTO: Zur Bevölkerungs- und Wirtschaftsgeschichte des Fürstbistums Bamberg im Zeitalter des Absolutismus (Erlanger Abhandlungen zur mittleren und neueren Geschichte, Neue Folge 3), Erlangen 1940

MÜLLER 1751/2002 - MÜLLER, JOHANN CASPAR: Grundriß der hochfürstlichen Brandenburgischen Hauptstadt Erlangen nach dem größesten Theil ihrer dermahligen Einwohner. Das erste Einwohnerbuch Erlangens von 1751, [Repr. der Ausg.], Erlangen 2002

MUlZer 1967/68 - MUlZER, ERICH: Der Nürnberger Fachwerkbau, in: MVGN 55, Nürnberg 1967/68

MUlZer 1981 - MulZer, ERICH: Dem Unschlittplatz auf der Spur, in: Nürnberger Altstadtberichte 6, Nürnberg 1981, S. 35-82

NÜRMBERGER 2015 - NÜRMBERGER, BERND (Bearb.): Das Lagerbuch der Neustadt Erlangen von 1745. Eine bebilderte Hausgeschichte (Erlanger Bausteine zur fränkischen Heimatforschung, Sonderbd. 5), Erlangen 2015

OSTENRIEDER 1993 - OstenRIEDer, PetRA: Wohnen und Wirtschaften in Oettingen 1600 - 1800. Untersuchungen zur Sozialtopographie und Wirtschaftsstruktur einer bikonfessionellen Residenzstadt (Materialien zur Geschichte des bayerischen Schwaben 19), Augsburg 1993

OTT 1907 - OTT, CASPAR: Bevölkerungsstatistik in Stadt und Landschaft Nürnberg in der 1. Hälfte des 15. Jahrhunderts, Berlin 1907 
PeTERS 1994 - PeTERS, LAMBeRT F.: Der Handel Nürnbergs am Anfang des Dreißigjährigen Krieges. Strukturkomponenten, Unternehmen und Unternehmer; eine quantitative Analyse (Vierteljahresschrift für Sozial- und Wirtschaftsgeschichte, Beiheft 112), Stuttgart 1994

Peuschel 2015 - Peuschel, Hans: Die Leonische Fabrik und der Rother Drahtzug, in: Wüst, Wolfgang (Hrsg.): Regionale Wirtschafts- und Industriegeschichte in kleinstädtisch-ländlicher Umgebung (Mikro und Makro - vergleichende Regionalstudien 1), Erlangen 2015, S. 27-35

PFISTER 1994 - PFISTER, CHRISTIAN: Bevölkerungsgeschichte und historische Demografie. 1500-1800 (Enzyklopädie deutscher Geschichte 28), München 1994

PIRSON 1961 - PIRSON, DiETRICH: Das Baurecht des fürstlichen Absolutismus im hohenzollernschen Franken, Düsseldorf 1961

PLODECK 1971/72 - PLODECK, KARIN: Hofstruktur und Hofzeremoniell in Brandenburg-Ansbach vom 16. bis zum 18. Jahrhundert, in: Jahrbuch des Historischen Vereins für Mittelfranken 86, 1971/1972, S. 1-260

PRÖVE 1995 - PRÖVE, RALF: Stehendes Heer und städtische Gesellschaft im 18. Jahrhundert. Göttingen und seine Militärbevölkerung 1713-1756, München 1995

Porzelt 2000 - Porzelt, CARolin: Die Pest in Nürnberg. Leben und Herrschen in Pestzeiten in der Reichsstadt Nürnberg (1562-1713), St. Ottilien 2000

PuChner 1975 - PuChner, OtTo: Das Register des Gemeinen Pfennigs (1497) der Reichsstadt Nürnberg als bevölkerungsgeschichtliche Quelle, in: Jahrbuch für fränkische Landesforschung 34/35, 1975, S. 934-946

RECHTER 1990 - RECHTER, GERHARD: Bevölkerungsstatistische Quellen Frankens. Bestand und Probleme, dargestellt am Beispiel des Fürstentums Brandenburg-AnsbachKulmbach, in: Andermann, Kurt (Hrsg.): Bevölkerungsstatistik an der Wende vom Mittelalter zur Neuzeit, Sigmaringen 1990, S. 65-78

RECKNAGEL 1998 - RECKNAGEL, HANS: Die Nürnbergische Universität Altdorf und ihre großen Gelehrten, Feucht 1998

RECKNAGEL 2001 - RECKNAGEL, HANS: Geschichte und Geschichten. Historische Skizzen von Altdorf und Nürnberg, Feucht 2001

RECKTENWALD 1990 - RECKTENWALD, HORST ClAus: Die fränkische Universität Altdorf, Nürnberg 1990

REILING 1989 - REILING, MARTINA: Bevölkerung und Sozialtopographie Freiburgs i. Br. im 17. und 18. Jahrhundert. Familie, Gewerbe und sozialer Status (Veröffentlichungen aus dem Archiv der Stadt Freiburg im Breisgau 24), Freiburg i. Br. 1989 
REINDERS-DÜSELDER 1993 - REINDERS-DÜSELDER, CHRISTOPH: Haushaltsstrukturen, Heirat und soziale Ungleichheit. Das Beispiel der Dammer Berge 1650 bis 1850, in: Schlumbohm, Jürgen (Hrsg.): Familie und Familienlosigkeit. Fallstudien aus Niedersachsen und Bremen vom 15. bis 20. Jahrhundert (Quellen und Untersuchungen zur Wirtschafts- und Sozialgeschichte Niedersachsens in der Neuzeit 17), Hannover 1993, S. 181-193

REUTER 1961 - REUTER, ORTULF: Die Manufaktur im fränkischen Raum: eine Untersuchung großbetrieblicher Anfänge in den Fürstentümern Ansbach und Bayreuth als Beitrag zur Gewerbegeschichte des 18. und beginnenden 19. Jahrhunderts (Forschungen zur Sozial- und Wirtschaftsgeschichte 3), Stuttgart 1961

RICHTER 1881 - RICHTER, OTTO: Zur Bevölkerungs- und Vermögensstatistik Dresdens im 15. Jahrhundert, in: Neues Archiv für sächsische Geschichte 2, 1881, S. $273-289$

RIEDLE 1933 - RIEDLE, AlFRED: Wirtschaft und Bevölkerung Heilbronns zur Zeit des Dreißigjährigen Krieges, Würzburg 1933

RIEHL 1854 - RIEHL, WILHELm HeINRICH: Die Familie (Naturgeschichte des deutschen Volkes als Grundlage einer deutschen Social-Politik, 3. Bd), 2. überarb. Aufl., Stuttgart und Augsburg 1854

RoeCK 1989-RoeCK, BeRnd: Eine Stadt in Krieg und Frieden. Studien zur Geschichte der Reichsstadt Augsburg zwischen Kalenderstreit und Parität (Schriftenreihe der Historischen Kommission bei der Bayerischen Akademie der Wissenschaften 27), Göttingen 1989

ROECK 1993 - ROECK, BeRnd: "Arme" in Augsburg zu Beginn des 30jährigen Krieges: Untersuchung zu Wohn- u. Vermögensverhältnissen d. städtischen "Unterschicht" und zur Sozialtopographie der Reichsstadt anhand einer Getreideverteilungsliste aus dem Jahr 1622, in: Zeitschrift für bayerische Landesgeschichte 46, Heft 3, 1993, S. $515-558$

ROller 1907 - Roller, OTTO KOnRAD: Die Einwohnerschaft der Stadt Durlach im 18. Jahrhundert: in ihren wirtschaftlichen und kulturgeschichtlichen Verhältnissen dargestellt aus ihren Stammtafeln, Karlsruhe 1907

RosSEAUX 2006 - ROSSEAUX, UlRICH: Städte in der frühen Neuzeit, Darmstadt 2006 SACHSE 1985 - SACHSE, WIELAND: Wohnen und soziale Schichtung in Göttingen im 18. Jahrhundert, in: TEUTEBERG 1985, S. 131-15

SACHSE 1987 - SACHSE, WIELAND: Göttingen im 18. und 19. Jahrhundert. Zur Bevölkerungs- und Sozialstruktur einer deutschen Universitätsstadt, Göttingen 1987 
SAKUMA 1993 - SAKUma, HiRonobu: Die Nürnberger Tuchmacher, Weber, Färber und Bereiter vom 14. bis 17. Jahrhundert (Nürnberger Werkstücke zur Stadt- und Landesgeschichte 51), Nürnberg 1993

SANDeR 1902 - SANDeR, PAUl: Die reichsstädtische Haushaltung Nürnbergs, dargestellt auf Grund ihres Zustandes von 1431 bis 1440, Leipzig 1902

SCHANZ 1884 - SCHANZ, GEORG VON: Zur Geschichte der Colonisation und Industrie in Franken, Erlangen 1884

SCHEFTEL 1988 - SCHEFTEL, MiCHAEL: Gänge, Buden und Wohnkeller in Lübeck. Bau-und sozialgeschichtliche Untersuchungen zu den Wohnungen der ärmeren Bürger und Einwohner einer Großstadt des späten Mittelalters und der frühen Neuzeit (Häuser und Höfe in Lübeck 2), Neumünster 1988

SCHILling/EHRENPREIS 2015 - SCHILling, HEInZ/EHRENPREIS, STEFAN: Die Stadt in der Frühen Neuzeit (Enzyklopädie deutscher Geschichte 24), 3., um einen Nachtrag erw. Aufl., Berlin/Boston 2015

SCHIMMELPFENNIG 1964 - SCHIMMELPFENNIG, BERNHARD: Bamberg im Mittelalter. Siedelgebiete und Bevölkerung bis 1370 (Historische Studien 391), Lübeck/ Hamburg 1964

SCHLÜPFINGER 1977 - SCHLÜPFINGER, HEINRICH: Bauliche Entwicklung und Bevölkerungswachstum der Stadt Schwabach, in: Ders. (Hrsg.): Schwabach. Beiträge zur Stadtgeschichte und Heimatpflege, Schwabach 1977, S. 1-82

SCHLÜPFINGER 1986 - SCHLÜPFINGER, HEINRICH: Schwabach - Zur Stadtgeschichte von 1648 bis zur Gegenwart, Schwabach 1986

SCHLÜPFINGER 1994 - SCHLÜPFIngER, HeINRICH: Die Stadt Schwabach und ihre Landesherren, Handwerk und Gewerbe, Handel und Industrie im Wandel der Zeiten in Wort und Bild, Schwabach 1994

SCHMÖLZ-HÄBERLEIN 2014 - SCHMÖLZ-HÄBERLEIN, MiCHAELA: Von Häusern und Menschen Plädoyer für eine neue Betrachtungsweise des christlich-jüdischen Zusammenlebens und der historischen Bausubstanz in Bamberg, in: Bericht des Historischen Vereins für die Pflege der Geschichte des Ehemaligen Fürstbistums Bamberg 150, Bamberg 2014, S. 127-149

SCHNABEL 1992 - SCHNABEL, WERNER WILHELM: Österreichische Exulanten in oberdeutschen Reichsstädten - zur Migration von Führungsschichten im 17. Jahrhundert (Schriftenreihe zur bayerischen Landesgeschichte 101), München 1992

SCHNAPPER-ARNDT 1915 - SCHNAPPER-ARNDT, GOTTLIEB: Studien zur Geschichte der Lebenshaltung in Frankfurt a. M. während des 17. und 18. Jahrhunderts, Teil 1: Darstellung, Teil 2: Quellen und Material, Frankfurt 1915 
SCHNEIDAWIND 1791 - SCHNEIDAWIND, FRANZ ADOLPH: Chronik der täglichen Begebenheiten zu Bamberg durch die acht letzten Monate d. J. 1790, in: Journal in und für Deutschland 8/9 (1791), S. 760-781

SCHNEIDER 1967 - SCHNEIDER, LOTHAR: Der Arbeiterhaushalt im 18. und 19. Jahrhundert: dargestellt am Beispiel des Heim- und Fabrikarbeiters (Beiträge zur Ökonomie von Haushalt und Verbrauch, Heft 4), Berlin 1967

SCHNYDER 1925 - SCHNYDER, WERNER: Die Bevölkerung der Stadt und Landschaft Zürich vom 14. bis zum 17. Jahrhundert - eine methodologische Studie, Diss. Universität Zürich, 1925

SCHUBERT 1982 - SCHUBERT, ERNST: Erlangen als Fabrikstadt des 18. Jahrhunderts, in: Sandweg, Jürgen (Hrsg.): Erlangen: von der Strumpfer- zur Siemensstadt, Beiträge zur Geschichte Erlangens vom 18. zum 20. Jahrhundert, Erlangen 1982, S. $13-58$

SCHUBERT 1990 - SCHUBERT, ERNST: Arme Leute, Bettler und Gauner im Franken des 18. Jahrhunderts, 2., durch ein Nachw. und ein Reg. erg. Aufl, Neustadt a. d. Aisch 1990

SCHUler 1978 - SCHUler, PeTER-Johannes: Die Erhebungslisten des „Gemeinen Pfennigs" von 1496-1499. Eine demographische, wirtschafts- und sozialgeschichtliche Untersuchung, in: Irsigler, Franz (Hrsg.): Quantitative Methoden in der Wirtschafts- und Sozialgeschichte der Vorneuzeit, Stuttgart 1978, S. 127-145

SCHUlin 1920 - SCHULIN, PAUL: Beitrag zur Geschichte der mittelalterlichen Miete in west- und süddeutschen Städten, in: Zeitschrift der Savigny-Stiftung für Rechtsgeschichte, Germanistische Abteilung 41 (1920), S. 127-209

SCHUltheiss 1969 - SCHUltheISS, WeRner: Stadterweiterung und Vororte der Reichsstadt Nürnberg, in: Maschke, Erich/Sydow, Jürgen (Hrsg.): Stadterweiterung und Vorstadt (Veröffentlichungen der Kommission für geschichtliche Landeskunde in Baden-Württemberg, Reihe B, Forschungen 51), Stuttgart 1969, S. $125-126$

SCHUltheiss 1972 - SCHUlTHEISS, WeRneR: Das Bürgerrecht der Königs- und Reichsstadt Nürnberg, Beiträge zur Verfassungsgeschichte der deutschen Städte, in: Max-Planck-Institut für Geschichte (Hrsg.): Festschrift für Hermann Heimpel zum 70. Geburtstag am 19. September 1971 (Veröffentlichungen des Max-PlankInstituts für Geschichte 36/II), Göttingen 1972, S. 159-194

SEBERICH 1960 - SEBERICH, FRANZ: Die Einwohnerzahl Würzburgs in alter und neuer Zeit, in: Mainfränkisches Jahrbuch für Geschichte und Kunst : Archiv des 
Historischen Vereins für Unterfranken und Aschaffenburg 12, Baunach 1960, S. $49-68$

SPINDLER 1979, MAX: Handbuch der Bayerischen Geschichte, dritter Band, erster Teilband. Franken, Schwaben, Oberpfalz bis zum Ausgang des 18. Jahrhunderts, 2. verbesserte Aufl., München 1979

SPRANDEL 1979 - SPRANDEL, ROLF: Neue Forschungen über Vermögensverhältnisse in hansischen Städten, in: Ehbrecht, Wilfried (Hrsg.): Voraussetzungen und Methoden geschichtlicher Städteforschung, Köln/Wien 1979, S. 129-138

SPRANDEL 2003 - SPRANDEL, ROLF: Das Würzburger Ratsprotokoll des 15. Jahrhunderts. Eine historisch-systematische Analyse (Veröffentlichungen des Stadtarchivs Würzburg 11), Würzburg 2003

STIEWE 1996 - STIEWE, HeINRICH: Hausbau und Sozialstruktur einer niederdeutschen Kleinstadt. Blomberg zwischen 1450 und 1870 (Schriften des Westfälischen Freilichtmuseums Detmold, Landesmuseum für Volkskunde 13), Detmold 1996 Hansestadt Strahlsund 2008 - Hansestadt Strahlsund (Hrsg.): Hus un Hoff. Wohnbauten im mittelalterlichen Stralsund (Schriftenreihe Stralsunder Denkmäler, Heft 3), Stralsund 2008

StubenVoll 1990 - StubenVoll, Willi: Die deutschen Hugenottenstädte, Frankfurt a. M. 1990

TEUTEBERG 1985 - TEUTEBERG, HANS JÜRGEN: Homo habitans: zur Sozialgeschichte des ländlichen und städtischen Wohnens in der Neuzeit, Stuttgart/Leipzig 1985

UHLICH 2001 - UHLICH, WERNER: Feuchtwangen - Geschichte einer fränkischen Stadt, Feuchtwangen 2001

VITS 1993 - VITS, BRIGITTA: Hüfner, Kötter und Beisassen. Die Wirtschafts- und Sozialstruktur ländlicher Siedlungen in Nordhessen vom 16. bis zum 19. Jahrhundert (Hessische Forschungen zur geschichtlichen Landes- und Volkskunde, Heft 25), Kassel 1993

WeISS - WeIsS, HeRMANN: Bevölkerungs- und Vermögensverhältnisse der Stadt Mühlhausen i. Th. in der Zeit von 1563-1614, Diss. Universität Wittenberg-Halle 1926

Weltrich 1838 - Weltrich, Johann APOLLONiUs PETER: Beitrag zur Bevoelkerungs-Statistik des Vaterlandes, insbesondere des königlich bayerischen Landgerichts Culmbach in Ober-Franken, Culmbach 1838 
WENDEROTH 2012/13 - WENDEROTH, THOMAS: Das barocke Mietzinshaus. Zinshäuser als Grundelement barocker Stadtstrukturen, in: Bayerisches Landesamt für Denkmalpflege (Hrsg.): Jahrbuch der Bayerischen Denkmalpflege 66/67, München 2015, S. 73-102

Wenderoth 2019a - Wenderoth, ThOMAs: Mietshaus und Mietwohnung auf dem Land. Verbreitung, Entwicklung und Typologie am Beispiel des Nürnberger Umlandes 1500 bis 1800 (Schriftenreihe des Bayerischen Landesamtes für Denkmalpflege 18), München 2019

WENDEROTH 2019b - WeNDEROTH, THOMAS: Typenhaus und serielles Bauen - Der Erfolg des „Erlanger Hauses“, in: ONNEN/SPOHN 2019, S. 119-128

Wenderoth 2021 - Wenderoth, Thomas: Mietwohnen in der Stadt vor 1800. Verbreitung, Entwicklung und Typologie am Beispiel Frankens, Habil. Bauhaus-Universität Weimar 2021

Wiesand 1762 - Wiesand, Georg Stephan: Juristisches Hand-Buch ..., Hildburghausen 1762

WIEST 1968 - WIEST, EKKEHARD: Die Entwicklung des Nürnberger Gewerbes zwischen 1648 und 1806, Stuttgart 1968

WILL 1771 - WILL, GEORG ANDREAS: Gedächtniß des vor zweyhundert Jahren, den 30 September 1571. gelegten Grundes des vortreflichen Collegien-Gebäudes der Universität Altdorf, Altdorf 1771

WILL 1780/81 - WILL, GEORG ANDREAS: Historisch-diplomatisches Magazin für das Vaterland und angrenzende Gegenden, Band I., Nürnberg 1780/81

WILl 1796 - WiLl, GeORG ANDREAS: Geschichte und Beschreibung der Nürnbergischen Landstadt Altdorf, Altdorf 1796

WilletT 2001 - WilleTt, OlaF: Sozialgeschichte Erlanger Professoren 1743-1933, Göttingen 2001

Wischermann 1997 - Wischermann, Clemens: Mythen, Macht und Mängel: Der deutsche Wohnungsmarkt im Urbanisierungsprozess, in: Reulecke, Jürgen (Hrsg.): Geschichte des Wohnens 3, 1800-1918: Das bürgerliche Zeitalter, Stuttgart 1997, S. 333-502

WOZNIAK 2013 - WOZNIAK, THOMAS: Quedlinburg im 14. und 16. Jahrhundert, ein sozialtopographischer Vergleich, Berlin 2013

ZOBEL 1731 - Zobel, ERNST FRIEDERICH: Das unter einem Hochlöbl. Magistrat des H. R. R. Freyen Republique Nürnberg jetzt-lebende Altdorff : wie sich dasselbe bey Anfang des Jahrs 1731 befindet, Altdorf 1731 


\section{Abkürzungen}

\begin{tabular}{|c|c|}
\hline Abb. & Abbildung \\
\hline Anm. & Anmerkung \\
\hline Aufl. & Auflage \\
\hline $\mathrm{BA}$ & Bamberg \\
\hline Bd. & Band \\
\hline $\mathrm{Bl}$. & Blatt \\
\hline Bearb. & Bearbeiter \\
\hline Brdbg. Lit. & Brandenburgische Literarien \\
\hline $\mathrm{d}$ & Pfenning \\
\hline Ebd. & Ebenda \\
\hline ER & Erlangen \\
\hline Fasz. & Faszikel \\
\hline $\mathrm{fl}$ & Gulden \\
\hline $\mathrm{fl}(\mathrm{rh})$ & Gulden rheinisch \\
\hline Fm AN & Fürstentum Ansbach \\
\hline fol. & Folio \\
\hline GÖ & Göttingen \\
\hline HG & Herdgemeinschaft \\
\hline $\mathrm{HH}$ & Haushalt \\
\hline Jg. & Jahrgang \\
\hline Kap. & Kapitel \\
\hline lb & Pfund \\
\hline MVGN & Mitteilungen des Vereins für Geschichte der Stadt Nürnberg \\
\hline $\mathrm{N}$ & Nürnberg \\
\hline NLA & Niedersächsisches Landesarchiv \\
\hline pag. & Pagina (Seite) \\
\hline Prot. & Protokoll \\
\hline Reg. & Regierung \\
\hline Rep. & Repertorium \\
\hline Rst. & Reichsstadt \\
\hline $\mathrm{SC}$ & Schwabach \\
\hline StA & Staatsarchiv \\
\hline StadtA & Stadtarchiv \\
\hline Tab. & Tabelle \\
\hline UBER & Universitätsbibliothek Erlangen \\
\hline Vgl. & Vergleiche \\
\hline $\mathrm{xr}$ & Kreuzer \\
\hline
\end{tabular}




\section{Verzeichnis der Grafiken}

Grafik 1 Die räumliche Hierarchie des Wohnens

Grafik 2 Städte im Fürstentum Brandenburg-Ansbach 1603-1723 mit geringem Mieteranteil

Grafik 3 Städte im Fürstentum Brandenburg-Ansbach 1603-1723 mit starkem Mieteranstieg ab dem letzten Drittel des 17. Jhs.

Grafik 4 Verhältnis Stadtgröße und prozentualer Anteil der Mietbevölkerung anhand der Angaben des Alphabetischen Verzeichnisses 1818

Grafik 5 Gliederung der Städte in Gruppen, 1818

Grafik 6 Anteil der in den Quellen erfassten Miethaushalte in fränkischen Städten, 1407-1818

Grafik 7 Ansbach, Anzahl der Haushalte 1668-1723 (nur Bürgergemeinde), nach den Angaben aus den Huldigungslisten

Grafik 8 Schwabach, Mieteranteil nach den Angaben in den Huldigungslisten

Grafik 9 Schwabach, Anzahl der Haushalte 1497-1818

\section{Verzeichnis der Tabellen und Tabellenanhang}

Tabelle 1 Dreigenerationenhaushalte in Städten des Fürstentums Brandenburg-Ansbach-Kulmbach, nach dem Reichssteuerregister 1497

Tabelle 2 Anteil der Frauenhaushalte unter den Hausgenossen/ (Unter-)Mietern im Jahr 1497

Tabelle 3 Anteil der weiblichen Miethaushalte in ausgewählten Städten Frankens

Tabelle 4 Anteil der weiblichen Miethaushalte in Oettingen

Tabelle 5 Altdorf 1717, Miethaushalte, sortiert nach Geschlecht und Anzahl der Kinder

Tabelle 6 Altdorf 1717, Mieter und die Anzahl ihrer Kinder

Tabelle 7 Nürnberg, Anzahl der im Grabenbuch 1430 angegebenen Bewohner pro Haus/Einheit 
Tabelle 8 Nürnberg, Anzahl der Haushalte pro Anwesen im Jahr 1431 in 3 von 6 Stadtteilen

Tabelle 9 Nürnberg, männliche Mitglieder eines Wohnverbundes im Barfüßerviertel 1431

Tabelle 10 Nürnberg, Lorenzer Seite, Anzahl der im Reichssteuerregister 1497 angegebenen Personen, die über 15 Jahre alt sind pro Herdgemeinschaft

Tabelle 11 Nürnberg, Sebalder Seite, Haushaltsanzahl pro Haus 1797/98

Tabelle 12 Regensburg, Belegungsdichte der Wohnhäuser 1436 (FORNECK 2000, Tab. 8)

Tabelle 13 Augsburg, Belegung der Häuser mit Haushalten 1550-1646

Tabelle 14 Kitzingen, Veränderungen der Hausbesitzeranteile im 16. Jahrhundert

Tabelle 15 Einwohnerzahlen und Wohnsituation in den mittelfränkischen Mittelstädten 1818

Tabelle 16 Einwohnerzahlen zu den kursächsischen Mittelstädten 1550-1811

Tabelle 17 Hausgenossenhaushalte („Untermieter“) in Städten des Fürstentums Brandenburg-Ansbach-Kulmbach, nach dem Reichssteuerregister 1497

Tabelle 18 Hausgenossen - alleinstehende Frauen und Ehepaare (Familien) in Städten des Fürstentums Brandenburg-Ansbach-Kulmbach, nach dem Reichssteuerregister 1497

Tabelle 19 Anteil der Miethaushalte in den ansbachischen Städten anhand der Angaben in den Huldigungslisten 1603-1723

Tabelle 20 Durchschnittliche Mieteranteil der kleinen bis sehr kleinen Landstädte im Fürstentum Ansbach 1603-1723

Tabelle 21 Anteil der Miethaushalte in den Nürnberger Landstädten 1716/17

Tabelle 22 Prozentuale Anteil der Miethaushalte in den Gebirgsämtern des Hochstiftes Bamberg 1674

Tabelle 23 Mieteranteil mittelfränkischer Städte im Jahr 1818

Tabelle 24 Anteil Miethaushalte in mittelfränkischen Residenzstädten 1818

Tabelle 25 Kassel, rechtlicher Status der Haushalte um 1723, Anzahl Militär 1731, Herrenlose 1724 
Tabelle 26 Schwabach, Haushaltsgrößen 1497

Tabelle 27 Altstadt Erlangen, Anteil der Miethaushalte 1497-1752

Tabelle 28 Neustadt Erlangen, Anteil der Miethaushalte 1696-1818

Tabelle 29 Anteil der Miethaushalte in den Exportgewerbestädten Mittelfrankens Anfang des 18. Jhs.

Tabelle 30 Anteil der Miethaushalte in den Exportgewerbestädten Mittelfrankens 1818

Tabelle 31 Mieterzahlen in den Nürnberger Vortorten 1538, 1548 und $1716 / 17$

\section{Verzeichnis der Tabellen im Anhang:}

Tabelle I.1 Mieter in den städtischen Huldigungslisten des Fürstentums Brandenburg-Ansbach 1603

Tabelle I.2. Mieter in den städtischen Huldigungslisten des Fürstentums Brandenburg-Ansbach 1639/41

Tabelle 1.3. Mieter in den städtischen Huldigungslisten des Fürstentums Brandenburg-Ansbach 1668

Tabelle 1.4. Mieter in den städtischen Huldigungslisten des Fürstentums Brandenburg-Ansbach 1673

Tabelle I.5. Mieter in den städtischen Huldigungslisten des Fürstentums Brandenburg-Ansbach 1686

Tabelle I.6. Mieter in den städtischen Huldigungslisten des Fürstentums Brandenburg-Ansbach 1695

Tabelle I.7. Mieter in den städtischen Huldigungslisten des Fürstentums Brandenburg-Ansbach 1703

Tabelle I.8. Mieter in den städtischen Huldigungslisten des Fürstentums Brandenburg-Ansbach 1723 


\begin{tabular}{|c|c|c|c|c|c|c|c|c|c|c|c|c|}
\hline \multicolumn{3}{|c|}{ м хәџә!W \% } & 요 & ㅇㅇㅇ & & वे & & ○̊ & ๖े & & ㅇํㅇ & \\
\hline \multicolumn{3}{|c|}{ хәңә!以 \% } & ○̊ & 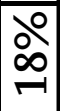 & & วิ & & $\begin{array}{l}\text { ก̊ } \\
\text { ஸे }\end{array}$ & ஓे & & 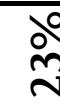 & \\
\hline \multicolumn{3}{|c|}{ [Чеzuళ גәғә!W } & $a$ & นn & & r & & $\hat{a}$ & $\approx$ & & $\stackrel{\infty}{+}$ & \\
\hline \multicolumn{3}{|c|}{ 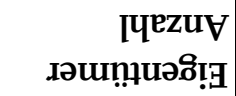 } & $\mid \begin{array}{l}0 \\
\text { m } \\
\end{array}$ & $\tilde{\sigma}$ & & 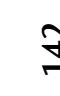 & st & $\frac{\infty}{m}$ & $\xi$ & & กิ & \\
\hline \multicolumn{3}{|c|}{ 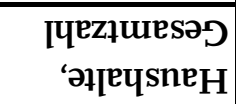 } & 蒚 & $\stackrel{\infty}{\sim}$ & ๖े & $\bar{\alpha}$ & - & $\frac{n}{7}$ & 8 & & 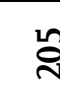 & \\
\hline \multicolumn{3}{|c|}{ рų̈sıәəт/po } & & & & & & & & & & \\
\hline$\frac{\tilde{d}}{\tilde{\Xi}}$ & 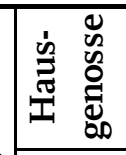 & $\frac{3}{\xi}$ & & -7 & & & & & & & & \\
\hline \multirow{6}{*}{ : } & \multirow{2}{*}{ 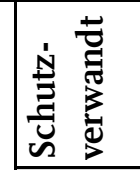 } & 3 & & & & & & & & & & \\
\hline & & $\xi$ & & & & & & & & & & \\
\hline & 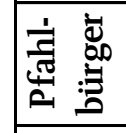 & $\Xi$ & $a$ & & & & & & & & & \\
\hline & 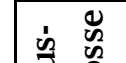 & 3 & & 0 & & 0 & & & 0 & & 0 & \\
\hline & 胥 总 & $\Xi$ & & $\dot{m}$ & & $\tilde{n}$ & & a & ㅇ & & $\stackrel{\infty}{+}$ & \\
\hline & 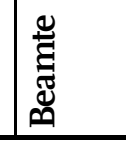 & $\Xi$ & & & & & & & & & & \\
\hline \multirow{2}{*}{ 离 } & \multirow{2}{*}{ 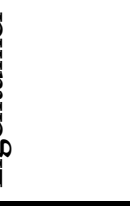 } & 3 & 인 & & $m$ & 0 & 0 & & $\stackrel{\operatorname{Ln}}{\sim}$ & $\nabla$ & $\wedge$ & 0 \\
\hline & & $\Xi$ & 古 & $\tilde{\sigma}$ & $\stackrel{\tilde{o}}{-1}$ & 8 & $\stackrel{\mathcal{F}}{+}$ & $\stackrel{\infty}{m}$ & $\underset{\exists}{ت}$ & $a$ & $\stackrel{\infty}{=}$ & $\tilde{m}$ \\
\hline $\begin{array}{l}z \\
\vdots \\
n\end{array}$ & & 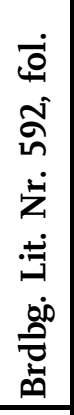 & 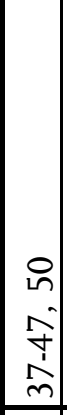 & $\begin{array}{l}a \\
\hat{\alpha} \\
\alpha \\
\end{array}$ & 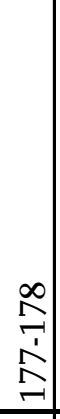 & 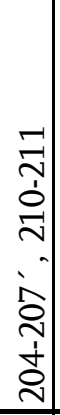 & 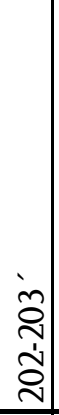 & $\tilde{\approx}$ & 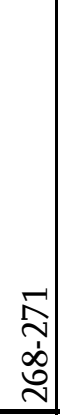 & & 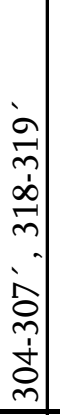 & \\
\hline 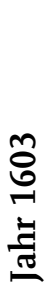 & & 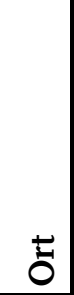 & 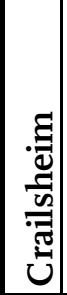 & 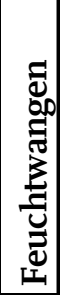 & 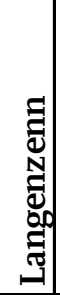 & 离 & 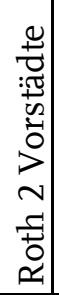 & 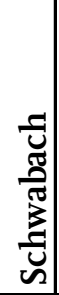 & 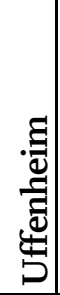 & $\begin{array}{l} \\
\overrightarrow{7} \\
\vec{\pi} \\
0 \\
0 \\
0 \\
>\end{array}$ & 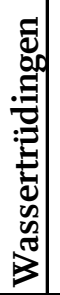 & 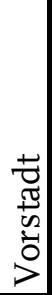 \\
\hline
\end{tabular}

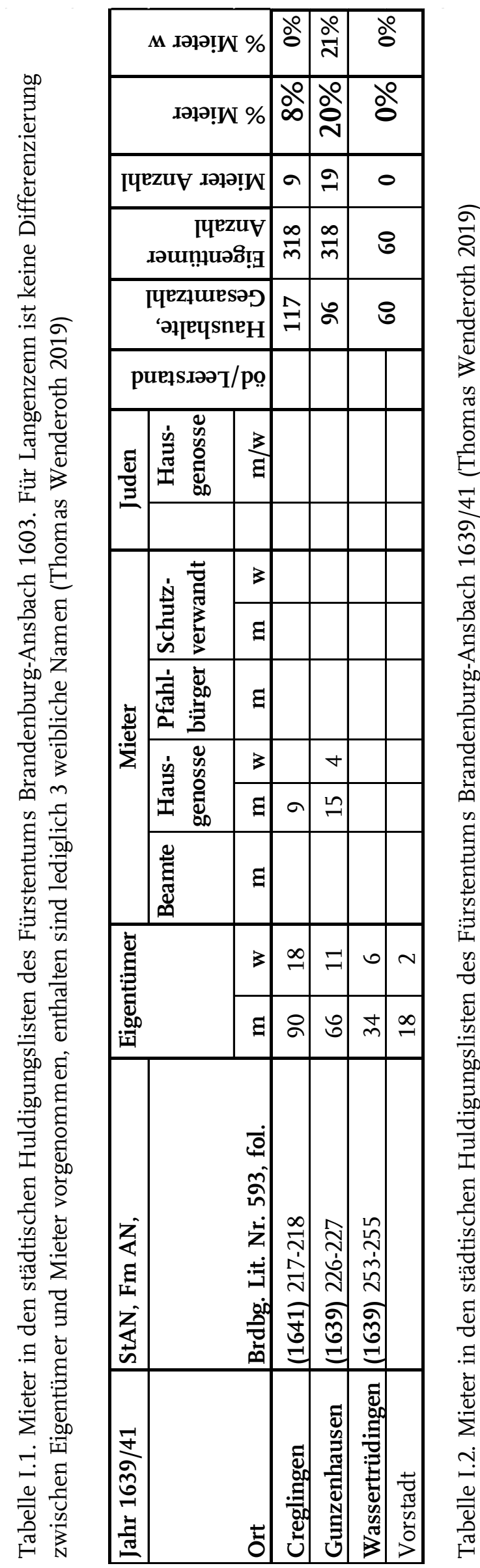




\begin{tabular}{|c|c|c|c|c|c|c|c|c|c|c|c|c|c|c|c|c|c|c|c|c|c|c|}
\hline \multicolumn{3}{|c|}{ м хәңә! К \% } & & ه̊ & ஃ̊ & $\frac{1}{7}$ & 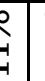 & 。̊ & g̊ & & $s^{\circ}$ & ठ̊ & ठ̊ & dे & غे & $\stackrel{0}{\circ}$ & @̊ & ذ్రి & 。ㅇ & 。ㅇ & ১̊ & : \\
\hline \multicolumn{3}{|c|}{ Іәџว!К \% } & & ֵొ & ㅇำ & ơ & $\frac{1}{-1}$ & ○े & 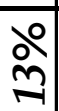 & & o & $\stackrel{2}{1}$ & ஹ & ơ & o & $\begin{array}{l}0 \\
f\end{array}$ & â & 인 & ํํ & ฉ̊ & ภ̊ & $\begin{array}{r}\infty \\
\infty \\
0 \\
0 \\
\overrightarrow{0}\end{array}$ \\
\hline \multicolumn{3}{|c|}{\begin{tabular}{|l|} 
IYEZü \\
دәңә!W \\
\end{tabular}} & & $\stackrel{\infty}{\sim}$ & $\stackrel{m}{7}$ & 今 & v & $\stackrel{n}{n}$ & $\tilde{\nu}$ & & C & 0 & $\stackrel{\sim}{\sim}$ & $\infty$ & r & $n$ & $\tilde{\sim}$ & $\widehat{\infty}$ & $m$ & $\infty$ & 6 & $\vec{E}$ \\
\hline \multicolumn{3}{|c|}{ 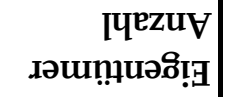 } & & 논 & $m$ & 5 & 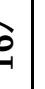 & $\tilde{\sigma}$ & $\overline{\mathrm{N}}$ & & น & aे & $\hat{\curvearrowright}$ & 2 & g & की & $\stackrel{\mathcal{N}}{ \pm}$ & กิ & $\stackrel{\widetilde{\vartheta}}{\rightarrow}$ & $\stackrel{m}{=}$ & $\infty$ & 离 \\
\hline \multicolumn{3}{|c|}{$\begin{array}{r}\text { Iчеz]üesəつ } \\
\text { 'әү[ечsnен }\end{array}$} & & $\stackrel{\infty}{\approx}$ & F & $\stackrel{\alpha}{\infty}$ & 6 & $\widehat{\widehat{N}}$ & $\stackrel{N}{N}$ & & 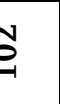 & $\infty$ & ำ & 崩 & $\hat{\imath}$ & v & $\hat{n}$ & $\stackrel{\infty}{m}$ & 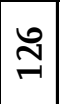 & L & $\mathscr{\sigma}$ & 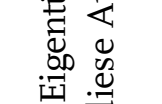 \\
\hline \multicolumn{3}{|c|}{ риетs.əәТ/pọ } & & & & $\sigma$ & 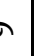 & กี & กิ & & & 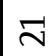 & m & & & & $\underset{\sim}{\infty}$ & $\overline{0}$ & & in & $\stackrel{-}{-}$ & 运 \\
\hline \multirow[t]{2}{*}{ 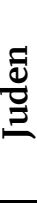 } & 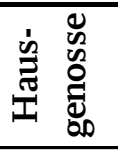 & $\frac{z}{z}$ & & & & $r$ & & & 0 & & & & & & & & & & & & & 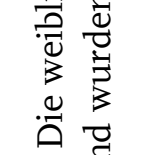 \\
\hline & & & $\approx$ & 0 & $\infty$ & ร & j & $\wedge$ & 0 & $\curvearrowright$ & & & $\infty$ & & & & & in & -1 & & & $\infty$ \\
\hline \multirow{6}{*}{ } & \multirow{2}{*}{ 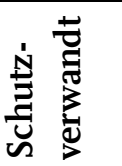 } & 3 & & & & $\sim$ & & 0 & 0 & & & & & & & & 0 & 0 & & & & \\
\hline & & $\Xi$ & & & & $\sigma$ & & $\sim$ & $\tilde{\nu}$ & & & & & & & & n & $\sim$ & & & & 岂. \\
\hline & 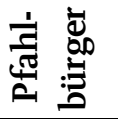 & $\Xi$ & & & & & & & & & & & & & & & & & & & & 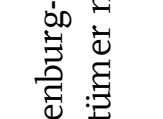 \\
\hline & $\dot{m}$ & 3 & 0 & & 0 & c & D & 0 & & 0 & & 0 & 0 & 0 & c & D & 0 & $\approx$ & 0 & 0 & 0 & \\
\hline & 茎 & $\xi$ & $\stackrel{\infty}{-}$ & & $m$ & $\alpha$ & 0 & $m$ & & $\wedge$ & & 6 & $\stackrel{\sim}{\sim}$ & $\alpha$ & $r$ & $n$ & $\infty$ & $\widetilde{\sigma}$ & $m$ & $\infty$ & ○ & \\
\hline & 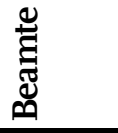 & $\Xi$ & & & & & & $\bar{\sim}$ & & & & & & & & & & & & & & 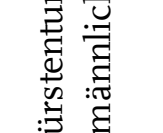 \\
\hline \multirow{2}{*}{ 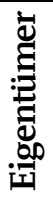 } & & 3 & $\approx$ & & t & 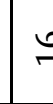 & to & 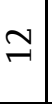 & & $\stackrel{ \pm}{二}$ & & 0 & $\stackrel{n}{n}$ & $a$ & c & b & ○ & $\stackrel{\vartheta}{\curvearrowright}$ & 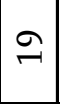 & 0 & 0 & $\begin{array}{l}1 \\
0 \\
0 \\
0 \\
0 \\
0\end{array}$ \\
\hline & & $\Xi$ & $\stackrel{ }{1}$ & $\stackrel{\tau}{\tau}$ & 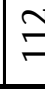 & & ? & 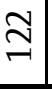 & $\stackrel{q}{ \pm}$ & $\stackrel{\infty}{+}$ & $\stackrel{2}{2}$ & $\mathfrak{N}$ & $\stackrel{\text { 을 }}{\rightleftharpoons}$ & 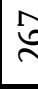 & g & לे & $\begin{array}{l}\infty \\
= \\
\end{array}$ & $\widehat{ત}$ & $\tilde{\theta}$ & $\pi$ & $\widetilde{\sigma}$ & \\
\hline 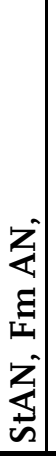 & & 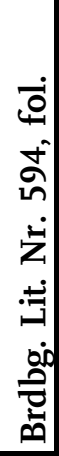 & 官 & & مُ & 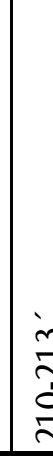 & 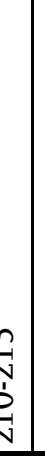 & 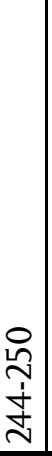 & 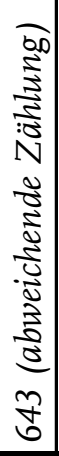 & $\begin{array}{l}n \\
\tilde{n} \\
\tilde{n} \\
\tilde{m}\end{array}$ & & $\begin{array}{l}\hat{m} \\
\tilde{d} \\
\infty \\
m \\
m\end{array}$ & $\begin{array}{l}\stackrel{0}{0} \\
\text { î̀ } \\
\text { లn }\end{array}$ & 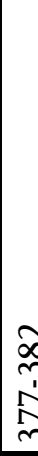 & 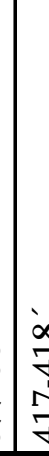 & & 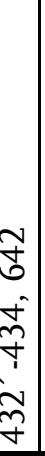 & 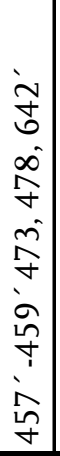 & 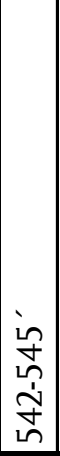 & 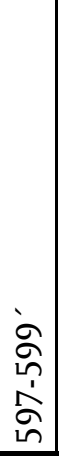 & 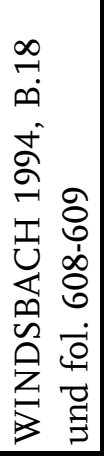 & 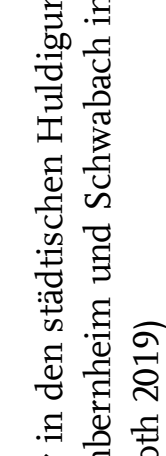 \\
\hline 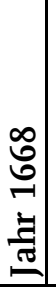 & & 5 & 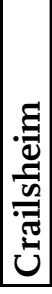 & 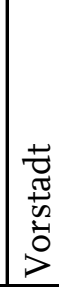 & Ũ & s & לָ. & 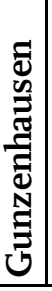 & 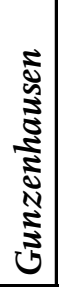 & 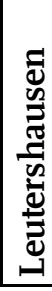 & $\begin{array}{l}\overrightarrow{7} \\
\tilde{\pi} \\
0 \\
0 \\
0 \\
0 \\
>\end{array}$ & 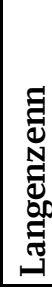 & 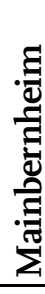 & $\frac{5}{8}$ & 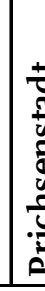 & 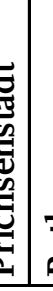 & 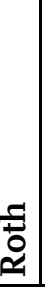 & 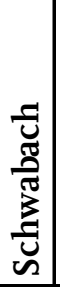 & 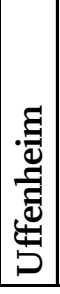 & 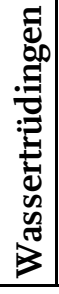 & 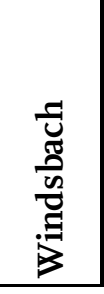 & 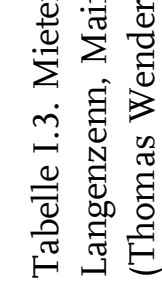 \\
\hline
\end{tabular}




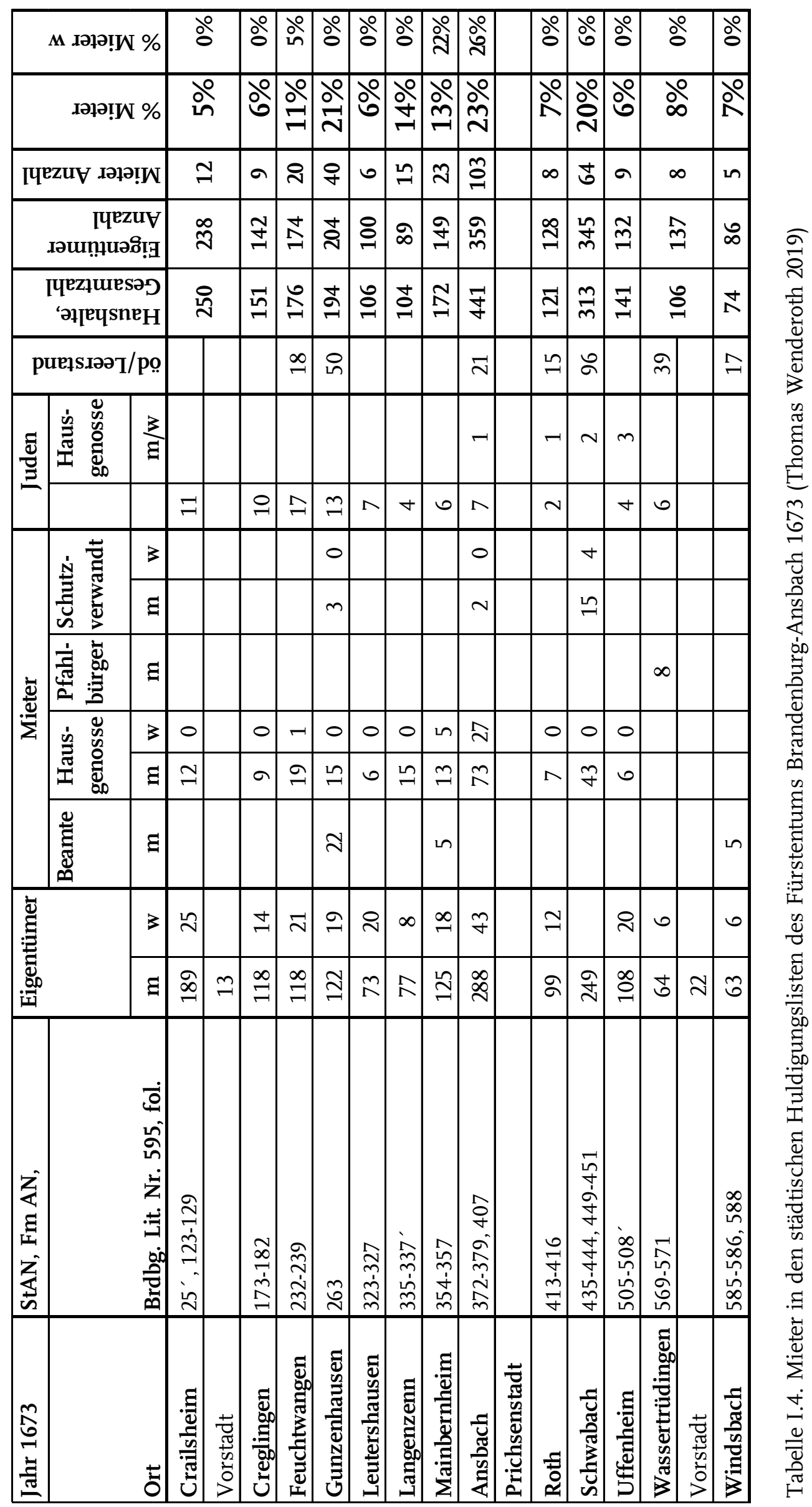




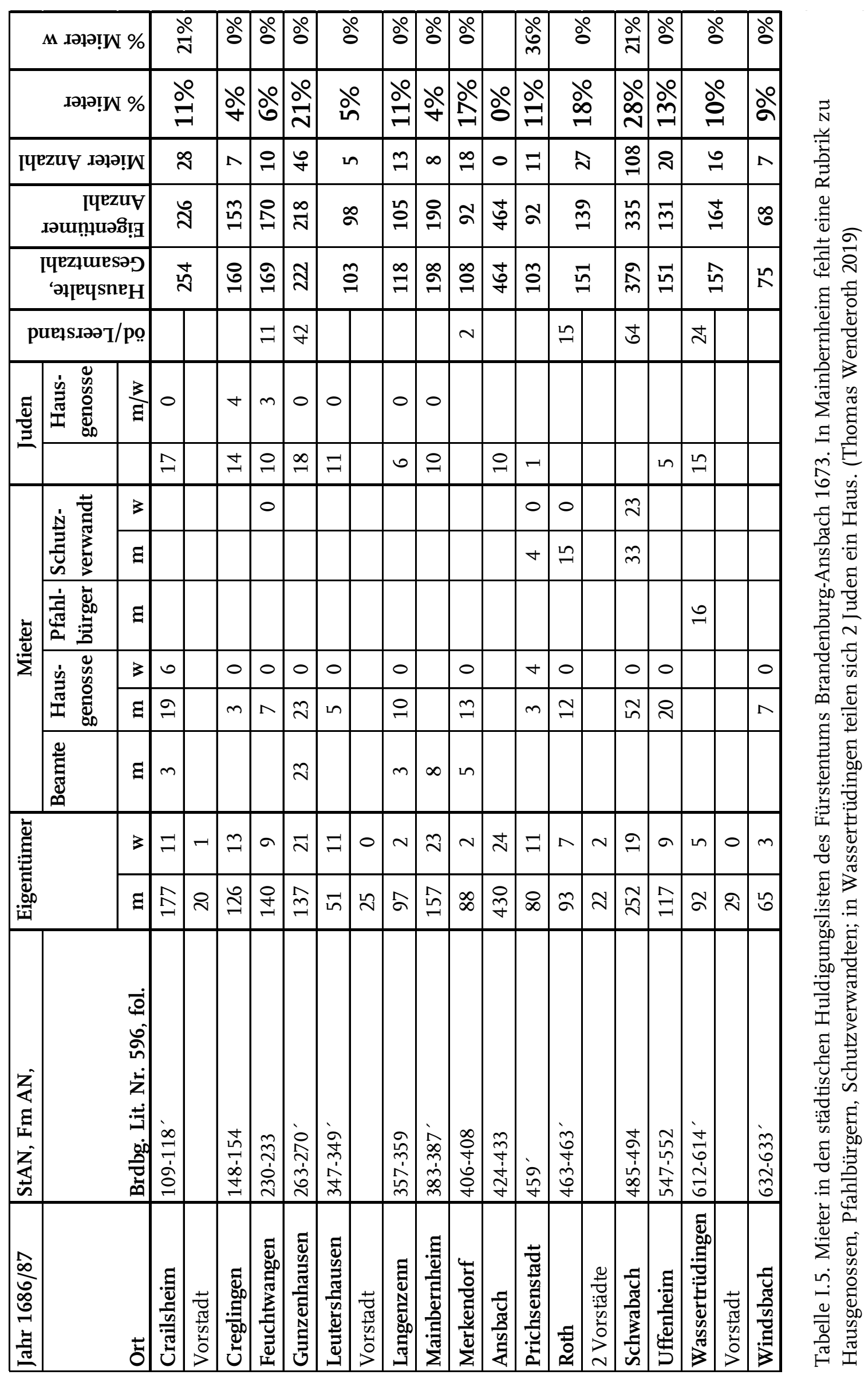




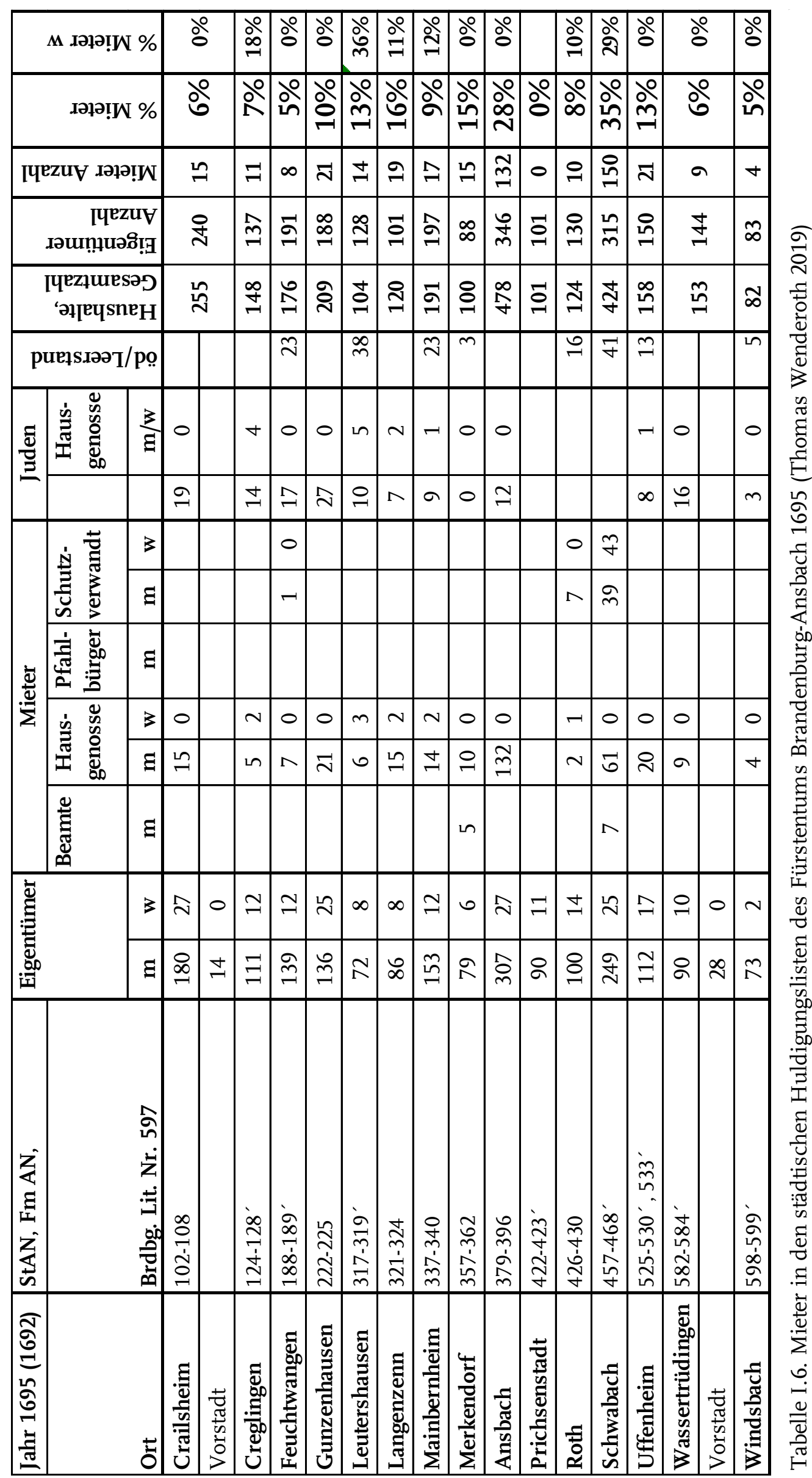




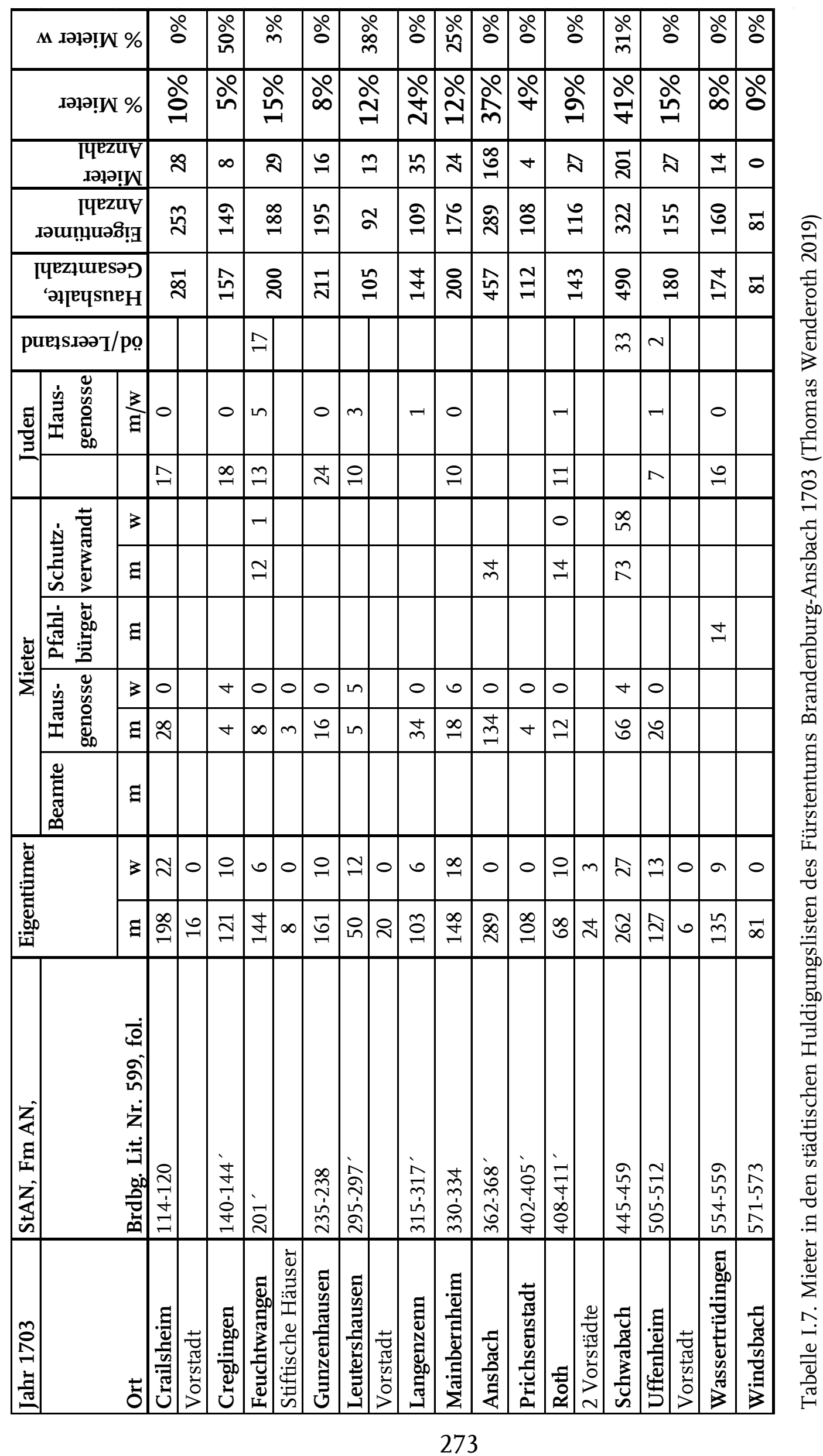




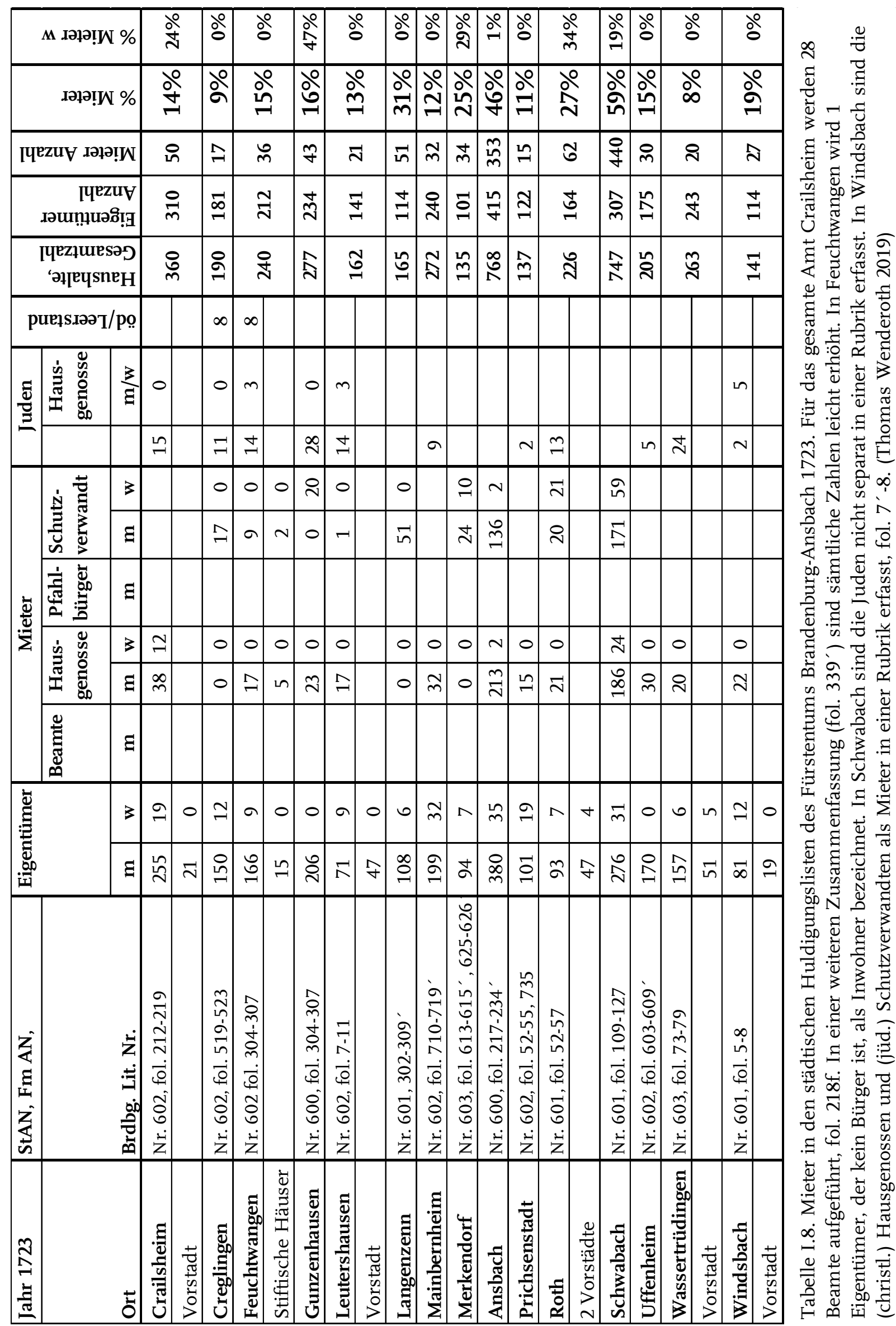




\section{Karte zu den fränkischen Städten}

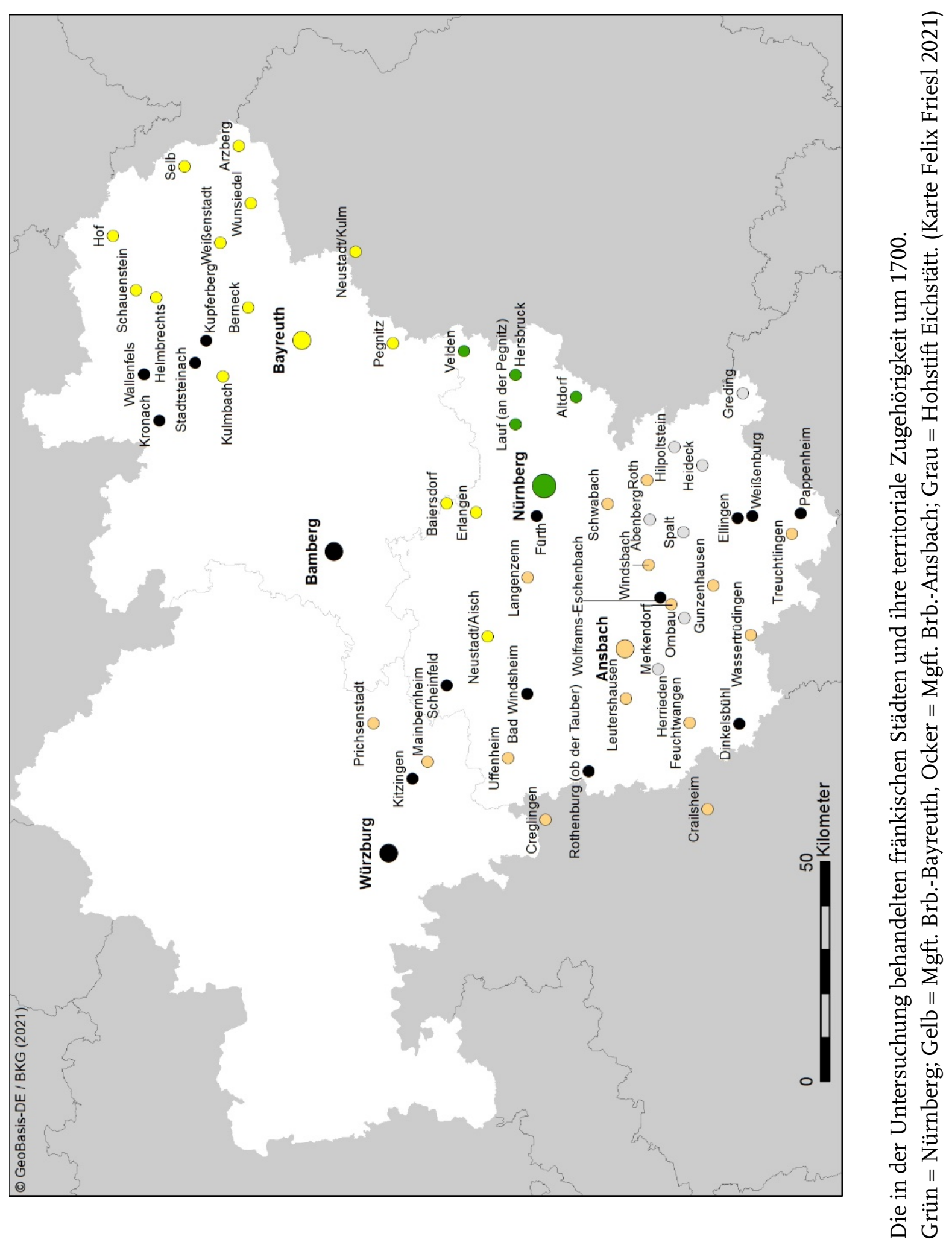




\section{University of Bamberg Press}

Die Bevölkerungs- und Stadtgeschichtsschreibung wird dominiert vom Blick auf die Eigentümer, eigentumslose Einwohner bleiben damit zumeist unsichtbar. Bei einer genauen Quellenanalyse lassen sich Mieter jedoch seit dem Mittelalter nachweisen, sie waren ein selbstverständlicher Teil der ländlichen und städtischen Bevölkerung. Anhand verschiedener Einwohnerverzeichnisse vom ausgehenden 15. bis zum beginnenden 19. Jahrhundert wird die Bevölkerungsentwicklung erstmalig aus der Perspektive der eigentumslosen Haushalte untersucht. Die Untersuchung erfolgt exemplarisch anhand von fränkischen Städten. Die politische Zersplitterung der Region erlaubt dabei den Vergleich von Städten unterschiedlicher Herrschaftsgebiete. Ergänzend dazu werden auch Städte und Regionen außerhalb Frankens mit in die Diskussion einbezogen.

Trotz einer disparaten Quellenlage lassen sich allgemeine Tendenzen nachweisen: Der Anteil der eigentumslosen Haushalte stieg seit dem Mittelalter stetig. Der Dreißigjährige Krieg führte zu einem deutlichen Rückgang der eigentumslosen Haushalte, ihr Anteil nahm erst ab dem späten 17. Jahrhundert wieder rasant zu. Die Größe einer Stadt war nicht allein maßgebend für den Mieteranteil. Neben der territorialen Zugehörigkeit erweisen sich städtische Sonderfunktionen, wie Residenz und Exportgewerbe, als wichtige Faktoren für den Anteil der Miethaushalte. Dieser lag bereits in der vorindustriellen Zeit nicht selten über 50\%. Unter den eigentumslosen Haushalten waren Kleinhaushalte und verwitwete Frauenhaushalte überdurchschnittlich stark vertreten.

Die Arbeit leistet einen wichtigen Beitrag für die Prosopographie und die Sozialgeschichte der Vormoderne. Sie stellt zudem Grunddaten für eine erweiterte Diskussion in der Architektur- und Stadtbaugeschichte zur Verfügung.
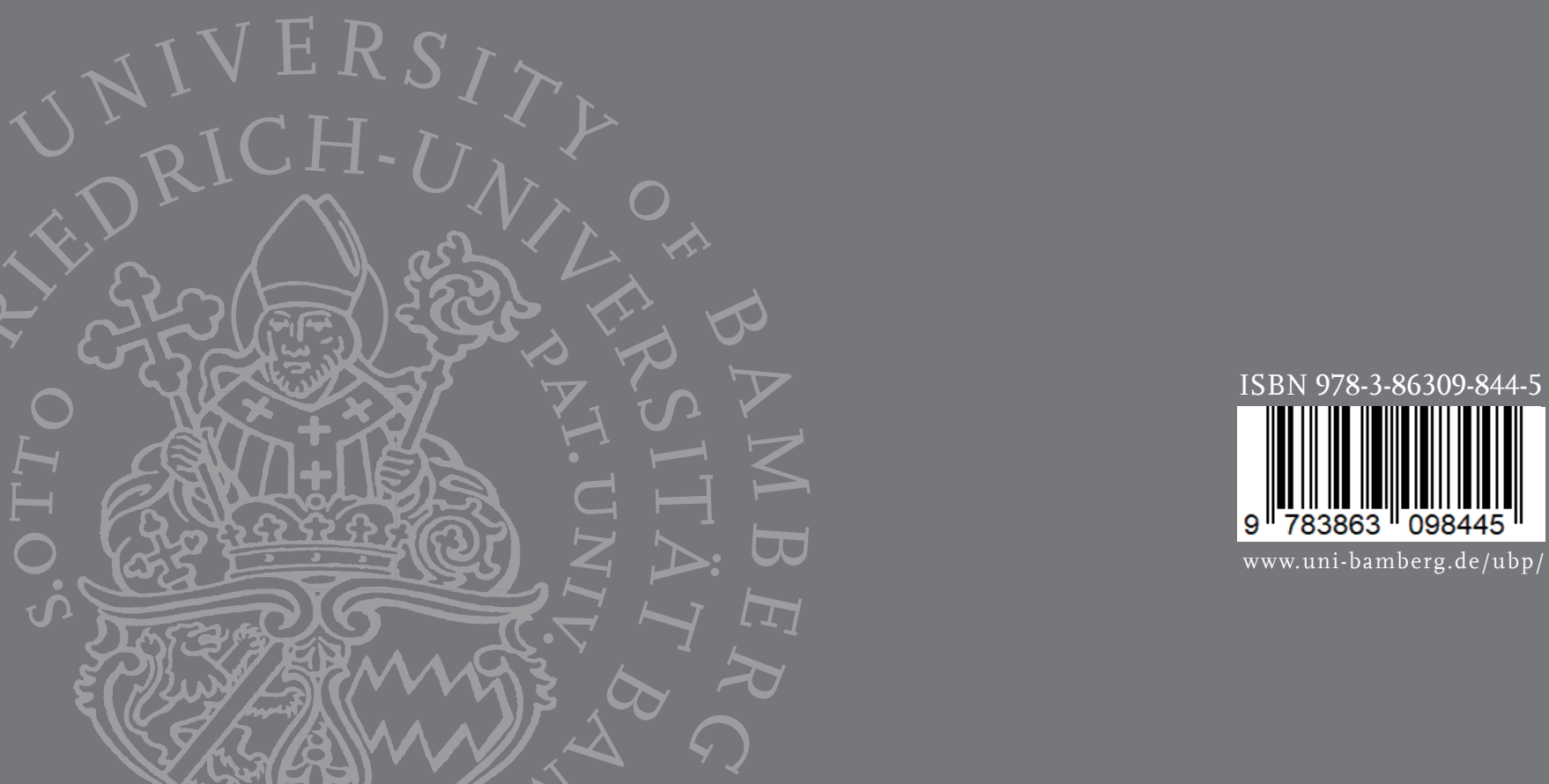